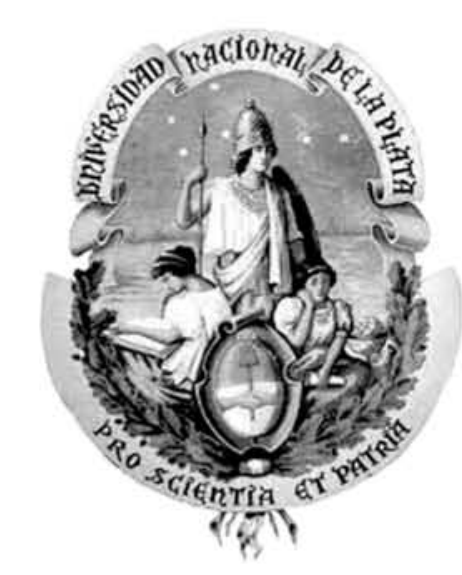

UNIVERSIDAD NACIONAL DE LA PLATA

Facultad de Ciencias Naturales y Museo

\title{
"Evaluación de los límites genéricos y evolución morfológica de las Calyceraceae sobre la base de un análisis filogenético combinado (molecular-morfológico)"
}

\author{
Lic. Lucio Martín Zavala Gallo \\ Director: Dr. Raúl E. Pozner \\ Directora: Dra. Silvia S. Denham \\ Instituto de Botánica Darwinion \\ Buenos Aires, 2013
}

Trabajo de Tesis Doctoral para optar al título de Doctor en Ciencias Naturales 


\section{AGRADECIMIENTOS}

Quiero expresar mi más profundo agradecimiento a mi querida familia, con todas sus luces y sombras, mi primera experiencia de mundo.

A mis directores de tesis Raúl Pozner y Silvia Denham, por haberme guiado tan pacientemente en el desarrollo de este trabajo, por darme la oportunidad de iniciarme en la investigación científica, por enseñarme todo lo referido a sistemática, taxonomía, filogenia y morfología, por sus consejos, paciencia y por todo el apoyo brindado durante estos años.

Al director del instituto Darwinion, Fernando Zuloaga, por haberme brindado desde el primer momento un lugar agradable de trabajo, facilitándome siempre todo lo necesario para llevar a cabo mi tesis.

A los becarios del Darwinion, los que generaron siempre un ambiente cordial para que mi estadía resulte agradable y enriquecedora.

Al personal técnico de biblioteca, Liliana Mallo, Horacio Illarraga y Erika Werner, el cual siempre se mostró muy atento y colaborador conmigo. También quisiera agradecerles por el apoyo afectivo que supieron darme, y hacerlo extensivo a sus familias.

A Francisco Rojas y Marcelo Moreno por su paciencia y su bello trabajo en dibujar las láminas para este trabajo.

A todo el personal de Instituto de Botánica Darwinion, que me brindó su ayuda incondicional durante todo este período y por los buenos momentos vividos.

A la Academia Nacional de Ciencias Exactas, Físicas y Naturales y al CONICET, por haberme brindado la posibilidad de llevar a cabo mi carrera de doctorado, financiando mi estadía en Buenos Aires y mis estudios con sus becas de postgrado.

A Aníbal Prina, Graciela Alfonso, Walter Muiño y el plantel de guardaparques de la Reserva Provincial "Laguna del Diamante", por haberme enriquecido con las experiencias y momentos compartidos en los dos viajes de colección que llevamos a cabo entre diciembre de 2009 y febrero de 2010.

A Leigh Johnson, por su calidez humana y por haber confiado en mí, dándome la posibilidad de recorrer juntos los ambientes de Chile central durante el viaje de 
colección que realizamos en diciembre de 2010, más allá de las diferencias culturales y el idioma.

A Sabina Donadío, Natalia Álvarez y Horacio Illarraga, por el viaje a San Juan, La Rioja y Catamarca; mi primer viaje de colección, que atesoro en la memoria por todos los buenos momentos que vivimos juntos.

A los curadores y las curadoras de los herbarios que gentilmente facilitaron el material de estudio necesario para realizar este trabajo y siempre me recibieron cordialmente.

A todas las personas que estuvieron en estrecho contacto con mi existencia en estos años y me ayudaron a crecer. Sin nombrarlas a todas, las saludo y las abrazo con el corazón. 


\section{RESUMEN}

La familia Calyceraceae es un pequeño grupo ca. 54 especies endémicas Sudamérica austral. La mayoría de ellas se distribuye a lo largo de la cordillera de los Andes y Patagonia a partir del sur de Perú, aunque algunas especies presentan distribución chaqueña, alcanzando la costa atlántica y algunos enclaves de las selvas paranaense y atlántica en Brasil. Las Calyceraceae están estrechamente relacionadas con las Asteraceae, una de las cinco familias más ricas en especies de Angiospermas y una de las familias botánicas con mayor importancia económica. El subclado Calyceraceae + Asteraceae comparte varias sinapomorfías: las flores dispuestas en inflorescencias capituliformes rodeadas por brácteas involucrales, las anteras connadas en un anillo que rodea el estilo, con dehiscencia introrsa y presentación secundaria de polen, el polen espinulado o liso con concavidades intercolpares, ovarios uniloculares con un único óvulo, y cipselas con un cáliz persistente modificado. Recientes análisis filogenéticos han demostrado que el subclado Calyceraceae + Asteraceae queda incluido en el clado MGCA (Menyanthaceae + Goodeniaceae + Calyceraceae + Asteraceae), un grupo monofilético con alto nivel de soporte. Las Calyceraceae se distinguen de las Asteracae principalmente por su óvulo péndulo (erecto en las Asteraceae), el estigma indiviso, punctiforme o capitado (dividido en dos ramas en las Asteraceae, que a veces no se separan simiulando un estigma indiviso), y la presencia de un tubo estaminal más o menos desarrollado (muy raro en las Asteraceae). Si bien las Calyceraceae se distinguen claramente como familia, no sucede lo mismo con sus géneros. Diversos problemas de interpretación de su morfología, más la variación morfológica de algunas de sus especies con amplia distribución geográfica, han desdibujado los límites entre los géneros (todos ellos basados en taxonomía alfa). Así, la familia Calyceraceae necesita una revisión de su morfología, de la taxonomía de sus especies y una reevaluación de sus géneros dentro de un marco filogenético, que permita proponer una clasificación natural y desarrollar hipótesis de evolución morfológica.

De este modo, se propuso realizar una revisión taxonómica detallada de todas las especies de esta familia, más un análisis filogenético exploratorio combinando caracteres morfológicos y moleculares. Para la revisión taxonómica detallada, se efectuó una recopilación y revisión exhaustiva del material bibliográfico relacionado con la diversidad y taxonomía de la familia (catálogos, floras, tratamientos taxonómicos 
parciales y protologos), se procedió a la búsqueda y reconocimiento de ejemplares tipo, se estudiaron plantas vivas en su hábitat; se examinaron especímenes de varios herbarios, se observaron caracteres vegetativos y reproductivos de los ejemplares de herbario y de plantas vivas en su hábitat de las especies conocidas de la familia Calyceraceae.

Como resultados de la revisión taxonómica, se reconocieron 47 especies distribuidas en los 6 géneros tradicionales. Cada especie se delimitó claramente, actualizando las listas sinonímicas, efectuando las lectotipificaciones necesarias y realizando descripciones ampliadas y detalladas para cada una de ellas. Además, se señalaron sus respectivas áreas de distribución geográfica con comentarios acerca de su hábitat y se presentaron discusiones acerca de los caracteres morfológicos entre especies similares y cómo diferenciarlas. Se publicaron dos especies nuevas para ciencia y una nueva combinación; se establecieron 25 nuevas sinonimias y se realizaron 21 lectotipificaciones y una neotipificación. Finalmente se confeccionó una clave artificial para identificar las 47 especies reconocidas de Calyceraceae.

Para el análisis filogenético exploratorio, se realizó un el análisis cladístico de las 47 especies de la familia Calyceraceae reconocidas en esta tesis (con algunas excepciones dependiendo de la disponibilidad de datos para unas pocas especies raras). La elección de los grupos externos se basó en la selección de taxones hermanos de Calyceraceae, pertenecientes al clado MGCA. Se identificaron 37 caracteres exomorfológicos variables entre las especies y se definieron sus estados homólogos. Para los datos moleculares se trabajó con material coleccionado en sílica gel o material de herbario de 35 taxones, representando todos los géneros de Calyceraceae. También se obtuvieron secuencias de Genbank. Se amplificaron, mediante la reacción en cadena de la polimerasa (PCR), secuencias nucleotídicas correspondientes a espaciadores intergenes del cloroplasto: trnS-trnG y trnH-psbA. El análisis cladístico se basó en el criterio de máxima parsimonia. Se realizaron análisis con datos morfológicos, moleculares y combinados. Para cada análisis se obtuvieron árboles de consenso estricto y de mayoría (50\%). Para evaluar la homoplasia de los caracteres en los árboles se obtuvieron los índices de consistencia y de retención. Para calcular el soporte de las ramas en los árboles óptimos se usó Jackknife Parsimony. Para calcular el soporte de las 
ramas en los árboles óptimos resultantes del análisis morfológico se usó Bremer support.

De acuerdo con los resultados del análisis filogenético, la estructura de la inflorescencia de tipo cefalodio se agrega a las sinpomorfías de ls Calyceraceae. De los seis géneros tradicionalmente aceptados, sólo Acicarpha es monofilético; Boopis, Calycera, Gamocarpha, Moschopis y Nastanthus son géneros polifiléticos. La mayoría de los caracteres morfológicos son altamente homoplásicos. Con excepción de la forma columnar del receptáculo y la presencia de flores estaminadas en el centro de la inflorescencia (sinapomorfías de Acicarpha), los caracteres utilizados en taxonomía clásica para delimitar los géneros de Calyceraceae no permiten delimitar grupos naturales. Sin embargo, surgieron algunos pocos nuevos caracteres morfológicos con valor taxonómico: la presencia de pelos (especialmente en el pedúnculo y el involucro), la ubicación de los nectarios, la presencia de una secreción glutinosa en los tallos y las hojas, y en menor medida la forma de la base de las anteras, la nervadura central de los sépalos. Otro caracteres con valor taxonómico en ls Asteraceae, como el ápice de las anteras, las brácteas del receptáculo, la proporción del hipanto y de la porción lobada de la corola (estos dos últimos como indicadores de la estructura general de la corola), resultaron altamente homoplásicos y no permitieron delimitar grupos naturales.

Se reconocen dos grandes grupos monofiléticos dentro de Calyceraceae, especies con glándulas nectaríferas estaminales (clado A) y especies con glándulas nectaríferas hipantiales (clado B). Dentro del clado A se reconocen dos grupos monofiléticos: el clado 1, integrado por el género Acicarpha junto con su especie hermana Boopis anthemoides; y el grupo de plantas formado por las especies "Glutinosas" del clado 2 junto con su especie hermana Moschopis leyboldi y las dos variedades de Boopis gracilis (en su mayoría de distribución austral). Dentro del clado B se reconocen tres grupos monofiléticos: el grupo de especies "Pubescentes", que incluye especies de los géneros Boopis y Calycera (clado 3); el clado 4, que incluye a Moschopis monocephala y algunas especies del género Calycera; y el clado 5, que incluye varias especies de los géneros Nastanthus, Boopis y Gamocarpha. 
Todos los clados del análisis combinado, inclusive los de bajo soporte, presentan coherencia en la distribución geográfica de sus especies. Los resultados del análisis filogenético sugieren que son necesarios cambios profundos en la taxonomía de la familia, para delimitar nuevamente y enmendar los géneros que la integran.

Palabras clave: Acicarpha, Boopis, Calycera, Calyceraceae, Filogenia, Gamocarpha, Moschopis, Nastanthus, Taxonomía.

\begin{abstract}
Calyceraceae is a small family with ca. 54 species from southern South America. Most of them grow in the Patagonian steppe and along the Andes up to southern Peru, but a few species grow in the "Chaqueña" Dominion, reaching the Atlantic sea shore, and some spots in "Paranense" and "Atlantica" rainforests from Brazil. Calyceraceae is closely realted to the Asteraceae, one of the five largest families of angiosperms, with well known economic importance. The synapomorphies of the subclade Calyceraceae + Asteraceae are: tiny flowers crowded in head-like inflorescences surrounded by involucre bracts, anthers connated into a ring that surrounds the style, introrse dehiscence and secondary pollen presentation, pollen spinulate or smooth with intercolpus cavities, 1-locular ovary with one ovule, and cypselae with a persistent, modified calyx.
\end{abstract}

Recent phylogenetic analysis have revealed that the subclade Calyceraceae + Asteraceae belongs to MGCA clade (Menyanthaceae + Goodeniaceae + Calyceraceae + Asteraceae), a monophyletic group with high level of support. Calyceraceae differ from the Asteraceae by the apical, descendent ovule (basal, ascendant in Asteraceae), punctiform or capitate stigma (split in two branches within Asteraceae, secondarily undivided when those branches do not diverge from each other), and a more or less developed staminal tube (very rare in Asteraceae). Although Calyceraceae is clearly distinct as a family, its genera are not. Interpretative problems in Calyceraceae morphology and structural variation in taxa with wide geographic distribution, have blurred boundaries among genera (all of them based on alpha taxonomy). That is why Calyceraceae needs a morphological and taxonomical revision of its species, and a 
reevaluation of its genera within a phylogenetic context, to suggest a natural classification of the species and develop hypotheses of morphological evolution.

This $\mathrm{PhD}$ thesis developed a detailed, extensive taxonomic revision of every species of Calyceraceae, plus an exploratory phylogenetic analysis combining molecular and morphological characters. The taxonomic revision included a complete revision of bibliographic material related to diversity and taxonomy of Calyceraceae (catalogues, floras, partial taxonomic studies and protologues), search and identification of type material, the study of herbarium collections and living plants in the field, observing vegetative and reproductive characters for every single species of this family. Every species was described in detail, including synonyms, geographic distribution, commentaries about habitat and key characters. This taxonomic revision concluded that Calyceraceae includes 47 (two of them new) clearly defined species primarily distributed among the six traditional genera, 25 new synonyms were proposed, 21 lectotypes and one neotypification were designated. In addition, a key to the 47 species of Calyceraceae produced.

The exploratory phylogenetic analysis included most of the 47 species, only a few ones were excluded because of the lack of material. Outgroup selection was based on the sister taxa according to the MGCA clade. Morphological evidence was organized in 37 external characters, defining the primary homology of their states. Molecular data were obtained from silica gel preserved material or herbarium vouchers of 35 taxa representing the six genera of Calyceraceae. Some sequences were downloaded from the Genbank. Nucleotidic sequences from trnS-trnG y trnH-psbA were amplified by PCR. The cladistic analysis was based on maximum parsimony, using morphological characters, molecular characters, and both combined. Strict concensus and mayority $(50 \%)$ trees were obtained for every analisis (morphological, molecular and combined). Consistency and retention index were used to evaluate honoplasy. Branch support in molecular trees was calculated with Jackknife Parsimony. Branch support in morphological trees was calculated with Bremer support.

According to the phylogenetic analysis, the general structure of the inflorescence (a cephaloid) is also a synapomorphy of the family Calyceraceae. Concerning genera, 
only Acicarpha is monophyletic; Boopis, Calycera, Gamocarpha, Moschopis and Nastanthus are polyphyletic. Most morphological characters are highly homoplasic. Except columnar receptacle and presence of central (distal) staminate flowers (both synapomorphies of Acicarpha) the remaining characters used in alpha taxonomy to define Calyceraceae genera, do not support natural groups. However, a few new morphological characters with taxonomic importance were revealed: presence of trichomes (particularly on the peduncle and involucres), presence of glutinose secretion in stems and leaves, shape of the anther base, and presence of a central vein in the sepals. Other characters with taxonomic value within Asteraceae (its closest sister group), like the anther apex (connective appendage), receptacle bracts (paleae), and general morphology of the corolla, are highly homoplasic within Calyceraceae, and dot support natural groups.

Two main natural groups can be distinguished within Calyceraceae: species with staminal nectaries (clade A) and species with hipanthial nectaries (clade B). Within clade $\mathbf{A}$ there are two monophyletic groups: clade 1 (Acicarpha and its sister species Boopis anthemoides) and clade 2 (the "Glutinose" species group plus their sister species Moschopis leyboldi and the varieties of Boopis gracilis, most of them with southern distribution). Within clade B, there are three monophyletic groups: the "Pubescent" species group or clade 3 (with species of Boopis and Calycera); clade 4 (with Moschopis monocephala and some spcies of Calycera), and clade 5 (with several species of Nastanthus, Boopis and Gamocarpha).

All clades in the combined analysis, even those with low support, have a coherent geographic distribution. The phylogenetic analysis of Calyceraceae suggests deep taxonomical changes to build natural genera.

Key words: Acicarpha, Boopis, Calycera, Calyceraceae, Phylogeny, Gamocarpha, Moschopis, Nastanthus, Taxonomy. 


\section{CONTENIDO}

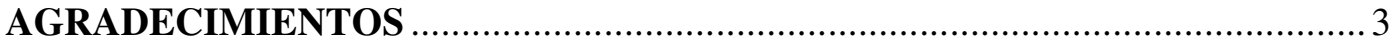

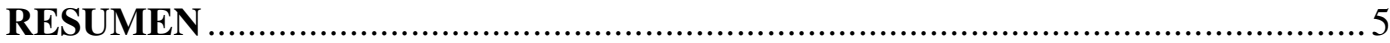

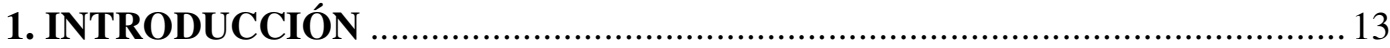

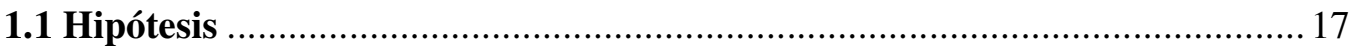

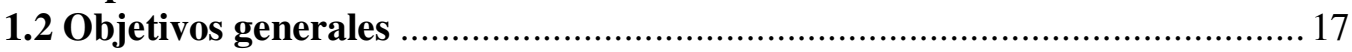

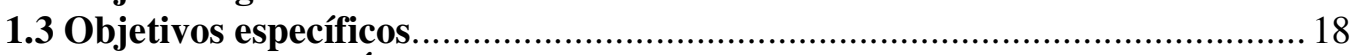

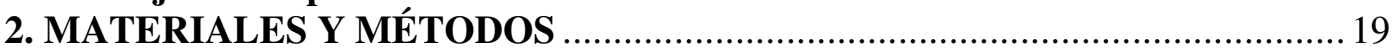

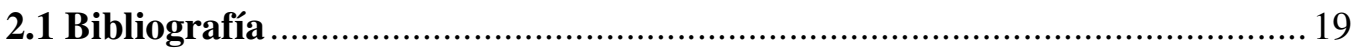

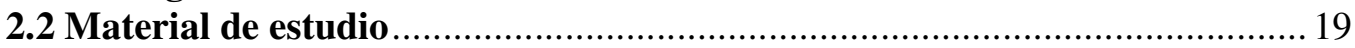

2.3 Estudios morfológicos ................................................................... 19

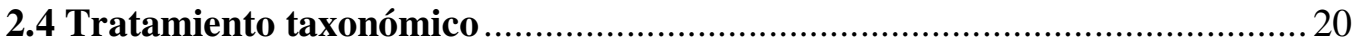

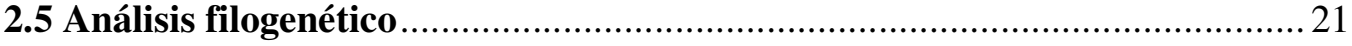

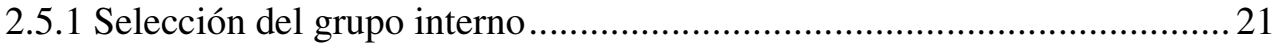

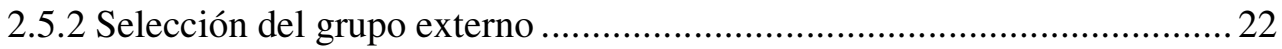

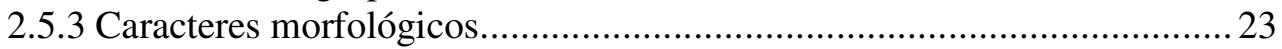

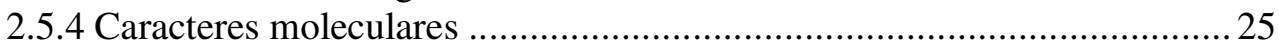

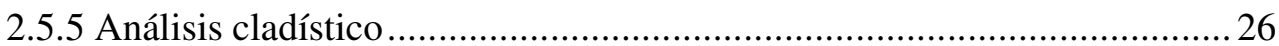

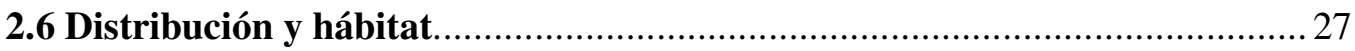

Apéndice 1. Ejemplares examinados.............................................................. 28

Apéndice 2. Matriz de los caracteres morfológicos utilizados. ................................ 70

Apéndice 3. Tabla de ejemplares utilizados para la obtención de caracteres

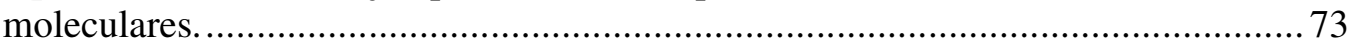

Apéndice 4. Protocolo de extracción..................................................................... 75

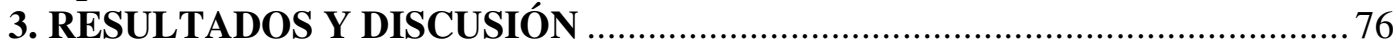

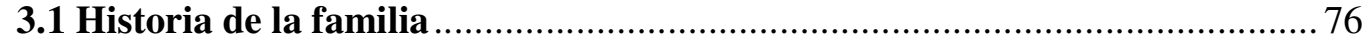

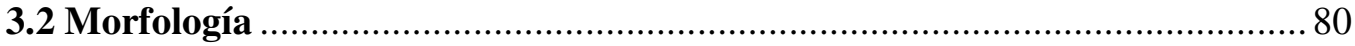

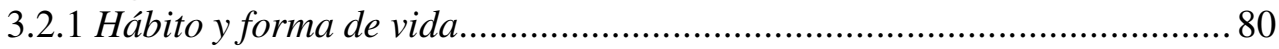

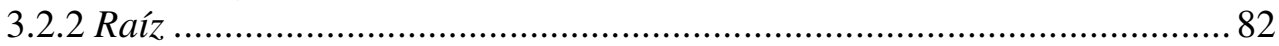

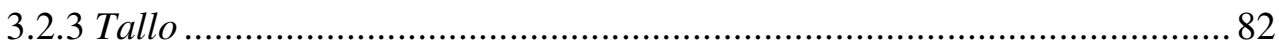

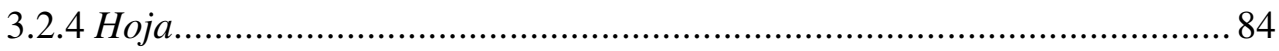

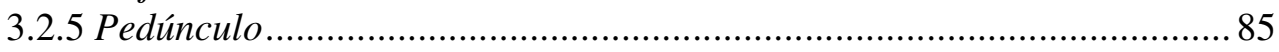

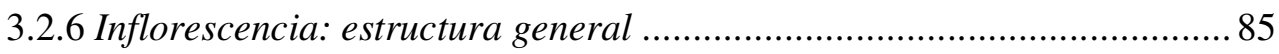

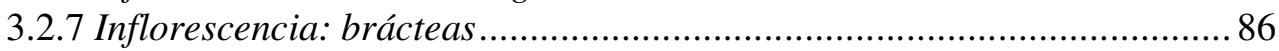

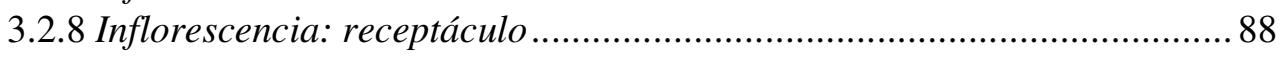

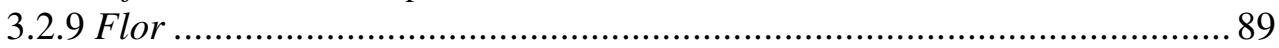

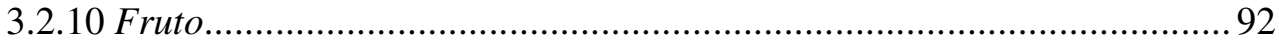

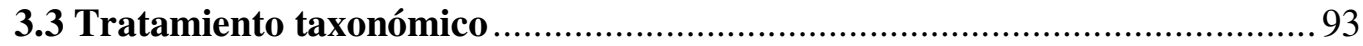

3.3.1 Clave artificial para las especies de la familia Calyceraceae .................. 93

3.3.2 Revisión nomeclatural de la familia Calyceraceae............................... 106

3.3.3 Resumen de la revisión nomeclatural de la familia Calyceraceae...........238

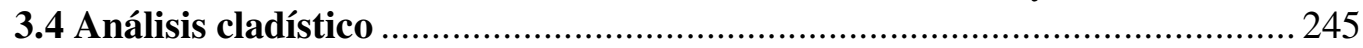

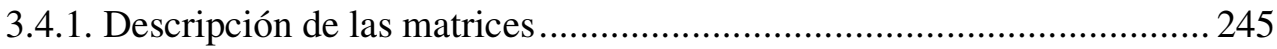

3.4.2. Descripción y discusión de los árboles obtenidos. Sinapomorfías y soporte

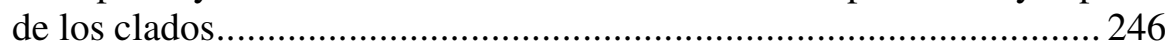

3.4.3. Discusión de los caracteres morfológico-taxonómicos usados en Calyceraceae para distinguir géneros............................................. 251

3.4.4. Descripción y discusión de otros caracteres morfológicos relevantes.... 257 
3.4.5. Discusión de las relaciones filogenéticas de los taxones no incluidos en el análisis combinado. 262

3.4.6. Apéndice 5. Optimización en uno de los AMP de los caracteres discutidos.

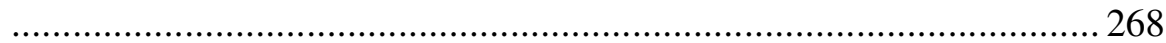

3.5 Distribución y Hábitat ................................................................................ 273

3.5.1 Comentarios acerca de la distribución geográfica de las especies de Calyceraceae ................................................................................... 273

3.5.2 Comentarios acerca de la distribución geográfica de los clados discutidos

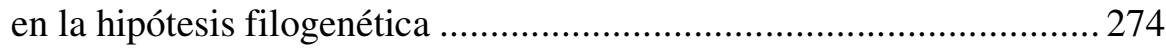

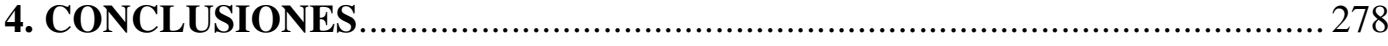

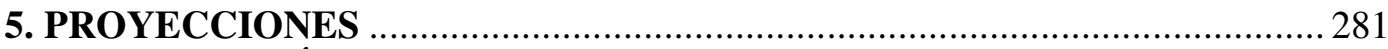

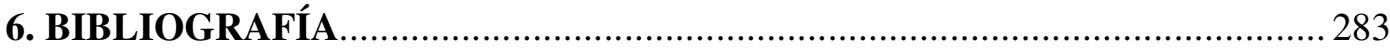




\section{INTRODUCCIÓN}

La familia Calyceraceae incluye tradicionalmente ca. 54 especies herbáceas de bajo porte, caulescentes o rosuladas, a veces subleñosas, anuales o perennes, endémicas de Sudamérica austral (sur de Brasil, sur de Perú, Bolivia, Paraguay, Uruguay, Chile y Argentina; DeVore, 1994; Chiapella, 1999a y b; Zanotti \& Pozner, 2008). La mayoría de las especies crecen en ambientes áridos y semiáridos de los Andes y la Patagonia, pocas especies habitan ambientes más húmedos (Chiapella, 1999a y b; Galvão Magenta \& Pirani, 2002; Zanotti \& Pozner, 2008). Alrededor del 90\% de las especies de Calyceraceae crecen a lo largo de los Andes de Argentina y Chile (Zanotti \& Pozner, 2008). Las Calyceraceae no se destacan por su frecuencia, abundancia o caracterización de comunidades vegetales. Tampoco son conocidas por su riqueza de especies ni por ser vistosas. Sin embargo son extremadamente valiosas por su relación filogenética con las Asteraceae, una de las cinco familias de Angiospermas más rica en especies y una de las más importantes para el hombre. En efecto, el grupo hermano de Asteraceae es la pequeña familia Calyceraceae (Lundberg, 2009). Entre las sinapomorfías del grupo Calyceracae + Asteraceae se cuentan las inflorescencias capituliformes rodeadas por brácteas involucrales, las anteras parcial o totalmente connadas, con dehiscencia introrsa y presentación secundaria de polen, el polen espinulado o liso con concavidades intercolpares y presencia de cemento polínico (Pollenkit), el ovario unilocular, uniovulado, y aquenios (cipselas) con un cáliz persistente modificado. Asimismo, una clara diferencia morfológica entre las dos familias es la orientación del óvulo en el ovario: mientras que en Asteraceae es erecto y basal, en Calyceraceae es péndulo (Lundberg, 2009). Los análisis filogenéticos de los últimos 20 años apoyan fuertemente la inclusión del subclado Calyceraceae + Asteraceae dentro del clado denominado MGCA (Gustafsson \& Bremer, 1995; Lundberg \& Bremer, 2003). El clado MGCA es un grupo monofilético bien soportado por caracteres moleculares y morfológicos, integrado por las familias Menyanthaceae, Goodeniaceae, Calyceraceae y Asteraceae (Lundberg \& Bremer, 2003), las cuales comparten varias sinapomorfías: la presencia de venas laterales en los pétalos, la ausencia de haustorios endospermáticos micropilares, óvulos con un tegumento grueso y multiestratificado (Inoue \& Tube, 1999); elementos de vaso con placas perforadas escalariformes, la ocurrencia frecuente de idioblastos 
esclerosados, polen binucleado, células del tapete multinucleadas, y la fusión (al menos parcial) de las venas laterales de los pétalos adyacentes (Lundberg, 2009).

La información más completa conocida hasta el momento sobre sistemática y taxonomía de la familia Calyceraceae fue publicada por Pontiroli (1963), Chiapella (1999a y b), Galvão Magenta \& Pirani (2002), Zanotti \& Pozner (2008, y la bibliografía allí citada). Según diferentes autores, se pueden distinguir seis o cuatro géneros de Calyceraceae. La propuesta de seis géneros (cf. Pontiroli, 1963; Chiapella, 1999a y b; Zanotti \& Pozner, 2008) distingue: Acicarpha Juss. (5 especies de Argentina, Bolivia, Brasil, Perú, Uruguay); Boopis Juss. (13 especies y 5 variedades de Brasil, Paraguay, Uruguay, Argentina y Chile); Calycera Cav. (14 especies, 3 variedades y 2 formas de Bolivia, Perú, Chile y Argentina); Gamocarpha DC. (6 especies y 2 variedades de Chile y Argentina); Moschopis Phil. (7 especies de Chile y Argentina) y Nastanthus Miers (9 especies y 4 variedades de Chile y Argentina). La propuesta de cuatro géneros varía según los autores: de acuerdo con Reitz (1988) deberían fusionarse Nastanthus y Gamocarpha bajo Boopis, mientras que Hellwig (2007) incluye Nastanthus y Moschopis dentro de un criterio amplio de Boopis.

Una de las principales dificultades a la que se han enfrentado los botánicos es la de definir con claridad los caracteres diagnósticos adecuados que permitan una delimitación clara de los géneros propuestos. La causa principal de los problemas en la delimitación de los géneros y las especies de Calyceraceae es la escasa información (muchas veces superficial) que se tiene de la morfología y el desarrollo de estas plantas. Muy poco se conoce sobre las formas de vida (Carlquist \& DeVore, 1998), la estructura floral (Erbar, 1993; Pesacreta et al., 1994; Gustafsson, 1995) y el polen (Belem, 1977; Skvarla et al., 1977; Avetisjan, 1980; Hansen, 1992) y del fruto (Reiche, 1900 [1901]; Muradian, 1991; Zanotti \& Pozner, 2007). La interpretación de la estructura de la inflorescencia ha sido uno de los aspectos más conflictivos de la morfología de esta familia (Reiche, 1900; Carolin, 1967; Troll, 1964; Hellwig, 2007) y sólo recientemente se ha revelado su estructura básica y sus posibles homologías con el capítulo de las Asteraceae (Pozner et al., 2012), pero aún es necesario evaluar su diversidad dentro de las Calyceraceae y su valor taxonómico a nivel de género. Los caracteres que han sido utilizados para delimitar los géneros de Calyceraceae son: la presencia de flores estaminadas en el centro (ápice) de las inflorescencias, la fusión al receptáculo del ovario de las flores periféricas (basales), la forma del receptáculo, la diferenciación del 
involucro, la fusión de las brácteas del receptáculo (páleas), la presencia de un cáliz fructífero espinescente y la presencia de alas o carenas en los frutos. Sin embargo, llama la atención que otros caracteres reproductivos, claramente visibles en las ilustraciones de los trabajos taxonómicos o florísticos (cf. Pontiroli, 1963; Chiapella, 1999 a, b), no se hayan considerado para la delimitación de los géneros (ni de las especies), tales como: la longitud del hipanto, la longitud del tubo y lóbulos corolinos, la longitud de los filamentos y del tubo estaminal, y la presencia y posición de las glándulas nectarígenas. Otras preguntas básicas de la morfología de estas plantas parecen no haber sido consideradas hasta ahora, como por ejemplo: ¿es posible que podamos observar en las Calyceraceae de manera incipiente algunos rasgos que se han desarrollado ampliamente en las Asteraceae (como el dimorfismo floral y las anteras sagitadas)?

La escasa información sobre la morfología de las Calyceraceae y la variabilidad en algunos de sus taxones con amplia distribución, se traduce en descripciones y claves poco precisas y ambiguas. Por esta razón, los ejemplares depositados en los herbarios carecen de valor de referencia a menos que hayan sido identificados por Pontiroli (1963), Chiapella (1999) o por los autores de las especies. La consecuencia inmediata de este problema, es la incertidumbre que surge de los resultados publicados en otros aspectos (anatomía, palinología, citogenética, etc.) de esta familia, pues es lógico dudar de la buena identificación del material estudiado. Se analizan a continuación los problemas de delimitación de cada uno de los géneros restantes.

Acicarpha se caracteriza por sus verdaderos capítulos (DeVore, 1994), con receptáculo alargado, flores periféricas perfectas y centrales estaminadas, y ovario de las flores periféricas parcialmente soldado al receptáculo, más evidente durante la maduración de los frutos (Pontiroli, 1963). Sin embargo A. tribuloides y A. procumbens poseen flor terminal (y por lo tanto cefalodios), y sépalos espiniformes en los frutos, como las especies de Calycera.

Calycera se reconoce por poseer frutos dimórficos sobre el mismo receptáculo: cipselas con sépalos poco acrescentes o vestigiales (inermes) y cipselas con sépalos desarrollados, espiniformes. Sin embargo, algunas especies de Calycera no tienen un dimorfismo neto sino una variación gradual desde flores y frutos con sépalos espiniformes hasta flores y frutos con sépalos escuamiformes, reducidos (p. ej. $C$. leucanthema). Dentro de las especies de Calycera con frutos netamente dimórficos, algunas poseen sépalos aciculares $(C$. herbacea $)$ y otras foliosos y pungentes $(C$. 
crassifolia). Además, las flores con sépalos espiniformes tienen diferente posición dentro de la inflorescencia según cada especie (Denham et al., enviado). Esta variabilidad en la forma de los sépalos espiniformes, en la ubicación de las flores y frutos con sépalos espiniformes dentro de la inflorescencia, más el hecho que especies de otros géneros también tienen sépalos espiniformes (p. ej. Boopis gracilis y $B$. castilloni) sugiere que el desarrollo de sépalos espiniformes ha ocurrido más de una vez durante la evolución de las especies de Calyceraceae, y que no es un buen carácter para delimitar un género, contrariamente a los sugerido por Hellwig (2007).

Nastanthus se define sobre la base de dos caracteres diagnósticos: presencia de inflorescencias agregadas en densas masas compactas y cipselas costadas. Sin embargo, estos caracteres no son exclusivos de este género, ya que la presencia de inflorescencias agregadas en densas masas compactas también se da en Gamocarpha selliana y en Boopis australis, y la presencia de cipselas costadas también puede verificarse en Calycera herbacea y Acicarpha obtusisepala. Además, no todas las especies del género poseen cipselas costadas, como es el caso de Nastanthus patagonicus donde las carenas son delgadas y rugosas, o N. compactus donde las carenas están reducidas a su mínima expresión.

El género Gamocarpha se define por la presencia de brácteas del receptáculo (= páleas) soldadas formando celdas. Sin embargo, se incluye en este género a G. dentata y G. angustifolia, que no poseen dicho carácter, pero se excluye a Boopis multicaulis a pesar de que presenta el mismo carácter diagnóstico que el resto de Gamocarpha.

Moschopis se define por la ausencia de un involucro diferenciado, que sólo puede verificarse sin dudas en tres especies: $M$. leyboldi, M. monocephala y $M$. trilobata. Sin embargo, este carácter no se aplica a la mayoría de las especies incluidas en el género: $M$. ameghinoi, $M$. caleofuensis, $M$. rosulata y $M$. subandina presentan involucros bien diferenciados. Del mismo modo, se excluyen de Moschopsis especies en las que la ausencia de un involucro diferenciado es evidente como Nastanthus caespitosus o Calycera pulvinata, que sólo forman involucros poco diferenciados en inflorescencias secundarias.

Finalmente, el género Boopis ha quedado más definido por la ausencia de los caracteres hallados en los otros géneros que por la presencia de caracteres propios. Así, las especies que no poseen dimorfismo de frutos con cipselas espinosas (propio de Calycera), que no poseen inflorescencias agregadas en densas masas compactas y 
cipselas costadas (propio de Nastanthus), que no poseen brácteas del receptáculo soldadas formando celdas (propio de Gamocarpha), que no poseen flores estaminadas y flores perfectas sobre el mismo receptáculo (propio de Acicarpha) y con un involucro bien diferenciado (típicamente ausente en Moschopsis), son incluidas dentro del género Boopis, grupo que incluye especies tan dispares como Boopis anthemoides y Boopis pterocalyx.

En resumen, la familia Calyceraceae necesita una revisión de su morfología, de la taxonomía de sus especies, y una reevaluación de sus géneros dentro de un marco filogenético, que permita proponer una clasificación natural, y desarrollar hipótesis de evolución morfológica, especialmente de aquellos caracteres con valor taxonómico y de otros valiosos para comprender el origen evolutivo de la morfología de las Asteraceae.

\subsection{Hipótesis}

1) Los géneros de Calyceraceae son para- o polifiléticos.

2) Los caracteres diagnósticos usados hasta ahora en taxonomía clásica para definir los géneros (estructura del fruto y la inflorescencia) son homoplásicos y no permiten definir grupos naturales.

3) Algunos caracteres florales no utilizados en la taxonomía tradicional de Calyceraceae y Asteraceae, como la posición de las glándulas nectarígenas, permiten definir distintos linajes dentro de las Calyceraceae.

4) Algunos caracteres florales con valor taxonómico dentro de las Asteraceae, como la forma de base y el ápice de las anteras, las brácteas del receptáculo y la morfología de la corola, también tienen valor taxonómico en las Calyceraceae.

\subsection{Objetivos generales}

1) Realizar una revisión taxonómica completa y exhaustiva de todos los taxones de Calyceraceae, junto con un análisis detallado de la morfología externa que permita precisar la comprensión y descripción de los caracteres utilizados en su taxonomía, y proponer nuevos caracteres aún no evaluados.

2) Desarrollar el primer análisis filogenético exploratorio de la familia Calyceraceae basado en caracteres morfológicos y moleculares para poner a prueba la monofilia de los géneros. Sin embargo, las combinaciones necesarias 
para establecer géneros naturales no son objetivos de esta tesis (véase capitulo 5. Proyecciones).

3) Analizar la evolución de los principales caracteres de la inflorescencia, de la flor y del fruto para evaluar los caracteres utilizados en taxonomía de esta familia, e identificar sinapomorfías morfológicas que puedan definir los principales grupos naturales.

\subsection{Objetivos específicos}

1) Establecer los terminales para el análisis filogenético, para lo cual se realizará una revisión de la nomenclatura y la delimitación morfológica de cada una de las especies de Calyceraceae; se revisarán las descripciones originales y los materiales tipo, y se reevaluarán los sinónimos.

2) Reevaluar los caracteres morfológicos utilizados en taxonomía e investigar nuevos caracteres con posible valor taxonómico, particularmente de la inflorescencia, la flor y el fruto.

3) Establecer homologías primarias para los caracteres morfológicos.

4) Desarrollar un análisis filogenético de Calyceraceae sobre la base de caracteres morfológicos.

5) Desarrollar un análisis filogenético de las Calyceraceae sobre la base de dos marcadores molecular plastidiales.

6) Realizar un análisis filogenético sobre la base de la evidencia parcial y total (morfológica y molecular).

7) Utilizar la filogenia para evaluar los límites de los géneros.

8) Analizar la optimización de los caracteres morfológicos en la filogenia para evaluar el valor taxonómico de los caracteres tradicionalmente utilizados en taxonomía de Calyceraceae (de la inflorescencia y el fruto), frente a los nuevos caracteres morfológicos utilizados (florales); y así proponer sinapomorfías morfológicas para definir los principales grupos de especies. 


\section{MATERIALES Y MÉTODOS}

\subsection{Bibliografía}

Se efectuó una recopilación y revisión exhaustiva del material bibliográfico relacionado con la diversidad y taxonomía de la familia (catálogos, floras, tratamientos taxonómicos parciales y protologos). Esta información posibilitó el desarrollo de los apartados 3.1 (Historia de la familia) y 3.3 (Tratamiento taxonómico).

\subsection{Material de estudio}

Se procedió a la búsqueda y el reconocimiento de ejemplares tipo por medio de visitas personales a herbarios, solicitudes de préstamo, fotografías, imágenes digitales y bases de datos en línea. Se confeccionó un banco de imágenes digitales con los ejemplares tipo y los protólogos correspondientes.

Se estudió un elevado número de ejemplares de herbario y de plantas vivas en su hábitat de todas las especies conocidas de la familia Calyceraceae. Se examinaron especímenes de los siguientes herbarios: BA, BAB, CONC, CORD, CTES, LIL, LP, MERL, SGO y SI (Thiers, 2013), con cuyos datos de colección y observaciones de identificación se confeccionó una base de datos de aproximadamente 1270 ejemplares. Se realizaron 6 viajes de colección en busca de material y para observar la variación fenotípica en el campo. Se coleccionaron muestras de herbario y semillas, se tomaron fotografías digitales de los individuos, se conservó material en sílica gel para estudios moleculares y se conservó material en FAA para estudios morfológicos y futuros estudios anatómicos.

\subsection{Estudios morfológicos}

Se observaron caracteres vegetativos y reproductivos de material de herbario, fijado y fresco, a ojo desnudo y con lupa WILD Heerbrugg Switzerland M5-85943, y de plantas vivas en su hábitat de todas las especies conocidas de la familia Calyceraceae excepto Boopis jürgensii y Nastanthus falklandicus. 
Los caracteres y medidas florales se obtuvieron mediante diafanización del material de herbario y del conservado en FAA. El proceso de diafanización se realizó calentando las flores en baño térmico a $60^{\circ} \mathrm{C}$ durante 3 a 5 minutos y luego sumergiéndolas en solución de $\mathrm{NaOH} 5 \%$ a temperatura ambiente durante 2 a 10 minutos, dependiendo de la especie y el estado de conservación del material. A continuación, se procedió a la disección de las flores. Las mismas fueron extendidas sobre portaobjetos, hidratadas con solución de glicerol $50 \%$ y teñidas con Fucsina Básica. Una vez añadido el colorante, las muestras se dejaron reposar durante 30 minutos. Las muestras de flores se montaron en preparados semipermanentes, usando alcohol polivinílico como medio de montaje. Se obtuvieron imágenes de los preparados usando una lupa estereoscópica Nikon SMZ 800 y cámara fotográfica Nikon con sistema NIS elements del Laboratorio de Anatomía y Desarrollo del Instituto Darwinion (LAD-IBODA). La descripción de las estructuras florales se basó en la interpretación de Erbar (1993).

Para el análisis de la estructura de la inflorescencia se diseccionaron bajo lupa estereoscópica yemas rehidratadas o conservadas en FAA. La interpretación de la estructura de la inflorescencia se realizó sobre la base de Pozner et al. (2012).

Para el estudio de la exomorfología de los frutos se eligieron sólo frutos maduros con su semilla bien desarrollada, y se observaron secos con lupa estereoscópica.

\subsection{Tratamiento taxonómico}

Se siguió la metodología clásica para los tratamientos taxonómicos. Se citan las especies asignadas a los seis géneros tradicionales, porque uno de los propósitos principales de la revisión taxonómica de la familia fue establecer los terminales para el análisis filogenético exploratorio. Cada especie, independientemente del género al que sea asignada en trabajos futuros, queda aquí claramente delimitada, con una descripción ampliada y detallada. Se actualizan las listas sinonímicas, se efectúan lectotipificaciones, se presentan discusiones acerca de los caracteres morfológicos entre especies similares y cómo diferenciarlas y se confecciona una clave artificial para identificar todas las especies de la familia. Sin embargo, las combinaciones necesarias para establecer géneros naturales no son objetivos de esta tesis (véase capitulo 5: Proyecciones). 
Los géneros fueron descriptos teniendo en cuenta la variación morfológica de todas las especies que le fueron asignadas. Bajo cada género de describen las especies ordenadas alfabéticamente.

Las novedades nomenclaturales presentadas en este trabajo no están efectivamente publicadas de acuerdo al Artículo 30.8 del Código Internacional de Nomenclatura (McNeill et al., 2012). Algunas de las novedades que surgieron de este estudio fueron efectivamente publicadas en Zavala-Gallo et al. $(2010 ; 2011)$ y otras están siendo preparadas para su publicación.

Se seleccionaron lectotipos cuando fue posible estudiar detalladamente abundante material tipo. Cuando no se consideró apropiado seleccionar lectotipos, se citaron todos los sintipos o isosintipos hallados.

El material de herbario examinado se muestra en el Apéndice 1.

\subsection{Análisis filogenético}

\subsubsection{Selección del grupo interno}

Se incluyeron en el análisis las especies de la familia Calyceraceae consideradas taxones válidos de acuerdo a los resultados obtenidos en la revisión nomenclatural de esta tesis (capítulo 3). Sin embargo, para algunas especies no fue posible obtener datos moleculares y no fueron incluidas en el análisis molecular. Para los taxones sin secuencias se asignaron datos faltantes en la matriz de datos moleculares cuando sólo se obtuvo uno de los dos marcadores o en la matriz de datos combinados. En el análisis de datos morfológicos se consideraron todas las especies, excepto $N$. falklandicus y $B$. jürgensii, especies de las que no se obtuvo material.

El muestreo de los taxones se detalla en la siguiente tabla: 


\begin{tabular}{|l|c|c|c|}
\hline \multicolumn{1}{|c|}{ Género } & $\begin{array}{c}\text { Nro. de } \\
\text { especies } \\
\text { válidas }\end{array}$ & $\begin{array}{c}\text { Nro. de especies incluidas en el } \\
\text { análisis molecular y combinado } \\
\text { (trnS-trnG / trnH-psbA) }\end{array}$ & $\begin{array}{c}\text { Nro. de especies } \\
\text { incluidas en el } \\
\text { análisis morfológico }\end{array}$ \\
\hline Acicarpha & 4 & $3 / 3$ & 4 \\
\hline Boopis & 16 & $9 / 9$ & 15 \\
\hline Calycera & 9 & $7 / 8$ & 9 \\
\hline Gamocarpha & 5 & $4 / 4$ & 7 \\
\hline Moschopis & 7 & $5 / 5$ & 5 \\
\hline Nastanthus & 6 & $3 / 3$ & 5 \\
\hline
\end{tabular}

\subsubsection{Selección del grupo externo}

Debido a que la monofilia de la familia Calyceraceae ya ha sido corroborada en análisis filogenéticos previos (Gustafsson \& Bremer, 1995; Hansen, 1997; Lundberg \& Bremer, 2003), y no es este un objetivo del presente trabajo, la elección de los grupos externos se basó en la selección de taxones hermanos de Calyceraceae, pertenecientes al clado MGCA. De este modo se incluyeron representantes de las siguientes familias:

Menyanthaceae: se estableció un taxón hipotético con los caracteres morfológicos de la familia y fue designado como raíz, de acuerdo con Lundberg \& Bremer (2003). No se hallaron datos de secuencias de los marcadores de cloroplasto usados en este estudio para Menyanthaceae.

Goodenciaceae: se estableció un taxón hipotético con los caracteres morfológicos de la familia. No se hallaron datos de secuencias de los marcadores de cloroplasto usados en este estudio para Goodeniaceae.

Asteraceae: las especies incorporadas al análisis como representantes de la familia fueron: Barnadesia lehmani Hieron., Fulcaldea laurifolia (Bonpl.) Poir. y Dasyphyllum brevispinum Sagást. \& M. O. Dillon, porque se contaba con las secuencias de marcadores de cloroplasto de trabajos previos de filogenia (Pozner et al., 2012). Las secuencias fueron obtenidas de GenBank (véase Apéndice 2.1). Se incorporaron los datos correspondientes a la matriz morfológica. 


\subsubsection{Caracteres morfológicos}

A partir de la revisión del material de herbario detallado más arriba, se determinaron los caracteres morfológicos variables entre las especies y sus estados homólogos. Los caracteres vegetativos fueron explorados pero no fueron utilizados para la filogenia porque no fue posible codificarlos con precisión, ni encontrar hipótesis de homologías primarias. En efecto, en algunos casos se halló que los caracteres vegetativos varían en gran medida de acuerdo a las condiciones ambientales en que se desarrolla cada población y se obtenían estados polimórficos para la mayoría de las especies; en otros casos se observaron transiciones continuas entre un estado de carácter y otro, no pudiendo establecer estados definidos para su codificación para el análisis filogenético (véase apartado 3.2). Sin embargo, el carácter relacionado a la presencia de exudados glutinosos se pudo codificar para cada taxón de manera precisa. En consecuencia, en la matriz de caracteres morfológicos, 36 de 37 caracteres corresponden a estructuras reproductivas.

Los caracteres que permanecen fijos en Calyceraceae pero varían en el clado MGCA, tales como: anteras connadas, placentación de los óvulos, posición del ovario, tipo de fruto, número de semillas, entre otros, no se incorporaron en la matriz de caracteres morfológicos, ya que no es el objetivo del presente trabajo poner a prueba la monofilia de Calyceraceae.

No se codificaron autapomorfías, solo se usaron caracteres informativos. Se asignó dato faltante cuando no se observó el carácter morfológico o éste era no codificable o aplicable para algún taxón.

La matriz morfológica (Apéndice 2) comprendió 54 taxones y 37 caracteres morfológicos: 1 vegetativo, 19 florales, 12 de las inflorescencias y 5 del fruto:

\section{Caracteres vegetativos}

1. Hojas glutinosas: ausentes (0); presentes (1)

\section{Caracteres florales}

2. Ápice de las anteras: no apiculado (0); apiculado (1)

3. Forma de la base de las anteras: obtusa (0); sagitada (1)

4. Caudículas en las anteras: ausentes (0); presentes (1) 
5. Nectarios estaminales: ausentes (0); presentes (1)

6. Nectarios hipantiales: ausentes (0); presentes (1)

7. Tubo estaminal: ausente (0); presente (1)

8. Longitud del tubo estaminal: reducido (0); desarrollado (1)

9. Filamentos estaminales: ausentes (0); presentes (1)

10. Longitud de los filamentos estaminales: reducidos (0); desarrollados (1)

11. Forma de los lóbulos corolinos: planos (0); cuculados (1)

12. Forma del ápice de los lóbulos corolinos: recto (0); uncinado (1)

13. Ángulo del ápice de los lóbulos corolinos: agudo (0); obtuso (1); escotado (2)

14. Nervadura central de los sépalos: ausente (0); presente (1)

15. Posición del ovario de las flores perfectas: externo y libre sobre el receptáculo (0); hundido y fusionado en el receptáculo (1)

16. Hipanto: ausente (0); presente (1)

17. Proporción del hipanto: subnulo (0); de igual longitud que la corola (1); más largo que la corola, 1.5 veces o más (2)

18. Proporción de la porción lobada de la corola: fida (0); partida (1); secta (2)

19. Flores estaminadas en el centro de la inflorescencia: ausentes (0); presentes (1)

20. $\mathrm{N}^{\mathrm{o}}$ de piezas por ciclo floral: flores pentámeras (0); flores tetrámeras (1)

\section{Caracteres de la inflorescencia}

21. Pubescencia en el pedúnculo: ausente (0); presente (1)

22. Brácteas del receptáculo: ausentes (0); presentes (1)

23. Estado de las brácteas del receptáculo: libres en la base (0); soldadas por uno de sus bordes (1); soldadas delimitando celdas (2)

24. Forma del receptáculo florífero: punctiforme (0); globoso (1); columnar (2)

25. Forma del receptáculo fructífero: punctiforme (0); globoso (1); columnar (2); discoideo (3)

26. Flores terminales: ausentes (0); presentes (1)

27. Flores solitarias: ausente (0); presente (1)

28. Grupos cimosos internos al involucro: ausentes (0); presentes (1)

29. Grupos cimosos externos al involucro: ausentes (0); presentes (1)

30. Diferenciación del involucro: ausente (0); presente (1) 
31. Involucelos en los grupos cimosos externos al involucro: ausentes (0); presentes (1)

32. Brácteas del involucro: libres (0); soldadas (1). [Este carácter también puede interpretarse como porción fusionada del involucro ausente (0); presente (1). Véase el punto 3.2.7, pág. 81].

\section{Caracteres del fruto}

33. Cáliz fructífero espinoso: ausente (0); presente (1)

34. Fruto compuesto (infrutescencia como unidad de dispersión): ausente (0); presente (1)

35. Polimorfismo de frutos (frutos multiformes sobre el mismo receptáculo): ausente (0); presente (1)

36. Pared del fruto: lisa (0); carenada (1)

37. Cipselas periféricas fusionadas al receptáculo: ausente (0); presente (1)

\subsubsection{Caracteres moleculares}

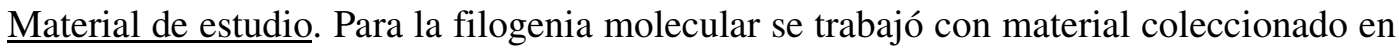
sílica gel o material de herbario de 35 taxones (Apéndice 3), representando todos los géneros de Calyceraceae. También se obtuvieron secuencias de Genbank, particularmente para el grupo externo.

Extracción de ADN y amplificación y secuenciación de marcadores. Para material conservado en sílica gel se realizó la extracción de ADN utilizando el buffer CTAB según el método de Doyle \& Doyle (1987, modificado por Silvana Sede y Jorge Hurst, véase Apéndice 4). Para material de herbario, se siguió el Protocolo de DNeasy Quiagen. Se amplificaron, mediante la reacción en cadena de la polimerasa (PCR), secuencias nucleotídicas correspondientes a espaciadores intergenes del cloroplasto: trnS-trnG y trnH-psbA. Se utilizaron los primers: trnH ${ }^{\text {GUG }}$ (CGC GCA TGG TGG ATT CAC AAT CC) (Tate \& Simpson, 2003), psbA (GTT ATG CAT GAA CGT AAT GCT C) (Sang et al., 1997), $\operatorname{trnS}^{\text {GCU }}$ (AGA TAG GGA TTC GAA CCC TCG GT) (Shaw et al., 2005) y 5'trnG2S (TTT TAC CAC TAA ACT ATA CCC GC) (Shaw et al., 2005). Estos marcadores han sido seleccionados por presentar suficiente variación e 
información sobre la base de una exploración previa de marcadores en 14 especies de Calyceraceae.

Para la purificación del producto de PRC y secuenciación se solicitó utilizó el servicio de Macrogen Inc., Corea (www.macrogen.com). Se secuenciaron ambas cadenas del ADN.

El alineamiento de las secuencias se realizó mediante el programa MUSCLE (Edgar, 2004) y corregidos manualmente mediante el programa BioEdit (Hall, 1999). Este paso fundamental en la construcción de una filogenia no está estandarizado, en particular para secuencias de regiones no codificantes del genoma, por lo tanto, se examinaron distintos métodos de alineamiento e incorporación de "gaps" que necesariamente se introducen en secuencias de distinta longitud para establecer homologías. Se identificaron gaps no ambiguos e informativos siguiendo a Simmons \& Ochoterena (2000). Se trataron como datos faltantes en la matriz pero luego se codificaron como ausencia (0) y presencia (1).

Los datos obtenidos fueron reunidos en una matriz única para los dos marcadores plastidiales y la codificación de gaps.

\subsubsection{Análisis cladístico}

El análisis cladístico se basó en el criterio de máxima parsimonia (Farris, 1983) y se utilizó el programa TNT versión 1.1 (Goloboff et al., 2003) bajo pesos iguales de los caracteres. Debido al alto número de terminales se realizaron búsquedas heurísticas. Inicialmente se realizó una búsqueda tradicional para obtener árboles de Wagner con random seed $=0$ y 10000 réplicas como parámetros. Se utilizó TBR (tree bisection reconection) como algoritmo de swappeo, salvando 10 árboles por réplica. Con el total de árboles resultantes de la búsqueda tradicional, se efectuaron rearreglos usando nuevas tecnologías (sectorial searchs, tree fusing, ratchet, drift) para obtener árboles más cortos.

Se realizaron búsquedas de árboles más parsimoniosos (AMP) con tres matrices diferentes: 1) con datos morfológicos, 2) con datos moleculares (incluidos los gaps) y 3) con datos combinados morfológicos-moleculares. Para cada análisis se obtuvieron árboles de consenso estricto y de mayoría (50\%). Para evaluar la homoplasia de los caracteres en los árboles se obtuvieron los índices de consistencia (CI) y de retención 
(RI) (Farris, 1989). Para calcular el soporte de las ramas en los árboles óptimos se usó Jackknife Parsimony (J) (Farris et al., 1996), con 10000 réplicas, eliminando el 36\% de los datos cada vez. Para calcular el soporte de las ramas en los árboles óptimos resultantes del análisis morfológico se usó Bremer support (B) (Bremer, 1994) buscando árboles subóptimos hasta de 6 pasos más que los óptimos, donde colapsaba completamente el árbol consenso de los subóptimos.

\subsection{Distribución y hábitat}

Se realizaron seis viajes de colección abarcando gran parte del área de distribucón de las especies de la familia que permitieron corroborar y actualizar los datos de distribución de los taxones. Se realizaron los viajes de colección a: San Luis, San Juan, La Rioja, Catamarca y Córdoba, 3 al 18 de enero de 2009; Reserva Pcial. "Laguna del Diamante", pcia. de Mendoza, 4 al 12 de diciembre de 2009 y 1 al 6 de febrero de 2010; sur de la pcia. de Mendoza y pcia. de Neuquén, 19 de noviembre al 3 de diciembre de 2010; V, VII, VIII, IX Regiones y Región Metropolitana, Chile, 5 al 15 de diciembre de 2010; Patagonia andina, Neuquén - Santa Cruz, 2 al 17 de enero de 2011). Para completar los datos de distribución geográfica y hábitat de cada una de las especies se usaron las notas de etiqueta de los materiales de herbario.

Todas las áreas fitogeográficas mencionadas en esta tesis siguen el criterio de clasificación de Cabrera \& Willink (1980) y Cabrera (1994).

En el capítulo 3.5 se señala la distribución y hábitat de cada especie o variedad y se analiza también la correspondencia en la distribución geográfica de los clados obtenidos en el análisis filogenético. 
Apéndice 1. Ejemplares examinados

\section{Acicarpha obtusisepala}

URUGUAY. Rocha: Punta del Diablo. Yendo desde la playa de Punta del Diablo hacia Santa Teresa (hacia el norte), 17-I-2011, Zanotti 160 (SI).

\section{Acicarpha procumbens}

ARGENTINA. Buenos Aires. Pdo. Ensenada: Punta Lara, bosques salobres antes de llegar a los bosques cost., 9-XII-1962, Burkart 23915 (SI). Pdo. La Plata: La Plata, 19XII-1939, Burkart s.n. (SI 12673); La Plata- alrededores, lugar llamado "El Bañado", 6XI-1914, Ceppi 2 (SI); Tolosa, F.C.S., XI-1916, Scala s.n. (SI 12617). Pdo. San Fernando: San Fernando, 1-XI-1942, Burkart 12967 (SI); Bancalari, 17-XII-1931, Burkart 4320 (SI). Pdo. San Vicente: San Vicente F.C.S., 6-XI-1943, Burkart 13465 (SI). Pdo. Tigre: Los Troncos del Talar, 12-X-1954, Lanfranchi 1221 (SI). Corrientes. Depto. Paso de los Libres: Ea. "El Recreo", 21 km E de Bonpland, costa río Uruguay, A Bonpland, 19-XI-1973, Lourteig et al. 2799 (CONC, CTES). Entre Ríos. Sine loco accurat, sin fecha indicada, sin coleccionista indicado, Ex Herb. Min. Agr. 9772 (SI). Depto. Concepción del Uruguay: Río Gualeguaychú, 27-XII-1941, Nicora 3353 (SI); Estación Experimental Concepción del Uruguay, 9-XI-1965, Burkart 26065 (SI). Depto. Federación: Estancia "Buena Esperanza", 14-XI-1964, Pedersen 7140 (SI). Depto. Gualeguay: Gualeguay, La Verde, pajonales del R. Gualeguay, 21-X-1949, Burkart 18055 (SI). Depto. La Paz: distrito Banderas, Estancia La Esperanza, en campo salobre, 19-I-1960, Burkart \& Bacigalupo 21436 (SI).

\section{Acicarpha spathulata}

BRASIL. Paraná. Guaratuba in litora, 24-XII-1911, Dusén 13673 (SI). Municipio Paranagua: Matinhos, 20-IX-1946, Hatsbach 401 (LIL). Rio de Janeiro. Municipio Rio de Janeiro: Barra de Tijuca, 20-VII-1956, Castellanos 21694 (LIL). Rio Grande do Sul. Municipio Igrejinha: Igrejinha, 27-II-1928, Schreiter 29 (LIL). Santa Catarina. Municipio Florianopolis: Canasvieiras, Ilha de Santa Catharina, 7-III-1942, Rohr 42 (LIL); Ilha de Santa Catharina: Canasvieiras, 23-XI-1945, Rohr s.n. (LIL 159130); Florianopolis, VI-1938, Anchieta 3104 (LIL). Municipio Laguna: Ponta da Barra, Playa 
Gravatá, 14-I-2010, Zanotti \& Vitali 152 (SI). Sâo Paulo. Santos, litoral, 20-XI-1902, Puttemans s.n. (SI 126718).

\section{Acicarpha tribuloides}

ARGENTINA. Buenos Aires. Isla Martín García, 8-20-XII-1900, P.L. Spegazzini s.n. (Ex Herb. Min. Agr. 24051) (SI). Pdo. Campana: Reserva Natural Otamendi, 26-X2007, Morrone et al. 5808 (SI); Campana, 27-X-1935, Nicora 538 (SI). Pdo. Ensenada: Punta Lara, 17-X-1949, Crespo s.n. (SI 25374). Pdo. La Plata: Isla Santiago, 27-X1935, Cabrera 3373 (SI). Pdo. Quilmes: Quilmes, habita terrenos altos, arenosos, 10XI-1912, Rodríguez 54 (SI). Pdo. San Fernando: Pacheco, orilla río Las Conchas, 13XI-1952, Boelcke 6677 (SI). Pdo. San Isidro: Punta Chica, XI-1907, sin coleccionista indicado (SI 12625); San Isidro, X-1899, Bettfreund 80 (SI); San Isidro, barrancas, 18XII-1941, Mendoza s.n. (SI). Pdo. San Nicolás de los Arroyos: Delta del Paraná.- Islas de San Nicolás, 29-XII-1945, Burkart 15583 (SI). Pdo. San Pedro: San Pedro, 1-XII1939, Cabrera 5598 (SI). Pdo. Tigre: Tigre, 28-XI-1943, Lanfranchi 14 (SI); 9-XI1958, Taphanel 68 (SI). Pdo. Zárate: Islas Lechiguanas, 11-I-2008, Morrone et al. 5818 (SI). Chaco. Depto. 1 de Mayo: Colonia Benítez, IX-XI-1930, Schulz 128 (SI). Depto. Bermejo: Las Palmas, XI-1917, Jörgensen 2351 (SI). Depto. San Fernando: Fontana, Resistencia, sin fecha indicada, Meyer 115 (SI). Ciudad Autónoma de Buenos Aires: Barracas al Sud B. Aires, 12-III-1902, Venturi 47 (SI); Palermo, 27-II-1897, Hicken 1071 (SI 12620); Bosquecillo bajo Belgrano, 30-X-1924, Millán 145 (SI). Córdoba. Depto. Calamuchita: Sierra Chica (Falda O): La Cruz, rumbo a Amboy, 570 m, 25-XI1962, A.T. Hunziker \& A.E. Cocucci 16097 (SI); Valle de Los Reartes, margen derecha del río de Los Molinos, 7-IV-1917, Castellanos 179 (SI); Villa Alpina, 3158'08"S, 64²7'21"W, 11-X-2009, Larsen 69 (SI). Depto. Capital, Quebrada de las Rosas, camino a La Calera, márgenes del río Primero, 3-III-1954, Lanfranchi 1181 (SI). Depto. Colón: Ascochinga, 16-XII-1935, Giardelli 237 (SI), en la cascada, 13-XI-1936, Mendoza 1286 (SI). Depto. Punilla: San Esteban, 3-I-1938, Nicora 1548 (SI); Pampa de Achala, 15-I1940, Burkart 10450 (SI); Sierra de Achala: Cuesta del Tránsito, 6-13-I-1895, Kurtz 8385 (SI); Copina, 29-XII-1935, Burkart 7441 (SI). Depto. Río Primero: Río Primero, XI-1895, Bodenbender s.n. (SI 19946). Depto. San Alberto: Cercanías de Hotel "La Posta", 31³6'30,6"S, 6452'38,3"W, 2137 m, 2-XII-2006, Bonifacino et al. 2809 (SI). Corrientes. Depto. Capital: Campus Libertad de la Universidad Nacional del Nordeste, 
22-IX-2007, Zanotti 143 (SI). Depto. Goya: sin localidad indicada, 5-XI-1987, Cordo et al. 88-A-58 (SI). Depto. Mburucuyá: P.N. Mburucuyá, Potrero 1 grande, 2801'24"S, 5806'09"W, 18-X-2006, Arbo et al. 9359 (SI); Estancia Santa Teresa, 24/10/1976, Cabrera 28195 (SI). Entre Ríos. Depto. Diamante: Diamante, 15-XII-1960, Burkart 22318 (SI). Depto. Gualeguaychú: Carbó a Las Mercedes, 24-X-1971, Burkart 28929 (SI). Depto. La Paz: puerto Soto, sobre el Arroyo Aragua, Estancia La Invernada, 16-I1960, Burkart \& Bacigalupo 21435 (SI); R.P. 1, $15 \mathrm{~km}$ al NE de La Paz rumbo a Feliciano, 3043'S, 59³0'W, 31-X-1973, Boelcke et al. 5145 (SI); distrito Tacuaras, estancia Santa Cruz Cué, 7-XI-1965, Burkart 26066 (SI). Depto. Paraná: Crespo, 2-XI1970, Burkart \& Troncoso 28056 (SI). Formosa. Sin localidad indicada, X-1918, Jörgensen 3177 (SI). Jujuy. Depto. Dr. Manuel Belgrano: Camino de Reyes a lagunas de Yala, 16-XI-1988, Cabrera et al. 34548 (SI); Laguna de Yala, 16-I-1988, Zuloaga \& Deginani 3536 (SI); cerros de Yala, campos graminosos, 19-II-1940, Burkart \& Troncoso 11291 (SI). Depto. Ledesma: Parque Nacional Calilegua, 19-XI-1980, Cabrera 32118 (SI). Depto. Santa Bárbara: Subida al Centinela, 12-XII-1983, Rotman 955 (SI, LIL). Misiones. Depto. Candelaria: Santa Ana, 29-X-1909, Jörgensen 413 (SI); Bonpland, X-1910, Jörgensen 413 (SI); Depto. Capital: Posadas, 1912, Schrottky s.n. (SI 12627), verano 1907, Issouribehere s.n. (Ex Herb. Minist. Agr. 24134) (SI). Depto. Concepción: Ruta Provincial 2, cerca de Italcanaré, 275' S, 55²5'W, 180 m, 18-IX2000, Múlgura et al. 2053 (SI). Salta. Depto. Candelaria: Cumbre de la Sierra de la Candelaria, 2500 m, 21-IV-1925, Venturi 3813 (SI). San Luis. Depto. Junín: Ruta Provincial 5, de Merlo a mirador Los Cóndores, pastizal de altura cerca del límite con Córdoba, 32²1'51"S, 6456'23"W, 2130 m, 29-XI-2008, Biganzoli et al. 2089 (SI). Santa Fe. A ${ }^{\circ}$ Frias, 21-XII-1949, Morello 4055 (SI). Depto. Castellanos: $200 \mathrm{~m}$ al S del puente Río Salado, 27-IX-1956, Martínez Achenbach 277 (SI). Depto. Gral. Obligado: Mocoví, sin fecha indicada, Venturi 43 (SI). Depto. La Capital: Rincón Norte, 20-X1957, Martínez Achenbach 162 (SI); Alto Verde, 30-XII-1970, Donnet 2086 (SI). Tucumán. Depto. Chicligasta: Alpachiri, 27²0'24"S, 6545'23"W, 500 m, 2-XI-2009, Larsen \& Ulibarri 73 (SI). Depto. Famaillá: Río Colorado, 300 m, IX-1919, Venturi 425 (SI). Depto. Río Chico: La Colina, 17-XI-1903, Monetti 1495 (SI). Depto. Tafí del Valle: La Quebradita (Tafí del Valle), 27-XI-1966, Ahumada \& Jerez s.n. (LIL 517887); Sala Ancajulio, 14-XII-1944, Olea 84 (SI); Tacanas, playas, 2000 m, 17-XII1908, Lillo 8763 (SI); Abra del Infiernillo, 2604'07"S, 6546'24"W, 3040 m, 2-II-2005, 
Zuloaga et al. 8432 (SI); Cerro Muñoz, 3000 m, 26-V-1906, Monetti 285 (SI); subida al Infiernillo, 7-XI-1978, Cabrera et al. 29697 (SI).

BRASIL. Rio Grande do Sul. S. Salvador, in prato paludoso, 15-X-1946, Sehnem 2208 (SI). Municipio Sâo Francisco de Paula: Cambará, in humidis umbrosis, 2-II-1948, Rambo 36350 (SI). Santa Catarina. Municipio Laguna: Ponta da Barra, Playa Gravatá, 14-I-2010, Zanotti \& Vitali 151 (SI). Municipio Xanxerê: Rio Xanxerê, Xanxerê and vicinity, 265'S, 52²4'W, 700 m, 14-XI-1964, Smith \& Klein 13271 (SI).

PARAGUAY. Sin localidad indicada, sin fecha indicada, Jörgensen 3964 (SI). De la

Cordillera: San Bernardino, X-1915, Hassler 1496 (SI). Guairá: Villa Rica (Villarrica), dans les rues, X-1874, Balansa 2386 (SI). Misiones: Santiago. Estancia "La Soledad", 27-X-1959, Pedersen 5241 (SI).

PERÚ. Cuzco. Pcia. Cuzco: Alrededores de Cuzco, 3500 m, 15-II-1922, Herrera 15 (SI 12048), Herrera 18 (SI 12050).

URUGUAY. Montevideo: Santiago Vasquez, X-1924, Herter 71403 (SI). Salto: Salto, in campis et ad vias, 9-X-1910, Osten 5249 (SI). San José: Barra, XII-1926, Herter 81545 (SI).

\section{Boopis anthemoides}

ARGENTINA. Buenos Aires. Pdo. Bahía Blanca: Bahía Blanca, 2-I-1952, Boelcke \& Correa 5200 (SI); Bahía Blanca, 22-XII-1899, C. Spegazzini 958 (SI). Pdo. Campana: Campana, 27-X-1928, Burkart 3088 (SI). Pdo. Patagones: Patagones, 21-XI-1950, Fabris 483 (SI); Patagones, en terreno baldío, 12-IV-1945, Krapovickas s.n. (LIL 434544); Carmen de Patagones, 20-XI-1944, Meyer s.n. (LIL 122039); Ruta 130. Campo Fiscal, 12-X-1937, Ricci Peralta 3 (SI); Ruta 131. Campo Fiscal, 14-X-1937, Ricci Peralta 26 (SI); Ruta 126. Médano, 16-X-1937, Ricci Peralta 30a (SI). Pdo. Pehuajó: Alagón, III-1946, Cabrera 10270 (SI). Pdo. Pigüé: Pigüé, F.C.S., 9-XI-1932, Burkart 4810 (SI). Pdo. Saavedra: Sierra de Curamalal. A Cochen-leufú, 4-XI-1939, Cabrera 5492 (SI); Estancia Cura Malal, 10-XII-1899, C. Spegazzini 971 (SI). Pdo. Tornquist: Sierra de la Ventana, Estancia "Leiva", 21-XI-1895, Alboff 151 (CORD, SI); Sierra de la Ventana, 6-XII-1964, Burkart 25643 (SI); Sierra de la Ventana. Río Sauce Grande, 3-XI-1941, Cabrera 7310 (SI); Sierra de la Ventana, 6-XI-1938, Cabrera 4667 (SI); Sierra de la Ventana, XI-1904, Dusén 6310 (SI); Sierra de la Ventana, 25-XII1905, Hauman-Merck s.n. (SI 12633); Sierra de la Ventana, 9-XI-1907, Hicken 1 (SI); 
Sierra de la Ventana (Estancia del Sr. Funke), 9-II-1901, Hicken 2 (SI); Saldungaray, 15-XII-1984, Villamil 3324 (SI). Pdo. Villarino: Road to Bahía Blanca, 60km west of Rio Colorado; in pure sand, half buried, low, rolling hills, shrub formation, $50 \mathrm{~m}$, 16XII-1938, Eyerdam et al. 23446 (SI); Arroyo Saladillo Dulce, ruta 3, al oeste de Bahía Blanca, 1-XI-1972, Del Puerto \& Marchesi 11535 (MVFA). Pdo. Zárate: Las Palmas, al N de Zárate, 13-X-1946, Krapovickas 3096 (SI). Catamarca. Sin datos de localidad, I-II-1910, P. L. Spegazzini s.n. (BAB 33421); Sin datos de localidad, I-II-1910, P. L. Spegazzini 33285 (SI, ex Herb. Min. Agr.). Depto. Ambato: El Rodeo, Sierra de Ambato, 12-I-1957, Calderón 1264 (SI); Valle del Rodeo, 2-XII-1908, Castillón 9058 (LIL, SI); 2km al NNE de El Bolsón. Finca de Cebil, 1250 m, 31-III-1995, SaraviaToledo et al. 13236 (CTES); El Rodeo, 1200 m, 8-XII-1959, Villa Carenzo s.n. (LIL 454022). Pdo. Andalgalá: Andalgalá, 15-X-1966, Schulz 9569 (CTES). Depto. El Alto: El Alto, 7-XI-1948, Argañaraz s.n. (LIL 551281). Depto. Paclín: Amadores, El Molino, 17-XI-1946, Dimitri \& Piccinini s.n. (BAB 68176, CORD); Balcosna, 1250 m, 19-I1928, Venturi 7145 (SI). Chaco. Depto. 12 de Octubre: Gral. Capdevila, 22-IX-1972, Schulz 18354 (CTES). Depto. $1^{\circ}$ de Mayo: Colonia Benítez, E. Urien F.C.S.Fe, XI1930, Schulz 129 (SI). Depto. Campo del Cielo: Capdevila, 12-XI-1946, Schulz s.n. (LIL 187840). Depto. Mayor Luis J. Fontana: Enrique Urien, IX-XII-1939, Schulz 1654 (CTES). Chubut. Travesía Kel-la, XII-1900, Burmeister s.n. (BAB 960). Patagonia, 1III-1899, Illín 978 (SI). Travesía de Rawson a Las Cordilleras, 1-30-XI-1903, Illín 26 (SI). Depto. Biedma: 40km S de Arroyo Verde, Ruta 3, 8-XI-1965, Correa 3217 (BAB); Puerto Madryn, 13-XII-1970, Crespo \& Troncoso 1799 (SI); Puerto Madryn, 9XI-1928, Donat 7 (SI); Puerto Madryn, 29-XI-1982, Villamil 2678 (leg. P.M. Hermann) (SI); Caleta Valdés, 24-I-1982, Wulff \& Simone 69 (SI); Alrededores de Puerto Madryn. Cerca del Co Avanzado, 6-XI-2006, Zanotti 60 (SI); Ruta Nac. 3, de Puerto Madryn a

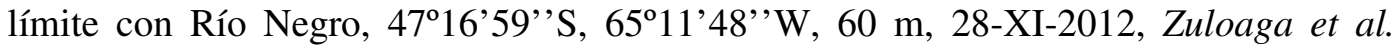
14155 (SI). Depto. Escalante: Comodoro Rivadavia. Punta Borja, 8-II-1903, Pennington 54 (SI); Comodoro Rivadavia, 13-XI-1952, Ruiz Leal 14772 (MERL). Depto.Florentino Ameghino: Pto. Lobos, 5-I-1967, Boelcke et al. 12114 (SI); Punta Tombo (Pingüinera), 17-I-1977, Lotti de Stange s.n. (LIL 554561).Depto. Gaiman: Las Chapas, 43²9'S, 66²2'W, 27-XI-1976, Arroyo et al. 14 (SI). Depto. Languiñeo: 64 kms E of El Molle (Junction Route 62) on Route 25. Patagonian steppe. Sandy soil. (Extremely windy!), 600 m, 8-XII-1984, Stuessy et al. 6923 (SI). Depto. Paso de Indios: Ruta 24, al S de 


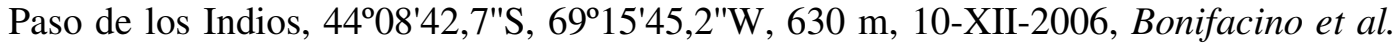
2960 (SI). Depto. Rawson: Ruta Pcial. 53, ca 1km al SW de Intersección con Ruta 25, 4346'28,1"S, 67²9'26,2"W, 26-XI-2002, Bonifacino \& Donato 689 (SI); Trelew Puerto Madryn, XII-1970, Crespo \& Troncoso 1789 (SI); Bahía Cracker, 23-X-1943, Soriano 2743 (SI); $52 \mathrm{kms} \mathrm{N}$ of Trelew on Route 3, $40 \mathrm{~m}, 10-X I I-1984$, Stuessy et al. 6951 (SI); Trelew, Cima Castela, 11-XI-1897, Mus. Nac. Bs. As. 4 (SI). Depto. Río

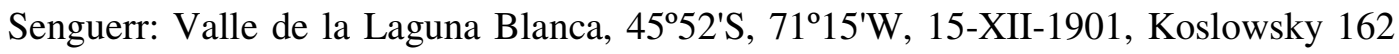
(SI). Ciudad Autónoma de Buenos Aires: Barracas al Sud de B. Aires, 26-I-1902, Venturi 20 (SI). Córdoba. Sierra Chica de la Prov. de Córdoba, 6-X-1897, Stuckert 3244 (CORD). Depto. Calamuchita: Embalse de Río 3º 30-X-1949, Krapovickas 6465 (SI); Villa Gral. Belgrano, 21-I-1969, Krapovickas A. \& C.L. Cristóbal 14656 (CTES). Depto. Capital: Ciudad de Córdoba, calles de San Vicente, 29-IX-1918, Castellanos 643 (SI); Córdoba, 30-IX-1936, Giardelli 446 (SI); Córdoba, 27-XI-1877, Hieronymus s.n. (CORD); Córdoba: San Vicente, 14-XI-1963, A.T. Hunziker 16564 (CORD); Córdoba: en el río Primero, 14-XI-1951, A.T. Hunziker 9387 (CORD); Córdoba: Excursión Altos del Observatorio, 3-XI-1971, A.T. Hunziker \& T. Di Fulvio 21394 (CORD); Quebrada de las Rosas, XII-1891, Kuntze s.n. (SI 12632); Altos Sur de Córdoba, 30-X-1897, Stuckert 3470 (CORD); General Paz (Córdoba), 14-I-1897, Stuckert 1454 (CORD). Depto. Colón: Calera (Sierra Chica), 13-XI-1897, Stuckert 3799 (CORD); La Reducción (al W de Villa Allende), 27-XII-1935, Burkart 7440 (SI); Río Ceballos; Sierra de Córdoba, XII-1891, Kuntze s.n. (SI 12630); Ascochinga, 13-XI, Kurtz 648 (CORD), X-1936, Nicora 1056 (SI). Depto Cruz del Eje: Cruz del Eje, 1-III-1941, Nicora s.n. (SI). Depto. Gral Roca: Ruta 35: entre Huinca Renancó y el límite con La Pampa, 5-X, Kurtz 650 (CORD); Entre las estaciones Burmeister y Onagoity, (F.C. Gral. S. Martín), a lo largo del terraplén ferroviario, o en sus inmediaciones, 28-IX1885, Kurtz 2444 (CORD). Depto. Gral. San Martín: Ruta Nac. No 9: entre James Craik y Villa María, 2-XI-1972, Subils \& Anton 1597 (CORD). Depto. Marcos Juarez: Marcos Juarez, 22-X-1907, Stuckert 17849 (CORD). Depto. Pocho: Camino entre CTM y Salsacate, 6-II-1938, Nicora 1843 (SI). Depto. Punilla: Los Cocos, 12-XI-1908, Stuckert 19277 (CORD), 24-X-1906, Stuckert 16085 (CORD); Capilla del Monte (Sierra Chica), X-1907, Kurtz 14879 (CORD); Dolores, 8-I-1938, Nicora 1676 (SI). Depto. Río Cuarto: Río Cuarto, 10-XII-1908, Stuckert 19458 (CORD). Depto. Río Primero: Estancia "San Teodoro", 1-IX-1900, Stuckert 9522 (CORD). Depto. Río 
Segundo: Estación Oncativo entre Rosario y Córdoba, 8-9-III-1887, Kurtz 4802 (CORD); Ruta Nac. N 9: Pilar, próximo a las vías del Ferrocarril Gral. Bartolomé Mitre, 20-X-1963, Subils 635 (CORD). Depto. San Alberto: Mina Clavero, XII-1901, Stuckert 11308 (CORD). Depto. San Javier: Cumbre de Achala (Falda O.): Villa de Las Rosas, camino a San Javier, 12-X-1961, Subils \& Artico 295 (CORD). Depto. San Justo: Sacanta, 1-XII-1898, Stuckert s.n. (CORD); El Tío, 8-XII-1946, Balegno s.n. (LIL 171957). Depto. Santa María: Ochoa, 31-XII-1902, Stuckert 12505 (CORD). Depto. Tercero Arriba: Salto, 27-XI-1897, Stuckert 894 (CORD). Depto. Totoral: Ruta Nac. No 9: entre Las Peñas y Simbolar, 6-X-1973, Subils 1782 (CORD); Las Cañadas, 8-10-III-1887, Kurtz 4788 (CORD); Laguna de Los Molles, 5-II-1955, Lanfranchi 1231 (SI). Entre Ríos. Depto. Concepción del Uruguay: Ruta 14 a Concordia, km 249, A Isletas, 21-IX-1961, Burkart 22766 (SI); Concepción del Uruguay, 7-XII-1967, Burkart 26760 (SI). Depto. La Paz: Ombú a Paso Yunque, 22-X-1971, Burkart 28900 (SI). La Pampa. Sin localidad indicada, 1925, Williamson s.n. (SI 14629); II-1931, Monticelli H-45 (SI). Depto Guatraché: Guatraché, 18-II-1948, Burkart 17495 (SI). Depto. Lihué Calel: Sierra de Lihué Calel, cañadón de la Cruz, 5-XII-1981, Cabrera et al. 32803 (SI); Lihué Calel, 2-XI-1974, Cano 4765 (BAB). Depto. Loventué: Telén, 17-X-1979, De Azkue et al. s.n. (BAB 91320, SI). Depto. Toay: 40 km al sur de Santa Rosa, 4-XI-1953, Orbea s.n. (SI 18716). La Rioja. Cuesta de la Puerta de Piedra (Cuesta de Sigu); Sierra de Velasco, 8-11-I-1879, Hieronymus \& Niederlein 35 (CORD). La Hoyada, 2500 m, 27-31-I-1908, Kurtz 15025 (CORD). Depto. Chamical: Ciudad de Chamical, alrededores de la Universidad, 467 m, 23-III-1988, Biurrun 2263 (CORD). Depto. Chilecito: Camino de acceso a Mina "El Oro", 1850 m, 2906'41,7"S, 67³7'42,4"W, 3XII-2006, Bonifacino et al. 2834 (SI); Cuesta de Catinzaco, 30-I-1906, Kurtz 13364 (CORD); Chilecito, estafeta Santa Florentina, 19-XII-1951, von Rentzell s.n. (SI). Depto. Famatina: Sierra de Famatina. Cerca de Cañadón Amarillo, 3000 m, 2-III-1979, Cei s.n. (MERL 28001); Velazco (Sierra de Velasco?), 2300-2500 m, 6-III-1944, Soriano 929 (dupl. SI); Camino a la mina La Mejicana, 2856'24''S, 67\%41'09'”W, 1223 m, 11-I-2009, Donadío et al. 129 (SI). Depto. Gral. Belgrano: Ruta Nac. No 38, entre Chamical y Chañar a $20 \mathrm{~km}$ de la primera, campo del INTA "Las Vizcacheras", cultivo de Cenchrus ciliaris, 22-III-1989, Biurrun et al. 2739 (CTES). Depto. Rosario Vera Peñaloza: Entre Totoral y Mina San Juan, 1780-3050 m, 20-21-II-1906, Kurtz 13478 (CORD). Mendoza. Depto. Capital: Parque San Martín, 1-XII-1942, Covas 709 
(SI). Depto. Gral. Alvear: Cochico, sin fecha indicada, Monticelli 8 (SI). Depto. La Paz: Desaguadero, inmediaciones del Paso de las Tropas, 9-IV-1944, Ruiz Leal 9073 (MERL). Depto. Las Heras: Quebrada de la Mina La Atala, 24-XII-1937, Ruiz Leal 4703 (MERL); La Crucecita, en el Co Verde, 1500-1600 m, 20-XI-1938, Ruiz Leal 5444 (MERL); Puerto La Obligación, 20-XI-1943, Covas 2748 (SI). Depto. Malargüe: Distrito Río Grande. Carta "El Manzano" 1:100.000 (hoja 3769-2). Aprox. 1,5km al S del Pto. Ojo de Agua, 1690 m, 3603'S, 69³7'W, XI-1981, Ambrosetti \& Del Vitto s.n. (MERL 30859); Calmuco, cerros, 13-II-1942, Burkart et al. s.n. (SI 14316); Pte. Al borde de la barda de la Meseta basáltica de Malal Chico, 1350 m, 7-X-1980, Méndez \& Wuilloud s.n. (MERL 30669); Mina Ethel, 1700 m, 3600'49"S, 6848'41"W, 23-XI2004, Prina et al. 2552 (SI); Entre Ramblón de la Bandera y Portezuelo del Milico, 17XII-1960, Ruiz Leal 21511 (MERL). Depto. San Rafael: Las Picasas, 1000-1500 m, 23I-1941, Ruiz Leal 7312 (MERL); Sierra del Nevado, puesto Los Zainos, laderas al este del puesto, 3541,5'S, 68 $17^{\circ} \mathrm{W}, 4-\mathrm{XII}-1973$, Boelcke et al. 15579 (SI); 25 de Mayo (al Oeste), 1600 m, 21-XI-1986, H. A. L. 7721 (SI); Cuesta de los Terneros, 24-X-1991, Lutz 49 (CTES). Neuquén. Piedra Chenque, XII-2001, Cony s.n. (MERL 53995). Parque Industrial, 5-XI-2005, Winter 1 (SI). Depto. Confluencia: El Chocón, 5-XI1972, Correa 4732 (BAB); Zona árida de la confluencia del Neuquén con el Limay, 3905'S, 68¹0'W, 6-XI-1956, Roig s.n. (MERL 18094); Ruta 237, al sudoeste del Chocón, 5-XI-1972, Del Puerto et al. 11672 (MVFA). Depto. Pehuenches: Ruta Provincial 1, a 9,3km S empalme Ruta Provincial 7, 3740'20"S, 6944'31"W, 1239 m, 4-XII-2007, Ávila et al. s.n. 9 (SI); Pampa Tril [R 11 (Eduardo M.C.)], 18-XII-1997, Dalmasso s.n. (MERL 58319). Depto. Zapala: Ruta Nac. 40, unos $11 \mathrm{~km}$ al S de Zapala, junto al acceso a El Manzano, 3900'14"S, 7005'18"W, 1080 m, 23-I-2006, Belgrano et al. 472 (SI). Río Negro. Rincón del Palo, I-1916, Scala 23 (SI). Depto. 25 de Mayo: Ruta Pcial. $\mathrm{N}^{\circ} 8$, a 11,9km N de Los Menucos, 4046'08"S, 68²10'59"W, 874 m, 4-XI2006, Zanotti \& Ávila 34 (SI). Depto. Adolfo Alsina: Ruta Nac. 250, C Campanín, 9XI-1988, Correa 9581 (BAB). Depto. Gral. Roca: Gral. Roca, XII-1959, Bahamonde s.n. (MERL 21002). Depto. Pichi Mahuida: Ruta 22, 117 km al este de Choele Choel, 1XI-1972, Del Puerto \& Marchesi 11589 (MVFA). Depto. San Antonio: Sierra Grande. Camino próximo a la antena de transmisión, 11-XII-2003, Bartoli \& Tortosa 42/03 (SI);

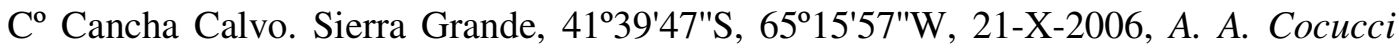
3930 (SI); Ruta 3; 16 km antes de La Porfía, entre San Antonio y Sierra Grande, 24-XI- 
1979, Roig \& Méndez s.n. (MERL 43861). Depto. Valcheta: 70 km al N de Valcheta, 120 m, 14-XI-1968, Ruiz Leal 26122 (MERL); Cañadón Cortaderas a Laguna Reimundo, 5-I-2003, Troiani \& Steibel 15519 (SI). Salta. Depto. Candelaria: entre Ruiz de los Llanos y Rosario de la Frontera, 28-I-1947, Schulz 6557 (CTES). Depto. Rosario de la Frontera: sin datos en la etiqueta, 11-XI-1974, Burkart et al. 30655 (SI). San Juan. Cuesta Vieja, 28-II-1921, Hosseus s.n. (CORD). Depto Angaco: Sierra de Pie de Palo: subiendo por el camino al Mogote Los Corralitos, en la Quebrada del Molle: La Aguada, 2150 m, 19-20-XII-1980, A. T. Hunziker et al. 23867 (CORD), camino a Mogote de los Corralitos, 2600 m, 20-I-1981, Kiesling 3140 (SI), Pie de Palo, aguada del caño, 31²3'08"S, 6758'48"W, 2060 m, 7-II-2000, Kiesling et al. 9392 (SI). Depto. Sarmiento: Sierra de Ansilta. Quebrada Santa Clara, unos 40km al SW de Pedernal, 2350 m, 21-III-1999, Biurrun et al. 5801 (SI); Río Bachongo, 23-I-1986, Guaglianone et al. 1447 (SI). Depto. Zonda: Estancia "Maradona", Agua Pinto, 2300 m, 9-II-1986, Kiesling et al. 6066 (SI). San Luis. Depto. Ayacucho: Entre San Francisco y Balde de Retamito, 28-XII-1885, Kurtz 3247 (CORD). Depto. Belgrano: Socoscora, Ea. Lomas Blancas, 20-I-1979, Del Vitto \& Belgrano s.n. (MERL 27679); Ruta Nac. No 147: entre El Médano y San Antonio, 700-750 m, 30-31-I-1963, A. T. Hunziker \& P. Maldonado 16221 (CORD); 5 km de San Antonio, 17-XII-1974, Roig s.n. (MERL 43880). Depto. Chacabuco: La Toma, 16-II-1944, Varela 558 (SI). Depto Gral. Pedernera: Mercedes, al S del camino a Las Isletas, 20-XI-1981, Elena B. Rosa 107 (SI); Villa Mercedes, X1947, Nicora 4830 (SI). Depto. La Capital: Ruta 147, desvío a Villa de la Quebrada, 20IV-1980, Rotman et al. 292 (SI); Alto Pencoso, II-1914, Bruch \& Carette 62 (SI); San Luis, 5-XI-1940, Burkart 10690 (SI); San Jerónimo, ruta 147, 20-I-1986, Guaglianone et al. 1336 (SI); Ruta 7: Río Desaguadero, muy cerca del límite con Mendoza, 13-XI1956, A. T. Hunziker 13056 (CORD); Entre Desaguadero y Estación Jarilla, 16-19-II1944, Ruiz Leal 9110 (MERL); Entre Pescadores y Chosmes, 30-X-1945, Ruiz Leal 10432 (MERL). Depto. San Martín: Santa Bárbara, 10-III-1882, Galander s.n. (CORD). Santa Fe. Fortín Chilcas, Ea. "Los Charabones", 18-XII-1975, Carnevali 3613 (CTES). Ruta 13 - 20km al N de Ruta 32, 15-II-1978, Pire \& Nisensohn 352 (SI). Depto. 9 de Julio: 3 km al N de Tostado, 14-XI-1988, Pensiero \& Faurie 3365 (SI). Depto. Gral. López: Rufino, Estancia "San Miguel", 11-XI-1900, C. Spegazzini 970 (SI). Depto. Las Colonias: Zona río Salado - Ruta, 24-XI-1957, Martínez Achenbach 349 (SI). Depto. Vera: Las Gamas, 16-X-1979, Lewis et al. 865 (SI), Potrero Las Gamas, 12-III-1983, 
Stofella 73 (SI); Vera F.C.S.F., 13-XI-1933, Burkart 5817 (SI); $5 \mathrm{~km}$ al E de $\mathrm{A}^{\mathrm{o}}$ Golondrinas, camino a Fortín Olmos, 2904'S, 60³0'W, 23-IX-1983, Cristóbal et al. 2019 (CTES); La Tigra, 17-XI-1981, Franceschi 492 (SI); Ruta 13, 22km al N de la 32, 17-XI-1981, Pire \& Prado 910 (SI). Santiago del Estero. Depto. Guasayán: El Cimbolar, 29-III-1944, Pierotti (h) s.n. (SI 26904). Depto. Hondo: Est. Tacana, 25-XI1908, Chaves 35215 (SI). Depto. Moreno: La Paloma, 9-IV-1972, Schulz 18089 (CTES). Tucumán. Depto. Burruyacú: Tusca y Taruca - Pampa cerca de La Cruz, 2228-XII-1872, Lorentz \& Hieronymus 105 (CORD). Depto. Chicligasta: Estancia "Rumicocha", XII-1911, Padilla 17 (BAB), Padilla 18 (BAB). Depto. Leales: Los Puertos, 400 m, 1-X-1913, Monetti 1205 (SI); Chañar Pozo, 300 m, X-1919, Venturi 474 (SI). Depto. Trancas: Las Trancas, 23-X-1948, Ruiz Leal 12324 (MERL); Ruta vieja desde Trancas a Salta, 802 m, 16-III-2004, Slanis et al. s.n. (LIL 607880); Tapia, 750 m, 30XII-1923, Venturi 2318 (SI).

URUGUAY. Paysandú: Escuela Fac. Agron. Paysandú, V-1975, Rosengurtt 12484 (MVFA).

\section{Boopis australis}

ARGENTINA. Chubut. Depto. Florentino Ameghino: Ruta 3, a $33 \mathrm{~km} \mathrm{~W}$ de Camarones, Ea. "Lochiel", 16-I-1978, Romanczuk 905 (BAB). Depto. Languiñeo: río Corcovado, 2-III-1900, Illín 982 (SI). Depto. Río Senguerr: Vuelta del río Senguerr. Aguada Quemada, 27-XII-1902, Koslowsky 12526 (SI); Cañadón Tacho, 24-V-1958, sin coleccionista indicado 5339 (BAB); Río Guenguel, 30XII-1902, Koslowsky 12528 (BAB). Neuquén. Depto. Catan Lil: Entre La Negra y Las Coloradas, 27-I-1961, PérezMoreau 3121 (BAB). Depto. Chos Malal: Ruta Prov. 43, 40km al Oeste de Chos Malal hacia Andacollo; \pm 1570 m, 10-II-1985, Rossow 2522 (BAB). Depto. Collón-Curá: Laguna Carilafquen Grande, 27-I-1956, Castellanos 3548 (LIL). Depto. Minas: Curso inferior del río Varvarco. Campamento arroyo Aguas Calientes, $36^{\circ} 42^{\prime} \mathrm{S}, 70^{\circ} 37^{\prime} \mathrm{W}$, II1970, Boelcke et al. 14342 (BAB); Aguas Calientes, 21-II-1985, Rossow 3006 (BAB); Curso inferior del río Varvarco. Campamento arroyo Aguas Calientes, 36 $42^{\prime} \mathrm{S}$, 70³7'W, 4-II-1970, Boelcke et al. s.n. (LIL 566440); Curso inferior del río Varvarco. Campamento arroyo Aguas Calientes, 36 42'S, 70³7'W, 4-II-1970, Boelcke et al. 14342 (SI); Desvío de la Ruta Provincial 43, Aguas Calientes, 3640'47"S, 70³6'28"W, 1750 m, 27-XI-2010, Zuloaga et al. 12543 (SI). Dpto. Norquín: Ea. "El Sol de 
Singone", frente a Copahue, 19-II-1954, Castellanos s.n. (LIL 442194). Depto. Picunches: Las Lajas, 1000 m, 3-III-1935, R.A. Spegazzini 56692 (BAB). $\quad$ Depto. Zapala: Parque Nacional Laguna Blanca. Límite W del PN, cerca de la casa de las hermanas Quilaqueo, 27-X-2008, Villamil et al. 10778 (SI, BBB); El Sauce, 11-XII1952, Cabrera 11174 (LP); El Sauce, 11-XII-1952, Cabrera s.n. (LIL 517544); Zapala, II-1932, Monticelli I-104 (SI), 2-XII-1944, Descole 2121 (LIL), 3-XII-1944, Descole 2129 (LIL), 14-XII-1944, O'Donell 2045 (LIL), 4/12/1944, Descole 2171 (LIL); Parque Nacional Laguna Blanca. Vega del Llano Blanco, 18-XII-1999, Villamil et al. 9181 (SI, BBB), Laguna Antiñir, 12-XII-1999, Villamil et al. 10943 (SI, BBB), Estanque hermanas Quilaqueo, 15-XII-1999, Villamil et al. 11256 (SI, BBB), Cruce La Vega Laguna Jabón, 2-II-2010, Villamil et al. 11380 (SI, BBB), Vega de Llano Blanco, 3859'36"S, 70²2'35"W, 15-I-2008, Villamil et al. 10583 (SI, BBB), Laguna Verde, 4XII-2010, Testoni 97 (SI, BBB). Río Negro. Depto. Ñorquinco: Ñorquinco, cerros, 3-II1944, Nicora 3802 (SI). Depto. Valcheta: Orilla del A Valcheta, 3-XII-1974, Piccinini et al. 2426 (BAB). Santa Cruz. Río Santa Cruz, I-III-1900, Burmeister 975 (SI). Tehuelches, 200 m, 10-XII-1928, Donat 96 (SI). Depto. Corpen Aike: Laguna Grande, II-1949, Grondona 2302 (BAB); Monte León, 27-I-1914, Hicken \& Hauman 465 (Iter Patagonicum) (SI); Ruta Provincial 27, a 82km SE de Gobernador Gregores, en el puesto fijo de vialidad, en el borde del arroyo, 49²0'41"S, 6952'37"W, $196 \mathrm{~m}$, 6-I2007, Zanotti 68 (SI). Depto. Deseado: Puerto Deseado, XII-1901, Burmeister s.n. (BAB 4154), XII-1901, Burmeister s.n. (SI 12636), camino Cabo Blanco-Tellier. Cañadón del Comisario, 13-XI-1965, Correa 3357 (BAB); a 11kn de la salida de Puerto Deseado hacia Fitz Roy; Ruta Prov. 281, 26-II-1990, Correa et al. 10266 (BAB). Depto. Güer Aike: Ruta Nacional n 3, unos 9 km SW de Río Gallegos, adyacencias del río Chico, 51²42'33"S, 69¹7'13"W, 8 m, 16-I-2003, Belgrano et al. 2 (SI); Camino Lemarchand. Pto. Coig; cañadón en cruce del río Coig, 24-XI-1963, Correa 2774 (BAB); 29 km south of Puerto Coyle, road to Río Gallegos, 20 m, 2-I-1939, Eyerdam et al. 24048 (SI); río Coyle, 16-III-1914, Hicken 788 (Iter Patagonicum) (SI); Estancia "Las Vegas"; Río Coyle, Brazo Norte; Subsección Al-Aike, 51¹9'S, 70²0'W, 24-XII1967, Moore 1301 (BAB); Chimen Aike, río Chico, 30 m, 26-XI-1950, Sleumer s.n.

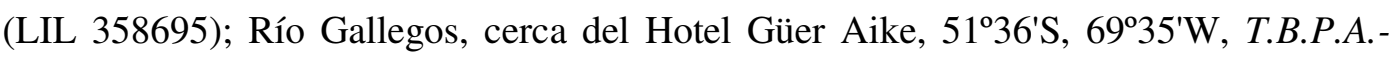
FIT 204 (BAB); Ea. "La Angelina", 51²6'S, 6907'W, 6-XII-1975, T.B.P.A.-FIT 39 1/2 (BAB); Río Gallegos, laguna La Leona, 513'ㄴ, 69²4'W, 7-XII-1975, T.B.P.A.-FIT 
253 (BAB); Ruta Provincial 1, Cabo Vírgenes, en frente de planta de petróleo, 52²3'21"S, 68²5'09"W, 18 m, 8-I-2007, Zanotti 75 (SI); Güer Aike, 15-XII-1945, O'Donell 4077 (hojas a-b) (LIL). Depto. Lago Argentino: Ea. "Los Lagos", costa N del lago Viedma, entre $\mathrm{A}^{\circ}$ Blanco y río Las Vueltas, 49²6'S, 72²4'W, 21-I-1967, Boelcke et al. 12685 (BAB, SI); El Calafate, orilla SW laguna del lago, 9-XII-1963, Correa 3086 (BAB); Estancia "Los Baguales". Sierra de los Baguales (al E del Brazo Sur del Lago Argentino), II-1900, Hauthal 66 (SI); Lago Argentino, 12-II-1914, Hicken 1033 (SI); Ruta Provincial 9, a 51,3km E de la Ruta Nacional 40. Cerca de Estancia "La Leonor", 50¹7'07"S, 7101'44"W, 266 m, 12-I-2007, Zanotti 88 (SI); 1km N Estancia "La Lucila", a 54,8km N Tres Lagos, C Cordón, 49¹3'49"S, 71²0'31"W, 650-700 m, 16-I-2007, Zanotti 106 (SI). Depto. Lago Buenos Aires: Ruta Nac. 40, a 39km al S Perito Moreno, 46 $55^{\prime} \mathrm{S}, 7^{\circ} 43^{\prime} \mathrm{W}, 6-\mathrm{II}-1975$, Boelcke et al. 16139 (BAB); Lago Buenos Aires, 200 m, 28-I-1903, Hicken 5. (leg. Platen \& Greiner) (SI). Depto. Magallanes: San Julián, 13-XII-1944, Blake 386 (SI). Depto. Río Chico: Gob. Gregores, cerro NE de INTA, 4850'S, 70 $\mathrm{W}, 1-\mathrm{II}-1965$, Ancibor \& Vizinis s.n. (BAA 4572, CTES). Tierra del Fuego. Depto. Río Grande: Cabo Domingo, 17km N de Río Grande, 18-XI-1971, Boelcke et al. 15053 (BAB); Ea. "Cullen", arroyo Alfar, near to the sea, 20-V-1972, Goodall 4300 (BAB); Punta Yopper from Frigorifico to mouth of Río Grande, 18-XII1972, Goodall 4401 (BAB); Región del río del Fuego, 7-IV-1902, Holmberg \& Calcagnini 4086 (SI).

CHILE. XI Región de Aysen. Pcia. Aisén: Pescadores (prope Santa Cruz), 25-XII1904, Dusén 5437 (SI). XII Región Magallanes. Pcia. Magallanes: Ea. "Laguna Blanca", Sección Cabeza del Mar, 5-XII-1971, Goodall 4071 (BAB); Punta Arenas, 19-

IX-1910, Herb. Salesiano servatae 291 (SI); Bahía Inútil, 150 m, 28-XI-1930, Donat 328 (LIL, SI).

\section{Boopis bupleuroides}

BRASIL. Paraná. Curitiba in fruticetis humidis, 30-XI-1903, Dusén 2346 (SI).

\section{Boopis castilloni}

ARGENTINA. Catamarca. Depto. Capayán: Huillapima [localidad dudosa], XII-1909, P. L. Spegazzini s.n. (SI). Depto. Ambato: Sierra de Ambato (Falda E): subiendo desde El Rodeo hacia el cerro Manchao, 2900-3100 m, 23/25-II-1967, A. T. Hunziker 19193 
(SI); Sierra de Ambato (Falda E): subiendo desde El Rodeo hacia el cerro Manchao, 2900-3100 m, 23/25-II-1967, A. T. Hunziker 19269 (CORD); Sierra de Ambato (Falda E): subiendo desde El Rodeo hacia el cerro Manchao: alrededores de Los Cajones, 3000 m, 28/30-I-1968, A. T. Hunziker \& T. Di Fulvio 19712 (CORD); Sierra de Ambato (Falda E): hacia Casa de Cubas desde El Crestón del cerro Manchao, 3100-3500 m, 28/30-III-1968, A. T. Hunziker \& A. E. Cocucci 20064 (CORD); Sierra de Ambato (Falda E, bajando hacia El Rodeo): desde Pampa de las Minas hacia Campo Grande, rumbo al primer campo, 3800-3900 m, 21-II-1971, A. T. Hunziker 20903 (CORD); Sierra de Ambato (Falda E): subiendo desde El Rodeo hacia el cerro Manchao, cerca de la Casa de Piedra del primer campo, ca. 3500 m, 22-II-1971, A.T. Hunziker 20985 (CORD, SI).

\section{Boopis chubutensis}

ARGENTINA. Chubut. Río Aysen, XII-1900, Burmeister 962 (BAB por triplicado). Depto. Languiñeo: Cerca Lago Gral. Paz, 44 S, 12-II-1902, Gerling 210 (SI). Depto. Paso de Indios: Ruta 25, a 42km S Paso de Indios, 43 44' S, 69 41' W, 29-XI-1976, Arroyo et al. 73 (BAB). Depto. Río Senguerr: Estancia "La Pepita", 4-II-1949, Grondona 2354-a (BAB); Río Mayo, Est. Zootecnia. Mallín "El Cacho", 4-II-1954, Grondona 3540 (BAB), Grondona 3542 (BAB); Est. Zoot. de Río Mayo - Cañadón "El Tacho", 8-II-1954, Soriano 4575 (BAB). Depto. Sarmiento: Ruta provincial 23, 87.8 km SE empalme Ruta Provincial 20, 4444'22'’S, 69³6'29'’W, 891 m, 17-XII-2007, Nicola s.n. (SI). Depto. Tehuelches: Ruta Nac. 40, a $31 \mathrm{~km} \mathrm{~S} \mathrm{de} \mathrm{Gobernador} \mathrm{Costa,} 44^{\circ}$ 16' S, 70² 24' W, 3-II-1975, Boelcke et al.16066 (BAB); Valle de la Laguna Blanca, 15XII-1901, Koslowsky 70 (BAB 12527 por duplicado); Valle del Lago Blanco, XII-1902, Koslowsky 55 (BAB 13753 por duplicado).

\section{Boopis filifolia}

ARGEnTINA. Patagonia, 1904, Tessleff 5836 (BAB). Santa Cruz. Depto. Corpen Aike: Ruta Nac. 3, 54 km al sur del río Chico, 28-XII-1992, Botta \& Miconi 500 (SI). Depto. Güer Aike: Monte Aymond. Proximidades de Gendarmería Nacional, 28-XI1976, Ambrosetti 28624 (BAB); Ruta 293, a 140km W de Río Gallegos, camino a El

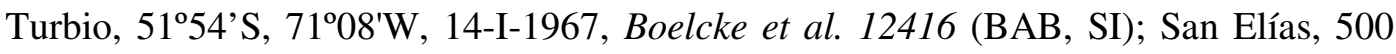
m, 19-I-1978, Roig 11630 (MERL 43879); Ea. "Punta Loyola Sur", 5151'S, 6902'W, 
5-XII-1976, T.B.P.A.-FIT 846 (BAB); Ea. "La Carlota", sección San Elías, 51²4'S, 71³2'W, 19-I-1978, T.B.P.A.-FIT 2809 (BAB). Depto. Lago Argentino: El Calafate, laguna del lago, 30-XI-1963, Correa 2948 (BAB); Lago Argentino ad Kare-Aike, 13-II1905, Dusén 5836 (SI); Lago Argentino, Médano Punta E Lago Argentino, 28-XII2006, Guerrido et al. 722 (SI); Bajada del Petizo, 11-III-1914, Hicken 793 (SI); Lago Argentino, colina en la punta este del lago, entre Charles Fuhr y Paso Biggieri, 15-I1987, Leuenberger \& Arroyo 3699 (BAB); C Huyliche, Ea. "Huyliche". Cima del cerro, 50²2'50"S, 72¹6'59"W, 877 m, 12-I-2011, Zavala-Gallo et al. 219 (SI); Médano Punta E Lago Argentino, 15-XII-2007, Guerrido et al. 763 (SI). Depto. Lago Buenos Aires: Meseta del lago, 2km NO casco Ea. "La Vizcaína", 9-XII-1986, Sánchez 463 (BAB). Depto. Río Chico: Ruta Nacional 40, a 99,6km N de Tres Lagos, 3 km N de Estancia "La Siberia", arenal costa E lago Cardiel, 48 57'15"S, 7101'57"W, 399 m, 16I-2007, Zanotti 110 (SI); Ruta Nacional 40, a 37km N del empalme R.P. 37, a P.N. "Perito Moreno", pasando entrada a Estancia "La Fe", 4758'13"S, 7058'21"W, 618 m, 18-I-2007, Zanotti 119 (SI).

CHILE. XII Región de Magallanes. Pcia. Magallanes: Depto. Tierra del Fuego. Manantiales, I-1956, Magens 1129 (CONC); San Gregorio, Parque Nacional Pali Aike, sector bajos los guanacos, 5204'S, 69²46'W, 100 m, 18-XII-2000, Domínguez \& Elvebakk 23 (CONC).

\section{Boopis gracilis var. gracilis}

ARGENTINA. Chubut. Depto. Languiñeo: Tecka, Estancia "Quichaura", 11-XII-1946, Soriano 2262 (SI). Depto. Paso de Indios: 50 km de Los Altares, 9-XI-1972, Correa 4853 (BAB). Depto. Río Senguerr: Estancia "La Pepita", 4-II-1949, Grondona 2354-b (BAB). Mendoza. Vega del Loro, 2400 m, I-1921, Carette s.n. (SI 12670); Paso Cruz Cordillera, $34^{\circ} \mathrm{S}, 1500 \mathrm{~m}, \mathrm{I}-1892$, Kuntze 19 (CORD). Depto. Malargüe: $20 \mathrm{~km} \mathrm{NE}$ Valle Hermoso, camino a Los Molles, 2250 m, 28-I-1963, Boelcke et al. 10328 (SI); Los Molles, 2000-2400 m, 14-II-1944, Burkart 14853 (SI); Cerros al N de Calmuco, 1900 m, 16-II-1942, Burkart et al. (SI 14323); Valle del río Salado, 18-I-1982, Cabrera et al. 33372 (SI); Co de la Olla - La Payunia, 8-XII-1994, H. A. L. 8680 (SI); El Infiernillo -Valle del Salado-, 1800 m, 24-XII-1984, H. A. L. 6643 (SI); río Salado superior, Los Molles, sin fecha indicada, Kurtz s.n. (SI 12641); Sierras de Chachahuén, Base del C $C^{\circ}$ Ureta, 3704'11"S, 68 53'17"W, 1565 m, 13-XII-2001, Prina et al. 1500 
(SI); Sierras de Chachahuén, río seco entre Pto. "Los Álamos" y Pto. "Chachahuén", 3704'53"S, 68 53'16"W, 20-XI-2001, Prina et al. 1212 (SI); Los Molles, en la cuesta del "Cuchillo", 2350 m, 30-XII-1949, Sleumer s.n. (LIL 302312); Pasando Castillos de Pincheira, 35³1'42"S, 6950'15"W, 1900 m, 21-XI-2010, Zuloaga et al. 12305 (SI). Depto. San Carlos: Refugio Gral. Alvarado, 2400 m, 26-I-1950, Cuezzo \& Barkley s.n. (LIL 294585); Valle del río Salinillas, frente al volcán San José, 2600 m, II-1937, Grandjot s.n. (SGO 57213); Real de “Agua del Medio", 34¹0'39.5', S, 69¹8'55' W, 2270 m, 7-XII-2009, Zavala-Gallo 22 (SI). Depto. San Rafael: El Sosneado, 24-I-1943, Bartlett 19523 (SI); Portezuelo del Choique, 2050 m, 7-II-1950, Boelcke 4208 (BAB); RP 190, La Estrechura al SE del $C^{\circ}$ Carrizo, 35²43.5'S, 68 $12^{\prime} \mathrm{W}, 1500$ m, 7-XII-1973, Boelcke et al. 15645 (SI); Sa. del Nevado, 1,5- 3,5km al W de la Cienaguita, 35³7'S, 68²1'W, 2320-2400 m, 22-I-1974, Boelcke et al. 15892 (SI); El Sosneado, cerca del hotel, 17-II-1944, Burkart 14902 (SI); Arroyo La Manga, 1900 m, I-1921, Carette 397 (SI); Cerca del Valle del Río Grande: Minacar, 10-II-1945, Covas 19992 (SI); Los Molles, 1700 m, 14-II-1944, Covas 2786 (SI); Cerca del Valle del Río Grande: Minacar, 10-II-1945, Covas 3261 (SI); Qda. de la Manga, entre C ${ }^{\circ}$ Bayo y A de la Manga, 2290 m, 8-I-1985, Marques \& Gómez-Sosa 30 (SI); N de El Sosneado, camino secundario al N-NE del cruce con Ruta Pcial. 220 (que bordea el río Atuel), 3444'00"S, 69³9'18"W, 2694 m, 20-I-2006, Pozner et al. 565 (SI); N de El Sosneado, camino secundario al NNE del cruce con Ruta Pcial. 220 (que bordea el río Atuel), 3446'27"S, 6940'19"W, 2457 m, 20-I-2006, Pozner et al. 543 (SI); San Rafael, sin fecha indicada, sin datos del coleccionista (SI 12647); San Rafael, sin fecha indicada, sin datos del coleccionista (Plantae Argentinae) (SI 12680); Hotel Termas de El Sosneado, 3446'12"S, 7003'33"W, 2180 m, 22-XI-2010, Zuloaga et al. 12382 (SI). Depto Tunuyán: Paso de las Nieves Negras, Cordillera de Santiago, volcán San José, 2600 m, II-1937, Grandjot 2659 (SI). Neuquén. Depto. Añelo: Chihuido Medio, R.P. 7, 9-XII-1982, Mallo et al. s.n. (LIL 580478). Depto. Chos Malal: A 14kmde Chos Malal camino a Andacollo, 37²3'S, 70²17'W, 820 m, 12-I-1964, Boelcke et al. 10666 (SI). Depto. Huiliches: Junín de los Andes, 14-XII-1969, Eskuche 959 (CTES, CORD, SI). Depto. Lácar: Lago Villarino, 1896, Roth s.n. (SI 12682, SI 12643). Depto. Los Lagos: Ruta Pcial. 63, unos $22 \mathrm{~km}$ W de Confluencia, camino a Paso Córdoba, 40³5'31"S, 7108'45"W, 1314 m, 27 I-2006, Belgrano et al. 553 (SI); Parque Nacional "Nahuel Huapi", Fortín Chacabuco, 8-II-1952, Boelcke \& Correa 6323 (SI); Paso del Córdoba, 3-XII-1990, Correa et al. 
10459 (BAB); Camino a Paso Córdoba ca. 20km. Aproximadamente mitad de camino

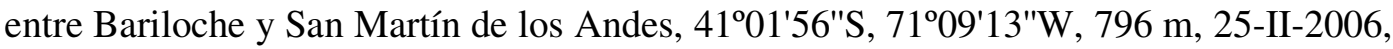
Zanotti et al. 18 (SI). Depto. Minas: Pichi-Neuquén, puesto de Gendarmería, valle del río Pichi-Neuquén, 36³6'S, 7049'W, 21-I-1970, Boelcke et al. s.n. (LIL 568666); Baños Calientes, río Varvarco, ladera rocosa al E, 36² $42^{\prime} \mathrm{S}, 70^{\circ} 37^{\prime} \mathrm{W}, 1550-1650 \mathrm{~m}, 31-$ I-1964, Boelcke et al. 11426 (SI); Pichi-Neuquén, puesto de Gendarmería, $\quad 36^{\circ} 36^{\prime S}$, 7049'W, 21-I-1970, Boelcke et al. 13563 (SI). Depto. Norquín: Ruta Provincial 4, entre Naunauco y Colipilli, 37³9'59"S, 70¹4'35"W, 1050 m, 28-XI-2010, Zuloaga et al. 12574 (SI); Trolope, 26-XII-1944, O'Donell s.n. (LIL 116090). Depto. Pehuenches: Cerca del río Barrancas, 2-XI-1956, Ruiz Leal 17999 (MERL). Río Negro. F. C. S., 17XII-1913, Fischer 38 (SI). Depto. Bariloche: San Carlos de Bariloche, $41^{\circ} \mathrm{S}, 850 \mathrm{~m}, 16-$ II-1905, Buchtien s.n. (SI 12642). Depto. Pilcaniyeu: Comallo, 5-I-1945, Meyer s.n. (LIL 112870); 6 km de Pilcaniyeu, camino a Bariloche, 28-I-1944, Nicora 3687 (SI). Santa Cruz. Depto. Lago Buenos Aires: 20km de Bajo Caracoles a Perito Moreno, 47²3'S, 7058'W, 28-I-1967, Boelcke et al. 12909 (SI); Ruta Nacional 40, $1 \mathrm{~km}$ N Casa de Piedra, 4707'07"S, 7051'40"W, 710 m, 8-I-2008, Paiaro s.n. 1 (SI); Ruta 40 entre Perito Moreno y Bajo Caracoles, 4707'07"S, 7051'40"W, 703 m, 4-XII-2009, Biganzoli et al. 2368 (SI), entre Telken y Bajo Caracoles, 4707'02'’S, 7051'16' 'W, 710 m, 21-XI-2012, Zuloaga et al. 13975 (SI); Ruta Nacional 40, tranquera Ea. "La Vizcaína", antes de cruzar el río Ecker, 702 m, 10-I-2011, Zavala-Gallo et al. 206 (SI).

CHILE. VII Región de Maule. Pcia. Linares. Depto. Linares: Valle Gualquivilo, Los Cipreses, 36²' $12^{\prime} \mathrm{S}, 70^{\circ} 55^{\prime} \mathrm{W}, 1100 \mathrm{~m}, 23-\mathrm{I}-1961$, Schlegel 3615 (CONC).

\section{Boopis gracilis var. lazulina}

ARGENTINA. Chubut. Depto. Futaleufú: Esquel, aeropuerto, 12-I-1951, Sleumer s.n. (LIL 355221); a 20km de Esquel sobre la ruta a Bariloche, 830 m, 14-XII-1965, Ruiz Leal 24302 (MERL); Ruta 40 de Tecka a Esquel, 4305'07"S, 7055'44"W, 607 m, 6XII-2009, Biganzoli et al. 2403 (SI); Ruta 40, a $50 \mathrm{~km}$ de Tecka poco antes de Arroyo Pescado, 850 m, 5-I-1966, Ruiz Leal 26638 (MERL). Depto. Languiñeo: RN 25 (por ripio) entrada a Aldea Epulef, 12 km S Colán Conhué, 43ำ19'42"S, 6952'25"W, 830 m, 5-XI-2008, Nicola 83 (SI); Camino a Establcimiento 6 Hermanos, entre RN 25 y Ruta Colán Conhué, 25 km N Pampa de Agnia, 43³6'03"S, 6950'39"W, 718 m, 5-XI-2008, Nicola 79 (SI). Neuquén. Camino Paso Limay a Piedra del Águila, sin fecha indicada, 
Diem 3197 (SI). Depto. Collón Curá: RN 237, entre Piedra del Águila y Huayquimil, 4008'37'’S, 70¹4'44'’W, 843 m, 3-I-2011, Zavala-Gallo et al. 62 (SI), de Piedra del Águila a empalme con Ruta Nac. 40, 4007'54'’S, 70¹3'08', W, 790 m, 16-XI-2012, Zuloaga et al. 13795 (SI). Depto. Picunches: Paso Pino Hachado. Primeros Pinos, 1500 m, 8-XII-1990, Roig et al. s.n. (MERL 58316); a 2km al N de Codihue, 11-I-1982, Rossow 811 (BAB); alrededores de Pino Hachado. Borde de ruta, 2024 m, 11-I-2000, Vanni et al. 4457 (CTES). Depto. Zapala: Zapala, 3-XII-1944, Descole 2150 (LIL); Entre Ruta 22 y Bosque Petrificado, 10km al S sobre Ruta Prov. 34, desde el empalme con Ruta Nac. 22 (km 1357), 5-XI-1972, Krapovickas et al. 22484 (CTES); Laguna Blanca, 1420 m, 1-XII-1965, Ruiz Leal 23869 bis (MERL); de Zapala a Primeros Pinos, 7-XII-1981, Cabrera et al. 32860 (SI); Parque Nacional Laguna Blanca. Cerro Mellizos Sur, pie de la ladera NW, 16-XII-1999, Villamil et al. 9081 (SI, BBB); Zapala, 7 km sobre el camino a Primeros Pinos, 3853'41"S, 7008'57"W, 14-XII-2009, Villamil et al. 11218 (SI, BBB); Parque Nacional Laguna Blanca. Bajada del Choique, 2-II-2010, Villamil et al. 11415 (SI, BBB); Parque Nacional Laguna Blanca. Tranquera E, 15-XII2009, Villamil et al. 11284 (SI, BBB); PN laguna Blanca, límite E, cerca de la laguna, 14-XII-2008, Villamil et al. 10990 (SI, BBB); Ruta Prov. 46, Parque Nacional Laguna Blanca, a $23 \mathrm{~km}$ del desvío de la Ruta Nac. 40, El Mirador, 3902'32"S, 70¹8'33"W, 1315 m, 8-XII-2008, Morrone et al. 6187 (SI). Río Negro. Depto. 25 de Mayo: Laguna Cari Laufquen Chica y RP 6, 10 km N empalme RN 23, 41²13'52"S, 69²4'56"W, 834 m, 6-XI-2008, Nicola 91 (SI). Depto. 9 de Julio: Meseta de Somuncurá: Laguna Raimundo, sector NNE, 1350 m, 24-25-II-1980, Cei s.n. (MERL 39921); Meseta de Somuncurá, 1050 m, 15-XI-1968, Ruiz Leal 26158 (MERL), entre Laguna Raimundo y Laguna Chasa, 1270 m, 17-XI-1968, Ruiz Leal 26235 (MERL); El Rincón: a Laguna Raimunda, 680 m, 19-XII-1967, Ruiz Leal 25549 (MERL). Depto. El Cuy: 10km al N de Aguada Guzmán, 6-XI-1972, Correa et al. 4772 (BAB); RP 74, 33,2 km N Chasicó (camino a Aguada Guzmán), 33,2 km N empalme RP6, 4002'38"S, 6854'53"W, 876 m, 6-XI-2008, Nicola 97 (SI). Depto. Pilcaniyeu: 20 km al S de Paso Flores, 20-I-1966, Eskuche 1306 (CTES); Ruta Nac. 40, km 2145, camino de Paso Flores a Bariloche, 40³4'29"S, 7051'15"W, 726 m, 7-XII-2008, Morrone et al. 6166 (SI). 


\section{Boopis graminea}

ARGENTINA. Neuquén. Depto. Aluminé: Pampa de Lonco Luán, 1540 m, 6-I-1968, Ruiz Leal 25852 (MERL); Región del río Aluminé, 1-IV-1902, Asp 136 (SI). Depto Minas: Sierra de Cochicó, cajón de la Botica, 36²21'-22'S, 70³4'-36'W, 2500-2700 m, 29-I-1970, Boelcke et al. 14122 (BAB); Sierra de Cochicó, cajón de la Botica, 36²1'22'S, 70³4'-36'W, 2500-2700 m, 29-I-1970, Boelcke et al. 14122 (SI); Laguna Varvarco Campos, A ${ }^{\circ}$ Enfermera, 36²3'S, 70³7'W, 28-I-1970, Boelcke s.n. (LIL 566441); Extremo norte de la laguna Varvarco Campos, cajón Benítez, paso Puerta Vieja, 36²17'S, 7041'W, 2600-2750 m, 1-II-1970, Boelcke et al. 14315 (BAB); Extremo sur de la laguna Varvarco Campos, 36²17'S, 70³9'W, 28-I-1970, Boelcke et al. 13973 (BAB); Extremo norte de la laguna Varvarco Campos, arroyo Benítez curso inferior, 36² $17^{\prime} \mathrm{S}, 7^{\circ} 39^{\prime} \mathrm{W}, 31-\mathrm{I}-1970$, Boelcke et al. 14221 (SI); Extremo norte de la laguna Varvarco Campos, cajón Benítez, paso Puerta Vieja, 36²17'S, 7041'W, 26002750 m, 1-II-1970, Boelcke et al. 14315 (SI); Laguna Varvarco Campos extremo sur, borde de la laguna, 36²2'S, 70³7'W, 28-I-1970, Boelcke et al. 13973 (SI); Laderas al Oeste del destacamento Cerrillos, al pie del cerro Morado, 2300 m, 11-II-1985, Rossow 2574 (BAB); Cajón de la Botica, 16-II-1985, Rossow 2856 (BAB). Depto. Ñorquín: Copahue. Las Maquinitas, 22-II-1972, Kuhnemann s.n. (LP); Copahue, 2000 m, 23-I1980, Ezcurra 147 (SI); Copahue, alrededores del pueblo, 2000 m, 22-I-2002, Ezcurra et al. 3174 (SI); Copahue, 1900 m, 11-I-1965, Schajovskoy 107/IV (SI); Copahue, cerros al Oeste de las termas, 12-I-1982, Rossow 875 (BAB); Copahue, 1970 m, 14-I1963, Roig s.n. (MERL 22458). Depto. Picunches: Próximo al Paso Pino Hachado, 1800 m, 28-I-1978, Gentili 709 (SI). Depto. Zapala: arriba de los Primeros Pinos cerca de Zapala, 5-II-1976, Fiedler 155 (SI).

CHILE. VIII Región del Bio Bio. Pcia. del Bio Bio: Cupulhué, sin fecha indicada, Volkmann s.n. (SGO 43603). Pcia. Nuble: Termas de Chillán, 36º54'S, 71²3'W, 2150 m, 29-I-1990, Gardner et al. 4635 (SGO); Baños de Chillán, Nieblas, 2000 m, III-1927, Werdermann 1358 (SI). IX Región de la Araucanía. Pcia. Malleco: Dpto. Victoria. El Saltillo, 2 km antes del Paso Pino Hachado, 1800 m, 27-XII-1968, Ricardi \& Marticorena 5684/1845 (CONC). 


\section{Boopis itatiaiae}

BRASIL. Rio de Janeiro. Itatiaia. Abrigo Robouças, 30-XII-1966, Strang 745 \& Castellanos 25739 (LP).

\section{Boopis multicaulis}

ARGENTINA. Neuquén. Depto. Minas: Confluencia de los ríos Pichi-Neuquén y Neuquén, cerro de las Yeguas, 36 35'S, 7045'W, 23-I-1970, Boelcke et al. 13753 (BAB, SI). Depto. Norquín: Trolope, 26-XII-1944, O'Donell 2224 (LIL); Ruta Provincial 22, a Caviahue, 3749'19"S, 7055'08"W, 1520 m, 28-XI-2010, Zuloaga et al. 12591 (SI).

CHILE. VII Región del Maule. Cordillera de Linares, 1873-1874, F. Philippi 2053 (SGO). IX Región de la Araucanía. Pcia. Gral Carrera: Comuna Chile Chico, Reserva Nacional Lago Jeinimeni, 46º3'41"S, 7202'03"W, 1300-1600 m, 15-I-2003, Rojas \& Saldivia s.n. (SGO 153284).

\section{Boopis necronensis}

ARGENTINA. Catamarca. Depto. Tinogasta: Negro Muerto a Reales Blancos, 4300 m, 2-II-1930, Schreiter 6111 (LIL89019, LIL89281). La Rioja. Depto. Vinchina: Laguna Brava. Cordillera, 4200 m, 4-II-1947, J. H. Hunziker 2163 (CORD, SI); Refugio de Mulas Muertas, alrededores del refugio, 15-XII-1996, Biurrun \& Molina 4565 (SI), dentro del corral de pircas, 28 $16^{\prime} 28^{\prime \prime} \mathrm{S}, 68^{\circ} 44^{\prime} 43^{\prime \prime} \mathrm{W}, 4200 \mathrm{~m}, 7-\mathrm{I}-2009$, Donadío et al. 66 (SI).

\section{Boopis patagonica}

ARGEnTINA. Patagonia, 1904, Tessleff 6030 (BAB, SI). Santa Cruz. Depto. Güer Aike: Ea. "La Angelina", 29-I-1976, Mendez 1190 (BAB). Depto. Río Chico: Al sur de Lago Cardiel, 4908'44,8"S, 71²17'15,1"W, 607 m, 12-XII-2006, Bonifacino et al. 2995 (SI); Ruta Nacional 40, al S del lago Cardiel. En un bajo llano de la estepa. Muy ventoso, 4908'46"S, 71¹7'19"W, 579 m, 11-I-2011, Zavala-Gallo et al. 216 (SI).

CHILE. XII Región de Magallanes. Pcia. Última Esperanza: Parque Nacional Torres del Paine, sector Laguna Amarga, al N de la Laguna Blanquillos, 5059'S, 7245' W, 180 m, 23-XI-2001, Domínguez 345 (CONC). 


\section{Boopis pterocalyx}

ARGENTINA. Mendoza. Depto. San Rafael: Sierra del Nevado, portezuelo del Blanco al E-NE del Cerro Perro Atado, 35³7'S, 68³3’W, 2300 m, 11-XII-1973, Boelcke et al. 15819 (SI); subida desde Ruta Provincial 180 a Cerro El Nevado, 35³7'41'’S, 68³1'52'’W, 2440 m, 20-XI-2010; Zuloaga et al. 12253 (SI); Zuloaga et al. 12259 (SI).

\section{Boopis pusilla}

CHILE. Región Metropolitana. Valle del Clarillo, 1200 m, XI-1933, Grandjot s.n. (SGO 057207). Pcia. Santiago: Puente Alto. El Manzano, 18-X-1969, Montero-O. 8296 (LP). Pcia. Cordillera: Cajón del Maipo. El Toyo: ladera exp. norte, 33 44' S, 70²0' W, 1350 m, 22-I-2000, Teillier 4637 (CONC).

\section{Boopis raffaellii}

ARGENTINA. Chubut. Depto. Gastre: Gastre, 1-I-1948, Krapovickas 3823 (BAB); Gastre, 1-I-1948, Soriano 2793 (BAB); A 20km de Gastre, 1270 m, 31-XII-1967, Ruiz Leal 25784 (MERL). Depto. Río Senguerr: Estancia "Pepita". Alto Río Senguerr, 15-II1947, Soriano 2602 (SI). Río Negro. Depto. Pilcaniyeu: Ea. "Pilcañeu", 4-I-1965, Vallerini 543 (BAB).

\section{Calycera calcitrapa}

ARGENTINA. Catamarca. Recolección de germoplasma en Prosopis, sin localidad indicada, 17-I-1990, sin coleccionista indicado (MERL 52825); I-II-1910, P.L. Spegazzini s.n. (SI 160345). Depto. Andalgalá: Campo del Arenal, 4-XII-1917, Jörgensen 1845 (SI). Depto. Belén: Belén, 30-I-1973, Ulibarri 303 (SI). Depto. Santa María: El Cajón. Saladillo, 13-I-1914, Castillón 3339 (LIL, SI). Depto. Tinogasta: Ruta Nac. No 45, entre Alpasinche y Tinogasta, a 35 km de la primera, 29-XI-1997, Biurrun et al. 4938 (SI). La Rioja. Depto. Arauco: Aimogasta, 1-III-1944, A. T. Hunziker 5001 (SI). Depto. Gral. F. Varela: Talampaya, al S de Villa Unión, 20-XI-1982, Kiesling \& Saenz 4270 (SI). Depto. Independencia: Guayapa (15 km al SW de Patquía), 16-IX1947, Lourteig 1195 (SI). Salta. Depto. Cafayate: Cafayate, 16-I-1976, Cabrera et al. 27277 (SI). Depto. Guachipas: Quebrada de Guachipas, XII-1896, C. Spegazzini s.n. (SI 12667). Depto. San Carlos: Amblayo, I-1897, C. Spegazzini s.n. (SI 12666). San Juan. 
Depto. Caucete: al Este de Caucete, médanos, 20-III-2005, Kiesling 10227 (SI); Entre Caucete y Difunta Correa, 12-X-1978, Cabrera et al. 29572 (SI); Al pie de la Sierra Pie de Palo, 2-II-1908, Herb. Min. Agr. 23730 (SI). Depto. Ullún: entre la Estación y el Dique de Ullún, 11-II-1984, Kiesling 4366 (SI); cercanías de la represa, 17-II-1981, Nicora et al. 8110 (SI). Depto. Valle Fértil: San Agustín del Valle Fértil, 23-XI-1986, Haene 475 (SI); Camino a Usno, 13-XI-1980, Kiesling 3075 (SI); huella entre Los Baldes de Astica y el río del Valle por Puesto "La Lata", 20-IV-1995, Biurrun et al. 4085 (SI). Tucumán. Depto. Tafí del Valle: Amaicha al Bañado, 1840 m, 12-II-1913, Castillón 2636 (SI).

\section{Calycera crassifolia var. crassifolia}

ARGENTINA. Buenos Aires. Pdo Cnel. de Marina L. Rosales: Pehuén Co, 14-X-1960, Verettoni 1912 (SI). Pdo. Monte Hermoso: Monte Hermoso, pasando el Camping Americano hacia la boya, desde Monte Hermoso, 12-I-2007, Deginani 2022 (SI). Pdo. Gral. Alvarado: Miramar, I-1949, Burkart 17824 (SI). Pdo. Gral. Pueyrredón: Mar del Plata, 2-II-1937, Nicora 1335 (SI). Pdo. de la Costa: San Clemente del Tuyú, 30-I-1939, Cabrera 4923 (SI). Pdo. Mar Chiquita: Mar Chiquita del Sud, XII-1905, Fablet s.n. (SI). Pdo. Necochea: Necochea, playa, 9-XII-1964, Boelcke et al. 11989 (SI). Pdo. Tres Arroyos: San Francisco de Bellocq, 14-XI-1942, Mollura 1008 (SI). Pdo. Villa Gesell: Mar Azul, a $15 \mathrm{~km}$ al S de Villa Gesell. Autocamping Mar Azul (Avenida Mar del Plata y calle 36),15-I-2008, Zanotti \& Donadio 144 (SI). Chubut. Depto. Biedma: Puerto Madryn, en las dunas playeras enfrente del CENPAT, 6-XI-2006, Zanotti 61 (SI); Penísula Valdés, I-1971, Cabrera 21202 (LP). Río Negro. Depto. Adolfo Alsina: Embouchure der Río Negro, II-1912, Hauman-Merck 259 (SI).

URUGUAY. Maldonado: Piriápolis, I-1911, Hicken 181 (SI). Rocha: Isla Gorriti y Cabo Polonio, I-1922, Felippone 4941 (SI); La Pedrera, I-1981, Cabrera 32314 (SI); Barra de Valizas. Costa de la desembocadura del $\mathrm{A}^{\mathrm{o}}$ Valizas, camino a Cabo Polonio, 3 m, 19-II-2013, Zavala-Gallo 245 (SI). San José: Estancia Pascual [Playa Pascual], 28II-1931, Herter 86707 (SI).

\section{Calycera crassifolia var. spinulosa}

ARGEntinA. La Pampa. Depto. Chical-Co: About $5 \mathrm{~km} \mathrm{~S}$ of Cerro Centinela, 1-I1982, Pedersen 13329 (LP). Depto. Puelén: Puelén, III-1932, Durango s. n. (SI 
107617). Mendoza. Depto. Malargüe: Sa. del Nevado, portezuelo de los Cos. de Mondaca al SE del Co. Negro, 1760 m, 12-XII-1973, Boelcke et al. 15857 (SI). Depto. San Carlos: Cerro Guanacos, 2600 m, I-1921, Carette 393 (SI). Depto. San Rafael: Ruta Provincial 180 entre cruce de la Ruta Provincial 184 y El Nihuil, 3457'05's, 68'40'15'’W, 1300 m, 20-XI-2010, Zuloaga et al. 12271 (SI). Depto. Tupungato: Los Álamos, 2300 m, 27-XII-1949, Paci \& Melis 49 (LIL). Neuquén. Depto. Confluencia: Plaza Huincul, 3-XII-1943, Platnick 54 (LP). Depto. Pehuenches: Along route \# 40 generally 1-2 kms N of Barrancas, 3200 ft, 14-I-1985, King \& Heinz 9421 (SI). Depto. Añelo: Ruta Provincial 17, entre Aguada Pichana y Añelo, 38²6'05'’S, 6857'20'’W, 420 m, 1-XII-2010, Zuloaga et al. 12671 (SI). Depto. Collón Curá: Ruta Nacional 237, entrada Dique Piedra del Águila, 3,6 km S Piedra del Águila, 4004’27’S, 7007’18’’ W, 544 m, 16-XII-2008, Nicola 115 (SI). Río Negro. Depto. Avellaneda: Chelforó, 6-XII1981, Cabrera et al. 32822 (SI). Depto. Gral. Roca: Paso Córdoba, en médano al pie de las bardas, 6-XI-1972, Bacigalupo \& Nicora s.n. (BAA 11619, SI).

\section{Calycera eryngioides}

CHILE. IV Región de Coquimbo. Pcia. Limarí: Cordillera Ovalle.- La Hualtata, 2300 m, 29-X-1949, Jiles-P. 1558 (LIL). VI Región del Libertador Gral. Bernardo O'Higgins. Pcia. Cachapoal: Cordillera de la Compañía, XI-1853, R. A. Philippi s.n. (SI 12662). Región Metropolitana. Pcia. Santiago: Piuquencillos, Valle del Río Colorado, 2000-3500 m, 8-10 -XII-1942, Pisano-V. et al. 1646 (CONC).

\section{Calycera herbacea var. herbacea}

ARGENTINA. Mendoza. Depto. Malargüe: altos valles de El Sosneado, 2200 m, 19-II1942, Burkart et al. s.n. (SI 14321). Depto. San Carlos: Reserva "Laguna del Diamante". Quebrada del Paso Cruz de Piedra. Camino al refugio, 34¹3'50'S, 69²5'19'’W, 2646 m, 6-XII-2009, Zavala-Gallo 21 (SI); Las Ánimas, río Salado, 20II-1946, Rossi 427 (LIL). Depto. San Rafael: Hotel Termas del Sosneado, 3446'12'’S, 7003'33'’W, 2180 m, 22-XI-2010, Zuloaga et al. 12381 (SI); Sa. del Nevado, zanjón del Plateado frente a los Cos. Morados, 3540'S, 68²3'W, 1650 m, 8-XII-1973, Boelcke et al.15732 (SI); Termas de La Huencó, 1950 m, 30-XII-1949, Sleumer 396 (LIL); Los Molles, en la cuesta del "cuchillo", ca. 2200-2300 m, 30-XII-1949, Sleumer 596 (LIL). Depto. Tupungato: XI-1981, Wingenroth et al. 381 (SI). 
CHILE. Región Metropolitana. Pcia. Cordillera: Along the Embalse El Yeso, near the upstream end, along the access road $6 \mathrm{~km}$ upstream from the dam. Rock fields and alluvial fans, 33³5'40"S, 70¹0'15"W, 2520 m, 14-I-1993, Taylor \& Gereau 10924 (CONC, SI); Cajón río Maipo. Confluencia con río Cruz de Piedra, 3406’ S, 7003’ W, 2800 m, 20-I-2000, Teillier 4539 (CONC). VII Región del Maule. Pcia. Talca: Laguna del Maule, orilla NO, 3600’S, 70³3’W, 2040 m, 18-I-1995, A. Marticorena et al. 250 (CONC).

\section{Calycera herbacea var. sinuata}

ARGENTINA. Catamarca. Depto. Andalgalá: am Fusse der Capillitas [al pie de Capillitas], I-1891, Berg 292 (SI); Cerros del Aconquija, 4500 m, 20-II-1917, Jörgensen 1676 (SI); Cuesta de Minas Capillitas, 11-III-1961, Cabrera et al. 14113 (LP); Rio Potrero sup., 3900 m, 28-II-1951, Sleumer 1908 pp (LIL); Quebrada del río Pisauil, 2500 m, 20-XI-1948, Filipóvich Urquiza 172 (LIL); Capillitas; Las Negritas, 3000 m, 2-III-1952, Sparre 9786 (LIL). Depto. Antofagasta de la Sierra: Real Grande. Crece en las laderas de la quebrada, 4100 m, 25-I-1987, Haber 92 (SI). Depto Belén: al pie de la cuesta de Randolfo, 3200 m, 23-II-1981, Cabrera et al. 32470 (SI); Al pie de la cuesta de Randolfo, 3200 m, 23-II-1981, Cabrera et al. 32470 (SI). Depto. Santa María: Quebrada de Santa María a San Antonio, I-1915, Castillón s.n. (LIL 89446). Depto. Tinogasta: La Crispita a Vallecito, 3100 m, 6-II-1930, Schreiter 6254 (LIL 89293A, B). Jujuy: Depto. Cochinoca: Miraflores, INTA, I-1975, Cabezas 48 (SI); Abra Pampa, 3700 m, 21-II-1929, Venturi 9358 (SI); Abra Pampa, Cerro Huancar, 3500 m, II-1963, Cabrera et al. 15426 (LP); Casabindo, 18-I-1948, Cabrera 9316 (LP); Espinazo del Diablo, 3900 m, 17-I-1971, sin datos de coleccionista (LIL 566443, BAA 7108); Abra Pampa, 3700 m, 21-II-1929, Venturi 9358 (LIL). Depto. Humahuaca: Tres Cruces, 21-I1976, Cabrera et al. 27452 (SI); Mina Aguilar. Cerro Aguilar, arriba de la Mina, ca. Toma de Agua, 4670-4730 m, 4-III-1983, J. H. Hunziker et al. 10583 (SI); Mina Aguilar, Veta, 4800 m, 14-I-1972, Ruthsatz 266 (SI); Subida al cerro Aguilar, 4700 m, 17-I-1953, Sleumer 3437 (LIL). Depto. Rinconada: Mina Pirquitas, Cerro Granadas Ladera Sud, III-1970, Fabris \& Zuloaga 7739 (LP); Mina Pirquitas, 4300 m, 1-III-1964, Schwabe et al. 652 (LP), Schwabe et al. 1114 (LP), camino a Cusi-Cusi, 1-III-1964, Schwabe et al. 1154 (LP); Alrededores lag. Vilama, 4600 m, 5-12-III-1964, Schwabe et al. 1026 (LP). Depto. Susques: Cerro Tuzgle, 4700-4900 m, 10-II-1946, Cabrera 9101 
(LP). Depto. Tilcara: Maimará: Laguna Colorada, 4000 m, 22-I-1906, Lillo 4894 (SI); Tilcara: arriba de San Gregorio, 3900 m, 27-I-1953, Sleumer 3585 (LIL). Depto. Valle Grande: subida a Cerro Amarillo, 3000 m, 2-I-1978, Kiesling et al. 1593 (SI); Caspalá, cumbres, 1-III-1940, Burkart \& Troncoso 11872 (SI). La Rioja. Depto. Famatina: Cueva de Pérez, camino a la mina La Mexicana, 2859'50"S, 67²43'59"W, 3818 m, 10-I2009, Donadio et al. 121 (SI); Sierra de Famatina, La Mesada, 3500 m, 29-IV-1951, Sparre 8881 (LIL); Entre Los Corrales y Cueva de Pérez. Cueva de Pérez, 3800 m, 13 I-1976, Cabrera et al. 27169 (SI); camino a la mina La Mexicana. Cueva de Pérez, 3750 m, 20-II-1986, Kiesling et al. 6394 (SI), 3800 m, 26-IV-1951, Sparre 8807 (LIL 423480, LIL 423251). Mendoza. Depto. Las Heras: Puente del Inca, 11-I-1914, Sanzin 345 (SI), 20-I-1908, Hauman-Merck 120 (Herb. Andino) (SI), 2750 m, 18-I-1969, Meyer 22877 (LIL), 2800 m, 3-I-1950, Mellis \& Paci 181 (LIL), 24-II-1945, Lourteig 659 (LIL), 2700 m, 19-XII-1946, Sparre 1510 (LIL); Punta de Vacas, 4-II-1934, Burkart 9311 (SI); Paramillo de Uspallata, 6-IV-1945, Covas 18527 (SI), 3000 m, 6-V1945, Semper 676 (LIL), 2900-3000 m, 21-XI-1937, Ruiz Leal 3480 (LIL); Cruz de los Paramillos, 3000 m, 3-I-1950, Sleumer 384 (LIL), 3000 m, 3-I-1958, Cáceres \& Paci 273 (LIL), 3000 m, 21-I-1944, O'Donell 1154 (LIL), 3000 m, 3-I-950, Barkley 20Mz010 (LIL), 2900 m, 24-I-1950, Cuezzo \& Balegno 1892 (LIL), (Cumbre), 3000 m, 25-I-1950, Cuezzo \& Balegno 1977 (LIL), Cuezzo \& Balegno 2006 (LIL); Quebrada del Toro (El Centinela), 2000 m, 25-I-1950, Cuezzo \& Balegno 2002 (LIL), 1-I-1948, Garcia 476 (LIL 234079); Uspallata, 6-I-1941, Castellanos s.n. (LIL 18481); Uspallata Mountains, along route 52 at Cruz de Paramillos, 29,5 km east of Uspallata, 32²8'56"S, 6906'49"W, 2959 m, 21-I-2002, Bayer \& Chandler ARG-02062 (LP); Garganta del Diablo, 2000 m, 21-XII-1949, Araque-M. \& Barkley s.n. (LIL 334382). Depto. Tunuyán: El Guindo (valle del arroyo Manzano), 10-III-1945, Covas 6980 (SI); 16,8 km W of Manzano Histórico, W of Tunuyán, along Arroyo Grande, 33³6'33"S, 69³1'02"W, 2894 m, 20-I-2002, Chandler \& Bayer 1104 (LP); Valle del Alto Tunuyán, en los cerros cercanos al Real de Contreras, 27-XII-1933, Ruiz Leal 2007 (LIL); Mesillas del Rincón Colorado, 7-III-1933, Ruiz Leal 1311 (LIL); Camino al Paso del Portillo, Cuesta de los Afligidos, 24-XII-1933, Ruiz Leal 1873 (LIL); Quebrada Capitán Lemos, 2300 m, 1-II-1950, Palacios \& Cuezza 4427 (LIL), Palacios \& Cuezza 4471 (LIL). Depto. Tupungato: Tupungato, 1910, Hauman-Merck s.n. (SI 12664). Salta. Depto. Cachi: Valle Encantado 5 km del desvío de la Cuesta del Obispo, 28-IV-1977, 
Abbiatti \& García 4520 (LIL); Cuesta del Obispo, 3720 m, 21-I-1947, Gardera-Romero s.n. (LIL 186935). Depto. Chicoana: Camino a Cachi, Piedra del Molino, 3500 m, 27III-1979, Cabrera et al. 30739 (SI). Depto. La Poma: Mina Esperanza, 12-II-1960, Hernández 18 (LP). Depto. Los Andes: desvío de la ruta 51 hacia Viaducto La Polvorilla, 241' $15^{\prime \prime S}, 66^{\circ} 23^{\prime} 43^{\prime \prime} \mathrm{W}, 3950 \mathrm{~m}, 13-\mathrm{II}-2007$, Zuloaga et al. $9306 b$ (SI); Viaducto Polvorilla, 26-I-1940, Schulz 2671 (LP). Depto. Rosario de Lerma: Puente del Diablo, 5 km al sur de Tres Cruces, III-1970, Fabris \& Zuloaga 7811 (LP). Depto. San Carlos, Camino a Mina Don Otto, 6 km de ruta prov. 33, 23-II-1987, Nicora et al. 9082 (SI); Camino de Abra de Fundición a Nazareno, 22²7'49"S, 650805"W, 4370 m, 21-II2011, Zuloaga et al. 13180 (SI). Depto. Santa Victoria: Ruta Prov. 145, de Nazareno a

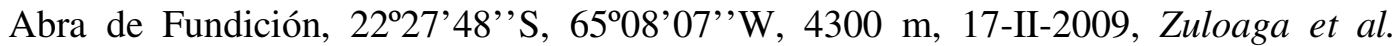
10882 (SI). San Juan. Depto. Calingasta: de confluencia a Alojo Las Minitas, 25003000 m, 17-II-1988, Kiesling et al. 6856 (SI); Manantiales, 7-XII-1994, Kiesling 8569 (SI), I-1972, Fabris \& Zuloaga 8464 (LP), IV-1972, Volponi 256 (LP); Sa. del Tontal, 3500-3700 m, 22-I-1987, Kiesling \& Meglioli 6550 (SI). Depto. Iglesia: Camino a Valle del Cura. Quebrada de la Vicuñita, 3200 m, 23-I-1981, Kiesling 3283 (SI); 2 km al N de Las Vicuñitas, 3000'53"S, 69³5'05"W, 3792 m, 22-II-1998, Herrera \& Jiménez 880 (SI); Reserva de San Guillermo: camino a Los Caserones, vega Los Corrales, borde de la vega, 23-II-1981, Nicora et al. 8324 (SI) Reserva de San Guillermo, Río Macho Muerto, a más o menos 7km del refugio, 14-I-1983, Nicora et al. $8507 b$ (SI); Quebrada del Agua Negra, 3900 m, 10-I-1976, Cabrera et al. 27032 (SI), 15-II-1986, Kiesling et al. $6188 b$ (SI) Ruta Nacional 150, Paso de Agua Negra, pasando Arrequintín hacia límite con Chile, 30²1'46"S, 6940'40"W, 3600 m, 11-II-2011, Zuloaga et al. 12771 (SI); sobre ruta nacional 150, a $3 \mathrm{~km}$ al Sur de Vialidad Nacional, Pircas Negras, a 59,4 km al oeste de Las Flores, 30¹9'49"S, 6943'52"W, 3869 m, 27-II-1998, Herrera \& Jiménez 933 (SI). Depto. Ullún: del refugio de la Ea. "Don Carmelo" hacia el W, con. En la quebrada de la Sa. del Tigre, 3055'01"S, 6908'18"W, 3455 m, 10-II-2000, Kiesling et al. 9430 (SI). Tucumán. Depto. Chicligasta: Estancia Santa Rosa, 4000 m, 13-I-1927, Venturi 4720 (SI, LIL 89451, LIL 89388); Estancia Las Pavas, Puesto El Bayo, 3200 m, 13-III-1924, Venturi 3132 (SI, LIL). Depto. Lules: Cumbre de Mala Mala, 3400 m, 6-IV-1904, Lillo 3432 (SI), Malamala, 21-XII-1907, Castillón 127a (LIL). Depto. Tafí del Valle: sin localidad indicada, II-1913, Castillón 2714 (LIL); Cuesta del Infiernillo, 3-XII-1960, Burkart 22075 (SI); Infiernillo, 1-II-1947, O'Donell 
4604 (LIL), 3100 m, 23-II-1949, Sparre 5982 (LIL), 3100 m, 22-III-1951, Sparre 8643 (LIL), Carapunco; Infiernillo, 3000 m, 29-I-1952, Sparre 9406 (LIL); Tafí, 16-I-1908, Lillo 8309 (SI); Calchaquíes, 4200 m, 29-XI-1965, Hernández 10 (LP); Quebrada del Barón, Los Planchones, 3300 m, 7-II-1958, Fabris 1376 (LP); Cerro Negrito- Cumbres Calchaquíes, 4500 m, 17-II-1990, Ayarde 344 (LIL); Calchaquíes; Quebrada Honda, 3100 m, 24-I-1952, Sparre 9264 (LIL); Valles Calchaquíes: Amaicha, II-1914, Castillón 3431 (LIL); Nevados del Aconquija: Cerro Áspero, falda O, 4000-4400 m, 25II-1941, Rohmeder s.n. (LIL 63987); Cumbres Calchaquíes. El Negrito, 4700 m, 3-II1903, Lillo 3075 (LIL); Campo de la Flora (San José), 3500 m, XII-1931, Schreiter 6774 (LIL); Cumbres Calchaquíes; El Alazán, 4100-4200 m, 23-III-1951, Sparre 8579 (LIL); Cumbres Calchaquíes, 27-XII-1913, Castillón 16247 (LIL); Las lagunas del Negrito, 4300 m, 6-II-1926, Schreiter 4403 (LIL 89286). Depto. Tafí Viejo: La HoyadaQda. del Zarzo- Sierra Aconquija, 4580 m, 20-XII-1933, Peirano s.n. (LIL 89170). Depto. Trancas: Cumbres Calchaquíes, 27-XII-1913, Castillón s.n. (SI, ex LIL 16247). CHILE. II Región de Antofagasta. Pcia. El Loa: Tatio, 22²1'S, 6802'W, 4300 m, 9II-1969, Martin 458 (SI); Camino a Portezuelo del Cajón. Cerro Toco. Ladera N, 2255'S, 6746’W, 4700-4850 m, 3-IV-1997, Arroyo et al. 97034 (CONC).

\section{Calycera horrida}

ARGENTINA. Neuquén: Norquín, Cerro Huaile, ladera NO, 3704'22"S, 7007'14"W, 2550 m, 8-I-2001, Biganzoli 1186 (SI); Pehuenches, inter Aguas Calientes et Laguna del Tromen, 2125 m, 24-II-1888, Kurtz 6142 (SI); Pque. Prov. Tromen, a 45 km del cruce de ruta 40, 2100 m, 28-XII-1999, Ezcurra et al. 2592 (BCRU).

\section{Calycera leucanthema}

CHILE.VII Región del Maule. Pcia. Curicó: Hacienda Monte Grande, 700 m, XII1924, Werdermann 552 (SI); Cordillera de los Andes - Curicó, II-1896, Rivera s.n. (SI 12655). Pcia. Linares: East and a little south of Linares along the Río Ancoa, along the road to Melado and Medina $38.2 \mathrm{~km}$ upstream from the intersection with the road to Peñasco, 3550-52’S, 71¹0-20’W, 750-900 m, 21-I-1993, Taylor \& Gereau 10981 (CONC, MO). Pcia. Talca: Cordillera de Talca, El Picazo, 28-XII-1936, Barros 2982 (SI); Roadside $10 \mathrm{~km}$ south of Vilches Alto and $1 \mathrm{~km}$ south of village of Vilches, 35³4’S, 71¹1’W, 700 m, 19-I-1991, DeVore 1486 (CONC). VIII Región del Bío Bío. 
Pcia. Nuble: Cordillera de Chillán, XII-1855, R. A. Philippi s.n. (SI 12656); Off Route N59 between Yungay and Pemuco, east side of road about $2 \mathrm{~km}$ south of ChillanYungay bridge, 3700'44',S, 7204'15'’W, 232 m, 7-XII-2010, Johnson \& Zavala 10105 (BRY, SI); Los Lleuques, río Diguillín, Los Molinos, 3652'S, 71³8’'W, 670 m, 21III-2001, Baeza \& Finot 3715 (CONC); Chillán: Cordillera de los Andes:El Cerrillo, 36²51'40,8"S, 71³4'41,5"W, 1244 m, 26-XII-2003, Gardner \& Knees (GAK) 6798 (CONC); San Antonio. Al Noroeste de Yungay, 3701'S, 7200'W, 275 m, 5-II-1992, Klee s.n. (CONC 118622); Recinto, up N from Hotel Lleuques toward small hill.

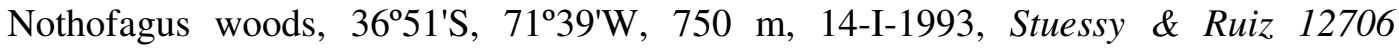
(CONC).

\section{Calycera pulvinata}

ARGENTINA. Catamarca. Depto. Andalgalá: Río Potrero superior, 3900 m, 28-II1951, Sleumer 1908 (LIL); Andalgalá, En las cumbres: C. Aconquija, 4500 m, 20-II1917, Jörgensen $1676 b$ (SI); subida al cerro Yutuyaco desde Capillitas, 3800 m, 3-III1952, Sleumer 2717 (LIL). Depto. Tinogasta, Reales Blancos, 4000 m, 2-II-1930, Schreiter 6114 (LIL). Jujuy. Depto. Dr. Manuel Belgrano: Refugio Militar, 2402'13'’S, 6542'58'’W, 4610 m, 26-I-2012, Zanotti \& Suescún 258 (SI). Depto. Tumbaya: de Purmamarca al Abra de Lipán, 4000 m, 11-III-1982, Kiesling et al. 3534 (SI); en el camino entre El Moreno y el Abra de Pibes, 3600 m, 7-I-1953, Sleumer 3306 (LIL). Salta. Depto. San Carlos: Cerros del Cajón, 4500 m, 28-II-1914, Rodriguez 1315 (SI). Depto. Los Andes: ca. 15 km al N de Abra del Gallo, ruta Mina La Poma.- Santa Rosa de los Pastos Grandes, 4400 m, 2-XII-1986, Charpin \& Novara 20773 (SI). Tucumán. Depto. Tafí del Valle: Cumbres Calchaquíes, Huaca Huasi en lomadas, 26ํํ'S, 6544'W, 4300 m, 13-III-1984, Gómez-Sosa \& Múlgura 173 (SI); habita los valles de "El Pelado", 4000 m, 17-III-1912, Rodriguez 438 (LIL, SI); Cerro Negrito, 4600 m, 9-II-1958, Fabris 1433 (LP); Cumbres Calchaquíes, I-1912, Castillón 2204 (LIL), 4400 m, 31-I-1907, Lillo 5518 (LIL), EL Alazán, 4200 m, 23-III-1951, Sparre 8598 (LIL 423612, LIL 423618), Quebrada del Matadero, 4200 m, I-1963, de la Sota 2720 (LP), El Negrito, 4700 m, 3-II-1903, Lillo 3075pp (LIL); Cerro Muñoz, 3900 m, I1907, Lillo 5919 (LIL), Quebrada Honda, 4000 m, 23-II-1905, Lillo 4189 (LIL, SI); Cumbre del Chaquivil, 11-I-1945, Olea 219 (LIL); Campo del Alazán a Las Lagunas, 4000 m, 6-II-1926, sin datos del coleccionista (LIL 89287). 
BOLIVIA. La Paz: Pacajes, Rosario, 6-I-1921, Shepard 233 (LIL).

PERÚ. Junín. Pcia. Huancayo: Huancayo, 4200 m, 19-XII-1960, Kunkel 845 (LP).

\section{Calycera sessiliflora}

CHILE. IV Región de Coquimbo. Pcia. Limarí: Tulahuen, 1200 m, 10-X-1948, Jiles-

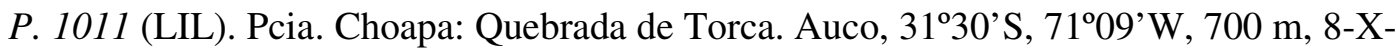
1993, Arancio 92852 (CONC). V Región de Valparaíso. Pcia. San Felipe de Aconcagua: Inter Mendoza et Santa Rosa, 1868-69, R. A. Philippi s. n. (SI 12672). Pcia. Marga Marga: Las Vizcachas, ca. 10 km from La Dormida; steep, rocky, west-facing slopes, 1900 m, 8-XII-1938, Goodspeed 16784 (SI), cuesta La Dormida vicinity, off

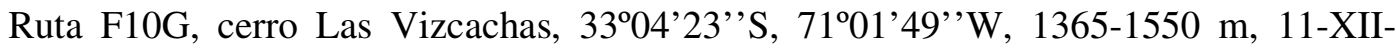
2010, Johnson \& Zavala 10-153 (BRY, SI). Región Metropolitana. Pcia. Chacabuco: Cordillera de Tiltil, XI-1865, sin datos del coleccionista (SI 20196). Pcia. Santiago: Comuna de La Reina. Quebrada de Ramón, 33²6'S, 70³0’W, 1600 m, 10-IX-2000, Tomé-R. 64 (CONC), Salto de Ramón, 1550 m, XI-1933, Grandjot s.n. (SGO 057208, SI 20197); Salto de Conchalí, IX-1876, R. A. Philippi s.n. (SI 12671); Santuario de la Naturaleza "Yerba Loca". Laderas del O del estero Manzanito, 3321'S, 70²0'W, 1550 m, 6-II-1999, Arroyo \& Humaña 995311 (CONC), Cordón entre estero Manzanito y estero Yerba Loca, 33²0’S, 70²1'W, 1530 m, 20-X-1999, Arroyo \& Humaña 993603 (CONC), Ladera rocosa al NE de la Qda. del Lunes, 33²0'S, 70¹8'W, 1990 m, 15-XI1999, Arroyo et al. 995043 (CONC).

\section{Calycera sympaganthera}

CHILE. IX Región de la Araucanía. Pcia. Malleco: Vega de Chanleo. Cordillera de Nahuelbuta, I-1877, R. A. Philippi s.n. (SI 12665); Angol, Parque Nacional Nahuelbuta, ruta Cerro Anai, 37²4'28"S, 7300'04"W, 1315 m, 20-I-2008, Zapata \& Sede 376 (SI); Parque Nacional Nahuelbuta; Sector Piedra del Águila, 3747's, 7259’W, 1300 m, 9II-1991, De Vore \& Baeza 1611 (CONC); Parque Nacional Nahuelbuta. Near to main camping area, 37\%48'26"S, 7300'60"W, 1232 m, 8-II-2003, Gardner et al. DCI $n^{\circ} 589$ (CONC); Parque Nacional Nahuelbuta. Camino a Cerro Anay. Sendero para subir al cerro, 3748'07"S, 7300'52"W, 1239 m, 17-II-2012, Larsen \& De Gennaro 206 (SI). 


\section{Gamocarpha alpina}

ARGENTINA. Neuquén. 3-III-1974, Fiedler 139 (SI). Depto. Aluminé: Volcán Batea Mahuida (Antenas), 3850'55.3"S, 71¹1'44.1"W, 4-II-2010, Villamil et al. 11470 (SI, BBB); Parque Nacional Lanín. Co Bolsico del Diablo, 1500 m, 17-I-1970, Eskuche 957 (SI). Depto. Chos Malal: Ruta Provincial 37, entre Agua Caliente y Los Barros, 3702'43"S, 7002'38"W, 2300 m, 25-XI-2010, Zuloaga et al. 12461 (SI). Depto. Minas: Arroyo Memanque, Cordillera del Viento, 18-IV-1912, Pastore 122 (SI); Reserva Prov. Domuyo, camino geotérmico hacia la picada a la base $S$ del Domuyo, a aprox. $3 \mathrm{~km} \mathrm{del}$ río Covunco, 2300 m, 27-I-2001, Ezcurra 3156 (SI); Cordillera del Viento, filo norte, 36² 48'S, 70³1'W, 2550 m, 2-II-1964, Boelcke et al. 11545 (SI). Depto. Norquín: Copahue. Las Maquinitas, 22-II-1972, Kuhnemann s.n. (LP); Caviahue, sendero a la Cascada Escondida, 24-I-2006, Winter 2 (SI), 1670 m, 21-II-2006, Zanotti et al. 4 (SI); Copahue, $1 \mathrm{~km}$ antes del pueblo, 14-I-2004, Troiani \& Steibel 15849 (SI, SRFA); Copahue, 25-II-2004, Troiani \& Steibel 16036 (SI, SRFA); Cerca de Caviahue. Suelo

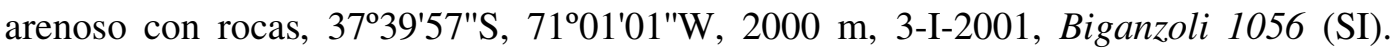
Depto. Picunches: Pino Hachado, en el hito, 2800 m, 6-III-1939, Burkart 9637 (SI).

CHILE. VIII Región del Bío Bío. Pcia. Bío Bío: Paso Pichachén, hito fronterizo, 37²7'S, 71 ${ }^{\circ} 08^{\prime} \mathrm{W}, 2100$ m, 23-I-2001, Baeza et al. 2976 (CONC). Pcia. Nuble: Termas de Chillán, desde Valle Hermoso a Aguas Calientes, 36º54'S, 71²23'W, 1600 m, 20-III2001, Baeza \& Finot 3702 (CONC), Termas de Chillán, 3654'S, 71²4'W, 1700, XII2001, Cavieres et al. 3741 (CONC), Chillán: Cordillera de los Andes: Termas de Chillán. Slopes below thermal springs, 36054' 19.4"S, 71²4'13.4"W, 907 m, 27-XII2003, Gardner \& Knees 6838 (CONC), Baños de Chillán, Nieblas, 2000 m, III-1927, Werdermann 1345 (LIL, SI), Baños de Chillán, 1800 m, 10-II-1949, Kausel 2770 (LIL), Supra baños de Chillán, I-1877, sin datos de coleccionista (SI 12702); Andes de Chillán i Talca, sin fecha indicada, Fuentes s.n. (SI 12704). IX Región de la Araucanía. Pcia. Malleco: Volcán Lonquimay, 1600 m, 25-III-1954, Sparre \& Constance 10903 (CONC).

\section{Gamocarpha angustifolia}

ARGENTINA. Neuquén. Depto. Minas: valle superior del A Atreuco, $36^{\circ} 45^{\prime} \mathrm{S}, 70^{\circ}$ 33’W, 2010-2050 m, 2-II-1964, Boelcke et al. 11519 (SI); sierra de Cochicó, cajón de la Botica, 36 21'-22' S, 70 34'-36' W, 2500-2700 m, 29-I-1970, Boelcke et al. 14092 
(SI); extremo norte de la laguna de Varvarco Campos, cajón Benítez, paso Puerta Vieja, $36^{\circ} 17^{\prime} \mathrm{S}, 70^{\circ} 41^{\prime} \mathrm{W}, 2600-2750 \mathrm{~m}, 1-\mathrm{II}-1970$, Boelcke et al. 14307 (LIL, SI).

CHILE. VIII Región del Bío Bío. Ñuble: Termas de Chillán. Potrero El Sol, 2300 m, 6I-1949, Pfister s.n. (CONC 8768); Termas de Chillán, 2000 m, 4-I-1973, Zöllner 6618 (CONC); Termas de Chillán, 16-I-1986, Pedersen 14242 (SI).

\section{Gamocarpha dentata}

ARGENTINA. Neuquén. Depto. Aluminé: C ${ }^{o}$ Ruca Choroi, lago Ruca Choroi, 2000 m, 20-XII-1969, Eskuche 958 (SI). Depto. Ñorquín: Copahue, camino al Paso Copahue, 2100-2200 m, 9-II-1994, Ezcurra et al. 906 (BCRU), Copahue, serranías al NW del

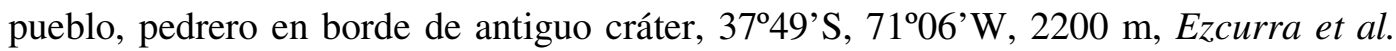
3209 (BCRU); Parque Provincial Caviahue-Copahue, camino a 2 km de Copahue, 2050 m, 29-I-2000, Ezcurra et al. 2902 (BCRU). Depto. Picunches: Pino Hachado, en el hito, 2800 m, 6-III-1939, Burkart 9647 (SI); Ruta Prov. 13 de Zapala a Aluminé, pasando Primeros Pinos. Camino a la cumbre del cerro Atravesada, 2200-2400 m, 12-XII-1999, Ezcurra et al. 2527 (BCRU).

CHILE. VIII Región del Bío Bío. Pcia. Bío Bío: Paso Pichachén, hito fronterizo, 37²7' S, 7108' W, 2100 m, 23-I-2001, Baeza et al. 2975 (CONC); Faldeos del volcán Antuco, cerca de Los Barros, 1500 m, 22-I-1969, Ricardi \& Marticorena 5792/1953 (CONC). IX Región de la Araucanía. Pcia. Malleco: Victoria. Paso Pino Hachado, 1800 m, 10-I-1965, Pfister s.n. (CONC 8128, LP).

\section{Gamocarpha gilliesii}

ARGENTINA. Mendoza. Depto. Malargüe: Atuel Valley half-way between Campamento Atuel and Laguna Atuel, 2800 m, 30-XII-1955, Böcher, Hjertimg \& Rahn 1955 (LP); Del Mirador de Valle Hermoso a Las Leñas, 3505'36"S, 7006'34"W, 2600 m, 22-XI-2010, Zuloaga et al. 12374 (SI); Pasando Castillos de Pincheira, 35³2'43"S, 6956'44"W, 2150 m, 21-XI-2010, Zuloaga et al. 12324 (SI); Portezuelo del Choique, 36²2'29"S, 6948'14"W, 2450 m, 31-I-2003, Prina et al. 1918 (SI, SRFA). Depto. San Carlos: Reserva Pcial. Laguna del Diamante, ladera de derrubios frente al Refugio Cruz de Piedra, del otro lado del arroyo, 34 ${ }^{\circ} 14^{\prime} 20.4^{\prime}$ 'S, 69²7'25.3' ' W, 2606 m, 11-XII2009, Zavala-Gallo 29 (SI), A ' El Gaucho, 34¹2'16'’S, 69³1'28'’ W, 3422 m, 6-II2010, Zavala-Gallo 52 (SI). Depto. San Rafael: Valle del río Atuel, Planta Mina de 
Azufre a Laguna Atuel, $80 \mathrm{~km} \mathrm{~W}$ de El Sosneado, 25-I-1963, Boelcke et al. 10227 (LP); El Sosneado.- Los Enganches, 3200 m, 24-I-1972, Lagiglia 1166 (LP); Al Occidente de la Cuchilla de la Tristeza, cerro China Muerta, 23-II-1953, Castellanos s.n. (LIL 476795); Valle del río Atuel, Planta Mina de Azufre a Laguna Atuel, 80 km W El Sosneado a laguna de Atuel, 25-I-1963, Boelcke et al. 10227 (SI); Vega del Loro, 2400 m, I-1921, Carette 391 (SI); Tres Esquinas, 2200 m, I-1921, Carette 392 (SI); El Angulo- El Sosneado, 3450 m, 19-I-1993, H. A. L. 8360 (SI); Valle de El Sosneado, 2250 m, 19-II-1942, Burkart et al. s.n. (SI 14434); N de El Sosneado, camino secundario al N-NE del cruce con Ruta Pcial. 220 (que bordea el río Atuel), 3444'00"S, 69³9'18"W, 2694 m, 20-I-2006, Pozner et al. 559 (SI). Depto. Tunuyán: Los Chacayes, Cuesta de los Aflijidos, 33³6'46"S, 69³1'24"W, 2858 m, 16-I-2007, Méndez 10121 (SI, MERL), Real de las Yaretas, 3337'15.8"S, 69³4'03.2"W, 3626 m, 14-I-2007, Méndez 9992 (SI, MERL).

\section{Gamocarpha selliana}

ARGENTINA. Chubut: río Aysén, XII-1900, Buermeister 963 (SI). Depto. Escalante: Comodoro Rivadavia- Pico Salamanca- ruta 3, 12-XII-1970, Crespo \& Troncoso 1763

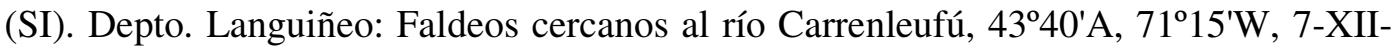
1946, Soriano 2237 (SI). Depto. Río Senguerr: Pampa de Chalía, Ea. La Media Luna, 3XII-1981, Villamil et al. 2206 (SI). Neuquén. Depto. Ñorquín: Puerta del Trolope. Caviahue, 3749'28"S, 7057'52"W, 5-XII-2006, Sérsic \& Cosacov s.n. (SI 069096). Río Negro. Depto. Bariloche: Arroyo Cascada (Bariloche a Catedral), 24-XII-1944, Meyer 7603 (LIL); Cerro Catedral, al pie, 1000 m, 14-I-1946, Montiel s.n. (LIL 246769); San Carlos de Bariloche (lago Nahuel Huapi, 41 s. Br.) Cerro Gutierrez, 1400 m, 25-II1905, Buchtien s.n. (SI 12698); Lago Moreno, 800 m, 15-I-1942, Neumeyer 598 (SI); Parque Nacional Nahuel Huapi, cima del cerro Challhuaco, "Mirador Nirihuau", acceso

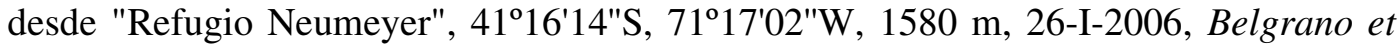
al. 543 (SI). Depto. Pilcaniyeu: Ruta 23, entre Bariloche y Pilcaniyeu.Valle del arroyo Pichilenfu, XI-2005, Picca 324 (SI). Santa Cruz: in valle rivuli Arroyo Palque in terra nuda subuliginosa, 17-I-1905, Dusén 5604 (SI). Depto. Corpen Aike: Ruta Nac. 3, 54 km al sur del río Chico, 28-XII-1992, Crespo \& Miconi 495 (SI); Ruta Nacional 3, empalme Ruta Provincial 63, entrada al Parque Nacional Monte León, 50¹4'35"S, 6900'29"W, 345 m, 13-I-2008, Paiaro s.n. 8 (SI). Depto. Lago Argentino: Ruta 
Nacional 40, Hotel La Leona, debajo del puente que cruza el río La Leona, 4948'26"S, 7203'05"W, 272 m, 14-I-2007, Zanotti 96 (SI); Ruta nacional 40, Laguna de los Escarchados, cordón de los Escarchados, a 29,4 km SE empalme R.P. 11, 50²1'06"S, 71³0'36"W, 812 m, 13-I-2007, Zanotti 92 (SI); Lago Argentino, verano 1958-1959, James 186 (SI); Los Laberintos, C $C^{o}$ Huiliche, Ea. Anita, El Calafate, 10-I-2001, Guerrido et al. 330 (SI), Cerro Huiliche Laberinto. Ea. Anita. También acompaña Mirabet, 12-I-2009, Guerrido \& Fernández 828 (SI); Ruta Provincial 65, 43,5 km W empalme Ruta Provincial 17, 1 km S cerro Mank Aike, 4946'17"S, 7043'48"W, 807 m, 14-I-2008, Paiaro s.n. 9 (SI); PN Los Glaciares, Brazo Norte del lago Argentino, Estancia Cristina, desde el casco hacia Base Cristina del Instituto del Hielo Continental Patagónico, 29-XI-2001, Guerrido \& Fernández 406 (SI); Centinela, 5045'S, 72³0'W, 24-II-1968, Anliot 6177 (SGO); El Calafate, cerro Huiliches, 50²2'50',S, 72¹6'59'’W, 865 m, 23-XI-2012, Zuloaga et al. 14022 (SI). Depto. Lago Buenos

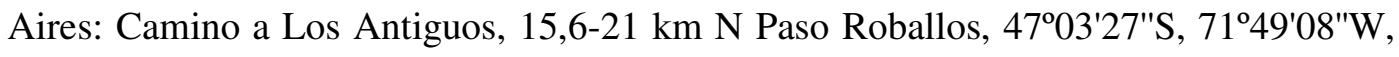
829-1501 m, 19-I-2007, Zanotti 128 (SI); Camino a Paso Roballos-Los Antiguos, a 49,1 km N de puente metálico sobre el río Ghio, 4650'47"S, 71²2'16"W, 845 m, 20-I-2007, Zanotti 136 (SI); Al S de Perito Moreno, 46º59'36.2"S, 7041'54,5"W, 692 m, 11-XII2006, Bonifacino et al. 2979 (SI); camino fronterizo, al N de Paso R. Roballos, rumbo a Los Antiguos, 24-I-2003, Belgrano et al. 72 (SI); Región entre lago Buenos Aires norte y codo río Mayer, 48 $15^{\prime} \mathrm{S}, 71^{\circ}-72^{\circ} 20^{\prime} \mathrm{W}, 200$ m, 28-I-1903, von Platen \& Greiner s.n. (SI); camino fronterizo, al N de Paso R. Roballos, rumbo a Los Antiguos, 24-I-2003, Belgrano et al. 82 (SI); Laguna de los Gendarmes. Ruta Provincial 45, camino a El Portezuelo, 87,6 km NW Perito Moreno, 4605'58"S, 7140'58"W, 1088 m, 10-I-2008, Paiaro s.n. 6 (SI); Puesto Lebrún (ahora Puesto Viejo), 27,3 km N casco Estancia La Vizcaína. Meseta del Lago Buenos Aires, 4657'52"S, 7106'27"W, 1353 m, 8-I-2008, Paiaro s.n. 3 (SI).

CHILE. XI Región de Aysen. Pcia. Coihaique: Ruta 7 entre Villa Cerro Castillo y Puerto Tranquilo, 46 07'39"S, 72²12'16"W, 430 m, 28-XI-2009, Biganzoli et al. 2250 (SI).

\section{Moschopis ameghinoi}

ARGENTINA. Santa Cruz. Depto. Lago Buenos Aires: meseta del lago, 7 km NO casco estancia “La Vizcaína”, 1100 m, 9-XII-1986, Sánchez et al. 516 (BAB); Meseta 
del lago Buenos Aires. Laguna del Cervecero, leg. M.L. Iribarren, 4709'24'’S, 71²16’37’’W, 1265 m, 5-V-2012, Zavala-Gallo 244 (SI).

\section{Moschopis caleofuensis}

ARGENTINA. Neuquén. Depto. Lácar: Fuentes del río Caleufu, 2100 m, 1896, Roth s.n. (SI 12692); Lago Lácar, 1896, Roth s.n. (SI 12707); Chapelco, San Martín de los Andes, 1800 m, 10-II-1973, Fiedler 20 (SI). Río Negro. Depto. Bariloche: Cima del C

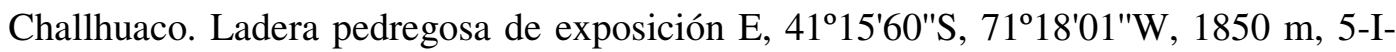
2011, Zavala-Gallo et al. 117 (SI); Alrededores del lago Nahuel Huapi, 1896, Roth s.n. (SI 12706, SI 12685, SI 12691); Cerro al NE de Puerto Manzano, costa oriental del Nahuel Huapi, 1600 m, 25-XII-1946, Diem 991 (SI); Cerro Meta. Filo. Pedrero, entre rocas pequeñas, 1950 m, 14-III-1993, Ferreyra 119 (BCRU).

\section{Moschopis leyboldi}

ARGENTINA. Mendoza. Depto. Las Heras: Valle de Horcones sup. Plaza de Mulas, 4300 m, 18-II-1940, Ruiz Leal 6694 (LIL); Valle Horcones sup., 4300 m, II-III-1940, Ruiz Leal 207 (SI). Depto. San Carlos: Laguna Diamante, 3500 m, 2-II-1950, Soriano 4086 (SI), Est. alta Perón, 18-I-1951, Hueck s.n. (SI 18159); Pampa de los Avestruces,

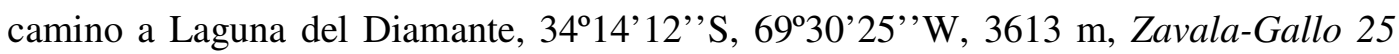
(SI). Depto. San Rafael: Los Molles, arriba del "Cuchillo", 2850 m, 31-XII-1949, Sleumer 661 (LIL); sin localidad indicada, sin fecha indicada, sin coleccionista indicado (SI 12710). Depto. Tunuyán: Valle del Alto Tunuyán, cumbre del cerro Las Delinas, 6II-1934, Ruiz Leal 2148 (LIL), sobre las cuchillas en inmediaciones del Cajón Chico, 9II-1934, Ruiz Leal 2161 (LIL). Neuquén. Depto. Chos Malal: filo SE del macizo del Domuyo, en pedregales, 3642'S, 70²2'W, 3030 m, 27-I-1964, Boelcke et al. 11292 (SI). San Juan. Depto. Calingasta: Portezuelo La Fría, 3360 m, I-II-1950, Roig F. (H) s.n. (LIL 370638, LIL 367551, MERL 13003).

CHILE. Región Metropolitana. Pcia. Santiago: Las Condes, leg. J. Morales, 2200 m, II-1950, Gunckel 21791 (CONC); Cordillera de Santiago, leg. V. Barrientos, II-1949, Gunckel 21255 (CONC). 


\section{Moschopis monocephala}

ARGENTINA. Catamarca. Depto. Antofagasta de la Sierra: Real Grande. Crece en las laderas de la quebrada, 4100 m, 25-I-1987, Haber 92 pp (SI); Ruta 53. Falda de Ciénaga, 19-II-1980, Cabrera et al. 31855 (SI). Jujuy. Depto. Rinconada: Alrededores lag. Vilama, 4600 m, 5-12-III-1964, Schwabe et al. 1080 (LP). Depto. Susques: Ruta Nacional 40, límite con Salta a Sey, 2405'26"S, 66³0'24"W, 4450 m, 17-II-2011, Zuloaga et al. 13030 (SI); Alto Chorrillos, subida, 21-II-1980, Cabrera et al. 31871 (SI); Abra de Taire, 27,5 km W of Susques on Rt. 16, 233' $\mathrm{S}, 66^{\circ} 32^{\prime} \mathrm{W}, 4244 \mathrm{~m}, 22-\mathrm{II}-$ 2007, Olmstead et al. 2007-47 (SI); Abra Los Chorrillos, 4500 m, 16-II-1980, Cabrera et al. 31766 (SI). Salta. Depto. Cachi: Quebrada del río Las Cuevas, 5000 m, 23-II1966, Cisin s.n. (LIL 535259). Depto. Los Andes: Huaytiquina, 27-II-1972, Cabrera et al. 22562 (LP); Vega de Tocomar, 4300 m, 27-II-1972, Cabrera et al. 22528 (LP); Hombre Muerto, 4000 m, 1927, Catalano 18 (SI).

CHILE. I Región de Tarapacá. Pcia. El Tamarugal: Along road, between Salar de Huasco and Collacagua. In flat, altiplano-like habitat, $20^{\circ} 10.40^{\prime} \mathrm{S}, 68^{\circ} 49.76^{\prime} \mathrm{W}, 3852 \mathrm{~m}$,

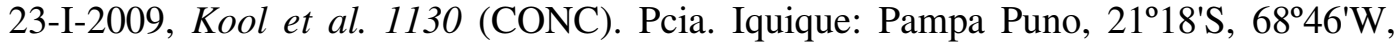
4000 m, 19-I-2000, Peñaloza 20016 (CONC). II Región de Antofagasta. Pcia. Antofagasta: Dep. Taltal. Cord. Volcán Llullaillaco, 4400 m, II-1926, Werdermann 1016 (LIL, SI). Pcia. El Loa: Sector N del Salar de Aguas Calientes, 230' S, 67 $22^{\prime} \mathrm{W}$, 4250 m, I-1997, Arancio 10693 (CONC); Camino de San Pedro de Conchi a Toconce, cerca de Aiquina, 3650 m, 5-IV-1961, Ricardi et al. 464 (CONC); 4 km E Volcán San Pablo, 14,400 ft, XI-1969, Martin 558 (SI); Alto antes de Lejía, 4300 m, 6-III-1967, Martin 278 (SI). III Región de Atacama. Pcia. Atacama: Quebrada de Colalasta, 4000 m, 20-XII-1897, Gerling s.n. (SI 12712, SI 12677). Pcia. Copiapó: Rio Frio, 31-I-1901, Reiche s.n. (SI 12714). XV Región de Arica y Parinacota. Pcia. Parinacota: Bofedal de Parinacota, 18 $8^{\circ} 3^{\prime} \mathrm{S}, 6^{\circ} 14^{\prime} \mathrm{W}, 4680$ m, 4-V-2000, Belmonte 20049 (CONC); Camino de Putre a Chucuyo. Km 17, 4250 m, 12-II-1964, Marticorena et al. 204 (CONC).

\section{Moschopis rosulata}

ARGENTINA. Santa Cruz. Depto. Lago Argentino: Lago San Martín, C ${ }^{\circ}$ Pana, 1500 m, 19-II-1945, Rohmeder s.n. (LIL 114897); margen de la meseta arriba de la Ea. Perez (rio de las Vueltas), 1200 m, 28-XII-1950, Sleumer 1383 (LIL); Sierra Buenos Aires, 1020 m, 5-III-1914, Hauman-Merck \& Hicken s.n. (SI 19963); Lago Argentino, XII- 
1908, Hauthal 8837 (SI), verano 1958-1959, James 85 (SI), II-1914, Hauman-Merck \& Hicken 782 (Iter Patagonicum) (SI); Lago Viedma. Cerro Solo, 1600-2500 m, I-1916, Witte 59 (SI). Tierra del Fuego. Depto. Ushuaia: Lago Escondido. C ${ }^{\circ}$ Garibaldi, 1000 m, 26-II-1961, Gamundi s.n. (SI 25373).

CHILE. XI Región de Aysen. Pcia. Capitán Prat: Cerro Mocho (PN Bernardo O'Higgins), 1300 m, 5-I-1931, Donat 445 (SI). XII Región de Magallanes. Pcia.

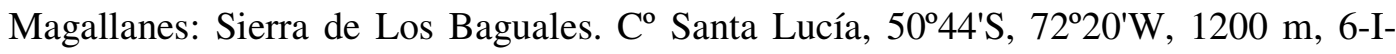
1985, Arroyo 850053 (CONC); Sierra de Los Baguales. Estancia La Cumbre. Campo de La Tropilla. Base Co Santa Lucía, 5046'S, 72²5'W, 1300 m, 8-I-1987, Landero 738 (CONC), Campo Estancia Vieja. Lado norte C ${ }^{\circ}$ Sin Nombre, 5043'S, 72º'19'W, $1600 \mathrm{~m}$, 7-I-1987, Landero 731 (CONC); Sierra Cazador. C Castillo, 850-900 m, 9-II-1962, Ricardi \& Mathei 480 (CONC).

\section{Moschopis subandina}

ARGENTINA. Neuquén. Depto. Lácar: Cordón del Chapelco -Nacientes. $A^{\circ}$ Chapelco Grande. En el Distrito Altoandino Austral. En los faldeos de acumulación morénica que contienen al pequeño lago La Kika, 1800 m, 23-I-1979, Gentili 753 (SI). Chubut. Depto. Tehuelches: Camino lago Vintter- lago Los Niños, campo de G. Alemán, puesto Chenkit, 23-I-1992, Nicora 9632 (SI).

CHILE. XIV Región de Los Lagos. Pcia. Valdivia: Volcán Choshuenco, leg. A. Hollermayer, 1600 m, 28-I-1927, Gunckel 5740 (CONC).

\section{Moschopis trilobata}

ARGEnTINA. Santa Cruz. Depto. Lago Argentino: El Calafate. Ea. Anita. Los Laberintos. C $C^{o}$ Huiliche. Excursión 4x4. Laberinto 1, 10-I-2001, Guerrido et al. 347 (SI), Co Huyliche, Ea. "Huyliche". Cima del cerro, 50²2'50"S, 72016'59"W, 877 m, 12I-2011, Zavala-Gallo et al. 221 (SI); El Calafate, cerro Huiliches, 50²2'50'’S, 72 ${ }^{\circ} 16$ '59'’ W, 865 m, 23-XI-2012, Zuloaga et al. 14026 (SI).

CHILE. XII Región de Magallanes. Pcia. Magallanes: Cerro Donoso. Sector Río de las Chinas, 5044'S, 72³1'W, 1000 m, 9-11-II-1987, Arroyo et al. 870260 (CONC); Dpto. Última Esperanza. Las Cumbras. Baguales, 550-850 m, 6-II-1962, Ricardi \& Mathei 402 (CONC), Ricardi \& Mathei 388 (CONC). 


\section{Nastanthus caespitosus}

CHILE. II Región de Antofagasta. Pcia. Antofagasta: Vega Incahuasi, 14-I-1898, Gerling s.n. (SI 12674, SI 12687, SI 12719); Valles de Río Frío, 3200 m, 15-I-1944, Muñoz-P. 3891 (SGO); Río Frío, 3500 m, I-1926, Werdermann 1028 (LIL, SI). III Región de Atacama. Pcia. Copiapó: Pastos Largos, I-1885, F. Philippi 1009-6 (SGO); Camino al salar de Maricunga. Aguada Caballo Muerto. Km 134, 4100 m, 31-I-1963, Ricardi et al. 612 (CONC). Pcia. Chañaral: Camino de Potrerillos al Salar de Maricunga, 38 km al interior del Tranque La Ola, 3900 m, 14-II-1966, Ricardi et al. 1618 (CONC). Pcia. Huasco: Quebrada Cantarito, entre Quebrada Marancel y Portezuelo de Cantarito, 2839'S, 69²4'W, 3500-4300 m, 12-II-1981, Arroyo 81623 (CONC), entre el extremo oeste de la Laguna Grande y la Quebrada Marancel, 28 $44^{\prime} \mathrm{S}$, $69^{\circ} 51^{\prime} \mathrm{W}, 3100-3500$ m, 22-I-1983, Marticorena et al. 83429 (CONC)

\section{Nastanthus compactus}

ARGENTINA. Mendoza. Depto. San Rafael: Camino a Mina Volcán Overo, 30003200 m, III-1945, Christoffel s.n. (LIL 114198); Cajón del arroyo Infiernillo, afl. río Grande, 10-II-1946, Rossi s.n. (LIL 163580). Depto. Tunuyán: Valle del Alto Tunuyán, 27-XII-1933, Ruiz Leal s.n. (LIL 214652); Rincones del Cerro Morado, 3700 m, 21-III1935, Ruiz Leal 3177 (MERL). Depto. Luján de Cuyo: Luján de Cuyo, Placetas Bayas (Loma Blanca), 3000-3300 m, 5-I-1937, Semper s.n. (MERL 4301).

CHILE. Región Metropolitana. Pcia. Cordillera: Valle Nevado última pista, trayecto entre Alto Tres Puntas hasta Piedra Numerada, camino a El Plomo, 3000-3500 m, I1993, Solervicens s.n. (SGO 130759); Cajón del Maipo. Vega Los Chorreados, 3406'S, 700' 'W, 3100 m, 20-I-2000, Teillier 4588 (CONC 147883). Pcia. Santiago: Farellones. Valle de Las Llaretas, 33²0'S, 70¹5'W, 3200 m, 4-III-1956, Schlegel 1067 (CONC). VII Región de Maule. Pcia. Curicó: Andes de Curicó, Santa Elena, II-1902, Rivera s.n. (SI 12722); Dpto.Curicó. A orillas de la Laguna de Teno, 2500 m, 10-III-1967, Marticorena \& Matthei 924 (CONC 32292), Alrededores de la Laguna de Teno, 35²10'S, 70³3'W, 2560 m, 29-III-1973, Marticorena et al. 42 (CONC 39336). Pcia. Talca: Laguna de Aguas Calientes, Andes, II-1879, F. Philippi 2049 (SGO). 


\section{Nastanthus patagonicus}

ARGENTINA. Neuquén. Depto. Minas: Cordillera del Viento, cruzada de Triaco Malal al Cajón de Butaló, portezuelo, 3658'S, 70³1'W, 2300 m, 3-II-1964, Boelcke et al. $11624 a$ (SI). Depto. Collón-Curá: \pm 10km de Rinconada, 18-XII-1985, Correa et al. 9387 (BAB). Depto. Lácar: C Chapelco, faldeo NW, 2000 m, 26-I-1966, Eskuche 6037 (CTES); San Martín de los Andes, cerro Chapelco, 1900 m, 26-I-1982, Rossow 1502 (BAB); C Chapelco, al S de San Martín de los Andes, 2000 m, 8-III-1964, Schajovskoy s.n. (SI); Cerro Chapelco, del refugio Greef hacia arriba. 3-I-1983, Villamil et al. 2790 (SI). Depto. Aluminé: Espinazo del Zorro, 1500 m, 6-XII-1964, Schajovskoy 55/IV (SI). Río Negro. Depto. Pilcaniyeu: Ruta 40 cerca cruce Ruta 23, 10-X-1981, Cabrera et al. 33027 (SI). Depto. Bariloche: Ñireco, 1600 m, 7-XII-1941, Neumeyer 545 (SI). Depto. 9 de Julio: Ruta Prov. 60, a 65,5 km SW del Puesto Policial, cerca del $\mathrm{C}^{\mathrm{o}}$ Corona (e/ $\mathrm{C}^{\mathrm{o}}$ Corona Grande y Chico), 41'23'41"S, 6657'33"W, 1425 m, 3-XI-2006, Zanotti \& Ávila 26 (SI). Chubut. Depto. Río Senguerr: Ruta 22, 2 km N Río Mayo, 45³9'S, 70¹5’W, 7-XII-1976, Arroyo et al. 353 (SI). Depto. Rawson: Ruta Prov. 23, 4505'53,3"S, 69²2'51,9"W, 28-XI-2002, Bonifacino \& Donato 734 (SI). Depto. Languiñeo: $C^{\circ}$ Quichaura, Estancia "Quichaura", 1500 m, 19-XII-1946, Soriano 2297 (SI); 41 km E of Tecka. Estancia Quichaura, on Route Prov. 62, to the right of the road going W-E., 900 m, 6-XII-1984, Stuessy et al. 6871 (SI). Depto. Escalante: Comodoro Rivadavia, sin fecha indicada, Wilensky s.n. (SI). Depto. Cushaman: Ruta Pcial. 13, a 17,7 km S del límite Chubut-Río Negro, Sierra de Calcatapul, 4204'25"S, 69³0'54"W, 1219 m, 5-XI2006, Zanotti \& Ávila 57 (SI). Santa Cruz. Depto. Deseado: Ruta Prov. 12, Estancia "Sierras Blancas", 47¹7'26,5"S, 68²32'21"W, 2-XII-2002, Bonifacino \& Donato 769 (SI); Monumento Natural "Bosques Petrificados", cercanías Aguada del Sauce, 20-XII1994, Montenegro 37 (CTES). Depto. Lago Buenos Aires: Lago Ghio, 400 m, 28-I1930, Donat s.n. (LIL); Ruta Nac. 40; 8,4 km al S de Estancia "Telken", 4652'01"S, 7043'58"W, 619 m, 9-I-2008, Paiaro s.n. (SI); Estancia "El Cerrito", Ruta Prov, 45, 7,7 km SE empalme Ruta Prov. 72,6,3 km NW Estancia "Bahía", 46¹5'46"S, 71²2'39"W, 634 m, 10-I-2008, Paiaro s.n. (SI); Ruta Nac. 40, 59 km S Perito Moreno, 8-XII-1986, Sánchez 448 (BAB); Ruta Prov. 39, a 84,9 km E Bajo Caracoles, 17,8 km E Estancia "Santa Rita", 47¹7'20"S, 7002'12"W, 828 m, 21-I-2007, Zanotti 141 (SI); Entre Telken y Bajo Caracoles, Ruta Nacional 40, 4707'02'’S, 7051'16' 'W, 710 m, 21-XI-2012, Zuloaga et al. 13974 (SI). Depto. Río Chico: 22 km al W de Bajo Caracoles hacia Lago 


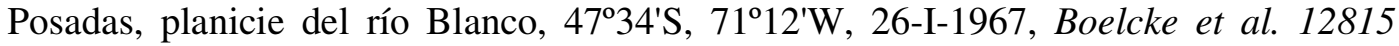
(SI).

\section{Nastanthus scapigerus}

ARGENTINA. Mendoza. Depto. Las Heras: Cuevas, Quebrada de Matienzo, 11-II1982, Andrada et al. s.n. (SI); Quebrada de Matienzo, 3200-3800 m, 26-II-1962, Capitanelli s.n. (MERL); Las Cuevas, Refugio Militar "Gral. Lamadrid", 10-I-1963, Boelcke et al. 9730 (SI); Puente del Inca, 22-I-1914, Sanzin 361 (SI). Depto. Malargüe: Alto valle de El Sosneado, 2700 m, 19-II-1942, Burkart et al. s.n. (LIL, SI); Vega del Azufre, 15-I-1941, Castellanos s.n. (LIL); Valle Hermoso, 2800 m, 19-II-1974, Fiedler 98 (SI); Valle Hermoso, I-1897, Stuckert 3017 (CORD); Valle de Las Leñas. 25-XII1989, Kiesling \& Donath 7265 (SI); Portezuelo del Choique, 36²2'38"S, 6948'21"W, 23-XI-2001, Prina et al. 1399 (SI); Sobre la ruta 40 vieja, 36²2'38"S, 6945'47"W, 2550 m, 14-XII-2001, Prina et al. 1547 (SI); Alto Valle del Atuel, Quebrada Nield, 917-I-1954, Roig 254 (MERL); Quebrada del A Nield, 2800-2900 m, 9-17-I-1954, Ruiz Leal \& Roig 15793 (MERL). Depto. San Carlos: Frente al volcán Maipo, 3500 m, I1921, Carette 396 (SI). Depto. San Rafael: Distrito El Sosneado: Alto Valle del Atuel, entre Sominar y Laguna Atuel, 2800 m, 6-7-II-1955, Ruiz Leal 16796 (MERL). Depto. Tunuyán: Valle del Alto Tunuyán: Las Salinillas, cerca del cerro Marmolejo, 1-II-1934, Ruiz Leal 2068 (MERL). Neuquén. 1-II-1900, Asp 1010 (SI). Dpto. Norquín: Co Huaile, ladera NO, 3704'22"S, 7007'14"W, 2550 m, 8-I-2001, Biganzoli 1193 (SI). Dpto. Huiliches: Estancia "Las Breñas", de Mallín Escondido a Paso Cerrado, ladera Oeste, 39³1'27"S, 7102'29"W, 1625 m, 17-I-2001, Biganzoli 1211 (SI). Depto. Minas: Lagunas Epu-Lauquén, pedregales próximos al $\mathrm{A}^{\circ}$ Pincheira, 36 $50^{\circ} \mathrm{S}, 71^{\circ} 03^{\prime} \mathrm{W}, 1300 \mathrm{~m}$, 18-I-1964, Boelcke et al. 11006 (SI); Cajón del Portillo, 36²12'S, 70³6'W, 2560 m, 31I-1970, Boelcke et al. 14163 (SI); Reserva Prov. "Domuyo", camino geotérmico al sur de Aguas Calientes, río Covunco, comienzo de la picada sur al Domuyo, 2400-2600 m, 27-I-2001, Ezcurra 3113 (SI). Depto. Los Lagos: sobre la Ruta Prov. 63, cerca del Paso Córdoba, 1200 m, 30-I-1997, Chiapella et al. s.n. (SI). Depto. Loncopué: Chenque Pehuen, 14-I-1965, Schajovskoy 131/IV (SI). Río Negro. Depto. Bariloche: Ñireco, 1100 m, 7-XII-1941, Neumeyer 544 (SI). Santa Cruz. Depto. Lago Buenos Aires: 26 km SO de Los Antiguos hacia Monte Zeballos, 11-XII-1986, Sánchez et al. 600 (BAB); Ruta Nac. 40, 8,4 km al S de Estancia "Telken", 4652'01"S, 7043'58"W, 619 m, 9-I- 
2008, Paiaro s.n.4 (SI); de Perito Moreno a Bajo Caracoles, 4654'06'’S, 7043'04', W, 650 m, 21-XI-2012, Zuloaga et al. 13971 (SI).

CHILE. V Región de Valparaíso. Pcia. Los Andes: Cerca de Río Blanco, camino a 3 Lagunas, 2800 m, 15-IV-1973, Zöllner 6630 (CTES); 15 km W of Portillo, between Portillo and Juncal, at roadside, 2550 m, 17-XI-1991, Eggli \& Leuenberger 1681 (SGO); Caracoles F.C.T.C., 3-I-1931, King 413 (BAB); Cordillera Río Blanco, Cajón de Los Hornillos y Cajón del León, I-1936, Mandujano s.n. (SGO). Región Metropolitana. Pcia. Santiago: Río Yeso, Lag. Piuquenes, 2500 m, 13-I-1945, Biese s.n. (LIL); Lag. Negro, 2700 m, 13-I-1945, Biese s.n. (LIL); Fierro Carrera, valle de San Francisco (Cordillera de Las Condes), 28-I-1930, Looser 1176 (SI); Cerca de La Parva, 3000 m, 6-I-1979, Muñoz-S. \& Meza-P. 1332 (SGO); Cerca de La Parva, 3000 m, 6-I1979, Muñoz-S. \& Meza-P. 1346 (SGO). Pcia. Cordillera: dpto. de Puente Alto, Comuna de San José de Maipo, Valle del Yeso, Ladera SO del embalse, 2600 m, 7-I1970, Vargas \& Farah 46 (SGO); Cordillera, Cajón del Maipo, Baños Colina, 2400 m, 30-XII-1986, Solervicens s.n. (SGO); Cajón del Maipo, Hito Paso Internacional Maipo, 3325 m, 17-II-1995, Villagrán et al. 8461 (SGO). VII Región de Maule. Pcia. Linares: Termas de Longaví, Laguna de los Huesos, 17-I-1938, Castellanos 21678 (SGO). Pcia. Talca: por Ruta Nac. 115, a ca. $3 \mathrm{~km}$ de la Laguna de Maule viniendo desde San Clemente, 3600'23"S, 70³3'36"W, 2171 m, 9-II-2007, Chiapella et al. 1668 (SI); Laguna del Maule, 3601'S, 70³3'W, 2250 m, 25-I-1990, Gardner et al. 4559 (SGO); Cuenca de las arenas, Andes, II-1879, F. Philippi 1010-d (SGO); Cuesta de las Arenas, 15-II-1879, sin coleccionista indicado (SI 12725). Pcia. Curicó: Andes de Curicó, A ${ }^{\circ}$ de Curicó, 2000 m, 20-II-1902, Ravena s.n. (SI); Cord. de Peteroa, 1891, Vidal s.n. (SGO); Andes prov. Curicó, 1.d. Baños, 1889-1890, Vidal s.n. (SGO).

\section{Nastanthus ventosus}

ARGENTINA. La Rioja. Vegas de Descubrimiento Viejo, 3000 m, 22-XI-1915, Hosseus 1534 (CORD). Depto. Vinchina: Entre Jagüé y Salina del Leoncito, a $61 \mathrm{~km}$ del primero, $11 \mathrm{~km}$ al oeste de la Puerta de la Quebrada del Leoncito, 3400 m, 7-II-1998, Biurrun et al. 5171 (CTES); "Reserva Laguna Brava", Laguna Brava, costa oeste de la laguna, 28¹6’21'’S, 6849'50'’W, 4281 m, 8-I-2009, Donadío et al. 75 (SI). San Juan. Depto. Iglesia: Reserva de San Guillermo, Nacimiento del Río La Sal: Rincón de las Flechas, 3900 m, 4-I-1984, Beorchia 6 (SI); de la Laguna del Macho Muerto a Las 
Caranchas, 3700-4200 m, 22-II-1984, Kiesling 4622 (SI); Llanos de San Guillermo, 3300 m, 10-I-1997, Kiesling et al. 8783 (SI); Cerca de San Guillermito, Bajada la Olorosa, 3650 m, 2-I-1984, Meglioli 11 (SI); Mina Las Carachas, 4050 m, 20-I-1981, Nicora et al. 8228 (SI); Río Macho Muerto, refugio de Macho Muerto, 4030 m, 14-I1983, Nicora et al. 8498 (SI); Río Macho Muerto, a más o menos 7 km del refugio, 14I-1983, Nicora et al. 8507 (SI); Refugio del Macho Muerto, 4050 m, 13-XII-1981, Pujalte 42 (SI); Veguita en la cordillera de San Guillermo, 4100 m, 17-XII-1982, Pujalte 168 (SI), Quebrada del Agua Negra, 4000 m, 21-II-1979, Cabrera 30079 (SI); Quebrada del Agua Negra, 3800 m, 10-XII-1979, Cabrera et al. 31124 (SI); Quebrada del Agua Negra, 15-II-1986, Kiesling et al. 6188 (SI); A 10km al Oeste del Portezuelo de Conconta, 2957'49"S, 6943'05"W, 4052 m, 22-II-1998, Herrera \& Jiménez 865 (SI); Cerca del Paso del Agua Negra, entre Arrequintín y el límite con Chile, ca. Km 125/130, 3700 m, 28-II-1983, A. T. Hunziker \& Subils 24580 (CORD); Valle del Cura, Alojo Los Catres, 3850 m, 23-I-1981, Kiesling 3194 (SI); Valle del Cura, 4000 m. 26XI-1980, Múlgura \& Deginani 165 (SI); Zona del río Las Taguas, Quebrada Potrerillos, 29²1'S, 6958'W, 3700 m, 1-II-2000, Teillier \& Márquez 4496 (SI). Depto. Ullúm: Del refugio de la Estancia "Don Carmelo" hacia el S, entre las Sierras de la Invernada y del Tigre, 3058'39'’S, 6905'13"W, 3100 m, 9-II-2000, Kiesling et al. 9407 (SI). Depto. Calingasta: Reserva Natural Estricta "El Leoncito", Ciénaga de las Cabeceras, 22-I1995, Apochian et al. 175 (SI); Ciénaga Las Cabeceras, 2780 m, 14-I-1995, Kiesling et al. 8579 (SI); Quebrada del A ${ }^{\circ}$ de las Cabeceras, 3000 m, 19-XI-1997, Haene et al. 1778 (SI); Quebrada Arroyo Portezuelo, 3730 m, 9-IV-1999, Haene 1995 (SI); Estancia "El Leoncito", Ciénaga de las Cabeceras, 2730 m, 8-I-1993, Haene 1164 (SI); Sierra del Tontal, C Casa de Piedra, 3400 m, 20-II-1984, Kiesling 4529 (SI); Sierra del Tontal, 2800 m, 22-I-1987, Kiesling \& Meglioli 6518 (SI); Cordillera de Ansilta, Quebrada del Fierro, 3900-4000 m, 20-II-1988, Kiesling et al. 6930 (SI); Oeste de Barreal: El Pachón: laguna, 3800 m, 9-15-II-1977, Kiesling \& Sáenz 1317 (SI); El Pachón: casilla Naranja, 9-15-II-1977, Kiesling \& Sáenz 1447 (MERL, SI); Laguna Pachón y Laguna Pachón Alta, 3610 m, 28-II-1975, Luti \& Barrera 5482 (CORD); Yunque, entre El Molle y Erizos, camino de Barreal a Pachón, 3100 m, 9-I-1976, Luti et al. 5547 (CORD); Arroyo Blanco, 3950 m, 19-31-I-1950, Roig 12179 (MERL); Cordillera del Espinacito, Los Portillos, 3435 m, 10-II-1897, Kurtz 9678 (SI). Mendoza. Depto. Las Heras: Puente del Inca, faldeo S, C' Banderita Norte, 3150 m, 12-I-1973, Boelcke et al. 9808 
(BAB, SI); Puente del Inca, sin fecha indicada, Autran 61 (SI); C Banderita sur, 11-II1940, Ruiz Leal 6611 (MERL); Puente del Inca, III-1901, C. Spegazzini 959 (BAB, SI); Puente del Inca, III-1908, C. Spegazzini 24360 (SI); Laguna Los Horcones, 2800 m, 27III-1954, J. H. Hunziker 6340 (BAB); Ladera limoso-pedregosa ca. Río Los Horcones, 3030 m, 16-I-1985, J. H. Hunziker et al. 11160 (SI); Alrededores Laguna, 3180 m, 16-I1985, J. H. Hunziker et al. 11173 (SI); Laguna de Los Horcones, 14-XII-1964, Ruiz Leal 23417 (MERL); Laguna de Los Horcones, 10-II-1908, C. Spegazzini s.n. (BAB); Sierra de Bonilla, IV-1906, Looser 15014 (SI); Pampa de Canota, 3000 m, 20-XII-1982, Martínez-Carretero s.n. (MERL); Precordillera: Agua de las Cerrajas, 22-XI-1957, Roig s.n. (MERL); Co El Platita, 4150 m, 21-I-1983, Roig 11055 (MERL); Sierra de Uspallata: El Jagüel, 9-II-1982, Roig 10822 (MERL); Paramillo de Uspallata: Pampa Fría, 5-IV-1967, Ruiz Leal 25260 (MERL); Las Cuevas, 8-VIII-1901, C. Spegazzini 1009 (SI); Quebrada de Matienzo, 3510 m, 30-I-1980, Wingenroth 30 (SI); Quebrada de Matienzo, 14-II-1981, Wingenroth 90 (SI). Depto. Luján de Cuyo: Río Mendoza, Altas Cordilleras de Mendoza, 2700-3500 m, 20-I-1908, Hauman-Merck 114 (SI); Vallecitos cerca del refugio Club Regatas Mendoza, 3258'48,3"S, 69²1'19,8'W, 3020 m, 11-I2003, A. Cocucci et al. 2218 (CORD, SI); Quebrada del Rincón de los Vallecitos, 3700 m, 21-24-I-1939, Vadés s.n. (MERL). Depto. San Carlos: Laguna del Diamante, 3300 m, 28-I-1950, Balegno \& Palacios s.n. (LIL); Laguna del Diamante, 3200 m, 20-I-1941, Ruiz Leal 7236 (MERL); Laguna del Diamante, 3-II-1950, Soriano 4106 (SI); Ruta 98, Pampa de los Avestruces, 3780 m, 25-I-1985, J. H. Hunziker et al. 11307 (MERL, SI); Inmediaciones de las vegas del Llaucha, II-1942, Patiño s.n. (MERL); Estancia "Llaucha": A de Los Leones, 3200 m, 16-I-1949, Ruiz Leal 11760 (MERL); Arroyo de los Gauchos y Pampa de los Avestruces, 3370 m, 20-I-1965, Ruiz Leal 23507 (MERL); Entre la aduana y Potrero de los Zorros, 16-I-1965, Ruiz Leal 23609 (MERL). Depto. San Rafael: Cajón del Burro, II-1913, Gerth 120 (SI); El Ángulo - El Sosneado, 19-I1993, H. A. L. 8393 (SI); Distrito Cuadro Benegas: El Ángulo. Nacientes del A ${ }^{\circ}$ Las Yeseras, 3020 m, 16-II-1984, Méndez \& Wuilloud s.n. (MERL). Depto. Malargüe: Baños del Azufre, 19-I-1941, Castellanos s.n. (LIL); Sierra del Nevado, lomas al SSE expuesta al S, 2450-2500 m, 22-I-1974, Boelcke et al. 15918 (BAB); Co Nevado, 35³5'43"S, 68³0'21"W, 3055 m, 14-XII-2005, Prina et al. 2938 (SI); Portezuelo Ancho, 2900 m, 8-XII-1985, H. A. L. 7008 (SI); Cord. de Mendoza (Río Salado sup.): Cerro de Los Molles, 3000 m, 8-I-1890, Kurtz 7513 (SI); Los Molles, arriba del 
"Cuchillo", 2600 m, 31-XII-1949, Sleumer s.n. (LIL). Neuquén. Depto. Minas: Sierra de Cochicó, cumbre, 36²18'S, 70³0'W, 2500-2700 m, 29-I-1970, Boelcke et al. 14066 (BAB, SI); La Corona, Cordillera del Viento, 6-IV-1912, Pastore 96 (SI).

CHILE. III Región de Atacama. Pcia. Copiapó: Cord. Río Turbio, Co. Cadillal, 3500 m, I-1926, Werdermann 946 (LIL, SI); Laguna Negro Francisco, 27²7'S, 69²13'W, 4150 m, 1-II-1997, Teillier 4183 (SGO); Laguna Negro Francisco, Agua Dulce, 20-III1993, Torres-Mura s.n. (SGO). V Región de Valparaíso. Pcia. Los Andes: Laguna del Inca, 29-I-1886, Kurtz s.n. (SI). Región Metropolitana. Pcia. Cordillera: Nacimientos del Maipo, 3100 m, I-1921, Carette 68 (SI). 
Apéndice 2. Matriz de los caracteres morfológicos utilizados.

Se indican los estados polimórficos entre corchetes y los datos faltantes o inaplicables con "?".

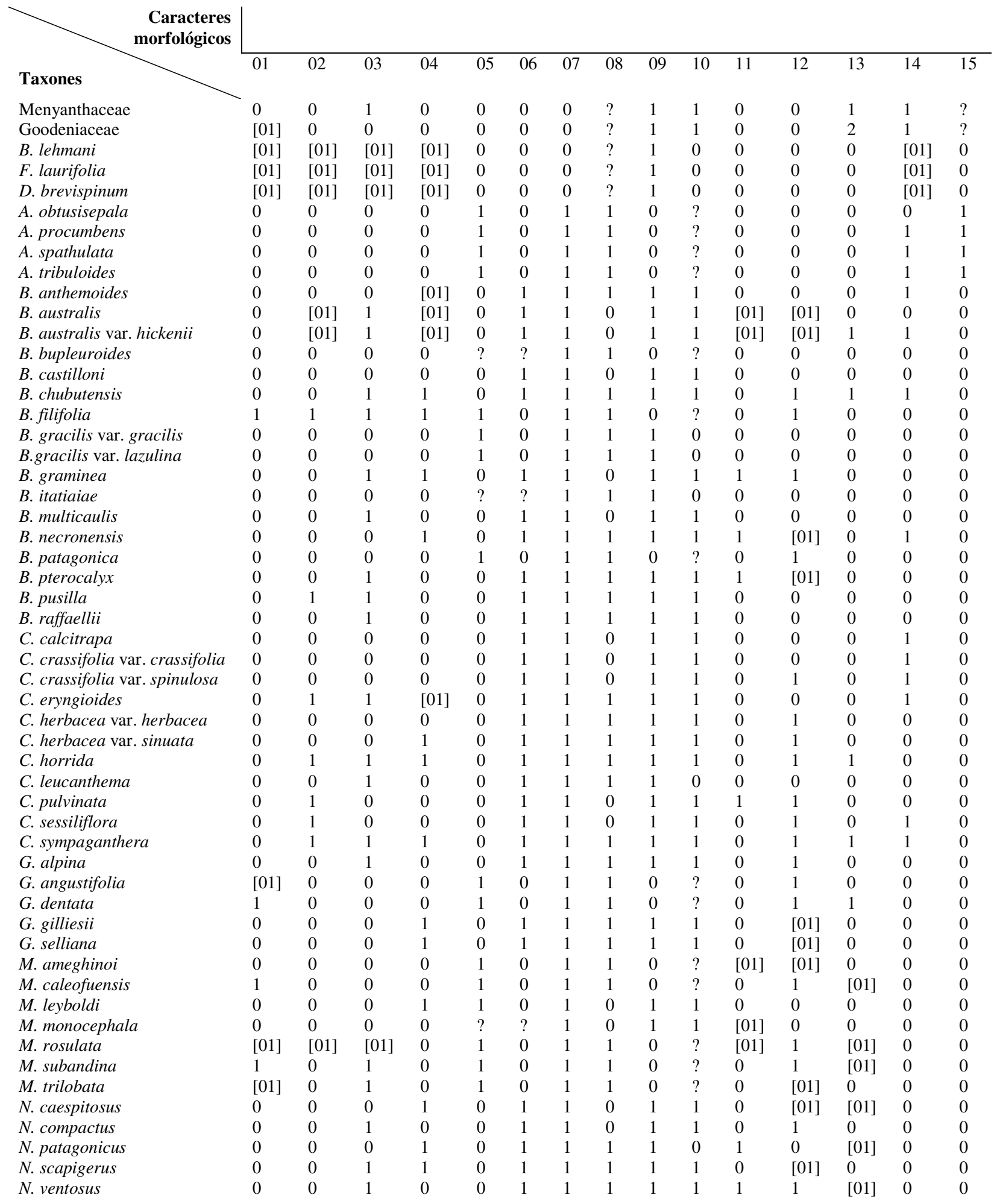


Apéndice 2. Continuación.

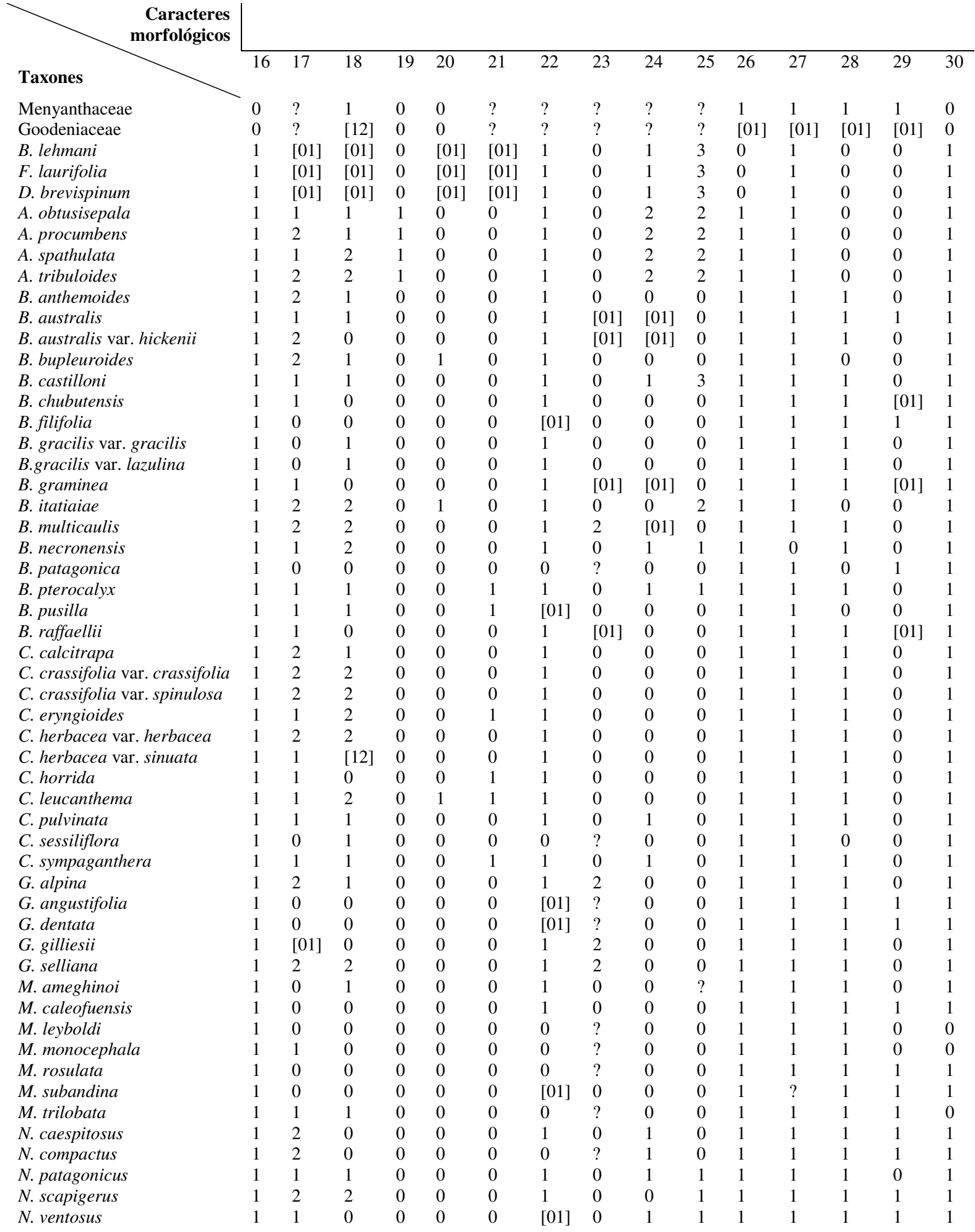


Apéndice 2. Continuación.

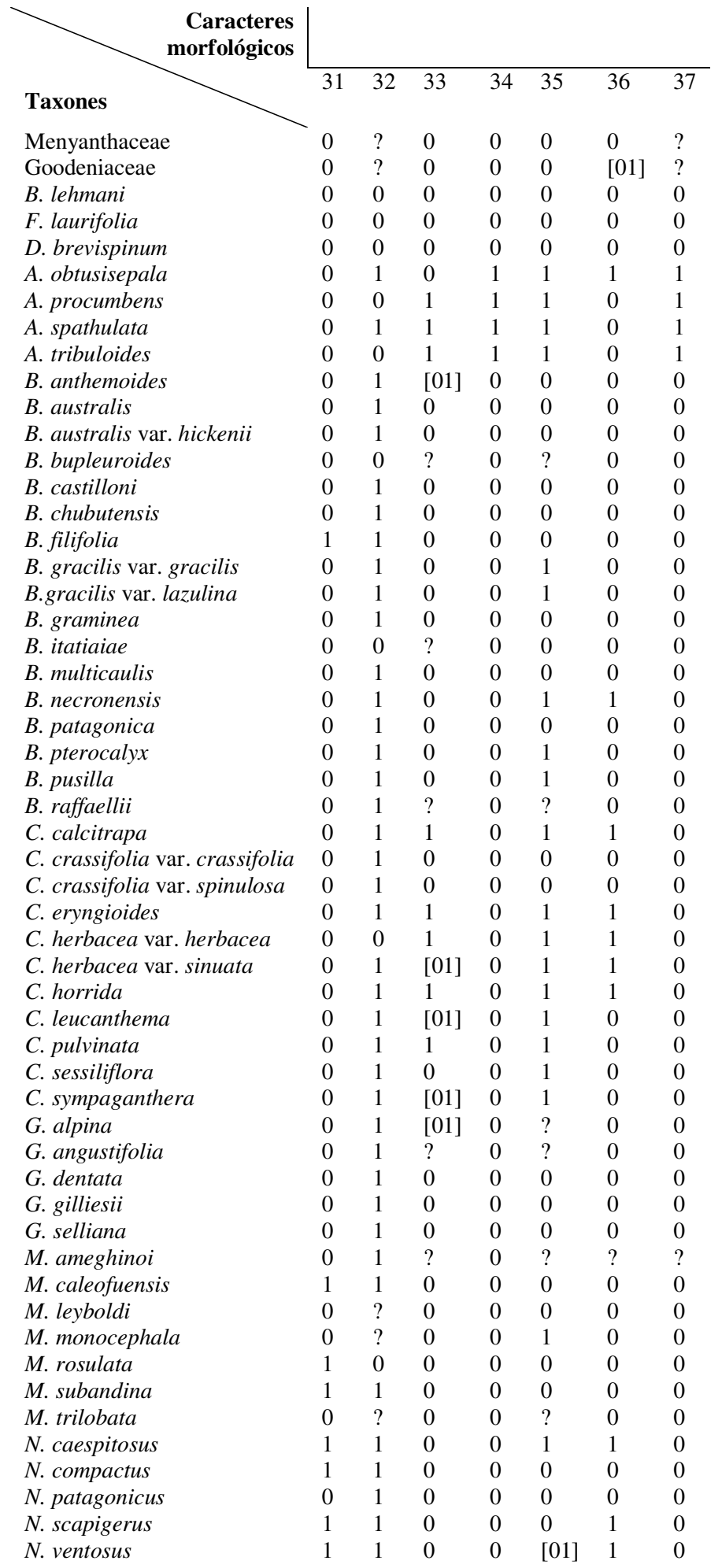


Apéndice 3. Tabla de ejemplares utilizados para la obtención de caracteres moleculares.

\begin{tabular}{|c|c|c|c|c|c|}
\hline Género & Especie & País & $\begin{array}{c}\text { 2da división } \\
\text { administrativa }\end{array}$ & $\begin{array}{l}\text { Colector y } \\
\text { número }\end{array}$ & $\begin{array}{c}\mathbf{N}^{\circ} \text { GenBank } \\
\text { / Herbario }\end{array}$ \\
\hline Barnadesia & B. lehmani & Sin datos & Sin datos & Sin datos & EU841142 \\
\hline Fulcaldea & F. laurifolia & Sin datos & Sin datos & Sin datos & EU841298 \\
\hline Dasyphyllum & D. brevispinum & Sin datos & Sin datos & Sin datos & EU841290 \\
\hline \multirow[t]{3}{*}{ Acicarpha } & A. obtusisepala & Uruguay & Rocha & Zanotti 160 & SI \\
\hline & A. spathulata & Brasil & Santa Catarina & Zanotti 152 & SI \\
\hline & A. tribuloides & Argentina & Buenos Aires & Morrone 5818 & SI \\
\hline \multirow[t]{10}{*}{ Boopis } & B. anthemoides & Argentina & Chubut & Zanotti 60 & SI \\
\hline & B. australis & Argentina & Santa Cruz & Zanotti 106 & SI \\
\hline & $\begin{array}{l}\text { B. australis var. } \\
\text { hickenii }\end{array}$ & Argentina & Neuquén & Zuloaga 12543 & SI \\
\hline & B. filifolia & Argentina & Santa Cruz & Zanotti 119 & SI \\
\hline & $\begin{array}{l}\text { B. gracilis var. } \\
\text { gracilis }\end{array}$ & Argentina & Neuquén & Belgrano 553 & SI \\
\hline & $\begin{array}{l}\text { B. gracilis var. } \\
\text { lazulina }\end{array}$ & Argentina & Chubut & Biganzoli 2403 & SI \\
\hline & B. multicaulis & Argentina & Neuquén & Zuloaga 12591 & SI \\
\hline & B. patagonica & Argentina & Santa Cruz & Zavala 216 & SI \\
\hline & B. pterocalyx & Argentina & Mendoza & Zuloaga 12253 & SI \\
\hline & B. pusilla & Chile & Metropolitana & Teillier 4637 & CONC \\
\hline \multirow[t]{10}{*}{ Calycera } & C. calcitrapa & Argentina & Salta & Zuloaga 11854 & SI \\
\hline & $\begin{array}{l}\text { C. crassifolia var. } \\
\text { crassifolia }\end{array}$ & Argentina & Buenos Aires & Zanotti 144 & SI \\
\hline & $\begin{array}{l}\text { C. crassifolia } \text { var. } \\
\text { spinulosa }\end{array}$ & Argentina & Neuquén & Zuloaga 12671 & SI \\
\hline & $\begin{array}{l}\text { C. herbacea var. } \\
\text { herbacea }\end{array}$ & Argentina & Mendoza & Zavala 21 & SI \\
\hline & $\begin{array}{l}\text { C. herbacea var. } \\
\text { sinuata }\end{array}$ & Argentina & Jujuy & Zanotti 261 & SI \\
\hline & C. horrida & Argentina & Neuquén & Zuloaga 12465 & SI \\
\hline & C. leucanthema & Chile & VIII (Bío Bío) & Johnson 10-105 & SI-BRY \\
\hline & C. pulvinata & Argentina & Jujuy & Zanotti 258 & SI \\
\hline & C. sessiliflora & Chile & V (Valparaíso) & Johnson 10-153 & SI-BRY \\
\hline & $\begin{array}{l}\text { C. } \\
\text { sympaganthera }\end{array}$ & Chile & IX (Araucanía) & Johnson 10-103 & SI-BRY \\
\hline
\end{tabular}




\begin{tabular}{|l|l|l|c|c|c|}
\hline Gamocarpha & G. alpina & Argentina & Neuquén & Zanotti 4 & SI \\
\cline { 2 - 6 } & G. dentata & Chile & VIII (Bío Bío) & Baeza 2975 & CONC \\
\cline { 2 - 6 } & G. gilliesii & Argentina & Mendoza & Pozner 559 & SI \\
\cline { 2 - 6 } & G. selliana & Argentina & Santa Cruz & Zanotti 96 & SI \\
\hline Moschopis & M. caleofuensis & Argentina & Río Negro & Zavala 117 & SI \\
\cline { 2 - 6 } & M. leyboldi & Argentina & Mendoza & Zavala 25 & SI \\
\cline { 2 - 6 } & M. monocephala & Argentina & Jujuy & Zuloaga 13030 & SI \\
\cline { 2 - 6 } & M. rosulata & Chile & XII & Arroyo 850053 & CONC \\
\cline { 2 - 6 } & M. subandina & Argentina & Chubut & Nicora 9632 & SI \\
\cline { 2 - 6 } & M. trilobata & Argentina & Santa Cruz & Zavala 221 & SI \\
\hline Nastanthus & N. compactus & Argentina & Mendoza & Zavala 41 & SI \\
\cline { 2 - 6 } & N. patagonicus & Argentina & Chubut & Zanotti 57 & SI \\
\cline { 2 - 6 } & N. scapigerus & Argentina & Santa Cruz & Paiaro s.n. 4 & SI \\
\hline
\end{tabular}


Apéndice 4. Protocolo de extracción

Basado en Doyle \& Doyle (1987) y modificado por Silvana Sede y Jorge Hurst (para 6 muestras):

- Preparar $5 \mathrm{ml}$ de solución buffer: disolver 0.2 g de CTAB 2x y 0.1 g de PVP en 20 $\mu \mathrm{l}$ de $\beta$-mercaptoetanol. Calentar la solución obtenida en baño térmico a $37^{\circ} \mathrm{C}$ durante 5 minutos. Agitar hasta disolver.

- Rotular 2 tubos por muestra. Moler las muestras para extracción de ADN con $\mathrm{N}_{2}$ líquido en mortero, agregando el pellet obtenido en cada uno de los tubos rotulados.

- Agregar $600 \mu \mathrm{l}$ de la solución buffer por tubo y agitar.

- Calentar las muestras en baño térmico a $37^{\circ} \mathrm{C}$ durante 60-90 minutos. Retirar del baño térmico y dejar que las muestras tomen temperatura ambiente.

- Agregar a cada tubo $600 \mu 1$ de SEVAG (24 cloroformo : 1 alcohol isoamílico).

- Agitar mediante vortex. Centrifugar las muestras 2 minutos a $12000 \mathrm{rpm}$.

- Retirar la fase acuosa (fase superior) de la mezcla contenida en cada tubo. Transferir a un tubo rotulado. Si se observa la fase acuosa turbia, se repiten los tres pasos anteriores.

- Agregar 0,5 vol. de solución $5 \mathrm{M}$ de CINa (200 $\mu$ l, aprox.).

- Agregar 0,4 vol. de isopropanol (250 $\mu 1$, aprox.).

- Agitar por inversión lenta 3 veces.

- Colocar los tubos en freezer a $-20^{\circ} \mathrm{C}$. Dejar reposar por 12 horas.

- Centrifugar 5 minutos a $12000 \mathrm{rpm}$.

- Descartar el sobrenadante cuidadosamente. Si se observa precipitado en el sobrenadante se centrifuga nuevamente, también se puede retirar el sobrenadante con pipeta.

- Agregar $500 \mu \mathrm{l}$ de alcohol de $70^{\circ}$, lavar el pellet despegándolo completamente de la pared del tubo. Dejar 5 minutos a temperatura ambiente.

- Centrifugar 2 minutos a $12000 \mathrm{rpm}$.

- Descartar el sobrenadante cuidadosamente. Repetir los dos pasos previos.

- Secar el pellet bajo lámpara incandescente.

- Resuspender en $20 \mu 1$ de buffer. 


\section{RESULTADOS Y DISCUSIÓN}

\subsection{Historia de la familia}

A fines del siglo XVIII, Cavanilles (1797) publicó la descripción de Calycera herbacea, coleccionada en la cordillera del Portillo en los Andes chilenos, siendo éste el primer registro para una especie de la familia. El ejemplar, perteneciente a la colección realizada por Louis Née durante la expedición Malaspina, actualmente se conserva en el herbario del Real Jardín Botánico de Madrid (MA).

Ruiz \& Pavón (1798) publicaron la descripción de la segunda especie de Calyceraceae encontrada en su expedición por el territorio chileno (Scabiosa sympaganthera = Calycera sympaganthera), ubicándola dentro de la familia Dipsacaceae.

A. L. de Jussieu (1803), publicó la descripción de dos nuevas especies y creó sendos géneros: Acicarpha tribuloides y Boopis anthemoides (los ejemplares pertenecían a la colección de Commerson de los alrededores de Buenos Aires y Montevideo). Reconoció la afinidad de estas especies con Calycera herbacea y reasignó Scabiosa sympaganthera al recién creado género Boopis (= Boopis balsamitaefolia). Reconoció las similitudes entre los géneros Acicarpha y Gundelia, con lo que ubicó al nuevo grupo como cercano a las Compositae. Se refirió a este grupo como "falsas Cynarocephalae" y consideró que podría tratarse de una nueva familia o de una nueva sección dentro de Cynarocephalae.

Robert Brown publicó en 1817 (Hardwicke, 1867) su descripción de la segunda especie reconocida para el género Acicarpha, Acicarpha spathulata, en base a un ejemplar coleccionado en Brasil por Sellow. A su vez, realizó una breve discusión acerca de las especies de Calyceraceae conocidas hasta ese momento y, por primera vez, otorgó al grupo la categoría taxonómica de familia con el nombre Calycereae, situándolo entre Dipsaceae y Compositae.

A. H. Cassini publicó en 1819 (King \& Dawson, 1975) la primera descripción completa del grupo y de las especies conocidas hasta ese momento. Propuso el nombre Boopideae para la familia, describió sus caracteres diagnósticos vegetativos y florales, señaló sus diferencias y similitudes con Synanthereae (Asteraceae) y con Dipsaceae 
(Dipsacaceae). Propuso el nombre Cryptocarpha en reemplazo de Acicarpha, en atención a su revisión de los caracteres diagnósticos del género.

Richard (1820) realizó la segunda revisión y descripción de la familia, incluyendo todas las especies conocidas hasta ese momento. Describió detalladamente las estructuras morfológicas vegetativas y reproductivas a las que acompañó una lámina por especie. Escogió el nombre Calycereae (ya utilizado por R. Brown) con preferencia a Boopideae (propuesto por Cassini en 1818) por ser el nombre adoptado por De Candolle en su serie de familias, por ser el nombre derivado del género más antiguo y por ser más confiable la etimología de Calycera que la de Boopis (además, en ese momento los frutos de Boopis eran desconocidos).

Lessing (1831a, b), describió dos nuevas especies del género Boopis para el territorio chileno (Boopis alpina, Boopis leucanthema) y dos especies del género Acicarpha para Brasil (Acicarpha bupleuroides, Acicarpha procumbens), a partir de ejemplares pertenecientes a las colecciones de Poeppig y Sellow que la expedición Romanzoff consiguiera a su paso por Sudamérica.

Meyen (1834), describió una nueva especie de Calycera para los Andes Chilenos, en su viaje al volcán San José, remontando la cuenca del río Volcán (Domeyko, 1859).

De Candolle (1836) realizó la tercera revisión de la familia incluida en el Prodromus Systematis Naturalis, donde describió el nuevo género Gamocarpha, basándose en el caracter diagnóstico "presencia de páleas (brácteas del receptáculo) fusionadas formando celdas".

Turczaninow (1848) creó el género Leucocera, en base a un ejemplar de Boopis leucanthema (sensu Miers en Contr. Bot., vol. 2: 28. 1860-1869) coleccionado por T. Bridges en Colchagua (Chile), siendo los caracteres diagnósticos más sobresalientes la tetramería floral y la abundante pubescencia villosa de la planta. Posteriormente, Reiche (1900) recategorizaría Leucocera como un subgénero de Calycera, criterio aceptado actualmente por Hellwig (2007).

La primera revisión de Calyceraceae de Chile realizada por E. J. Rémy (1848) en el marco del atlas dirigido por Claudio Gay, reportó otras dos nuevas especies: Boopis scapigera y Calycera eryngioides. Otro aporte importante a la taxonomía del grupo en Chile fue el trabajo realizado por R. A. Philippi durante la segunda mitad del siglo XIX (1856, 1860, 1861, 1862a, 1862b, 1864, 1865, 1870, 1872, 1891, 1894). Philippi 
describió gran parte de los taxones de Calyceraceae presentes en ese país, incluyendo el género Moschopis, después nombrado erróneamente Moschopsis [Philippi en Anales Univ. Chile 27 (3): 334. 1865].

Examinando el ejemplar tipo de Boopis australis, un nuevo taxón del extremo austral de Sudamérica, Grisebach (1854) creó el género Acarpha basado en el número de brácteas del involucro (10-12 brácteas), la ausencia de brácteas del receptáculo, las cipselas prismáticas, rugosas en la base y las glándulas del tubo estaminal (aunque estos dos últimos caracteres no son exclusivos de Acarpha).

La cuarta revisión general de la familia fue llevada a cabo a principios de la década de 1860 por J. Miers y publicada en el segundo volumen de su obra Contributions to Botany (Miers, 1860-1869). En ella, se realizó una breve reseña de la historia de Calyceraceae, se discutieron sus afinidades taxonómicas y sus similitudes y diferencias con Asteraceae, se analizaron los caracteres de dos nuevos géneros (Anomocarpus, Nastanthus) y se describieron e ilustraron los taxones existentes hasta el momento, así como nuevas especies. Debido a la inconsistencia de los caracteres diagnósticos se sinonimizó Acarpha bajo Boopis, postura que aceptó la mayoría de los sucesivos revisores (Bentham \& Hooker, 1873; Müller, 1885; Philippi, 1894; Reiche, 1900; Hicken, 1919; Pontiroli, 1963; Reitz, 1988; Chiapella, 1999b; Zanotti \& Pozner, 2008). Sin embargo, Stapf (1929 [1932]) distinguió el género Acarpha como un taxón correcto. Argumentó que los caracteres de Boopis australis, basónimo de la especie tipo Acarpha australis, son equivalentes a los presentados por Miers (1860-1869) como caracteres diagnósticos de Nastanthus y, por lo tanto, Nastanthus caería bajo la sinonimia de Acarpha, que tiene prioridad. De acuerdo a Stapf, Miers tuvo acceso al material tipo de Acarpha, pero malinterpretó los caracteres. Stapf propuso, entonces, transferir todas las especies que hasta el momento pertenecían al género Nastanthus al género Acarpha. Este criterio nomenclatural es el que actualmente siguen varios herbarios europeos, entre los que se cuentan el del Real Jardín Botánico de Edimburgo (E) y el de los Reales Jardines Botánicos de Kew (K), en el Reino Unido.

Durante el siglo XIX aparecieron, además, algunas publicaciones que aportaron otros puntos de vista a los criterios nomenclaturales convencionales, señalando datos interesantes, comentando información complementaria y, a veces, produciendo confusiones. Cabe mencionar los trabajos de: a) Weddell (1857 [1858]) acerca de la flora andina (ilustrada), donde describió algunas de las especies conocidas para Chile, 
Perú y Bolivia, b) Asa Gray (1861) acerca de una nueva descripción de Acicarpha crassifolia, con comentarios acerca de nuevos caracteres y la nueva combinación Boopis crassifolia, y c) Hieronymus (1881) quien describió dos nuevos taxones de Calyceraceae e hizo notar la plasticidad morfológica del género Nastanthus: [...] "es muy posible que esta especie; como igualmente todas las de Nastanthus propuestas por Miers, no sean más que diversas formas de una misma especie muy variable, que en tal caso debería tener el nombre de Boopis scapigera [...] por ser éste el más antiguo dado a una de las formas. El señor Weddell [...] que es de esta opinión une a ella como variedad el Nastanthus ventosus (Miers) syn. Calycera ventosa (Meyen). Los señores Bentham y Hooker [...] están conformes en este sentido.” [...] [Hieronymus en Bol. Acad. Nac. Córdoba 4 (1): 32. 1881].

La revisión de Reiche (1900), fue la primera para la flora de Chile en presentar una clave para la identificación de las especies. Además de definir nuevos taxones (Gamocarpha selliana, Calycera foliosa, Boopis graminea var. bracteata) recategorizó al género Leucocera como un subgénero de Calycera. La misma revisión fue reimpresa unos años más tarde (Reiche, 1902).

La primera revisión de Calyceraceae para Argentina fue presentada por Hicken (1919) en el marco de una conferencia dictada en la Primera Reunión Nacional de la Sociedad Argentina de Ciencias Naturales.

La segunda revisión de la familia para Argentina estuvo a cargo de Pontiroli (1963), quien fue la primera en confeccionar una clave para las especies de este país. Además de contar con novedades nomenclaturales y una nueva variedad (Boopis australis var. hickenii), el trabajo fue uno de los primeros en presentar un compendio de los conocimientos que, hasta ese momento, se tenían acerca de la morfología general, la inflorescencia, la flor, el polen y la anatomía de Calyceraceae. Otras revisiones importantes relacionadas con la flora argentina, fueron las que corresponden a Flora de Jujuy y Flora Patagónica, editados por el INTA (Pontiroli, 1993; Chiapella, 1999a, respectivamente), y la lista florística publicada en el Catálogo de las Plantas Vasculares de la República Argentina (Chiapella, 1999b). Por su parte, Zanotti \& Pozner (2008) ampliaron y revisaron esta lista florística en el marco de la Flora del Cono Sur.

La última revisión a cargo de Hellwig (2007), ofrece un compendio ampliado de las características de la familia en lo referente a: morfología, anatomía, inflorescencia y estructura floral, anatomía floral, embriología, morfología de polen, cariología, 
polinización, fruto, semilla y dispersión, fitoquímica, distribución y hábitat, relaciones taxonómicas y filogenia, e importancia económica. Además, se discute la posición taxonómica de Calyceraceae en relación al resto de la familia del clado Asterales y se ofrece una revisión nomenclatural de los géneros. De acuerdo a Hellwig (2007), Calyceraceae cuenta con sólo cuatro géneros aceptados: Acicarpha, Boopis, Gamocarpha y Calycera. Los géneros Nastanthus y Moschopsis quedan sinonimizados bajo Boopis porque no existen caracteres diagnósticos que los diferencien.

\subsection{Morfología}

\subsubsection{Hábito y forma de vida}

Las especies de Calyceraceae son principalmente plantas herbáceas, sólo dos especies, Boopis anthemoides Juss. y Calycera calcitrapa Griseb., llegan a desarrollar portes subarbustivos. Las diversidad de formas de vida adoptadas por la familia varía entre caméfitos, terófitos, geófitos y hemicriptófitos, siendo estas dos últimas las más frecuentes de las Calyceraceae, incluyendo aproximadamente el $60 \%$ de las especies [sólo en Boopis castilloni (Hicken) Pontiroli y Boopis australis Decne. pueden hallarse alternativamente geófitos y hemicriptófitos]. En segunda instancia se hallan los caméfitos y los terófitos con aproximadamente el $20 \%$ de las especies cada uno. Si bien el hábito de cada especie puede variar con la altitud o una distribución geográfica amplia (por ej., de hemicriptófito rosulado a ramoso), la forma de vida es una característica estable para cada especie.

Las formas de vida geófita y hemicriptófita están presentes en su mayoría en especies distribuidas por el Dominio Andino-Patagónico, asociadas con suelos pobres, poco desarrollados (prácticamente sin materia orgánica), arenoso-pedregosos, bien drenados, creciendo en ambientes de altura, expuestas a fuertes vientos, bajas temperaturas y sometidas a nevadas prolongadas en ciertos periodos del año. Muchas de ellas están asociadas a vegas y cursos de agua, o bien se hallan irrigadas indirectamente por agua de deshielo. A modo de excepción, Boopis jürgensii es el único geófito rizomatoso que crece en suelos bien desarrollados (ricos en materia orgánica), en los campos anegados de la selva paranaense, por debajo de los $100 \mathrm{~m} \mathrm{~s}$. m., en clima tropical sin estación seca. 
La forma de vida caméfita está representada, en mayor medida, por especies distribuidas en dos áreas fitogeográficas diferentes: el Dominio Chaqueño, y el Dominio Andino-Patagónico (Distritos Subandino de la Provincia Patagónica y Austral de la Provincia Altoandina). Las especies andino-patagónicas crecen en suelos similares a los anteriores, pero en climas más húmedos. Las plantas desarrollan tallos pseudoleñosos postrados y ascendentes con raíces adventicias que permiten el crecimiento cespitoso del vástago y lo sujetan al suelo suelto, desagregado. Los brotes de renuevo forman tallos verticales a ascendentes, cortos, poco ramificados, que, generalmente, rematan en una inflorescencia terminal. Las especies chaqueñas (Boopis anthemoides y Calycera calcitrapa) también se desarrollan en suelos pobres, con poca en materia orgánica, pero más maduros, con mayor contenido de limos y arcillas. No están sometidas al clima extremo del Dominio Andino-Patagónico, aunque soportan periodos de helada y sequía. Las plantas maduras poseen una base subleñosa a partir de la cual se desarrollan los tallos anuales a partir de los meristemas de renuevo. Por lo general son plantas más ramificadas. Un tercer ambiente relacionado con la forma de vida caméfita se da en los pastizales asociados con los bosques mixtos del norte de la Provincia Subantártica en Chile, donde crece Calycera sympaganthera, caméfito endémico de hábito similar al de las especies chaqueñas, aunque completamente herbáceo. Por otro lado, Acicarpha spathulata y A. obtusisepala son caméfitos herbáceos, endémicos del sur de Brasil y Uruguay, con tallos decumbentes ramificados que crecen en dunas costeras, en un ambiente tropical sin estación seca.

La forma de vida terófita está representada, en su mayor parte, por especies asociadas con suelos lateríticos y ambientes sometidos a periodos de sequía extrema y prolongada. El ejemplo más representativo esta dado por las especies endémicas de la Provincia Chilena Central (Boopis pusilla, Calycera sessiliflora, C. eryngioides y $C$. leucanthema), que se desarrollan sobre alfisoles, vertisoles e inceptisoles, en un clima de estacionalidad marcada con inviernos húmedos y veranos secos. Esto expone a los suelos a ciclos alternados de expansión y contracción, dejando a las raíces en condiciones de escasa ventilación. Sin embargo, existen otras especies que escapan a esta norma. Boopis gracilis, terófito prácticamente exclusivo de Argentina, se distribuye por la meseta patagónica y las primeras estribaciones de los Andes de Mendoza y Neuquén, en los mismos suelos y ambientes descriptos para geófitos y hemicriptófitos. Por otro lado, Acicarpha tribuloides es un terófito arrosetado de amplia distribución en 
el Cono Sur. Debido a su amplio rango de aclimatación puede hallársela en toda el área comprendida entre la llanura pampeana, los Andes del sur de Perú, el Gran Chaco y las selvas paranaense y atlántica. Sus órganos vegetativos adoptan diversas formas dependiendo del suelo y el clima en los que se desarrolla (un acercamiento al estudio fenotípico de estas formas fue desarrollado por DeVore, 1994). Por último, Boopis bupleuroides y $B$. itatiaiae son terófitos con hábito caulescente de distribución restringida a la selva paranaense y las cumbres de la sierras de Itatiaia y Mantiqueira, habitando suelos con alto contenido de materia orgánica, en clima tropical sin estación seca.

\subsubsection{Raíz}

La presencia de raíces contráctiles es una de las características más difundidas dentro de la familia. Es muy probable que estén presentes en todas las especies geófitas y hemicriptófitas, aunque en algunos casos hace falta corroborar este supuesto con nuevas observaciones de campo.

El desarrollo de raíces adventicias es otra característica difundida, especialmente en las especies rizomatosas y en la mayoría de los caméfitos herbáceos.

Se detectó la presencia de raíces gemíferas en Calycera horrida.

\subsubsection{Tallo}

El tallo es una estructura muy variable, incluso dentro de una misma especie o variedad, lo que demuestra la capacidad de varias especies para aclimatarse a los diferentes ambientes en los que puede desarrollarse. Casi siempre es de consistencia herbácea, aunque algunas especies caméfitas pueden desarrollar una base subleñosa. Generalmente son glabros, aunque existe un grupo de especies en los que pueden desarrollar pelos uniseriados de aspecto aplanado. No presentan glándulas externas ni cavidades secretoras (Hansen, 1992). De acuerdo con Carlquist \& DeVore (1998), no existen sustancias resinosas en el tallo. Sin embargo, Hellwig (2007) observó la presencia de una sustancia resinosa en los tallos y las brácteas involucrales de Boopis gracilis. Esta misma sustancia se observó en las hojas de varias especies de Gamocarpha, Boopis y Moschopis, aunque no ha sido posible verificar su presencia en los tallos. 
En las especies ramosas, que engloban a los caméfitos y los terófitos, el tallo va ramificándose simpódicamente desde la base en numerosas ramas secundarias que dan un aspecto más o menos frondoso a la planta. Las ramas principales y secundarias que forman el cuerpo del vástago no suelen crecer erectas desde la base, es más común ver un crecimiento ascendente, procumbente o incluso decumbente en las ramas surgidas de los brotes de renuevo. En los caméfitos subandinos, la inflorescencia terminal probablemente agote por completo el crecimiento de la rama en la que se origina. En Acicarpha (y probablemente en el resto de las especies ramosas) las inflorescencias también son terminales, pero el sistema de ramificación es continuado por un eje secundario que se superpone al precedente (Hellwig, 2007).

Todas las especies geófitas se distinguen por la presencia de rizomas. Estos pueden ser horizontales o verticales. Los rizomas horizontales se presentan en especies que se propagan formando tapices de brotes aéreos rosulados o caulescentes más o menos cespitosos. En Boopis castilloni, B. australis, B. graminea y B. chubutensis, son estructuras irregulares, poco ramificadas, con catafilos triangular-lanceolados, y entrenudos visibles por la presencia de la cicatriz foliar de los catafilos. En otras especies, los rizomas están mucho más ramificados: Boopis filifolia se caracteriza por poseer abundantes rizomas muy delgados, lineares, con catafilos poco distinguibles, cubiertos por un exudado glutinoso que deja parte del sustrato adherido a la superficie; B. patagonica posee rizomas similares pero gruesos y blancos, sin exudado glutinoso; Gamocarpha alpina y G. gilliesii poseen numerosos rizomas horizontales que brotan de la base de los brotes aéreos más desarrollados, y desarrollan un gran número de raíces adventicias. Por su parte, Calycera crassifolia (en sus dos variedades) posee extensos rizomas delgados, poco ramificados, que desarrollan numerosos brotes aéreos y pueden cubrir distancias considerables. Los rizomas verticales se presentan en especies que habitan ambientes de alta montaña (Moschopis leyboldi, M. monocephala, M. trilobata, Boopis necronensis, B. pterocalyx) en general asociadas a suelos sueltos pedregosos o arenosos, y laderas de acarreo. Son rizomas que brotan a patir de una base caulinar gruesa enterrada a varios centímetros $(\mathrm{ca} .10 \mathrm{~cm}$ ) de la superficie del suelo. Por lo general, dicha base caulinar descansa sobre una gruesa raíz contráctil. En Calycera pulvinata, no fue posible hallar la base caulinar en ninguno de los ejemplares de herbario examinado. C. pulvinata y $M$. monocephala son las únicas especies en las que pudieron observarse raíces adventicias brotando a partir de los rizomas verticales. 
Sólo se conocen tres especies que desarrollan estolones: Gamocarpha alpina, G. gilliesii y G. selliana. Esta característica sumada a la capacidad de desarrollar numerosos rizomas, posibilita que G. alpina y G. gilliesii lleguen a formar grandes masas cespitosas.

\subsubsection{Hoja}

Al igual que el tallo, es un órgano muy variable. Todas las especies tienen filotaxis espiralada. La hoja es gruesa y a veces algo coriácea, ya que la mayoría de las especies habitan ambientes secos y abiertos, expuestas a pleno sol y sometidas a fuertes vientos. En las pocas especies que habitan ambientes húmedos, la hoja es membranácea. Algunas especies (Boopis pusilla, B. pterocalyx, Calycera leucanthema, C. eryngioides, C. horrida, C. sympaganthera) presentan una pubescencia de tipo villosa o lanosa con pelos aplanados en las hojas; en Boopis pusilla y Calycera eryngioides el grado de pubescencia es muy laxo.

El extremo distal de la hoja es más ancho y se distingue del extremo proximal más estrecho, por lo que se acepta como válido que la lámina se atenúa en un pecíolo, aunque el límite entre ambos no siempre está claramente definido. En algunas especies la hoja es sésil. En las espécies que poseen hojas membranáceas y sésiles, la base foliar suele ser amplexicaule.

La forma básica de la hoja es espatulada para la mayoría de las especies de la familia, aunque lámina puede adoptar diversas formas, que pueden variar incluso dentro de la misma especie. El contorno puede ser entero, oblongo, linear, lanceolado, panduriforme, oval, laciniado, lobado a pinnatisecto o escuarroso-laciniado, con margen entero, dentado o crenado, dientes mucronados o no, y con ápice agudo, obtuso o emarginado, mucronado o no mucronado.

En Moschopis caleofuensis, M. rosulata, M. subandina, M. trilobata, Boopis filifolia, Gamocarpha angustifolia y $G$. dentata lámina se va adelgazando gradualmente hacia el extremo proximal, de manera que la hoja adopta una forma cuneiforme con la base foliar punctiforme. En estas especies el tallo queda cubierto por las bases foliares persistentes. Las hojas de estas especies están cubiertas por un exudado glutinoso.

Algunas especies, como Calycera crassifolia y Acicarpha tribuloides, poseen dos tipos de hojas: las basales pecioladas y las superiores sésiles. 


\subsubsection{Pedúnculo}

Pueden diferenciarse dos tipos básicos de pedúnculos: los más o menos foliosos, poco diferenciados de las ramas distales del tallo, presentes en las especies caulescentes ramosas (caméfitos y terófitos), y los pedúnculos escapiformes, en especies geófitas, hemicriptófitas y algunos caméfitos (Moschopis caleofuensis, M. ameghinoi, Gamocarpha angustifolia). Calycera sessiliflora es la única especie de Calyceraceae con pedúnculos nutantes. Existen especies con pedúnculos pubescentes (Boopis pterocalyx, B. pusilla, Calycera leucanthema, C. horrida, C. sympaganthera, $C$. eryngioides).

\subsubsection{Inflorescencia: estructura general}

La inflorescencia de Calyceraceae ha tenido diversas interpretaciones a lo largo de la historia de la familia. Diversos autores la han descripto como cabezuela, capítulo, agregado de unidades cimosas o capítulo terciariamente condensado (cf. Pozner et al., 2012; y la bibliografía allí citada). Debido a que la inflorescencia adopta una gran diversidad de formas dentro de la familia, las primeras generalizaciones no alcanzaron para dar una explicación unívoca de la estructura, y las interpretaciones alternativas posteriores fueron referidas a un género particular o a un grupo de géneros, con lo que quedaron reducidas a explicaciones parciales.

Pozner et al. (2012) lograron aunar criterios al proponer una nueva interpretación de la estructura de la inflorescencia para Calyceraceae, basados en la evidencia filogenética obtenida a partir del clado MGCA. Dicha interpretación plantea que la inflorecencia de Calyceraceae, el cefalodio, es una forma derivada de la inflorescencia de tipo tirsoide basal, ya que el meristema apical se agota en una flor terminal, a diferencia de la inflorescencia de tipo tirso cuyo meristema apical permanece abierto. Esta forma tirsoide, se interpreta a la vez como un botrioide, debido a que las ramas laterobasales de la inflorescencia se encuentran muy reducidas o suprimidas. El cefalodio de las Calyceraceae es un tirsoide/botrioide que combina una porción terminal de flores solitarias (en la que queda incluida la flor terminal) con un conjunto de grupos cimosos laterobasales (cada uno de los cuales posee una flor terminal propia) sobre una estructura axial fuertemente condensada, conocida como receptáculo.

Este patrón básico varía dentro de las distintas especies de la familia, dependiendo de las partes constitutivas que se manifiesten o no en la construcción de la 
inflorescencia. En todo el género Acicarpha, algunas especies del género Boopis (B. bupleuroides, B. itatiaiae, B. gracilis, B. pusilla) y Calycera sessiliflora los grupos cimosos no se expresan y el cefalodio queda reducido a la porción terminal de flores solitarias y a la flor terminal. En Moschopis caleofuensis, M. rosulata, M. subandina, M. trilobata, Boopis filifolia, B. patagonica, Gamocarpha angustifolia y G. dentata lámina y en casi todo el género Nastanthus los grupos cimosos son muy numerosos y pueden distinguirse los periféricos, internos al involucro central, de los externos al involucro central, los cuales pueden desarrollar un involucelo propio. Es sencillo interpretar los grupos de flores más externos del cefalodio terminal como grupos cimosos pues son muy similares a los desarrollados sobre el receptáculo y no poseen un involucelo. A medida que se alejan del cefalodio terminal, los grupos cimosos desarrollan su propio involucelo y producen numerosas flores (hasta 80) y pueden confundirse con nuevos cefalodios independientes que repetirían el patrón del cefalodio terminal. Aunque este es un aspecto difícil de discernir, se mantiene aquí la interpretación de grupo cimoso, pues no se les observa una zona central de flores solitarias.

En algunas especies como casi todos los Moschopis, Boopis patagónica, B. filifolia y Gamocarpha dentata, entre otras, los grupos cimosos periféricos internos están ausentes y sólo se manifiestan los externos al involucro. En el género Nastanthus y en varias especies de Gamocarpha la porción terminal de flores solitarias está representada por unas pocas flores, mientras que en Boopis castilloni queda reducida a la flor terminal. En Boopis necronensis la porción terminal de flores solitarias está totalmente suprimida y el cefalodio sólo está formado por grupos cimosos [si bien no se ha observado la flor teminal en inflorescencias con grado de desarrollo avanzado, existe la posibilidad que la flor terminal exista pero sea visible sólo durante las etapas tempranas del desarrollo de la inflorescencia, como sucede en Acicarpha tribuloides (Pozner et al., 2012)]. En Moschopis subandina tampoco se ha confirmado la presencia de una flor terminal, pero el material disponible para estudio ha sido insuficiente.

\subsubsection{Inflorescencia: brácteas}

Las brácteas que acompañan a las flores solitarias y a los grupos cimosos pueden estar morfológicaent muy diferenciadas, o no, de las hojas vegetativas o nomofilos. De acuerdo a la posición que ocupen y su diferenciación morfológica, pueden reconocerse 
dos tipos: las brácteas del involucro, y las brácteas del receptáculo (también conocidas como páleas).

El involucro es una estructura verticilada o espiralada, uniseriada, formada por las brácteas tectrices de los grupos cimosos más periféricos (o de las flores solitarias más periféricas cuando faltan los grupos cimosos) del cefalodio, que nacen de un mismo nudo o de nudos muy próximos entre sí y rodean a las flores pertenecientes a un mismo receptáculo. Esta estructura adopta numerosas formas dependiendo de la especie. Las brácteas del involucro pueden estar libres entre sí o presentar una porción fusionada o gamófila basal reducida a desarrollada, de modo que las brácteas del involucro se observan como lóbulos laciniados, rectangulares o triangulares, y suelen describirse en la literatura taxonómica como "brácteas involucrales fusionadas", si bien la porción fusionada del involucro y los lóbulos no son completamente homólogos. La porción fusionada puede ser rotácea, pateliforme, cupuliforme o campanulada. En algunas especies, como Moschopis subandina y M. caleofuensis, la transición de nomofilos a brácteas es tan progresiva que resulta imposible delimitar un involucro bien diferenciado. La misma dificultad sucede cuando no hay una diferencia morfológica neta entre brácteas del involucro y del receptáculo, como sucede en Moschopis monocephala, Calycera pulvinata y Nastanthus caespitosus (si bien en las dos últimas especies pueden distinguirse involucros parciales en inflorescencias secundarias).. En estos casos es común que la porción gamófila falte por completo y las brácteas se vean libres entre sí desde su base (como en Moschopis leyboldi y M. trilobata), aunque puedan desarrollar una porción fusionada durante la maduración de los frutos (como en Acicarpha y de Calycera herbacea var. herbacea).

En especies con grupos cimosos externos al involucro central, cada grupo está acompañado por su bráctea tectriz. Dichos grupos cimosos pueden quedar fusionados a la cara externa del involucro central, en cuyo caso la bráctea tectriz también está fusionada delimitando una celda. Estos grupos cimosos externos pueder estar pecunculados o no, y pueden presentar un involucelo (presumiblemente de origen profilar). Ejemplos de este último caso se encuentran en algunas especies de los géneros Moschopis (M. caleofuensis, M. subandina, M. rosulata) y Nastanthus (N. compactus, N. scapigerus, $N$. ventosus)

Por dentro del perímetro que define el involucro pueden desarrollarse brácteas conocidas como páleas o brácteas del receptáculo. Por lo general, corresponden a las 
brácteas tectrices de los grupos cimosos internos y de las flores solitarias centrales del cefalodio, que a veces también pueden desarrollar un profilo (Pozner et al., 2012). Las brácteas del receptáculo pueden adoptar distintas formas dependiendo de la especie, aunque las más comunes son linear, espatulada y triangular. Los profilos adoptan forma subulada y su tamaño disminuye centrípetamente. En Gamocarpha alpina, G. gilliesii, G. selliana y Boopis multicaulis las brácteas del receptáculo están fusionadas por sus bordes entre sí, y con las brácteas del involucro, delimitando celdas. En Boopis australis, B. graminea, B. chubutensis, B. raffaellii existen brácteas periféricas fusionadas por uno de sus bordes a las brácteas del involucro, aunque sin llegar a delimitar celdas.

En especies con pedúnculos pubescentes, las brácteas involucrales y del receptáculo, por lo general, también presentan el mismo indumento (Boopis pterocalyx, Calycera leucanthema, $C$. horrida, $C$. sympaganthera), aunque existen excepciones ( $B$. pusilla, C. eryngioides).

\subsubsection{Inflorescencia: receptáculo}

Es la estructura soporte sobre la que se desarrollan las flores y las brácteas. Se forma por supresión completa de la elongación de los entrenudos de los ejes de la inflorescencia, como sucede en Acicarpha, aunque podría ser el resultado de modificaciones más profundas ápice meristemático de la inflorescencia (cf.Pozner et al., 2012).

El receptáculo adopta distintas formas y, en mayor o menor medida, es acrecente durante la madurez de los frutos. Por esta razón se distingue en esta tesis entre la forma del receptáculo en el momento de la antesis, de la forma del receptáculo durante la madurez de los frutos. Durante la antesis, la forma más frecuente del receptáulo es la punctiforme, más o menos cónico o esférico, aunque muy reducido, que está presente en casi todas las especies de Calyceraceae. El receptáculo columnar en la antesis es un caracter distintivo del género Acicarpha.

Durante la fructificación, un receptáculo punctiforme puede elongarse y volverse columnar, globoso o discoideo. El receptáculo se vuelve durante la fructificación en Boopis itatiaiae (y es probable que ocurra lo mismo en B. jürgensii; sin embargo, no es posible definir el estado del receptáculo durante la antesis para esta especie, ya que sólo se conoce por su colección tipo en estado fructificado). 
En las especies del género Nastanthus y algunas especies del género Boopis como B. pterocalyx y B. necronensis, el receptáculo punctiforme es muy acrescente y adopta una forma globosa. En Boopis castilloni, el receptáculo es punctiforme en la antesis, tornándose discoideo durante la madurez de los frutos. Es la única especie de la familia en la que el receptáculo sólo crece en diámetro y no en longitud.

\subsubsection{Flor}

La flores son actinomorfas y pentámeras en la mayoría de la familia (ocasionalmente hexámeras como una teratología), aunque existen tres especies con flores tetrámeras (Boopis bupleuroides, B. itatiaiae y Calycera leucanthema). Las flores de Calyceraceae poseen dos partes bien diferenciables (Erbar, 1993): 1) un perianto epígino, que contiene un hipanto corolino (usualmente denominado tubo corolino, tanto en Calyceraceae como en Asteraceae), la corola y el androceo, está y rodeando al estilo; 2) y un ovario ínfero coronado por el cáliz (desde un punto de vista formal, el cáliz también forma parte del perianto; sin embargo en las Calyceraceae, el cáliz está tan íntimamente relacionado al ovario, y suele ser tan poco desarrollado en la antesis, que en esta tesis se considerará perianto al conjunto de hipanto y corola).

El hipanto puede ser tubuloso o filiforme, de longitud variable, que nace del extremo superior-central del ovario, y se continúa en una corola simpétala. En la corola puede distinguirse un tubo basal de forma y longitud variables, coronado por los lóbulos corolinos lanceolados o triangulares, planos o cuculados, con el ápice agudo u obtuso, recto o uncinado, con estivación valvar. El androceo nace de la unión entre el hipanto y la corola, con los estambres inclusos o semi exsertos, isómeros, alternos a los lóbulos corolinos. Los filamentos pueden ser largos hasta subnulos, y surgen de un tubo estaminal reducido o bien desarrollado. Las anteras son oblongas con dehiscencia longitudinal introrsa, lateral y basalmente connadas. Las tecas pueden tener una base obtusa o sagitada, y el conectivo puede estar apiculado o no. Existen cinco glándulas nectarígenas $(=$ nectarios $)$ ovoideas que pueder estar ubicadas en tubo estaminal (generalmente en la base), o bien entre el nacimiento del tubo estaminal y el hipanto. Por esta razón, el tejido glandular de los nectarios puede ser de origen estaminal o hipantial, y estar desarrollado en mayor o menor medida, de modo que las glándulas sean apenas visibles, visibles individualmente o sean casi coalescentes formando un engrosamiento contínuo. Existen especies en las que las glándulas nectarígenas no 
fueron detectadas, como en Boopis bupleuroides. El perianto y el androceo rodean por completo al estilo terete que remata en un estigma simple, punctiforme a levemente capitado, indiviso, papiloso. El estilo y la estructura del androceo están adaptados a la presentación secundaria del polen (Leins \& Erbar, 1990; Erbar \& Leins, 1995), de modo que al comienzo de la antesis, el polen se libera en el espacio delimitado por el anillo de anteras connadas, justo por encima del estigma. A medida que la antesis progresa, el estilo se alarga y el estigma empuja el polen a través del anillo formado por las anteras a modo de pistón, hasta que el estilo queda completamente exserto portando el contenido de polen de la flor sobre el estigma (no receptivo) bien expuesto por encima del nivel de las flores.

Sobre la base de las diferentes proporciones del hipanto y la corola, y la posición de las glándulas nectarígenas, pudieron identificarse dos tipos florales extremos, con algunos casos intermedios (Fig. 1): el tipo floral A, con hipanto reducido, corola bien desarrollada y nectario estaminal; y el tipo floral B con hipanto desarrollado, corola menor y nectario hipantial.
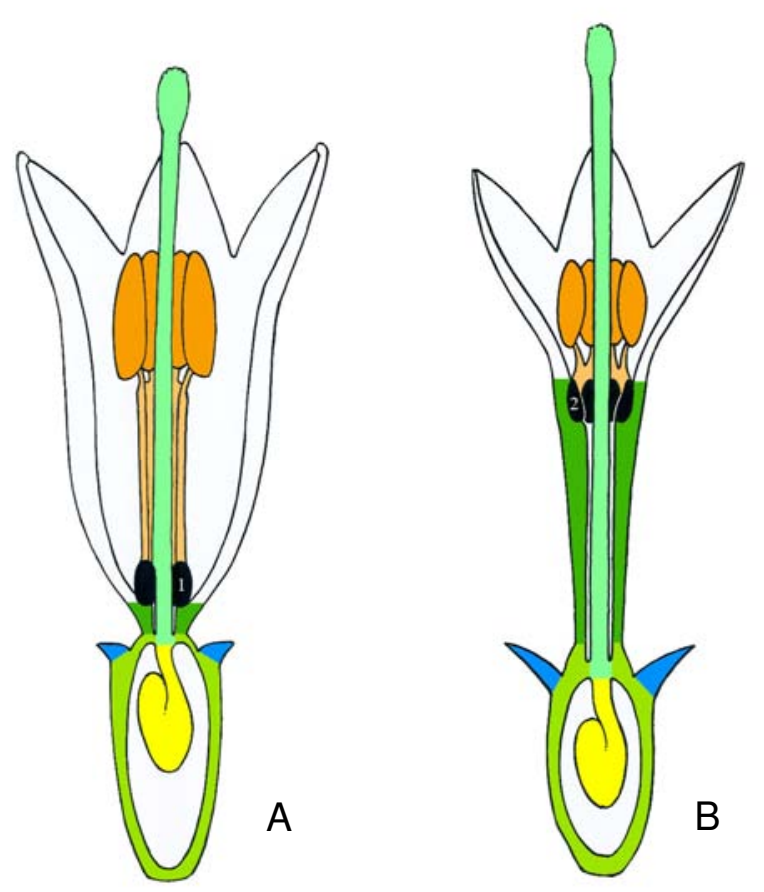

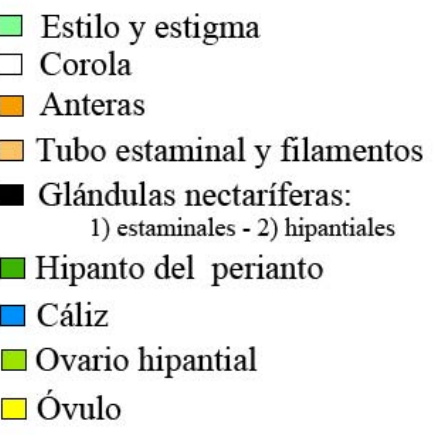

Figura 1. Esquema comparativo de las formas florales básicas. Los órganos homólogos figuran con el mismo color. 
Algunas especies de Calyceraceae presentan un dimorfismo o polimorfismo floral incipiente, que puede clasificarse en dos grandes tipos: funcional y estructural. Dentro del polimorfismo funcional se encuentran las especies de Acicarpha (Miers, 1860-1869; Pontiroli, 1963; Chiapella, 1999a) que presentan flores perfectas y funcionalmente estaminadas en la misma inflorescencia. Las flores estaminadas poseen un gineceo completo, pero el óvulo aborta tempranamente, auque sin embargo el estilo se alarga para permitir la presentación del polen. Este mismo tipo de dimorfismo funcional parece presentarse en algunas especies de Nastanthus (Zavala-Gallo et al., 2010) pero aún necesita ser comprobado.

El polimorfismo o dimorfismo estructural se observa especialmente en las flores de las especies de Calycera. Algunas especies de este género poseen flores con cáliz reducido y otras con cáliz espiniforme en la misma inflorescencia. Dentro de las especies de Calycera con flores netamente dimórficas, algunas poseen sépalos aciculares (C. herbacea) y otras foliosos y pungentes (C. crassifolia). Otras especies de Calycera no tienen un dimorfismo neto sino una variación gradual desde flores con sépalos espiniformes hasta flores con sépalos escuamiformes, reducidos (p. ej. $C$. leucanthema). Además, los diferentes morfos florales tienen una posición fija dentro de la inflorescencia según cada especie (Denham et al, enviado).

Si bien el perianto de la mayoría de las especies se marchita y cae durante la madurez de los frutos, existen casos excepcionales donde es persistente. En Nastanthus compactus, el perianto es acrescente, debido al desarrollo de un tejido esponjoso similar al de las carenas de los frutos, y persiste sobre la cipsela. En Boopis gracilis var. lazulina, el perianto también es acrescente, inflándose durante la madurez de los frutos, aunque la fragilidad del hipanto hace que se desprenda con facilidad de la cipsela.

El hipanto suele ser más o menos verdoso, mientras que la corola es usualmente blanca. En algunos casos, el color de la corola puede ser un caracter diagnóstico, como ocurre en Calycera herbacea var. herbacea, C. herbacea var. sinuata y Nastanthus scapigerus.

El ovario unicarpelar, unilocular, sésil, es de origen hipantial (Leins \& Erbar, 2010) y contiene un único óvulo anátropo, péndulo (Pontiroli, 1963). El ovario puede ser cilíndrico a lacrimiforme, con el extremo superior-central atenuado en el estilo y la base del perianto. Su superficie se encuentra recorrida, desde su base hasta la base del ápice, por cuatro o cinco carenas longitudinales, desarrolladas en mayor o menor 
medida y rematadas por el cáliz. Los sépalos poseen estivación abierta, valvar o imbricada, y pueden ser suborbiculares, lanceolados, dentiformes, o espiniformes; planos, cuculados o inflados; membranáceos, foliosos, corchosos o endurecidos; con margen entero, dentado o sinuado, y ápice obtuso, agudo o emarginado, mucronado o no mucronado. Tanto el cáliz como las carenas pueden ser acrescentes durante la maduración de los frutos.

\subsubsection{Fruto}

Todas las especies de Calyceraceae producen un fruto seco, indehiscente y uniseminado derivado de un ovario ínfero, razón por la cual se lo denomina cipsela.

Las cipselas de las Calyceraceae son prismáticas en la mayoría de las especies, con el pericarpio recorrido por tres, cuatro o cinco carenas longitudinales, que pueden estar muy reducidas hasta bien desarrolladas como alas o costillas, de dimensión y consistencia variables, coronadas por los sépalos persistentes.

La superficie de las cipselas puede ser lisa o estar ligeramente arrugada en forma transversal, en cuyo caso los pliegues del pericarpio son provocados por el desecamiento durante la última etapa de la maduración (Reiche, 1900 [1901]) y coinciden con grandes espacios aéreos de origen ezquizógeno (Zanotti \& Pozner, 2007).

De acuerdo con Pontiroli (1963), los sépalos acrescentes son aciculares en Boopis, obtusos en Nastanthus, y en Calycera y Acicarpha crecen y asumen la forma de espinas rígidas, a veces de longitud desigual y muy prolongadas en Calycera. Sin embargo, la forma y el grado de desarrollo que alcanzan los sépalos no son uniformes para cada género, pudiendo variar entre frutos de un mismo receptáculo. Este último es el caso de Calycera, originalmente interpretado como un dimorfismo de frutos (Miers, 1860-1869; Reiche, 1900, 1902), con cipselas internas (centrales o apicales) prismáticas, arrugadas e inermes (con sépalos poco acrescentes o vestigiales) y cipselas exteriores (periféricas o basales) turbinadas y espinosas (con sépalos muy acrescentes, espiniformes), sobre el mismo receptáculo. Autores recientes (Pontiroli, 1963; Chiapella 1999a; Hellwig, 2007) han seguido el mismo criterio. Sin embargo, Denham et al. (enviado) han observado que algunas especies de Calycera no tienen un dimorfismo neto sino una variación gradual desde flores y frutos con sépalos espiniformes hasta flores y frutos con sépalos escuamiformes, reducidos (p. ej. C. leucanthema). 
De acuerdo con Reiche (1900 [1901]), la estrategia de dispersión de los frutos de Calyceraceae es de dos tipos. Por un lado los frutos de Nastanthus están especialmente dotados, algo menos los de Boopis, con costillas o alas que actuarían como órganos de vuelo y, dado el caso, también facilitan la dispersión por el agua (Hellwig, 2007); por lo menos de aquellas cipselas equipadas con un pericarpio esponjoso y alas anchas que actúan como flotadores. Otro medio de dispersión está constituido por el cáliz espiniforme (como en Calycera) adecuado para la epizoocoria.

\subsection{Tratamiento taxonómico}

Se revisó la nomenclatura de todas las especies de Calyceraceae, con un total de 192 binomios revisados. Sobre la base de caracteres de la inflorescencia, de la flor y del fruto se reconocieron 47 especies y 6 variedades que constituyen las terminales para los estudios filogenéticos.

Se encontraron y describieron dos nuevas especies endémicas de Argentina: Boopis pterocalyx (de Sierra del Nevado, Pcia. de Mendoza) y Boopis necronensis (de la laguna Mulas Muertas, Reserva Pcial. "Laguna Brava”, Pcia. de La Rioja), publicadas en Brittonia (Zavala-Gallo et al., 2011). Se seleccionaron 21 lectotipos (propuestos o publicados) y un neotipo (propuesto). Se lectotipifica un género (Anomocarpus, propuesto). Se propone una nueva combinación. Se establecen 25 nuevos sinónimos (propuestos o publicados), se enmienda una especie y se establecen dos nombres dudosos. Para seis nombres se citan los sintipos hallados o previamente citados. Véase apartado 3.3.3: Resumen de la revisión nomenclatural.

Se hallaron un total de 35 ejemplares tipo nomenclaturales de Calyceraceae inadvertidos dentro de la colección general de los herbarios consultados y en páginas web (bases de datos en línea).

3.3.1 Clave artificial para las especies de la familia Calyceraceae

0) Flores tetrámeras. (1)

0’) Flores pentámeras. (3)

1) Hierbas laníferas. Cefalodios con grupos cimosos y flores solitarias. 
1’) Hierbas glabras. Cefalodios sin grupos cimosos, únicamente con flores solitarias. (2)

2) Hierbas con hojas panduriformes. Brácteas involucrales con la base ensanchada. Receptáculo no acrescente durante la maduración de los frutos.

\section{Boopis bupleuroides}

2') Hierbas con hojas espatuladas, oblanceoladas o elípticas. Brácteas involucrales sin base ensanchada. Receptáculo columnar acrescente $(5 \mathrm{~mm})$ durante la maduración de los frutos.

\section{Boopis itatiaiae}

3) Subarbustos glabros, ramosos, hasta de 50-60 cm de altura. (4)

3') Hierbas glabras o pubescentes (vilíferas o lanosas), generalmente menores de $50 \mathrm{~cm}$ de altura. (5)

4) Sépalos espinescentes endurecidos durante la antesis. Hojas lanceolado-oblongas con margen dentado-mucronado. Involucro rotáceo con lóbulos triangulares. Cefalodios con aproximadamente 15 grupos cimosos 2-4 floros. Cipselas polimórficas.

Calycera calcitrapa

4') Sépalos espinescentes (cuando están presentes) no endurecidos durante la antesis. Hojas lineares a pinnatifidas o pinnatisectas, laciniadas, con margen entero. Involucro rotáceo, pateliforme o crateriforme y lóbulos triangulares, a veces laciniados. Cefalodios con 5-6 grupos cimosos 3-5 floros. Cipselas uniformes.

Boopis anthemoides

5) Presencia de dimorfismo floral (flores perfectas y flores estaminadas con ovario vestigial o abortado). Cipselas agregadas en infrutescencias como unidad de dispersión. (6)

5') Ausencia de dimorfismo floral. Cipselas libres no agregadas en infrutescencias como unidad de dispersión. (9)

6) Caméfitos herbáceos caulescentes, rastreros, con raíces adventicias. Hojas espatuladas o lineares con margen entero. Infrutescencias sin cipselas caedizas. (7) 
6') Hierbas anuales rosuladas con brotes aéreos a partir del periodo de floración. Hojas basales espatuladas, y hojas caulinares sésiles subamplexicaules; lámina oblonga a lanceolada, con margen entero, sinuado, crenado, dentado, pinnatifido, pinnatipartido o runcinado, mucronado, con ápice obtuso y mucronado. Cipselas centrales asimétricas, caedizas.

\section{Acicarpha tribuloides}

7) Flores suculentas. Cipselas con sépalos acrescentes no espiniformes, con ápice obtuso.

\section{Acicarpha obtusisepala}

7’) Flores no suculentas. Cipselas con sépalos acrescentes espiniformes cónicos. (8)

8) Hierbas con tallos decumbentes y erectos. Hojas espatuladas con lámina obovada a elipsoidal y margen entero. Involucro con lóbulos espatulados. Cipselas con surcos intercarinales poco visibles, agregados en una unidad de dispersión cónica.

\section{Acicarpha spathulata}

8') Hierbas con tallos procumbentes. Hojas lineares a espatuladas con lámina oblanceolada y margen entero o paucidentado. Involucro con lóbulos estrechamente triangulares. Cipselas con surcos intercarinales muy visibles, agregados en una unidad de dispersión esférica.

\section{Acicarpha procumbens}

9) Hierbas pubescentes (vilíferas o laníferas). (10)

9') Hierbas glabras. (14)

10) Hierbas con pubescencia abundante, detectable a ojo desnudo. Cefalodios multifloros con grupos cimosos internos. (11)

10') Hierbas con pubescencia muy escasa en tallos, hojas y pedúnculos, no detectable a ojo desnudo. Cefalodios paucifloros hasta con 10 flores solitarias, sin grupos cimosos.

Boopis pusilla

11) Hierbas rizomatosas, con brotes aéreos rosulados o con entrenudos cortos. A veces con raíces gemíferas. (12) 
11') Hierbas caulescentes, ramosas. Raíces gemíferas ausentes. (13)

12) Hierbas laníferas con raíces gemíferas. Hojas pinnatisectas. Involucro rotáceo con lóbulos rectangulares. Cipselas multiformes con 3-5 carenas lisas o rugosas, algunos con sépalos acrescentes gruesos, espiniformes, recurvados.

\section{Calycera horrida}

12’) Hierbas vilíferas sin raíces gemíferas. Hojas espatuladas enteras. Involucro pateliforme con lóbulos triangulares. Cipselas uniformes con 5 carenas fusionadas rugosas, con sépalos acrescentes foliáceos, cartáceos, orbiculares, nunca espiniformes.

Boopis pterocalyx

13) Hierbas anuales, pubescencia laxa en órganos vegetativos maduros y abundante en órganos vegetativos en desarrollo. Hojas espatuladas, con lámina lanceolada, margen entero, ligeramente sinuado o crenado-mucronado. Involucro rotáceo, acrescente durante la maduración de los frutos. Cipselas espinosas con sépalos acrescentes cónicoespiniformes, aciculados.

\section{Calycera eryngioides}

13') Hierbas perennes, pubescencia abundante uniformemente distribuida. Hojas espatuladas, con lámina lanceolada, partida con lóbulos triangulares, mucronados, y margen entero. Involucro pateliforme, no acrescente durante la maduración de los frutos. Cipselas espinosas con sépalos acrescentes lanceolado-espiniformes.

Calycera sympaganthera

14) Receptáculo columnar. Hierbas rizomatosas con brotes aéreos simples, de 50-70 cm de altura. Hojas sésiles, lanceoladas a estrechamente ovado-lanceoladas, margen entero a escasamente dentado.

Boopis jürgensii

14') Receptáculo punctiforme, globoso o discoideo; nunca columar. Sin formas exclusivas. (15)

15) Hierbas sin involucro diferenciado (ocasionalmente en cefalodios secundarios de Calycera pulvinata y Nastanthus caespitosus). Exclusivamente rizomatosas, una única especie con raíz napiforme (N. caespitosus). En su mayoría, propios del área andina de 
Chile, Argentina, Bolivia y Perú; sólo una especie del extremo austral de Patagonia. (16)

15') Hierbas con involucro bien diferenciado. Sin formas exclusivas. (20)

16) Hierbas con brotes aéreos rosulados. (17)

16') Hierbas con brotes aéreos erectos a ascendentes, con entrenudos breves, nunca rosulados, escasamente ramificados. (19)

17) Hierbas con láminas foliares casi pinnatisectas, con lóbulos desiguales, obtusos. Raiz napiforme. Longitud del hipanto 2 veces (o más) la longitud de la corola. Lóbulos corolinos planos. Cipselas prismáticas, asimétricas debido al desarrollo desigual de las carenas, 5 carenas longitudinales, gruesas, infladas, frecuentemente 1 a 2 muy reducidas. Sépalos a menudo muy reducidos sobre el fruto. Endémica de la Provincia del Desierto en el norte de Chile.

\section{Nastanthus caespitosus}

17') Hierbas con láminas foliares obovadas u oblongas, con margen dentadomucronado. Raiz no napiforme, a menudo raíces adventicias sobre el rizoma. Longitud del hipanto aprox. igual o menor a la longitud de la corola, nunca el doble. Lóbulos corolinos acinaciformes. Cipselas prismáticas simétricas, con carenas uniformes. Sépalos reducidos o acrescentes sobre el fruto. (18)

18) Cipselas prismáticas multiformes, con 5 carenas connadas rugosas (frutos inermes) o lisas (frutos espinosos), con sépalos vestigiales o acrescentes suborbiculares, triangulares a espiniformes.

\section{Calycera pulvinata}

18') Cipselas uniformes cilíndricas a prismáticas, rugosas, con 5 carenas vestigiales o planas, coronadas por sépalos vestigiales a triangulares, nunca espiniformes.

\section{Moschopis monocephala}

19) Hierbas con hojas espatuladas, membranosas; lámina lanceolada, con margen entero y ápice acuminado. Corola blanca, campanulada, lobada hasta $1 / 4$ de su parte superior; lóbulos ligermante recurvados. Cipselas cilíndricas, lisas. Sépalos dentiformes en el fruto. 
Moschopis leyboldi

19’) Hierbas con hojas espatuladas, coriáceas en el extremo distal; lámina partida, trilobada, con lobos lanceolados a lineares con ápice acuminado. Corola verde, rotácea, partida; tubo reducido; lóbulos recurvados durante la antesis (incurvados durante la maduración de los frutos). Cipselas prismáticas a elipsoidales, rugosas. Sépalos acrescentes lanceolados, de textura membranácea en el fruto. Endémica del extremo austral de Patagonia.

\section{Moschopis trilobata}

20) Flores con hipanto de longitud inferior $(<1 / 5-1 / 3)$ a la de la corola. Corola campanulada, hipocrateriforme o urceolada, con lóbulos de longitud menor o igual a la del tubo. (21)

20') Flores con hipanto de longitud igual o superior a la de la corola. Corola de aspecto rotáceo, con lóbulos de longitud mayor o igual a la del tubo. (31)

21) Hierbas con hojas glutinosas, cuneiformes y cicatrices foliares en los tallos. Tallos ascendentes radicantes o rizomas lineares con brotes aéreos rosulados a erectos. (22)

21') Hierbas con hojas no glutinosas, espatuladas, a veces con la lámina sésil no atenuada en un pecíolo, y sin cicatrices foliares en los tallos. Tallos erectos o ascendentes no radicantes o rizomas gruesos con brotes aéreos caulescentes procumbentes. (26)

22) Hierbas con láminas foliares no lobadas. (23)

22’) Hierbas con láminas foliares lobadas. (24)

23) Hierbas cespitosas bajas de 1-3-(8) $\mathrm{cm}$ de altura, con brotes aéreos rosulados a caulescentes erectos. Rizomas lineares. Láminas foliares plegadas. Cefalodios sésiles con 2-5 grupos cimosos periféricos, los menores portando 1-2 flores pequeñas.

\section{Boopis filifolia}

23') Hierbas de 10-15 cm de altura, con tallos postrados radicantes y ramas ascendentes o erectas. Rizomas ausentes. Láminas foliares de contorno orbicular a elíptico, no plegada. Pedúnculos erectos, terminales, hojosos. Cefalodio con 6-7 grupos cimosos periféricos, los menores portando aproximadamente 12 flores pequeñas. 
24) Hierbas con lámina foliar orbicular a elíptica, plegada, con margen crenulado a pentalobulado. Pedúnculos nulos. Cefalodios sésiles, terminales, con grupos cimosos periféricos, unifloros, sin involucelos profilares.

Gamocarpha dentata

24') Hierbas con lámina foliar partida, plegada, digitada o trilobada a pedada. Cefalodios sobre pedúnculos terminales, con grupos cimosos periféricos multifloros, con involucelos profilares. (25)

25) Hierbas con lámina foliar partida, plegada, trilobada a pedada, con margen revoluto. Cefalodios sobre pedúnculos erectos, terminales, hojosos, largos; con 10-15 grupos cimosos periféricos 1-6 floros.

\section{Moschopis caleofuensis}

25') Hierbas con lámina foliar partida, plegada, digitada, 5-7-lobada, con margen involuto. Cefalodios sobre pedúnculos terminales, hojosos, reducidos, de $0.5-1 \mathrm{~cm}$; con 5-10 grupos cimosos periféricos 8-9 floros.

\section{Moschopis subandina}

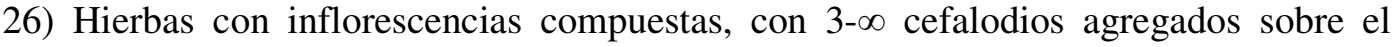
mismo pedúnculo. (27)

26’) Hierbas con inflorescencias simples, pedúnculos monocéfalos. (28)

27) Hierbas crasas, cespitosas, glaucas, caulescentes, de hasta $20 \mathrm{~cm}$ de altura. Inflorescencias sobre pedúnculos teretes. Hojas alternas, espatuladas; lámina suborbicular a oblonga, con margen dentado a escarroso-laciniado y ápice obtuso. Endémica de los afloramientos rocosos del oeste y noroeste de la pcia. de Santa Cruz.

Moschopis ameghinoi

27') Hierbas pequeñas con tallos carnosos, sólidos, más amplio hacia el ápice, rematado en una inflorescencia compuesta, estrechándose hacia la base en una estructura más o menos horizontal. Hojas densamente imbricadas, estrechamente oblongas a oblanceoladas o subespatuladas. Endémica del extremo sudoeste de la isla Gran Malvina. 
28) Hierbas perennes rizomatosas. Cefalodios con grupos cimosos. Endémicas de la pcia. de Santa Cruz. (29)

28') Hierbas anuales caulescentes sin rizomas. Cefalodios sin grupos cimosos. (30)

29) Hierbas glaucas, gruesas, cespitosas, con brotes aéreos caulescentes procumbentes de 2-8 cm de altura. Hojas espatuladas con lámina oblonga, plegada. Pedúnculos teretes breves. Involucro campanulado. Cefalodios laterales y terminales, con grupos cimosos externos ocasionales, 1-2 floros.

\section{Boopis patagonica}

29') Hierbas gruesas, con brotes aéreos ascendentes a rosulados, de 1-8 cm de altura. Catafilos oblongos. Hojas densamente imbricadas, lámina suborbicular. Pedúnculos nulos. Involucro ausente; brácteas del cefalodio libres. Cefalodios terminales, centrales, con 10-15 grupos cimosos 12-floros, rodeados por sendos involucelos profilares con la base soldada.

\section{Moschopis rosulata}

30) Hojas con pecíolo breve y lámina amplia lanceolada, margen entero o dentadomucronado. Pedúnculos breves, nutantes. Involucro campanulado. Cefalodios paucifloros 5-15 floros. Cipselas multiformes, con sépalos lanceolado-pungentes y con sépalos vestigiales. Endémica del centro de Chile.

\section{Calycera sessiliflora}

30') Hojas sésiles o con la lámina atenuada en un pecíolo estrecho de igual longitud; lámina incisa a pinnatifida. Pedúnculos erectos o ascendentes, nunca nutantes. Involucro pateliforme a crateriforme. Cefalodios $20-\infty$ floros. Cipselas uniformes, con sépalos vestigiales o triangulares, nunca pungentes. Andes de Maule (Chile) y Mendoza (Argentina) y Patagonia, hasta el norte de Santa Cruz.

\section{Boopis gracilis}

31) Hierbas con receptáculos punctiformes, ligeramente acrescentes durante la madurez de los frutos. (32)

31') Hierbas con receptáculos más desarrollados, muy acrescentes durante la madurez de los frutos (discoide-meniscoide o globoso). (44) 
32) Brácteas del receptáculo fusionadas entre sí por el margen, formando celdas. Hemicriptófitos rosulados, en su mayoría cespitosos. (33)

32’) Brácteas del receptáculo libres. (36)

33) Lámina foliar oblonga o suborbicular, abierta o plegada, atenuada en el pecíolo, con margen entero. (34)

33’) Lámina foliar oblonga, siempre abierta, con margen partido y lóbulos oblongos, o suborbiculares con margen entero, dentado o crenado. (35)

34) Involucro con lóbulos anchamente triangulares, margen entero y ápice mucronado. Lámina foliar oblonga.

Gamocarpha alpina

34') Involucro con lóbulos triangulares a panduriformes, recurvados, margen entero y ápice mucronado. Lámina foliar suborbicular.

Gamocarpha gilliesii

35) Hierbas cespitosas estoloníferas. Ramificaciones secundarias escasas. Forma caulescente poco conspicua. Ampliamente distribuida en la Provincia Patagónica.

Gamocarpha selliana

35') Hierbas no cespitosas, nunca estoloníferas. Ramificaciones secundarias numerosas. Exclusivamente caulescente. Endémica del pedemonte andino de Chile y Argentina, entre $37^{\circ}-46^{\circ}$ de latitud sur, en la Provincia Subantártica.

Boopis multicaulis

36) Geófitos rizomatosos con brotes aéreos rosulados o caulescentes y raíces delgadas.

36') Hemicriptófitos rosulados, no rizomatosos, con tallos reducidos y raíces napiformes. (40)

37) Rizomas gruesos. Brotes aéreos con base rosulada. Hojas lineares o acintadas a pinnatifidas o pinnatipartidas. Pedúnculos escapiformes monocéfalos. Ocasionalmente, brácteas de los grupos cimosos externos fusionadas al involucro central formando celdas periféricas. Brácteas del receptáculo libres o parcialmente fusionadas al 
involucro por uno de los bordes (sin formar celdas completas). Lóbulos corolinos cuculados. Cipselas prismáticas con sépalos de ápice agudo, nunca pungente ni espinuloso. Plantas de distribución exclusivamente patagónica. (38)

37') Rizomas delgados. Brotes aéreos caulescentes. Hojas basales espatuladas, hojas superiores sésiles, con lámina oblonga a ovales con margen dentado-mucronado a dentado-aristado. Pedúnculos no escapiformes. Cefalodios sin grupos cimosos externos. Brácteas del receptáculo siempre libres. Lóbulos corolinos planos. Cipselas prismáticas, muy estrechos en la base, con sépalos suborbiculares o triangulares, con ápice pungente o espinuloso. Exclusivas de suelos arenosos del Distrito de la Payunia (Provincia Patagónica) y médanos costeros de la costa atlántica argentino-uruguaya.

Calycera crassifolia

38) Hojas lineares o acintadas a pinnatifidas o pinnatipartidas de $0.5-15 \mathrm{~cm}$ de longitud. Brotes aéreos uni o multipedunculados. Cefalodios pedunculados o sésiles. Hipanto de longitud igual o mayor a la de la corola. Endémicas de la Provincia Patagónica. (39) 38') Hojas lineares a estrechamente lanceoladas, de 2-6 cm de longitud. Brotes aéreos unipedunculados. Cefalodios nunca sésiles. Hipanto de longitud menor o igual a la de la corola. Asociada a vegas y cursos de agua en espacios abiertos. Endémica de los Andes de Chile (VIII y IX Región) y Argentina (Neuquén), en el Distrito Austral de la Provincia Altoandina.

Boopis graminea

39) Hojas lineares a pinnatifidas, de $0.5-6 \mathrm{~cm}$ de longitud. Involucro pateliforme. Sépalos lanceolados con ápice acuminado. Oeste de la pcia. de Chubut.

Boopis chubutensis

39') Hojas lineares o acintadas a pinnatifidas o pinnatipartidas de $0.5-15 \mathrm{~cm}$ de longitud. Involucro rotáceo a cupuliforme. Sépalos orbiculares a aciculares. En toda la Patagonia, desde la costa hasta las primeras estribaciones de los Andes.

Boopis australis

40) Sépalos no espinescentes. Cipselas uniformes. (41) 
40’) Sépalos espinescentes endurecidos durante la antesis. Cipselas multiformes, con formas intermedias progresivas.

\section{Calycera herbacea}

41) Corola lobada hasta $1 / 2$ de su parte superior. Cipselas con carenas vestigiales o poco desarrolladas, comprimidas. (42)

41') Corola lobada hasta su base con tubo breve. Cipselas con carenas bien desarrolladas, expandidas.

Nastanthus scapigerus

42) Hojas lineares o acintadas a pinnatifidas o pinnatipartidas. Pedúnculos escapiformes monocéfalos. Ocasionalmente, brácteas de los grupos cimosos externos fusionadas al involucro central formando celdas periféricas. Brácteas del receptáculo libres o parcialmente fusionadas al involucro por uno de los bordes (sin formar celdas completas). Cipselas prismáticas con sépalos de ápice agudo, nunca pungente ni espinuloso. (43)

42') Hojas espatuladas con lámina lobada de margen dentado. Cefalodios sin grupos cimosos externos al receptáculo. Brácteas del receptáculo siempre libres. Cipselas rugosas, con carenas y sépalos vestigiales.

Nastanthus patagonicus

43) Lóbulos foliares oblongos a laciniados con ápice acuminado. Involucro pateliforme con base fusionada y lóbulos triangulares, dentados con margen entero o dentadofimbriado. Perianto hasta de $3 \mathrm{~mm}$ de longitud. Corola lobada hasta 1/4 de la longitud del tubo.

Boopis raffaellii

43’) Lóbulos foliares breves a laciniados con ápice obtuso y mucronado. Involucro con una base fusionada rotácea a cupuliforme, y lóbulos triangulares, laciniados con margen entero o dentado-mucronado, con mucrones cónicos a filiformes. Perianto de $3-7 \mathrm{~mm}$ de longitud. Corola lobada hasta 1/3 - 1/2 de la longitud del tubo corolino.

Boopis australis 
44) Grupo de flores solitarias del cefalodio, ausente o reducido a una única flor terminal. (45)

44’) Grupo de flores solitarias del cefalodio, presente con más de una flor solitaria. (46)

45) Receptáculo discoideo-meniscoide, acrescente en diámetro pero no en volumen. Cefalodios con flor solitaria terminal única, rodeada de numerosos grupos cimosos. Hierba de aspecto caulescente con tallos tendidos o ascendentes y entrenudos largos. Endémica de la sierra de Ambato, pcia. de Catamarca $\left(\mathrm{C}^{\circ}\right.$ Manchao).

Boopis castilloni 45') Receptáculo globoso, acrescente en diámetro pero no en volumen. Cefalodios sin flores solitarias terminales, formados sólo por numerosos grupos cimosos. Hierbas rosulada con entrenudos cortos, escasamente ramificada. Endémica de la cima de los Andes de La Rioja (Reserva Pcial. "Laguna Brava”) y Catamarca.

Boopis necronensis

46) Plantas rosuladas, hojas espatuladas. Inflorescencias terminales y laterales, simples, con pedúnculos escapiformes, diferenciadas, agregadas o parcialmente coalescentes, pero nunca formando inflorescencias compuestas; inflorescencia terminal igual o más grande que las laterales. Base fusionada del involucro desarrollada, 5-12 lóbulos. Plantas de los Andes de Chile y Argentina, y la estepa Patagónica. (47)

46') Plantas no rosuladas, hojas densamente dispuestas a lo longitud del tallo, oblongas a oblanceoladas o subespatuladas. Inflorescencia terminal, compuesta, constituida por cabezuelas basales pedunculadas, que se acortan progresivamente, sésiles y coalescentes a lo longitud del ápice. Base fusionada del involucro reducida, 3 lóbulos. Plantas endémicas de las islas Malvinas.

\section{Nastanthus falklandicus}

47) Lóbulos corolinos cortos, triangular-dentiformes. Tallos e inflorescencias usualmente agregadas, cespitosas. Receptáculos con brácteas nulas o subnulas. (48) 47') Corolas partidas, lóbulos estrechamente triangulares a oblongos. Tallos e inflorescencias usualmente no agregadas ni cespitosas. Receptáculos con brácteas lineares, espatuladas o rectangulares. (50) 
48) Perianto infundibuliforme sin membrana longitudinal, no persistente sobre fruto.

48') Perianto cilíndrico con una membrana longitudinal, hialina, gruesa, acrescente y persistente sobre el fruto.

Nastanthus compactus

49) Perianto de 5.5-7.5 mm de longitud. Glándulas nectaríferas estaminales en posición distal. Cipsela con carenas desiguales. Hojas partidas, casi pinnatisectas. Plantas endémicas de la II Región de Antofagasta y la III Región de Atacama, Chile.

Nastanthus caespitosus

49’) Perianto de 4-5 mm de longitud. Glándulas nectaríferas estaminales de posición mediana. Cipsela con carenas iguales. Hojas cortamente lobadas, pinnatifidas. Plantas de los Andes de Argentina y Chile.

Nastanthus ventosus

50) Cipselas con carenas estrechas laminares, rugosas. Sépalos reducidos, no distinguibles en el fruto. Pedúnculo central grueso, de 1-3 $\mathrm{cm}$ de diámetro, portador de una inflorescencia grande, de 1-6 cm de diámetro, rodeada por pedúnculos delgados portadores de inflorescencias más pequeñas. Hipanto de 2.5-3 mm de longitud, corola de 1.6-1.8 mm de longitud.

Nastanthus patagonicus

50’) Cipselas con carenas expandidas laminares lisas. Sépalos distinguibles en el fruto. Muchos pedúnculos, de 0.3-1 cm de diámetro, regularmente distribuidos en la planta, todos similares en longitud y grosor, portadores de inflorescencias de tamaño similar, de 0.6-3.5 cm de diámetro. Hipanto de 4-4.5 mm de longitud, corola de $2.5 \mathrm{~mm}$ de longitud.

Nastanthus scapigerus 


\subsubsection{Revisión nomeclatural de la familia Calyceraceae}

CALYCERACEAE R. Br. ex Rich., Mem. Mus. Hist. Nat. 6: 74, 1820, nom. cons.

Boopideae Cass., Bull. Sci. Soc. Philom. Paris. 1817

Caméfitos subarbustivos o herbáceos con tallos radicantes, hemicriptófitos herbáceos rosulados con o sin brotes caulescentes, geófitos herbáceos con rizomas alargados y brotes aéreos caulescentes o rosulados a veces estoloníferos, o terófitos caulescentes con o sin base rosulada; glabros o pubescentes, a veces resinosos. Raíces axonomorfas o fibrosas, a veces gemíferas. Hojas sin estípulas, alternas, distantes o imbricadas, sésiles o con pecíolo poco diferenciado (lámina atenuada en un pecíolo rectangular), base foliar punctiforme, a veces amplexicaule, persistente en la senescencia foliar, o caduca y entonces deja una cicatriz foliar notoria, lámina membranácea o gruesa, y a veces algo coriácea, contorno entero, oblongo, linear, lanceolado, oval, laciniado, lobado a pinnatisecto o escuarroso-laciniado, con margen entero, dentado o crenado, dientes mucronados o no, ápice agudo,obtuso o emarginado, mucronado o no mucronado. Flores reunidas en cefalodios compuestos por grupos cimosos periféricos rodeando un grupo central de flores solitarias que culmina en una flor terminal (Pozner et al., 2012). Cefalodios solitarios o agregados en inflorescencias compuestas, sobre pedúnculos teretes, escapiformes, erectos o nutantes, a veces nulos. Involucro indiferenciado, diferenciado con brácteas libres, o diferenciado con una base fusionada y lóbulos marginales; a veces acrescente durante la maduración de los frutos. Receptáculo punctiforme o columnar; a veces acrescente (discoideo o hemisférico) durante la maduración de los frutos. Brácteas del receptáculo (denomidas genéricamente páleas, pero que pueden incluir no sólo la bráctea tectriz de cada grupo cimoso sino también uno o más profilos internos y la bráctea tectriz de cada flor, solitaria), libres o fusionadas, de formas variadas o ausentes. Profilos de los grupos cimosos más externos, a veces presentes formando involucelos. Flores pentámeras o tetrámeras, dimórficas o polimórficas. Sépalos con estivación valvar o imbricada, suborbiculares, lanceolados, dentiformes, o espiniformes; planos, cuculados o inflados; membranáceos, foliosos, corchosos o endurecidos; con margen entero, dentado o sinuado, y ápice obtuso, agudo 
o emarginado, mucronado o no mucronado; acrescentes durante la maduración de los frutos. Hipanto tubuloso o infundibuliforme. Corola actinomorfa con estivación valvar, tubular a campanulada; lóbulos oblongos o lanceolados, planos o cuculados inflados, con ápice agudo a obtuso, recto o uncinado. Androceo con un tubo estaminal y filamentos libres en el extremo apical, de desarrollo variable. Anteras oblongas con dehiscencia longitudinal introrsa, basalmente connadas por la, tecas con base obtusa o sagitada; conectivo apiculado o no apiculado, polen con presentación secundaria (Leins \& Erbar, 1990). Glándulas nectaríferas interestaminales oblongas, ubicadas en la base del tubo estaminal (estaminales) o justo por debajo de la unión con el hipanto (hipantiales). Ovario cilíndrico a lacrimiforme, unilocular, uniovulado; óvulo solitario, anátropo y péndulo. Estilo filiforme, exerto. Estigma simple, punctiforme a levemente capitado, indiviso, papiloso. Cipselas homomórficas, dimórficas o polimórficas, prismáticas, cilíndricas, obcónicas o turbinadas con 3-5 carenas coronadas por los sépalos acrescentes que rodean a un apículo central. Semillas péndulas, con albumen carnoso, embrión recto y cotiledones elípticos o hemielípticos (Pontiroli, 1963).

ACICARPHA Juss., Ann. Mus. Natl. Hist. Nat. 2: 347. 1803.

Especie tipo: Acicarpha tribuloides Juss.

Cryptocarpha Cass., Bull. Sci. Soc. Philom. Paris vol. 1-3: 34. 1817.

Especie tipo: Cryptocarpha tribuloides Cass., nomen superfluum (= A. tribuloides Juss.).

Sommea Bory, Ann. Gen. Sci. Phys. 6: 94. t. 87. 1820.

Especie tipo: Sommea calcitrapa Bory (= A. spathulata R. Br.).

Acanthosperma Vell., Fl. Flumin. Icon. 8: t. 152. 1831 [1827 publ. 29 Oct 1831].

Especie tipo: Acanthosperma littorale Vell. (= A. spathulata R. Br.).

Echinolema J. Jacq. ex DC., Prodr. [A. P. de Candolle] 5: 3. 1836 [1-10 Oct 1836].

Especie tipo: Echinolema arenarium J. Jacq. ex DC. (= A. spathulata R. Br.). 
Acicarpa R. Br. emend. Miers, Ann. Mag. Nat. Hist. ser. 3, 6(36): 401. 1860 [Dec 1860] Especie tipo: Acicarpa tribuloides R. Br. emend. Miers, nomen superfluum $(=A$. tribuloides Juss.).

Caméfitos herbáceos con tallos decumbentes radicantes o terófitos rosulados con brotes aéreos erectos, decumbentes o ascendentes, glabros. Raíces axonomorfas. Hojas distantes, sésiles, a veces subamplexicaules, o con pecíolo poco diferenciado (base de lámina atenuadas en un pecíolo rectangular y entonces la hoja es espatulada), membranáceas o gruesas, a veces algo coriáceas; lámina entera o partida, oblonga, lanceolada u oval, con margen entero o dentado, no mucronado, y ápice agudo, obtuso o emarginado, mucronado o no. Cefalodios simples sobre pedúnculos teretes. Involucro diferenciado, brácteas libres, o base fusionada con lóbulos libres. Receptáculo columnar; acrescente durante la maduración de los frutos. Grupos cimosos ausentes, flores solitarias numerosas, flor terminal a veces reducida. Brácteas del receptáculo libres. Flores pentámeras, dimórficas, las externas (o inferiores) perfectas, las superiores (o centrales) funcionalmente estaminadas (aunque el gineceo se forma y el estilo se alarga para presentar el polen, el óvulo no completa su desarrollo). Sépalos suborbiculares, dentiformes o gibosos, con margen entero, y ápice obtuso o agudo, mucronado o no. Hipanto tubuloso o infundibuliforme. Corola con tubo corto, cilíndrico, y lóbulos oblongos, planos, con ápice agudo y recto. Tubo estaminal desarrollado, filamentos nulos. Anteras sentadas sobre el tubo estaminal, oblongas, con base obtusa, sin caudículas; conectivo no apiculado. Glándulas nectaríferas estaminales o hipantiales. Ovario cilíndrico a lacrimiforme, desde completamente embebido en el receptáculo (en las flores más externas/basales), progresivamente más expuesto en las flores perfectas internas, y totalmente expuesto en las flores estaminadas (Erbar, 1993). Cipselas prismáticas, con 5 carenas coronadas por los sépalos acrescentes, gibosos, aciculares o cónico-espinosos, que rodean a un apículo central, generalmente agregados en una única unidad de dispersión (infrutescencia) por su relación con el receptáculo.

Acicarpha obtusisepala Marchesi, Bol. Invest. Fac. Agron. Montevideo 5: 4. 1987. TIPO: Uruguay. Rocha. Parque Fortaleza Santa Teresa, 9-II-1967, B. Rosengurtt 10839 (holotipo MVFA no visto; isotipo SI 105961!). Fig. 2. 
Caméfitos herbáceos hasta de $15 \mathrm{~cm}$ de altura. Tallos erectos y decumbentes radicantes de ca. $40 \mathrm{~cm}$ de largo. Hojas gruesas, algo coriáceas, espatuladas; lámina obovada a suborbicular, con margen entero, ápice emarginado, mucronado. Involucro con base fusionada rotácea y lóbulos espatulados, patentes, con margen entero y ápice emarginado. Brácteas del receptáculo espatuladas. Flores flores perfectas con sépalos gibosos, de margen entero y ápice obtuso no mucronado. Flores estaminadas con sépalos suborbiculares, foliáceos, con margen entero, y ápice obtuso, no mucronado. Hipanto y corola suculentos, de 3,0-3,5 mm en las flores perfectas y ca. 4,5 $\mathrm{mm}$ en las flores estaminadas. Hipanto tubuloso. Corola con tubo y lóbulos de igual longitud, lóbulos imbricados, oblongos, planos, con ápice agudo y recto. Tubo estaminal de igual longitud que el tubo corolino. Anteras, de aproximadamente 1,7 mm. Glándulas nectaríferas estaminales. Ápice del ovario de las flores perfectas a veces visible y prismático; ovario lacrimiforme, hasta de $1,35 \mathrm{~mm}$ en las flores estaminadas. Infrutescencia cónica con cipselas prismáticas con 5 carenas coronadas por los sépalos gibosos que rodean a un apículo central.

Distribución. Microendémica de la costa atlántica uruguaya. Sólo ha sido coleccionada en la playa de Fortaleza Santa Teresa (Depto. Rocha, Uruguay).

Discusión. A. obtusisepala es la única especie de su género que posee cipselas con sépalos no espinosos. Por su porte y aspecto general de la planta, podría ser confundida con A. spathulata; sin embargo, esta última se diferencia por poseer cipselas con sépalos cónicos espinosos, flores externas y centrales con el perianto de igual longitud, y hojas espatuladas con margen entero o dentado y ápice obtuso mucronado.

Acicarpha procumbens Less., Linnaea 6: 527. 1831. TIPO: Brasil [Brasilia], sin fecha indicada, F. Sellow s.n. (holotipo B 1840†; isotipos K 000588768 no visto, foto!; HAL 113258 no visto, P 00852254 no visto, W 0032077 no visto, W 0032078 no visto). Fig. 3 (A-E). 
Acicarpha procumbens Less. var. viridiflora C. A. Muell., Fl. Bras. (Martius) 6, pt. 4: 358. 1885. TIPO: Argentina. Entre Ríos. Concepción del Uruguay, en bajadas al río Gualeguaychú, Estancia de Galarza, 31-X-1877, P. Lorentz 1178 (holotipo BM 000947756 no visto, foto!; isotipos BAF!, SI!, UPS no visto).

Caméfitos herbáceos. Tallos procumbentes, radicantes. Hojas membranosas, lineares a espatuladas; lámina oblanceolada con margen entero o paucidentado, ápice obtuso y mucronado. Involucro con base fusionada rotácea y lóbulos estrechamente triangulares, con margen entero y ápice obtuso y mucronado. Brácteas del receptáculo lanceoladas a espatuladas, con margen entero y ápice cuspidado. Flores perfectas con sépalos suborbiculares a espatulados, con margen entero a sinuado, y ápice obtuso, no mucronado. Flores estaminadas con sépalos suborbiculares a obovados, con margen entero y ápice obtuso, no mucronado. Perianto de las flores perfectas de 4,75-6,00 mm. Perianto de las flores estaminadas ca. 5,00 mm. Hipanto tubuloso. Tubo y lóbulos corolinos de igual longitud; lóbulos oblongos, planos, con ápice agudoy recto. Tubo estaminal de 3/4 la longitud del tubo corolino. Anteras sentadas sobre el tubo estaminal, de aproximadamente 1,0 mm. Glándulas nectaríferas estaminales. Ovario elipsoidal en las flores perfectas y estaminadas; hasta de $0,65 \mathrm{~mm}$ en las flores estaminadas. Infrutescencia esférica, con cipselas prismáticas con 5 carenas lisas, planas, con surcos visibles, carenas coronadas por los sépalos cónico-espinosos con base pulviniforme, que rodean a un apículo central.

Distribución. Sur de Brasil, Uruguay y noreste de Argentina, desde la Mesopotamia hasta el sur de la provincia de Buenos Aires (Pontiroli, 1963). Habita en las praderas húmedas de las provincias fitogeográficas Pampeana y del Espinal (Cabrera \& Willink, 1980).

Discusión. Por su porte y el aspecto general de la planta, podría ser confundida con A. spathulata; sin embargo, esta última se diferencia por poseer hojas espatuladas con lámina obovada a elipsoidal, lóbulos del involucro espatulados, cipselas con surcos intercarinales poco visibles, agregados en una unidad de dispersión cónica. 
Acicarpha spathulata R. Br., Trans. Linn. Soc. London 12(1): 129. 1818 [25 Feb 1818]. Cryptocarpha spathulata Cass., nomen superfluum, Dict. Sci. Nat., ed. 2. [F. Cuvier] 12: 85. 1819 [1818 publ. 9 Jan 1819]. TIPO: Brasil. "Brasilia prope Rio de Janeiro", sin fecha indicada, F. Sellow s.n. (holotipo BM 000947752 no visto, foto!). Fig. 3 (F-J).

Sommea calcitrapa Bory, Ann. Gen. Sci. Phys. 6: 94. t. 87. 1820. TIPO: Brasil, Rio de Janeiro, fecha desconocida, Herb. J. Gay s.n. (holotipo K 000588769 no visto, foto!).

Acanthosperma littorale Vell., Fl. Flumin. Icon. 8: t. 152. 1831 [1827 publ. 29 Oct 1831]. TIPO: Brasil, Sâo Paulo, fecha no indicada, Vellozo s.n. [holotipo probablemente perdido; material usado para Flora Fluminensis no ha podido ser localizado en LISC, P ni R (DeVore, 1994)].

Echinolema arenarium J. Jacq. ex DC., Prodr. [A. P. de Candolle] 5: 3. 1836 [110 Oct 1836]. TIPO: Brasil, localidad, fecha y coleccionista desconocidos [holotipo W no localizado (DeVore, 1994)].

Acicarpha spathulata R. Br. var. glauca DC., Prodr. [A. P. de Candolle] 5: 3. 1836 [1-10 Oct 1836]. TIPO: Brasil [Brasiliâ] (holotipo probablemente en G no localizado).

Caméfitos herbáceos. Tallos erectos y decumbentes radicantes. Hojas gruesas, algo coriáceas, espatuladas; lámina obovada a elipsoidal, con margen entero o dentadomucronado, ápice obtuso, mucronado (ocasionalmente emarginado). Involucro con base fusionada rotácea y lóbulos espatulados, patentes, con margen entero y ápice obtuso. Brácteas del receptáculo espatuladas con ápice mucronado. Flores perfectas con sépalos espinosos o suborbiculares, con margen entero o sinuado, y ápice obtuso, no mucronado. Flores estaminadas con sépalos espatulados, foliáceos, con margen entero, y ápice obtuso, no mucronado. Perianto de las flores perfectas de 3,40- 3,75 mm. Perianto de las flores estaminadas de ca. 3,40 mm. Hipanto tubuloso. Tubo y lóbulos corolinos de igual longitud; lóbulos oblongos, planos, con ápice agudoy recto. Tubo 
estaminal de igual longitud que el tubo corolino. Anteras sentadas sobre el tubo estaminal, de aproximadamente 1,55 mm. Glándulas nectaríferas estaminales. Ápice del ovario de las flores perfectas a veces visible y elipsoidal; ovario de las flores estaminadas terete a lacrimiforme, de ca. 1,30 $\mathrm{mm}$. Infrutescencia con cipselas prismáticas, con 5 carenas coronadas por los sépalos cónico-espinosos que rodean a un apículo central.

Distribución. Endémica de Brasil. Habita en la costa atlántica sur, desde Rio de Janeiro hasta Rio Grande do Sul.

Discusión. Por su porte y aspecto general de la planta, A. spathulata podría ser confundida con A. obtusisepala; sin embargo, esta última se diferencia por poseer cipelas con sépalos gibosos no espinosos, perianto de las flores centrales con mayor longitud que el de las flores externas, y hojas espatuladas con margen entero y ápice emarginado.

Acicarpha tribuloides Juss., Ann. Mus. Natl. Hist. Nat. 2: 348. 1803. Cryptocarpha tribuloides Cass., nomen superfluum, Dict. Sci. Nat., ed. 2. [F. Cuvier] 12: 85. 1819 [1818 publ. 9 Jan 1819]. TIPO: Uruguay. Montevideo, 1766, P. Commerson s.n. (holotipo P-JU 9546 no visto, foto!; isotipos P-LA no visto, foto!, BM 000947757 no visto, F no visto, LINN-HS1317-1 no visto,). Fig. 4.

Acicarpha pinnatifida Miers, Ann. Mag. Nat. Hist. ser. 3, 6(36): 404. 1860 [Dec 1860]. Acicarpha tribuloides var. pinnatifida (Miers) Kuntze, Revis. Gen. Pl. 3[3]: 126. 1898 [28 Sep 1898]. TIPO: Argentina. Buenos Aires, J. Tweedie 71 (holotipo BM 000947755 no visto, foto!).

Acicarpha runcinata Miers, Ann. Mag. Nat. Hist. ser. 3, 6(36): 404. 1860 [Dec 1860]. TIPO: Uruguay [Banda Oriental], J. Tweedie s.n. (holotipo K-000588772 no visto, foto!; isotipo BM 000947759 no visto). 
Acicarpha tribuloides Juss. var. dentata Kuntze, Revis. Gen. Pl. 3[3]: 126. 1898 [28 Sep 1898]. TIPO: Argentina, Buenos Aires, XII-1891, O. Kuntze s.n. (lectotipo aquí propuesto CORD 00005203 no visto, foto!; duplicado SI 12626!).

Acicarpha laxa R.E.Fr., Ark. Bot. 6 (11): 2. 1907. TIPO: Bolivia, “Tarija loco umbroso in ripa arenosa et humida fluminis", 6-II-1902, R. E. Fries 1233 (lectotipo aquí propuesto US 00147174 no visto, foto!, duplicados CORD 00005202 no visto, foto!, S05-2024, S05-2025).

Terófitos rosulados, con brotes aéreos a partir del periodo de floración. Tallos erectos o decumbentes. Hojas basales espatuladas, atenuadas en un pecíolo rectangular, y hojas caulinares sésiles subamplexicaules; lámina oblonga a lanceolada, con margen entero, sinuado, crenado, dentado, pinnatifido, pinnatipartido o runcinado, mucronado, con ápice obtuso y mucronado. Involucro con brácteas libres o base fusionada rotácea, acrescente durante la madurez de los frutos, y lóbulos oblongos a lanceolados, con margen entero o dentado-mucronado, con ápice obtuso y mucronado. Brácteas del receptáculo lanceoladas a espatuladas con ápice mucronado. Flores perfectas con sépalos espinosos o suborbiculares, con margen entero o sinuado, y ápice agudo mucronado o no. Flores estaminadas con sépalos suborbiculares, con margen entero, y ápice obtuso, no mucronado. Perianto de las flores perfectas de 4,00-4,75 mm. Perianto de las flores estaminadas de ca. 4,00 mm. Hipanto tubuloso. Corola lobada hasta 3/4 de su parte superior; lóbulos oblongos, planos, con ápice agudo y recto. Tubo estaminal de igual longitud que el tubo corolino. Anteras sentadas sobre el tubo estaminal, de aproximadamente 1,60 mm, oblongas, con base obtusa, sin caudículas; conectivo no apiculado. Glándulas nectaríferas hipantiales. Ápice del ovario de las flores perfectas a veces visible y elipsoidal, de ca. 1,80 mm; ovario de las flores estaminadas elipsoidal o vestigial, de 0,40 - 0,80 $\mathrm{mm}$. Infrutescencia con cipselas prismáticas. Cipselas centrales asimétricas, caedizas, con 5 carenas coronadas por los sépalos vestigiales y cónico-espinosos que rodean a un apículo central. Cipselas externas fusionadas, con 5 carenas coronadas por los sépalos cónico-espinosos que rodean a un apículo central. 
Distribución. Bolivia, sur de Perú, Brasil meridional, Uruguay, Paraguay y ampliamente difundida en la República Argentina, en el norte y centro del país (Pontiroli, 1963).

Discusión. Acicarpha tribuloides se diferencia del resto del género por su forma de vida terófita y su hábito rosulado caulescente durante la floración. Ocasionalmente pueden desarrollarse plantas persistentes a partir de una base pseudoleñosa (DeVore, 1994; Zavala-Gallo, obs. pers.).

Existen dos sintipos de A. tribuliodes var. dentata: Kuntze s.n. de Argentina (SI 12626 y CORD 00005203) y Kuntze s.n. de Uruguay (río Sa. Lucía, XI-1892, SI 12624). Se selecciona el ejemplar CORD 00005203 de Argentina porque presenta abundante material en buen estado y porque tiene un duplicado en SI.

Existen dos sintipos de A. laxa: R. E. Fries 271 (S05-2023) de Argentina (Jujuy: Sa. Barbara in regione Podocarpi, 11-VII-1901) y R. E. Fries 1233 de Bolivia. Se selecciona el ejemplar US 00147174 de Bolivia porque presenta material en buen estado y porque tiene duplicados.

BOOPIS Juss., Ann. Mus. Natl. Hist. 2: 350. 1803.

Especie tipo: Boopis anthemoides Juss.

Acarpha Griseb., Abh. Königl. Ges. Wiss. Göttingen 6: 125.1856 ; impresión previa, Syst. Bemerk. publicada en 1854.

Espcie tipo: Acarpha australis Griseb., pro. syn. (= Boopis australis Decne.).

Caméfitos subarbustivos, geófitos herbáceos rizomatosos o hemicriptófitos herbáceos rosulados con brotes aéreos caulescentes; glabros o vilíferos. Tallos alargados, erectos, postrados, procumbentes, ascendentes a veces radicantes, o erectos con entrenudos reducidos, sin cicatrices foliares notorias, a veces resinosos. Raíces contráctiles o no, a veces gemíferas. Hojas sésiles o con pecíolo poco diferenciado (base de la lámina atenuada en un pecíolo rectangular y entonces la hoja es espatulada); lámina membranácea o gruesa; contorno espatulado, oblongo, linear, oval, laciniado, pinnatifido o pinnatisecto, con margen entero, dentado, mucronado o no, y ápice agudo 
u obtuso, mucronado o no. Pedúnculos teretes, escapiformes o nulos. Cefalodios con grupos cimosos con 2 a numerosas flores, excepcionalmente ausentes en $B$. pusilla, flores solitarias pocas a numerosas, excepcionalmente ausentes en B. castilloni y $B$. necronensis, y flor terminal excepcionalmente ausente en en B. necronensis. Involucro diferenciado, con brácteas libres, o bien con una base fusionada y lóbulos libres. Excepcionalmente pueden aparecer grupos cimosos bracteados, exteriores al involucro, independientes (libres) o asociados por fusión de su bráctea tectriz a la periferia del involucro. Receptáculo punctiforme o columnar; a veces acrescente durante la maduración de los frutos. Brácteas del receptáculo libres o parcialmente fusionadas por uno o ambos bordes (formando o no celdas completas). Flores pentámeras o tetrámeras. Sépalos suborbiculares, lanceolados, dentiformes, foliosos o gibosos, con margen entero, dentado o sinuado, y ápice obtuso, agudo o emarginado, mucronado o no. Hipanto tubuloso o infundibuliforme. Corola con tubo corto o largo, cilíndrico; lóbulos oblongos o lanceolados, planos o cuculados inflados, con ápice agudo a obtuso, recto o uncinado. Tubo estaminal desarrollado con filamentos libres en el extremo apical, o nulos. Anteras oblongas, con base obtusa o sagitada; conectivo apiculado o no. Glándulas nectaríferas estaminales o hipantiales. Ovario cilíndrico a lacrimiforme. Cipselas prismáticas, cilíndricas o turbinadas con 5 carenas coronadas por los sépalos acrescentes o no, nunca espinescentes, vestigiales, subulados, orbiculares, suborbiculares, lanceolados, triangulares, pulviniformes o foliáceos que rodean a un apículo central.

Boopis anthemoides Juss., Ann. Mus. Natl. Hist. 2: 350. 1803. TIPO: Argentina. Buenos Aires, 1766, P. Commerson s.n. (holotipo P-JU 9545 no visto, foto!; isotipos BAA 3835 no visto, foto!, BM 000947735 no visto, foto!, P-LA no visto, foto!). Fig. 5.

Boopis rigidula Miers, Ann. Mag. Nat. Hist. ser. 3, 6 (34): 284. 1860 [Oct 1860]. Boopis anthemoides Juss. var. rigidula (Miers) Griseb., Symb. Fl. Argent.: 161. 1879. TIPO: Argentina. Mendoza. Near San Isidro, 13-XI-1824, J. Gillies s.n. (lectotipo aquí propuesto, K 000588748 no visto, foto!). 
Boopis rigidula Miers var. patagonica Hieron., Bol. Acad. Nac. Cs. Cba. 3: 352. 1879 [1880]. Boopis anthemoides Juss. var. rigidula (Miers) Griseb. f. patagonica (Hieron.) Hicken, Reun. Nac. Soc. Arg. Cienc. Nat. 1: 241. 1919. TIPO: Argentina. An einzelnen Hallen in Camp am Rio Negro ziemlich [...]. Meist auf Anhöhen, 17-XI-1874, C. Berg 167 (holotipo CORD no visto; isotipo SI!).

Boopis anthemoides Juss. var. andina Hieron., syn. nov. propuesto, Bol. Acad. Nac. Cs. Cba. 4(1): 32, 1881. TIPO: Argentina. Leoncito; Prov. de San Juan, II-1876, S. Echegaray s.n. (holotipo CORD 00005204!).

Boopis anthemoides Juss. var. subscandens Speg., Anales Soc. Ci. Argent. 48: 176. 1899. TIPO: Argentina. La Pantanosa- Rio Negro, Carmen de Patagones, II-1898, C. Spegazzini s.n. (holotipo LP ex LPS 11026!; isotipos: LP ex LPS 12924!, LP ex LPS $12934 !)$.

Boopis anthemoides Juss. var. subintegrifolia Hicken, syn. nov. propuesto, Reun. Nac. Soc. Arg. Cienc. Nat. 1: 241. 1919. TIPO: Argentina. Tucumán, Tapia, 700 m, 22-XII-1911, Rodriguez 215 (lectotipo aquí propuesto SI 063045!; isotipos: SI 348!, SI 076338!, SI 076339!).

Caméfitos subarbustivos con tallos erectos o ascendentes, hasta de $60 \mathrm{~cm}$ de altura; glabros. Raíces gemíferas. Hojas sésiles, lineares a pinnatifidas o pinnatisectas, laciniadas, de 2-6 cm de longitud, con margen entero. Pedúnculo terete. Cefalodios con 5-6 grupos cimosos, 3-5- floros. Involucro con base fusionada rotácea, pateliforme o crateriforme y lóbulos triangulares, a veces laciniados, con margen entero y ápice mucronado. Receptáculo punctiforme. Brácteas del receptáculo libres, linearespatuladas con limbo lanceolado. Flores pentámeras. Sépalos suborbiculares a lanceolados, con margen entero y ápice emarginado o cuspidado. Perianto de 3,5 - 6 $\mathrm{mm}$ de longitud. Hipanto verde, tubuloso. Corola blanca, tubo de igual longitud que los lóbulos; lóbulos oblongos, planos, recurvados, ápice agudo, recto. Tubo estaminal reducido, de igual longitud de los filamentos. Filamentos de 1/3-1/4 de la longitud de las anteras. Anteras oblongas, tecas basalmente obtusas sin caudículas, conectivo no apiculado. Glándulas nectaríferas hipantiales. Ovario cilíndrico a lacrimiforme, 1,4 3,3 mm de longitud. Cipselas prismáticas a cilíndricas, lisas, con 5 carenas planas, 
coronadas por sépalos suborbiculares, triangulares o lanceolados con margen aserrado o entero y ápice pungente, que rodean un apículo central.

Distribución. Uruguay, Argentina, entre 0 y 3500 m s.m. Habita en la llanura chaco-pampeana, las sierras pampeanas, la estepa patagónica y las primeras estribaciones de la cordillera de los Andes. Ocupa las provincias fitogeográficas Pampeana, del Espinal, Chaqueña, del Monte, Patagónica y Prepuneña. Es una de las especies de Calyceraceae con más amplia distribución y, aunque aún no se ha podido confirmar con registros de herbario, es probable su presencia en las áreas chaqueñas de Bolivia, Brasil y Paraguay.

Discusión. Los caracteres distintivos de Boopis anthemoides son: forma de vida caméfita, hábito subarbustivo y hojas sésiles, como en Calycera calcitrapa pero esta última posee hojas lanceolado-oblongas, simples, con margen dentado-mucronado y cipselas espinescentes con sépalos cónicos.

Boopis anthemoides es un taxón de amplia distribución en el cono sur de Sudamérica con una variación morfológica marcada que le permite aclimatarse a los distintos tipos de ambientes donde se desarrolla. Aunque hubo numerosos intentos de identificar variedades dentro de la especie, el análisis exhaustivo del material de herbario del que se dispone permite verificar una variación continua de sus caracteres vegetativos y reproductivos. La estructura de la inflorescencia, que es igual para todas las variedades, apoya la decisión taxonómica de sinonimizar a todas las variedades de $B$. anthemoides como un único taxón.

Boopis anthemoides var. andina posee los mismos caracteres distintivos que la var. anthemoides. La identificación entre ambos taxones es dificultosa y la mayoría de las veces sólo se logra verificando el sitio de colección. Por otra parte, Hieronymus (1881) notó que la variedad andina tenía mucha afinidad con la variedad rigidula, ya sinonimizada bajo Boopis anthemoides por Pontiroli (1963). Si bien B. anthemoides var. andina podría caracterizarse por poseer cipselas con sépalos lanceolados de margen entero y carenas reducidas, el material de herbario revisado muestra una variación contínua en la forma de las cipselas, variación que dificulta, en particular, la identificación de los ejemplares coleccionados en las sierras pampeanas centrales. Por esto, se propone sinonimizar B. anthemoides var. andina bajo B. anthemoides. 
Boopis anthemoides var. subintegrifolia también posee los mismos caracteres distintivos de la var. anthemoides. Se diferencia de la var. anthemoides por poseer las hojas inferiores simples con contorno inciso-pinnatifido y las superiores lineares, fenómeno de variación foliar que también se conoce para Calycera leucanthema. Sin embargo, sus caracteres reproductivos (flor y fruto) y la estructura de la inflorescencia coinciden en todo. Por esto, se propone sinonimizar B. anthemoides var. subintegrifolia bajo $B$. anthemoides.

Se elige el ejemplar Gillies s.n. (K 000588748) como lectotipo de Boopis rigidula, por ser el que conserva mayor cantidad de material reproductivo (flores) y permanece en mejor estado de conservación. El sintipo de King s.n. conserva mucho material en más de un herbario $(\mathrm{K}, \mathrm{BM})$ pero las estructuras reproductivas son escasas y están en mal estado de conservación.

El ejemplar Rodriguez 215 (SI 063045) es seleccionado como lectotipo de Boopis anthemoides var. subintegrifolia debido a que cuenta con mayor cantidad de material en buen estado de conservación y posee varios duplicados. Es el único ejemplar que cuenta con etiquetas del autor del taxón. Además, ha sido considerado el ejemplar tipo de referencia desde la revisión de Calyceraceae de Pontiroli (1963).

Boopis australis Decne., Voy. Pole Sud Bot. ii. 87. t. 18, B. 1853. Acarpha australis Griseb., Abh. Königl. Ges. Wiss. Göttingen 6: 126. 1856; impresión previa, Syst. Bemerk. publicada en 1854, pro. syn. TIPO: Chile. Magallanes: "in arenosis secus fretum Magellanicum; Port-Galant, Havre-Pecket”, XI-1852, W. Lechler 1143 (duplicados W 1889-0008588, K 000009714, SGO 043606!, SGO no visto, foto!, W 1889-0217603, W 0021367). Fig. 6.

Boopis squarrosa Miers, Ann. Mag. Nat. Hist. ser. 3, 6(34): 286. 1860 [Oct 1860]. Boopis australis Decne. var. squarrosa (Miers) Reiche, Anales Univ. Chile 106: 1037. 1900. TIPO: Strait of Magallanes. “Ad Fretum Magellanicum”, M. Le Guillou s.n. (duplicados K 000009715 no visto, foto!, P no visto).

Boopis leptophylla Speg., Anales Soc. Ci. Argent. 48: 177. 1899. Boopis autralis Decne. var. leptophylla (Speg.) Hicken, Reun. Nac. Soc. Arg. Cienc. Nat. 1: 242. 1919. 
TIPO: Argentina. Patagonia. Kmanaich (Rio Chico), aest. II-1898, C. Ameghino s.n. (holotipo LP ex LPS 11028!).

Boopis australis Decne. var. hickenii Pontiroli, Revista Mus. La Plata, Secc. Bot. 9 (4): 225. 1963. TIPO: Argentina. Neuquén. Zapala, 12-II-1919, C. M. Hicken 78 (holotipo SI 075712!; isotipo SI 075711!).

Hemicriptófitos o geófitos herbáceos, rizomatosos, con brotes aéreos rosulados a caulescentes tendidos o ascendentes, simples o ramosos, hasta de $30 \mathrm{~cm}$ de altura; glabros. Hojas lineares o acintadas a pinnatifidas o pinnatipartidas (lóbulos breves a laciniados con ápice obtuso y mucronado), de 0,5-15 cm de longitud, con margen entero y ápice obtuso, mucronado. Inflorescencia central sésil o pedunculada e inflorescencias laterales pedunculadas. Pedúnculos terminales o laterales, erectos o postrados, estriados, desnudos u hojosos. Involucro con una base fusionada rotácea a cupuliforme, y lóbulos triangulares, laciniados con margen entero o dentado-mucronado, con mucrones cónicos a filiformes. Ocasionalmente, brácteas de los grupos cimosos externos fusionadas al involucro central formando celdas periféricas. Receptáculo punctiforme. Cefalodio con 10-12 grupos cimosos, 2-7 floros. Brácteas del receptáculo filiformes o espatuladas, con ápice obtuso u obtuso acuminado; libres o parcialmente fusionadas al involucro por uno de los bordes (sin formar celdas completas). Flores pentámeras. Sépalos inflados, suborbiculares o aciculados con ápice acuminado. Perianto de $3-7 \mathrm{~mm}$ de longitud. Hipanto verde, infundibuliforme a tubuloso. Corola blanca, lobada hasta 1/3 - 1/2 de la longitud del tubo; tubo infundibuliforme; lóbulos lanceolados, planos o cuculados inflados, ligeramente recurvados, ápice agudo a obtuso, recto o uncinado. Tubo estaminal de 1/2 - 2 veces el largo de los filamentos. Filamentos de 1/5 - 1/3 del largo de las anteras. Anteras obtusas a oblongas, tecas basalmente sagitadas sin caudículas, conectivo apiculado o no. Glándulas nectaríferas hipantiales. Ovario elipsoidal a lacrimiforme, de 1-3 $\mathrm{mm}$ de longitud. Cipselas prismáticas, lisas, de 1-4 mm de longitud, con 5 carenas coronadas por sépalos vestigiales a triangulares con ápice agudo que rodean a un apículo central cuneiforme.

Distribución. Extremo austral de Chile y sur de Argentina, desde el norte de la isla de Tierra del Fuego y la costa del estrecho de Magallanes, hasta el norte de la 
provincia de Neuquén y sur de la provincia de Río Negro. Habita la estepa patagónica hasta las primeras estribaciones de la cordillera de los Andes, entre 0-1750 m s. n. m. Exclusivo de la provincia fitogeográfica Patagónica.

Boopis australis es una de las especies de Calyceraceae con distribución geográfica más amplia. Su alta variabilidad morfológica le otorga una gran capacidad de adaptación al medio, permitiéndole habitar ambientes muy diversos: mallines, plena estepa o inmediaciones de zonas urbanas; suelos salinos, arcillosos, arenosos o pedregosos; en las cercanías de la costa, en plena meseta o en áreas cordilleranas.

Discusión. Los caracteres distintivos de Boopis australis son: forma de vida geófita rizomatosa con raíces contráctiles, como en Boopis graminea, pero esta última posee brotes aéreos con hojas lineares, nunca pinnatifidas, portando un pedúnculo escapiforme único, monocéfalo; cefalodios pedúnculados, nunca sésiles, con grupos

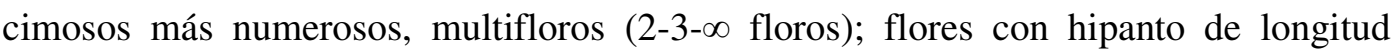
menor o igual a la de la corola (aunque este caracter es muy variable en B. australis); y sépalos lanceolados, algo inflados, con ápice agudo.

Boopis australis var. hickenii fue sinonimizado bajo B. australis en anteriores revisiones (Chiapella 1999a,b; Zanotti \& Pozner, 2008). Aunque los análisis filogenéticos llevados a cabo en este trabajo muestran una estrecha afinidad de la variedad hickenii con $B$. chubutensis, es muy dificultoso distinguir B. australis, $B$. chubutensis y B. raffaellii. Existen muchas similitudes en sus caracteres florales y en la estructura de sus inflorescencias, ya que los caracteres distintivos de B. chubutensis y $B$. raffaellii son continuos y están comprendidos dentro del rango de variación de $B$. australis o se acercan mucho a esos valores. Por su parte, los caracteres vegetativos son muy variables y probablemente estén sujetos a las presiones ambientales propias de cada localidad. Esto explicaría la distribución esporádica e irregular de B. chubutensis y B. raffaellii dentro del área de dispersión de B. australis. Además, estas tres especies aparecen relacionadas en el análisis filogenético. Probablemente formen parte de un mismo complejo de especies. Sin embargo, hasta no ampliar el muestreo de ADN, mantengo la individualidad de estas tres especies, distinguiéndolas morfológicamente por los detalles que se enumeran a continuación: B. chubutensis posee brácteas involucrales triangulares, de contorno entero a inciso-mucronado y margen entero; sépalos lanceolados con ápice acuminado; corola lobada hasta 1/4 - 1/5 de la longitud 
del tubo; y tubo estaminal de igual longitud que los filamentos; $B$. raffaellii posee forma de vida hemicriptófita; lóbulos foliares oblongos a laciniados con ápice acuminado; involucro pateliforme con base fusionada y lóbulos triangulares, dentados con margen entero o dentado-fimbriado; perianto más corto (hasta $3 \mathrm{~mm}$ ) y corola lobada hasta $1 / 4$ de la longitud del tubo. Prefiero ajustar la revisión a la taxonomía clásica y mantener la independencia de estos taxones hasta obtener más registros que permitan profundizar los análisis morfológicos y moleculares, y ampliar el conocimiento que se tiene de su distribución geográfica.

Boopis bupleuroides (Less.) C. A. Muell., Fl. Bras. (Martius) 6(4): 355. 1885. Acicarpha bupleuroides Less., Linnaea 6: 527. 1831. TIPO: Brasil, 1814, F. Sellow s.n. (holotipo probablemente en B†; isotipo HAL 0113258 no visto). Fig. 7.

Boopis bupleuroides (Less.) C.A. Muell. var. microphylla C.A. Muell., Fl. Bras. (Martius) 6(4): 356. 1885. TIPO: Brasil, sin fecha indicada, F. Sellow 4434 (holotipo probablemente en $\mathrm{B} \dagger$, no localizado).

Terófitos caulescentes, decumbentes, de $35-40 \mathrm{~cm}$ de altura, estriados; glabros. Hojas basales membranáceas, panduriformes, con la base largamente atenuada, $6-8$ x 1,5-2 $\mathrm{cm}$, margen irregularmente dentado, ápice obtuso a redondeado. Hojas caulinares membranáceas, espatuladas, con la base cordada amplexicaule, 2-3 x 0,6-2 cm, margen dentado, ápice redondeado a obtuso. Pedúnculos teretes de 1,5-3 cm de longitud. Cefalodios terminales o laterales, sin grupos cimosos. Involucro rotáceo, brácteas involucrales libres, oblongas, ensanchadas en la base, margen entero a dentado, 5,5-17 x 1-5 mm. Receptáculo punctiforme, de 4-9 mm de diámetro. Brácteas del receptáculo lanceoladas. Flores tetrámeras. Sépalos orbiculares u oblongos. Perianto de 3,7 - $4 \mathrm{~mm}$ de longitud. Hipanto verde, infundibuliforme. Corola blanca, lobada hasta la mitad; tubo cilíndrico; lóbulos oblongos, planos, ligeramente recurvados, ápice agudo, recto. Tubo estaminal de $2 / 3$ de la longitud del tubo corolino. Filamentos ausentes. Anteras oblongas, tecas basalmente obtusas, conectivo no apiculado. Glándulas nectaríferas indiferenciables. Ovario lageniforme, 0,8 - $1 \mathrm{~mm}$ de longitud. Cipselas prismáticas con 4 carenas compresas, coronadas por sépalos vestigiales que rodean un apículo central, de $3-3,5 \mathrm{~mm}$ de longitud. 
Iconografía. Magenta \& Pirani (2002, Prancha 1: A-D).

Distribución. Endémico del sudeste y sur de Brasil. Crece en terrenos pantanosos de la provincia fitogeográfica Paranaense (Cabrera \& Willink, 1980). Florece en primavera y fructifica en verano (Magenta \& Pirani, 2002)

Discusión. Boopis bupleuroides puede ser confundido con B. jürgensii por el hábito caulescente, las brácteas involucrales libres y la similitud de sus frutos. Sin embargo, B. jürgensii es una hierba rizomatosa, no ramificada, con hojas y brácteas involucrales lanceoladas sésiles de margen entero, receptáculo acrescente hasta de 30 mm y flores pentámeras.

Por su aspecto general, $B$. bupleuroides también podría ser confundido con $B$. itatiaiae. Sin embargo, B. itatiaiae se diferencia porsus hojas ligeramente dentadas, brácteas involucrales hasta de $50 \mathrm{~mm}$ de longitud y el receptáculo acrescente $(5 \mathrm{~mm})$ durante la madurez de los frutos.

Boopis castilloni (Hicken) Pontiroli, Revista Mus. La Plata, Secc. Bot. 9 (4): 223. 1963. Calycera castilloni Hicken, Physis (Buenos Aires) 1 (3): 386. 1912. TIPO: Argentina. Catamarca: El Rodeo, 15-I-1911, L. Castillón 2053 (holotipo SI 351!; isotipos CORD 00005208 no visto, LIL 89296!, LIL 89445!). Fig. 8.

Hemicriptófitos o geófitos herbáceos, rizomatosos, de aspecto rosulado, con brotes aéreos caulescentes, tendidos o ascendentes, simples o poco ramificados, glabros. Rizoma vertical con raíces adventicias y numerosos brotes en el extremo superior. Raíces contráctiles. Hojas sésiles; lámina incisa con margen entero, lóbulos y ápice obtusos, mucronados. Pedúnculos teretes, terminales. Cefalodios con 10- $\infty$ grupos cimosos, 2-3 floros. Involucro con base fusionada rotácea y lóbulos triangulares dentado-mucronados. Receptáculo acrescente en diámetro: punctiforme durante la antesis, discoideo-meniscoide durante la madurez de los frutos. Brácteas del receptáculo subuladas, libres. Flores pentámeras. Sépalos vestigiales o triangulares con ápice acuminado, inflados. Perianto de 2,70 - 3,24 mm de longitud. Hipanto verde, 
infundibuliforme. Corola blanca, lobada hasta la mitad del tubo; tubo infundibuliforme; lóbulos lanceolados, planos, ligeramente recurvados, con ápice obtuso y recto. Tubo estaminal del doble del largo de los filamentos. Filamentos de 1/6 del largo de las anteras. Anteras oblongas, tecas basalmente obtusas sin caudículas, conectivo no apiculado. Glándulas nectaríferas hipantiales. Ovario elipsoidal a lacrimiforme, de 0,9 1,1 mm de longitud. Cipselas prismáticas a piriformes, rugosas con 5 carenas vestigiales, coronadas por sépalos vestigiales u orbiculares que rodean a un apículo central grueso.

Iconografía. Pontiroli (1963, pág. 218, Fig. 9: D-F).

Distribución. Endémico de la sierra de Ambato, en la provincia de Catamarca, entre 3000-3500 m s.m., área que corresponde al ecotono existente entre el Distrito Serrano de la provincia Chaqueña y la provincia Altoandina.

Discusión. Las flores aparecen muy desordenadas sobre el cefalodio, con tamaños muy dispares. Pareciera que la infloresecencia está formada por muchos grupos cimosos paucifloros, con el grupo de flores solitarias reducido a una única flor central, terminal, muy desarrollada.

Boopis castilloni puede ser confundido con B. gracilis por los brotes aéreos caulescentes y la similitud de sus hojas y sus frutos. Sin embargo, B.gracilis es una hierba terófita que posee tallos ramificados erectos a postrados, sin rizomas, raíces no pivotantes ni contráctiles, involucro con lóbulos triangulares con margen entero, receptáculo no acrescente en diámetro durante la madurez de los frutos, brácteas del receptáculo lanceoladas, hipanto tubuloso, corola campanulada, glándulas nectaríferas estaminales y tubo estaminal de igual longitud que el tubo corolino.

Boopis castilloni es conocido localmente con el nombre vulgar de "roseta".

Boopis chubutensis Speg., Anales Mus. Nac. Buenos Aires 7: 300. 1902. TIPO: Argentina. Chubut. "In aridis saxosis secus Rio Chubut", aest.1899, N. Illín s.n. (holotipo LP ex LPS 12041!). Fig. 9 (A-D). 
Geófitos herbáceos, rizomatosos, con brotes aéreos rosulados a caulescentes con entrenudos breves, simples o poco ramificados, hasta de $15 \mathrm{~cm}$ de altura, glabros. Hojas lineares a pinnatifidas, de 0,5-6 cm de longitud, con margen entero o dentado, mucronado, y ápice obtuso, mucronado. Cefalodio central sésil o pedunculado a veces rodeado de varios cefalodios laterales pedunculados. Pedúnculos erectos, estriados, desnudos u hojosos. Cefalodios con aproximadamente 10 grupos cimosos, 2-5 floros. Involucro con base fusionada pateliforme y lóbulos triangulares, laciniados con margen entero o dentado-mucronado. Ocasionalmente pueden formarse 1-3 grupos cimosos por fuera del involucro, los bordes de las brácteas tectrices están fusionados externamente al involucro formando celdas periféricas aisladas. Receptáculo punctiforme. Brácteas del receptáculo filiformes o espatuladas, con ápice obtuso u obtuso acuminado; libres o parcialmente fusionadas al involucro por uno de los bordes (sin formar celdas completas). Flores pentámeras. Sépalos lanceolados con ápice acuminado. Perianto de 3,5 - 3,7 mm de longitud. Hipanto verde, tubuloso. Corola blanca, lobada hasta 1/4 - 1/5 de la longitud del tubo; tubo infundibuliforme; lóbulos lanceolados, planos, ligeramente recurvados, ápice agudo a obtuso, uncinado. Tubo estaminal de igual longitud que los filamentos. Filamentos de 1/5 del largo de las anteras. Anteras obtusas a oblongas, tecas basalmente sagitadas con caudículas, conectivo no apiculado. Glándulas nectaríferas hipantiales. Ovario lacrimiforme, de 1,5 mm de longitud. Cipselas prismáticas, lisas, de 2-4 mm de longitud, con 5 carenas coronadas por sépalos lanceolados con ápice agudo, que rodean a un apículo central cuneiforme.

Distribución. Endémico del oeste de la provincia de Chubut (Argentina). Habita en bordes de curso de agua y mallines en la estepa patagónica. Ocupa la provincia fitogeográfica Patagónica (Cabrera \& Willink, 1980).

Discusión. Algunos de los ejemplares examinados muestran que los brotes aéreos de esta especie son la continuación de ramificaciones verticales (que pueden estar, a su vez, ramificadas o no) que emergen de una estructura similar a un rizoma delgado más profundo. Algunos ejemplares se distinguen por la presencia de tricomas glandulosos en las brácteas involucrales y del receptáculo.

Los caracteres distintivos de Boopis chubutensis son: geófito herbáceo rizomatoso, involucro con brácteas laciniadas y hojas lineares a pinnatifidas, como en 
Boopis australis pero esta última posee hojas lineares con margen entero a dentadomucronado y sépalos orbiculares a aciculares.

Probablemente forme parte de un complejo de especies junto con B. australis y B. raffaelli. Para más detalles, véase el apartado Discusión para Boopis australis.

Boopis filifolia Speg., Anales Soc. Ci. Argent. 48: 176. 1899. TIPO: Argentina. Santa Cruz. Patagonia. Pan de Azúcar (Rio Chico), XII-1897, C. Ameghino s.n. (holotipo LP ex LPS 11027!). Fig. 9 (E-G).

Boopis prichardi S. Moore in Rendle, Jour. Bot. 42: 372. 1904. TIPO: Argentina. "Tops of mountains, Burmeister Peninsula; also shingle beach, Lake Argentino", I-1900, H. Prichard s.n. (holotipo BM no visto, foto!).

Geófitos herbáceos, rizomatosos, cespitosos, con brotes aéreos rosulados a caulescentes erectos, de 1-3-(8) cm de altura; glabros, glutinosos. Rizomas delgados, lineariformes, radicantes, con cicatrices foliares notorias en las ramificaciones verticales. Hojas lineares a linear-cuneiformes; lámina plegada, con margen entero y ápice obtuso, atenuada en un largo pecíolo. Pedúnculos teretes, cortos, erectos, terminales, hojosos, o bien ausentes. Cefalodio con 2-5 grupos cimosos periféricos y varios grupos cimosos externos al involucro, los mayores (2-15 flores) con involucelo profilar, los menores (12 flores) sin involucelo profilar o con involucelo profilar fusionado a la bráctea tectriz, brácteas tectrices externas lineares con base ensanchada. Involucro con base fusionada campanulada y lóbulos lanceolados, desiguales. Brácteas del receptáculo ocasionales, espatuladas o lanceoladas. Flores pentámeras. Sépalos dentiformes, reducidos, inflados, con ápice obtuso. Perianto de 4-5 mm de longitud. Hipanto tubuloso, de 0,40-0,55 mm de longitud. Corola blanca, campanulada, de 4-4,5 mm de longitud, lobada en 1/4-1/5 de su parte superior; tubo cilíndrico, lóbulos oblongos, recurvados, planos, ápice agudo, uncinado. Tubo estaminal de 1/2 de la longitud de la corola. Filamentos nulos. Anteras oblongas, tecas basalmente sagitadas, con caudículas, conectivo apiculado. Glándulas nectaríferas estaminales. Ovario cilíndrico a piriforme, de 2,8-3,3 mm de longitud. Cipselas prismáticas, rugosas, con 5 carenas rugosas, coronadas por sépalos membranáceos, suborbiculares de ápice obtuso que rodean un diminuto apículo central. 
Distribución. Endémica del centro-sur de la provincia fitogeográfica Patagónica. Se la encuentra vegetando en plena estepa, en la pcia. de Santa Cruz (Argentina), sobre suelos arenoso-pedregosos. Ha sido coleccionada esporádicamente en el sur de Chile (XII Región de Magallanes).

Discusión. Boopis filifolia puede ser confundida con B. patagonica, especie con la que comparte la forma de vida geófita rizomatosa, el aspecto general de los brotes aéreos, la forma de la inflorescencia y el área de distribución. Sin embargo, $B$. patagonica posee brotes aéreos glaucos, gruesos, no glutinosos; rizomas blancos, gruesos y sin cicatrices foliares notorias en las ramificaciones verticales; hojas espatuladas con lámina plegada y cefalodios sin grupos cimosos periféricos, con grupos cimosos externos con menor número de flores.

Boopis gracilis Phil., Linnaea 28: 707. 1856. TIPO: Chile. "In collibus ejusdem departamenti Linares ocurrit", 1856, P. Germain s.n. (lectotipo aquí propuesto W 18890008587 no visto, foto!; duplicados CONC 29655!, K 000588750 no visto, foto!, SI 12639!, W 0021370 no visto, foto!). Fig. 10.

Terófitos caulescentes, ocasionalmente rosulados, con ramas erectas o decumbentes, de 1-35 cm de altura, glabras, gruesas. Hojas sésiles o con la lámina atenuada en un pecíolo estrecho de igual longitud; lámina espatulada u oblonga a lanceolada, incisa a pinnatifida, con margen entero, y ápice obtuso, mucronado. Pedúnculos teretes, erectos o ascendentes. Cefalodio sin grupos cimosos. Involucro con base fusionada pateliforme a crateriforme y lóbulos triangulares a laciniados con margen entero o dentado y ápice mucronado. Receptáculo punctiforme. Brácteas del receptáculo libres, lineares y espatuladas. Flores pentámeras. Sépalos membranáceos, suborbiculares, con margen entero a sinuado y ápice obtuso o emarginado. Perianto infundibuliforme $\mathrm{o}$ hipocrateriforme, de $2-4 \mathrm{~mm}$ de longitud. Hipanto filiforme, de hasta $0.5 \mathrm{~mm}$ de longitud. Corola blanca y verde, lobada en $1 / 2-1 / 3$ de su parte superior; tubo infundibuliforme o cilíndrico; lóbulos oblongos, planos o inflados, ligeramente recurvados, con ápice agudo, recto. Tubo estaminal de igual longitud a 2/3 la longitud 
del tubo corolino. Filamentos subnulos. Anteras oblongas, tecas basalmente obtusas, sin caudículas, conectivo no apiculado. Glándulas nectaríferas estaminales. Ovario elipsoidal a cilíndrico, 1,0 - 1,7 mm de longitud. Cipselas prismáticas, con 5 carenas rugosas o lisas, coronadas por sépalos vestigiales o triangulares que rodean un apículo central.

Distribución. Especie ampliamente distribuida en el extremo sur de Sudamérica. Ocupa las provincias fitogeográficas del Monte, Patagónica y Chilena Central. Se desarrolla sobre suelos arenoso-pederegosos, a pleno sol, en ambientes áridos del sur argentino, desde los Andes de Mendoza hasta el sur de la pcia. de Santa Cruz. Ocasionalmente, puede hallársela en Chile central (VII Región de Maule), creciendo en los valles cordilleranos crecanos al límite con Argentina.

Los datos de distribución proporcionados por los ejemplares tipo de Boopis pozoaeformis y B. bicolor (= B. gracilis var. gracilis) se desestiman por no aportar información confiable y no haber colecciones posteriores que los corroboren.

Discusión. Boopis gracilis puede ser confundido con Boopis castilloni por el hábito caulescente y la similitud de sus hojas y sus frutos. Sin embargo, B.castilloni es una hierba criptófita rizomatosa que posee brotes aéreos caulescentes tendidos o ascendentes, raíces contráctiles, involucro con lóbulos triangulares con margen dentadomucronado, receptáculo acrescente en diámetro durante la madurez de los frutos, brácteas del receptáculo subuladas, hipanto infundibuliforme, corola con tubo corto, glándulas nectaríferas hipantiales y tubo estaminal de longitud muy reducida respecto al tubo corolino.

Se selecciona como lectotipo del nombre de la especie al ejemplar de W debido a que tiene mayor cantidad de material reproductivo y vegetativo en buen estado de conservación que los restantes duplicados.

La especie posee dos variedades que se distinguen por algunos de sus caracteres florales:

1. Perianto infundibuliforme.Tubo corolino verde. Lóbulos corolinos planos, blancos en el haz y en el envés.

B. gracilis var. gracilis 
1'. Perianto hipocrateriforme. Tubo corolino verde, blanco durante la madurez de los frutos. Lóbulos corolinos inflados, blancos en el haz y verdes en el envés.

B. gracilis var. lazulina

Boopis gracilis Phil. var. gracilis. Fig. 10 (A-D).

Boopis pozoaeformis Phil., Anales Univ. Chile 41 (1): 736. 1872. Boopis gracilis Phil. var. pozoaeformis (Phil.) Hicken, Reun. Nac. Soc. Argent. Cien. Nat. 1: 242. 1919. TIPO: Chile. Prov. Santiago (?), hac. Esmeralda, sin fecha indicada, J. Volkmann s.n. (lectotipo aquí propuesto SGO 043595 no visto, foto!; duplicados SGO 057240 no visto, foto!, SI 12648 !).

Boopis bicolor Phil., Anales Univ. Chile 85: 813. 1894. TIPO: Chile. Araucanía, aestate 1887, C. Rahmer s.n. (lectotipo aquí propuesto SGO 043590 no visto, foto!; duplicaods SGO 057206 no visto, foto!, SI 12640!)

Boopis gracilis Phil. var. decumbens Chodat \& Wilczek, Bull. Herb. Boissier ser. 2, 2: 541. 1902. TIPO: Argentina. Mendoza. Valle del Atuel, Arroyo Manga, 1800 $\mathrm{m}$, sin fecha indicada, Chodat 78 (holotipo no localizado, probablemente en G).

Calycera boopidea Hicken, syn. nov. propuesto, Reun. Nac. Soc. Argent. Cien. Nat. 1: 251. 1919. TIPO: Argentina. Neuquén. Confluencia Limay, 20-31-X-1897, coleccionista no indicado (holotipo SI 350!; isotipo LP!).

Perianto infundibuliforme, de $2-2,5 \mathrm{~mm}$ de longitud. Corola lobada en $1 / 2$ de su parte superior; tubo verde, cilíndrico; lóbulos blancos, oblongos, planos, ligeramente recurvados, con ápice agudo y recto. Tubo estaminal de igual longitud que el tubo corolino. Ovario elipsoidal a cilíndrico, 1,0 - 1,7 mm de longitud.

Discusión. Se selecciona el ejemplar de SGO 043595 como lectotipo de B. pozoaeformis por conservar mayor cantidad de inflorescencias en buen estado de 
conservación. Se selecciona el ejemplar de SGO 043590 como lectotipo de B. bicolor porque es el que tiene mayor cantidad de estructuras reproductivas conservadas en buen estado.

Se considera aquí a Calycera boopidea como sinónimo de B. gracilis var. gracilis debido a que todos sus caracteres diagnósticos caen dentro del rango de variación de dicha variedad.

Boopis gracilis Phil. var. lazulina Speg., Revista Argent. Bot. 1 (4): 220. 1926. TIPO: Argentina. Río Negro. N de Patagonia: Carri-lauquen, I-1926, A. Raffaelli s.n. (holotipo BAF no visto, foto!). Fig. 10 (E-G).

Perianto hipocrateriforme, de $3-4 \mathrm{~mm}$ de longitud, algo acrescente y persistente a la madurez de los frutos. Corola lobada en 1/3 de su parte superior; tubo verde, blanco durante la madurez de los frutos, cilíndrico; lóbulos blancos en el haz y verdes en el envés, oblongos, inflados, ligeramente recurvados, ápice agudo, recto. Tubo estaminal de $2 / 3$ de la longitud del tubo corolino. Ovario elipsoidal a cilíndrico, 1,5 $\mathrm{mm}$ de longitud.

Distribución. Esta variedad es endémica del noroeste de la Patagonia argentina. Se distribuye en mayor medida en la provincia fitogeográfica Patagónica, aunque se la puede hallar en el área de contacto con la provincia fitogeográfica del Monte. Es particularmente abundante en el Parque Nacional Laguna Blanca (Neuquén).

Boopis graminea Phil., Linnaea 28: 707. 1856. TIPO: Chile. "In Andibus ejusdem depart. Linares legit. orn. Germain”, I-1856, P. Germain s.n. (holotipo SGO 057212 no visto, foto!; isotipos K 000009703 no visto, foto!, W 1889-0008582 no visto, W 0021371 no visto). Fig. 11 (A-C).

Boopis graminea Phil. var. bracteata Reiche, syn. nov. propuesto, Anales Univ. Chile 106: 1035. 1900. TIPO: Argentina. Chubut. "Hoya del Rio Ftaleufu (Cholila)" 
Exped. Reñihue, sin fecha indicada, C. Reiche s.n. (holotipo SGO no visto, foto!; isotipo SI 12645!).

Geófitos herbáceos, rizomatosos, brotes aéreos caulescentes, simples, erectos o ascendentes, hasta de $35 \mathrm{~cm}$ de altura, glabros, con entrenudos breves. Hojas lineares a estrechamente lanceoladas, de 2-6 cm de longitud, margen entero, excepcionalmente dentado, ápice agudo. Cefalodio central pedunculado, único, con pedúnculo

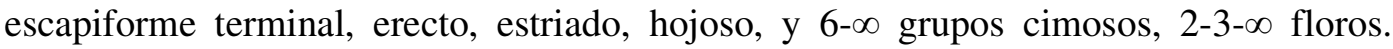
Excepcionalmente pueden aparecer 1-3 grupos cimosos bracteados, exteriores al involucro, independientes (libres, a veces distribuidos a lo largo de la porción superior del pedúnculo) o asociados por fusión de su bráctea tectriz a la periferia del involucro formando celdas periféricas. Involucro con base fusionada pateliforme; lóbulos triangulares, laciniados con margen entero y ápice agudo. Receptáculo punctiforme. Brácteas del receptáculo lanceoladas, espatuladas con limbo lanceolado o lineares; libres o parcialmente fusionadas por uno de los bordes (sin formar celdas completas). Flores pentámeras. Sépalos lanceolados, algo inflados, con ápice agudo. Perianto de 4 4,5 mm de longitud. Hipanto verde, infundibuliforme a tubuloso. Corola blanca, lobada hasta $1 / 3-1 / 2$ de la longitud del tubo; tubo infundibuliforme; lóbulos lanceolados, cuculados inflados, ligeramente recurvados, ápice agudo y uncinado. Tubo estaminal de igual longitud que los filamentos. Filamentos de 1/4 del largo de las anteras. Anteras obtusas a oblongas, tecas basalmente sagitadas con caudículas, conectivo no apiculado. Glándulas nectaríferas hipantiales. Ovario elipsoidal a lacrimiforme, de 2,5 $\mathrm{mm}$ de longitud. Cipselas prismáticas a turbinadas, lisas, de 3-4 mm de longitud, con 5 carenas coronadas por sépalos lanceolados que rodean a un apículo central.

Distribución. Endémico de los Andes de Chile (VIII Región del Bío Bío y IX Región de la Araucanía) y Argentina (Neuquén), en el Distrito Austral de la Provincia Altoandina. Crece en zonas húmedas cercanas a cursos y cuerpos de agua, entre 20002700 m s.m.

Discusión. Los caracteres distintivos de Boopis graminea son: forma de vida geófita rizomatosa con raíces contráctiles, como en Boopis australis, pero esta última posee brotes aéreos con hojas lineares o acintadas a pinnatifidas o pinnatipartidas, 
portando pedúnculos terminales y/o laterales, erectos o postrados, estriados, desnudos u hojosos; cefalodios a veces sésiles, con grupos cimosos menos numerosos, paucifloros (2-7 floros); y sépalos suborbiculares o aciculados, inflados, con ápice acuminado. Aunque es un caracter variable, B. australis se distingue, además, por sus flores con hipanto del doble de la longitud de la corola.

Todos los caracteres de la variedad bracteata están dentro del rango de variación morfológica de B. graminea, incluso el largo de las brácteas del involucro, carácter diagnóstico que se usó en la descripción original para distinguirla.

Boopis itatiaiae Dusén, Arq. Mus. Nac. Rio de Janeiro, 13: 24. 1903. TIPO: Brasil: "Serra do Itatiaya: In paludosis (sine loco speciali indicato)", sin fecha indicada, E. Ule s. $n$. (holotipo no localizado, probablemente en RB). Fig. 12.

Terófitos caulescentes decumbentes, con tallos simples, fistulosos, estriados, de 30-40 cm de altura; glabros. Hojas caulinares sésiles, membranáceas, espatuladas, oblanceoladas o elípticas, margen escasamente dentado, con la base decurrente, 10-15 x 0,5-3 cm, ápice redondeado, obtuso, agudo o acuminado. Pedúnculos teretes, terminales o laterales, de $1-5 \mathrm{~cm}$ de longitud. Cefalodios sin grupos cimosos. Involucro rotáceo, brácteas involucrales libres, lineares a oblanceoladas, margen entero o dentado, 20-50 x 2-4 mm. Receptáculo acrescente: punctiforme durante la antesis, columnar durante la madurez de los frutos (ca. $5 \mathrm{~mm}$ ). Brácteas del receptáculo lanceoladas o espatuladas, con el nervio central evidente. Flores tetrámeras. Sépalos orbiculares u oblongos, carenados abaxialmente, inflados. Perianto de 3,4 - $4 \mathrm{~mm}$ de longitud. Hipanto tubuloso. Corola blanca, partida; tubo reducido; lóbulos oblongos, planos, ligeramente recurvados, ápice agudo, recto, a veces ligeramente uncinado. Tubo estaminal del triple de la longitud de los filamentos (de igual longitud que el tubo corolino). Filamentos de 1/4-1/5 de la longitud de las anteras. Anteras oblongas, tecas basalmente obtusas, sin caudículas, conectivo no apiculado. Glándulas nectaríferas indiferenciables. Ovario lacrimiforme, 0,85 - 1,2 $\mathrm{mm}$ de longitud. Cipselas prismático-elipsoidales, con 4 carenas estrechas, coronadas por sépalos vestigiales a subulados que rodean un apículo central, de 3,5-4,5 mm de longitud. 
Iconografía. Magenta \& Pirani (2002, Prancha 1: H-I).

Distribución. Endémico de las sierras de Itatiaia y de Mantiqueira, en los estados de Rio de Janeiro y Sâo Paulo (Brasil), área correspondiente a la Provincia Atlántica. Crece en terrenos anegadizos de campos de altura; sobre turberas (Magenta \& Pirani, 2002).

Discusión. Boopis itatiaiae puede ser confundido con B. bupleuroides por el hábito caulescente, las brácteas involucrales libres, la presencia de flores tetrámeras y la similitud de sus frutos. Sin embargo, B.bupleuroides posee brácteas involucrales con la base ensanchada y receptáculo no acrescente durante la madurez de los frutos.

Por el aspecto general de la planta, también puede ser confundido con B. jürgensii. Sin embargo, B. jürgensii es un geófito rizomatoso con hojas lanceoladas, brácteas involucrales lanceoladas con margen entero, receptáculo acrescente de mayor longitud (hasta $30 \mathrm{~mm}$ ) y flores pentámeras.

Boopis jürgensii Pilg., Notizbl. Bot. Gat. Berlin 11: 101. 1931. TIPO: Brasil. Rio Grande do Sul. Municipio Rio Pardo: Campo do Sobrado, 70 m, XII-1929, C. Jürgens 378 (holotipo B 100002920 no visto, foto!). Fig. 38.

Geófitos herbáceos, rizomatosos, brotes aéreos simples, erectos surcado-estriados, de 50-70 cm de altura; glabros. Hojas caulinares ligeramente coriáceas, sésiles, amplexicaules, erectas, lanceoladas a estrechamente ovado-lanceoladas, margen entero a escasamente dentado, con la base decurrente, $8-12 \times 1,5-2,5 \mathrm{~cm}$, ápice redondeado, obtuso, agudo o acuminado. Pedúnculos terminales, de $2 \mathrm{~cm}$ de longitud. Brácteas involucrales libres, $15 \mathrm{~mm}$ de longitud, lanceoladas, margen entero. Receptáculo probablemente acrescente, columnar durante la madurez de los frutos (hasta $30 \mathrm{~mm}$ ). Brácteas del receptáculo subulado-lanceoladas. Flores pentámeras. Sépalos estrechamente oblongos, crasos, 0,75 $\mathrm{mm}$ de longitud. Perianto de $4-5,3 \mathrm{~mm}$ de longitud. Hipanto tubuloso. Corola blanco-verdosa, partida; tubo reducido; lóbulos erectos, ovales, algo crasos, ápice no muy estrecho, $2 \mathrm{~mm}$ de longitud. Filamentos de 1 
mm de longitud. Anteras oblongas. Ovario cilíndrico. Cipselas elipsoideas, de 4-5 mm de longitud, con carenas algo crasas, sépalos decurrentes no muy acrescentes.

Distribución. Boopis jürgensii sólo es conocido por su colección tipo. Probablemente sea endémico de los campos anegados del sur de Brasil (Pilger, 1931), en la provincia fitogeográfica Paranaense.

Discusión. Se considera que el receptáculo es probablemente acrescente porque sólo se conoce el ejemplar tipo con inflorescencias con frutos inmaduros y algunas flores residuales, y no se ha podido comparar con inflorescencias en plena floración. Boopis jürgensii puede ser confundido con B. bupleuroides por el hábito caulescente, las brácteas involucrales libres, el receptáculo acrescente y la similitud de sus frutos. Sin embargo, B.bupleuroides posee hojas basales y caulinares panduradas con margen dentado, hojas caulinares con base cordada, brácteas involucrales con la base ensanchada, flores tetrámeras y receptáculo punctiforme no acrescente durante la madurez de los frutos.

Por el aspecto general de la planta, también puede ser confundido con $B$. itatiaiae. Sin embargo, B. itatiaiae es un terófito con hojas espatuladas, oblanceoladas o elípticas, brácteas involucrales lineares a oblanceoladas, margen entero o dentado, receptáculo acrescente de menor longitud (ca. $5 \mathrm{~mm}$ ) y flores tetrámeras.

Boopis multicaulis Phil., Linnaea 28: 706. 1856. TIPO: Chile. "Cordil. de Maule", 1856, P. Germain s.n. (duplicados K 000009706 no visto foto!, P 00852229 no visto, P 00852230 no visto, W 0021372 no visto, W 1889-0008585 no visto, W 0021373 no visto). Fig. 11 (D-E).

Hemicriptófitos rosulados a caulescentes con tallos tendidos o ascendentes, ramificados, hasta de $20 \mathrm{~cm}$ de altura. Raíz pivotante, contráctil. Hojas espatuladas, con la base atenuada en un peciolo rectangular notorio; lámina partida de contorno oblongo y lóbulos oblongos, con margen entero y ápice obtuso no mucronado. Inflorescencia central pedunculada o sentada, inflorescencias laterales pedunculadas, terminales o

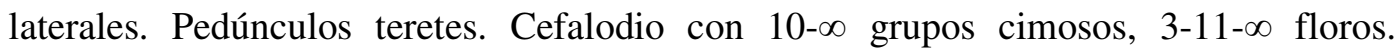


Involucro rotáceo con base fusionada y lóbulos triangular-oblongos con margen entero. Receptáculo punctiforme, algo acrescente y globoso durante la madurez de los frutos. Brácteas del receptáculo lineares, espatuladas de limbo lanceolado, o triangularlaciniadas; las periféricas fusionadas por ambos bordes entre sí y con el involucro (formando celdas completas), las interiores libres. Flores pentámeras. Sépalos pulviniformes. Perianto de $5-7 \mathrm{~mm}$ de longitud. Hipanto verde, tubuloso. Corola blanca, partida, tubo breve, lóbulos lanceolados, planos, ligeramente recurvados, con ápice agudo y recto. Tubo estaminal del doble del largo de los filamentos. Filamentos de 1/8 del largo de las anteras. Anteras oblongas, tecas basalmente sagitadas sin caudículas, conectivo no apiculado. Glándulas nectaríferas hipantiales. Ovario elipsoidal a lacrimiforme, de $2 \mathrm{~mm}$ de longitud. Cipselas prismáticas, lisas con 5 carenas, coronadas por sépalos orbiculares que rodean a un apículo central.

Distribución. Endémico del pedemonte andino de Chile y Argentina, entre $37^{\circ}$ $46^{\circ}$ de latitud sur, en la Provincia Subantártica. Ha sido coleccionada muy esporádicamente.

Discusión. B. multicaulis muestra una semejanza muy estrecha con Gamocarpha selliana en todos sus caracteres. G. selliana sólo se diferencia por desarrollar estolones poseer entrenudos más cortos, una forma caulescente menos conspicua y menor número de inflorescencias. La morfología de la flor y el fruto es idéntica para los dos taxones, aunque en $B$. multicaulis el hipanto está más desarrollado y existe un mayor número de flores por inflorescencia. La mayoría de las diferencias en los caracteres vegetativos es de tipo cuantitativo. Es muy probable que ambos taxones formen parte de una misma especie con alta variabilidad morfológica que ocupa un área de distribución amplia. Sin embargo, es preferible mantener la independencia taxonómica hasta que puedan coleccionarse más ejemplares que permitan desarrollar análisis moleculares y morfológicos más detallados y concluyentes.

Boopis necronensis Zav.-Gallo, S. Denham \& Pozner, Brittonia 63(1): 115. 2011. TIPO: Argentina. La Rioja: Reserva Laguna Brava, $200 \mathrm{~m}$ al oeste del refugio de Mulas 
Muertas, 3775 m, 10-II-1998, F. Biurrun, J. Molina \& Ruiz 5218 (holotipo CTES 307622!). Fig. 13.

Geófito herbáceo, rizomatoso, con brotes aéreos rosulados, escasamente ramificados, de $4 \mathrm{~cm}$ de altura y $11 \mathrm{~cm}$ de diámetro, brotando de un rizoma vertical; glabros. Raíces contráctiles. Hojas gruesas espatuladas, 2-5,5 cm de longitud; pecíolo 1-2,5×0,5 cm; lámina oval, 1,2-3×1-2 cm, margen crenado-mucronado, con base cuneada-atenuada, ápice obtuso; superficie adaxial de la lámina ligeramente aciculada, y nerviación fuertemente marcada sobre la superficie abaxial. Pedúnculos más cortos que las hojas, 1-2 cm de longitud, teretes, surcados, monocéfalos; usualmente con un único pedúnculo central rodeado por 1-5 pedúnculos laterales, o inflorescencia central sésil. Cefalodio

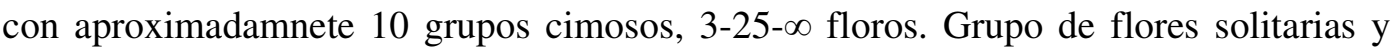
flor terminal ausentes. Involucro pateliforme con la base fusionada, con 5-6 lóbulos amplios triangulares, $0,3-0,7 \times 0,25-0,85 \mathrm{~cm}$; lóbulos con ápice agudo a obtuso, mucronado. Receptáculo punctiforme, convexo, 1-4 cm de diámetro, acrescente (globoso) durante la maduración de los frutos. Brácteas del receptáculo escasas, muy dispersas, linear-espatuladas, mucronadas, carnosas, $0,3 \mathrm{~cm}$ de longitud en receptáculos con flores, a $0,5 \mathrm{~cm}$ de longitud en receptáculos con frutos, libres. Flores verdes, pentámeras, 100-150 por inflorescencia. Sépalos con estivación imbricada, foliáceos, membranáceos, desiguales, con margen undulado, 1-2×1-1,15 mm. Perianto infundibuliforme, 4-4.5 $\mathrm{mm}$ de longitud. Hipanto tubuloso, 1,85-2,2×0,7-1,2 $\mathrm{mm}$. Corola 2-2,5×1,2-2,35 mm, partida; tubo reducido; lóbulos cuculados inflados, 1,6$2 \times 0,8 \mathrm{~mm}$. Tubo estaminal de igual longitud que los filamentos. Anteras connadas, 1,5 $\mathrm{mm}$ de longitud oblongas, tecas basalmente obtusas con caudículas, conectivo no apiculado. Glándulas nectaríferas hipantiales, de $1 \times 0,25 \mathrm{~mm}$. Estilo de $2 \mathrm{~mm}$ de longitud (fase estaminada) a 5,5 $\mathrm{mm}$ de longitud (fase pistilada). Ovario elipsoide a cilíndrico, 2-2,5×1-1,4 mm. Cipselas prismáticas, 4,5×2,5 mm, glabras, lisas, o con desarrollo eventual de algunas rugosidades; apículo 0,4-0,6 $\mathrm{mm}$ de longitud, con 5 carenas longitudinales de sección transversal romboide y margen agudo, coronado por los sépalos acrescentes $(1,5-2,6 \times 1,1-1,25 \mathrm{~mm})$, suborbiculares a lanceolados, plegados, corchosos, con margen undulado y ápice obtuso-mucronado. Semilla $3 \times 1 \mathrm{~mm}$. 
Distribución. Boopis necronensis habita en el Parque Provincial Laguna Brava (La Rioja) creciendo en terrenos de altura, planos y áridos con suelos pedregosos y rocas sueltas. Además, se conoce un ejemplar de los Andes de Catamarca (Depto. Tinogasta).

Etimología. El epíteto específico hace referencia al sitio donde fue coleccionado el holotipo, el refugio "Mulas Muertas" (del griego, necrós = muerto; ónos = mula, asno).

Discusión. Boopis necronensis es similar a B. pterocalyx, pero el primero se distingue por sus hojas espatuladas más grandes, con margen crenado-mucronado, y flores más grandes con un hipanto expandido (debido a las glándulas estaminales) y lóbulos corolinos profundamente partidos. Las cipselas de B. necronensis también son diferentes por poseer un apículo reducido, de textura lisa, y coronado por sépalos más pequeños, plegados, obtuso-mucronados, y corchosos. Observaciones de campo mostraron que la corola es verde oscura con el margen de los lóbulos blancos.

Los caracteres florales (forma, coloración y proporciones) de B. necronensis muestran una estrecha semejanza con los de Nastanthus patagonicus. Sin embargo, el resto de los caracteres y sus respectivas áreas de distribución hacen que ambas especies sean perfectamente distinguibles.

Boopis patagonica Speg., Anal. Mus. Nac. Buenos Aires 7: 301. 1902. TIPO: Argentina. Santa Cruz: San Julián - Rio Deseado, verano 1899-1900, C. Ameghino 234 (lectotipo aquí propuesto LP ex LPS!; duplicados BA 28893!). Fig. 14 (A-C).

Geófitos herbáceos, rizomatosos, glaucos, gruesos, cespitosos, con brotes aéreos caulescentes procumbentes de $2-8 \mathrm{~cm}$ de altura; glabros. Rizomas blancos, gruesos, radicantes. Hojas espatuladas, atenuadas en pecíolo; lámina oblonga, plegada, con margen entero a sinuado (ocasionalmente dentado), y ápice obtuso. Pedúnculos teretes breves. Cefalodios sin grupos cimosos periféricos internos, pero con grupos cimosos externos ocasionales, con involucelos profilares fusionados a su bráctea tectriz, portando 1-2 flores. Involucro con base fusionada campanulada y lóbulos triangulares, 
plegados con ápice obtuso, rodeando al grupo de flores solitarias del cefalodio. Brácteas del receptáculo ausentes. Flor terminal del cefalodio pequeña. Flores pentámeras. Sépalos dentiformes, inflados con ápice obtuso. Perianto de 3,8-4,0 mm de longitud. Hipanto tubuloso, de 0,7-0,9 mm de longitud. Corola campanulada, de 2,90-3,30 mm de longitud, lobada en 1/3 de su parte superior; tubo largo; lóbulos oblongos, planos, con ápice agudo u obtuso, uncinado. Tubo estaminal de 1/2 de la longitud de la corola. Filamentos nulos. Anteras oblongas, tecas basalmente obtusas, sin caudículas, conectivo no apiculado. Glándulas nectaríferas estaminales. Ovario cilíndrico a elipsoidal, de 2,2 2,7 mm de longitud. Cipselas prismáticas, rugosas, con 5 carenas rugosas, coronadas por sépalos membranáceos, suborbiculares de ápice obtuso que rodean un diminuto apículo central.

Distribución. Endémica de Patagonia austral. La mayoría de las colecciones provienen de la Pcia. de Santa Cruz (Argentina). Sin embargo, existe un registro para el sur de Chile. Especie exclusiva de la provincia fitogeográfica Patagónica. Ha sido coleccionada muy esporádicamente.

Discusión. Boopis patagonica puede ser confundida con B. filifolia, especie con la que comparte: la forma de vida geófita rizomatosa, el aspecto general de los brotes aéreos, la forma de la inflorescencia y el área de distribución. Sin embargo, B. filifolia posee brotes aéreos resinosos; rizomas lineares, con catafilos aciculares y cicatrices foliares notorias en las ramificaciones verticales; hojas lineraes a linear-cuneiformes y grupos cimosos más frecuentes con mayor número de flores.

De los dos sintipos de Boopis patagonica: Argentina. Patagonia. Santa Cruz: Chonkenk-aik (Rio Chico), II-1898, C. Ameghino s.n. (LP ex LPS 12039) y Santa Cruz: San Julián - Rio Deseado, verano 1899-1900, C. Ameghino 234 (LP ex LPS, BA 28893), se selecciona como lectotipo al ejemplar Ameghino 234 de LP ex LPS (cartulina sin número de herbario) ya que conserva mayor cantidad de estructuras reproductivas que el duplicado de BA. El ejemplar Ameghino s.n. (LP ex LPS 12039) es estéril. 
Boopis pterocalyx Zav.-Gallo, S. Denham \& Pozner, Brittonia 63(1): 113. 2011. TIPO: Argentina. Mendoza: base de la Sierra del Nevado por ruta provincial 180, 35³7'16"S, 68 31'40”'W, 2340 m, 12-XII-2004, A. Prina, G. Alfonso \& E. Morici 2627 (holotipo SI 124298!; isotipos: CTES 0405304!, SRFA no visto). Fig. 15.

Geófitos herbáceos, rizomatosos, brotes aéreos rosulados a caulescentes con entrenudos breves de $4 \mathrm{~cm}$ de altura y $10 \mathrm{~cm}$ de diámetro, ramificados o apenas ramificados, brotando de un rizoma vertical; vilíferos. Catafilos escuamiformes. Raíces contráctiles. Hojas parcialmente carnosas, espatuladas con la base atenuada en un pecíolo, de 1-5 cm de longitud; las superiores villosas en el margen y particularmente en la base del pecíolo; pecíolo 0,5-3×0,1-0,5 cm, surcado; lámina con superficie aciculada, oval a suborbicular, 0,5-1.5×0,5-2 cm, con margen entero, sinuado y ápice redondeado no mucronado. Pedúnculos más cortos que las hojas, teretes, surcados; plantas usualmente con 1 ó 2 pedúnculos centrales, gruesos $(1,5 \times 0,5 \mathrm{~cm})$ portando una inflorescencia más grande, y algunos pedúnculos laterales delgados $(2,5 \times 0,15 \mathrm{~cm})$, con inflorescencias pequeñas o de tamaño mediano. Cefalodio central con aproximadamente 20 grupos

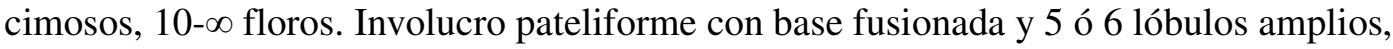
triangulares, de 0,3-0,7 cm de longitud, villosos en el margen. Receptáculo punctiforme, convexo, de 0,5-3 cm de diámetro, acrescente (globoso) durante la maduración de los frutos. Brácteas del receptáculo libres, lineares a linear-espatuladas, parcialmente carnosas, apenas villosas en el ápice, de 0,3-0,5 cm de longitud en receptáculos con flores, a $1 \mathrm{~cm}$ de longitud en receptáculos con frutos. Flores pentámeras, blanco-verdosas, 30-300 por inflorescencia. Sépalos con estivación imbricada, foliáceos, membranáceos, obovados, desiguales, margen sinuado, 0,85×0,6 $\mathrm{mm}$. Perianto infundibuliforme, de aproximadamente $4 \mathrm{~mm}$ de longitud. Hipanto tubuloso, 1,75-1,96× 0,6-0,8 mm. Corola campanulada, 2,2-2,35×1,6-1,7 mm, con una ligera constricción en la base; lóbulos oblongos, cuculados inflados, con ápice obtuso, uncinado, 1,2× 0,8 $\mathrm{mm}$. Tubo estaminal de igual longitud que los filamentos. Filamentos libres de $0,5 \mathrm{~mm}$ de longitud. Anteras de $1 \mathrm{~mm}$ de longitud, tecas basalmente sagitadas sin caudículas, conectivo no apiculado. Glándulas nectaríferas hipantiales, reducidas. Estilo exserto, 4-5 mm (fase estaminada) a 5,7-5,8 $\mathrm{mm}$ de longitud (fase pistilada). Ovario elipsoidal a lacrimiforme, 1,3×0,5 $\mathrm{mm}$. Cipsela terete $5 \times 1,5 \mathrm{~mm}$, con 5 carenas longitudinales, obtusas, y con rugosidades transversales, 
rematado en un apículo cónico, conspícuo, de 1,2-1,8 $\mathrm{mm}$ de longitud, y coronado por sépalos foliáceos, acrescentes $(4 \times 2,5-3,3 \mathrm{~mm})$, truncados, orbiculares. Semilla 3,5×1 $\mathrm{mm}$.

Distribución. Boopis pterocalyx es endémico de la Sierra del Nevado (Pcia. de Mendoza, Argentina), en la provincia fitogeográfica Patagónica. Crece sobre suelos arenosos y pedregosos en la base de la montaña.

Etimología. El epíteto específico hace referencia a los sépalos foliosos, con forma de ala, persistentes en las cipselas.

Discusión. Esta especie puede ser reconocida fácilmente por los sépalos orbiculares foliáceos, imbricados en el fruto a manera de hélice, y por sus hojas, involucro y brácteas del receptáculo villosas.

Boopis pusilla Phil., Linnaea 33: 105. 1864. TIPO: Chile. Cordillera de Santiago, Cordillera de las Arañas, XI-1861, F. Philippi 1008 (holotipo SGO 043584 no visto, foto!; isotipos K 000588752 no visto, foto!, W no visto, foto 31052 del Field Museum of Natural History en SI!, W no visto, foto 31055 del Field Museum of Natural History en SI!). Fig. 16.

Terófitos caulescentes, hasta de $15 \mathrm{~cm}$ de altura; glabrescentes. Hojas espatuladas; lámina lanceolada, de 0,6-3 x 0,2-1 cm, con margen entero o dentado-mucronado (paucidentado) y ápice agudo, mucronado; pecíolo de 0,5-3,5 x 0,05-0,1 cm laxamente pubescente. Pedúnculos axilares, teretes, de 0,3-0,7 $\mathrm{cm}$ de longitud, laxamente pubescentes. Cefalodios paucifloros, hasta con 10 flores solitarias. Grupos cimosos ausentes. Involucro ligeramente acrescente durante la maduración de los frutos, con una base rotácea a tubular fusionada y lóbulos triangulares. Receptáculo punctiforme. Brácteas del receptáculo infrecuentes, estrechamente lanceoladas. Flores pentámeras. Sépalos vestigiales o suborbiculares, con margen sinuado, membranáceos. Perianto 1,9 - 3,8 mm long. Hipanto verde, infundibuliforme. Corola blanca, lobada hasta la mitad del tubo; tubo infundibuliforme; lóbulos lanceolados, planos, ligeramente recurvados, 
ápice agudo, a veces ligeramente uncinado. Tubo estaminal de 1/2-1/3 de la longitud del tubo corolino. Filamentos de $1 / 4$ del largo de las anteras. Anteras oblongas, tecas basalmente sagitadas, conectivo apiculado. Glándulas nectaríferas indistinguibles. Ovario cilíndrico a lacrimiforme, 1,8 - 2,2 $\mathrm{mm}$ de longitud. Cipselas turbinadas a cilíndricas, rugosas con 5 carenas vestigiales, coronadas por sépalos vestigiales a orbicular-foliáceos que rodean a un apículo central.

Distribución. Endémico de Chile, Región Metropolitana (cordillera de Santiago) a $1350 \mathrm{~m}$ de altitud, en la Provincia Chilena Central. Ha sido coleccionado muy esporádicamente.

Discusión. Boopis pusilla puede confundirse con Calycera sessiliflora por el aspecto general de la planta, ya que son muy similares y comparten parte de su área de distribución. Sin embargo, C. sessiliflora puede distinguirse por sus pedúnculos más cortos siempre glabros, su involucro campanulado, sus flores con sépalos lanceolados, su corola urceolada, y sus cipselas espinosas, lisas, con sépalos acrescentes lanceolados pungentes.

De acuerdo con la descripción original del protologo y la posterior de Reiche (1900), B. pusilla posee pedúnculos nutantes. Sin embargo, esto no pudo ser constatado en el material examinado.

Boopis raffaellii Speg., Rev. Argent. Bot. 1 (4): 221. 1926. TIPO: Argentina. Chubut. N de Patagonia: Sierra de Talagapa, I-1926, A. Raffaelli s.n. (holotipo BAF no visto, foto!; isotipo BA 27/705!). Fig. 14 (D-E).

Hemicriptófitos rosulados, con brotes aéreos caulescentes ascendentes, simples o poco ramificados, hasta de $15 \mathrm{~cm}$ de altura. Raíces contráctiles. Hojas acintadas a pinnatipartidas (lóbulos oblongos a laciniados con ápice acuminado), de 1-5 cm de longitud, con margen entero y ápice obtuso, acuminado. Cefalodios sésiles o pedunculados, uno central y varios laterales. Pedúnculos teretes, terminales erectos, desnudos; pedúnculos laterales sobre ramas postradas o ascendentes, hojosas, a veces varios pedúnculos axilares pequeños. Cefalodios con ca. 7 grupos cimosos periféricos 
internos, 3-4 floros. Involucro con base fusionada pateliforme, lóbulos triangulares, dentados con margen entero o dentado-fimbriado. Ocasionalmente, se forman algunos grupos cimosos externos con brácteas fusionadas al involucro central formando celdas periféricas. Receptáculo punctiforme. Brácteas del receptáculo filiformes, espatuladas o lanceoladas, con ápice agudo acuminado; libres o parcialmente fusionadas al involucro por uno de los bordes (sin formar celdas completas). Flores pentámeras. Sépalos aciculares con ápice acuminado, inflados en la base. Perianto de 2,8 $-3 \mathrm{~mm}$ de longitud. Hipanto verde, tubuloso. Corola blanca, lobada hasta 1/4 de la longitud del tubo; tubo infundibuliforme; lóbulos lanceolados, planos, ligeramente recurvados, con ápice agudo a obtuso, recto. Tubo estaminal 1/2-1/3 longitud que los filamentos. Filamentos de 1/5 del largo de las anteras. Anteras obtusas a oblongas, tecas basalmente sagitadas sin caudículas, conectivo no apiculado. Glándulas nectaríferas hipantiales. Ovario cilíndrico, de 1-1,2 mm de longitud. Cipselas prismáticos, lisos, de 1,5-2 mm de longitud, con 5 carenas coronadas por sépalos no espinescentes, que rodean a un apículo central.

Distribución. Endémico del norte de Patagonia (Argentina). Habita en bordes de curso de agua y mallines de la estepa. Ha sido coleccionado pocas veces en sitios aislados de las provincias de Chubut y Río Negro.

Discusión. Boopis raffaellii puede ser confundido con B. chubutensis debido al aspecto general de los brotes aéreos, sus pedúnculos escapiformes, involucro con brácteas triangulares y hojas pinnatifidas. Sin embargo, B. chubutensis posee forma de vida geófita rizomatosa, hojas lineares a pinnatifidas, con margen entero, mucronado, nunca fimbriado, brácteas del involucro triangular laciniadas no dentado-fimbriadas, tecas de las anteras basalmente sagitadas con caudículas y sépalos lanceolados.

Probablemente forme parte de un complejo de especies junto con B. chubutensis y B. australis. Para más detalles, véase el apartado Discusión para Boopis australis.

CALYCERA Cav., Icon. [Cavanilles] 4: 34, 1797.

Especie tipo: Calycera herbacea Cav. 
Leucocera Turcz., Bull. Soc. Imp. Naturalistes Moscou 21 (1): 583. 1848.

Especie tipo: Leucocera annua Turcz. [= Calycera leucanthema (Poepp. ex Less.) Kuntze].

Gymnocaulus Phil., Linnaea 28: 705. 1856.

Especie tipo: Gymnocaulus viridiflorus Phil. [= Calycera herbacea Cav. var. herbacea].

Anomocarpus Miers, Ann. Mag. Nat. Hist. ser. 3, 6(35): 351. 1860. [Nov 1860].

Especie tipo: aquí propuesta, Anomocarpus axillaris Miers [= Calycera sessiliflora Phil.].

Subarbustos o hierbas. Caméfitos, criptófitos, hemicriptófitos o terófitos con hábito caulescente, rosulado o columnar; glabros o laníferos. Tallos erectos, postrados, procumbentes, ascendentes, rizomatosos (con catáfilos) o bien acaules, potencialmente radicantes. Raíces axonomorfas, con o sin epidermis contráctil, a veces gemíferas. Hojas alternas o imbricadas, gruesas, algo coriáceas, enteras o pinnatisectas, sésiles o espatuladas (atenuadas en un pecíolo rectangular); lámina oblonga, lanceolada u oval, con margen entero, dentado, partido o escuarroso-partido, mucronado o no mucronado, y ápice agudo u obtuso, mucronado o no mucronado. Pedúnculos teretes, escapiformes o nulos, a veces nutantes. Cefalodios con flor terminal. Involucro diferenciado (brácteas con base fusionada) o bien indiferenciado; a veces acrescente durante la maduración de los frutos. Receptáculo punctiforme. Brácteas de los grupos cimosos (= páleas) libres o nulas, dispuestas sobre el receptáculo por dentro del perímetro del involucro. Flores pentámeras o tetrámeras. Sépalos suborbiculares, lanceolados o gibosos, con margen entero, dentado o sinuado, y ápice obtuso, agudo o emarginado, mucronado o no mucronado. Hipanto tubuloso o infundibuliforme. Corola con estivación valvar, tubular, lobada, actinomorfa; lóbulos oblongos o lanceolados, planos o cuculados inflados, con ápice agudo, recto o uncinado. Glándulas nectaríferas hipantiales. Tubo estaminal con filamentos libres en el extremo apical. Anteras oblongas con dehiscencia longitudinal introrsa, con base soldada, obtusa o sagitada; conectivo apiculado o no apiculado. Ovario cilíndrico a lacrimiforme. Cipselas desconocidas. 
Discusión. Se selecciona como tipo del género a la especie Anomocarpus axillaris debido a que el autor los publicó simultáneamente, sin mencionar especie alguna como tipo, y ésta coincide en todos sus caracteres con el protologo del género.

Calycera calcitrapa Griseb., Abh. Königl. Ges. Wiss. Göttingen 19: 163. 1874. TIPO: Argentina. Catamarca, "in convallibus arenosis inter Nacimiento et Laguna Blanca", I1872, P. Lorentz 432 (holotipo CORD 00006342!; isotipos BA 42208!, K 000588753 no visto, foto!). Fig. 17.

Caméfitos subarbustivos glabros, con tallos erectos hasta de $50-60 \mathrm{~cm}$ de altura. Hojas sésiles, lanceolado-oblongas, margen dentado-mucronado. Pedúnculos indiferenciados de los entrenudos superiores del tallo. Cefalodios con ca. 15 grupos cimosos (2-4 flores). Involucro con base fusionada estrecha, rotácea y lóbulos triangulares con margen entero o con dientes mucronados y ápice mucronado; brácteas del involucro claramente diferenciadas de las hojas superiores distales. Receptáculo punctiforme, con brácteas lineares y espatuladas. Flores pentámeras. Cáliz polimórfico: 2-3 sépalos desiguales, de más de $5 \mathrm{~mm}$, aciculares, con sección triangular, lignificados en la antesis (flor terminal central, flores terminales de los grupos cimosos periféricos); 5 sépalos desiguales, más cortos, plano-triangulares, no lignificados en la antesis (flor terminal de los grupos cimosos internos); 5 sépalos iguales, muy cortos, lanceolados, no lignificados en la antesis (flores de $2^{\text {do }}$ y $3^{\text {er }}$ orden de los grupos cimosos y flores solitarias). Perianto de $5-5.3 \mathrm{~mm}$ de longitud. Hipanto verde, tubuloso. Corola blanca, profundamente lobada; tubo reducido; lóbulos de 1- 1,2 mm, oblongos, planos, recurvados, ápice obtuso, no uncinado. Tubo estaminal reducido, de 3/4 de la longitud de los filamentos. Filamentos de 1/3 del longitud de las anteras. Anteras oblongas, de 1,3-1,4 mm; tecas basalmente obtusas, conectivo no apiculado. Glándulas nectaríferas hipantiales. Ovario cilíndrico a lacrimiforme, $1.45-1.60 \mathrm{~mm}$ de longitud. Cipselas polimórficas, prismáticas, estrechas en la base, con 3-5 carenas lisas, coronadas por sépalos cortos lanceolados, o triangulares a cónico-espiniformes, de acuerdo a la posición de la flor que les dé origen y el polimorfismo de sépalos descripto más arriba. 
Distribución. Endémica del noroeste de Argentina, desde San Juan, La Rioja, y Catamarca, al sur de Salta y noroeste de Tucumán, entre 400-1900 m de altitud. Los hábitats de Calycera calitrapa incluyen valles y pedemontes con entisoles y aridisoles de la provincia firogeográfica del Monte y su límite con la provincia fitogeográfica Prepuneña.

Discusión. Los caracteres distintivos de C. calcitrapa son: hábito subarbustivo, anteras oblongas sin apículo, y base obtusa sin caudículas. Hojas sésiles, como las hojas superiores en $C$. crassifolia, pero esta última es una geófita rizomatosa. La flor terminal puede tener, eventualmente, un cáliz espinoso intermedio, como en las flores de 2 do y 3er orden de los grupos cimosos, o no ser espinosas.

Calycera crassifolia (Miers) Hicken, Physis (Buenos Aires) 2: 117. 1916. Acicarpha crassifolia Miers, Ann. Mag. Nat. Hist. ser. 3, 6(36): 402. 1860 [Dec 1860]. Boopis crassifolia (Miers) A. Gray, Proc. Amer. Acad. Arts. 5: 321. 1861. TIPO: Uruguay. Maldonado, sin fecha indicada, J. Tweedie1068 (holotipo K 000588754 no visto, foto!; isotipo BM 000947754 no visto, foto!). Fig. 18.

Geófitos herbáceos con rizomas blancos y brotes aéreos erectos. Tallos erectos hasta de 15-75 cm de altura, glabros. Hojas basales espatuladas, con base atenuada en un corto pecíolo rectangular; lámina oval u oblonga, margen dentado-apiculado o dentadoaristado, ápice obtuso, apiculado o aristado. Hojas superiores sésiles. Pedúnculo indiferenciado de los entrenudos superiores del tallo. Involucro con base fusionada pateliforme, y lóbulos triangulares a rectangulares, con margen entero, dentadomucronado o dentado-apiculado, y ápice mucronado o aristado; brácteas del involucro claramente diferenciadas de las hojas superiores distales. Receptáculo puntctiforme, brácteas periféricas lanceoladas, brácteas internas espatuladas. Flores pentámeras. Cáliz dimórfico: 5 sépalos iguales, $0.7 \mathrm{~mm}$, orbiculares, con sección plana, no lignificados en la antesis (flor terminal central y todas las flores restantes, excepto las flores terminales de los grupos cimosos periféricos); 5 sépalos iguales, $1.5 \mathrm{~mm}$ ampliamente triangulares, con sección plano-navicular, y ápice pungente a espinuloso, no lignificados en la antesis (flores terminales de los grupos cimosos periféricos). Perianto de $5.4-7 \mathrm{~mm}$ de 
longitud. Hipanto de $4 \mathrm{~mm}$, verde, tubuloso. Corola blanca, profundamente lobada, tubo reducido de $1 \mathrm{~mm}$, lóbulos de $3 \mathrm{~mm}$, oblongos, planos, ápice obtuso, no uncinado. Tubo estaminal de $1 / 2$ o de igual longitud que los filamentos. Filamentos de $0.5 \mathrm{~mm}$. Anteras de $1.5 \mathrm{~mm}$, oblongas, tecas basalmente obtusas, conectivo no apiculado. Glándulas nectaríferas hipantiales. Ovario cilíndrico a lacrimiforme, de $1.5-1.9 \mathrm{~mm}$ de longitud. Cipselas dimórficas, prismáticas, muy estrechas en la base, con 4-5 carenas lisas, coronadas por sépalos suborbiculares o triangulares, de acuerdo con la posición de la flor que les dé origen y el polimorfismo de sépalos descripto más arriba.

Distribución. Calycera crassifolia típicamente crece en suelos arenosos (entisoles y aridisoles) en un rango geográfico amplio desde el pedemonte de Mendoza y Neuquén a la costa atlántica de Argentina y la costa atlántica y rioplatense de Uruguay.

Discusión. Calycera crassifolia es la única especie criptófita de este género. Otros caracteres distintivos son: cipselas prismáticas muy estrechas en la base con sépalos acrescentes, triangulares, pungente-espinulosos hacia el ápice, pero nunca espiniformes. $C$. crassifolia es similar a $C$. calcitrapa por la forma de las hojas, la inflorescencia y la flor, aunque esta última es un subarbusto y posee cipselas con sépalos espiniformes.

La especie posee dos variedades que se diferencian por la siguiente clave:

1. Láminas oblongas, margen dentado-apiculado, ápice apiculado. Involucro con lóbulos triangulares. Tubo estaminal de $1 / 2$ del longitud de los filamentos, filamentos de 1/3 del longitud de las anteras. Cipselas con sépalos pungentes.

C. crassifolia var. crassifolia

1'. Láminas ovales, margen dentado-aristado, ápice aristado. Involucro con lóbulos rectangulares. Tubo estaminal de igual longitud que los filamentos, filamentos de 1/5 de la longitud de las anteras. Cipselas con sépalos espinulosos.

C. crassifolia var. spinulosa 
Calycera crassifolia (Miers) Hicken var. crassifolia. Fig. 18 (A-G).

Boopis crassifolia (Miers) A. Gray var. spinuligera Speg., Anales Soc. Ci. Argent. 48: 174. 1899. Calycera crassifolia (Miers) Hicken var. spinuligera (Speg.) Hicken, Reun. Nac. Soc. Argent. Cien. Nat. 1: 244. 1919. TIPO: Argentina. Buenos Aires. Médanos de Punta Rubia, Bahía de San Blas, 3-II-1898, C. Spegazzini s.n. (holotipo LP ex LPS 11029!; isotipo CORD 00005205 no visto).

Brotes aéreos erectos hasta de 20-75 cm de altura. Lámina de las hojas basales oblonga, con margen dentado-apiculado, ápice obtuso, apiculado. Cefalodios con aproximadamente 10 grupos cimosos (2-8 flores). Brácteas del involucro con lóbulos triangulares con margen entero o dentado-apiculado, ápice mucronado. Ápice de los sépalos obtuso o emarginado, a menudo mucronado. Tubo estaminal de $1 / 2$ de la longitud de los filamentos. Filamentos de 1/3 la longitud de las anteras.

Distribución. Uruguay y Argentina. Crece en las dunas del Río de la Plata (costa uruguaya) y en dunas de la costa atlántica, desde Cabo Polonio (Depto. Rocha, Uruguay) hasta el Depto. Biedma (Pcia. de Chubut, Argentina).

Discusión. No existen diferencias morfológicas significativas en las estructuras reproductivas de ambas variedades. No existen caracteres diagnósticos cualitativos para diferenciarlas. Las diferencias morfológicas son cuantitativas y están polarizadas en los extremos de la distribución morfológica de la especie: el pedemonte andino y la costa atlántica. Probablemente, ambos taxones están conectados a lo largo de las cuencas de los ríos Colorado y Negro.

Calycera crassifolia (Miers) Hicken var. spinulosa (Gillies ex Miers) Zav.-Gallo, S. Denham \& Pozner. nov. comb. propuesta. Calycera spinulosa Gillies ex Miers, Ann. Mag. Nat. Hist. ser. 3, 6(36): 399. 1860 [Dec 1860]. TIPO: Argentina. Mendoza. On sandy soil, near El Totoral \& Los Arbolitos, 4-XI-1824, J. Gillies s.n. (holotipo K 000588765 no visto, foto!; isotipos BM 000947751 no visto, foto!, E 00392101 no visto). Fig. 18 (H-O). 
Calycera spinulosa Gillies ex Miers var. serratifolia Miers, Ann. Mag. Nat. Hist. ser. 3, 6(36): 399. 1860 [Dec 1860]. TIPO: Argentina. Mendoza. Near La Casa Pintada, sine data, J. Gillies s.n. (holotipo K 000677638 no visto, foto!).

Calycera foliosa Phil. ex Reiche, Anales Univ. Chile 106: 1046. 1900. TIPO: Argentina. Mendoza. Valle del Tunuyán, 1871, F. Leybold s.n. (holotipo SGO no visto; isotipo SI 12652!).

Calycera neuquenensis Suess., Repert. Spec. Nov. Regni Veg. 51: 197. 1942. TIPO: Argentina. Neuquén. Cobunco, $700 \mathrm{~m}$, fecha no indicada, E. Ammann 12 (holotipo M no visto).

Brotes aéreos erectos hasta de 15-30 cm de altura. Lámina de las hojas basales oval, con margen dentado-aristado, ápice obtuso, aristado. Cefalodios con ca. 12 grupos cimosos (hasta 10 flores). Brácteas del involucro con lóbulos triangulares a rectangulares, margen entero o dentado-mucronado, ápice aristado. Ápice de lod sépalos obtuso, mucronado. Tubo estaminal de igual longitud que los filamentos. Filamentos de 1/5 de la longitud de las anteras.

Distribución. Endémica de Argentina, creciendo en el pedemonte andino de Mendoza y Neuquén (Distrito de la Payunia) y extendiéndose hacia el oeste de La Pampa y norte de Río Negro, de 170 a 2500 m s.m. El sintipo de Calycera foliosa (Reiche, 1900, 1902), coleccionado en las "Cordilleras de Santiago" (y no hallado), es el único registro dudoso de Calycera crassifolia var. spinulosa para Chile.

Discusión. Calycera foliosa Phil. ex Reiche ha sido situada bajo la sinonimia de Calycera crassifolia (Miers) Hicken (Hicken, 1919). Sin embargo, los especímenes citados en el protologo fueron coleccionados en la Cordillera de Santiago y en Tunuyán, mientras que $C$. crassifolia var. crassifolia crece en las dunas de la costa atlántica de Argentina y Uruguay. Aunque el material tipo no posee flores ni frutos, las características distintivas y la descripción original de Reiche (1900) dejan claro que $C$. foliosa es co-específica con C. crassifolia var. spinulosa. 
Calycera eryngioides J. Rémy, Fl. Chil. [Gay] 3(3): 254, 1847 [1848]. Anomocarpus eryngioides (J. Rémy) Miers, Ann. Mag. Nat. Hist. ser. 3, 6(35): 353. 1860 [Nov 1860]. TIPO: Chile. Prov. de Santiago, fecha no indicada, C. Gay 1066 (holotipo P no visto; isotipos K 000588755 no visto, foto SI!, K 000588756 no visto, foto SI!, LIL 281133!, BM 000947744 no visto). Fig. 19.

Terófitos herbáceos caulescentes, hasta de $15 \mathrm{~cm}$ de altura. Hojas espatuladas, con la base atenuada en un pecíolo estrecho, lámina lanceolada, margen entero, ligeramente sinuado o crenado-mucronado, ápice obtuso, mucronado. Pedúnculos lanígeros, indiferenciados de los entrenudos superiores del tallo. Cefalodio con ca. 5 grupos cimosos (2-10 flores). Involucro con base fusionada, rotácea, lanígera, y lóbulos acrescentes triangulares con margen entero y ápice mucronado.Brácteas del involucro claramente diferenciadas de las hojas superiores distales. Receptáculo punctiforme, brácteas lineares y espatuladas. Flores pentámeras. Cáliz polimórfico: 5 sépalos iguales, de más de $1 \mathrm{~mm}$, suborbiculados, emarginados, margen crenado, no lignificados en la antesis (flor central terminal, flores solitarias, y flores de los grupos cimosos internos); 2-5 sépalos desiguales, de más de $2 \mathrm{~mm}$, estrechamente triangulares, no lignificados en la antesis (flores terminales de los grupos cimosos intermedios), y 2-5 sépalos desiguales, de más de $3 \mathrm{~mm}$, aciculares con sección triangular, lignificados en la antesis (flores terminales de los grupos cimosos periféricos). Perianto de $5-6 \mathrm{~mm}$ de longitud. Hipanto verde, tubuloso. Corola blanca, profundamente lobada; tube reducido; lóbulos oblongos, planos, ligeramente recurvados, ápice obtuso, recto. Tubo estaminal de igual longitud que los filamentos. Filamentos de $1 / 2$ de la longitud de las anteras. Anteras lanceoladas, tecas basalmente sagitadas, conectivo apiculado. Ovario lacrimiforme, 1.3 $1.35 \mathrm{~mm}$ de longitud. Cipselas prismáticas, con 3-5 carenas rugosas (frutos inermes) o planas (frutos espinosos), coronadas por sépalos suborbicular-plegados, triangulares o espiniformes, rodeando a un apículo cental, de acuerdo con la posición de la flor que les dé origen y el polimorfismo de sépalos descripto más arriba.

Distribución. Aunque esta especie ha sido registrada para Argentina (Hicken, 1919; Pontiroli, 1963) no hay evidencia física para sospechar que crezca fuera de Chile debido a que el ejemplar identificado por Hicken (Kurtz 5786) corresponde a Calycera 
herbacea var. herbacea. Se considera que Calycera eryngioides es endémica de la provincia Chilena Central, desde la IV Región de Coquimbo hasta la VI Región del Libertador Gral. Bernardo O’Higgins, creciendo sobre suelos argílicos (alfisoles y vertisoles).

Discusión. Calycera eryngioides puede ser confundida con C. calcitrapa por la similitud de las inflorescencias y el hábito erecto, caulescente (Pontiroli, 1963). Sin embargo, C. calcitrapa es un subarbusto caméfito endémico del noroeste de Argentina, completamente glabro, con hojas sésiles.

Calycera herbacea Cav., Icon. [Cavanilles] 4: 34. 1797. Calycera cavanillesi Rich., nomen superfluum, Mem. Mus. Hist. Nat. 6: 34. 1820. TIPO: Chile. Ex Cordillera del Portillo in Regno Chilensi, fecha no indicada, L. Née s.n. (holotipo MA 475481 no visto, fotos!; isotipo LINN-HS1361-1 no visto). Fig. 20 y Fig. 21.

Hemicriptófitos herbáceos rosulados o caulescentes, con tallos erectos o postrados, glabros, de más de $30 \mathrm{~cm}$ de altura, con raíces contráctiles. Hojas espatuladas, con la base atenuada en un pecíolo rectangular, lámina oblonga, margen sinuado, dentado o escarroso-laciniado, dientes mucronados. Pedúnculos escapiformes, largos, claramente diferenciados del tallo. Cefalodios con 10- $\infty$ grupos cimosos (3-7- $\infty$ flores). Involucro con o sin base fusionada pateliforme, brácteas involucrales claramente diferenciadas de las hojas superiores distales, rectangulares o triangulares, con margen entero o dentadomucronado, ápice obtuso, mucronado. Receptáculo punctiforme, brácteas lineares, espatuladas y rectangulares, dispuestas irregularmente. Flores pentámeras. Cáliz dimórfico: 2-5 sépalos desiguales, de más de $8 \mathrm{~mm}$, aciculares, con sección triangular, lignificados en la antesis (flor terminal central, flores terminales de los grupos cimosos); 5 sépalos iguales, triangulares, cortos, planos, de 0,3-0,5 mm, no lignificados en la anthesis (flores solitarias, flores de $2^{\mathrm{do}}$ y $3^{\text {er }}$ orden de los grupos cimosos). Perianto de 2,7 - 5,2 mm de longitud. Hipanto de 3-4 mm, verde, tubuloso. Corola verde o blanca, profundamente lobada, tubo reducido, de $1 \mathrm{~mm}$, lóbulos de 1,8- $2 \mathrm{~mm}$, oblongos, planos, ligeramente recurvados, ápice obtuso, uncinado. Tubo estaminal reducido, de ca. igual longitud que los filamentos $(0,2-0,3 \mathrm{~mm})$. Filamentos de 1/3-1/2 de la longitud de 
las anteras (0,3-0,4 mm). Anteras 1- 1,6 mm, oblongas, tecas basalmente obtusas, con o sin caudículas, conectivo no apiculado. Glándulas nectaríferas hipantiales. Ovario cilíndrico a lacrimiforme, 0.8 - $1.8 \mathrm{~mm}$ de longitud. Cipselas usualmente dimórficas, prismáticas, con 3-5 carenas, libres (surcos visibles) o connadas (surcos ocultos): frutos inermes con carenas usualmente rugosas y sépalos vestigiales o triangulares; frutos espinosos con carenas lisas y sépalos curvos espiniformes, de acuerdo con la posición de la flor que les dé origen y el polimorfismo de sépalos descripto más arriba.

Discusión. Calycera herbacea puede ser confundida con C. horrida debido a la forma de vida hemicriptófito y al hábito rosulado, el aspecto de las inflorescencias y la presencia de pedúnculos escapiformes. Sin embargo, C. horrida es una hierba con indumento lanoso; raíces gemíferas; hojas pinnatisectas; involucro lanífero, con una base amplia, fusionada, rotácea y lóbulos rectangulares, y flores con tubo corolino infundibuliforme.

Richard (1820) renombró el holotipo de C. herbacea como Calycera cavanillesi. Ambos nombres fueron usados indistintamente en la literatura hasta que Pontiroli (1963) sinonimizó Calycera cavanilliesi bajo Calycera herbacea.

Esta especie presenta dos variedades que se distinguen por caracteres reproductivos:

1. Flores con corola verde. Anteras sin caudículas. Brácteas involucrales libres, rectangulares, pequeñas, escondidas por las flores, con margen entero. Filamentos de 1/2 la longitud de las anteras. Cipselas espinosas con lóbulos calicinos recurvados, y 4-5 carenas connadas ocultando los surcos.

Calycera herbacea var. herbacea

1'. Flores con corola blanca. Anteras con caudículas. Brácteas involucrales fusionadas, rectangulares a triangulares, amplias, extendidas, con margen dentado-mucronado a entero. Filamentos de 1/3 - 1/2 la longitud de las anteras. Cipselas espinosas con lóbulos calicinos incurvados y 3-5 carenas libres (surcos visibles). 
Calycera herbacea Cav. var. herbacea. Fig. 20.

Gymnocaulus viridiflorus Phil., Linnaea 28: 706. 1856, syn. nov. propuesto, Calycera nudicaulis Phil. ex Miers, Ann. Mag. Nat. Hist. ser. 3, 6(36): 399. 1860 [Dec 1860]. Nom. nud. Calycera herbacea Cav. var. viridiflora (Phil.) Pontiroli, Revista Mus. La Plata, Secc. Bot. 9(41): 191. 1963. Calycera viridiflora (Phil.) Miers, Ann. Mag. Nat. Hist. ser. 3, 6(36): 399. 1860 [Dec 1860]. TIPO: Chile. Cordillera de Maule, 1856, P. Germain s.n. (holotipo SGO 057270 no visto; isotipos K 000588766 no visto, foto!, SI 090136!, SI 090137!).

Calycera squarrosa Miers, Ann. Mag. Nat. Hist. ser. 3, 6(36): 398. 1860 [Dec 1860], syn. nov. propuesto,. Calycera viridiflora (Phil.) Miers f. squarrosa (Miers) Hicken, Reun. Nac. Soc. Argent. Cien. Nat. 1: 247. 1919. TIPO: Argentina. On the Andes of Mendoza, fecha no indicada, J. Gillies s.n. (holotipo K 000588764 no visto, foto!; isotipo BM 000947747 no visto, foto!).

Hierbas rosuladas, hasta de $30 \mathrm{~cm}$ de altura. Brácteas involucrales pequeñas, libres, rectángulares, con ápice obtuso-mucronado, margen entero; acrescentes, lanceoladas, fusionadas por la base durante la madurez de los frutos. Brácteas del receptáculo lineares, espatuladas y rectangulares. Sépalos suborbiculares, emarginados, margen entero. Corola verde. Anteras sin caudículas. Cipselas con 4-5 carenas connadas ocultando los surcos, frutos espinosos con sépalos espiniformes recurvados.

Distribución. Endémica de los Andes del sur. Crece entre 2200-3000 m de altitud en la Región Metropolitana (Chile) y el centro y sur de los Andes de Mendoza (Argentina). Existen registros aislados a menores altitudes en las Sierras del Nevado $(1650 \mathrm{~m})$ al sur de la provincia de Mendoza.

Discusión. Las estructuras reproductivas de C. herbacea var. viridiflora y $C$. herbacea var. herbacea son idénticas, y los caracteres diagnósticos descriptos para var. viridiflora (tallos parcialmente ramificados, hojas ovado-oblongas, irregularmente pinnatifidas) son extremadamente variables dentro de la especie. Por lo tanto, $C$. herbacea var. viridiflora cae en la sinonimia de la variedad tipo. 
Calycera squarrosa fue considerada una variedad de $C$. viridiflora a partir de la revisión de Hicken (1919) y posteriormente fue sinonimizada bajo C. herbacea var. virdiflora (Chiapella, 1999; Zanotti \& Pozner, 2008). Considerando la sinonimia de $C$. herbacea var. viridiflora en este trabajo, y sobre la base del estudio delmaterial tipo, $C$. squarrosa queda sinonimizada bajo $C$. herbacea var. herbacea.

Calycera herbacea Cav. var. sinuata (Miers) Pontiroli, Revista Mus. La Plata, Secc. Bot. 9 (4): 191, 1963. Emend. Calycera sinuata Miers, Ann. Mag. Nat. Hist. ser. 3, 6(36): 398. 1860 [Dec 1860]. Calycera viridiflora (Phil.) Miers f. sinuata (Miers) Hicken, Reun. Nac. Soc. Argent. Cien. Nat. 1: 247. 1919. TIPO: Argentina. Mendoza. "Puente del Inca, altit. 8000 pedes", 1825, J. Miers s.n. (holotipo BM 000947748 no visto, foto!). Fig. 21.

Calycera intermedia Phil., syn. nov. propuesto, Anales Univ. Chile 36: 173. 1870. TIPO: Argentina. Mendoza, fecha no indicada, R. A. Philippi s.n. (holotipo K 000588763 no visto, foto SGO!).

Calycera involucrata Phil., Anales Univ. Chile 36: 174. 1870. TIPO: Argentina. Mendoza. Dpto. Tunuyán: El Guindo (valle del arroyo Manzano), 10-III-1945, G. Covas 6980 (neotipo aquí propuesto, SI!).

Calycera crenata R. E. Fr., syn. nov. propuesto, Nova Acta Regiae Soc. Sci. Upsal. ser. 4, 1(1): 98. 1905. Calycera pulvinata J. Rémy f. crenata (R. E. Fr.) Hicken, Reun. Nac. Soc. Argent. Cien. Nat. 1: 253. 1919. TIPO: Argentina. Jujuy: La Rinconada, 3800 m, 6-8-I-1901, F. Kurtz 11365 (lectotipo aquí propuesto, S 08-715 no visto, foto!; duplicados SI 353!, BAF no visto, foto!).

Calycera pulvinata J. Rémy f. cauligera Hicken, syn. nov. propuesto, Reun. Nac. Soc. Argent. Cien. Nat. 1: 253. 1919. TIPO: Argentina. Tucumán. Lara, 3200 m, 30-I-1912, D. Rodriguez 295 (lectotipo aquí propuesto, SI 358!; duplicados SI 104739!, LIL 89439!). 
Hierbas rosuladas o caulescentes, hasta de $20 \mathrm{~cm}$ tall. Involucro con base fusionada pateliforme y lóbulos triangulares o rectangulares, basalmente amplios, con margen entero o dentado-mucronado, y ápice obtuso, mucronado. Brácteas del receptáculo tanto lineares como espatuladas. Perianto de 2,7 - 3,7 mm. Sépalos suborbiculares, margen sinuado, ápice obtuso o agudo. Hipanto verde, tubuloso. Corola blanca. Anteras con caudículas. Cipselas con 3-5 carenas libres, con surcos visibles, frutos espinosos con sépalos espiniformes incurvados.

Distribución. Crece en el norte de Chile (Región de Antofagasta) y noroeste de Argentina, en una ámplia área de los cordones montañosos andinos y preandinos (Famatina, Aconquija, Calchaquíes), desde Salta y Jujuy hasta el noroeste de Mendoza, a lo largo de las provincias fitogeográficas Altoandina, Puneña y Prepuneña (Cabrera \& Willink, 1980), de 2500-4800 m de altitud.

Discusión. Calycera herbacea var. sinuata puede ser confundida con $C$. pulvinata por los brotes aéreos con hábito rosulado y el parecido de las cipselas. Sin embargo, C. pulvinata posee rizomas erectos con catáfilos, involucro indiferenciado, corola parcialmente lobada con tubo campanulado y lóbulos cuculados inflados, y anteras lanceoladas sin caudículas.

Previamente a la revisión nomenclatural de Hicken (1919), Hauman-Merck (1918) ya había sugerido la sinonimia de $C$. involucrata bajo $C$. sinuata, y discutido la posibilidad de reducir $C$. sinuata, $C$. intermedia y $C$. viridiflora a la sinonimia de $C$. herbacea. Aunque los caracteres diagnósticos de C. herbacea var. sinuata no son confiables ("se distingue de la variedad herbacea por las ramificaciones que puede presentar el tallo y por las hojas sinuoso-dentadas", Pontiroli, 1963: 191), el análisis de las estructuras reproductivas (particularmente el involucro, la flor y el fruto) revela nuevos caracteres que soportan la validez del taxón. Por lo tanto, se establecen como nuevos caracteres diagnósticos de $C$. herbacea var. sinuata [Art. 47, Código Internacional de Nomenclatura (McNeill et al., 2012)] el limbo corolino blanco (vs. verde en var. herbacea), brácteas involucrales amplias, extendidas, triangulares a rectangulares, con margen dentado-mucronado a entero (vs. lineares, pequeñas y ocultas en var. herbacea), anteras con caudículas (sin caudículas en var. herbacea), lóbulos calicinos incurvados en cipselas espinosas (recurvadas en var. herbacea) y surcos 
visibles entre las carenas de las cipselas (vs. carenas fusionadas ocultando los surcos en var. herbacea).

Típicamente, las hojas de Calycera herbacea poseen un borde definido, estrecho y semi-translúscido alrededor del margen. Dicho borde foliar desarrolla tricomas glandulares abundantes y notables en individuos del noroeste argentino ( $C$. herbacea var. sinuata), volviéndose gradualmente escasos e imperceptibles en individuos del sur del área de distribución (C. herbacea var. herbacea).

La morfología de la flor y el fruto de Calycera intermedia Phil. no difiere del holotipo y la ilustración de Calycera herbacea var. sinuata. De acuerdo al protologo, las flores de $C$. intermedia poseen corola blanca. Los demás caracteres diagnósticos de $C$. intermedia pueden encontrarse en $C$. herbacea var. sinuata. Además, ambos taxones comparten su distribución geográfica.

Calycera crenata R. E. Fr. ha sido considerada como un sinónimo de $C$. pulvinata J. Rémy. Sin embargo, todos sus caracteres florales y del fruto coinciden con los de C. herbacea var. sinuata, y sus caracteres vegetativos concuerdan con la variabilidad morfológica de la var. sinuata. Por lo tanto, se considera $C$. crenata como sinónimo de $C$. herbacea var. sinuata.

Hicken (1919) estableció Calycera pulvinata f. cauligera basado en el hábito y la morfología de la cipsela. Sin embargo, esta forma difiere claramente de la forma típica por la corola partida con tubo reducido (vs. parcialmente lobada con tubo campanulado en C. pulvinata f. pulvinata), lóbulos corolinos planos (vs. cuculados inflados en $C$. pulvinata f. pulvinata), involucro pateliforme (vs. Involucro indeferenciado en $C$. pulvinata f. pulvinata), anteras oblongas con caudículas y sin apículo terminal (vs. anteras lanceoladas con apículo terminal y sin caudículas en $C$. pulvinata f. pulvinata) y tallos amplios acortados sin catafilos (vs. rizoma vertical con catafilos en C. pulvinata f. pulvinata). Por otro lado, la morfología de la flor y el fruto de $C$. pulvinata f. cauligera reúne las características de $C$. herbacea var. sinuata así como todos los caracteres diagnósticos de $C$. pulvinata f. cauligera. Ambos taxones sólo difieren por los lóbulos calicinos espinosos (más cortos en cipselas de C. pulvinata f. cauligera) y la posción de los tallos y los pedúnculos escapiformes (decumbentes en C. pulvinata f. cauligera); estos caracteres son variables dentro de la var. sinuata.

Debido a que no pudo hallarse el ejemplar tipo de Philippi en ninguno de los herbarios donde se halla depositada su colección (B, BAF, G, LE, P, SGO, W) (Stafleu 
\& Cowan, 1988), ni en CONC, se propone al ejemplar G. Covas 6980 (SI) como neotipo de Calycera involucrata ya que presenta todos los caracteres diagnósticos del taxón que figuran en el protologo. Además, el ejemplar de Covas fue coleccionado cerca de la terra typica.

Existen dos sintipos de C. crenata; uno de ellos, Fries 861 (S 05-2021), está compuesto por cuatro plantas immaduras, las cuales no muestran los caracteres diagnósticos florales ni los del fruto. El segundo ejemplar, Kurtz 11365 (S 08-715), es seleccionado como lectotipo debido a que las estructuras reproductivas están bien preservadas y se ajustan en mayor medida a la descripción original del taxón.

Entre todos los sintipos de Calycera pulvinata f. cauligera [Lillo 3432 (LIL 89436, SI 107634), Lillo 4894 (LIL 89437, SI 107636), Jörgensen 1178 (LIL 89295, SI 107635), Holmberg 972 (SI 107637), Dinelli 512 (SI 108045), Gerling 91 (SI 108046, SI 108047), Rodriguez 295 (LIL 89439, SI 358, SI 104739)], se designa como lectotipo al ejemplar Rodriguez 295. Este ejemplar posee tres pliegos: uno depositado en LIL (LIL 89439) y dos depositados en SI (SI 104739, SI 358). Se selecciona el pliego SI 358 como lectotipo original debido a su mejor estado de preservación, su gran cantidad de flores y frutos, y por llevar la etiqueta del coleccionista y dos etiquetas manuscritas de Hicken, incluyendo notas y dibujos.

Nota. Existen tres ejemplares con caracteres morfológicos intermedios entre $C$. herbacea var. herbacea y C. herbacea var. sinuata: Wingenroth et al. 381(con flores verdes, tecas sin caudículas, brácteas involucrales fusionadas y rectangulares), Covas 6890 (con flores blancas, tecas sin caudículas, brácteas involucrales fusionadas con margen dentado-mucronado), y Kiesling 8569 (con flores verdes, tecas con caudículas, brácteas involucrales fusiondas y rectangulares).

Calycera horrida Hicken, Physis (Buenos Aires) 1: 129. 1912. TIPO: Argentina. Neuquén: Arroyo Huinganco, Cordillera del Viento, 1900 m, 21-III-1912, F. Pastore 76 (holotipo SI 355!; isotipo SI 28580!). Fig. 22.

Geófitos herbáceos, laníferos, hasta de 10-20 cm de altura, con raíces gemíferas. Hojas pinnatisectas, con la base atenuada en un pecíolo rectangular, lanífero; raquis lanífero; 
lámina oblonga, folíolos partidos, lobos obtusos, mucronados. Pedúnculos escapiformes, largos, claramente distinguibles del tallo acortado. Brácteas involucrales claramente diferenciadas de las hojas superiores distales, laníferas, con una base fusionada amplia, rotácea y lobos rectangulares amplios, con margen entero o crenadomucronado, ápice obtuso, mucronado. Receptáculo punctiforme, con brácteas espatuladas, laníferas. Cefalodios con aproximadamente 10 grupos cimosos (hasta 4-6 flores). Flores pentámeras. Perianto con 6 - $6.6 \mathrm{~mm}$ de longitud. Cáliz polimófico: 1-2 sépalos desiguales, de más de $12 \mathrm{~mm}$, aciculares, con sección circular, lignificados en la antesis (flor terminal de los grupos cimosos periféricos); 5 sépalos desiguales, lanceolados, planos, de más de 4 mm, no lignificados en la antesis (flor central terminal, flores solitarias, y flor terminal de los grupos cimosos internos); 5 sépalos iguales, diminutos, orbiculares, no lignificados en la antesis (flores de $2^{\text {do }}$ y $3^{\text {er }}$ orden en todos losgrupos cimosos). Hipanto de $3 \mathrm{~mm}$, verde, tubuloso. Corola cortamente lobada, tubo de $4 \mathrm{~mm}$, infundibuliforme, lóbulos de $1 \mathrm{~mm}$, rectangulares, recurvados, planos, ápice obtuso, estrecho. Tubo estaminal de de 1/2 la longitud del tubo corolino (ca. $2 \mathrm{~mm}$ ). Filamentos de 1/6 de la longitud de las anteras (ca. $0.3 \mathrm{~mm}$ ), incurvados. Anteras de 2 $\mathrm{mm}$, lanceoladas, tecas basalmente sagitadas con caudículas, y conectivo apiculado. Glándulas nectaríferas en el tubo estaminal. Ovario cilíndrico a lacrimiforme, 1.3 - 2 $\mathrm{mm}$ de longitud. Cipselas polimórficas, prismáticas, con 3-5 carenas rugosas (frutos inermes) o lisas (frutos espinosos), coronadas por sépalos vestigiales, suborbiculares, lanceolados o espiniforme-recurvados de acuerdo com la posición de la flor y el dimorfismo de sépalos descriptos más arriba.

Distribución. Endémica del norte de Neuquén (Argentina). Crece en la Cordillera del Viento y el Parque Provincial Tromen, entre 2000-2500 m.

Discusión. Calycera horrida puede ser confundida con $C$. herbacea debido a su forma de vida hemicriptófito y su hábito rosulado, sus pedúnculos escapiformes y al aspecto general de su inflorescencia. Sin embargo, $C$. herbacea es totalmente glabra, sin raíces gemíferas, y posee hojas con margen entero a escarroso-partido.

En el protologo, Hicken citó un ejemplar de Franco Pastore como único material de referencia, sin detallar el número de coleccionista. Dicho ejemplar fue montado en dos pliegos. Uno de ellos (SI 355) posee las etiquetas originales de Pastore y Hicken, 
donde puede leerse "76", "F. Pastore 76" y "No 98”. El otro pliego (SI 28580) sólo lleva una etiqueta con la referencia de la publicación original, y el nombre de la especie y el número "98" manuscritos por Hicken. SI 28580 ha sido considerado un paratipo, asumiendo que "98” es el número de coleccionista. Sin embargo, Hicken (1912) no cita ningún ejemplar adicional. Esto se debe a que " 98 " corresponde en efecto al número de registro de C. horrida en el listado de especies de Hicken. Por lo tanto, SI 28580 es un duplicado de SI 355. Se considera a SI 355 el holotipo de $C$. horrida debido a que este pliego lleva la etiqueta del coleccionista, la etiqueta de Hicken, y notas publicadas en el protologo, así como una ilustración floral hecha por el autor.

Calycera leucanthema (Poepp. ex Less.) Kuntze, Revis. Gen. Pl. 3[3]: 127, 1898 [28 Sep 1898]. Boopis leucanthema Poepp. ex Less., Linnaea 6: 259. 1831. Anomocarpus leucanthemus (Poepp. ex Less.) Miers, Ann. Mag. Nat. Hist. ser. 3, 6(35): 355. 1860. [Nov 1860]. TIPO: Chile. Cordillera de Antuco, fecha no indicada, E. F. Poeppig 774 (holotipo M no visto, foto 20567 Field Museum of Natural History en SI!; isotipos HAL 113261, no visto, P 00852244 no visto, P 00852245 no visto, P 00852246 no visto). Fig. 23.

Leucocera annua Turcz., Bull. Soc. Imp. Naturalistes Moscou 21 (1): 583. 1848. TIPO: Chile. Sandy places on the Andes. "Prope Colchagua reipubl. Chilensis", fecha no indicada, T. Bridges 1186 (holotipo K 000588758 no visto, foto!).

Anomocarpus tenuis Miers, Ann. Mag. Nat. Hist. ser. 3, 6(35): 356. 1860. [Nov 1860]. TIPO: Chile. Province de Concepcion, fecha no indicada, C. Gay 1495 (holotipo P no visto; isotipo LP 907866!).

Anomocarpus tenuifolius Miers, Ann. Mag. Nat. Hist. ser. 3, 6(35): 356. 1860. [Nov 1860]. TIPO: Chile. Cordillera de Chillán, 1856-1857, P. Germain s.n. (holotipo K 000588759 no visto, foto!).

Boopis integrifolia Phil., Anales Univ. Chile 85: 814. 1894, syn. nov.propuesto. Calycera integrifolia (Phil.) Reiche, Anales Univ. Chile 106: 1044. 1900. TIPO: Chile. 
Maule: "Thermae Longavi”, I-1888, O. Schönemann s.n. (holotipo SGO 057269 no visto, foto!; isotipo SI 12653!).

Terófitos caulescentes, laníferos, hasta de 10-15 cm de altura. Hojas laníferas, con la base atenuada en un peciolo; lámina lanceolada, pinnatisecta (raramente entera), lóbos lineares, con margen entero, ápice obtuso. Pedúnculos laníferos, indistinguibles de los entrenudos superiores del tallo. Brácteas involucrales casi libres, claramente diferenciadas de las hojas superiores distales, fusionadas en la base, notoriamente reducidas; brácteas lanceoladas a linear-lanceoladas, ápice mucronado. Receptáculo punctiforme, con brácteas espatuladas. Cefalodio con 8-10 grupos cimosos (hasta 4 flores). Flores tetrámeras. Cáliz polimórfico: 3-4 sépalos desiguales, de más de 1,5 mm, aciculares, con sección triangular, no lignificados en la antesis (flor terminal central y flor terminal de cada grupo cimoso); 4 sépalos desiguales, muy reducidos $(0.5 \mathrm{~mm})$ a triangulares, planos, no lignificados en la antesis (flores solitarias y flores de $2^{\text {do }}$ y $3^{\text {er }}$ orden en todos los grupos cimosos). Perianto de $2-2.5 \mathrm{~mm}$ de longitud. Hipanto de 0,75 mm, green, tubulose. Corola blanca, profundamente lobada, tubo reducido, de 0,5 $\mathrm{mm}$, lóbulos 1,25 mm, oblongos, planos, ligeramente recurvados, ápice obtuso, no uncinado. Tubo estaminal de 3-4 veces la longitud de los filamentos (ca. 0,4 mm). Filamentos de 1/5 de la longitud de las anteras (ca. 0,1 mm). Anteras 0,7 mm, oblongas, tecas basalmente sagitadas, conective no apiculado. Glándulas nectaríferas estaminales. Ovario cilíndrico a lacrimiforme, $0.6-0.8 \mathrm{~mm}$ de longitud. Cipselas polimórficas, prismáticas, con el apículo terminal pubescente, a menudo estrechos en la base, con 4 carenas lisas, coronadas por sépalos compresos, reducidos a triangular-pungentes, de acuerdo con la posición de la flor que les dé origen y el polimorfismo de sépalos descriptos más arriba.

Distribución. Endémica de Chile central. Habita en la VII Región del Maule y el norte de la VIII Región del Bío Bío, entre 200-900 m de altitud.

Discusión. Calycera leucanthema es la única especie de Calycera con flores tetrámeras. Podría ser confundida con $C$. sessiliflora debido a la forma de vida terófita, el hábito herbáceo caulescente, y la morfología de la cipsela. Sin embargo, $C$. 
sessiliflora es completamente glabra, con hojas lanceoladas, flores pentámeras, e involucro campanulado ligeramanete acrescente.

Observaciones. La sinonimia de Anomocarpus tenuis, A. tenuifolius y Leucocera апnиa bajo Calycera leucanthema ya había sido propuesta por Reiche $(1900,1902)$ y Zanotti \& Pozner (2008).

El nombre Calycera integrifolia (Phil.) Reiche debe ser tratado como sinónimo de C. leucanthema ya que sólo se diferencia de ésta por las láminas foliares enteras, coincidiendo en todos los caracteres morfológicos restantes. El holotipo es el único ejemplar conocido de $C$. integrifolia, y fue coleccionado dentro del área de distribución de $C$. leucanthema, hecho que respalda la sinonimia propuesta.

Calycera pulvinata J. Rémy, Ann. Sci. Nat., Bot. sér. 3, 6: 352, 1846. Anomocarpus pulvinatus (J. Rémy) Miers, Ann. Mag. Nat. Hist. ser. 3, 6(35): 354. 1860 [Nov 1860]. TIPO: Bolivia. Carangas, Grand plateau des Andes, fecha no indicada, A. D'Orbigny s.n. (holotipo P no visto; isotipos MO no visto, BM 000947749 no visto, foto!). Fig. 24.

Hemicriptófitos (¿?) herbáceos glabros, rosulados, ca. 3-4 cm de altura, con rizomas verticales y raíces adventicias. Hojas espatuladas, con la base atenuada en un peciolo rectangular; lámina oblonga, margen crenado-mucronado; ápice obtuso. Pedúnculos escapiforme muy corto. Cefalodio central rodeado por varios cefalodios más pequeños aglomerados en el centro de la planta. Cefalodio con aproximadamente 6 grupos cimosos (hasta 8 flores, aproximadamente). Involucro indistinguible. Brácteas florales amplias, lanceoladas, ápice entero o 3-dentado, libres, aunque claramente diferenciables de las hojas normales, las periféricas no dispuestas en un involucro distinguible. Flores pentámeras. Cáliz dimórfico: 5 sépalos iguales, de $1 \mathrm{~mm}$, aciculares, con sección triangular, parcialmente lignificados (flor central terminal y flor terminal de los grupos cimosos periféricos); 5 sépalos iguales, reducidos, con sección triangular, planos (flores solitarias y flor terminal de los grupos cimosos internos). Perianto de $2.8-3 \mathrm{~mm}$ de longitud. Hipanto de 1,5 mm, verde, tubuloso. Corola blanca, lobada hasta la mitad de su longitud, tubo de $1 \mathrm{~mm}$, campanulado, lóbulos de $0,75 \mathrm{~mm}$, lanceolados, cuculados, inflados, ápice obtuso, uncinado. Tubo estaminal de igual longitud que los filamentos 
(ca. 0,2 mm). Filamentos de 1/3 de la longitud de las anteras (ca. $0.3 \mathrm{~mm}$ ). Anteras 1 mm, lanceoladas, tecas basalmente obtusas. Glándulas nectaríferas hipantiales. Ovario cilíndrico, 1.3 -1.45 mm de longitud. Cipselas claramente dimórficas: frutos espinosos con carenas lisas, y frutos inermes redondeados, lisos de acuerdo con la posición de la flor que le dé origen y el dimorfismo de sépalos descripto más arriba.

Distribución. Bolivia y noroeste de Argentina, donde esta especie alcanza la cima de las cumbres Calchaquíes, por sobre los $4000 \mathrm{~m}$ de altitud. Existen registros de C. pulvinata para los Andes del sur de Perú (DeVore, 1994; X. Gallegos, com. pers.).

Discusión. Calycera pulvinata podría confundirse con C. herbacea var. sinuata por la forma de vida hemicriptófita, el hábito rosulado, las flores con corola blanca y el aspecto de las cipselas. Sin embargo, C. herbacea var. sinuata carece de rizomas verticales con catáfilos escuamiformes y posee raíces gruesas contráctiles, involucro diferenciado, corola profundmente lobada con tubo reducido, lóbulos corolinos planos, ligeramente recurvados, y anteras oblongas con caudículas y conectivo no apiculado.

Calycera sessiliflora Phil., Linnaea 28: 706. 1856. Anomocarpus subsessiliflorus Miers, nomen superfluum, Ann. Mag. Nat. Hist. ser. 3, 6(35): 352. 1860. [Nov 1860]. TIPO: Chile. Prope Santiago, fecha no indicada, R. A. Philippi 737 (lectotipo aquí propuesto K 000588762 no visto, foto!; isotipos BAF no visto, foto!, CONC 29656!, G no visto, GOET 000508 no visto, foto!, P no visto, S 05-2022 no visto, foto!, SGO 057255 no visto). Fig. 25.

Anomocarpus axillaris Miers, Ann. Mag. Nat. Hist. ser. 3, 6(35): 352. 1860. [Nov 1860]. syn. nov.propuesto. Calycera sessiliflora Phil. var. axillaris (Miers) Reiche, Anales Univ. Chile 106: 1045. 1900. TIPO: Chile. Valparaíso, fecha no indicada, H. Cuming 664 (holotipo K 000588751 no visto, foto!).

Terófitos caulescentes, glabros, hasta de $15 \mathrm{~cm}$ de altura. Hojas espatuladas, con la base atenuada en un corto pecíolo, lámina lanceolada, margen entero o dentado-mucronado, ápice agudo, mucronado. Pedúnculos muy cortos, nutantes. Brácteas involucrales 
claramente diferenciables de las hojas distales superiores con la base fusionada, campanulada, ligeramente accrescente, y lóbulos triangulares con el ápice mucronado. Receptáculo punctiforme, brácteas ausentes. Cefalodios paucifloros con 5-15 flores. Grupos cimosos ausentes. Flores pentámeras. Cáliz dimórfico: 3-5 sépalos desiguales, de más de $2 \mathrm{~mm}$, aciculares, con sección triangular distalmente y plana basalmente, no lignificada en la antesis (flor central terminal); 5 sépalos iguales, cortos triangulares, planos, 0,5 mm, no lignificados en la antesis (flores solitarias). Perianto de 1,9-2,2 mm de longitud. Hipanto $0,5 \mathrm{~mm}$, verde, tubuloso. Corola blanca, lobada hasta la mitad de su longitud, tubo $0,5 \mathrm{~mm}$, ligeramente urceolada, lóbulos de $1,5 \mathrm{~mm}$, lanceolados, planos, ligeramente recurvados, ápice obtuso, uncinado. Tubo estaminal reducido, de 1/3 la longitud de los filamentos (ca. 0,1 mm). Filamentos de aproximadamente igual longitud que las anteras (ca. 0,3-0,4 mm). Anteras 0,6 mm, oblongas, tecas basalmente obtusas, conectivo apiculado. Glándulas nectaríferas hipantiales. Ovario cilíndrico, dilatado en su parte media, 1,45 - 1,8 mm de longitud. Cipselas dimórficas, prismáticas, con 5 carenas rugosas y sépalos vestigiales, triangulares (frutos inermes), o con 3-5 carenas lisas y sépalos espiniformes recurvados (frutos espinosos) de acuerdo con la posición de la flor que les dé origen y el dimorfismo de sépalos descripto más arriba.

Distribución. Endémica de Chile central. Crece desde la IV Región de Coquimbo hasta la Región Metropolitana, entre 700-1900 m de altitud.

Discusión. Calycera sessiliflora puede ser confundida con Boopis pusilla, por la forma de vida terófita, el hábito herbáceo caulescente, las láminas foliares lanceoladas y los cefalodios paucifloros. Sin embargo, B. pusilla se distingue por poseer hojas con pecíolos laxamente pubescentes, pedúnculos no nutantes (a veces laxamente pubescentes), involucro rotáceo a tubular, hipanto infundibuliforme, tubo corolino infundibuliforme, y cipselas turbinadas a cilíndricas, rugosas con 5 carenas vestigiales coronadas por sépalos vestigiales a orbicular-foliosos que rodean a un apículo central.

La variedad axillaris no difiere de la variedad sessiliflora. No pudieron hallarse caracteres diagnósticos en las descripciones originales de Miers (1860-1869) y en las descripciones posteriores de Reiche (1900, 1902), así como tampoco entre los holotipos.

Se considera conveniente designar lectotipo al sintipo de R. A. Philippi (K) debido a su gran cantidad de material, sus numerosas flores y frutos y su mejor estado 
de conservación. El otro sintipo, coleccionado por P. Germain (Germain s.n., K, SGO), posee pocos frutos y se encuentra mal conservado.

Calycera sympaganthera (Ruiz \& Pav.) Kuntze, Revis. Gen. Pl. 3[3]: 127. 1898 [28 Sep 1898]. Scabiosa sympaganthera Ruiz \& Pav., Fl. Peruv. [Ruiz \& Pavón] 1: 49. 1798. Calycera sympaganthera (Ruiz \& Pav.) Martic. \& Quezada, comb. superfl., Gayana Bot., 44(1-4): 40.1987 [1988]. Calycera balsamitaefolia (Juss.) Rich., Mem. Mus. Hist. Nat. 6: 38. 1820. Boopis balsamitaefolia Juss., nomen superfluum, Ann. Mus. Par. 2: 350. 1803. TIPO: Chile. "Chile Flora. Decembri in arenosis. Syngenesia", 1786, H. Ruiz \& J. Pavón s. n. (lectotipo aquí propuesto, MA no visto, foto!; duplicados MA no visto, foto 29275 del Field Museum of Natural History en SI!, P no visto). Fig. 26.

Caméfitos herbáceos, vilíferos, hasta de $15-20 \mathrm{~cm}$ de altura, con tallos erectos y prostrados. Hojas espatuladas, atenuadas en un pecíolo rectangular pubescente, lámina lanceolada, margen vilífero, partido, lóbos triangulares, mucronados; ápice obtuso. Pedúnculos indistinguibles de los entrenudos superiores del tallo. Brácteas involucrales claramente diferenciadas de las hojas superiores distales, con una base fusionada pateliforme, reducida, y lóbulos lanceolados, amplios basalmente, con margen entero y ápice mucronado. Receptáculo punctiforme, con brácteas lineares, espatuladas y rectangulares. Cefalodio con aproximadamente 10 grupos cimosos (2-4 flores). Flores pentámeras. Cáliz dimórfico: 4-5 sépalos desiguales, de más de $6 \mathrm{~mm}$, largamente triangulares, naviculados, no lignificados en la antesis (flor terminal central, flor terminal de los grupos cimosos periféricos); 5 sépalos iguales, de 1-3 $\mathrm{mm}$, cortamente triangulares (flores solitarias, flor terminal de los grupos cimosos internos, y flores de $2^{\text {do }}$ orden en los grupos cimosos). Perianto de 4,4-5 mm de longitud. Hipanto de 2-2,5 $\mathrm{mm}$, verde, tubuloso. Corola blanca, lobada hasta la mitad de su longitud, tubo amplio de 1,5 mm, lóbulos de 2,5 mm, oblongos, ligeramente recurvados, planos, ápice obtuso, uncinado. Tubo estaminal de igual longitud que los filamentos. Filamentos de 0,3-0,4 mm. Anteras 1,2 mm, oblongas, tecas basalmente sagitadas, con caudículas. Glándulas nectaríferas hipantiale. Ovario cilíndrico a lacrimiforme, $2.3-2.4 \mathrm{~mm}$ de longitud. Cipselas dimórficas, prismáticas, con 5 carenas rugosas y sépalos vestigiales, 
cortamente triangulares (frutos inermes), o 3-5 carenas lisas y sépalos espiniformes recurvados (frutos espinosos), de acuerdo con la posición de la flor que les dé origen y el dimorfismo de sépalos descripto más arriba, usualmente sin formas intermedias.

Distribución. Endémica de Chile. Crece en la "Cordillera de Nahuelbuta" (Pcia. de Malleco, IX Región).

Discusión. A pesar de tener una ilustración con referencias de Ruiz y Pavón, el ejemplar fotografiado por el Field Museum muestra sólo dos inflorescencias inmaduras. El ejemplar aquí designado como lectotipo posee la etiqueta original manuscrita por Ruiz y Pavón, frutos, dos inflorescencias inmaduras y dos inflorescencias maduras.

El nombre Calycera coronata no está efectivamente publicado de acuerdo con el artículo 30.8 (McNeill et al., 2012), aunque De vore (1994) realizó una descripción y una ilustración de este taxón en su tesis. Esta especie fue designada Calycera balsamitaefolia desde que Richard (1820) recategorizó Boopis balsamitaefolia Juss. Sin embargo, Jussieu no consideró el epíteto específico original de la descripción original (Ruiz \& Pavón, 1798). Calycera coronata es una variación local de C. sympaganthera.

GAMOCARPHA DC., Prodr. [A. P. de Candolle] 5: 2. 1836 [Oct 1836].

Especie tipo: Gamocarpha poeppigii DC. [= Gamocarpha alpina (Poepp. ex Less.) H. V. Hansen].

Caméfitos o hemicriptófitos con hábito caulescente o rosulado; glabros. Tallos postrados, procumbentes, ascendentes o estoloníferos, a veces glutinosos, a veces radicantes. Raíces axonomorfas, a veces contráctiles. Hojas imbricadas, gruesas, algo coriáceas, enteras, espatuladas (atenuadas en un pecíolo rectangular); lámina oblonga, linear u oval, dentada, lobada, partida o pinnatifida, con margen entero y ápice agudo u obtuso, no mucronado. En algunas especies, las bases foliares persisten y cubren las partes más viejas de los tallos. Pedúnculos teretes, foliosos o escapiformes, a veces muy cortos. Cefalodios con involucro diferenciado y receptáculo punctiforme. Dos grandes tipos: cefalodios con grupos cimosos periféricos (internos al involucro) con brácteas tectrices fusionadas formando celdas (una celda por bráctea y un grupo cimoso por 
celda), o cefalodios sin grupos cimosos periféricos (internos) pero con grupos cimosos externos rodeados de sendos involucelos profilares. Flores pentámeras. Sépalos lanceolados, aciculares o dentiformes, con margen entero y ápice obtuso o agudo, no mucronado. Hipanto tubuloso o infundibuliforme. Corola tubular hasta rotácea, lobada; lóbulos oblongos o lanceolados, planos, con ápice agudo, recto o uncinado. Glándulas nectaríferas estaminales o hipantiales. Tubo estaminal presente, filamentos estaminales presentes o ausentes. Anteras oblongas, con base obtusa; conectivo no apiculado. Cipselas prismáticas o cilíndricas con 5 carenas coronadas por los sépalos acrescentes ovados a triangulares, no espinescentes, que rodean a un apículo central.

Gamocarpha alpina (Poepp. ex Less.) H. V. Hansen, Nordic J. Bot. 12(1): 65. 1992. Boopis alpina Poepp. ex Less., Linnaea 6: 258. 1831. Gamocarpha poeppigii DC., nom. superfl., Prodr. [A. P. de Candolle] 5: 2. 1836 [Oct 1836]. TIPO: Chile, E. F. Poeppig 823 (holotipo M no visto, foto 20566 Field Museum of Natural History en SI!). Fig. 27.

Gamocarpha ligulata Miers, Ann. Mag. Nat. Hist. ser. 3, 6(34): 282. 1860 [Oct 1860]. TIPO: Chile. Prov. Arauco, W. Lechler 2891 (holotipo K 000009726 no visto, foto!).

Gamocarpha polycephala Phil., Anales Univ. Chile 27 (2): 329. 1865, syn. nov. propuesto. TIPO: Chile. "Prope Chillan invenit orn. Manuel Antonio de Solis", sin fecha indicada, Solis s.n. (holotipo SGO 43617 no visto).

Hemicriptófitos herbáceos estoloníferos y rizomatosos con brotes aéreos rosulados o caulescentes, cespitosos. Raíces contráctiles. Hojas coriáceas, enteras, espatuladas; lámina oblonga, abierta o plegada, atenuada en el pecíolo, con margen entero y ápice obtuso, no mucronado. Pedúnculos teretes, escapiformes. Involucro con base fusionada pateliforme y lóbulos anchamente triangulares, margen entero y ápice mucronado. Receptáculo punctiforme. Cefalodio con aproximadamente 12 grupos cimosos, 2-6floros, perífericos (internos) con brácteas tectrices soldadas delimitando celdas. Perianto 6-6,5 mm. Sépalos dentiformes a lanceolados. Hipanto verde, infundibuliforme. Corola blanca lobada hasta $1 / 2$ de su parte superior, lóbulos lanceolados, planos, con ápice 
agudo, uncinado. Glándulas nectaríferas hipantiales. Tubo estaminal de 1/3 de la longitud del tubo corolino. Filamentos incurvos, de 1/6 del largo de las anteras. Anteras oblongas, tecas basalmente sagitadas sin caudículas, conectivo no apiculado. Ovario elipsoidal, de aproximadamente 2,5 $\mathrm{mm}$ de longitud. Cipselas prismáticas, lisas, con 5 carenas coronadas por sépalos lanceolados, que rodean a un apículo central.

Distribución. Endémica de los Andes de Chile (VIII y IX Regiones) y Argentina (Pcia. de Neuquén). Crece a partir de los 1500 m s.m. Ocupa el área correspondiente al Distrito Austral de la Provincia Altoandina.

Discusión. Gamocarpha alpina puede ser confundida con G. gilliesii por el aspecto general de la planta y de las cipselas. Pero G. gilliesii posee hojas con lámina suborbicular plana $\mathrm{u}$ oblongo-plegada, lóbulos del involucro triangulares a panduriformes recurvados y flores con perianto de 4-5 $\mathrm{mm}$ e hipanto tubuloso grueso.

Aunque no fue posible examinar el material tipo de Gamocarpha polycephala Phil., es muy probable que este taxón haya sido creado en base a un ejemplar de Gamocarpha alpina con pedúnculos escapiformes policéfalos, como sugieren la descripción original de Philippi (1865) y el ejemplar Werdermann 1345 (LIL, SI), coleccionado en la terra typica (Baños de Chillán) y determinada en primera instancia como Gamocarpha polycephala. Además, de acuerdo con el protologo, el material tipo sólo consta de "tallos sin su rizoma" y de acuerdo a la diagnosis, "se diferencia de la G. Poeppigii (= G. alpina) [...] por el pecíolo mucho más largo i angosto, i por el tallo policephalo i folioso, [...] por las escamas del involucro i las del receptáculo". Dado que el material examinado originalmente es escaso, que los caracteres diagnósticos caen dentro del rango de variabilidad de $G$. alpina, que el área de distribución de $G$. polycephala queda completamente superpuesta a la de $G$. alpina y que el material coleccionado es muy escaso, parece conveniente proponer la sinonimia de $G$. polycephala bajo G. alpina.

Gamocarpha angustifolia Phil., Anales Univ. Chile 21 (2): 380. 1862. Gamocarpha poeppigii DC var. angustifolia (Phil.) Reiche, Anales Univ. Chile 106: 1041. 1900. TIPO: Chile. Nevado de Chillan, 2-III-1862, R. A. Philippi s.n. (holotipo SGO 043614 
no visto; isotipos SI 12703!, SI 12697!, W no visto, foto 31049 del Field Museum of Natural History en SI!).

Caméfitos glaucos, glutinosos, cespitosos, de 10-15 cm de altura, con tallos postrados radicantes y ramas ascendentes o erectas, las partes más viejas cubiertas por las bases foliares persisitentes. Hojas coriáceas, cuneiformes; lámina orbicular a elíptica, con margen entero y ápice obtuso, atenuada en un largo pecíolo linear. Pedúnculos erectos, terminales, con hojas lineares, monocéfalos. Cefalodios sin grupos cimosos periféricos (internos), pero con 6-7 grupos cimosos externos al involucro, con brácteas tectrices lineares, con base ensanchada fusionada al involucro, delimitando celdas. Grupos cimosos mayores, con involucelos de brácteas (profilos?) lanceoladas, libres. Grupos cimosos menores con involucelos parcialmente fusionados. Receptáculo reducido sin brácteas. Involucro con base fusionada tubular y lóbulos lanceolados. Flores pentámeras, verdosas o amarillentas. Perianto de 6-6,9 $\mathrm{mm}$ de longitud. Sépalos oblongos a lanceolados, ápice obtuso. Hipanto tubuloso, de 1,58 -2 $\mathrm{mm}$ de longitud. Corola campanulada, de 4,3-5 mm de longitud, lobada en $1 / 4$ de su parte superior; tubo amplio, lóbulos oblongos, planos, ápice agudo, uncinado. Glándulas nectaríferas estaminales. Tubo estaminal de $1 / 2$ de la longitud de la corola. Filamentos nulos. Anteras oblongas, tecas basalmente obtusas, sin caudículas, conectivo no apiculado. Ovario lacrimiforme, de 2,45-2,85 mm de longitud. Cipselas prismáticas, rugosas, con 5 carenas lisas, delgadas, coronadas por sépalos membranáceos, oblongos de ápice obtuso que rodean un diminuto apículo central.

Iconografía. Hoffmann et al. (1998: 183, fig. 5, sub Gamocarpha alpina).

Distribución. Especie endémica de la cordillera andina, en los alrededores del volcán Chillán (noreste de la VIII Región del Bío Bío, Chile) y de la laguna Varvarco Campos (noroeste de la provincia de Neuquén, Argentina). Ocupa las áreas que corresponden a la provincia fitogeográfica Altoandina.

Discusión. Gamocarpha angustifolia puede ser confundida con Moschopis caleofuensis o con Gamocarpha dentata por el aspecto general de la planta, la forma de las flores y de las cipselas. Pero M. caleofuensis posee hojas con ápice tridentado y 
cefalodios con 12 grupos cimosos, y G. dentata posee hojas pentalobadas e inflorescencias sésiles, sin pedúnculo floral.

Gamocarpha angustifolia fue recategorizada como variedad de G. poeppigii por Reiche (1900) y posteriormente sinonimizada bajo G. alpina (Zanotti \& Pozner, 2008). La presencia de tallos postrados radicantes y las hojas espatuladas con margen entero y pecíolos lineares, probablemente hayan sugerido cierta similitud entre ambos taxones. Pero el tallo con bases foliares persistentes (vs. tallo sin bases foliares persistentes en $G$. alpina), la presencia de una secreción glutinosa (ausente en G. alpina), la morfología de la flor con hipanto reducido (vs. hipanto alargado en $G$. alpina) y corola campanulada (vs. corola con tubo reducido y completamente lobada en G. alpina), la presencia de glándulas nectaríferas estaminales (vs. glándulas nectaríferas hipantiales en G. alpina), los filamentos nulos (vs. filamentos desarrollados en G. alpina), los pedúnculos hojosos (vs. pedúnculos desnudos en G. alpina), la presencia de grupos cimosos externos al involucro portando involucelos (vs. ausencia de grupos cimosos externos al involucro en G. alpina) y la ausencia de brácteas del receptáculo soldadas formando celdas (vs. presencia de brácteas del receptáculo soldadas formando celdas en G. alpina), demuestran que Gamocarpha angustifolia debe ser rehabilitado como taxón independiente de G. alpina.

Gamocarpha dentata Phil., Anales Univ. Chile 41 (1): 735. 1872. TIPO: Chile. Araucanía. Cupulhue, sin fecha indicada, J. Volkmann s.n. (duplicados K 000009730 no visto, foto!, SGO 043618 no visto, SGO 057245 no visto, SI 12699!). Fig. 28 (A-C).

Gamocarpha dentata Phil. var. glaucescens Speg. ex Hicken, Reun. Nac. Soc. Arg. Cienc. Nat. 1: 248. 1919. TIPO: Argentina. Neuquén, región del río Aluminé, 1IV-1902, O. Asp s.n. (holotipo LP ex LPS!; isotipos SI!, BAB 8038!).

Caméfitos cespitosos, glutinosos, glaucos, de aproximadamente $5 \mathrm{~cm}$ de altura, con tallos postrados radicantes y ramas ascendentes o erectas, las partes más viejas cubiertas por las bases foliares persisitentes. Hojas coriáceas, cuneiformes; lámina orbicular a elíptica, plegada, con margen crenulado a pentalobulado y ápice obtuso, atenuada en un largo pecíolo linear. Pedúnculos nulos. Inflorescencias sésiles, terminales. Involucro 
con base fusionada campanulada y lóbulos lanceolados, desiguales. Receptáculo reducido sin brácteas. Cefalodio con ca. 5 grupos cimosos externos (al involucro). Brácteas tectrices de los grupos cimosos 5-7-palmatilobadas, libres, con la base ensanchada; involucelos profilares con brácteas libres o más o menos fusionadas, lineares a 3-lobadas. Flores pentámeras. Perianto de 6,5-8 $\mathrm{mm}$ de longitud. Sépalos oblongos a triangulares, ápice obtuso. Hipanto tubuloso, de 2,2-2,5 $\mathrm{mm}$ de longitud. Corola campanulada, de 4,5-5,5 mm de longitud, lobada en 1/4-1/5 de su parte superior; tubo amplio, lóbulos oblongos, planos, ápice obtuso, uncinado. Glándulas nectaríferas estaminales. Tubo estaminal de $1 / 2$ de la longitud de la corola. Filamentos nulos. Anteras oblongas, tecas basalmente obtusas, sin caudículas, conectivo no apiculado. Ovario cilíndrico a lacrimiforme, de 3,6-3,8 mm de longitud. Cipselas prismáticas, rugosas, con 5 carenas lisas, delgadas, coronadas por sépalos membranáceos, lanceolado-oblongos de ápice obtuso que rodean un diminuto apículo central.

Distribución. Endémica de Chile y Argentina, en los Andes del centro y sur de la VIII Región del Bío Bío, del norte de la XI Región de Araucanía, y del centro de la provincia de Neuquén. Ocupa las áreas que corresponden a la provincia fitogeográfica Altoandina.

Discusión. Gamocarpha dentata puede ser confundida con Moschopsis subandina por el aspecto general de la planta, el aspecto de la inflorescencia, la forma de las flores y de las cipselas. Pero M. subandina posee hojas digitadas, 5-7 lobadas, y las brácteas de los grupos cimosos (externos) son de naturaleza hipofilar (vs. hiperfilares en $G$. dentata).

Gamocarpha gilliesii Miers, Ann. Mag. Nat. Hist. ser. 3, 6 (34): 281. 1860 [Oct 1860]. TIPO: Argentina. El cerro de la Polcura, Andes of Mendoza, J. Gillies s.n. (holotipo K 000009727 no visto, foto!). Fig. 28 (D-E).

Boopis breviscapa Phil., Anales Univ. Chile 85: 818. 1894. TIPO: Chile. Colchagua. Valle Hermoso, I-1872, P. Ortega s.n. (lectotipo aquí propuesto K 
000009728 no visto, foto!; duplicados SGO 057214 no visto, foto!, SGO 043613 no visto, foto!, K 000009729 no visto, foto!).

Gamocarpha gilliesii Miers var. gerthi Hicken, Darwiniana 1: 70. 1923, syn. nov. prpopuesto. TIPO: Argentina. Mendoza. Arroyo de las Minas, XII-1912, E. Gerth 88 (holotipo SI 1256!).

Hemicriptófitos herbáceos cespitosos, estoloníferos y rizomatosos, con brotes aéreos rosulados. Raíces contráctiles. Hojas coriáceas, gruesas, enteras, espatuladas; lámina suborbicular plana, u oblongo-plegada, atenuada un pecíolo rectangular, con margen entero y ápice obtuso o agudo, no mucronado. Pedúnculos teretes, escapiformes. Involucro con base fusionada pateliforme y lóbulos triangulares a panduriformes, recurvados, margen entero y ápice mucronado. Receptáculo punctiforme. Cefalodios

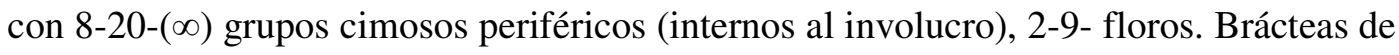
los grupos cimosos triangulares, anchas, fusionadas delimitando celdas. Brácteas de las flores solitarias más externas, fusionadas en celdas, y progresivamente libres y más angostas hacia el centro. Perianto 4-5 mm. Sépalos lanceolados a suborbiculares. Hipanto verde, tubuloso, grueso. Corola blanca lobada hasta 1/2 de su parte superior, lóbulos lanceolados, planos, con ápice agudo, recto o uncinado. Glándulas nectaríferas hipantiales. Tubo estaminal de 1/2 longitud del tubo corolino. Filamentos incurvos, de 1/4 del largo de las anteras. Anteras oblongas, tecas basalmente sagitadas sin caudículas, conectivo no apiculado. Ovario cilíndrico a elipsoidal, de 2,2-2,6 mm de longitud. Cipselas prismáticas, lisas, con 5 carenas coronadas por sépalos lanceolados, que rodean a un apículo central.

Distribución. Endémica de los Andes de la provincia de Mendoza. Crece a partir de los 2000 m s.m. El ejemplar tipo de Boopis breviscapa es el único registro dudoso para Chile, ya que el único paraje conocido como Valle Hermoso cercano a Colchagua (VI Región) se encuentra ubicado en la provincia de Cachapoal a ca. 450 m s.m.

Discusión. Gamocarpha gilliesii puede ser confundida con G. alpina por el aspecto general de la planta y de las cipselas. Pero G. alpina posee hojas con lámina oblonga abierta o plegada, lóbulos del involucro anchamente triangulares nunca 
panduriformes ni recurvados y flores con perianto de 6-6,5 $\mathrm{mm}$ e hipanto infundibuliforme.

Se selecciona como lectotipo de Boopis breviscapa el ejemplar K 000009728 ya que conserva mayor cantidad de inflorescencias en buen estado que los restantes duplicados.

La única característica distintiva usada por Hicken para la var. gerthi fue la ausencia de estolones. Los restantes caracteres del ejemplar tipo coinciden con la morfología de G. gilliesii, por lo tanto se considera aquí como su sinónimo.

Gamocarpha selliana Reiche, Anales Univ. Chile 106: 1040. 1900. TIPO: Argentina. Curso superior del Rio Ftaleufu (Valle de Cholila), 42²5'S, sin fecha indicada, $C$. Reiche s.n. (holotipo no localizado, probablemente en SGO). Fig. 28 (F-H).

Gamocarpha patagonica Speg., Anales Mus. Nac. Buenos Aires 7: 303. 1902. TIPO: Argentina. "Hab. in pratis siccis prope Colonia 16 de octubre", aest. 1901, $N$. Illín s.n. (holotipo LP ex LPS 12040!).

Gamocarpha selliana Reiche var. multicaulis Dusén, Ark. Bot. 7, no. 2: 40. 1907 [1908]. TIPO: Argentina. Chubut. "Die Art ist bisjetzt nur in dem nordpatagonischen Kordillera in dem Tale des Rio Cholila (lat. 42 ${ }^{\circ} 25^{\prime}$ ) gefunden”, 1904-1905, I. Koslowsky (holotipo no localizado, probablemente en S).

Hemicriptófitos herbáceos, cespitosos, estoloníferos, con brotes aéreos rosulados, glabras, caulescentes o acaules, de $1-20 \mathrm{~cm}$ de altura, con tallos tendidos o ascendentes, ramificados. Raíces contráctiles. Hojas espatuladas, con la base atenuada en un pecíolo rectangular, lámina oblonga con margen partido y lóbulos oblongos, o suborbicular con margen entero, dentado o crenado, ápice obtuso. Inflorescencia central pedunculada o sentada, inflorescencias laterales pedunculadas, terminales o laterales. Pedúnculos teretes. Involucro con una base rotácea fusionada y lóbulos triangular-oblongos o rectangulares a panduriformes, con margen entero. Receptáculo punctiforme. Cefalodios con aproximadamente 15 grupos cimosos periféricos (internos), 3-10floros). Brácteas de los grupos cimosos triangulares, fusionadas en la base formando 
celdas. Brácteas de las flores solitarias más externas, fusionadas en celdas, y progresivamente libres y más angostas hacia el centro. Perianto de $5-7 \mathrm{~mm}$ de longitud. Sépalos suborbiculares, margen entero, ápice agudo. Hipanto verde, tubuloso. Corola blanca, partida, tubo breve, lóbulos lanceolados, planos, ligeramente recurvados, ápice agudo, recto. Glándulas nectaríferas hipantiales. Tubo estaminal del doble del largo de los filamentos. Filamentos de 1/4-1/5 del largo de las anteras. Anteras oblongas, tecas basalmente sagitadas sin caudículas, conectivo no apiculado. Ovario elipsoidal a lacrimiforme, de 1-2 mm de longitud. Cipselas prismáticas, lisas con 5 carenas, coronadas por sépalos orbiculares que rodean a un apículo central.

Distribución. G. selliana es una de las especies más abundantes de la Patagonia argentina. Se distribuye desde el norte de Neuquén (depto. Ñorquín) hasta el sur de Santa Cruz. Se conoce un registro para Chile en la XI Región de Aysén (Pcia. Coihaique). Ocupa el área correspondiente a la Provincia Patagónica; existen registros de esta especie en las zonas de contacto con la Provincia Subantártica: Caviahue (Neuquén) y Paso Río Roballos (Santa Cruz). Existen registros para el Distrito Austral de la Provincia Altoandina: $C^{\circ}$ Challhuaco (Río Negro).

Discusión. Gamocarpha selliana muestra una semejanza muy estrecha con Boopis multicaulis en todos sus caracteres. Boopis multicaulis sólo se diferencia por no desarrollar estolones, poseer entrenudos más largos, una forma caulescente más conspicua y mayor número de inflorescencias. La morfología de la flor y el fruto es idéntica para los dos taxones. La mayoría de las diferencias en los caracteres vegetativos es de tipo cuantitativo. Es muy probable que ambos taxones formen parte de una misma especie con alta variabilidad morfológica que ocupa un área de distribución amplia. Sin embargo, es preferible mantener la independencia taxonómica hasta que puedan coleccionarse más ejemplares que permitan desarrollar análisis moleculares y morfológicos más detallados y concluyentes. 
MOSCHOPIS Phil., Anales Univ. Chile 27 (3): 334. 1865. ${ }^{(*)}$

Especie tipo: Moschopis leyboldi Phil.

Caméfitos o geófitos herbáceos, caulescentes o rizomatosos con brotes aéreos rosulados, ascendentes o erectos; glabros. Tallos procumbentes con o sin bases foliares persistentes, a veces glutinosos. Hojas imbricadas, gruesas, algo coriáceas, enteras, sésiles o espatuladas (atenuadas en un pecíolo rectangular); lámina oblonga, linear, lanceolada u oval, con margen entero, dentado, lobado o partido, mucronado o no mucronado, y ápice agudo u obtuso, mucronado o no mucronado. Pedúnculos teretes o nulos. En cefalodios con involucro diferenciado (con base fusionada y lóbulos), presencia de grupos cimosos externos al involucro, con o sin grupos cimosos periféricos internos al involucro, y con un grupo central de flores solitarias (ausente en Moschopis subandina). En cefalodios sin involucro diferenciado (brácteas tectrices y brácteas del receptáculo libres), presencia de grupos cimosos periféricos e internos rodeando un grupo central de flores solitarias, o bien inflorescencia formada exclusivamente por grupos de flores solitarias (grupos cimosos ausentes). A veces, involucelos presentes en la base de los grupos cimosos (¿de origen profilar?). Receptáculo punctiforme. Flores pentámeras. Sépalos lanceolados, dentiformes o lanceolados, con margen entero y ápice obtuso o agudo, no mucronado. Hipanto tubuloso o infundibuliforme. Corola con estivación valvar, tubular, lobada, actinomorfa; lóbulos oblongos o lanceolados, planos o cuculados inflados, con ápice agudo o agudo a obtuso, recto o uncinado. Glándulas nectaríferas estaminales o hipantiales. Tubo estaminal con filamentos libres en el extremo apical, o bien filamentos ausentes. Anteras oblongas, con base obtusa o sagitada, con o sin caudículas; conectivo apiculado o no apiculado. Ovario cilíndrico a lacrimiforme. Cipselas prismáticas o turbinadas con 5 carenas coronadas por los sépalos acrescentes ovados a triangulares, no espinescentes, que rodean a un apículo central.

Moschopis ameghinoi (Speg.) Dusén, in Scott, Rep. Princeton Univ. Exped. Patag. viii. Suppl. Bot. 253, 254. 1915. Boopis ameghinoi Speg., Anales Soc. Ci. Argent. 48: 175. 1899. Moschopis ameghinoi (Speg.) Hicken, comb. superfl., Reun. Nac. Soc. Arg.

${ }^{(*)}$ A partir de la revisión de Reiche (1900), el nombre de este género fue conocido y difundido erróneamente como Moschopsis. El nombre correcto dado por Philippi en el protologo es Moschopis (del gr. moschos $=$ ternero, ops= ojo), lo cual podría interpretarse como una variación de Boopis (del gr. boos= buey, ops= ojo). 
Cienc. Nat. 1: 249. 1919. Gamocarpha ameghinoi (Speg.) Speg., Anales Mus. Nac. Buenos Aires 7: 302. 1902. TIPO: Argentina. Santa Cruz. En pendientes desnudas aridísimas Karr-aik (Lago Argentino), III-1898, C. Ameghino s.n. (holotipo LP ex LPS!; isotipo SI 12705!). Fig. 29.

Hierbas, crasas, rizomatosas, cespitosas, glaucas, con brotes aéreos caulescentes, ascendentes, emergidos, con cicatrices foliares en la base, de hasta $20 \mathrm{~cm}$ de altura. Probablemente raíces contráctiles. Hojas alternas, espatuladas; lámina suborbicular a oblonga, con margen dentado a escarroso-laciniado y ápice obtuso, atenuada en un pecíolo rectangular. Inflorescencia compuesta, constituida por 3-4 cefalodios periféricos pedunculados con una bráctea foliar basal, rodeando a un gran cefalodio central terminal, todos agregados sobre un eje reducido. Pedúnculo de la inflorescencia escapiforme, erecto, ocasionalmente ramificado, portando otras inflorescencias compuestas laterales. Cefalodios periféricos sobre pedúnculos de ca. 1,5 mm de longitud, con 3-4 grupos cimosos externos al involucro 4-10-floros, sin involucelos, con brácteas tectrices rectangulares a oblongas, margen dentado-laciniado, ápice obtuso, con la base ensanchada, fusionadas al involucro formando celdas. Involucro con base fusionada pateliforme; lóbulos lanceolados, con margen entero o paucidentado, desiguales. Receptáculo punctiforme, con brácteas libres lanceoladas a linearlanceoladas. Grupos cimosos periféricos internos (9-10), 2-4-floros. Cefalodio central terminal sésil, sin grupos cimosos externos al involucro. Involucro con base fusionada pateliforme, lóbulos oblongos a lanceolados con margen dentado-laciniado y ápice obtuso. Receptáculo punctiforme, con brácteas libres lanceoladas o linear lanceoladas. Grupos cimosos periféricos internos (15-20), 2-5-floros. Perianto de 3,5-4,5 mm de longitud. Sépalos dentiformes a lanceolados, inflados. Hipanto verde, tubuloso, reducido. Corola campanulada, lobada en $1 / 2$ de su parte superior; tubo verdoso; lóbulos blancos, lanceolados, ligermante recurvados, planos a cuculados inflados, con ápice agudo, recto o uncinado. Glándulas nectaríferas estaminales. Tubo estaminal de igual longitud que el tubo corolino. Filamentos ausentes. Anteras oblongas, tecas basalmente obtusas sin caudículas, conectivo no apiculado. Ovario cilíndrico a lacrimiforme, de aproximadamente 1-2 mm de longitud. Cipselas desconocidas. 
Distribución. Endémica de la provincia de Santa Cruz. Crece entre las grietas de los afloramientos rocosos de la meseta. Sólo ha sido coleccionada en dos sitios: en las cercanías del lago Argentino (Karr-aik) y en la meseta del lago Buenos Aires, aunque también ha sido avistada en la meseta del lago Viedma (M. L. Iribarren, com. pers.). Ocupa el área correspondiente a la Provincia Patagónica (Distrito Central, Subdistrito Santacrucense).

Discusión. Esta especie puede ser reconocida fácilmente por las inflorescencias compuestas y las hojas con lámina suborbicular a oblonga y margen dentado a escarroso-laciniado. Probablemente se trate de un caméfito herbáceo; sin embargo, no es posible verificar esto hasta que se realicen nuevas colecciones y observaciones de campo.

Moschopis caleofuensis (Speg.) Dusén, in Scott, Rep. Princeton Univ. Exped. Patag. viii. Suppl. Bot. 254. 1915. Gamocarpha caleofuensis Speg., Anales Mus. Nac. Buenos Aires 7: 303. 1902. TIPO: Argentina. "In rupestribus andinis prope fontes Ca-leofú", I1898, O. Mauri s.n. (holotipo LP ex LPS 11031!). Fig. 30 (A-C).

Caméfitos herbáceos glabros, gruesos, glutinosos, cespitosos, glaucos, rizomatosos, de hasta $15 \mathrm{~cm}$ de altura, con tallos postrados radicantes y ramas ascendentes o erectas, cubiertas por las bases foliares persistentes. Hojas coriáceas, espatuladas a cuneiformes; lámina partida, trilobada a pedada, incurvada, atenuada en un largo pecíolo rectangular; lóbulos oblongos, con margen revoluto, entero o trilobado y ápice obtuso. Pedúnculos erectos, terminales, hojosos, monocéfalos. Cefalodio con receptáculo punctiforme sin brácteas receptaculares, sin grupos cimosos periféricos (internos), pero con 10-15 grupos cimosos externos (al involucro), 2-6-floros, asentados sobre brevísimos pedúnculos laterales, rodeados por un involucelo de brácteas (profilos?) con la base soldada. Brácteas tectrices de los grupos cimosos trilobadas, libres, con la base ensanchada, lóbulo central con ápice agudo. Involucro con base fusionada tubulosa, con lóbulos desiguales, trilobados o lanceolados con ápice agudo. Flores verdosas o amarillentas. Perianto de 4,8-5 mm de longitud. Sépalos oblongos a lanceolados, ápice obtuso. Hipanto tubuloso, de 0,85-1 mm de longitud. Corola campanulada, de 3,75-4 
mm de longitud, lobada en $1 / 4$ de su parte superior; tubo amplio, lóbulos oblongos, planos, ápice agudo a obtuso, uncinado. Glándulas nectaríferas estaminales. Tubo estaminal de 1/2 de la longitud de la corola. Filamentos nulos. Anteras oblongas, tecas basalmente obtusas, sin caudículas, conectivo no apiculado. Ovario elipsoidal, de 3,33,5 mm de longitud. Cipselas prismáticas, rugosas, con 5 carenas lisas, delgadas, coronadas por sépalos membranáceos que rodean un diminuto apículo central.

Distribución. Endémica de los Andes de Río Negro y el sur de Neuquén. Crece en terrenos pedregosos de las laderas de los cerros y adyacencias de los lagos de la región, ca. 1800 m s.m. Ocupa el área discontinua correspondiente a la Provincia Altoandina (Distrito Austral) que se intercala con la Provincia Subantártica. Ha sido coleccionada pocas veces. Hasta ahora no existen registros para Chile.

Discusión. Moschopis caleofuensis puede ser confundido con Gamocarpha dentata por el aspecto de la planta. Sin embargo, G. dentata posee hojas con margen crenulado a pentalobulado y cefalodios sésiles con brácteas tectrices de los grupos cimosos 5-7-palmatilobadas, libres, con la base ensanchada.

Moschopis leyboldi Phil., Anales Univ. Chile 27 (3): 335. 1865. TIPO: Chile. Cordillera de Santiago, cerro de La Polcura (Hac. de las Condes), sin fecha indicada, $F$. Leybold s.n. (duplicados SGO 043583 no visto, SGO 057230 no visto, SI 12709!, SI 12711!, W no visto, foto 31056 Field Museum of Natural History en SI!). Fig. 30 (D-F).

Geófitos herbáceos gruesos, rizomatosos, con brotes aéreos anuales ascendentes, de 2-5(7) $\mathrm{cm}$ de altura. Rizomas verticales. Raíces contráctiles. Hojas espatuladas; lámina lanceolada, con margen entero y ápice acuminado, atenuada en un pecíolo rectangular. Pedúnculos nulos. Cefalodio terminal, central, con ca. 10 grupos cimosos (2)-4-8-floros y un grupo central de flores solitarias paucifloro. Involucro ausente; brácteas del cefalodio libres. Receptáculo reducido. Brácteas de los grupos cimosos similares a nomófilos, lanceoladas, espatuladas o lineares. Perianto verdoso, de aproximadamente 4 mm. Sépalos foliáceos, lanceolados. Hipanto verde, tubuloso. Corola blanca, campanulada, lobada hasta 1/4 de su parte superior; lóbulos ligermante recurvados, lanceolados, planos, ápice agudo, recto. Glándulas nectaríferas estaminales. Tubo 
estaminal de igual longitud que los filamentos. Filamentos de $1 / 4$ de la longitud de las anteras. Anteras oblongas, tecas basamente obtusas con caudículas, conectivo no apiculado. Ovario terete a fusiforme, 3-3,4 mm de longitud. Cipselas cilíndricas, lisas, con 5 carenas vestigiales, coronadas por sépalos dentiformes que rodean un apículo central.

Distribución. Endémica de los Andes centrales de Chile (Región Metropolitana) y Argentina (sur de San Juan, Mendoza y norte de Neuquén). Habita en los suelos pedregosos desnudos de las cumbres a partir de los 2000 m s.m. en Chile y los $3000 \mathrm{~m}$ s.m. en Argentina. Ocupa el área correspondiente a la Provincia Altoandina (Distrito Cuyano).

Discusión. Moschopis leyboldi puede ser reconocida fácilmente por los brotes aéreos ascendentes, las brácteas libres del cefalodio (ausencia de involucro) y las hojas espatuladas con lámina lanceolada, margen entero y ápice acuminado.

Moschopis monocephala (Phil.) Reiche, Anales Univ. Chile 106: 1038. 1900. Boopis monocephala Phil., Anales Mus. Nac., Santiago de Chile 1891: 30. TIPO: Chile. Prope Antofagasta alta, 16-I-1885, F. Philippi 2052 (holotipo SGO 043589 no visto, foto!, isotipo SI 12715!). Fig. 31 (A-E).

Geófitos herbáceos gruesos, rizomatosos, con brotes aéreos anuales rosulados, de 1-3 cm de altura. Rizomas verticales. Catafilos amplexicaules similares a nomófilos. Probablemente raíces contráctiles. Hojas espatuladas; lámina obovada, con margen dentado-mucronado y ápice obtuso mucronado, atenuada en un pecíolo rectangular. Pedúnculos nulos. Cefalodios terminales, centrales, con ca. 15 grupos cimosos 8-floros y un grupo central de flores solitarias paucifloro. Involucro ausente; brácteas del cefalodio libres. Receptáculo reducido, con brácteas de los grupos cimosos obovadorectangulares, rectangulares, espatuladas o lineares y ligeramente acrescentes durante la madurez de los frutos. Perianto verdoso, de ca. 4,35-5,50 mm. Sépalos dentiformes, inflados. Hipanto verde, tubuloso. Corola blanca, tubulosa, lobada hasta 1/5 de su parte superior; lóbulos ligermante recurvados, lanceolados, planos o cuculados inflados, ápice 
agudo, recto. Glándulas nectaríferas indistinguibles. Tubo estaminal de $1 / 2-1 / 3$ de la longitud que los filamentos. Filamentos de 1/2-2/3 de la longitud de las anteras. Anteras oblongas, tecas basamente obtusas sin caudículas, conectivo no apiculado. Ovario cilíndrico a elipsoidal, 1,8-2,5 mm de longitud. Cipselas cilíndricas a prismáticas, rugosas, con 5 carenas vestigiales o planas, coronadas por sépalos vestigiales a triangulares que rodean un apículo central grueso.

Distribución. Andes del norte de Chile (I, II, III y XV Regiones) y noroeste de Argentina (Catamarca, Salta y Jujuy). Ocupa el área correspondiente a la Provincia Altoandina (Distrito Quichua), creciendo a partir de ca. 4000 m s.m., aunque ocasionalmente puede hallársela en el ecotono con la Provincia Puneña. Habita en vegas, bofedales y suelos húmedos irrigados por capilaridad.

Discusión. Moschopis monocephala puede ser confundido con Calycera pulvinata por el aspecto de los brotes aéreos, la forma de sus hojas, la ausencia de involucro diferenciado y la presencia de rizomas verticales. Pero, C. pulvinata posee cefalodios con brácteas tectrices lanceoladas con base amplia parcialmente fusionadas, flores más pequeñas (perianto 2,8 -3 mm, ovario 1,3-1,45 mm), anteras con conectivo apiculado y cipselas espinosas.

Moschopis rosulata (N. E. Br.) Dusén, Ark. Bot. 7(2): 42. 1907 [1908]. Acicarpha rosulata N. E. Br., Hooker's Icon. P1. 27: t. 2636 B. 1900. Gamocarpha rosulata (N. E. Br.) Skottsb., Kungl. Svenska Vetenskapsakad. Handl. 56(5): 311. 1916. TIPO: Patagonia australis. Cerro Toro, XII-1896, O. Nordenskjold s.n. (holotipo S no visto; isotipo K 000009719 no visto, foto!). Fig. 31 (F-I).

Moschopis spathulata Dusén, Ark. Bot. 7(2): 41. 1907 [1908]. TIPO: Argentina. Santa Cruz. "Rio Fósiles in montanis ad terram nudam in alt.", 1000-1600 m, sin fecha indicada, sin coleccionista indicado (holotipo no localizado, probablemente en S).

Geófitos herbáceos gruesos, rizomatosos, con brotes aéreos ascendentes a rosulados, de 1-8 cm de altura. Catafilos oblongos. Probablemente raíces contráctiles. Hojas densamente imbricadas, espatuladas, coriáceas en el extremo distal; lámina suborbicular 
a obovada, con margen entero o paucidentado (1-2 pares de dientes) y ápice obtuso, atenuada en un pecíolo rectangular. Pedúnculos nulos. Cefalodios terminales, centrales, con 10-15 grupos cimosos 12-floros, rodeados por sendos involucelos profilares con la base soldada. Involucro ausente; brácteas del cefalodio libres. Receptáculo reducido. Grupos cimosos periféricos con brácteas tectrices palmadas, con el lobo medio obovado y los laterales laciniados. Grupos cimosos centrales con brácteas tectrices rectangulares 5-lobado-laciniadas en el ápice .Grupo central de flores solitarias rodeado por brácteas similares a las de los grupos cimosos internos. Perianto de 4,80-5,30 mm. Sépalos foliáceos, lanceolados. Hipanto verde, tubuloso. Corola blanca, tubulosa, lobada hasta 1/3 de su parte superior; lóbulos ligermante recurvados, lanceolados, planos o cuculados inflados, ápice agudo u obtuso, uncinado. Glándulas nectaríferas estaminales. Tubo estaminal de igual longitud que el tubo corolino. Filamentos ausentes. Anteras oblongas, tecas basamente obtusas o sagitadas sin caudículas, conectivo apiculado o no apiculado. Ovario cilíndrico a elipsoidal, de 1-2 mm de longitud. Cipselas elipsoidales a prismáticas, lisas, con 5 carenas vestigiales, coronadas por sépalos vestigiales, de textura membranácea, que rodean un apículo central grueso.

Distribución. Endémico de Chile y Argentina, en la Patagonia austral, y la isla de Tierra del Fuego. Habita en pedreros y áreas rocosas de la meseta, cercanas a los lagos. Ocupa el área correspondiente a la Provincia Patagónica (Distrito Subandino).

Discusión. Moschopis rosulata puede identificarse por el aspecto de sus brotes aéreos ascendentes, sus hojas espatuladas coriáceas en el extremo distal, con lámina suborbicular a obovada, margen entero o paucidentado (1-2 pares de dientes) y ápice obtuso, y por la estructura de sus cefalodios multifloros con numerosos grupos cimosos 12-floros portando involucelos (¿profilares?).

Moschopis subandina (Speg.) Dusén, in Scott, Rep. Princeton Univ. Exped. Patag. viii. Suppl. Bot. 254. 1915. Gamocarpha subandina Speg., Anales Mus. Nac. Buenos Aires 7: 305. 1902. TIPO: Argentina. Chubut, Corcovado. "In montanis rupestribus secus Carren-leofú", 18/20-I-1901, N. Illín s.n. (holotipo LP ex LPS 11030!; isotipos SI!, BAF no visto, foto!). Fig. 32 (D-G). 
Caméfitos herbáceos glabros, glutinosos, cespitosos, glaucos, de ca. $5 \mathrm{~cm}$ de altura, con tallos postrados radicantes y ramas ascendentes o erectas, cubiertas por las bases foliares persistentes. Hojas coriáceas, espatuladas a cuneiformes; lámina digitada, plegada, con margen 5-7 lobado, involuto, y ápice obtuso, atenuada en un largo pecíolo linear. Pedúnculos terminales, hojosos, reducidos, de 0,5-1 cm. Cefalodio con receptáculo punctiforme sin brácteas receptaculares, con 5-10 grupos cimosos externos (al involucro) 8-9-floros y grupos cimosos periféricos (internos), pero sin flores solitarias. Grupos cimosos externos asentados sobre brevísimos pedúnculos laterales, rodeados por un involucelo de brácteas (profilos?) con la base soldada; brácteas tectrices similares a nomófilos, libres, con la base ensanchada y ápice lobulado. Involucro con base soldada tubular, lóbulos lanceolados, desiguales. Perianto de 5,3-5,8 $\mathrm{mm}$ de longitud. Sépalos inflados. Hipanto tubuloso, de 0,65-0,75 $\mathrm{mm}$ de longitud. Corola campanulada, de 4,7-5 mm de longitud, lobada en 1/4 de su parte superior; tubo amplio, lóbulos oblongos, planos, ápice agudo, uncinado. Glándulas nectaríferas estaminales. Tubo estaminal de $1 / 2-2 / 3$ de la longitud de la corola. Filamentos nulos. Anteras oblongas, tecas basalmente sagitadas, sin caudículas, conectivo no apiculado. Ovario cilíndrico a lacrimiforme, de 1,95-2,15 mm de longitud. Cipselas prismáticas, rugosas, con 5 carenas, coronadas por sépalos membranáceos, lanceolado-oblongos de ápice obtuso que rodean un diminuto apículo central.

Distribución. Endémico de los Andes australes de Chile ( $\mathrm{C}^{\mathrm{o}}$ MochoChoshuenco, XIV Región) y Argentina ( $C^{\circ}$ Chapelco, Neuquén; región lago Vintter- río Corcovado, Chubut). Posee una distribución discontínua. Ocupa dos áreas fitogeográficas diferentes dentro del Dominio Andino-Patagónico: la Provincia Patagónica (Distrito Subandino) y la Provincia Altoandina (Distrito Austral). Existen pocos registros de esta especie. Para el presente trabajo sólo se pudieron examinar tres ejemplares.

Discusión. La descripción desarrollada más arriba concuerda con la del holotipo y el protologo, y se ajusta a la de la forma de Moschopis subandina que habita en el Distrito Subandino de la Provincia Patagónica. Sin embargo, de acuerdo con el material examinado, M. subandina posee una variación regional propia del Distrito Austral de la Provincia Altoandina: lámina amplia, pecíolos acortados, entrenudos más espaciados, 
pedúnculos más desarrollados y ausencia de exudado glutinoso. El especímen Ferreyra 589 (BCRU), coleccionado en el sur de Neuquén (“Cuyín Manzano-NE ladera norte, vive en corridas de agua”) y determinado como Moschopis rosulata, probablemente se trate de un ejemplar de M. subandina de porte más reducido.

Moschopis trilobata Dusén, Ark. Bot. 7(2): 40. 1907 [1908]. TIPO: Argentina. Patagonia. Santa Cruz: "Rio Fósiles in montanis ad terram nudam in alt.", 800-1100 m, 1904, P. Dusén 5911 (holotipo no localizado, probablemente en S; isotipo SI 104740!). Fig. 32 (A-C).

Geófitos herbáceos gruesos, rizomatosos, con brotes aéreos rosulados a erectos, de hasta $3 \mathrm{~cm}$ de altura. Tallos cubiertos por las bases foliares persistentes. Catafilos aciculares y escuamiformes. Probablemente raíces contráctiles. Hojas densamente imbricadas, espatuladas, coriáceas en el extremo distal; lámina partida, trilobada, con lobos lanceolados a lineares con ápice acuminado. Pedúnculos nulos. Cefalodios terminales con receptáculo punctiforme, sin grupos cimosos, pero con más de 20 flores solitarias periféricas portando brácteas tectrices similares a nomófilos ensanchados en la base, y un grupo central numeroso de flores solitarias. Involucro ausente. Brácteas del receptáculo libres, linear-lanceoladas. Perianto verde, craso, de 4,80-5,50 mm. Sépalos membranáceos, lanceolados a triangulares. Hipanto verde, infundibuliforme. Corola verde, rotácea, partida; tubo reducido; lóbulos recurvados durante la antesis (incurvados durante la maduración de los frutos), lanceolados, planos, con ápice agudo, recto o uncinado. Glándulas nectaríferas hipantiales. Tubo estaminal de 1/2 la longitud de los lóbulos corolinos. Filamentos ausentes. Anteras oblongas, tecas basamente sagitadas sin caudículas, conectivo no apiculado. Ovario cilíndrico a lacrimiforme, de aproximadamente $3 \mathrm{~mm}$ de longitud. Cipselas prismáticas a elipsoidales, rugosas, con 5 carenas vestigiales, coronadas por sépalos lanceolados, de textura membranácea, que rodean un apículo central grueso.

Distribución. Endémica del extremo sur de la Patagonia, presente en Chile (XII Región) y Argentina (Santa Cruz). Habita en los suelos arenosos y pedregosos de las 
zonas próximas a los grandes lagos, junto a cursos de agua o en la cima de las mesetas. Ocupa el área correspondiente a la Provincia Patagónica (Distrito Subandino).

Discusión. Moschopis trilobata posee un aspecto muy particular que la diferencia del resto de las especies del género y de la familia: el aspecto erguido de sus brotes aéreos, sus hojas trilobadas, sus cefalodios con flores solitarias bracteadas en la periferia y ausencia de involucro, sus flores verdes con corola completamente partida y tubo estaminal prominente.

Por su aspecto general y la estructura floral, se asemeja a Nastanthus falklandicus, pero este taxón posee hojas con margen entero, cefalodios compuestos y flores con corola blanca y lóbulos corolinos breves, además de ocupar un área de distribución completamente diferente.

NASTANTHUS Miers, Ann. Mag. Nat. Hist. ser. 3, 6 (33): 184. 1860 [Sep 1860].

Especie tipo: Nasthanthus agglomeratus Miers [= Nastanthus ventosus (Meyen) Miers].

Hemicriptófitos rosulados con raiz napiforme, contráctil, y tallo usualmente no ramificado; excepcionalmente geófitos rizomatosos con brotes aéreos erectos $(N$. falklandicus); glabros. Hojas gruesas, oblongo-oblanceoladas a espatuladas (atenuadas en un peciolo rectangular); lámina suborbicular a oblonga, lobada o pinnatifida, con margen entero o dentado; ápice obtuso, no mucronado. Tirsoide terminal progresivamente condensado hacia el ápice, con grupos cimosos basales pedunculados e involucelados, que se vuelven sésiles y coalescentes hacia el ápice del tallo, agregándose hasta fusionarse en un cefalodio, con involucro infiferenciado, o diferenciado con base fusionada pateliforme (ausente en $N$. falklandicus). A veces los grupos cimosos basales faltan (o son muy pocos) y sólo desarrolla el cefalodio terminal (N. patagonicus). Brácteas involucrales 3-12, uniseriadas, oblongas, obtusas, sinuadas a trilobadas. Receptáculo globoso, carnoso, acrescente durante la madurez de los frutos. Brácteas receptaculares linear-espatuladas, o ausentes. Flores numerosas. Cáliz con sépalos suborbiculares de estivación abierta, valvar o imbricada. Perianto infundibuliforme o cilíndrico. Corola ciatiforme diferenciada, con lóbulos triangulares a oblongos, planos o cuculados, con ápice recto o uncinado. Glándulas nectaríferas 
hipantiales. Tubo estaminal presente, filamentos estaminales presentes. Anteras oblongas con base obtusa o sagitada, con o sin caudículas; conectivo apiculado o no. Estilo engrosado distalmente. Cipselas prismáticas, carenadas, corchosas, coronadas por los sépalos vestigiales, suborbiculares o aciculares que rodean un apículo central terminal.

Nastanthus caespitosus (Phil.) Reiche, Anales Univ. Chile 106: 1031. 1900. Boopis caespitosa Phil., Fl. Atacam. 26: 200. 1860. TIPO: Chile. "Rio Frio in deserto Atacamensis", II-1854, R. A. Philippi s.n. (lectotipo SGO 043609 no visto, foto! designado por Zavala-Gallo et al. 2010; duplicados SGO 057221 no visto, foto!, W 0021368 no visto, foto!, W no visto, foto 31051 del Field Museum of Natural History en SI!). Fig. 33.

Hemicriptófitos rosulados de 3,5 cm de altura y $10 \mathrm{~cm}$ de diámetro. Hojas espatuladas, de 4-5,5 cm de longitud, con la base atenuada en un pecíolo de 2,5-4 x 0,3-1 cm; lámina casi pinnatisecta, 0,5-1,5 x 0,3-0,5 cm, con lóbulos desiguales oblongos, obtusos. Tirsoide terminal progresivamente condensado hacia el ápice, con grupos cimosos basales pedunculados e involucelados, que se vuelven sésiles y coalescentes hacia el ápice del tallo, agregándose hasta fusionarse en un cefalodio. Grupos cimosos 5-30floros, los más basales con pedúnculos cilíndricos, frecuentemente ahorquillados, aglomerados, y con una bráctea tectriz foliosa distintiva, 0,8-1,5 x 0,25-0,4 cm. Involucelo de los grupos cimosos basales con o sin base fusionada rotácea, con 5 ó más lóbulos rectangular-elípticos, con ápice obtuso, apenas mucronados, 0,6 x 0,2-0,3 cm. Receptáculo convexo, 0,4-0,8 cm de diámetro, levemente acrescente durante la maduración de los frutos. Los grupos cimosos distales carecen de involucelos y coalescen en un único receptáculo junto con las flores solitarias y la flor central, formando un cefalodio sésil, las brácteas tectrices se transforman progresivamente en brácteas del receptáculo, carnosas, rectangular a linear-lanceoladas, ápice obtuso, $3 \mathrm{x}$ $0,8 \mathrm{~mm}$, y no se diferencia un involucro. Sépalos ampliamente triangulares o triangulares, obtusos, a veces inflados, 0,8 x 0,4 mm. Hipanto tubuloso, de 3-5 $\mathrm{mm}$ de longitud x 0,15 $\mathrm{mm}$ de ancho en la base a $0,5 \mathrm{~mm}$ distalmente. Corola crateriforme, de 2,5 mm de longitud x 1-2,5 mm de amplitud, lobada hasta 1/5 de su parte superior; 
lóbulos triangulares, planos, con ápice agudo a obtuso, recto o uncinado, de 0,3-0,6 x 0,25-0,5 mm, con estivación valvar. Glándulas nectaríferas hipantiales, oblongas, de 0,5 x 0,1-0,2 mm. Tubo estaminal de igual longitud que los filamentos. Filamentos apicales libres de 0,6 $\mathrm{mm}$ de longitud. Anteras oblongas, tecas basalmente sagitadas con caudículas, conectivo no apiculado, de 1,4 $\mathrm{mm}$ de longitud. Estilo de $6 \mathrm{~mm}$ (fase estaminada) a 10.5-12 $\mathrm{mm}$ de longitud (fase pistilada). Cipselas prismáticas, 2,5-3 x 2,5-3 mm, asimétricas, con 5 carenas, longitudinales, gruesas, infladas, con desarrollo desigual, frecuentemente 1 a 2 muy reducidas, raramente con rugosidades transversales sobre la porción media, coronadas por un apículo muy visible, 0,4-0,8 mm de longitud, sépalos a menudo muy reducidos sobre el fruto, o desarrollados y escariosos, mucronados. Semilla 1,7 x $0,6 \mathrm{~mm}$.

Distribución. Endémico de Antofagasta y Atacama (Chile), en la Provincia del Desierto.

Etimología. El epíteto específico hace alusión a la disposición agregada de la inflorescencia, que le confiere a la plata un aspecto cespitoso.

Discusión. Esta especie se identifica del resto del género Nastanthus por las láminas foliares casi pinnatisectas con lóbulos desiguales, flores con hipanto del doble de longitud que la corola y cipselas prismáticas asimétricas con apículo muy visible. También es posible confundir a N. caespitosus con Calycera pulvinata o con Moschopis monocephala por el aspecto general de la planta y estructura general de la inflorescencia. Sin embargo, C. pulvinata y M. monocephala se diferencian por ser plantas rizomatosas sin raíz napiforme, con láminas foliares enteras con margen dentado o crenado-mucronado.

Se seleccionó como lectotipo al ejemplar SGO 043609 porque es el único cuya etiqueta se encuentra en clara correspondencia con el espécimen. En SGO 057221 el material tipo fue montado junto con otros ejemplares, razón por la cual no puede distinguirse qué etiqueta corresponde a cada espécimen. 
Nastanthus compactus (Phil.) Miers, Ann. Mag. Nat. Hist. ser. 3, 6: 189. 1860. Boopis compacta Phil., Linnaea 28: 709. 1856. Gamocarpha pumila Miers, nomen superfluum, Ann. Mag. Nat. Hist. ser. 3, 6: 282. 1860. TIPO: Chile. "Cordillera de Linares”, I-1856, P. Germain s.n. (holotipo SGO 057224 no visto, foto!; isotipos: K 000009712 no visto, foto!, W no visto, foto 31050 del Field Museum of Natural History en SI!). Fig. 34.

Boopis diazi Phil., Anales Univ. Chile 21 (2): 396. 1862. Nastanthus diazi (Phil.) Phil. ex Reiche, Anales Univ. Chile 106: 1029. 1900. TIPO: Argentina. "Portillo, lado de Mendoza", 1861-1862, W. Diaz s.n. (holotipo SGO 057217 no visto, foto!; isotipo SI 12721 !).

Hemicriptófitos rosulados de $3 \mathrm{~cm}$ de altura y $10 \mathrm{~cm}$ de diámetro; glabros. Hojas gruesas, espatuladas, 3,5-4,5 cm de longitud, con la base atenuada en un pecíolo de 2-3 x 0,3 cm; lámina entera o pinnatifida, 1-1,5 x 0,7-1 cm, con lóbulos triangulares. Tirsoide terminal progresivamente condensado hacia el ápice, con grupos cimosos basales pedunculados e involucelados, que se vuelven sésiles y coalescentes hacia el ápice del tallo, agregándose hasta fusionarse en un cefalodio. Grupos cimosos 25-80floros, los más basales con pedúnculos cilíndricos, carnosos, 2-3 x 0,5 cm, involucelos con base fusionada pateliforme a rotácea, lóbulos ampliamente triangulares, de 0,3 $\mathrm{mm}$ de longitud x 0,4 mm de ancho. Receptáculo convexo, 0,5-3 cm de diámetro, acrescente durante la maduración de los frutos. Los grupos cimosos distales carecen de involucelos y coalescen en un único receptáculo junto con las flores solitarias y la flor central, formando un cefalodio sésil. Brácteas tectrices de los grupos cimosos y brácteas receptaculares ausentes. Involucro no visto. Flores blancas o verdosas. Sépalos suborbiculares, obtusos, 0,5 x 0,3 mm. Perianto de 4,5-5 mm de longitud. Hipanto 3 x 1 $\mathrm{mm}$. Corola ligeramente campanulada, de 2-2,5 $\mathrm{mm}$ de longitud $\mathrm{x}$ 1,4 $\mathrm{mm}$ de ancho, lobada en el 1/3 superior; lóbulos triangular-lanceolados, ápice agudo, recto, 0,7-1 x 0,5-0,8 mm, estivación valvar. Glándulas nectaríferas hipantiales oblongas $0,5 \times 0,2$ $\mathrm{mm}$. Tubo estaminal de igual longitud que los filamentos. Filamentos libres apicales de 0,3-0,5 mm. Anteras oblongas, tecas basalmente sagitadas, conectivo no apiculado, de 1,1 $\mathrm{mm}$ de longitud. Estilo 4,5 $\mathrm{mm}$ (fase estaminada) a $7 \mathrm{~mm}$ (fase pistilada). Cipselas blancas, prismáticas, 2,2-2,6 x 1-1,7 mm, con 5 carenas estrechas, longitudinales, lisas coronada por los sépalos, 0,5-0,7 x 0,35-0,8 mm, rodeando un apículo central 
cupuliforme, 0,2-0,4 $\mathrm{mm}$ de longitud; perianto acrescente sobre el fruto, verde-rojizo, hipanto 5-5,5 x 2-2,5 mm, corola 1,3 x 1,2-1,4 mm. Semilla 1,6-1,8 x 0,6 mm.

Distribución. Andes de Chile y Argentina, entre $32^{\circ}-36^{\circ} \mathrm{S}$, a partir de los $3000 \mathrm{~m}$.

Etimología. El epíteto específico hace alusión a las inflorescencias que crecen en una masa confluente, densamente agregada.

Discusión. Esta es la única especie de Calyceraceae con un perianto acrescente y persistente sobre el fruto (en el resto de las especies el perianto puede persistir, pero es no-acrescente y se marchita). El perianto tubuloso puede ser blanco o verde; durante la maduración de los frutos el hipanto se torna verdoso hacia el ápice y se hincha debido al desarrollo de un tejido esponjoso, similar al de las carenas de los frutos, tornándose rojiza la corola.

Por el aspecto general de la planta y sus flores, $N$. compactus puede ser confundida con $N$. ventosus. Ambos taxones tienen una afinidad morfológica muy estrecha y, de hecho, son sintópicas. Sin embargo, a pesar de que es difícil identificarlas durante el periodo de floración, los caracteres asociados al fruto marcan una diferencia por demás notable; en $N$. ventosus el perianto se marchita y desprende de la cipsela durante la maduración de los frutos, mientras que en $N$. compactus el perianto acrescente persiste sobre la cipsela durante la maduración de los frutos.

El análisis del material original revela que Boopis diazi está basado en un espécimen que debió ser asignado a $N$. compactus. Este espécimen posee todos los atributos diagnósticos de la especie (láminas foliares espatulado-pinnatifidas, pedúnculos cortos, flores con dientes corolinos rojizos, hipanto longitud y cilíndrico, perianto acrecente persistente sobre el fruto).

Gamocarpha pumila es un sinónimo nomenclatural de Boopis compacta debido a que el material tipo del primero, depositado en $\mathrm{K}$, es un isotipo del segundo. Esta conclusión se basa en el hallazgo de que ambos tipos son fragmentos de la misma planta. Además, las etiquetas de ambos pliegos especifican el mismo coleccionista y la misma fecha (Ph. Germain, 1856-1857), pero difieren en la localidad. El holotipo en SGO indica "Cordillera de Linares" y el tipo en K "Cordiliéres de Maule (sede orient)"; 
no obstante, desde 1826 a 1873, Linares fue parte de la subdivisión administrativa de la provincia de Maule.

Nastanthus falklandicus D.M. Moore, Bot. Not. 120: 18. 1967. TIPO: Islas Malvinas. "West Falkland (Gran Malvina): Port Stephens, Ten Shilling Bay Peninsula, west coast ca. 3 miles SE Stephens Peak, in gravel and sand between loose rock slabs near cliff top, also on bare cliff ledges", 90 m, 28-I-1964, D. M. Moore 707 (holotipo K 000634091 no visto, foto!; isotipos BAB!, GH 00283130 no visto, foto!, RNG no visto, foto!, S09-36193 no visto, foto!). Fig. 35 (A-D).

Geófitos rizomatosos con brotes aéreos erectos, carnosos, de 2-7 cm de altura y 0,5-1,8 $\mathrm{cm}$ de diámetro, rematados en una inflorescencia, estrechándose hacia la base en una estructura más o menos horizontal. Hojas densamente imbricadas, 1.2-4 cm de longitud x 0,2-0,4 cm de ancho, estrechamente oblongas a oblanceoladas o subespatuladas, con la base atenuada en un pecíolo; lámina entera, más carnosa que el pecíolo, con un surco longitudinal medio, ápice agudo a redondeado. Tirsoide terminal progresivamente condensado hacia el ápice, con grupos cimosos basales pedunculados e involucelados, que se vuelven sésiles y coalescentes hacia el ápice del tallo, agregándose hasta fusionarse en un cefalodio; pedúnculos basales usualmente simples, de $2 \mathrm{~cm}$ de longitud, monocéfalos, con una bráctea tectriz foliosa basal, la cual se vuelve cercana al involucro en los cefalodios superiores. Involucro con 3 brácteas estrechamente triangulares, 0,2-0,3 x 0,3-0,4 cm, basalmente connadas. Receptáculo de 0,4-0,8 cm de diámetro. Brácteas receptaculares (grupos cimosos), 6-10, estrechamente elípticas a oblanceoladas, 0,05 x 0,15-0,3 cm. Flores blancas. Sépalos obtusos a suborbiculares, 0,5 x 0,5 $\mathrm{mm}$. Perianto cilíndrico, $3 \mathrm{~mm}$ de longitud, hipanto de $2 \mathrm{~mm}$ de longitud, lóbulos agudos triangulares, cuculados, de $0,8 \mathrm{~mm}$ de longitud. Tubo estaminal inserto en la mitad basal del hipanto, con 5 glándulas nectaríferas, y filamentos libres, cortos. Anteras de 0,6 mm de longitud. Estilo de $1 \mathrm{~mm}$ de longitud. Cipselas inmaduras prismáticas, de $2,5 \mathrm{~mm}$ de longitud, con 5 carenas longitudinales coronadas por los sépalos. Cipselas maduras desconocidas. 
Distribución. Es endémica de la islas Malvinas, aparentemente restringida a la costa sudoeste de la isla Gran Malvina (Moore, 1967).

Etimología. El epíteto específico se refiere al sitio geográfico en donde esta especie está circunscripta (islas Malvinas/Falkland).

Discusión. La especie sólo se conoce por la colección tipo y un paratipo coleccionado en 1839, Robinson s.n. (K) (Moore, 1967). Como no fue posible estudiar el material tipo, la descripción se basó en la publicación original de Moore (1967). De acuerdo con este autor, N. falklandicus es protógino, un caracter muy improbable considerando el mecanismo de presentación secundaria de polen típico de Calyceraceae (Erbar \& Leins, 1995).

Esta especie se distingue por su hábito, con hojas oblongas a oblanceoladas o subespatuladas, densamente dispuestas a lo longitud del tallo, e inflorescencias compuestas. Las flores son blancas y despiden un fuerte olor a néctar.

Nastanthus patagonicus Speg., Anales Mus. Nac. Buenos Aires 7: 307. 1902. TIPO: Argentina. "Rarissime in aridissimis saxosis prope Teka-choique", XII-1899, N. Illín s.n. (holotipo LP ex LPS 11033!). Fig. 36.

Acarpha laciniata Stapf, Bot. Mag. 155: sub t. 9272. 1929 [1932]. TIPO: Argentina. «Croit sur les ou entre les cailloux sur les rives du Limay et du Chubut. Patagonie", 1865, G. Claraz 93 (holotipo K 000009720 no visto, foto!).

Hemicriptófitos rosulados de 2-25 cm de altura y 6-20 cm de diámetro. Raíz napiforme. Hojas espatuladas, de 2-7 cm de longitud, con la base atenuada en un pecíolo de 1,3-4 x 0,15-0,5 cm; lámina 0,8-3 x 0,8-3 cm, lobada, margen dentado, lóbulos obtusos a triangulares, mucronados. Cefalodio terminal grande, con pedúnculo de $15 \times 3 \mathrm{~cm}$ en especímenes maduros. Involucro con base fusionada rotácea, lóbulos, 0,5-1 x 0,35-0,8 $\mathrm{cm}$, variables en forma: triangular mucronados en el ápice; triangular, brevemente dentados, con ápice y dientes mucronados; o espatulados lobados con ápice y lóbulos mucronados. Receptáculo convexo, de 1-6 cm de diámetro, acrescente durante la 
madurez de los frutos. Brácteas del receptáculo gruesas, espatuladas a rectangulares, ápice obtuso mucronado, $0,35 \times 0,1 \mathrm{~cm}$ en el receptáculo florífero a 1 x $0,7 \mathrm{~cm}$ en el receptáculo con frutos. Flores verde blancuzco, más de 1.000 en el gran cefalodio central. Algunos ejemplares desarrollan grupos cimosos (o cefalodios) laterales, menores, con 20-500 flores, sobre pedúnculos cilíndricos, 2-20 x 0,1-0,5 cm. Perianto infundibuliforme, de 3,8-4,5 $\mathrm{mm}$ de longitud. Sépalos pequeños, suborbiculares, emarginados, translúcidos, 0,3-0,4 x 0,5-0,7 mm. Hipanto 2,5-3 mm de longitud x 0,81,2 $\mathrm{mm}$ de ancho. Corola campanulada, 1,6-1,8 x 0,9 mm, lobada en 1/2 de su parte superior; lóbulos triangular-lanceolados, cuculados inflados, ápice agudo a obtuso, recto, 1,4-1,8 x 0,8-0,9 mm, estivación valvar. Glándulas nectaríferas hipantiales oblongas, difusas, 0,6 x 0,2 mm. Tubo estaminal de $1 / 5$ de la longitud de la corola. Filamentos apicales libres 0,2-0,4 mm. Anteras oblongas, tecas basalmente obtusas con caudículas, conectivo no apiculado, $1 \mathrm{~mm}$ de longitud. Estilo exserto, $5 \mathrm{~mm}$ (fase estaminada) a $6 \mathrm{~mm}$ de longitud (fase pistilada). Ovario elipsoide a lacrimiforme, 1,4-2 x 0,8-1 mm. Cipselas prismáticas, rugosas, 2,4-2,7 x 1-1,6 mm, con 5 carenas longitudinales, delgadas, estrechas, laminares, coronadas por un apículo de 0,25-0,35 mm de longitud, con sépalos lanceolados reducidos o acrescentes ( 1 x $1 \mathrm{~mm}$ ). Semilla 2 x $0,7 \mathrm{~mm}$.

Distribución. Endémico del sur de Argentina, desde el norte de Neuquén a Santa Cruz, creciendo en la Provincia Patagónica. Ocasionalmente puede hallársela en los espacios abiertos del Distrito Austral (Provincia Altoandina), creciendo en los pisos de vegetación superiores a los bosques de la Provincia Subantártica.

Etimología. El epíteto específico hace alusión a la región geográfica donde esta especie crece exclusivamente.

Discusión. Esta especie se distingue por poseer las inflorescencias más grandes de la familia, con un cefalodio central de 1-6 cm de diámetro, el cual puede llevar alrededor de 1000 flores; las brácteas del receptáculo también son grandes. Las flores son verde blancuzcas.

De acuerdo con Chiapella (1999a), las individuos jóvenes de $N$. patagonicus pueden ser confundidos con N. scapigerus debido a la similitud de las hojas, el color de 
las flores, inflorescencias y hábito. La ausencia de un pedúnculo central más grande, y la presencia de inflorescencias de tamaño similar en individuos jóvenes de $N$. patagonicus aumentan la similitud entre ambas. Sin embargo, ambas entidades pueden ser diferenciadas por la morfología de las carenas del fruto y los sépalos, y por el tamaño floral.

Nastanthus scapigerus (J. Rémy) Miers, Ann. Mag. Nat. Hist. ser. 3, 6: 188. 1860. Boopis scapigera J. Rémy, Fl. Chil. [Gay] 3(3): 250. 1848. TIPO: Chile. Provincia de Colchagua, "In rupestris summ. andinum Talcaragone (Cajón del Azufre)", II-1833, C. Gay 375 (lectotipo P 00852232 no visto, foto 37100 del Field Museum of Natural History en SI! designado por Zavala-Gallo et al. 2010; duplicados P 00852231 no visto, P 00852233 no visto, P 00852234 no visto). Fig. 35 (E-G).

Boopis spathulata Phil., Linnaea 28: 708. 1856, syn. nov. publicado. Nastanthus spathulatus (Phil.) Miers, Ann. Mag. Nat. Hist. ser. 3, 6: 189. 1860. TIPO: Chile. "Cordillera de Linares", I-1856, R. A. Philippi s.n. (lectotipo SI 12723! designado por Zavala-Gallo et al. 2010; duplicado W no visto, foto 31053 del Field Museum of Natural History en SI!).

Nastanthus laciniatus Miers, Ann. Mag. Nat. Hist. ser. 3, 6: 187. 1860 syn. nov. publicado. Boopis laciniata (Miers) Ball, J. Linn. Soc., Bot. 21: 220. 1884 [1886 publ. 1884]. Nastanthus agglomeratus Miers var. laciniatus (Miers) Reiche, Anales Univ. Chile 106: 1030. 1900. Boopis agglomerata (Miers) Hauman var. laciniata (Miers) Hauman, An. Soc. Cient. Arg. 85: 312, 1918. TIPO: Chile. "Cordillera Chile", $H$. Cuming 326 (lectotipo K 000009721 no visto, foto! designado por Zavala-Gallo et al. 2010; duplicados BM 000947741 no visto, foto!, E 00259116 no visto, foto!, E 00259118 no visto, foto!).

Boopis araucana Phil., Anal. Univ. Chile 41: 736. 1872, syn. nov. publicado. Nastanthus araucanus (Phil.) Reiche, Anales Univ. Chile 106: 1033. 1900. TIPO: Chile. "San Lorenzo a orillas del río Duqueco", J. Volkmann s.n. (lectotipo SGO-057225 no 
visto, foto! designado por Zavala-Gallo et al. 2010; duplicados SGO 043594 no visto, foto!, K 000009716 no visto, foto!).

Boopis bellidifolia Phil., Anales Univ. Chile 41: 736. 1872, syn. nov. publicado. Nastanthus bellidifolius (Phil.) Reiche, Bot. Jahrb. Syst. 29: 114. 1900. Nastanthus spathulatus (Phil.) Miers var. bellidifolius (Phil.) Pontiroli, Revista Mus. La Plata, Secc. Bot. 9 (4): 238. 1963. TIPO: Chile. "Valle de las Damas, cordillera del Tinguiririca”, I1872, R.A. Philippi s.n. (holotipo SGO 057215 no visto, foto!; isotipo SI 12717!).

Boopis reichei Phil., Anales Univ. Chile 85: 815. 1894, syn. nov. publicado. TIPO: Chile. "In Andibus editioribus provinciae Santiago, v. gr. in Valle longitud”, II1892, C. Reiche s.n. (holotipo SGO 057216 no visto, foto!; isotipo SI 12724!).

Boopis dubia Phil., Anales Univ. Chile 85: 815. 1894, syn. nov. publicado. TIPO: Chile. "Cordillera de Peuco in provincia O’Higgins (Cádiz)”, sin coleccionista indicado (holotipo SGO 057218 no visto, foto!).

Boopis miersii Phil., Anales Univ. Chile 85: 817. 1894. TIPO: Chile. “Andes de Linares en las provincias de Curicó, Colchagua, Santiago", XI-1858, P. Germain s.n. (holotipo SGO 057238!).

Nastanthus chubutensis Speg., Anales Mus. Nac. Buenos Aires 7: 306. 1902, syn. nov. publicado. Nastanthus bellidifolius (Phil.) Reiche var. chubutensis (Speg.) Hicken, Reun. Nac. Soc. Arg. Cienc. Nat. 1: 250. 1919. TIPO: Argentina. "In rupestribus aridis prope Carren-leofú", I-1901, N. Illín s.n. (holotipo LP ex LPS $11034 !)$.

Boopis andicola Gand., Bull. Soc. Bot. France 59: 710. 1913 [1912 publ. 1913], syn. nov. propuesto. TIPO: Patagonia andina, barrancas del río Jeinemeni, 14-XII1908, C. Skottsberg 531 (holotipo probablemente en S; isotipo BA 28888!).

Hemicriptófitos rosulados de $3-10 \mathrm{~cm}$ de altura y $10-25 \mathrm{~cm}$ de diámetro. Hojas espatuladas de 5-10 cm de longitud, con la base atenuada en un pecíolo de 2-8 x 0,2-0,3 
cm; lámina 1-4 x 0,5-2,5 cm, entera con el margen entero a dentado, o irregularmente pinnatifido, y lóbulos obtusos a triangulares, brevemente mucronados, ápice obtuso.

Tirsoide terminal progresivamente condensado hacia el ápice, con grupos cimosos basales pedunculados e involucelados, que se vuelven sésiles y coalescentes hacia el ápice del tallo, agregándose hasta fusionarse en un cefalodio. Grupos cimosos basales 45-80-floros, con pedúnculos cilíndricos, carnosos, a veces ramificados, con una bráctea tectriz foliosa, 2-12 x 0,3-1 cm. Involucelo de los grupos cimosos basales con base fusionada pateliforme, y 5-7 lóbulos ampliamente triangulares, mucronados, 0,55 x 0,5 $\mathrm{cm}$ (a veces espatulados, 0,75 x 0,35 cm), margen entero o con pocos dientes. Receptáculo convexo, 0,6-3,5 cm de diámetro, acrescente durante la maduración de los frutos. Grupos cimosos distales ca. 11-floros, sin involucelos, que coalescen en un único receptáculo junto con las flores solitarias y la flor central, formando un cefalodio sésil, ocasionalmente con un pedúnculo central más grande, $6 \times 1 \mathrm{~cm}$. Las brácteas tectrices se transforman progresivamente en brácteas del receptáculo, escasas, carnosas, verdes, linear-espatuladas apicalmente mucronadas, 0,5 $\mathrm{mm}$ de longitud. Hay un involucro. Perianto infundibuliforme, de 7-8 $\mathrm{mm}$ de longitud. Sépalos suborbiculares, ápice usualmente emarginado, margen brevemente dentado, 1-1,4 x 1-1,4 mm. Hipanto infundibuliforme, 4-4,5 $\mathrm{mm}$ de longitud x 0,2-0,4 $\mathrm{mm}$ de diámetro en la base a 0,8 $\mathrm{mm}$ distalmente. Corola verde, a veces blanca, partida, de 2,5 mm de longitud x 0,5-1 mm de diámetro; lóbulos ligeranente recurvados, oblongos, planos, ápice agudo, recto o uncinado, 2,2 x 0,8 mm, con estivación valvar. Glándulas nectaríferas hipantiales, oblongas de 0,8 x 0,2 mm.Tubo estaminal de doble de la longitud de los filamentos. Filamentos apicales libres de 0,5 mm de longitud. Anteras oblongas, tecas basalmente sagitadas con caudículas, conectivo no apiculado, de $1.4 \mathrm{~mm}$ de longitud. Estilo $6 \mathrm{~mm}$ (fase estaminal) a $10 \mathrm{~mm}$ de longitud (fase pistilada). Cipselas prismáticas 3-5,5 x 2,5-4 $\mathrm{mm}$, con 5 carenas longitudinales, lisas, bien desarrolladas, expandidas, coronadas por los sépalos (1-2,15 x 1,2-2,8 mm), o bien, plegadas, delgadas, poco desarrolladas, apículo de 0,8 mm de longitud. Semillas 2-3,5 x 0,75 mm.

Distribución. Andes de Chile y Argentina entre los $32^{\circ}-42^{\circ}$ S. Existen registros de una población aislada en el noroeste de la pcia. de Santa Cruz (alrededores de la meseta de lago Buenos Aires). La mayor parte de su área de distribución abarca los Distritos Cuyano y Austral de la Provincia Altoandina, con excepción de la población 
aislada que ocupa el Distrito Central (Subdistrito Santacrucense) de la Provincia Patagónica.

Etimología. El epíteto específico hace alusión a los pedúnculos escapiformes que sostienen los grupos cimosos de la inflorescencia.

Discusión. Nastanthus scapigerus es una de las especies más comunes de la cima de los Andes centrales de Chile y Argentina. Por el aspecto general de la planta ha sido confundida usualmente con $N$. ventosus. Sin embargo, $N$. ventosus posee láminas foliares pinnatifidas, flores con hipanto y corola de igual longitud, y corola crateriforme, lobada hasta la mitad de su porción superior

Existen tres ejemplares de Boopis scapigera de la colección original de Claudio Gay con etiquetas del Herb. Mus. Paris; uno de ellos está depositado en P y los otros dos se conservan en $\mathrm{K}$. El ejemplar de $\mathrm{P}$ es elegido como lectotipo debido a que sus estrucruras vegetativas y reproductivas se encuentran bien preservadas y son las que mejor se ajustan a la descripción de Rémy. Uno de los ejemplares de K también se ajusta a la descripción original pero consiste en una planta fragmentada. El otro pliego de $\mathrm{K}$ incluye fragmentos pobremente preservados de una planta que no se ajusta al protólogo de Boopis scapigera. Este material fue identificado originalmente como "Boopis scapigera Remy”, muestra la inscripción en lápiz "non” sobre el nombre, y, en el margen del pliego, una inscripción incompleta donde puede leerse "Nastan...". Estas inscripciones aparecen en todos los ejemplares revisados por Miers para su obra “Contributions to Botany" (1860-1869). Este ejemplar de K es parte del material usado por Miers para tipificar Nastanthus pinnatifidus (= Nastanthus ventosus); sus referencias concuerdan con las del protólogo y ha sido reubicado sobre el mismo pliego con el sintipo Cuming 325.

El nombre Nastanthus spathulatus es uno de los mejores conocidos entre los botánicos chilenos y argentinos; está basado en Boopis spathulata. No obstante, todos los caracteres hallados en los ejemplares tipo de B. spathulata depositados en SI y W claramente coinciden con el material tipo de Boopis scapigera; la única diferencia es que el holotipo de $B$. spathulata está en flor y posee láminas foliares amplias, mientras que el lectotipo de B. scapigera (P) está en fruto. Miers (1860-1869) notó la similitud entre ambas especies pero no sacó conclusión alguna porque no había visto el holotipo 
de Philippi. Sin embargo, estas similitudes son evidentes en la ilustración de $N$. scapigerus en Miers (1860-1869), por lo que se propone su sinonimia. Se elige como lectotipo el ejemplar de SI porque es el mejor conservado y lleva la etiqueta de Philippi.

Miers (1860-1869) estableció la nueva especie Nastanthus laciniatus basado en tres ejemplares de diferentes coleccionistas. Luego, Reiche (1900) trató este taxón como una variedad N. agglomeratus. El análisis de los sintipos reveló que se corresponde con N. scapigerus, debido a que su corola está completamente partida. Considerando los tres sintipos disponibles en K, Cuming 326, de "Cordillera de Chile", se selecciona como lectitipo porque es el mejor preservado, conservando la forma de la planta con muchas inflorescencias y cipselas, y correspondiéndose perfectamente con la descripción de Miers. El resto de los sintipos: Gillies s.n. de "Los Palomares" y Bridges s.n. de "Ojos de Agua”, conservan más estructuras vegetativas (hojas y raíces), pero pocas estructuras reproductivas, que son requeridas para identificar las especies y que fueron especialmente usadas por Miers.

Algunos de las sinónimos aquí listados ya fueron citados como sinónimos con anterioridad: Boopis dubia y Boopis reichei bajo Nastanthus spathulatus (y aquí propuestos bajo N. Scapigerus); y Boopis miersii bajo Nastanthus scapigerus (Reiche 1900, Zanotti \& Pozner 2008). Todos ellos representan formas morfológicas relacionadas con diversas presiones ambientales que existen a lo longitud de la distribución andino-patagónica de la especie, incluyendo los rangos longitudinales, latitudinales y altitudinales.

Boopis araucana, conocida sólo por el material tipo, es un especímen con pedúnculos hojosos más longituds y más ramificados que en la condición típica de $N$. scapigerus, pero un estudio exhaustivo de la forma de las inflorescencias, flores y hojas, muestra que ambos taxones son conespecíficos. Se escoge como lectotipo el ejemplar SGO 057225 debido a que posee mayor cantidad de material reproductivo que SGO 043594.

Debido a la existencia de varias formas dentro de $N$. scapigerus y a la ausencia de caracteres diagnósticos entre ambos taxones se considera que Boopis bellidifolia es sinónimo de $N$. scapigerus.

Nastanthus chubutensis fue creado en base a dos especímenes del límite andino en la provincia de Chubut. Sus caracteres permiten asociarlo con la descripción de Rémy y su aspecto general también se asemeja al lectotipo de B. scapigera, ya que 
todos poseen frutos. Probablemente, estas similitudes fueron notadas con anterioridad por Hicken (1919), quien consideró a Nastanthus chubutensis como una variedad $N$. bellidifolius.

Boopis andicola fue creado en base a un ejemplar coleccionado en los alrededores de la meseta del lago Buenos Aires. Sus caracteres diagnósticos caen dentro del rango de variación esperado para $N$. scapigerus. El isotipo se asemeja a los de Boopis araucana y B. miersii. Es una hierba caulescente, ramosa, sin pedúnculos escapiformes, sin cefalodio central mayor y con numerosos cefalodios terminales que muestran el patrón arquitectural típico de la especie.

Además del ejemplar tipo, se conocen otros tres ejemplares atribuibles a B. andicola: Sánchez et al. 600, Paiaro s.n. 4 y Zuloaga et al. 13971, procedentes de la misma área de colección. La presencia de corola blanca, excepcional en individuos de la población altoandina (registrada sólo para el especímen Biganzoli 1193), y las cipselas con carenas delgadas, poco desarrolladas y algo plegadas, son caracteres distintitivos de este taxón. Es probable que $B$. andicola sea el representante de una variedad diferente de $N$. scapigerus que se mantiene reproductivamente aislada del resto de las poblaciones de la especie. No obstante, hasta obtener más registros que permitan efectuar análisis morfológicos y moleculares para tomar una decisión taxonómica respecto al cambio de categoría de $B$. andicola, prefiero tan sólo situarla en sinonimia bajo Nastanthus scapigerus.

La comparación entre $N$. scapigerus y sus taxones relacionados, tratados aquí como sinónimos, muestra que los caracteres morfológicos vegetativos son apenas variables, mientras que los reproductivos (caracteres florales) permanecen constantes.

La ilustración publicada por Chiapella (1999a: 514, Fig. 397) de N. scapigerus corresponde a $N$. patagonicus.

Nastanthus ventosus (Meyen) Miers, Ann. Mag. Nat. Hist. ser. 3, 6: 188. 1860. Calycera ventosa Meyen, Reise um die Erde 1: 356. 1834. Boopis scapigera J. Rémy var. ventosa (Meyen) Weddell, Chl. And. 2: 7. 1858. Nastanthus caespitosus (Phil.) Reiche var. ventosus (Meyen) Pontiroli, Revista Mus. La Plata, Secc. Bot. 9 (4): 233. 1963. TIPO: Chile. "Rio del Volcan [...] Am Ende des Thales hatten wir die Schneegrenze erreicht", F.J.F. Meyen s.n. [holotipo B $\dagger$, lectotipo H. A. Weddell, 
Chloris Andina 2: Planche 44A(1-6), sub Boopis scapigera. 1857(1858)! designado por Zavala-Gallo et al. 2010]. Fig. 37.

Boopis gayana Phil., Linnaea 28: 709. 1856. Nastanthus gayanus (Phil.) Miers, Ann. Mag. Nat. Hist. ser. 3, 6: 189. 1860. Nastanthus agglomeratus Miers var. gayanus (Phil.) Reiche, Anales Univ. Chile 106: 1030. 1900. TIPO: Chile. "In Andibus prov. Arauco ad Santa Barbara", XII-1838, C. Gay 1491 (holotipo SGO 057223 no visto, foto!).

Nastanthus agglomeratus Miers, Ann. Mag. Nat. Hist. ser. 3, 6: 186. 1860, syn. nov. publicado. Boopis agglomeratus (Miers) Hauman, An. Soc. Cient. Arg. 85: 311. 1918. Calycera andina Miers, Trav. Chil. 2: 531. 1826, nom. nud. Nastanthus andina (Miers) H.V. Hansen, Nordic J. Bot. 12 (1): 65. 1992, nom. inval. TIPO: Chile. Near the Cumbre on the descent to Las Cuevas elevation of 11500 feet above the level of the sea. Cordillera of Chile, I-1825, J. Gillies s.n. (holotipo BM 000947740 no visto, foto!; isotipos K 000009723 no visto, foto!, E 00259114 no visto, foto!).

Nastanthus pinnatifidus Miers, Ann. Mag. Nat. Hist. ser. 3, 6: 187. 1860, syn. nov. publicado. Nastanthus agglomeratus Miers var. pinnatifidus (Miers) Reiche, Anales Univ. Chile 106: 1030. 1900. Boopis agglomerata (Miers) Hauman var. pinnatifidus (Miers) Hauman, An. Soc. Cient. Arg. 85: 312. 1918. TIPO: Chile. "In Andibus Chilensibus", 1831, H. Cuming 325 (lectotipo K-000009718 no visto, foto! designado por Zavala-Gallo et al. 2010; duplicados E 00259115 no visto, foto!, E 00259117 no visto, foto!).

Nastanthus gilliesii Miers, Ann. Mag. Nat. Hist. ser. 3, 6: 188. 1860. Nastanthus agglomeratus Miers var. gilliesii (Miers) Reiche, Anales Univ. Chile 106:1030. 1900. TIPO: Chile. "In Andibus Chilensibus, Los Palomares", sin fecha indicada, J. Gillies s.n. (holotipo K 000009723 no visto, foto!).

Boopis sanjuanina Hieron., Bol. Acad. Nac. Cs. Cba. 4(1): 31. 1881, syn. nov. publicado. Boopis agglomerata (Miers) Hauman var. sanjuanina (Hieron.) Hauman, 
An. Soc. Cient. Arg. 85: 312, 1918. TIPO: Argentina. San Juan. Arroyo de la Ciénaga del Medio cerca del Leoncito, I-1876, S. Echegaray s.n. (holotipo CORD!).

Boopis breviflora Phil., Anales Univ. Chile 85: 814. 1894. Nastanthus agglomeratus Miers var. breviflorus (Phil.) Reiche, Anales Univ. Chile 106: 1031, 1900. TIPO: Chile. "Coquimbo: In Andibus Illapelinis 1. d. La Polcura”, I-1888, F. Philippi 2320 (lectotipo SGO 043591 no visto, foto! designado por Zavala-Gallo et al. 2010; duplicados SGO 057222 no visto, foto!, SI 12637!).

Boopis oocaulis Kuntze, Revis. Gen. Pl. 3(3): 127. 1898, syn. nov. publicado. TIPO: Argentina. Cordillera de Mendoza: Paso Cruz, 2800 m, I-1892, O. Kuntze 107 (holotipo CORD no visto, foto!; isotipo SI 070776!).

Hemicriptófitos rosulados de 2,5-10 cm de altura y 5-20 cm de diámetro. Raíz??? Hojas parcialmente carnosas, espatuladas, $3-12 \mathrm{~cm}$ de longitud, con la base atenuada en un pecíolo de 2-3 x $1 \mathrm{~cm}$; lámina pinnatífida, 1-6 x 1-3 cm, lóbulos obtusos a lanceolados, ápice obtuso. Tirsoide terminal progresivamente condensado hacia el ápice, con grupos cimosos basales pedunculados e involucelados, que se vuelven sésiles y coalescentes hacia el ápice del tallo, agregándose hasta fusionarse en un cefalodio. Grupos cimosos basales 10-plurifloros, aglomerados, con pedúnculos más cortos que las hojas, comprimidos, carnosos, con una bráctea tectriz foliosa. Involucelo de los grupos cimosos basales externos rotáceos a crateriformes con la base soldada, lóbulos amplios triangulares de 0,2-1 x 0,3-0,5 cm. Receptáculo convexo, de 0,5-3 cm de diámetro, acrescente durante la maduración de los frutos. Grupos cimosos distales ca. 2-8-floros, sin involucelos, que coalescen en un único receptáculo junto con las flores solitarias y la flor central, formando un cefalodio sésil. Brácteas del receptáculo ausentes, o 1-2 pequeñas, filiformes, a lo sumo de $0,5 \mathrm{~mm}$ de longitud en receptáculos con frutos. Hay un involucro. Perianto infundibuliforme, de 4-4,5 mm de longitud. Sépalos triangulares, obtusos, emarginados, inflados, 0,3-0,4 x 0,4-0,5 mm. Hipanto tubuloso, de 2,35 x 0,9 $\mathrm{mm}$. Corola blanca, a veces verde, crateriforme, 2 x 1,5 mm; lóbulos triangulares, ápice agudo a obtuso, uncinado, 1 x 0,7-1 mm, estivación valvar. Glándulas nectaríferas oblongas de 0,5 x 0,2 $\mathrm{mm}$ y posición media. Tubo estaminal de 0,3 mm. Filamentos apicales breves. Anteras oblongas, tecas basalmente sagitadas, conectivo no apiculado, 
de 1,3 $\mathrm{mm}$ de longitud. Estilo exserto, $5 \mathrm{~mm}$ (fase estaminada) a $7 \mathrm{~mm}$ (fase pistilada). Ovario cilíndrico, 2-2,5 x 1,2 mm. Cipselas prismáticas, 5-carenadas, 2-8,5 x 2-4 mm, apículo cónico ausente a $0,5 \mathrm{~mm}$ de longitud, y coronado por los sépalos persistentes, 1 2,5 x 0,5-1,5 mm, continuos a las carenas longitudinales. Semilla 1,25-4 x 0,2-0,8 mm.

Distribución. Andes de Chile y Argentina, entre los $27^{\circ}-37^{\circ} \mathrm{S}$, también crece en las cimas de la sierra del Nevado (Mendoza, Argentina).

Etimología. El epíteto específico, ventosus, es un término latino con muchos significados, todos ellos relacionados con el viento y sus atributos. En este caso, puede referirse al tipo de ambiente en el que se desarrolla esta especie (ventosus= ventoso), o a la amplia variabilidad morfológica que caracteriza a esta especie (ventosus= inconstante, variable). También puede referirse al aspecto que le confiere la disposición intensamente agregada de las inflorescencias (ventosus= hinchado).

Discusión. Estas plantas usualmente producen muchos pedúnculos escapiformes agregados en una masa hemisférica y cespitosa (Pontiroli 1963), semejando su aspecto general al de un coliflor (Reiche 1902). Tanto formas condensadas como laxas pueden observarse bajo diferentes condiciones ambientales a lo largo de su distribución andina. Las glándulas nectaríferas son más evidentes durante la fase estaminada. Las flores son verdes en el hipanto y blancas en la corola, aunque pueden encontrarse ejemplares con corola verde.

Miers (1860-1869) consideró Calycera ventosa Meyen bajo Nastanthus y estableció la combinación Nastanthus ventosus (Meyen) Miers. No obstante, Calycera ventosa fue considerada usualmente como un sinónimo de Boopis scapigera J. Rémy a partir de Weddell (1858), y luego como Nastanthus scapigerus (J. Rémy) Miers por Reiche (1901, 1902), Hicken (1919), Chiapella (1999a) y Zanotti \& Pozner (2008). El estudio del material tipo de Calycera ventosa y de Boopis scapigera provee la separación de la primera especie por sus sépalos más pequeños, hojas pinnatifidas, e inflorescencias muy condensadas (véase también Rémy, 1848). Además, el análisis de numerosos ejemplares de herbario correspondientes a Nastanthus agglomeratus y el material tipo de Calycera ventosa revelaron que comparten la mayoría de los carcteres de sus estructuras reproductivas, y que Calycera ventosa puede ser ubicada dentro de la 
variación morfológica considerada para N. agglomeratus, difiriendo sólo por sus pedúnculos más longituds y menos condensados. Por lo tanto, se considera que ambos taxones son conspecíficos, y el primer nombre legítimo es Nastanthus ventosus (Meyen) Miers.

El holotipo de Calycera ventosa Meyen, presuntamente depositado en B, fue destruido. Todos los herbarios donde todavía se conservan ejemplares de Meyen (BR, CAS, CGE, K, L y P; Stafleu \& Cowan, 1988) fueron consultados pero no pudo hallarse ningún isotipo de Calycera ventosa. Meyen (1834) no citó paratipos. Por lo tanto, se elige la ilustración de Weddell como lectotipo debido a la evidencia que sugiere que se basó en el material original de Meyen: "The drawings (probably based in part on sketches by Weddell) are by Alfred Riocreux ..." (Stafleu \& Cowan, 1988, vol. 7, p. 140), y Reiche (1902: 192) señaló: "La figura de Weddell se estableció sobre una muestra original".

Los isotipos de N. agglomeratus (Gillies s.n.), N. laciniatus (Cuming 326) y N. pinnatifidus (Cuming 325) están montados sobre el mismo pliego de herbario en E, pero las etiquetas no se corresponden con los ejemplares. Esto se hace evidente cuando se comparan los isotipos de $\mathrm{E}$, con los tipos de $\mathrm{K}$ y $\mathrm{BM}$, las ilustraciones y protologos (Miers, 1860-1869). Se concluye que el ejemplar de $N$. laciniatus está asociado con la etiqueta de Cuming 325 (E 00259116) (véase sinonimia de N. scapigerus), el ejemplar de $N$. pinnatifidus con la etiqueta de Gillies s.n. (E 00259115) (véase sinonimia de $N$. ventosus) y el ejemplar de N. agglomeratus con la etiqueta de Cuming 326 (E 00259114) (véase sinonimia de $N$. ventosus). Además, estos errores se encuentran repetidos en otras dos cartulinas de E, donde un ejemplar de $N$. pinnatifidus está etiquetado como Cuming 326 (E 00259117), y un ejemplar de N. laciniatus está etiquetado como Cuming 325 (E 00259118 ).

Nastanthus agglomeratus var. pinnatifidus no se considera una buena variedad debido a que la morfología foliar de $N$. ventosus incluye los caracteres usados para definir la variedad. Se elige como lectotipo el ejemplar de Cuming 325 debido a que es el que mejor describe la morfología de la planta y a que se encuentra mejor conservado que el sintipo Gay s.n. de "Cordillera de Coquimbo"; éste último se encuentra fragmentado y muy poco conservado.

Las variedades restantes de $N$. agglomeratus (N. agglomeratus var. gayanus, $N$. agglomeratus var. gilliesii and N. agglomeratus var. breviflorus) fueron consideradas 
previamente como sinónimos por Zanotti \& Pozner (2008). Se elige al ejemplar SGO043591 como lectotipo de Boopis breviflora, debido a que se encuentra en mejor estado de conservación y cuenta con mayor número de flores y frutos que SGO-057222.

El isotipo de Boopis oocaulis posee láminas cortas que consisten en cinco pequeños lóbulos, inflorescencias paucifloras con 20-30 flores, flores de corola corta con lóbulos blanquecinos breves, asociados estrechamente con $N$. ventosus. Las diferencias con $N$. ventosus (mayor cantidad de flores por inflorescencia y hojas con más de 5 lóbulos) podrían estar ocasionadas por condiciones ambientales tales como el desarrollo del suelo y la disponibilidad de agua. Además, B. oocaulis sólo es conocido por el ejemplar tipo, y otros autores (Pontiroli 1963) ya han señalado la dudosa validez de este taxón.

El ejemplar tipo de Boopis sanjuanina puede ser ubicado con seguridad dentro de la variación morfológica de Nastanthus ventosus; incluso Hieronymus (1881) remarcó su inseguridad al crear el taxón. Por otra parte, Pontiroli (1963) confundió $N$. agglomeratus con $N$. caespitosus y citó erróneamente a Boopis sanjuanina bajo sinonimia de esta última especie.

La comparación entre $N$. ventosus y taxones relacionados, tratados aquí como sinónimos, muestran que los caracteres morfológicos vegetativos varían, mientras que los reproductivos (caracteres florales) permanecen constantes.

\section{NOMBRES DUDOSOS}

Boopis acaulis Phil., nomen dubium, Anales Univ. Chile 18: 49, 1861. Nastanthus acaulis (Phil.) Reiche, Anales Univ. Chile 106: 1032, 1900. TIPO: Chile. "Huanta". Volckmann s.n. (holotipo SGO 057220, no visto, foto SI!).

Sólo se pudo ver una imagen digital del holotipo, y el especímen se encuentra tan depauperado, pobremente preservado y plegado que su morfología no puede evaluarse correctamente. Aunque algunos caracteres lo asemejan a N. ventosus, la decisión acerca de su sinonimia se pospone hasta que pueda efectuarse un examen directo del material tipo. 
Acicarpha lanata Lag. ex Pers., Syn. Pl. (Persoon) 2(2): 488. 1807 [Sep 1807], nomen dubium. Calycera lanata (Lag. ex Pers.) Benth. \& Hook. f., Gen. Pl. [Bentham \& Hooker f.] 2(1): 162. 1873 [7 Feb 1880]. Cryptocarpha lanata Cass., nomen superfluum, Dict. Sci. Nat. (ed. 2) ed. 2, 12: 86. 1819. TIPO: "N. Hispania”, fecha no indicada, L. Née s.n. (holotipo probablemente depositado en P, no localizado).

Bentham \& Hooker (1873) situaron Leucocera annua y Anomocarpus tenuifolius $($ ambas $=$ Calycera leucanthema) como sinónimos de Calycera lanata . Este nombre probablemente corresponde a Calycera leucanthema pero su brevísima descripción descarta su ubicación fidedigna como sinónimo de cualquiera de las especies tratadas en este trabajo. Richard (1820) mencionó que el holotipo probablemente estuviera depositado en $\mathrm{P}$, sin haber podido examinarlo. 


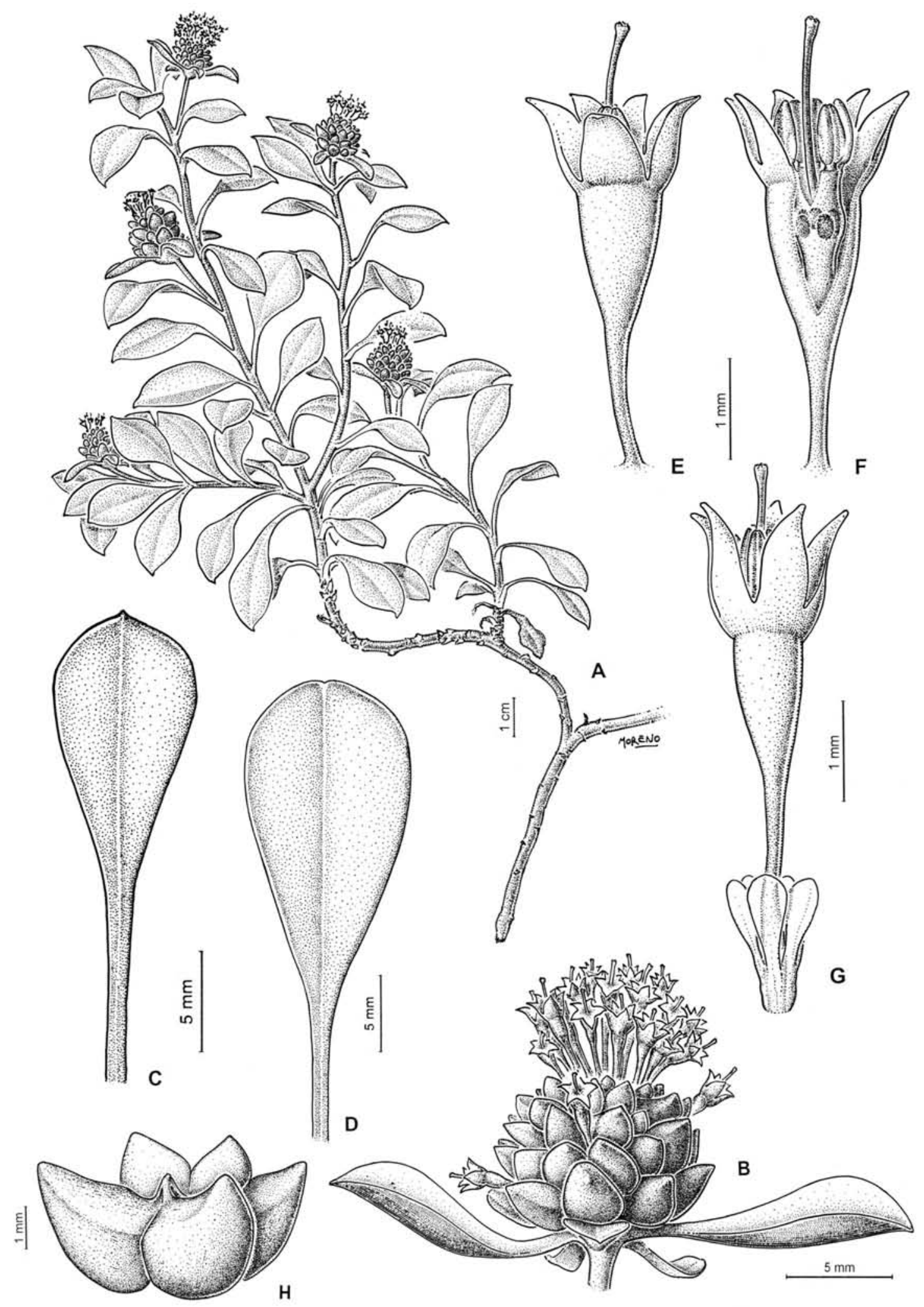

Figura 2. Acicarpha obtusisepala. A, hábito. B, cefalodio mostrando los sépalos acrescentes de las flores perfectas. C, hoja (haz). D, hoja (envés). E, flor perfecta (ovario removido). F, flor perfecta diseccionada, mostrando el androceo (ovario removido). G, flor estaminada. H, vista de la parte superior de la cipsela. Dibujado de C. A. Zanotti 160 (SI). 


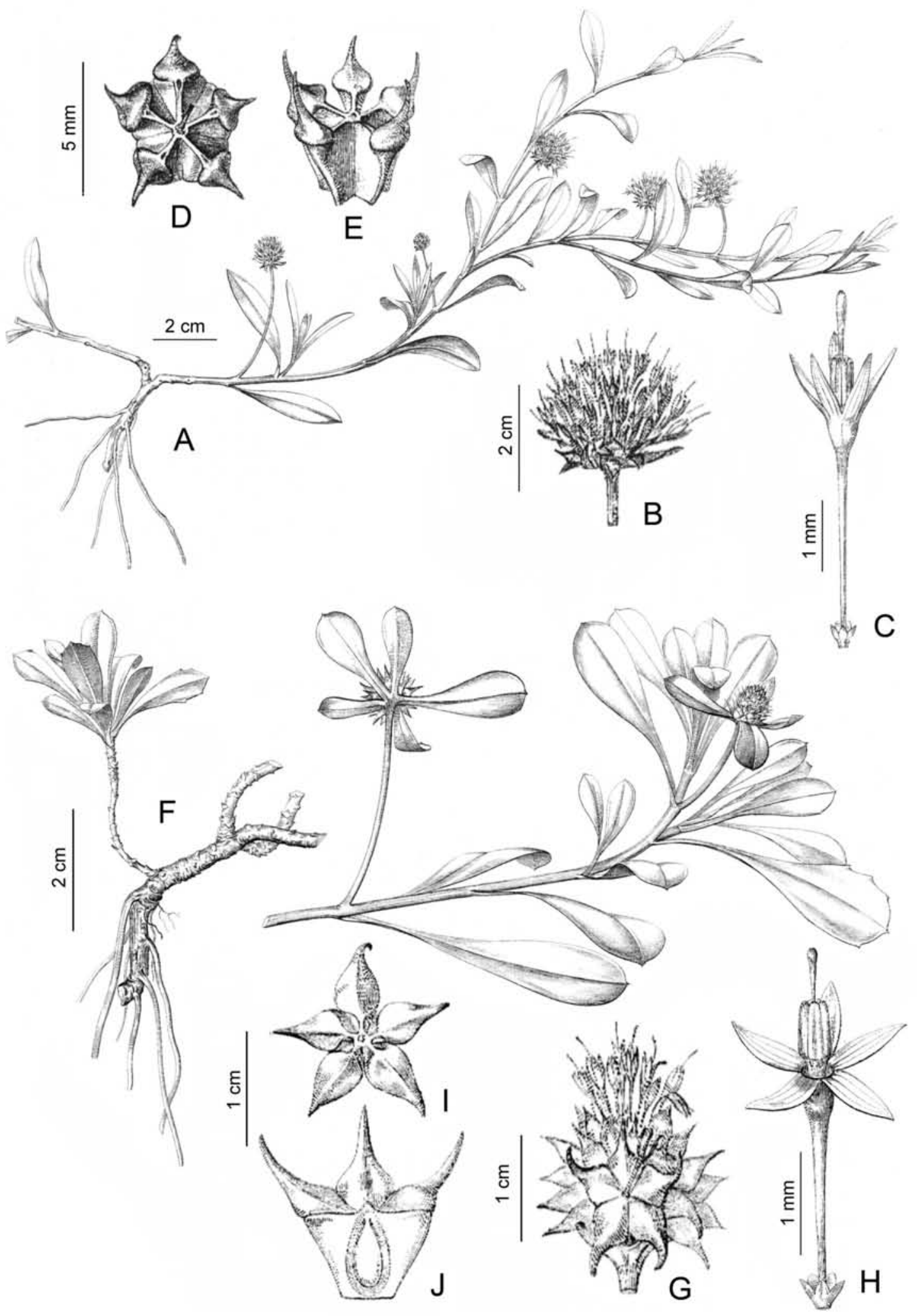

Figura 3. A-E. Acicarpha procumbens. A, hábito. B, cefalodio. C, flor estaminada. D, cipsela libre, vista superior. E, cipsela libre, vista general. F-J. A. spathulata. F, hábito. G, infrutescencia. $\mathrm{H}$, flor estaminada. I, cipsela libre, vista superior. J, cipsela libre, corte longitudinal. (Tomado de Müller, 1885). 


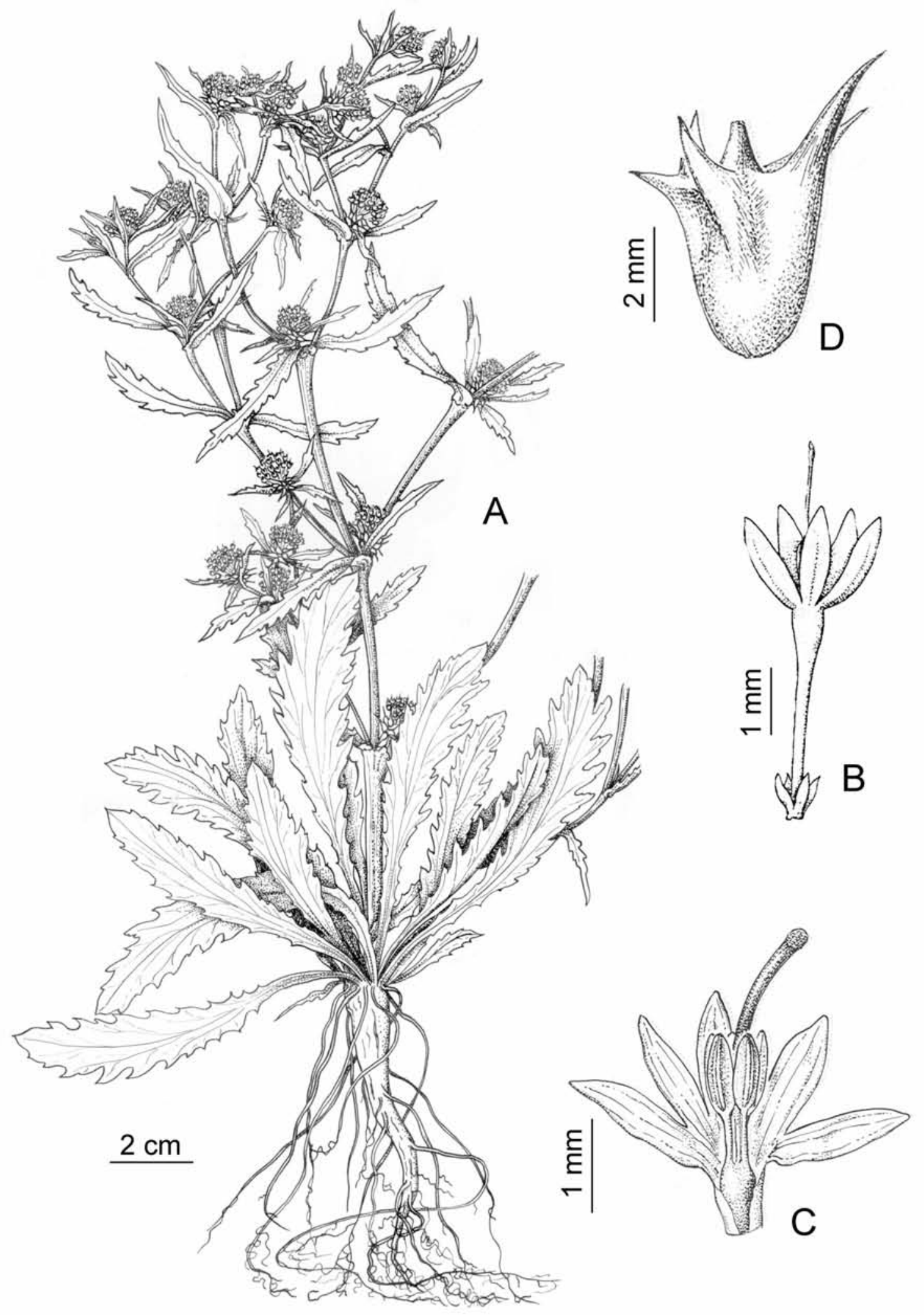

Figura 4. Acicarpha tribuloides. A, hábito. B, flor estaminada. C, flor perfecta diseccionada (parte inferior del hipanto y ovario removidos). D, cipsela libre. (A y B, tomado de Pontiroli, 1963; C y D, tomado de Chiapella, 1999a). 


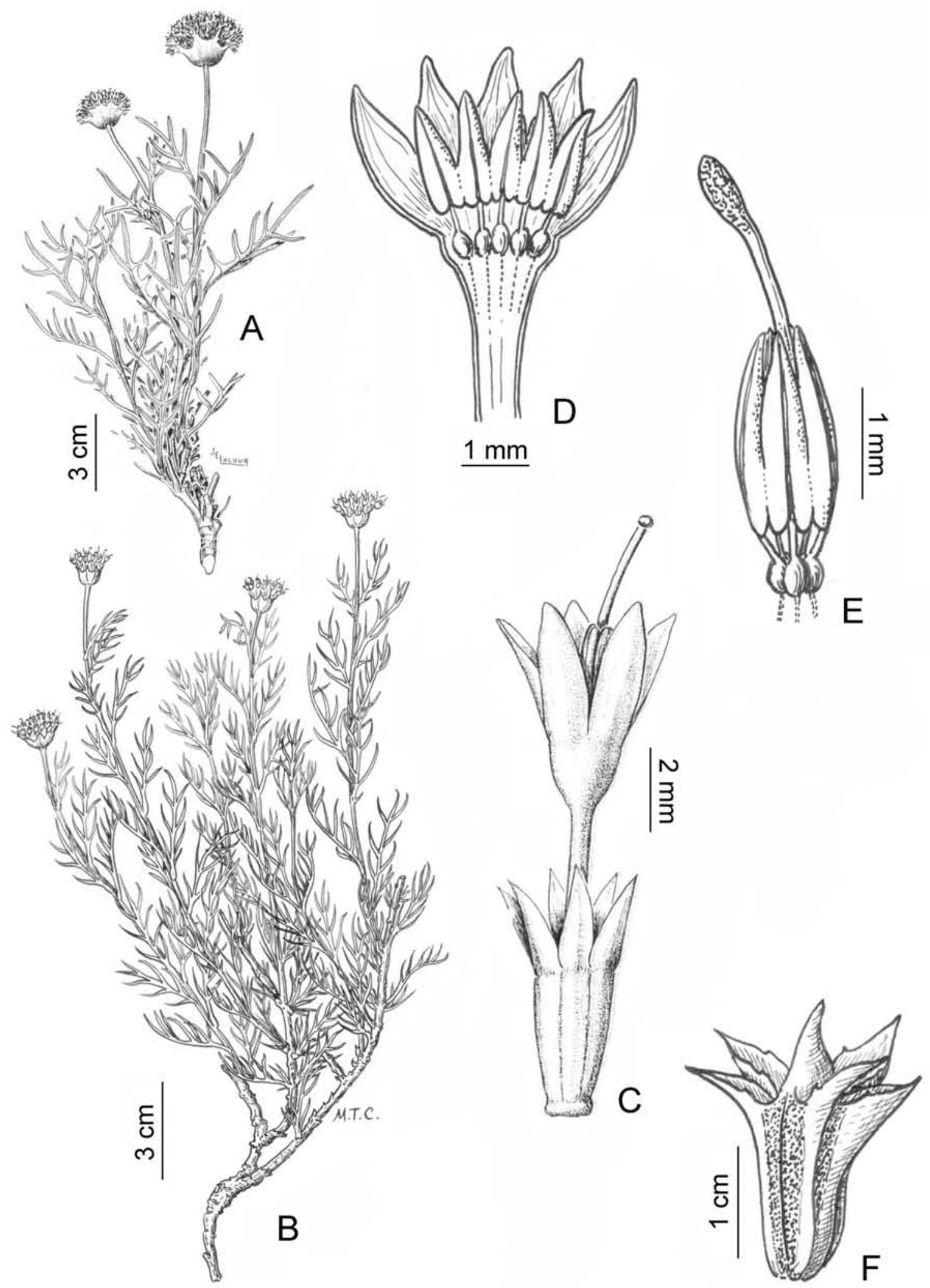

Figura 5. Boopis anthemoides. A-B, variación del hábito. C, flor. D, flor diseccionada (estilo y ovario removidos). E, androceo y estilo. F, cipsela (A y C, tomado de Chiapella 1999a; B, D, E y F, tomado de Pontiroli, 1993). 

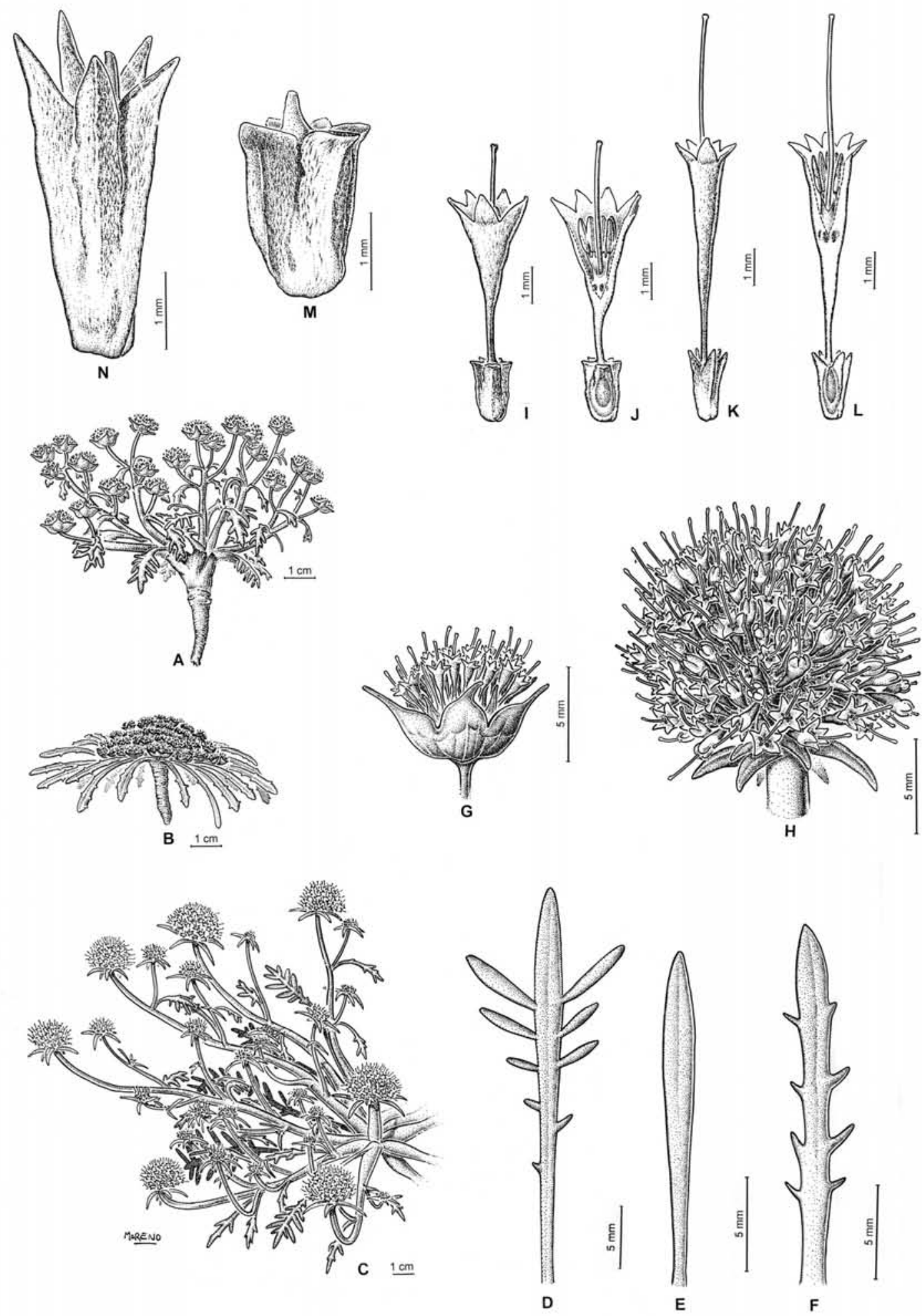

Figura 6. Boopis australis. A-C, variación del hábito. D-F, variación de la hoja. G-H, variación del cefalodio. I, K, variación de la flores. J, L, flores diseccionadas mostrando el androceo y parte del estilo. M-N, variación de la cipsela. A, G, E y M, dibujado de O. Boelcke 14342 (SI); B y F, dibujado de R.N. P. Goodall 4071 (BAB); C, D y H, dibujado de D. Testoni 97 (SI); I y J, dibujado de F. O. Zuloaga 12543 (SI); K, L y N, dibujado de M. J. Belgrano 2 (SI). 


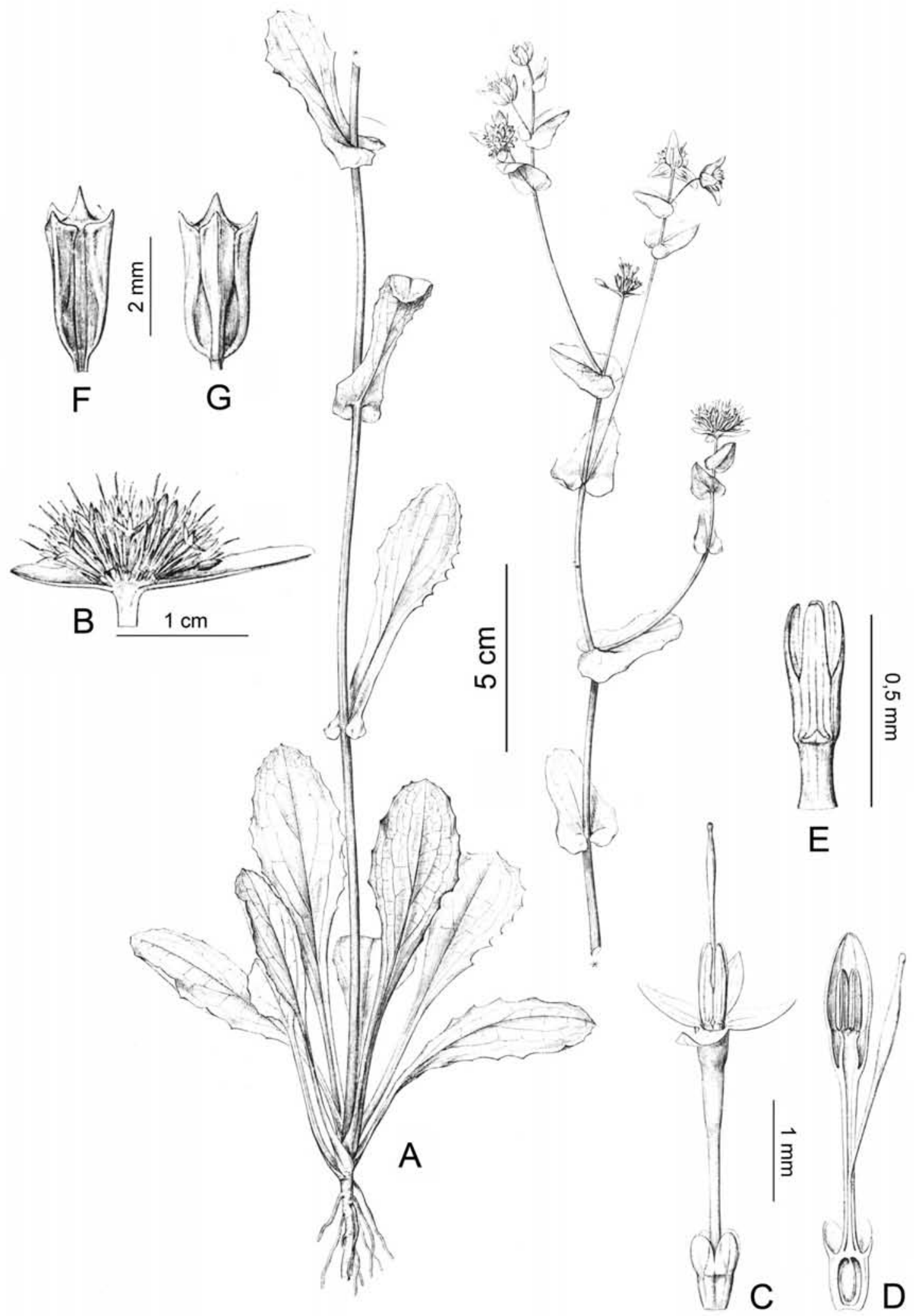

Figura 7. Boopis bupleuroides. A, hábito. B, cefalodio, corte transversal. C, flor. D, botón floral, corte transversal. E, vista de las anteras y la porción superior del tubo estaminal. F-G, cipsela. (Tomado de Müller, 1885) 


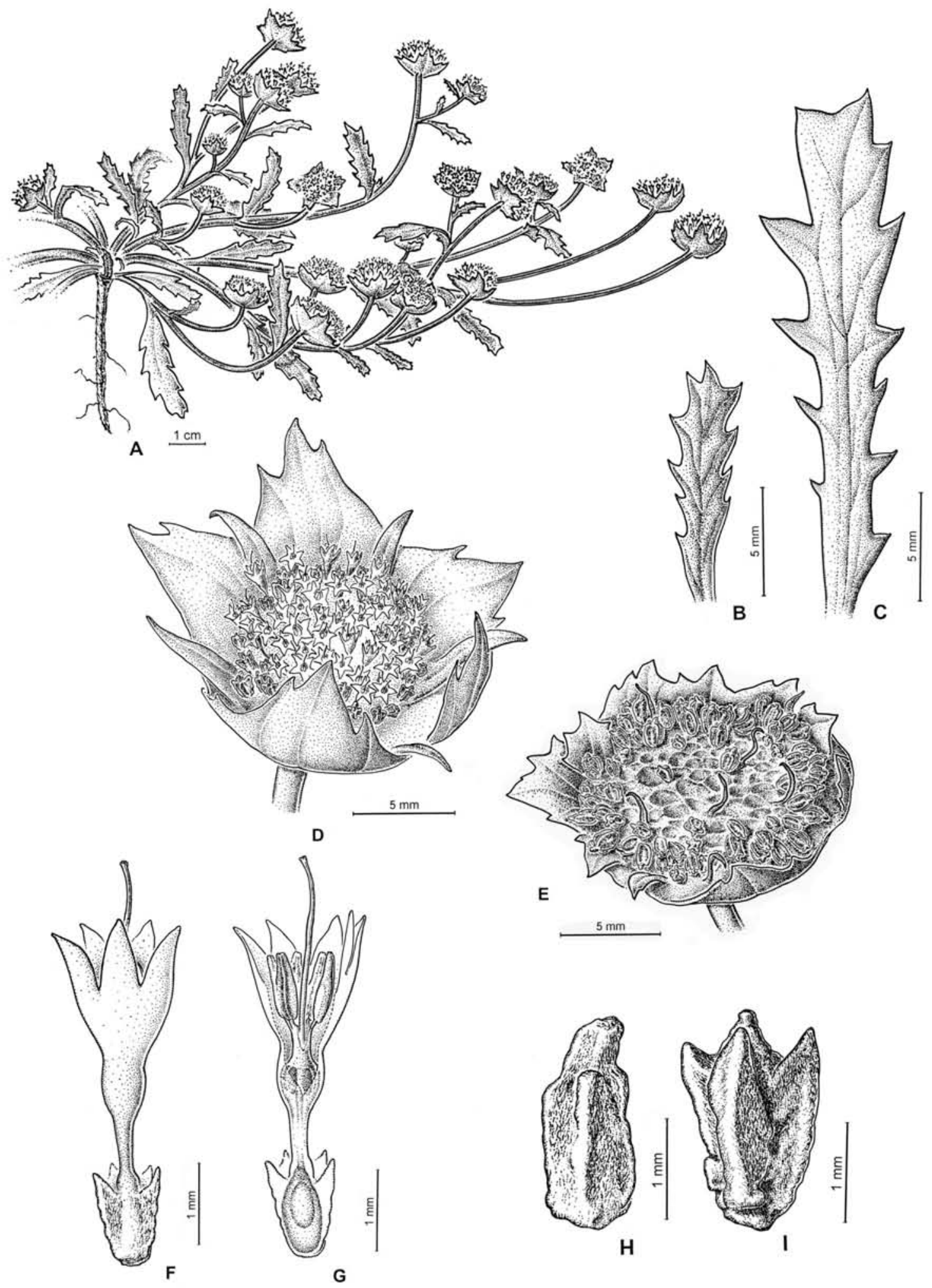

Figura 8. Boopis castilloni. A, hábito. B-C, variación de la hoja. D, cefalodio con receptáculo florífero. E, cefalodio con receptáculo fructífero. F, flor. G, flor diseccionada mostrando el androceo y parte del estilo. H-I, variación de la cipsela. A, B, C, D, F, G, H e I, dibujados de A.T. Hunziker 20985 (CORD); E, dibujado de A.T. Hunziker 20903 (CORD). 

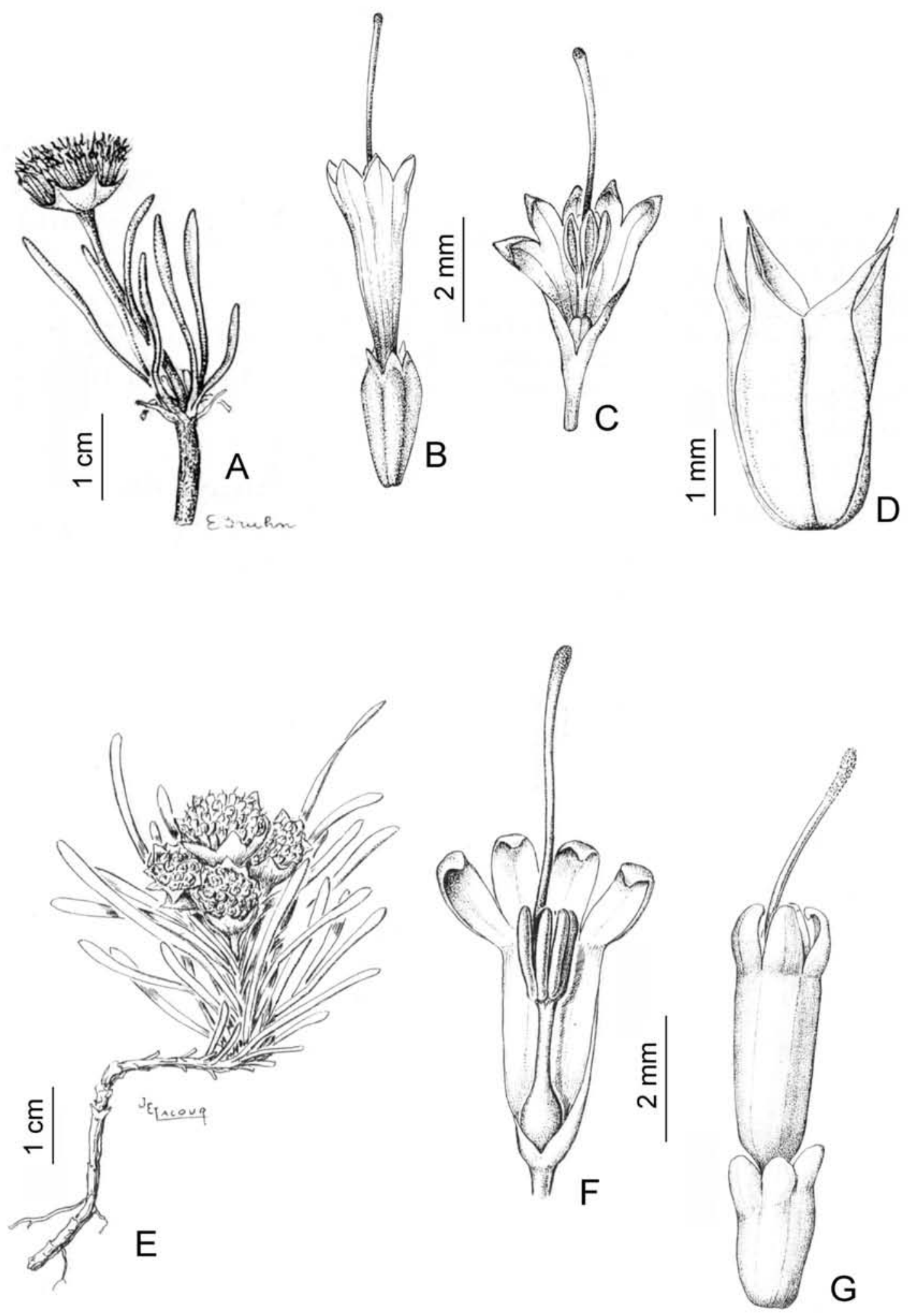

Figura 9. A-D. Boopis chubutensis. A, hábito. B, flor. C, flor diseccionada mostrando el androceo y parte del estilo (ovario removido). D, cipsela. E-G. Boopis filifolia. E, hábito. F, flor. G, flor diseccionada mostrando el androceo y parte del estilo (ovario removido). (Tomado de Chiapella, 1999a) 

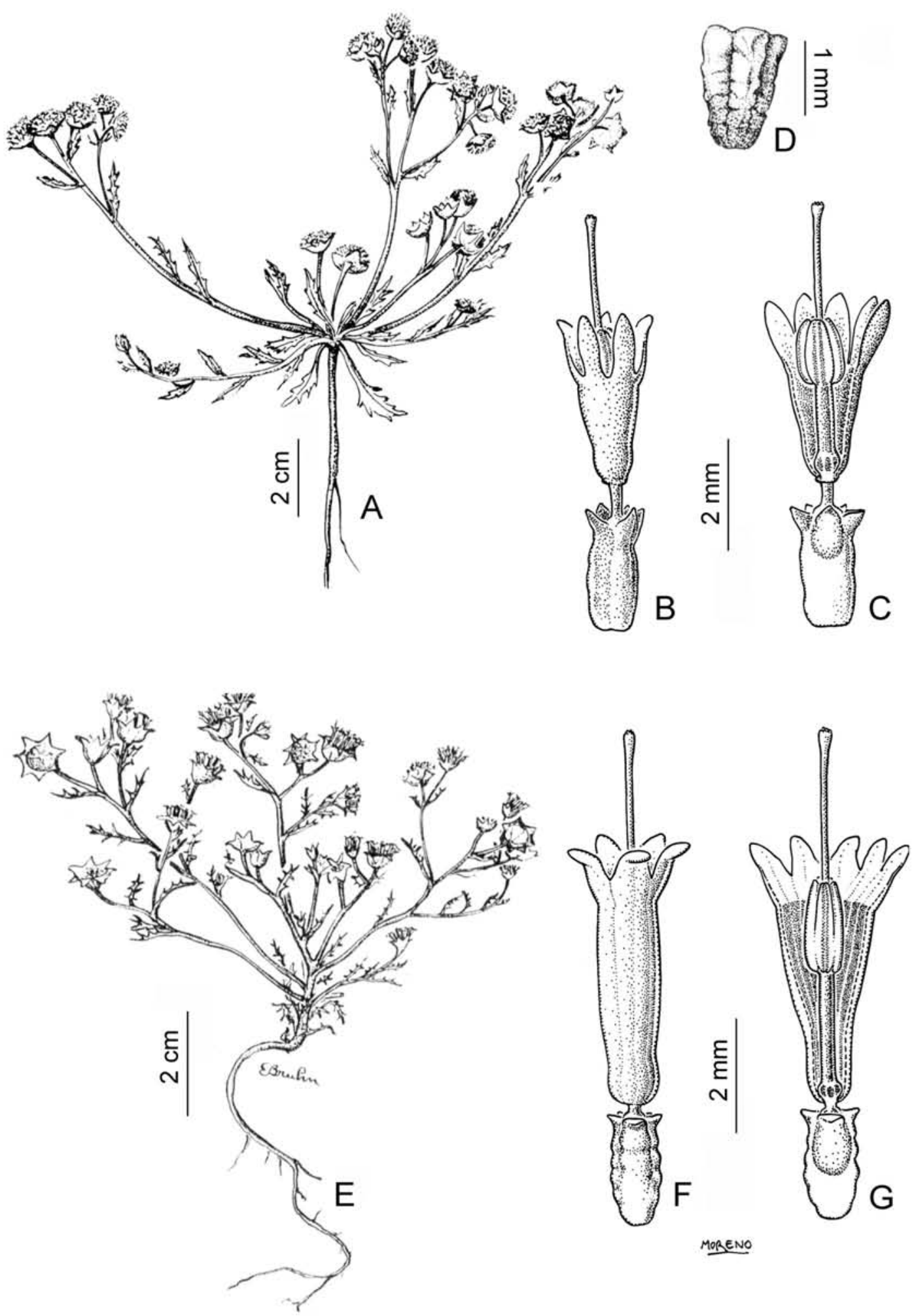

Figura 10. A-D. Boopis gracilis var. gracilis. A, hábito. B, flor. C, flor con la corola diseccionada mostrando el androceo. D, cipsela. E-G. B. gracilis var. lazulina. E, hábito. F, flor. G, flor con la corola diseccionada mostrando el androceo. B y C, dibujados de Zavala 22 (SI); F y G, dibujados de Morrone 6166 (SI). (A, D y E, tomado de Chiapella 1999a; A, sub. Calycera boopidea ). 


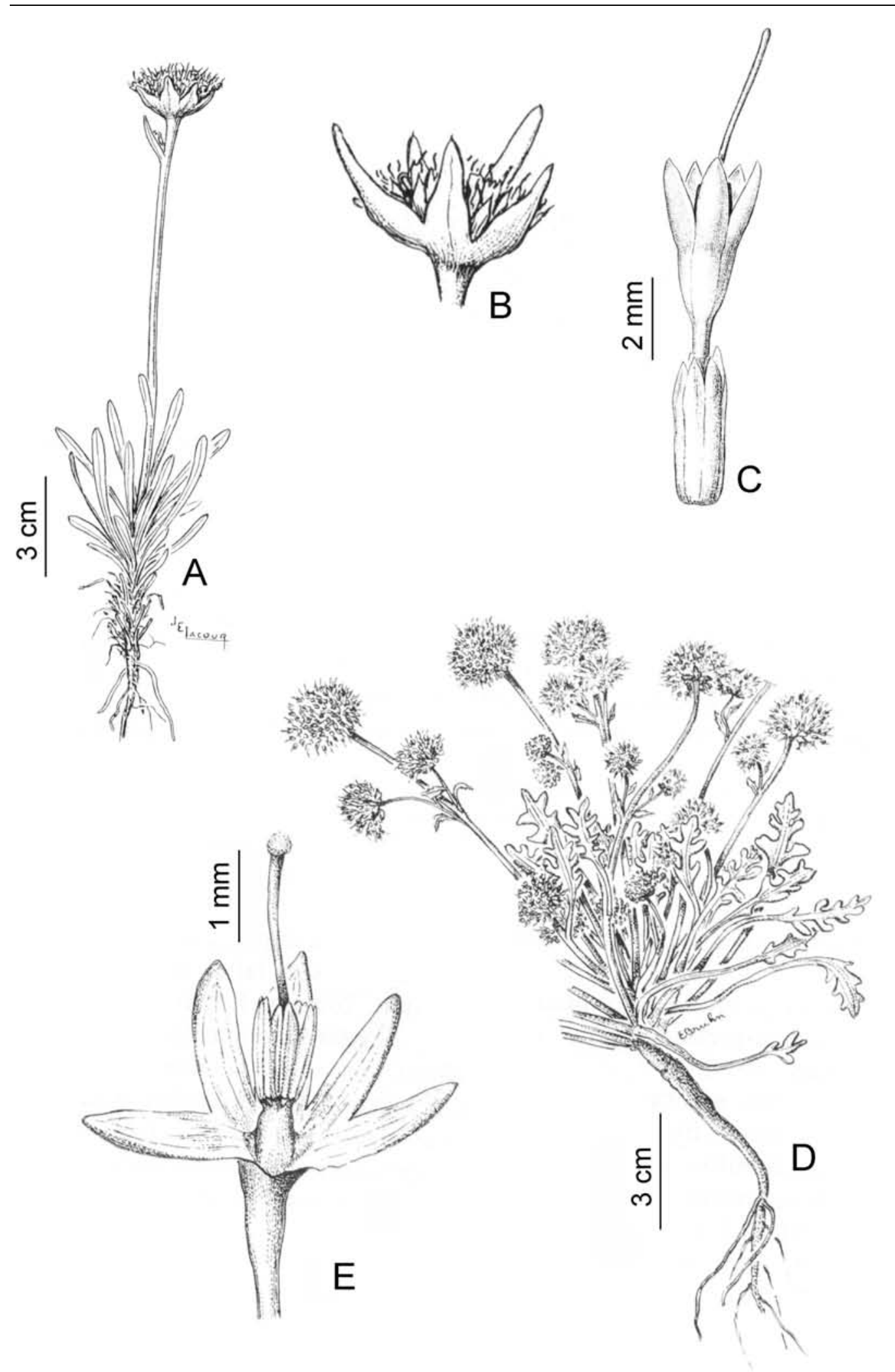

Figura 11. A-C. Boopis graminea. A, hábito. B, cefalodio. C, flor. D-E. B. multicaulis. D, hábito. E, flor diseccionada mostrando el androceo y parte del estilo (porción inferior del hipanto y ovario, removidos). (Tomado de Chiapella, 1999a: D y E, sub. Nastanthus agglomeratus var. pinnatifida). 


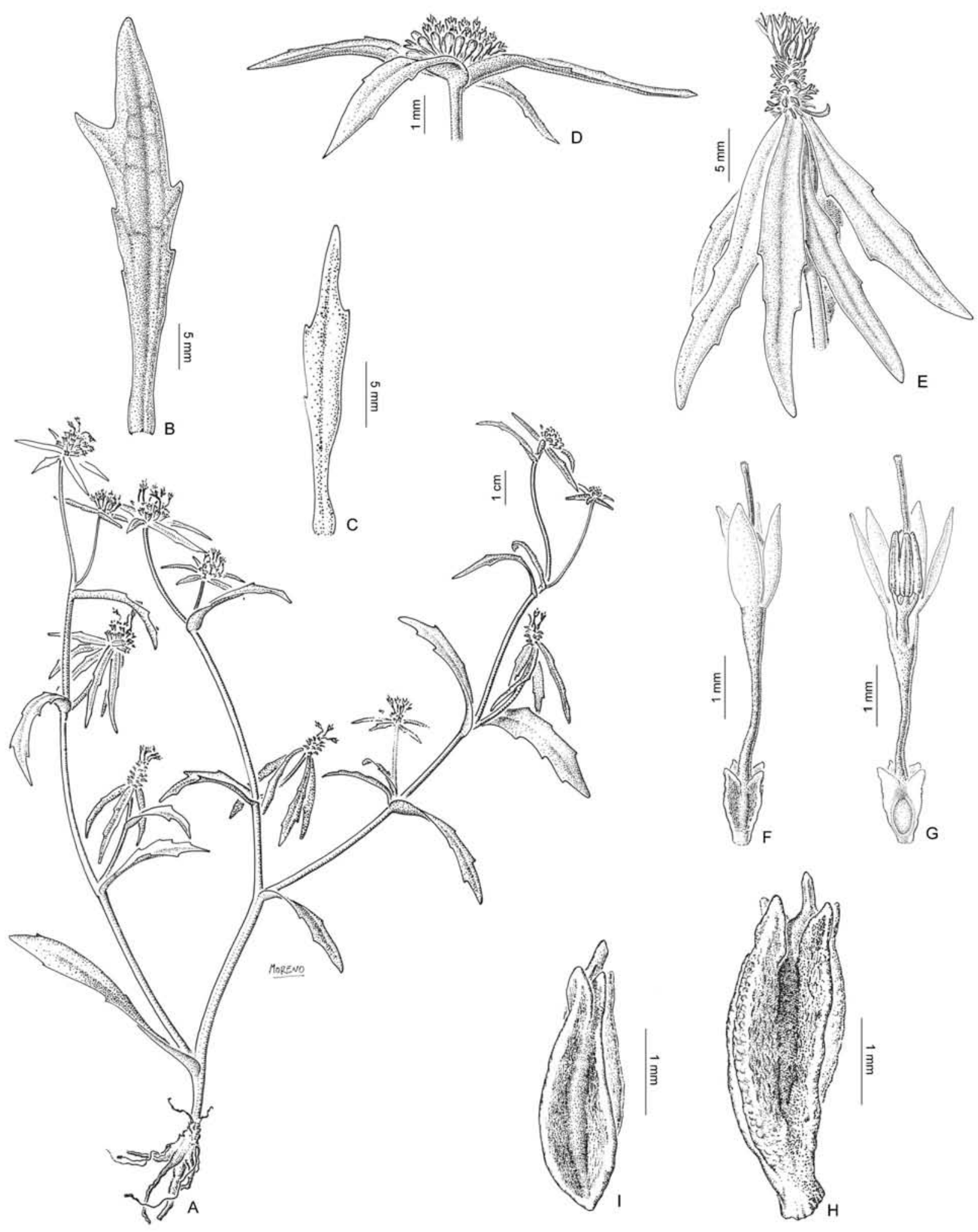

Figura 12. Boopis itatiaiae. A, hábito. B-C, variación de la hoja. D, cefalodio florífero. E, cefalodio fructífero. F, flor. G, flor diseccionada mostrando el androceo. H-I, variación de la cipsela. Dibujado de H. Strang 745 \& A. Castellanos 25739 (LP). 


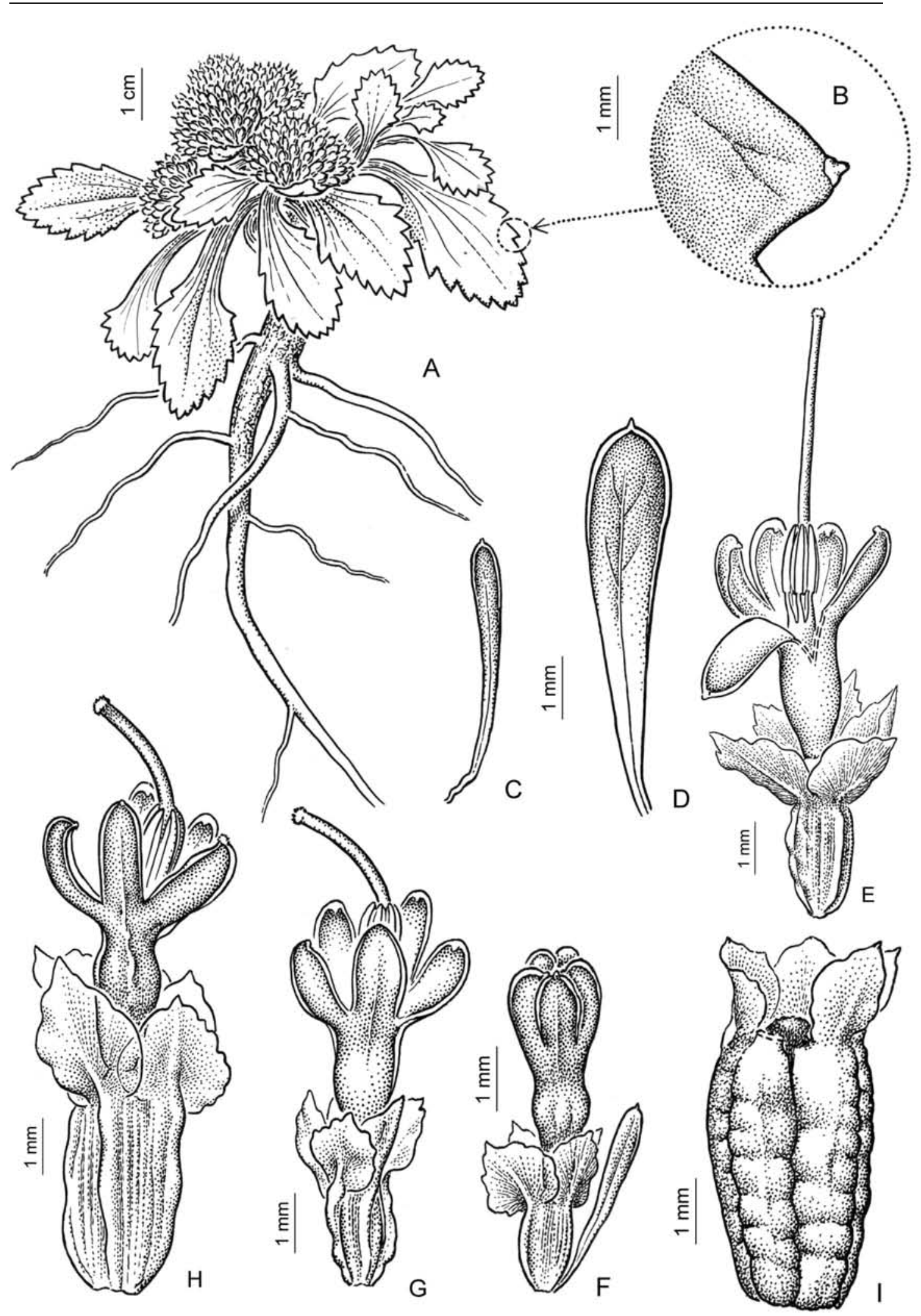

Figura 13. Boopis necronensis. A, hábito. B, detalle de un diente foliar. C, bráctea del receptáculo florífero. D, bráctea del receptáculo fructífero. E, flor en fase pistilada, parcialmente diseccionada, mostrando el androceo. F, botón floral. G, flor fertilizada. H, cipsela inmadura. I, cipsela madura. (Tomado de Zavala-Gallo et al., 2011). 


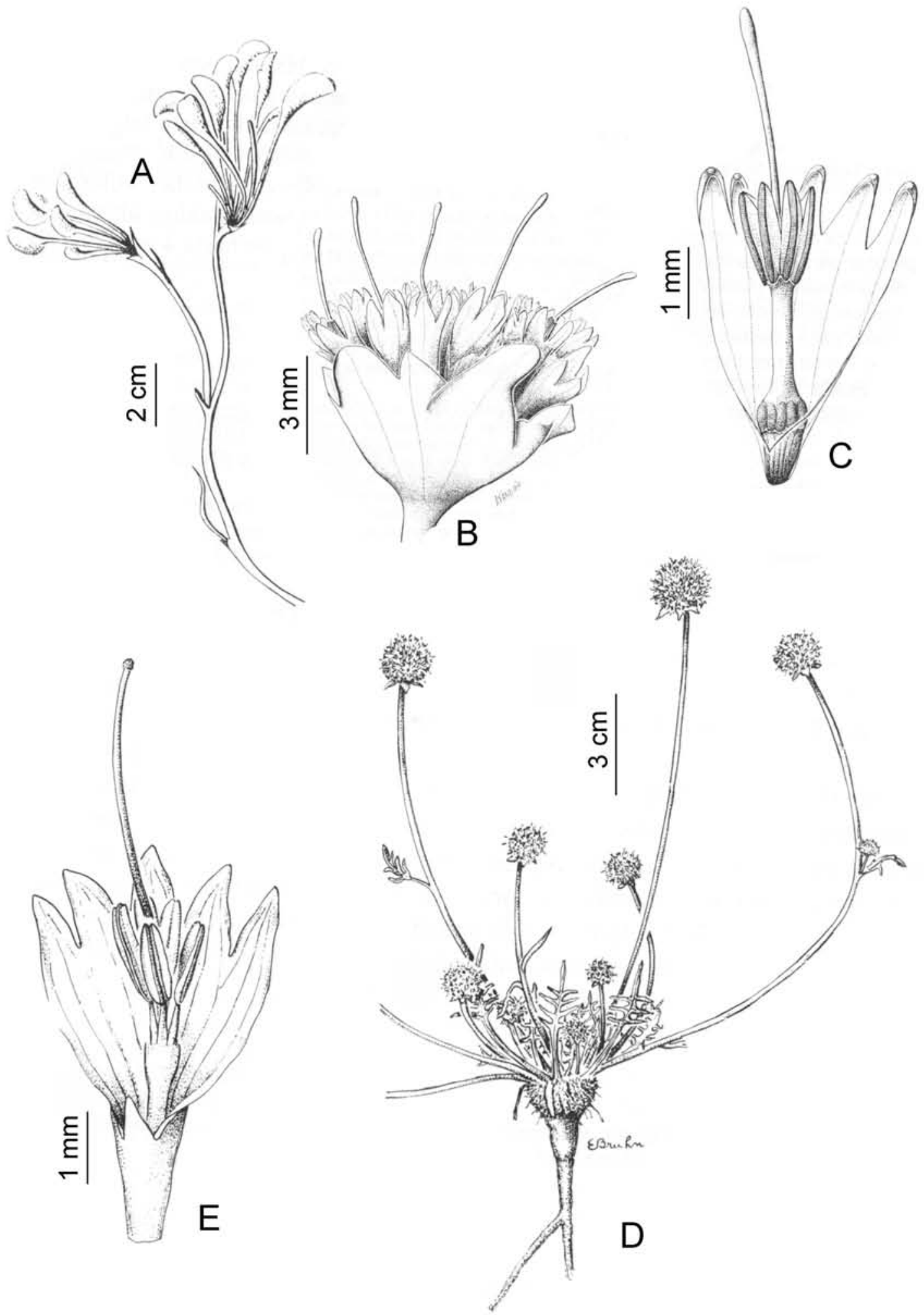

Figura 14. A-C. Boopis patagonica. A, hábito. B, cefalodio. C, flor con la corola diseccionada mostrando el androceo (ovario removido). D-E. B. raffaellii. D, hábito. E, flor con la corola y el hipanto diseccionados mostrando el androceo (el hipanto ha quedado diseccionado por debajo de su unión con la corola y el tubo estaminal). (Tomado de Chiapella 1999a). 

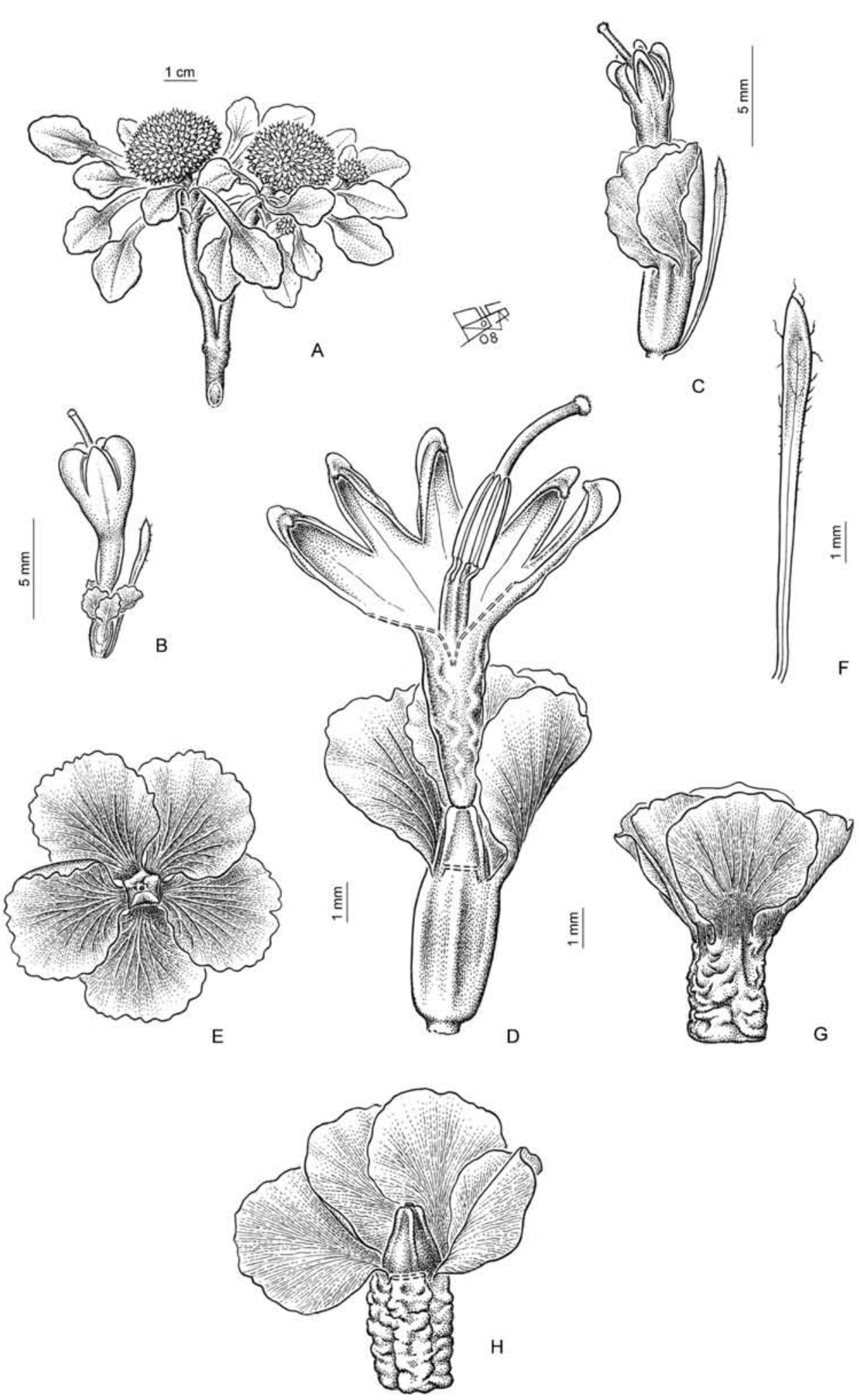

Figura 15. Boopis pterocalyx. A, hábito. B, flor en fase estaminada. C, flor fertilizada y cipsela inmadura. D, flor en el mismo estado que en C, parcialmente diseccionada, mostrando el androceo . E, vista superior de la cipsela mostrando los sépalos foliáceos acrescentes. F, bráctea del receptáculo. G, cipsela, vista lateral. H, ídem G, mostrando el apículo terminal . (Tomado de Zavala-Gallo et al., 2011). 


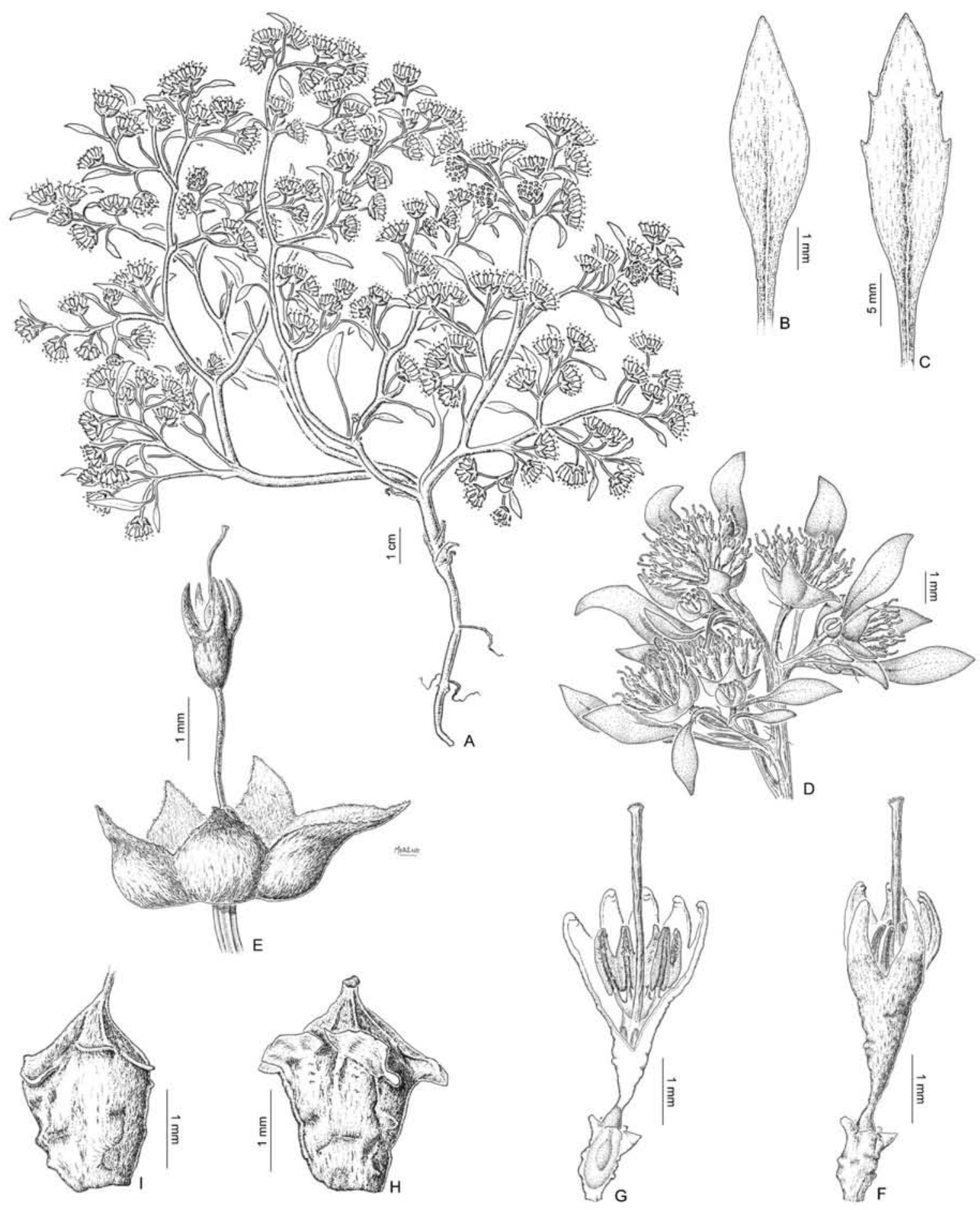

Figura 16. Boopis pusilla. A, hábito. B-C, variación de la hoja. D, grupo de cefalodios en el extremo de una rama. E, vista lateral de un receptáculo fructífero mostrando el perianto marchito sobre una cipsela (oculta por el involucro). F, flor. G, flor diseccionada mostrando el androceo y parte del estilo. H-I, variación de la cipsela. Dibujado de S. Teillier 4637 (CONC). 


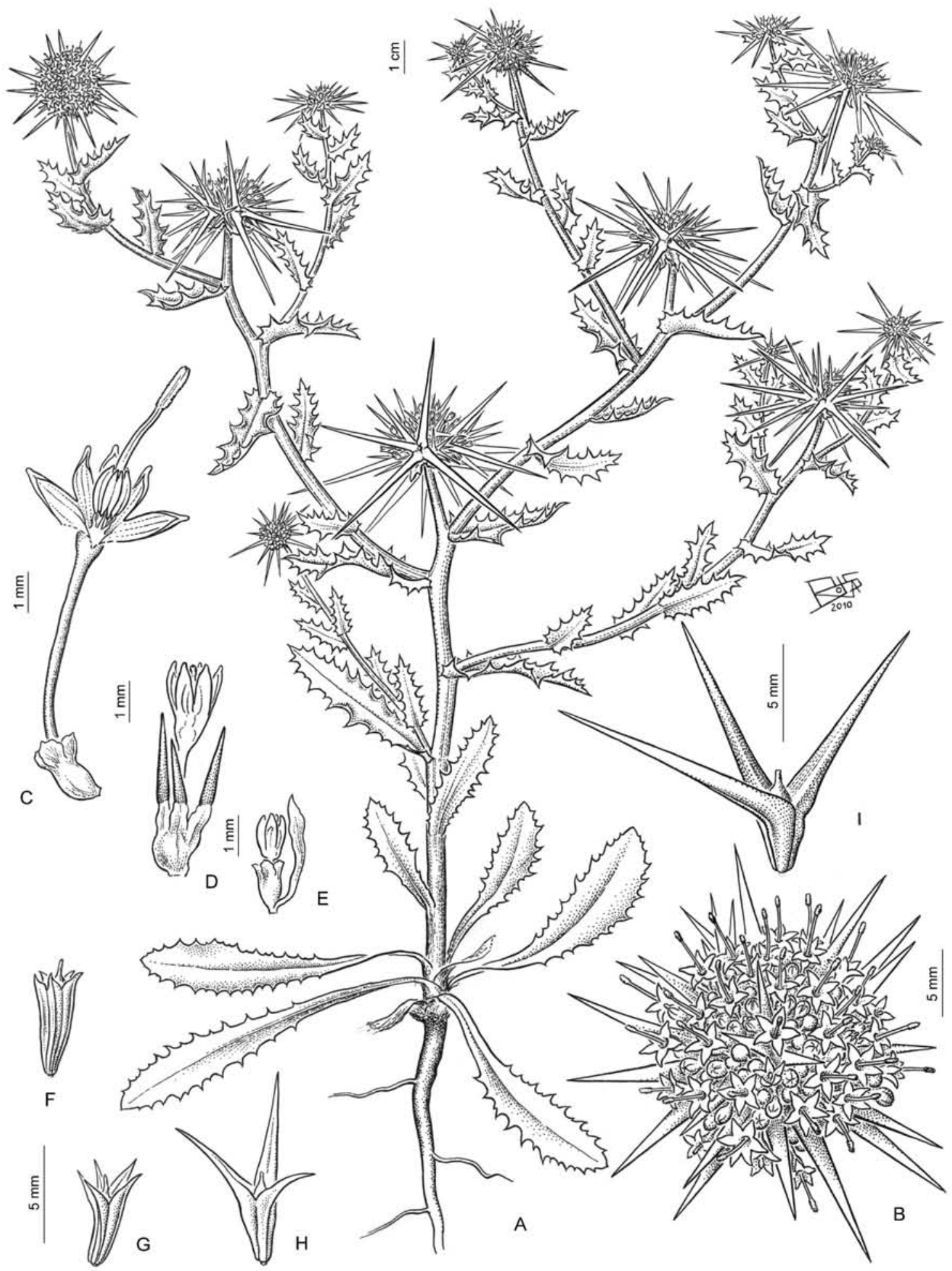

Figura 17. Calycera calcitrapa. A, hábito. B, vista superior del cefalodio mostrando los sépalos espinosos endurecidos durante la antesis. C, flor diseccionada mostrando el androceo; D, flor en fase estaminada, con sépalos espinosos ya endurecidos. E, flor pequeña. F-I, variación de la cipsela. Dibujado de R. Kiesling 3075 (SI). 


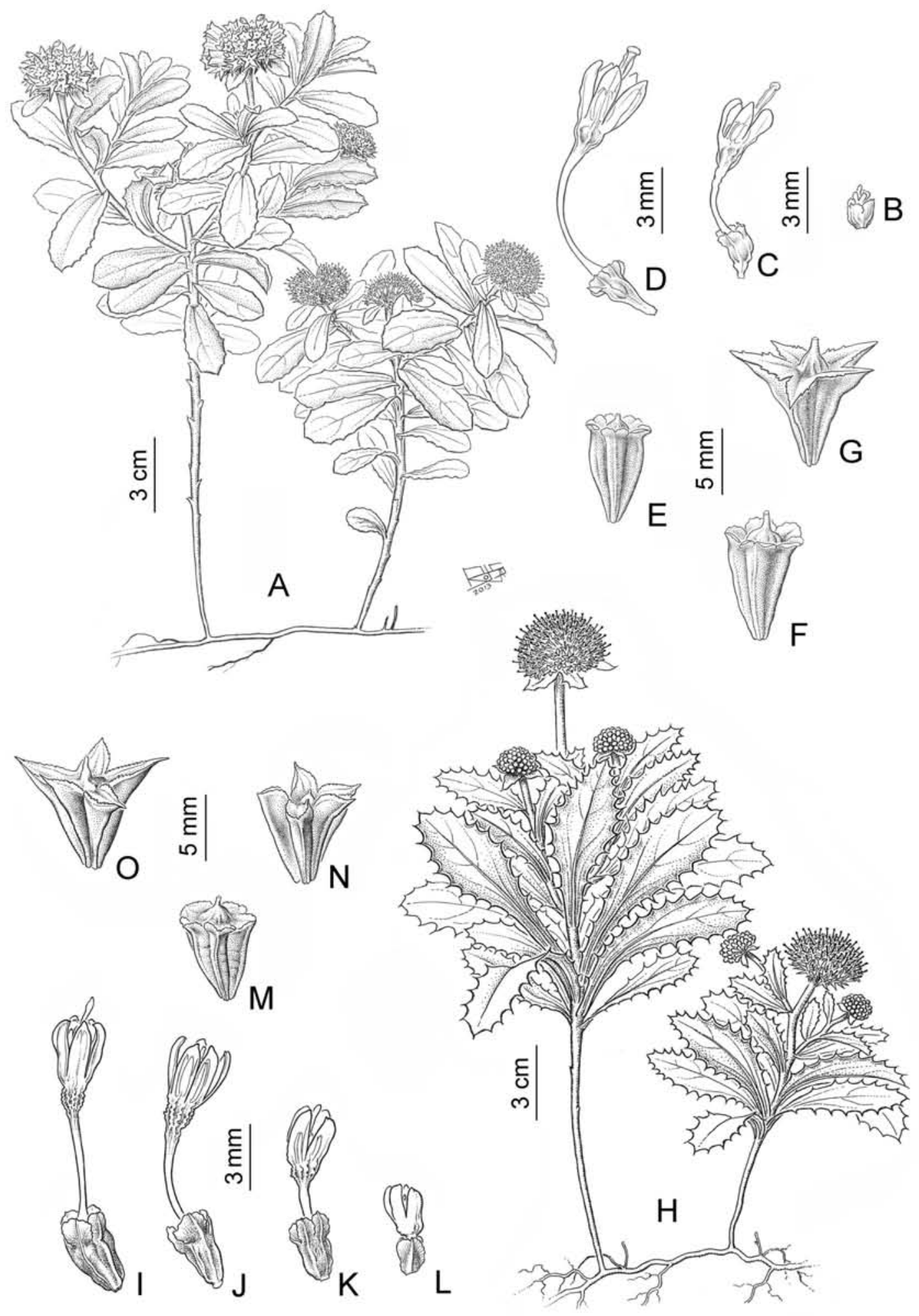

Figura 18. A-G. Calycera crassifolia var. crassifolia. A, hábito. B, flor pequeña. C-D, variación de la flor. E-G, variación de la cipsela. H-O. C. crassifolia var. spinulosa. H, hábito. I-L, variación de la flor. M-O, variación de la cipsela. A, dibujado de A. L. Cabrera 32314 (SI); B, C y D, dibujado de C. A. Zanotti 144 (SI); E, F y G, dibujado de E. G. Nicora 628 (SI) ; H, dibujado de F. O. Zuloaga 12271 (SI) I, J, K y L, dibujado de A. L. Cabrera 32822 (SI); M, N y O, dibujado de A. Burkart 14318 (SI). 


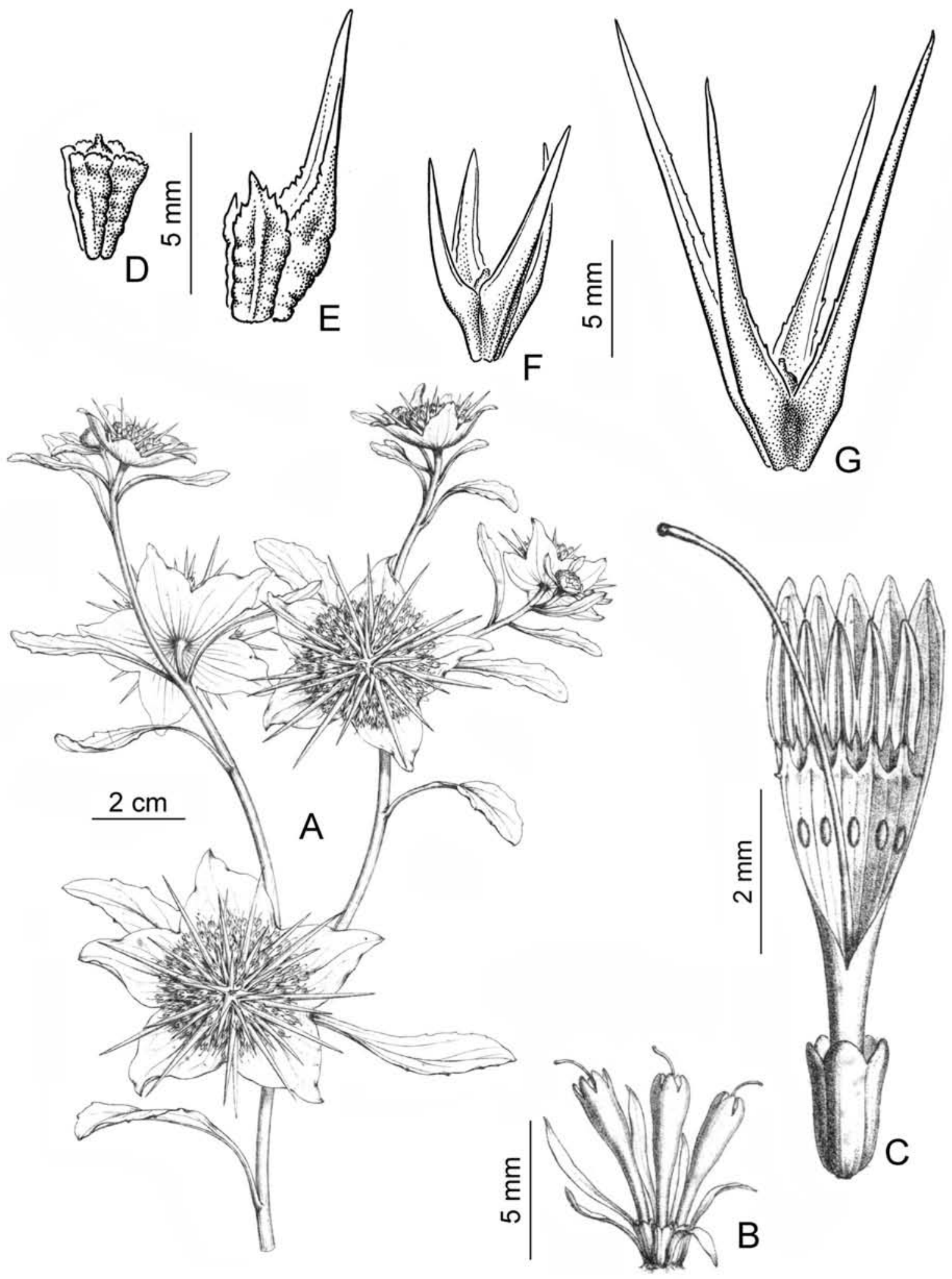

Figura 19. Calycera eryngioides. A, detalle del hábito. B, grupo de flores sobre el receptáculo. C, flor diseccionada mostrando el androceo, el interior del hipanto y el estilo. D, cipsela inerme. E, cipsela intermedia. F-G, cipselas espinosas. A, B y C, tomado de Weddell (1857 [1858]); D-G, dibujado de Jiles-P. 1558 (LIL). 


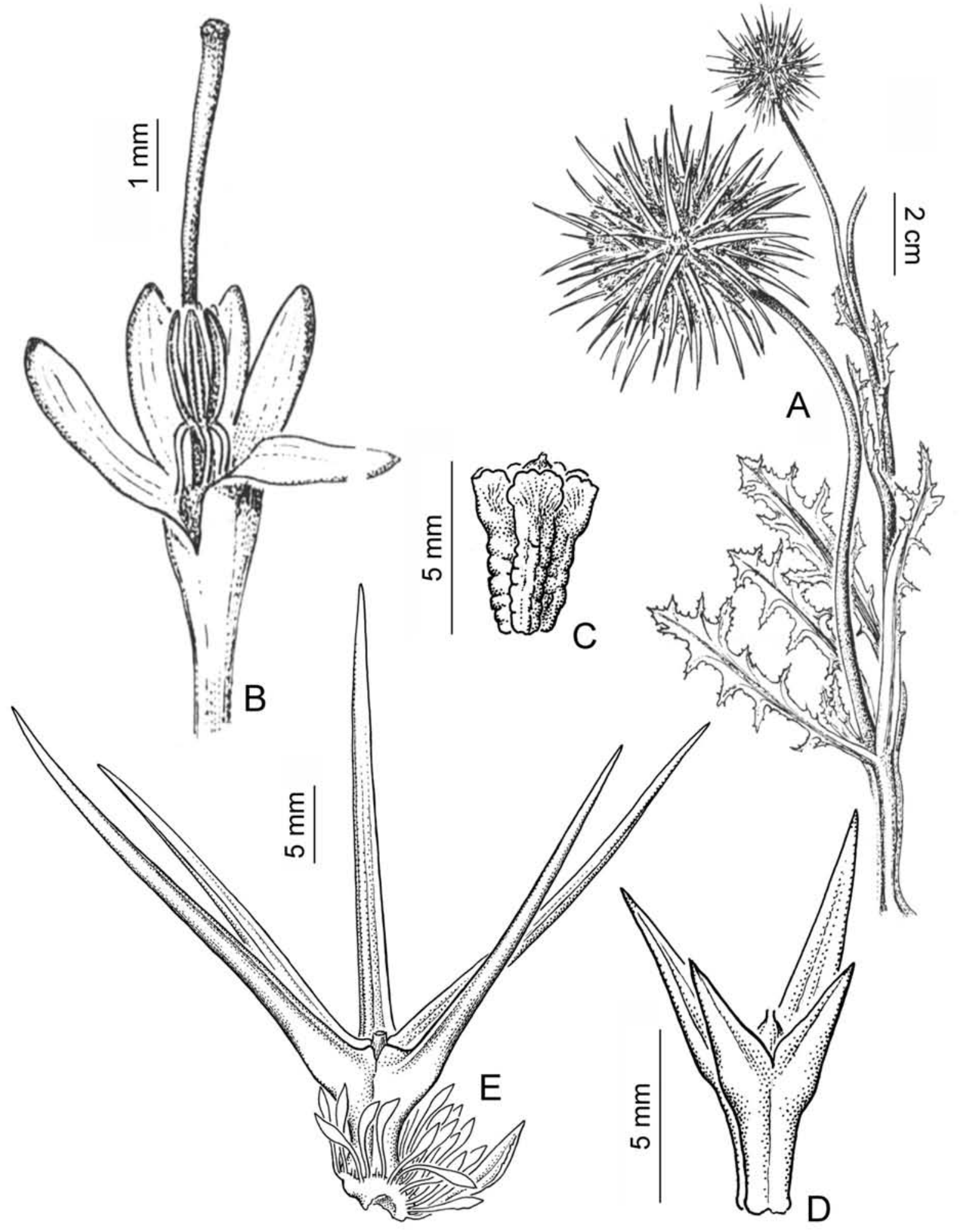

Figura 20. Calycera herbacea var. herbacea. A, detalle del hábito. B, flor diseccionada mostrando el androceo y el estilo (parte inferior del hipanto y ovario removidos).C-E, variación de la cipsela. A y B, tomado de Chiapella, 1999a; C, D y E, dibujado de R. Pozner et al. $563(\mathrm{SI})$. 


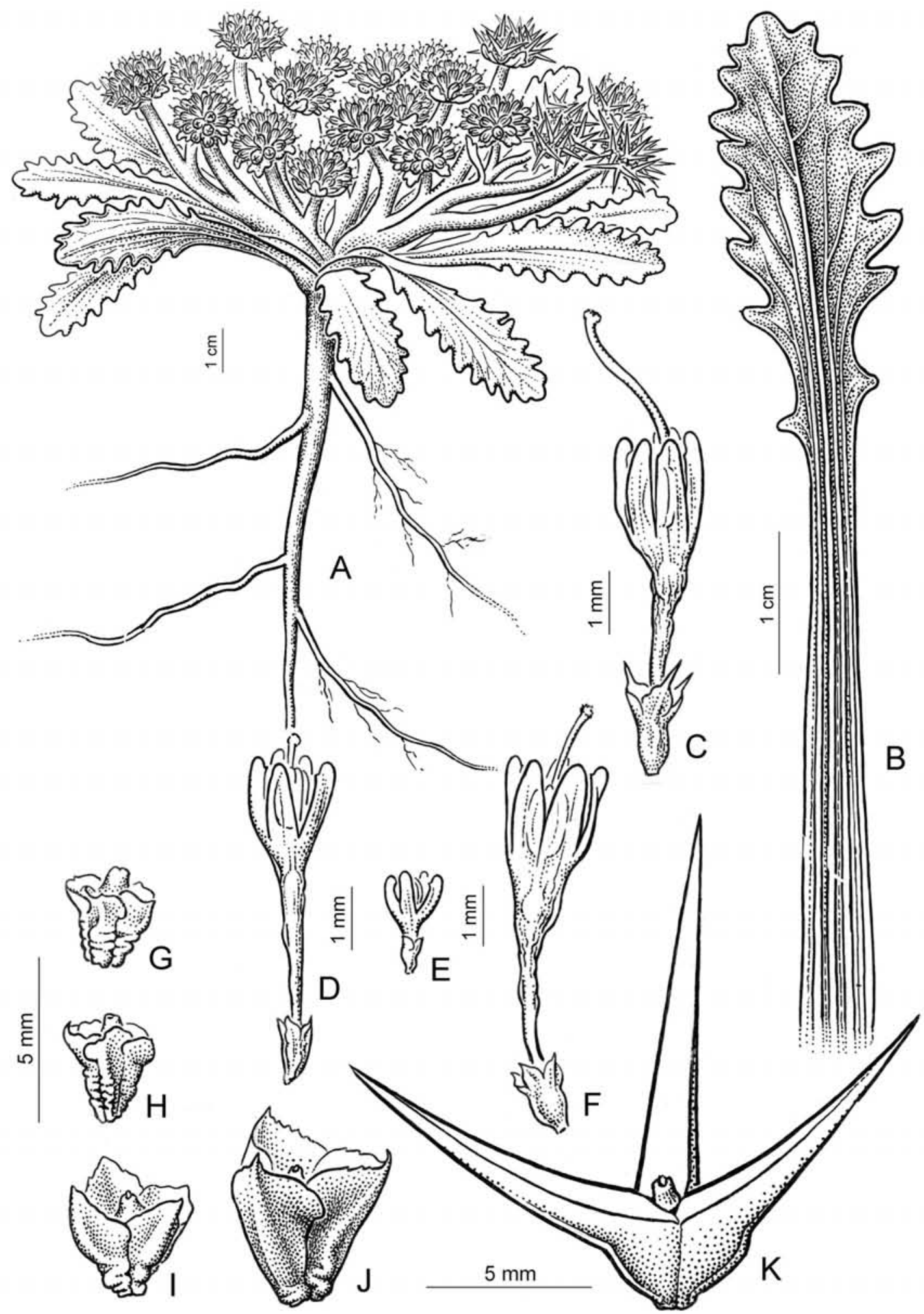

Figura 21. Calycera herbacea var. sinuata. A, hábito (forma típica de los Andes de San Juan). B, hoja. C, flor en fase pistilada. D-F, variación de la flor. G-H, cipselas inermes. I-J, cipselas con sépalos de acrescencia intermedia. K, cipsela espinosa. A y K, dibujados de R. Kiesling 9430 (SI); B, C, D, E y F, dibujados de R. Kiesling 3283 (SI); G, H, I y J, dibujados de R. Kiesling $6188 \mathrm{~b}(\mathrm{SI})$. 


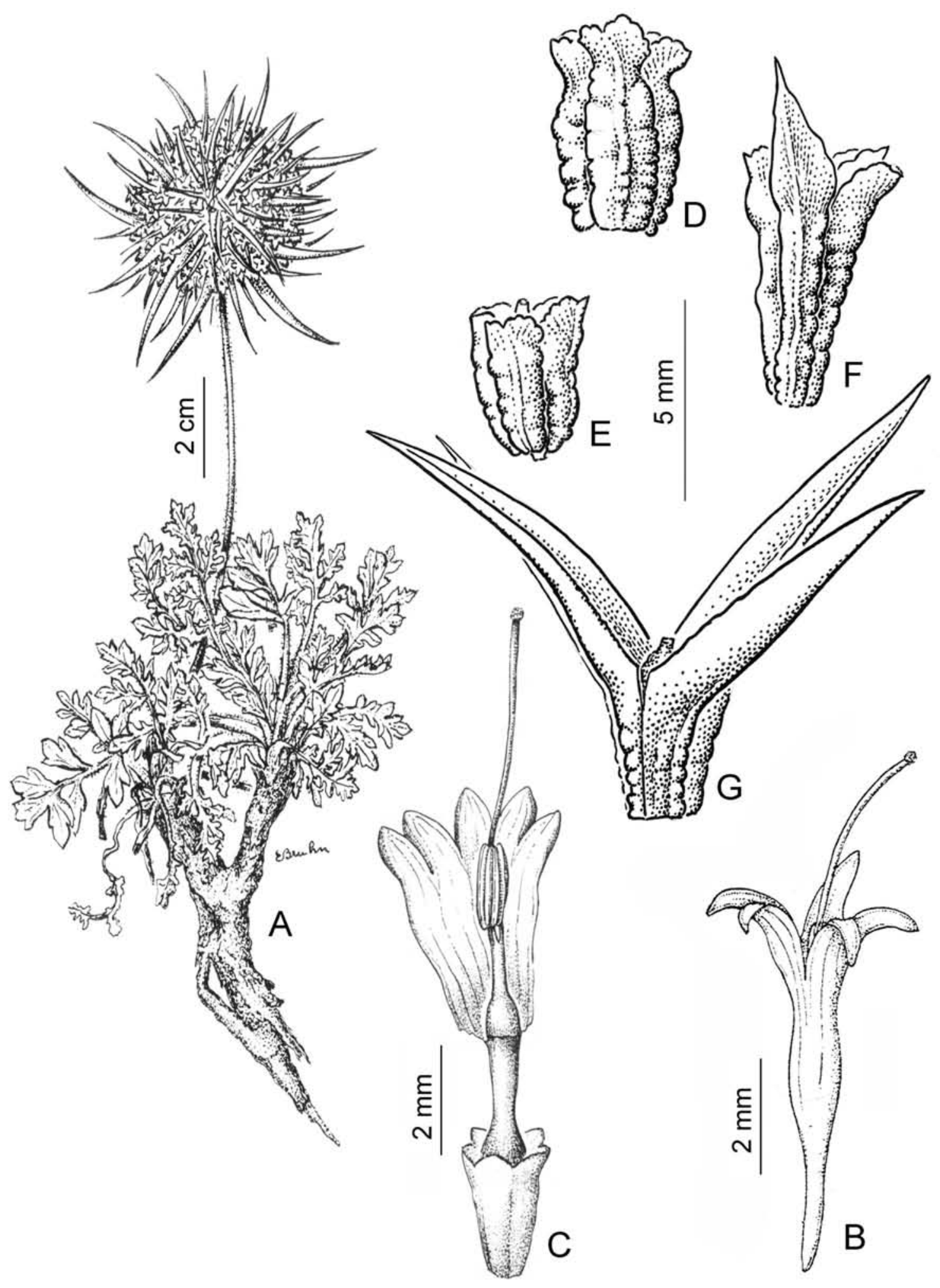

Figura 22. Calycera horrida. A, detalle del hábito. B, parte superior de la flor (ovario removido). C, flor diseccionada mostrando el androceo y el estilo. D-E, cipselas inermes. F, cipsela intermedia. G, cipsela espinosa. A y C, tomado de Chiapella, 1999a; B, tomado de Pontiroli, 1963; D, E, F y G, dibujado de F. Kurtz 6142 (SI). 

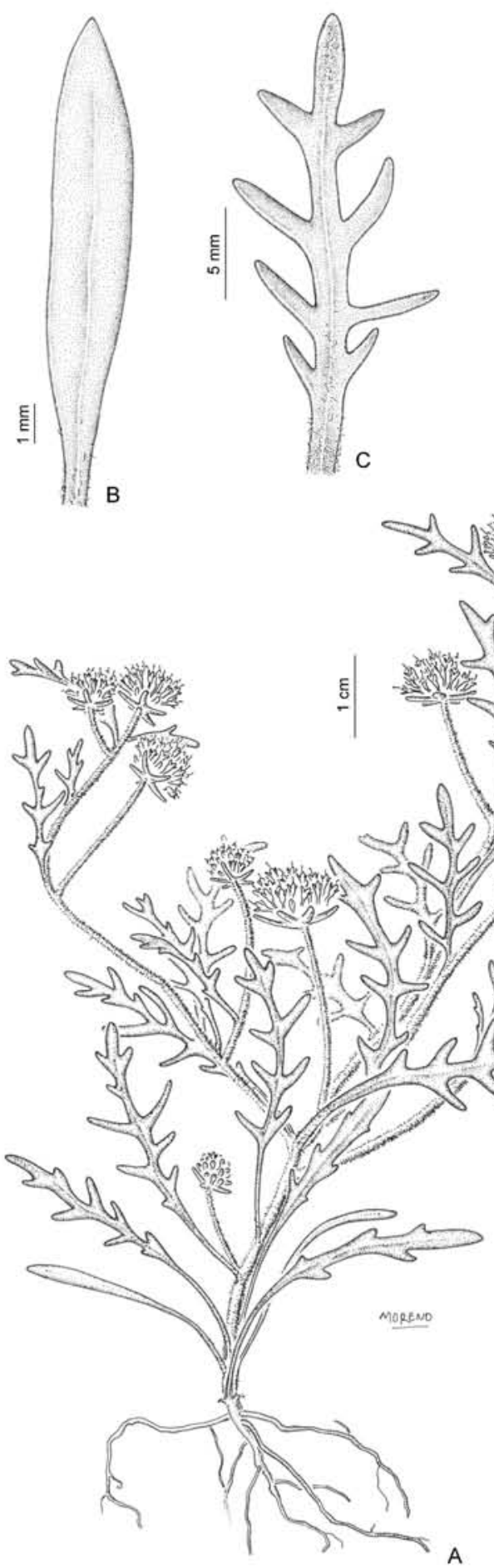
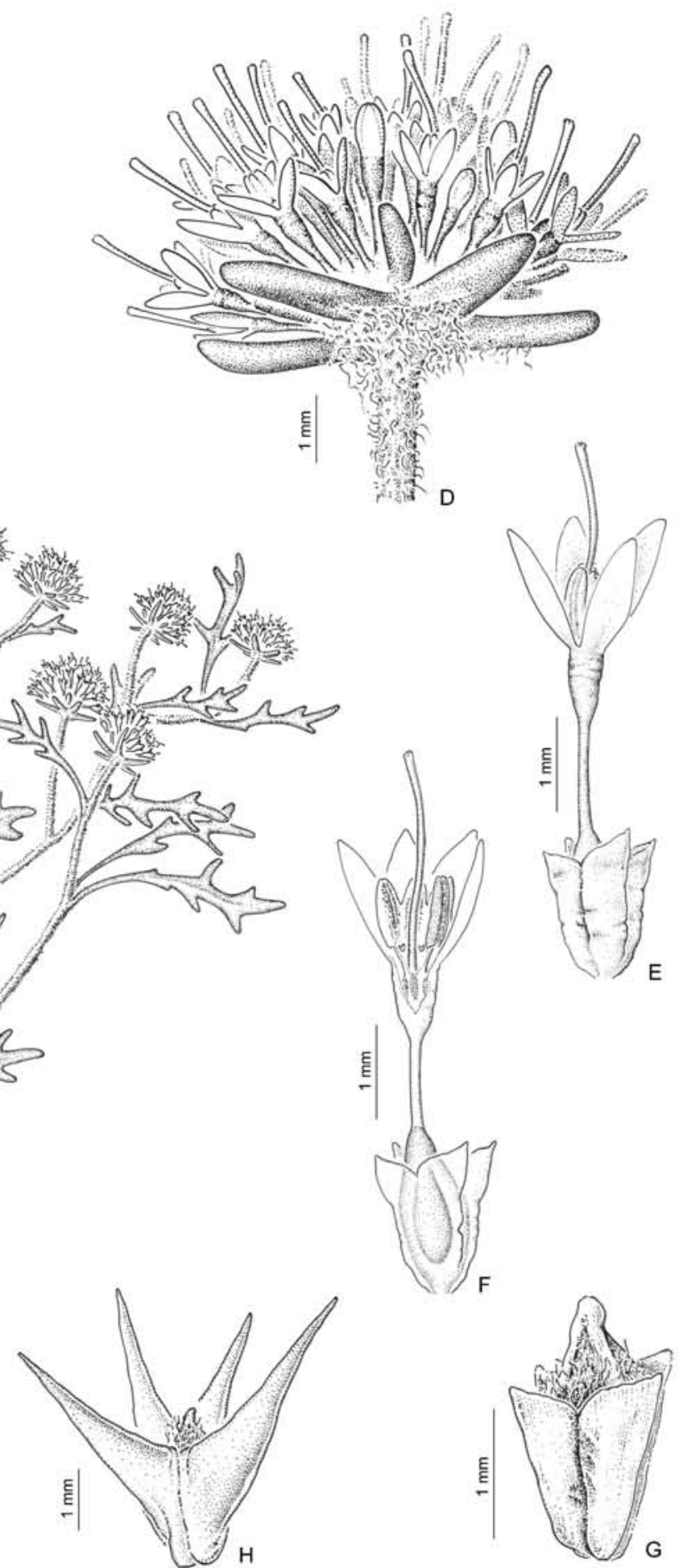

Figura 23. Calycera leucanthema. A, hábito. B-C, variación de la hoja. D, vista inferior del cefalodio. E, flor. F, flor diseccionada mostrando el androceo y parte del estilo. G, aquenio inerme. H, aquenio espinoso. Dibujado de Johnson \& Zavala 10-105 (SI). 


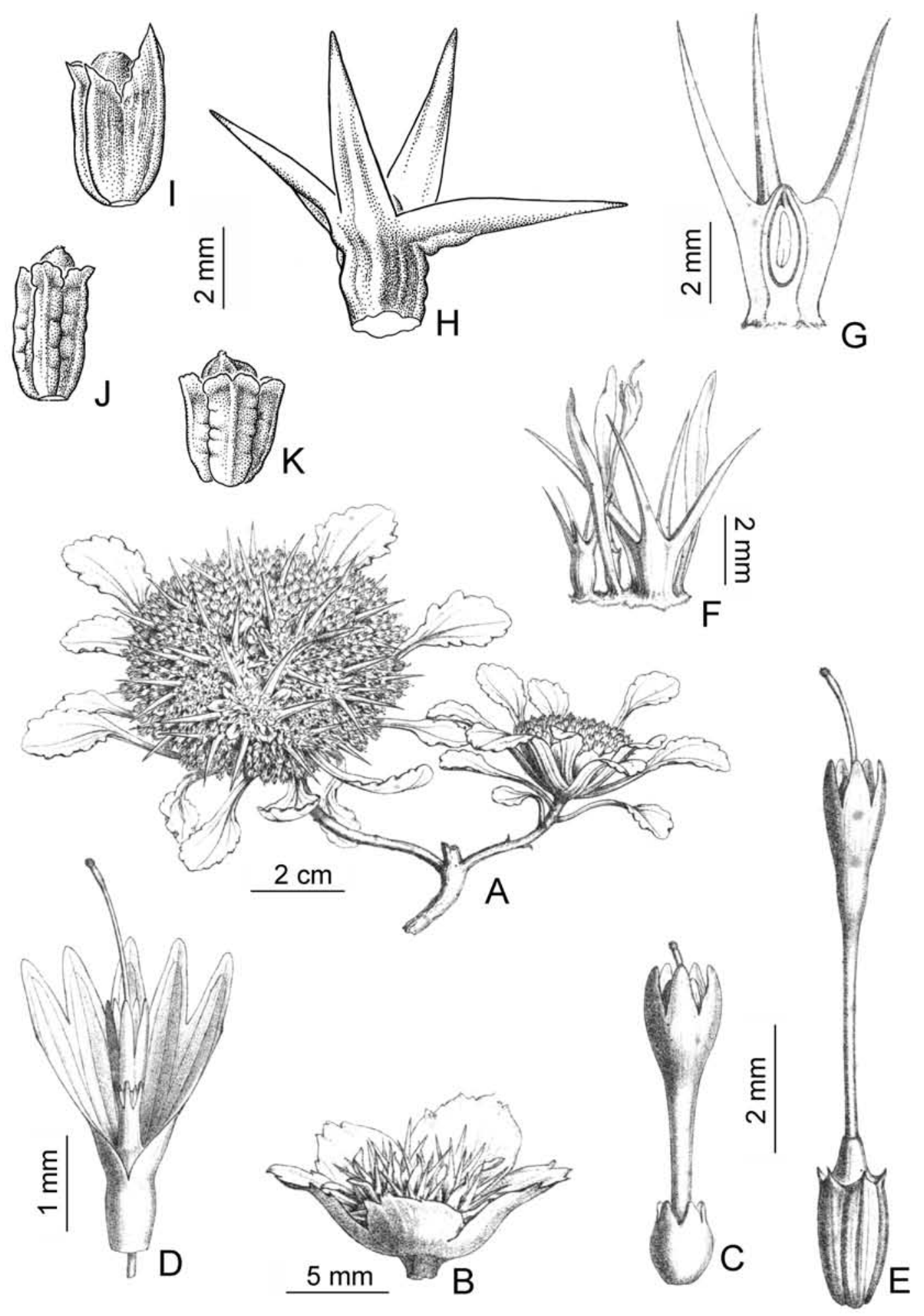

Figura 24. Calycera pulvinata. A, hábito. B, cefalodio secundario. C, flor. D, flor diseccionada mostrando el androceo (ovario removido). E, aquenio inmaduro con estructuras florales persistentes. F, aquenios maduros sobre el receptáculo. G, corte transversal de un aquenio espinoso. H, aquenio espinoso. I, aquenio intermedio. J-K, aquenios inermes. A-G, tomados de Weddell (1857 [1858]); G-K, dibujados de D. Olea 219 (LIL). 


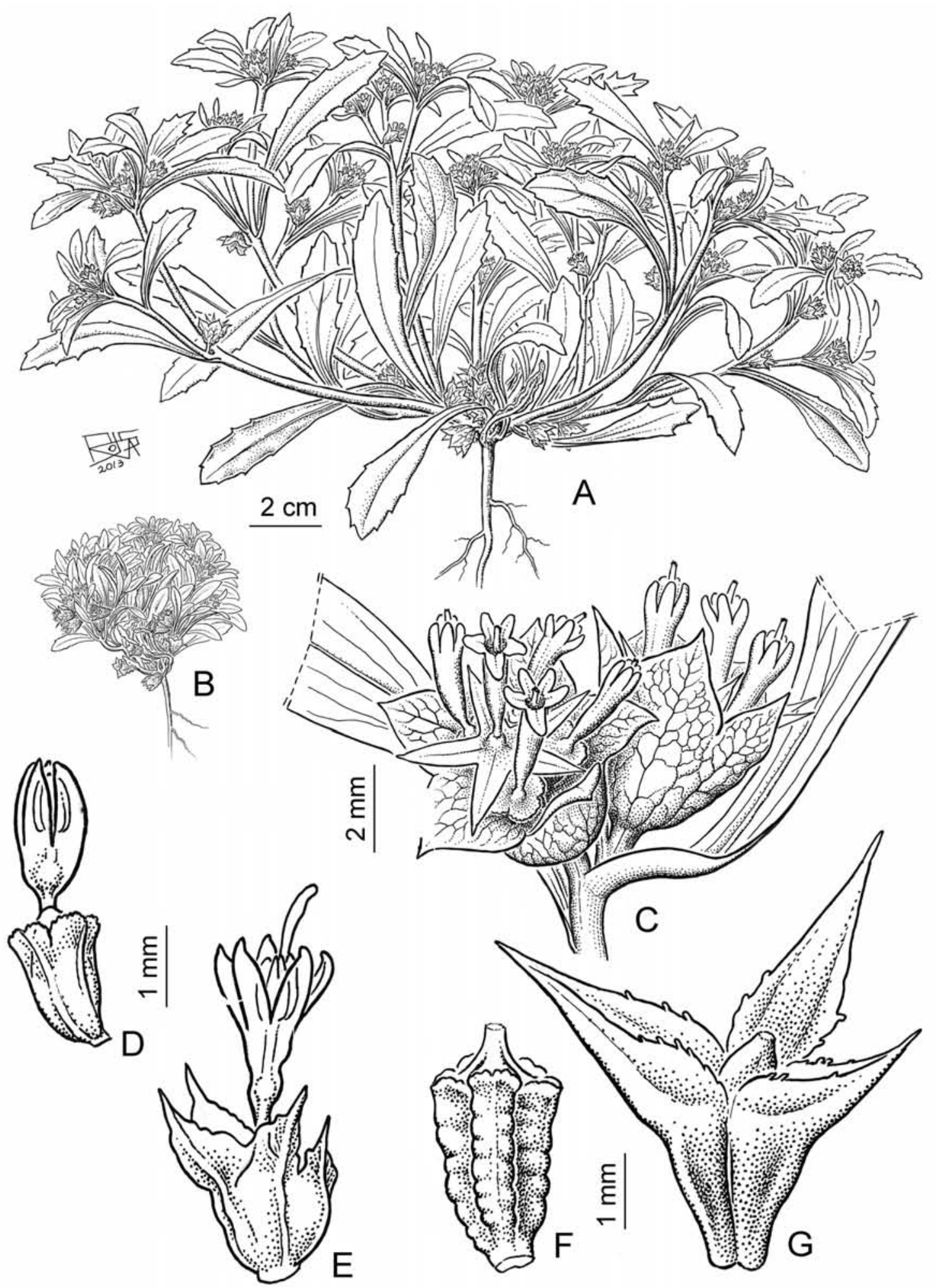

Figura 25. Calycera sessiliflora. A-B, variación del hábito (a igual escala). C, detalle de una rama florífera mostrando un par de cefalodios durante la maduración de los aquenios. D, botón floral. E, flor. F, aquenio inerme. G, aquenio espinoso. A, dibujado de Goodspeed 16784 (SI); B y C, dibujados de Johnson \& Zavala 10-153 (SI); D, E, F y G, dibujados de Jiles-P. 1011 (LIL). 


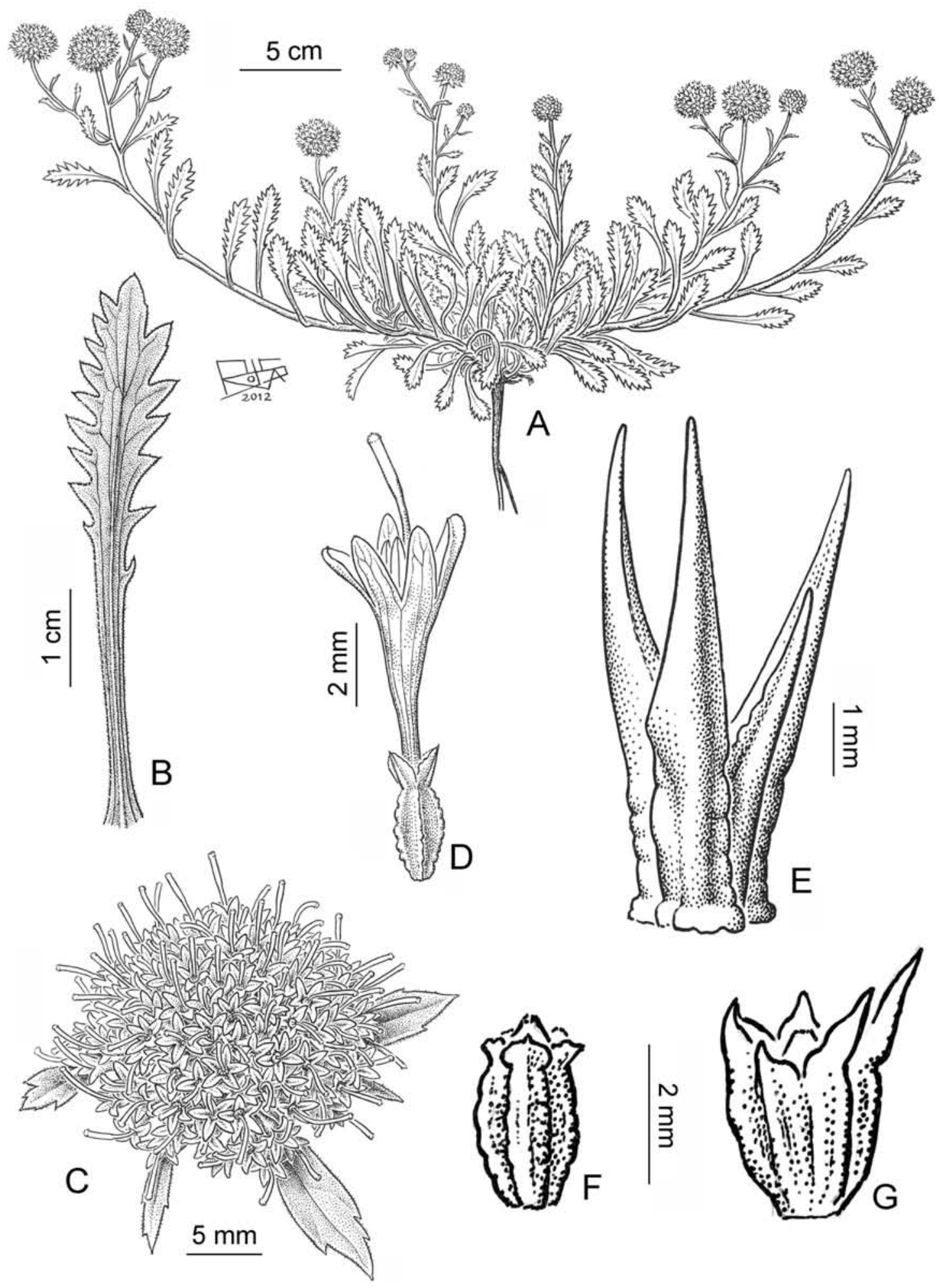

Figura 26. Calycera sympaganthera. A, hábito. B, hoja. C, cefalodio. D, flor. E, aquenio espinoso. F, aquenio inerme. G, aquenio intermedio. A y B, dibujado de Gardner et al. DCI n ${ }^{\circ}$ 589 (CONC); C y D, dibujados de Zapata \& Sede 376 (SI); E, F y G, dibujados de R. A. Philippi s.n. (SI 12665). 

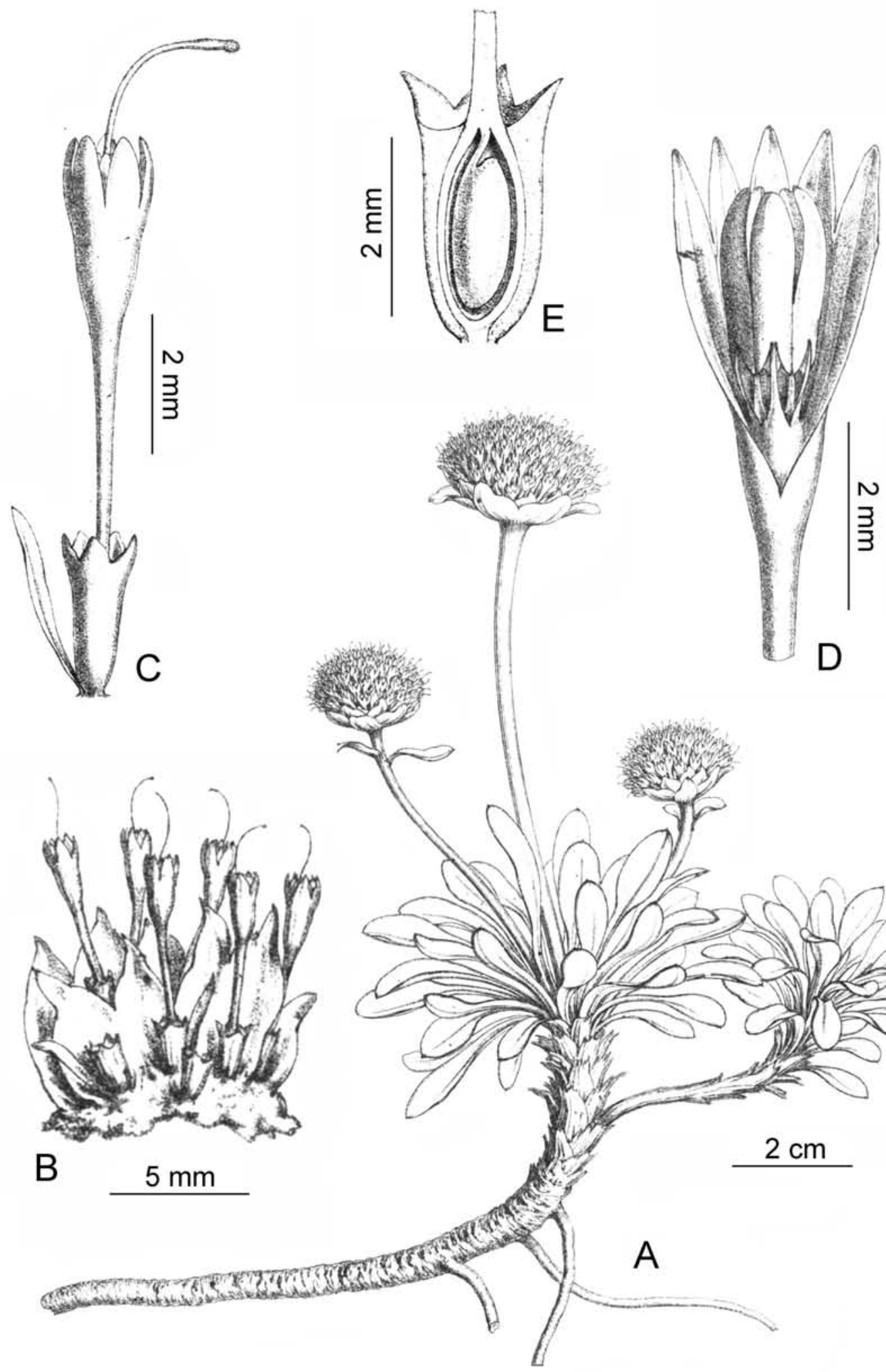

Figura 27. Gamocarpha alpina. A, hábito. B, detalle del cefalodio (flores y brácteas del receptáculo fusionadas). C, flor. D, flor con la corola diseccionada mostrando el androceo (estilo y ovario removidos). E, corte transcersal del ovario. (Tomado de Weddell, 1857 [1858]; sub, Gamocarpha poeppigii). 


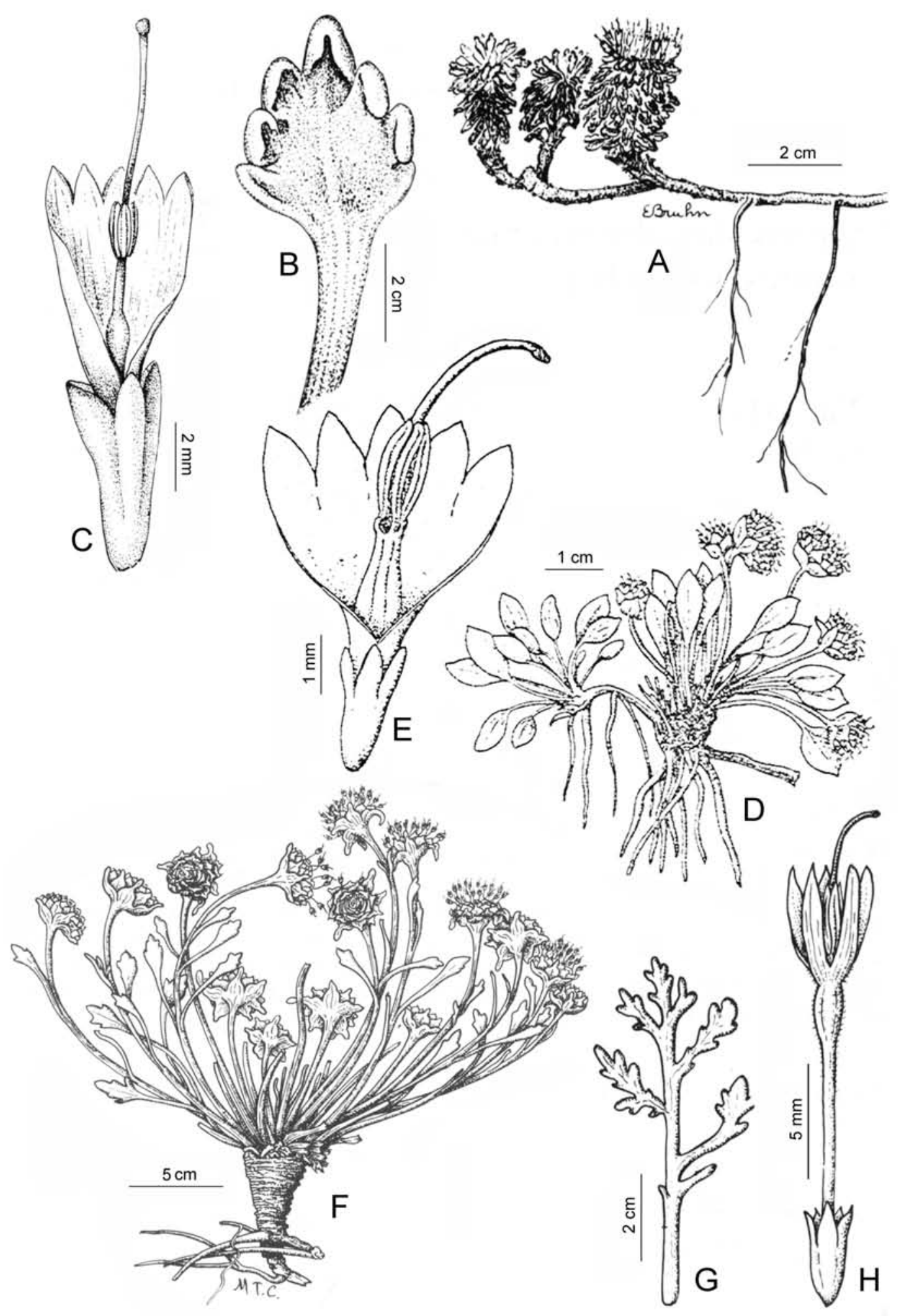

Figura 28. A-C. Gamocarpha dentata. A, hábito. B, hoja. C, flor con la corola diseccionada mostrando el androceo. D-E. G. gilliesii. D, hábito. E, flor con la corola y el hipanto diseccionados, mostrando el androceo y la porción interna tubular del hipanto por debajo del tubo estaminal. F-H. G. selliana. F, hábito. G, hoja. H, flor. (A,B,C, F, G y H, tomado de Chiapella, 1999a; D y E, tomado de Pontiroli, 1963). 


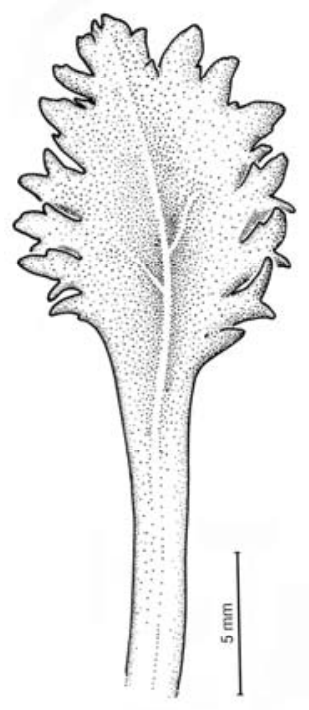

B

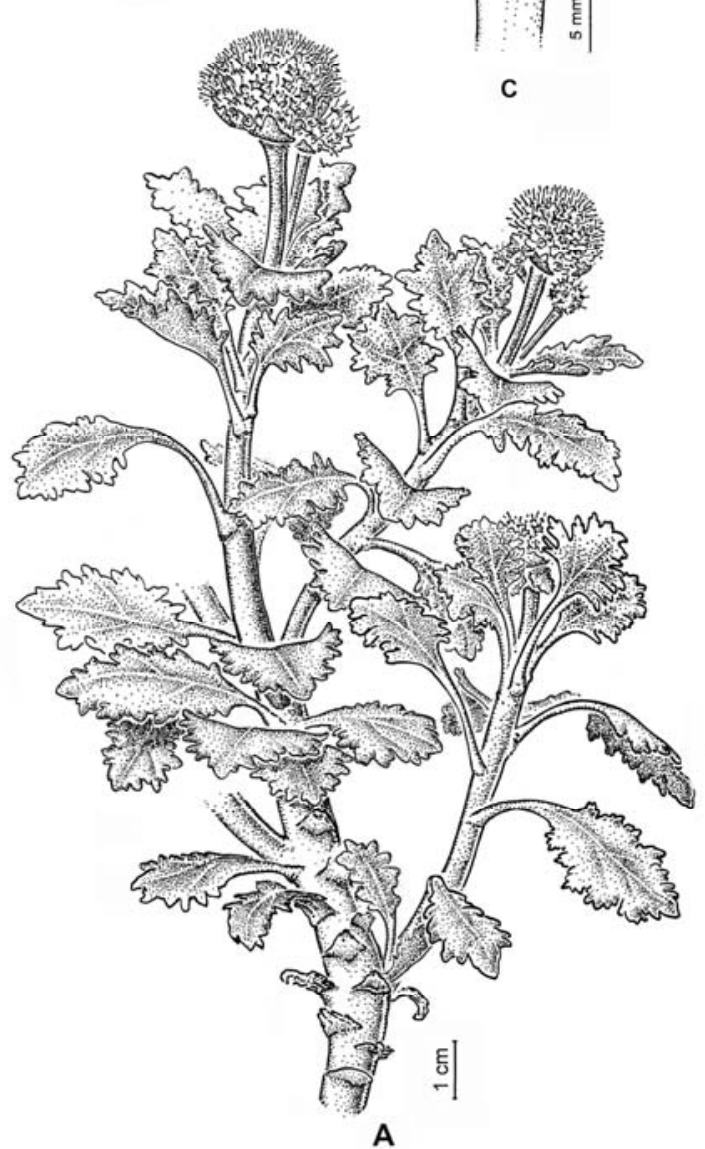

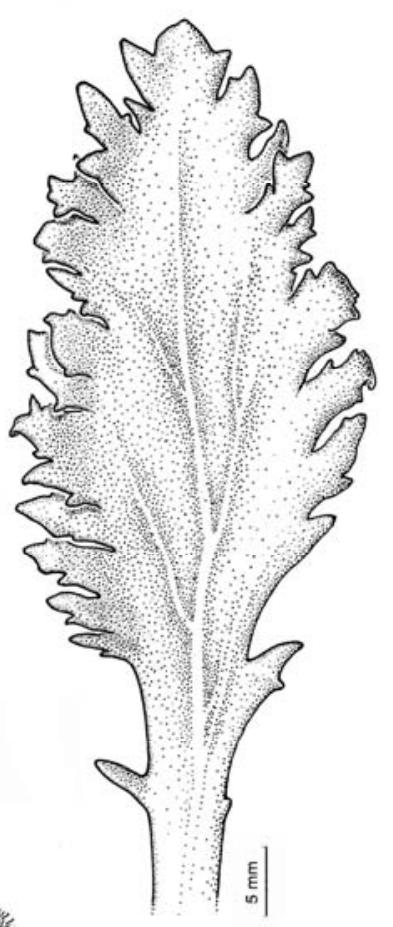
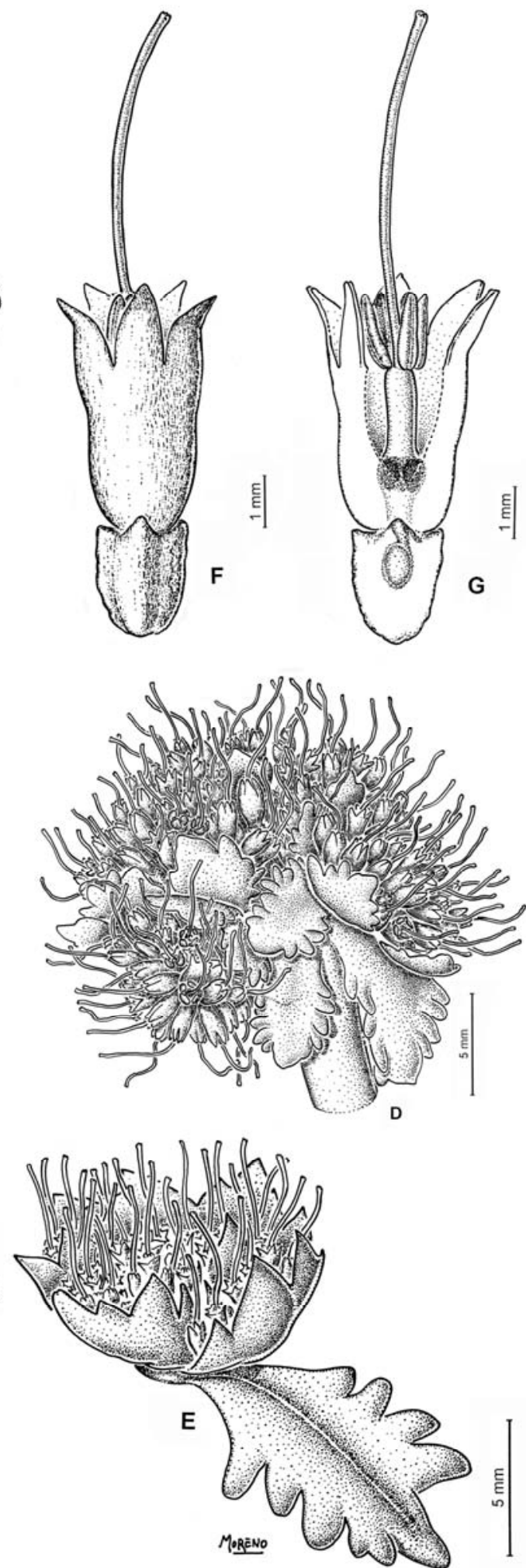

Figura 29. Moschopis ameghinoi. A, detalle del hábito. B-C, variación de la hoja. D, inflorescencia compuesta. E, cefalodio basal de la inflorescencia compuesta y bráctea tectriz. F, flor. G, flor con la corola diseccionada mostrando el androceo. Dibujado de M. I. Sánchez $516(\mathrm{BAB})$. 

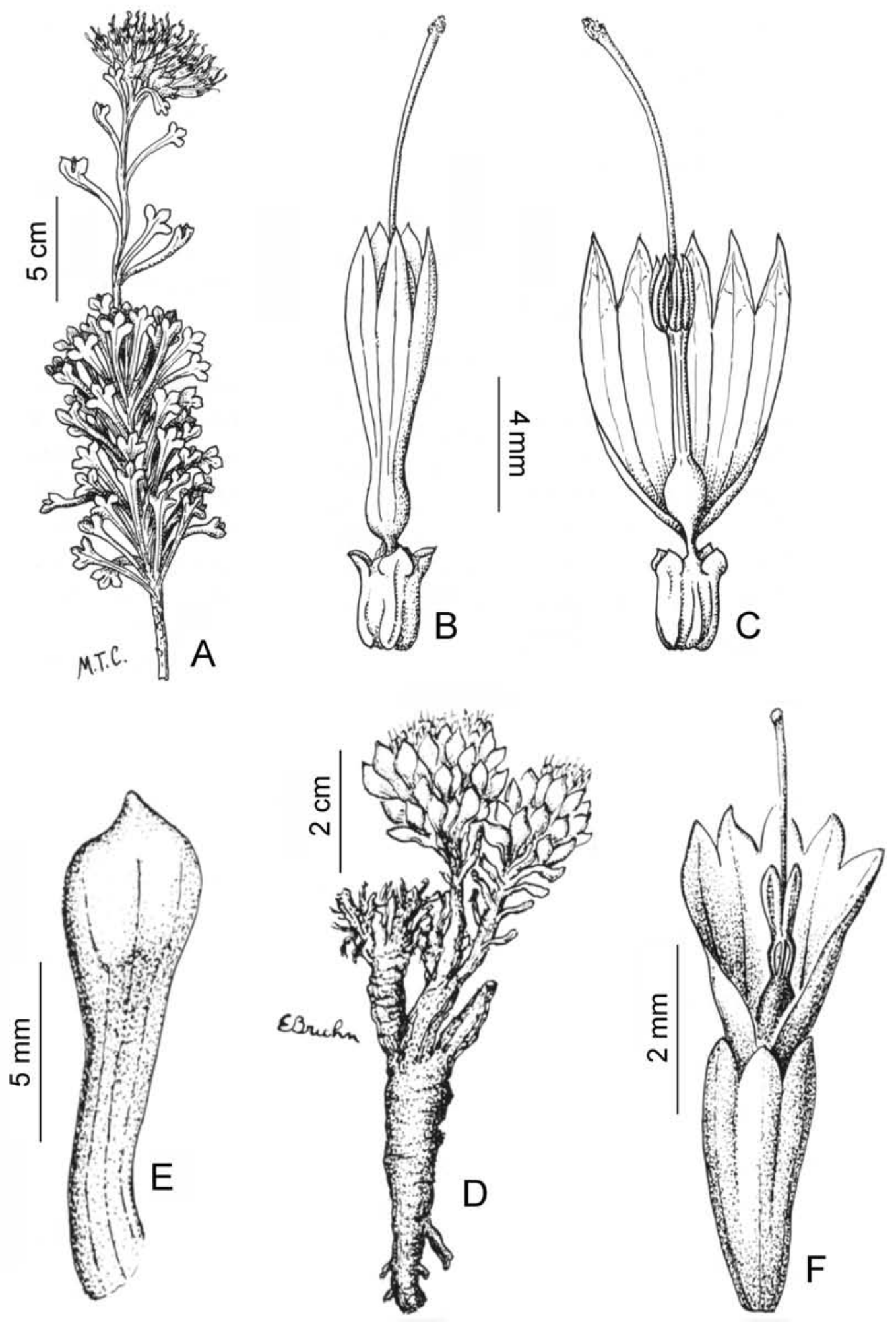

Figura 30. A-C. Moschopis caleofuensis. A, detalle del hábito. B, flor. C, flor con la corola diseccionada mostrando el androceo. D-F. M. leyboldi. D, hábito. E, hoja. F, flor con la corola y el hipanto diseccionados, mostrando el androceo y la porción interna del hipanto por debajo del tubo estaminal. (Tomado de Chiapella, 1999a). 


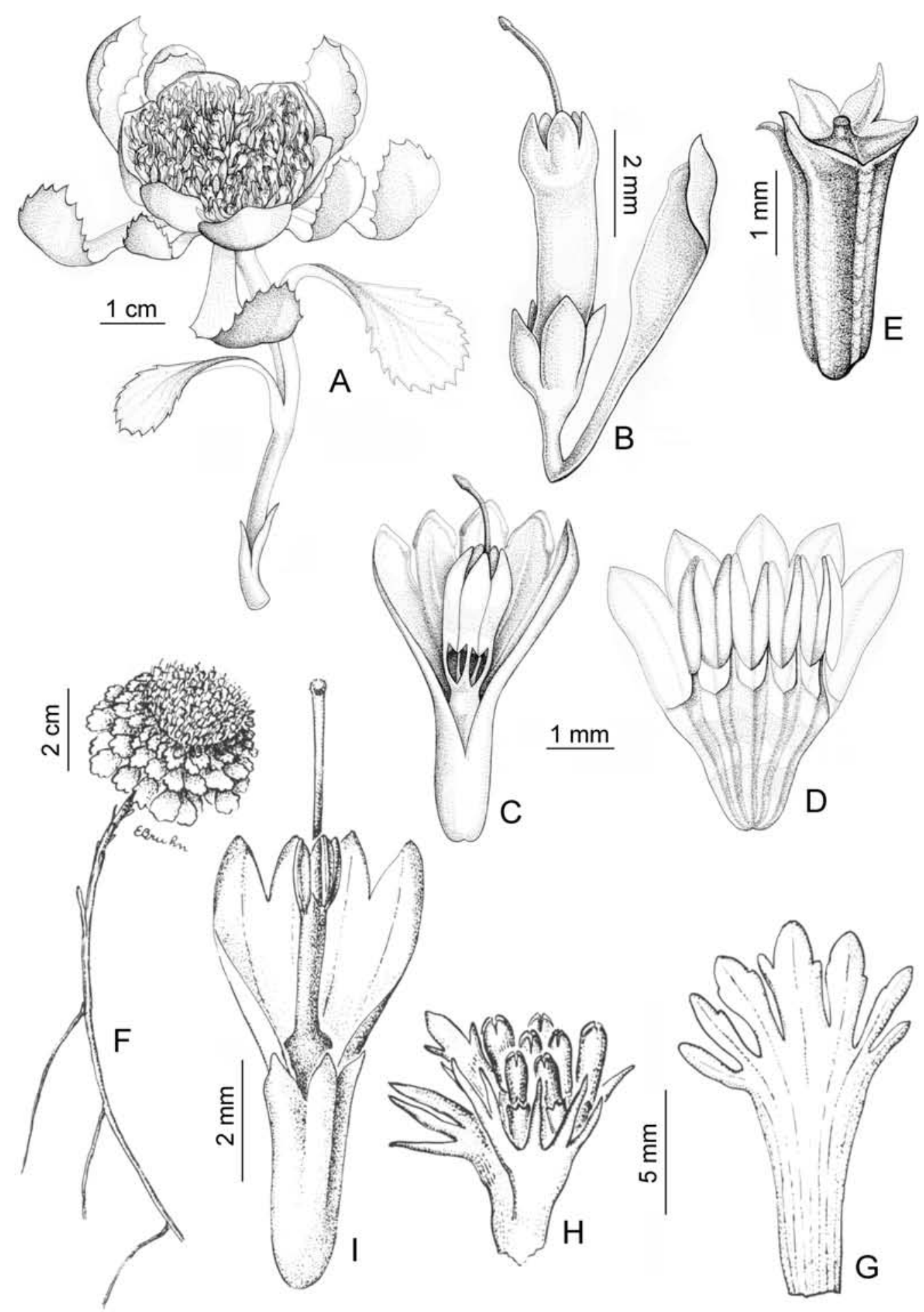

Figura 31. A-E. Moschopis monocephala. A, detalle del hábito. B, flor y bráctea del receptáculo asociada. $\mathrm{C}$, flor con la corola diseccionada mostrando el androceo (ovario removido). D, perianto y androceo desplegados mostrando la cara interna de las anteras, los filamentos, el tubo estaminal y el hipanto. E, aquenio. F-I. M. rosulata. F, hábito. G, hoja. H, grupo cimoso externo mostrando el involucelo y, por detrás, la bráctea tectriz asociada. I, flor con la corola diseccionada mostrando el androceo. (A-E, tomado de Pontiroli, 1993; F-I, tomado de Chiapella, 1999a). 


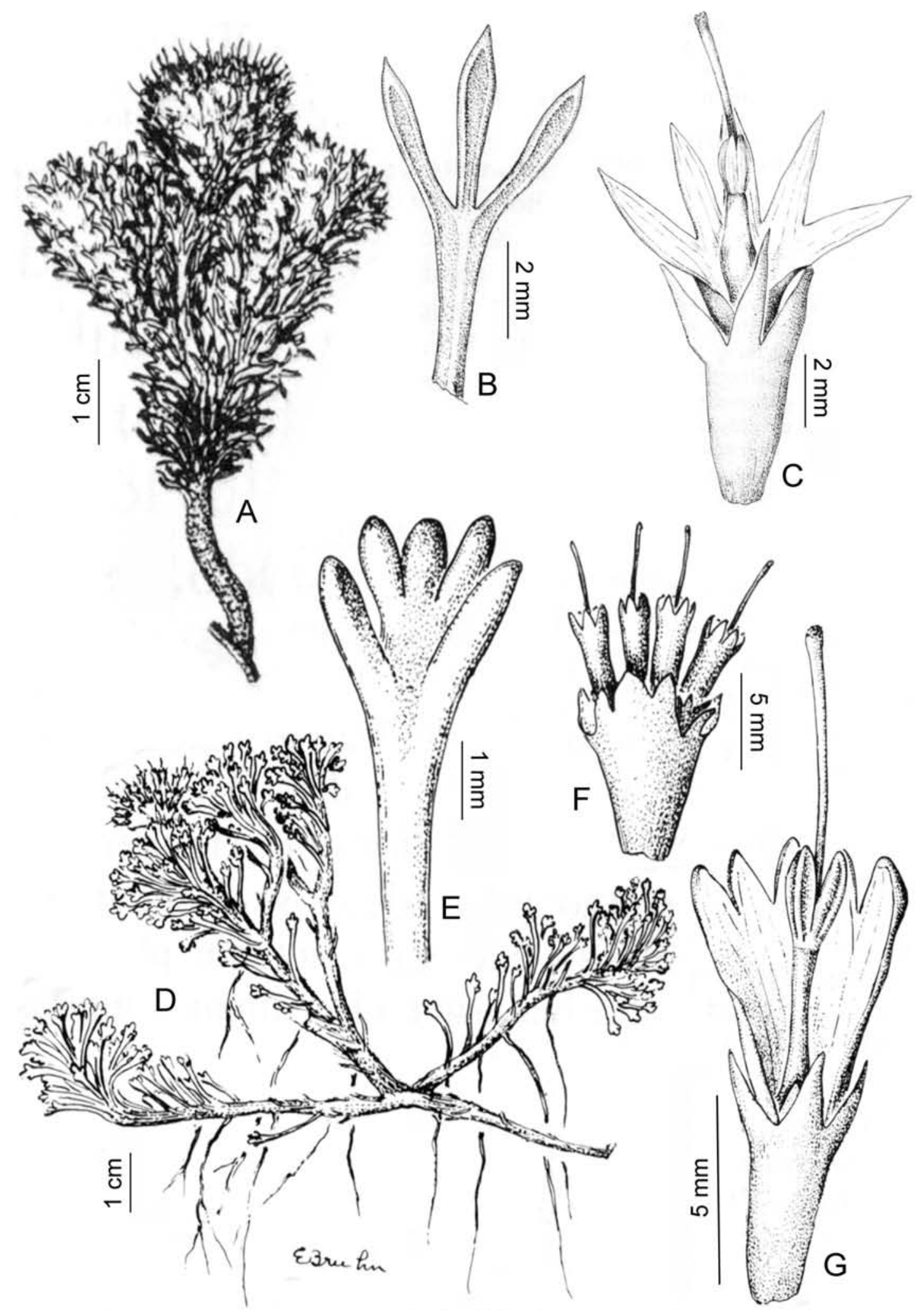

Figura 32. A-C. Moschopis trilobata. A, detalle del hábito. B, hoja. C, flor con la corola diseccionada mostrando el androceo. D-G. M. subandina. D, hábito. E, hoja. F, grupo cimoso externo mostrando el involucelo. G, flor con la corola y el hipanto diseccionados, mostrando el androceo. (Tomado de Chiapella, 1999a). 


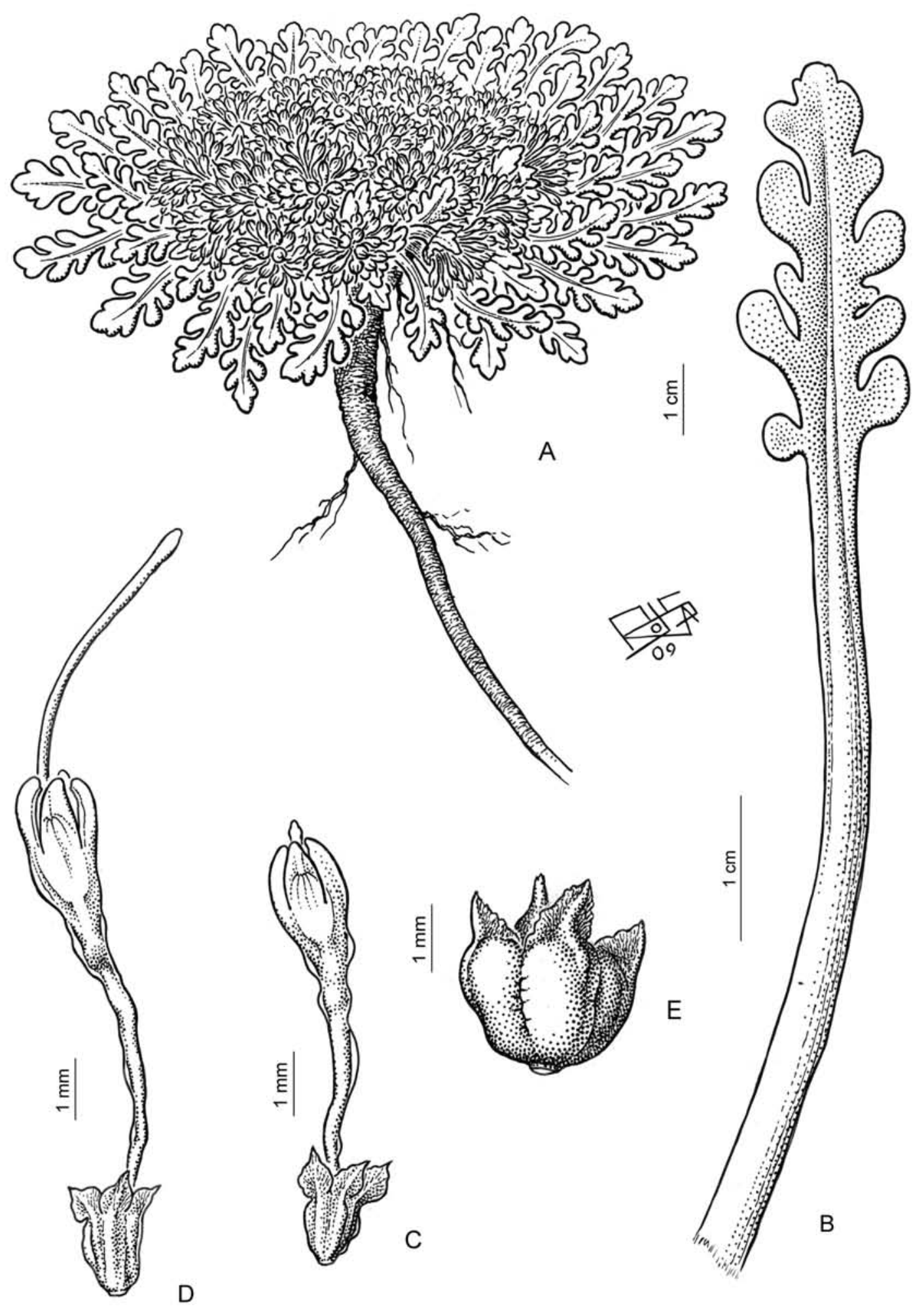

Figura 33. Nastanthus caespitosus. A, hábito. B, hoja. C, flor en fase estaminada. D, flor en fase pistilada. E, aquenio. Dibujado de C. Muñoz-P. 3891 (SGO). 


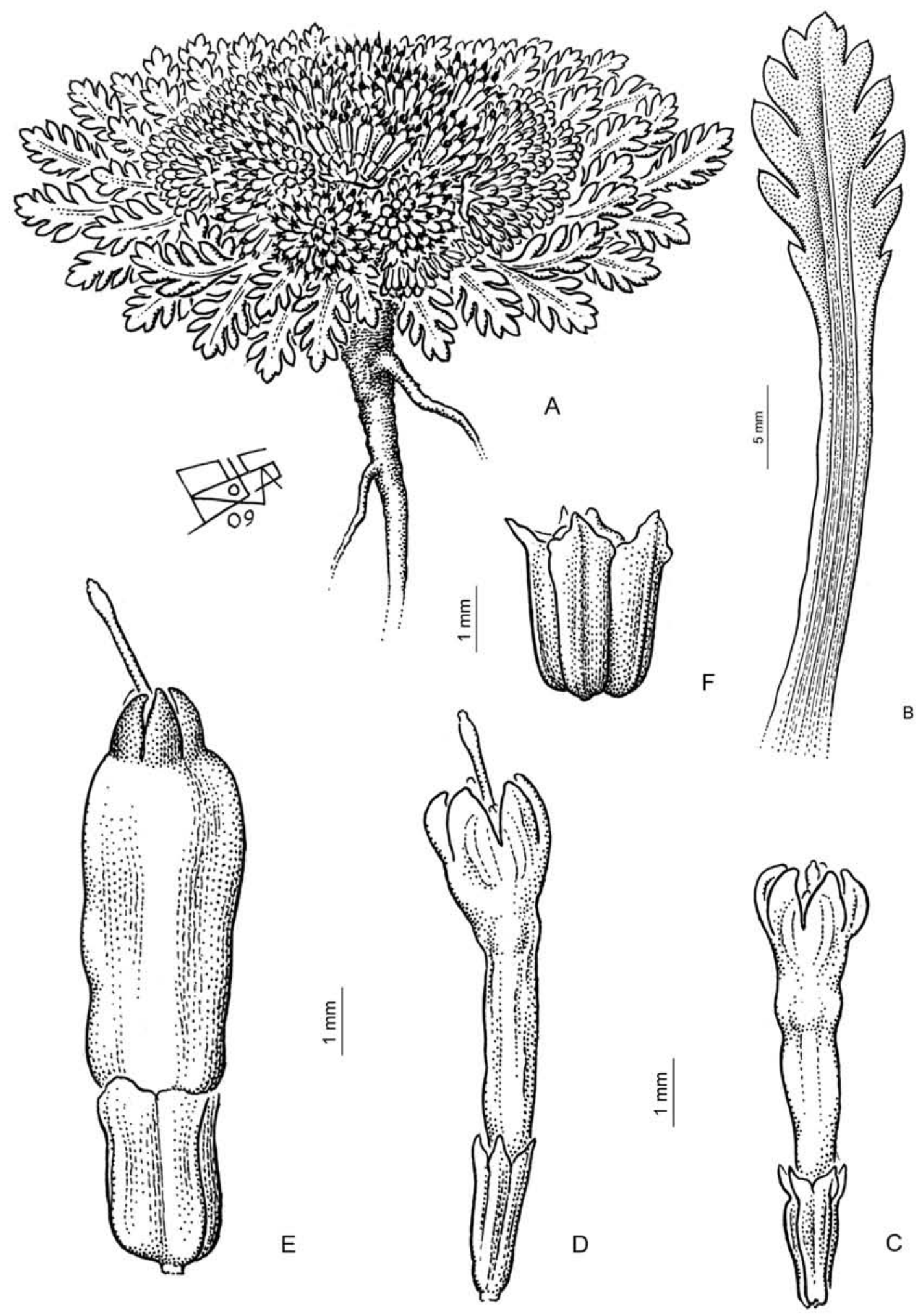

Figura 34. Nastanthus compactus. A, hábito. B, hoja. C, flor en fase estaminada. D, flor en fase pistilada. E, aquenio con corola persistente, acrescente. F, aquenio (corola removida). Dibujado de J. Solervicens s.n. (SGO-130759). 


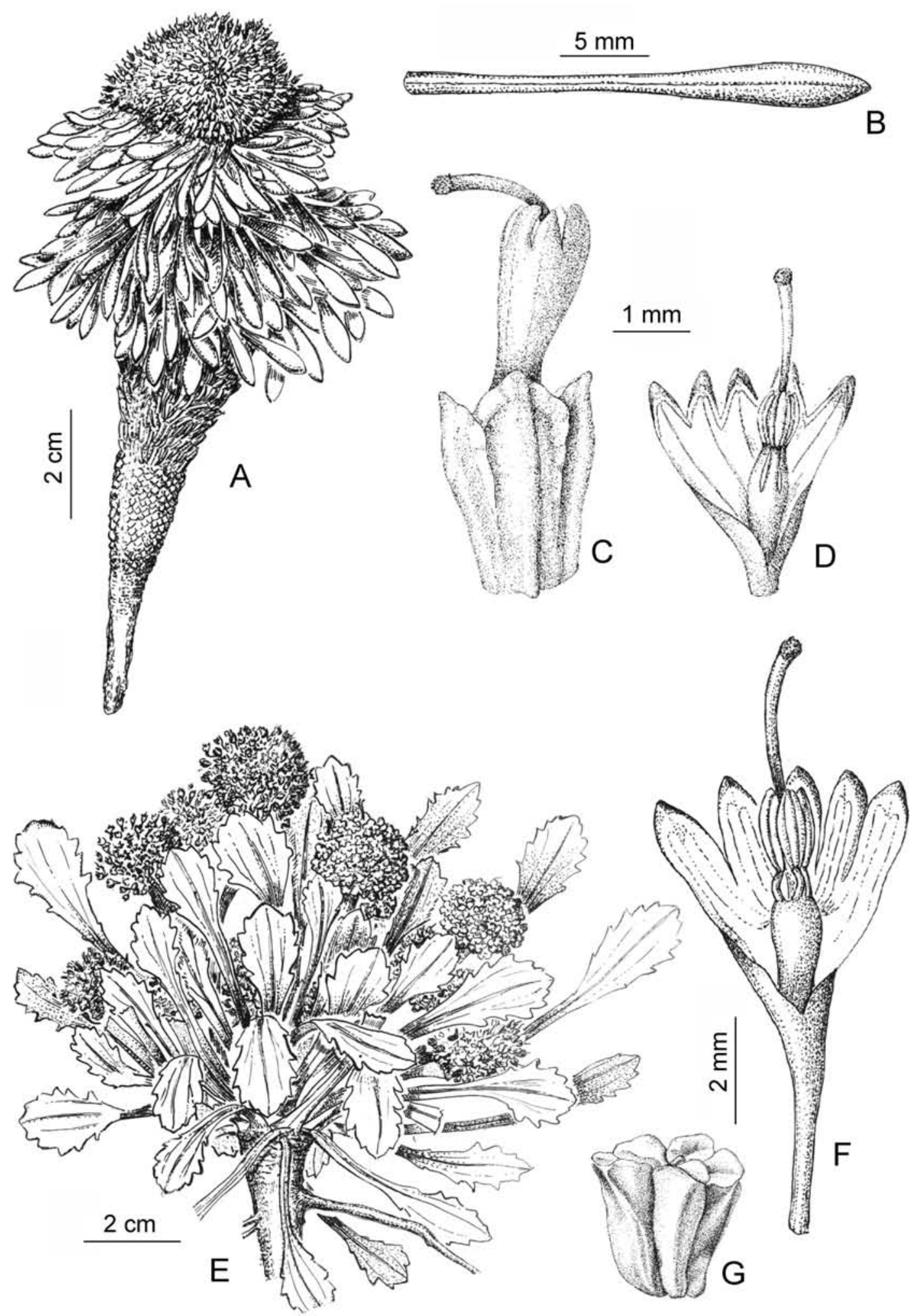

Figura 35. A-D. Nastanthus falklandicus. A, hábito. B, hoja. C, flor en fase pistilada. D, Corola diseccionada mostrando el tubo estaminal. E-G. N. scapigerus. E, hábito. F, perianto diseccionado mostrando el androceo y la continuación interna del hipanto por debajo del tubo estaminal. G, aquenio. (Tomado de Chiapella, 1999a). 


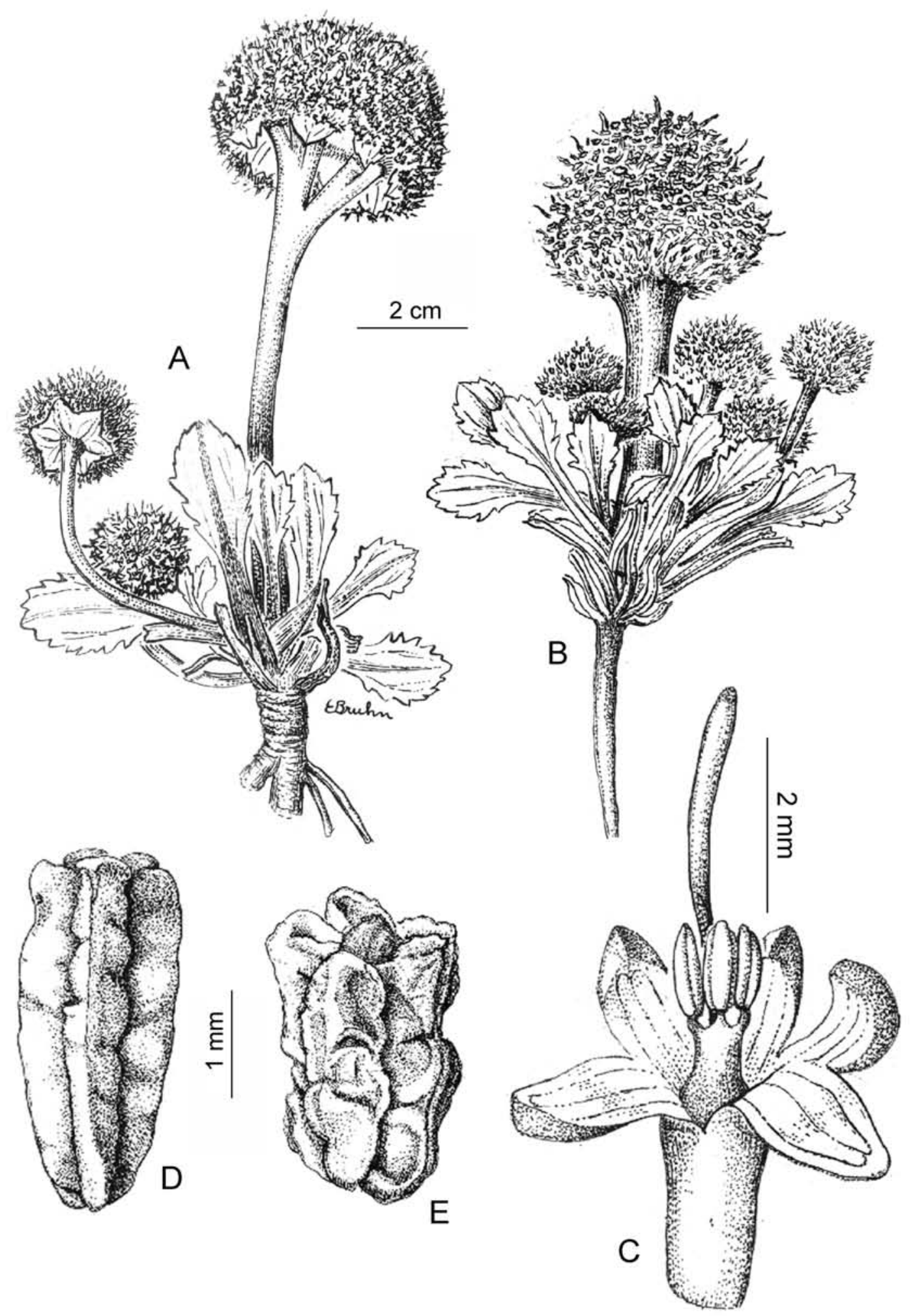

Figura 36. Nastanthus patagonicus. A-B, hábito. C, flor con el tubo corolino diseccionado dejando visible el tubo estaminal (ovario removido). D-E, variación del aquenio. (Tomado de Chiapella 1999a: A, C y D, sub N.patagonicus; B y E, sub N. Scapigerus). 


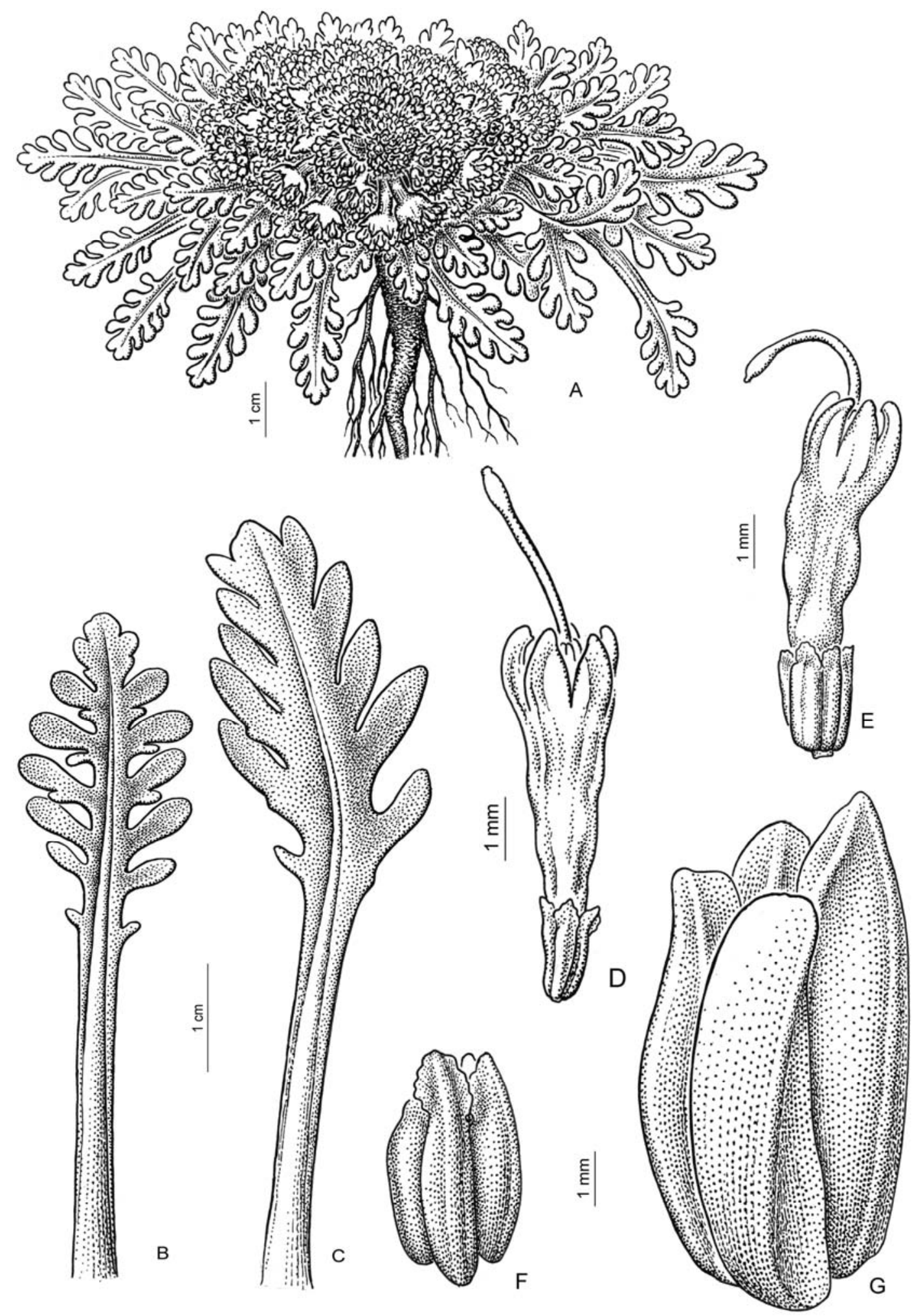

Figura 37. Nastanthus ventosus. A, hábito. B-C, variación de la hoja. D, flor en fase pistilada. E, aquenio inmaduro y corola marchita. F-G, variación del aquenio. A, B, D y E, dibujado de F. Roig s.n. (MERL 18719); C y F, de A. Ruiz Leal 23417 (MERL); G, de A. Ruiz Leal 6611 (MERL). 


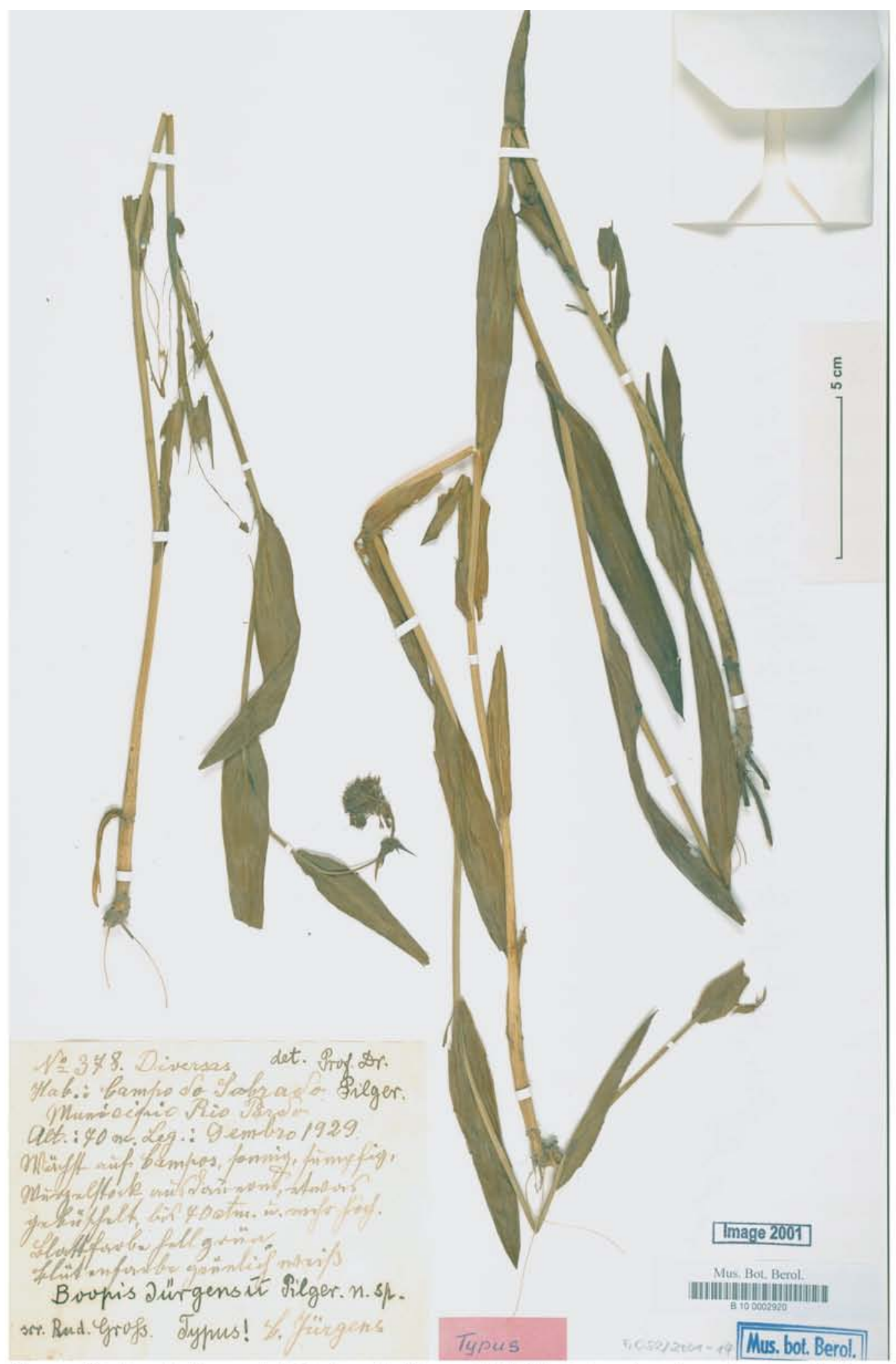

Figura 38. Boopis jürgensii. Holotipo, C. Jürgens 378 (B); único ejemplar coleccionado de esta especie en Rio Grande do Sul (Brasil) en 1929. 


\subsubsection{Resumen de la revisión nomeclatural de la familia Calyceraceae}

En el siguiente cuadro se resume la nomenclatura para las 47 especies aceptadas, sus sinónimos (nomenclaturales y taxonómicos) y las novedades para toda la familia. Se indica la presencia de sintipos cuando la selección de lectotipos no se consideró apropiada. 


\begin{tabular}{|c|c|c|c|c|}
\hline & Nombre aceptado & novedad & Sinónimos taxonómicos & novedad \\
\hline \multirow{9}{*}{$\begin{array}{l}\text { ACICARPHA Juss. } \\
\text { = Cryptocarpha } \\
\text { Cass. } \\
=\text { Sommea } \text { Bory } \\
=\text { Acanthosperma } \\
\text { Vell. } \\
=\text { Echinolema } \mathrm{J} . \\
\text { Jacq. } \text { ex DC. } \\
=\text { Acicarpa } \text { R. Br. }\end{array}$} & Acicarpha obtusisepala Marchesi & & & \\
\hline & \multirow{4}{*}{$\begin{array}{l}\text { Acicarpha spathulata } \mathrm{R} . \mathrm{Br} \text {. } \\
=\text { Cryptocarpha spathulata } \text { Cass., nom. superfl. }\end{array}$} & & Sommea calcitrapa Bory & \\
\hline & & & Acanthosperma littorale Vell. & \\
\hline & & & Echinolema arenarium J. Jacq. ex DC. & \\
\hline & & & Acicarpha spathulata $\mathrm{R}$. Br. var. glauca DC. & \\
\hline & \multirow{4}{*}{$\begin{array}{l}\text { Acicarpha tribuloides Juss. } \\
\text { = Cryptocarpha tribuloides } \text { Cass., nom. superfl. }\end{array}$} & & $\begin{array}{l}\text { Acicarpha pinnatifida Miers } \\
=\text { Acicarpha tribuloides var. pinnatifida } \text { (Miers) Kuntze }\end{array}$ & \\
\hline & & & Acicarpha runcinata Miers & \\
\hline & & & Acicarpha tribuloides Juss. var. dentata Kuntze & $\begin{array}{l}\text { lectotipo } \\
\text { propuesto }\end{array}$ \\
\hline & & & Acicarpha laxa R.E.Fr. & $\begin{array}{l}\text { lectotipo } \\
\text { propuesto }\end{array}$ \\
\hline \multirow{12}{*}{$\begin{array}{l}\text { BOOPIS Juss. } \\
=\text { Acarpha Griseb. }\end{array}$} & \multirow{5}{*}{ Boopis anthemoides Juss. } & & $\begin{array}{l}\text { Boopis rigidula Miers } \\
=\text { Boopis anthemoides Juss. var. rigidula (Miers) Griseb. }\end{array}$ & $\begin{array}{l}\text { lectotipo } \\
\text { propuesto }\end{array}$ \\
\hline & & & $\begin{array}{l}\text { Boopis rigidula Miers var. patagonica Hieron. } \\
=\text { Boopis anthemoides Juss. var. rigidula (Miers) Griseb. f. } \\
\text { patagonica } \text { (Hieron.) Hicken }\end{array}$ & \\
\hline & & & Boopis anthemoides Juss. var. andina Hieron. & $\begin{array}{l}\text { syn. nov. } \\
\text { propuesto }\end{array}$ \\
\hline & & & Boopis anthemoides Juss. var. subscandens Speg. & \\
\hline & & & Boopis anthemoides Juss. var. subintegrifolia Hicken & $\begin{array}{l}\text { lectotipo y syn. } \\
\text { nov. propuestos }\end{array}$ \\
\hline & \multirow{3}{*}{$\begin{array}{l}\text { Boopis australis Decne. } \\
=\text { Acarpha australis Griseb. }\end{array}$} & \multirow{3}{*}{ sintipos } & $\begin{array}{l}\text { Boopis squarrosa Miers } \\
=\text { Boopis australis } \text { Decne. var. squarrosa (Miers) Reiche }\end{array}$ & sintipos \\
\hline & & & $\begin{array}{l}\text { Boopis leptophylla Speg. } \\
=\text { Boopis autralis Decne. var. leptophylla (Speg.) Hicken }\end{array}$ & \\
\hline & & & Boopis australis Decne. var. hickenii Pontiroli & \\
\hline & $\begin{array}{l}\text { Boopis bupleuroides (Less.) C. A. Muell. } \\
=\text { Acicarpha bupleuroides Less. }\end{array}$ & & $\begin{array}{l}\text { Boopis bupleuroides (Less.) C.A. Muell. var. microphylla C.A. } \\
\text { Muell. }\end{array}$ & \\
\hline & $\begin{array}{l}\text { Boopis castilloni (Hicken) Pontiroli } \\
=\text { Calycera castilloni Hicken }\end{array}$ & & & \\
\hline & Boopis chubutensis Speg. & & & \\
\hline & Boopis filifolia Speg. & & Boopis prichardi S. Moore & \\
\hline
\end{tabular}




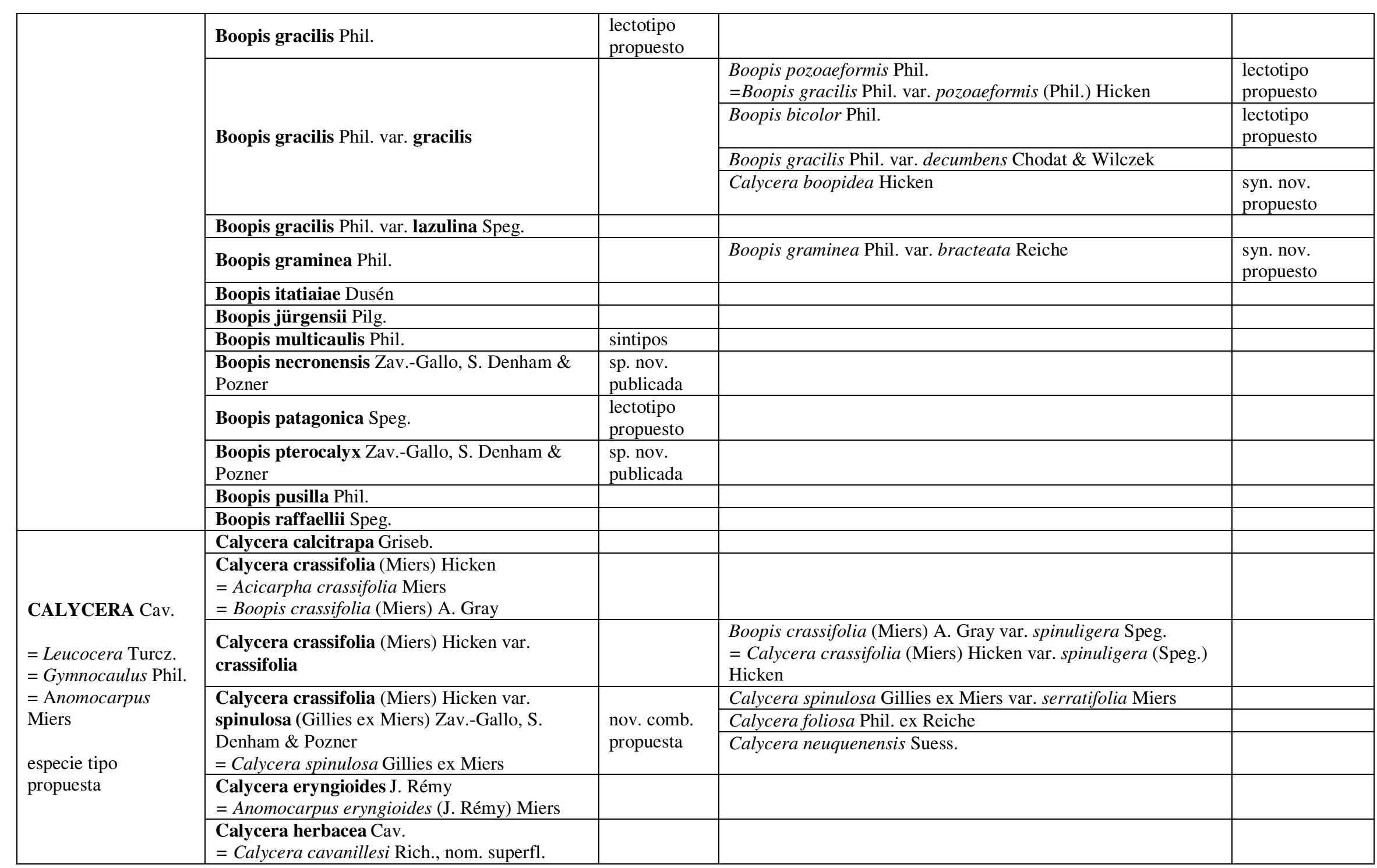




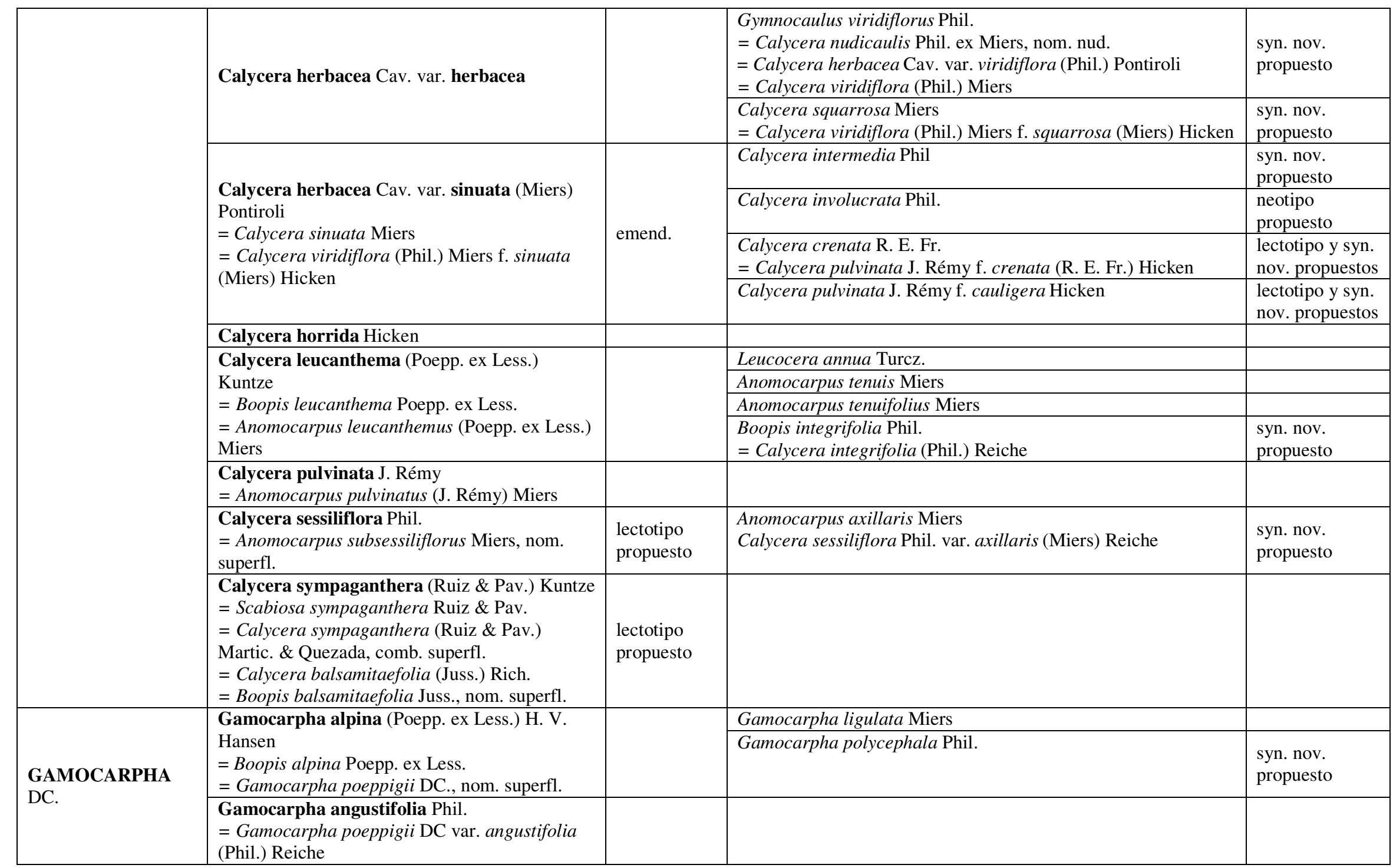




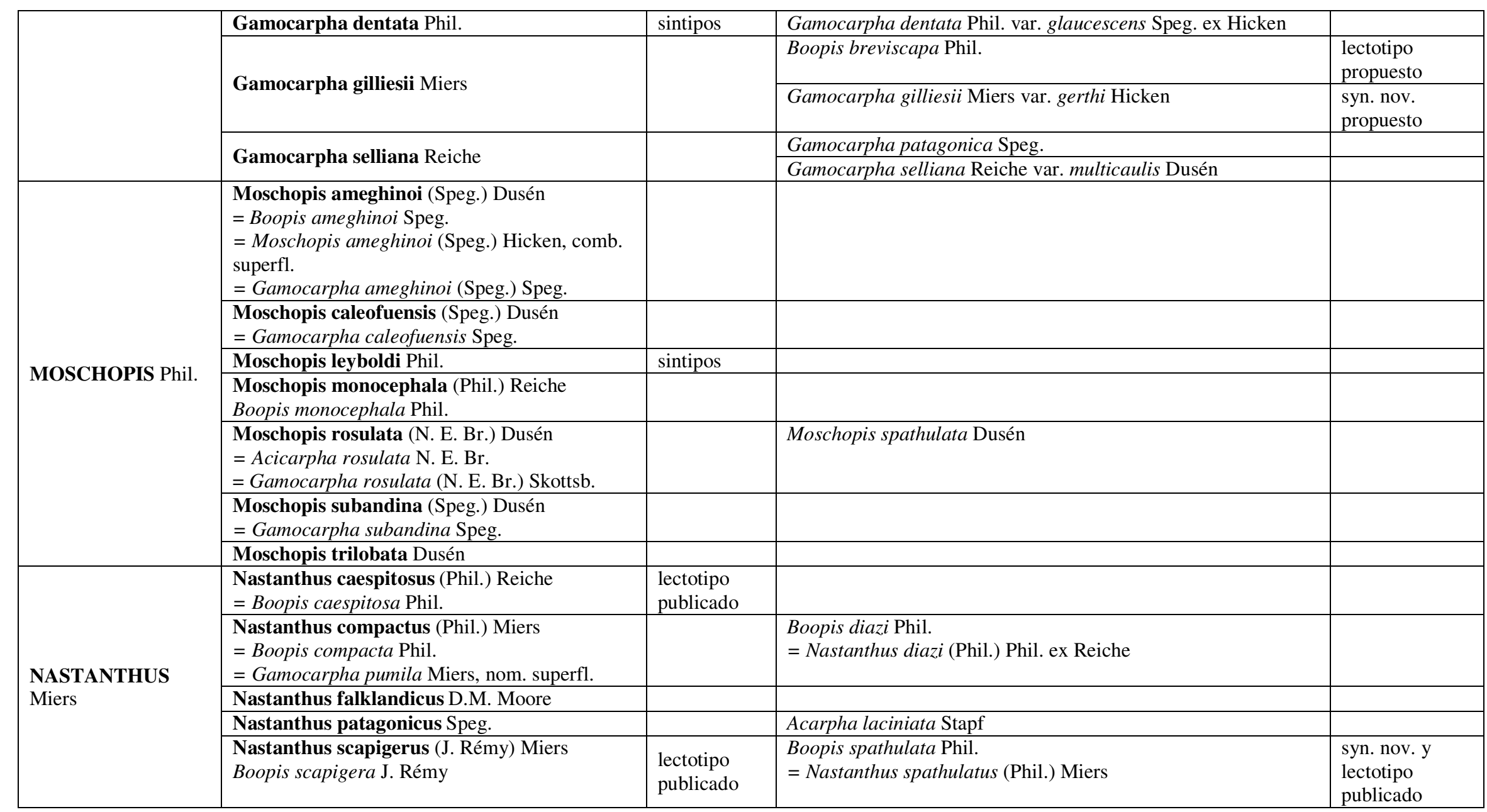




\begin{tabular}{|c|c|c|c|}
\hline & & $\begin{array}{l}\text { Nastanthus laciniatus Miers } \\
=\text { Boopis laciniata } \text { (Miers) Ball } \\
=\text { Nastanthus agglomeratus Miers var. laciniatus (Miers) } \\
\text { Reiche } \\
=\text { Boopis agglomerata (Miers) Hauman var. laciniata (Miers) } \\
\text { Hauman }\end{array}$ & $\begin{array}{l}\text { syn. nov. y } \\
\text { lectotipo } \\
\text { publicado }\end{array}$ \\
\hline & & $\begin{array}{l}\text { Boopis araucana } \text { Phil. } \\
=\text { Nastanthus araucanus (Phil.) Reiche }\end{array}$ & $\begin{array}{l}\text { syn. nov. y } \\
\text { lectotipo } \\
\text { publicado }\end{array}$ \\
\hline & & $\begin{array}{l}\text { Boopis bellidifolia } \text { Phil. } \\
=\text { Nastanthus bellidifolius } \text { (Phil.) Reiche } \\
=\text { Nastanthus spathulatus (Phil.) Miers var. bellidifolius (Phil.) } \\
\text { Pontiroli }\end{array}$ & $\begin{array}{l}\text { syn. nov. } \\
\text { publicado }\end{array}$ \\
\hline & & Boopis reichei Phil. & $\begin{array}{l}\text { syn. nov. } \\
\text { Publicado } \\
\end{array}$ \\
\hline & & Boopis dubia Phil. & $\begin{array}{l}\text { syn. nov. } \\
\text { publicado }\end{array}$ \\
\hline & & Boopis miersii Phil. & \\
\hline & & $\begin{array}{l}\text { Nastanthus chubutensis Speg. } \\
=\text { Nastanthus bellidifolius (Phil.) Reiche var. chubutensis } \\
\text { (Speg.) Hicken }\end{array}$ & $\begin{array}{l}\text { syn. nov. } \\
\text { publicado }\end{array}$ \\
\hline & & Boopis andicola Gand. & $\begin{array}{l}\text { syn. nov. } \\
\text { propuesto }\end{array}$ \\
\hline \multirow{4}{*}{$\begin{array}{l}\text { Nastanthus ventosus (Meyen) Miers } \\
=\text { Calycera ventosa Meyen } \\
=\text { Boopis scapigera } \mathrm{J} . \text { Rémy var. ventosa } \\
=(\text { Meyen) Weddell } \\
=\text { Nastanthus caespitosus (Phil.) Reiche var. } \\
\text { ventosus } \text { (Meyen) Pontiroli }\end{array}$} & \multirow{4}{*}{$\begin{array}{l}\text { lectotipo } \\
\text { publicado }\end{array}$} & $\begin{array}{l}\text { Boopis gayana Phil. } \\
=\text { Nastanthus gayanus (Phil.) Miers } \\
=\text { Nastanthus agglomeratus Miers var. gayanus (Phil.) Reiche }\end{array}$ & \\
\hline & & $\begin{array}{l}\text { Nastanthus agglomeratus Miers } \\
=\text { Boopis agglomeratus } \text { (Miers) Hauman } \\
=\text { Calycera andina } \text { Miers, nom. nud. } \\
=\text { Nastanthus } \text { andina } \text { (Miers) H.V. Hansen }\end{array}$ & $\begin{array}{l}\text { syn. nov. } \\
\text { publicado }\end{array}$ \\
\hline & & $\begin{array}{l}\text { Nastanthus pinnatifidus Miers } \\
=\text { Nastanthus agglomeratus Miers var. pinnatifidus (Miers) } \\
\text { Reiche } \\
=\text { Boopis agglomerata (Miers) Hauman var. pinnatifidus } \\
\text { (Miers) Hauman }\end{array}$ & $\begin{array}{l}\text { syn. nov. y } \\
\text { lectotipo } \\
\text { publicado }\end{array}$ \\
\hline & & $\begin{array}{l}\text { Nastanthus gilliesii Miers } \\
\text { Nastanthus agglomeratus Miers var. gilliesii (Miers) Reiche }\end{array}$ & \\
\hline
\end{tabular}




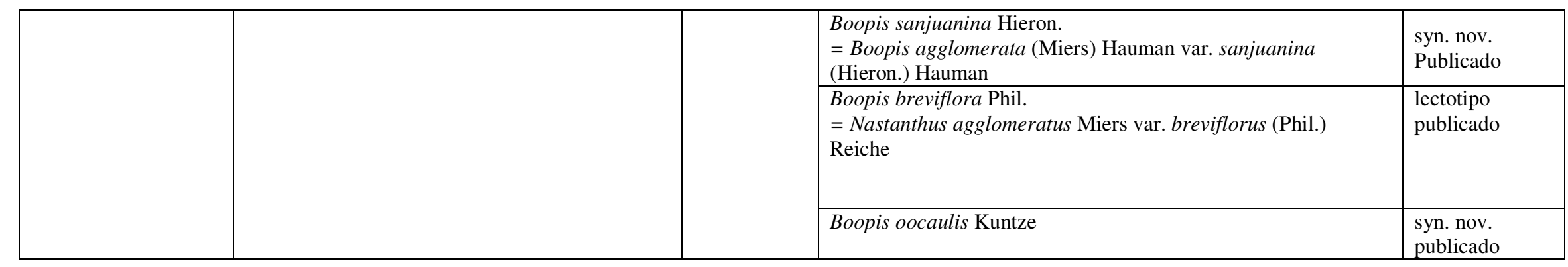

\section{NOMBRES DUDOSOS}

Boopis acaulis Phil.

= Nastanthus acaulis (Phil.) Reiche.

Acicarpha lanata Lag. ex Pers.

= Calycera lanata (Lag. ex Pers.) Benth. \& Hook. f.

$=$ Cryptocarpha lanata Cass., nom. superfl. 


\subsection{Análisis cladístico}

\subsubsection{Descripción de las matrices}

La matriz morfológica quedó conformada por 37 caracteres y 2,5\% de datos faltantes o no codificables. Todos los caracteres son informativos.

La matriz molecular alineada de los dos marcadores presentó 1378 pares de bases (pb), más 16 caracteres correspondientes a la codificación de gaps, con un total de 1394 caracteres; $30 \%$ de datos faltantes y 108 caracteres informativos, de acuerdo a las siguientes tablas:

\begin{tabular}{|l|c|c|c|c|}
\hline \multicolumn{1}{|c|}{ Marcador } & $\begin{array}{c}\text { Secuencia más } \\
\text { corta sin gaps }\end{array}$ & $\begin{array}{c}\text { Secuencia más } \\
\text { larga sin gaps }\end{array}$ & $\begin{array}{c}\text { Secuencia } \\
\text { alineada }\end{array}$ & $\begin{array}{c}\text { Caracteres } \\
\text { informativos }\end{array}$ \\
\hline trnS-trnG & $460 \mathrm{pb}$ & $703 \mathrm{pb}$ & $810 \mathrm{pb}$ & $25 \mathrm{pb}$ \\
\hline trnH-psbA & $360 \mathrm{pb}$ & $490 \mathrm{pb}$ & $568 \mathrm{pb}$ & $67 \mathrm{pb}$ \\
\hline Total & & & $1378 \mathrm{pb}$ & $92 \mathrm{pb}$ \\
\hline
\end{tabular}

Se identificaron 16 gaps no ambiguos e informativos (Simmons \& Ochoterena, 2000). Se trataron como dato faltante en la matriz pero luego se codificaron como presencia / ausencia, como se detallan en la siguiente tabla:

\begin{tabular}{|c|c|c|}
\hline GAP & Nro. de pb & $\begin{array}{c}\text { Ubicación del gap en el } \\
\text { marcador }\end{array}$ \\
\hline $1(\operatorname{trnS}-\operatorname{trnG})$ & 24 & $66-89$ \\
\hline $2(\operatorname{trnS}-\operatorname{trnG})$ & 17 & $180-196$ \\
\hline $3(\operatorname{trnS}-\operatorname{trnG})$ & 13 & $454-466$ \\
\hline $4(\operatorname{trnS}-\operatorname{trnG})$ & 4 & $467-470$ \\
\hline $5(\operatorname{trn} S-\operatorname{trnG})$ & 7 & $475-481$ \\
\hline $6(\operatorname{trnS}-\operatorname{trnG})$ & 1 & 347 \\
\hline $7(\operatorname{trnS}-\operatorname{trnG})$ & 5 & $654-658$ \\
\hline $8(\operatorname{trnH}-\mathrm{PSBA})$ & 25 & $90-114$ \\
\hline $9(\operatorname{trnH}-\mathrm{PSBA})$ & 7 & $138-144$ \\
\hline $10(\operatorname{trnH}-\mathrm{PSBA})$ & 5 & $133-137$ \\
\hline
\end{tabular}




\begin{tabular}{|c|c|c|}
\hline $11(\operatorname{trnH}-P S B A)$ & 16 & $168-183$ \\
\hline $12(\operatorname{trnH}-P S B A)$ & 7 & $330-336$ \\
\hline $13(\operatorname{trnH}-P S B A)$ & 3 & $335-337$ \\
\hline $14(\operatorname{trnH}-P S B A)$ & 4 & $334-336$ \\
\hline $15(\operatorname{trnH}-P S B A)$ & 1 & 476 \\
\hline $16(\operatorname{trnH}-P S B A)$ & 1 & 497 \\
\hline
\end{tabular}

La matriz combinada quedó conformada de la siguiente manera:

\begin{tabular}{|l|c|c|}
\hline \multicolumn{1}{|c|}{ Datos } & $\begin{array}{c}\text { Posición en la } \\
\text { matriz } \\
\text { combinada }\end{array}$ & $\begin{array}{c}\text { Nro. de } \\
\text { caracteres }\end{array}$ \\
\hline trnH-PSBA & $0-567$ & 568 \\
\hline trnS-trnG & $568-1377$ & 810 \\
\hline Gaps de trnS-trnG & $1378-1384$ & 7 \\
\hline Gaps de trnH-PSBA & $1385-1393$ & 9 \\
\hline Morfología & $1394-1430$ & 37 \\
\hline
\end{tabular}

3.4.2. Descripción y discusión de los árboles obtenidos. Sinapomorfías y soporte de los clados

Los resultados de los tres análisis realizados son los siguientes:

\begin{tabular}{|l|c|c|c|}
\hline & $\begin{array}{c}\text { Análisis } \\
\text { combinado }\end{array}$ & $\begin{array}{c}\text { Análisis } \\
\text { morfológico }\end{array}$ & $\begin{array}{c}\text { Análisis } \\
\text { molecular }\end{array}$ \\
\hline $\begin{array}{l}\text { Nro. de árboles más } \\
\text { parsimoniosos (AMP) }\end{array}$ & 2 & 10 & 7 \\
\hline Nro. de pasos de los AMP & 431 & 156 & 286 \\
\hline CI & 0.619 & 0.276 & 0.787 \\
\hline RI & 0.779 & 0.672 & 0.873 \\
\hline
\end{tabular}

En todos los análisis, la familia Calyceraceae es monofilética. Las sinapomorfías morfológicas establecidas en trabajos previos para definir a Calyceraceae son: anatomía nodal unilacunar, placentación apical (Gustafsson \& Bremer, 1995) y presencia de tubo estaminal (Lundberg \& Bremer, 2003). De acuerdo con el análisis 
de esta tesis puede agregarse: inflorescencia de tipo cefalodio [si bien la inflorescencia se desdobló en varios caracteres, la reconstrucción del tipo de inflorescencia en el ancestro de la familia es el de un cefalodio simple (cf. también Pozner et al., 2012)].

De acuerdo a los resultados del análisis filogenético, los géneros tradicionales de la familia Calyceraceae quedan, en la mayoría de los casos, segregados: Boopis, Calycera, Gamocarpha, Moschopis y Nastanthus son polifiléticos, mientras que Acicarpha es el único grupo cuya monofilia queda soportada.

A continuación, se discuten los resultados obtenidos a partir del árbol de consenso estricto de los dos árboles mas parsimoniosos del análisis combinado (Fig. 39); luego se comparan estos resultados con las topologías de los árboles de consenso obtenidas en los análisis de caracteres morfológicos (Fig. 40) y moleculares (Fig. 41) por separado.

En la Figura 39 pueden distinguirse dos grupos monofiléticos cuyas sinapomorfías están relacionadas con el origen de los nectarios (glándulas nectaríferas). El Clado A incluye especies con nectarios estaminales (clado 1 y clado 2+Boopis gracilis+Moschopis leyboldi); sin embargo, en este clado la presencia de nectarios estaminales es una sinapomorfía ambigua por su ausencia en Boopis anthemoides. El Clado B agrupa especies con nectarios hipantiales (Calycera sessiliflora+clado 3+clado 4+clado 5).

Clado A

El clado A posee bajos niveles de soporte: $\mathrm{J}=53 \%$ para el análisis de datos combinados y $\mathrm{J}=59 \%$ para el análisis de datos moleculares. En el árbol de consenso para caracteres morfológicos, el clado A aparece aunque con modificaciones: se agregan 12 taxones no incluidos en el análisis molecular (Boopis necronensis, B. castilloni, B. bupleuroides, B. itatiaiae, B. raffaellii, B. graminea, Acicarpha procumbens, Moschopis ameghinoi, Gamocarpha angustifolia, Nastanthus caespitosus, N. ventosus y Calycera eryngioides); queda excluido B. anthemoides y se incluye M. monocephala.

El clado 1 contiene todas las especies del género Acicarpha contempladas en este análisis (A. obtusisepala, A. spathulata, A. tribuloides; 3 de 4 especies) y Boopis anthemoides como taxón hermano. Este grupo no aparece soportado $(\mathrm{J}<50 \%)$ en el análisis combinado y queda segregado en todos los árboles resultantes del análisis de 
datos morfológicos por la exclusión de $B$. anthemoides. Sin embargo, queda formado en todos los árboles resultantes de la matriz de datos moleculares (las tres sinapomorfías que definen este grupo son sustituciones nucleotídicas).

Sin embargo, dentro del clado 1, puede reconocerse el grupo monofilético formado por las especies del género Acicarpha. Este grupo aparece formado en todos los árboles resultantes de las matrices morfológica, molecular y combinada, con un fuerte nivel de soporte $[\mathrm{B}=4 ; \mathrm{J}$ molecular $(\mathrm{Jm})=87 \%$, $\mathrm{J}$ combinado $(\mathrm{Jc})=99 \%]$. De acuerdo a la optimización de sinapomorfías comunes a todos los árboles obtenidos en el análisis combinado, se identificaron como sinapomorfías morfológicas los siguientes caracteres: 9 (filamentos estaminales, ausentes), 15 (posición del ovario de las flores perfectas, hundido en el receptáculo), 19 (flores estaminadas en el centro de la inflorescencia, presentes), 24 (forma del receptáculo florífero, columnar), 25 (forma del receptáculo fructífero, columnar), 28 (grupos cimosos internos al involucro, ausentes), 34 (fruto compuesto, presente), 37 (cipselas periféricas fusionadas al receptáculo, presentes).

El clado 2 agrupa varios taxones pertenecientes a los géneros Boopis, Gamocarpha y Moschopis (G. dentata, M. caleofuensis, B. patagonica, M. trilobata, M. rosulata, M. subandina, B. filifolia), muchas distinguibles por secretar un exudado glutinoso apreciable en la superficie de las hojas, por lo que será designado en adelante como grupo "Glutinosas". Este grupo aparece como monofilético en todos los árboles obtenidos a partir de las matrices morfológica, molecular y de datos combinados, aunque con bajos niveles de soporte $(\mathrm{B}=2 ; \mathrm{Jm}=59 \%, \mathrm{Jc}=53 \%)$. De acuerdo con la optimización de sinapomorfías morfológicas comunes, los caracteres que identifican a este grupo son: 9 (filamentos estaminales, ausentes), 12 (forma del ápice de los lóbulos corolinos, uncinada), 29 (grupos cimosos externos al involucro, presentes). El caracter 1 (hojas glutinosas) es una sinapomorfía ambigua para el grupo, debido a su ausencia en Boopis patagonica y a que en Moschopis trilobata y M. rosulata no todos los individuos lo expresan (codificado como polimórfico).

Moschopis caleofuensis y G. dentata forman el grupo más soportado dentro de las "Glutinosas" con un alto nivel de soporte para los análisis de datos moleculares y combinados $(\mathrm{Jm}=90 \%$ y $\mathrm{Jc}=91 \%)$. Siendo de géneros diferentes se relacionan por la similitud de sus secuencias de marcadores de cloroplasto, aunque en el árbol 
morfológico no sean hermanas. Además, la ausencia de los gaps 15 y 16 las diferencian del resto de los taxones incluidos en el clado "Glutinosas"

Por su parte, el subclado formado por M. trilobata, M. rosulata, M. subandina, B. patagonica y B. filifolia comparte la presencia de los gaps 15 y 16 como sinapomorfías.

Moschopis leyboldi aparece como un taxón hermano del grupo "Glutinosas” sólo en el análisis de datos combinados. Los análisis de datos morfológicos y moleculares por separado no definen la posición de $M$. leyboldi y de B. gracilis dentro del clado A (politomía).

Por su parte, Boopis gracilis aparece como grupo hermano del clado M. leyboldi + "Glutinosas", con el caracter 14 (nervadura central de los sépalos, ausente) como sinapomorfía del clado, de acuerdo con el árbol de consenso estricto del análisis combinado. Aunque este grupo aparece en todos los árboles resultantes del análisis de caracteres combinados, no posee soporte $(\mathrm{J}<50 \%)$ y aparece colapsado en los árboles de consenso estricto para datos morfológicos y moleculares por separado. Las dos variedades de B. gracilis forman un grupo bien soportado $(\mathrm{Jm}=\mathrm{Jc}=99 \%)$, con el caracter 10 (longitud de los filamentos estaminales, reducidos) y la presencia de los gaps 5,10 y 14 como sinapomorfías.

\section{Clado B}

Los clados 3, 4 y 5 forman un grupo monofilético con una sinapomorfía morfológica nervadura central de los sépalos (ausente), mientras que Calycera sessiliflora aparece como un taxón basal, relacionado con el conjunto de los clados 3, 4 y 5 por compartir el caracter 6 (nectarios hipantiales, presentes). El clado B no posee un nivel de soporte significativo $(\mathrm{J}<50 \%)$ y queda completamente segregado en los análisis de datos morfológicos y moleculares por separado. Sin embargo, en el árbol de consenso estricto molecular es posible identificar a los clados 3 y 4 por separado. En el árbol de consenso estricto morfológico sólo puede verificarse la presencia del clado 3, aunque con modificaciones (véase a continuación), y la persistencia del subclado formado por $N$. compactus+ $N$. scapigerus a los que se agregan $N$. ventosus y $N$. caespitosus, no incluidos en el análisis molecular.

El clado 3 contiene algunos taxones de los géneros Calycera y Boopis (B. pusilla, B. pterocalyx, C. leucanthema, C. horrida, C. sympaganthera) agrupados con 
un nivel de soporte medio $(\mathrm{Jc}=69 \%)$. El árbol de consenso estricto molecular no resuelve la posición de Boopis pusilla y el árbol de consenso estricto para el análisis de datos morfológicos, agrupa a casi todas las especies del árbol de consenso estricto combinado, aunque segrega a Boopis pterocalyx e incluye en el grupo al taxón Calycera herbacea con sus dos variedades. A pesar de estas incongruencias, los grupos internos del clado 3 están respaldados por altos niveles de soporte $(\mathrm{Jc}>80 \%, \mathrm{Jm}=93 \%)$. De acuerdo a la optimización de sinapomorfías comunes, el clado 3 es soportado por dos caracteres: 3 (forma de la base de las anteras, sagitadas) y 21 (pubescencia del pedúnculo, presente). Debido a esta característica particular y exclusiva dentro de la familia se mencionará al clado 3 como grupo "Pubescentes".

Dentro de las sinapomorfías moleculares, el grupo de las "Pubescentes" comparte la presencia del gap 3, mientras que el subclado formado por C. leucanthema, B. pterocalyx, C. horrida, C. sympaganthera muestra como sinapomorfías moleculares los gaps 4,6 y 8.

El subclado formado por $C$. horrida y $C$. sympaganthera, es un grupo sólido que aparece en todos los árboles de consenso y posee un alto nivel de soporte ( $\mathrm{Jc}=87 \%$ ). Comparten tres sinapomorfías morfológicas: 2 (ápice de las anteras, apiculado), 4 (caudículas en las anteras, presentes), 12 (forma del ápice de los lóbulos corolinos, obtusos); y la presencia del gap 7 como sinapomorfía molecular.

El clado 4, que incluye varios taxones del género Calycera $(C$. herbacea con sus dos variedades, C. calcitrapa, C. pulvinata, C. crassifolia con sus dos variedades) y a Moschopis monocephala, es un grupo sin soporte $(\mathrm{J}<50 \%)$. Aunque también se forma en el árbol de consenso estricto del análisis molecular, en el consenso estricto del análisis de datos morfológicos el grupo aparece completamente segregado. El carácter 33 (cáliz fructífero espinoso, presente) es una sinapomorfía del grupo en el análisis combinado. Sin embargo, este estado es ambiguo para la rama basal del clado ya que el estado es "ausente" en M. monocephala y C. crassifolia.

A pesar de la falta de soporte para el conjunto de de taxones que integran el clado 4, pueden reconocerse dos subclados con buen soporte, que corresponden a:

- Las dos variedades de C. herbacea (var. herbacea y var. sinuata) forman un grupo con altos niveles de soporte $(\mathrm{Jc}=95 \%, \mathrm{Jm}=93 \%)$ con el caracter 36 (pared del fruto, costada) como sinapomorfía morfológica. 
- Las dos variedades de C. crassifolia (var. crassifolia y var. spinulosa) forman otro grupo con altos niveles de soporte $(\mathrm{Jc}=88 \%, \mathrm{Jm}=81 \%)$, siendo sus sinapomorfías morfológicas: 18 (proporción de la porción lobada de la corola, secta) y $\mathbf{3 5}$ (polimorfismo de frutos, ausente).

Por otra parte, el grupo formado por C. calcitrapa + C. crassifolia (var. crassifolia y var. spinulosa) aparece en el árbol de consenso estricto para datos combinados $(\mathrm{Jc}=50 \%)$, pero colapsa en el consenso estricto para datos moleculares y queda segregado en el consenso estricto para datos morfológicos.

El clado 5 está formado por taxones de los géneros Gamocarpha, Boopis y Nastanthus (G. selliana, G. gilliesii, G. alpina, B. multicaulis, B. australis, B. australis var. hickenii, B. chubutensis, N. compactus, N. scapigerus) agrupados en una misma rama (politomía) con Nastanthus patagonicus como taxón hermano. Aunque la inconsistencia del grupo queda reflejada por la ausencia de soporte, el clado aparece formado en el árbol de consenso de mayoría para caracteres moleculares y todos sus taxones comparten la presencia del gap 12 para las secuencias de cloroplasto como sinapomorfía. Sin embargo, en el árbol de consenso estricto para caracteres morfológicos el grupo de las "Pubescentes" y Calycera herbacea quedan incluidos dentro del clado. De acuerdo con el mapeo de caracteres morfológicos, las sinapomorfías que definen el clado 5 son los caracteres 4 (caudículas en las anteras, presentes) y $\mathbf{3 5}$ (polimorfismo de frutos, ausente).

\subsubsection{Discusión de los caracteres morfológico-taxonómicos usados en Calyceraceae para distinguir géneros.}

A continuación se discuten todos los caracteres usados tradicionalmente para definir los géneros de la familia Calyceraceae, los cuales fueron usados en este análisis filogenético (se indica su número en la lista de caracteres). Como medida del grado de homoplasia para cada caracter se indica: $\mathrm{N}^{\circ}$ de pasos en uno de los AMP del análisis combinado : $\mathrm{N}^{\mathrm{o}}$ mínimo de pasos para el carácter. La optimización de los caracteres discutidos en uno de los AMP puede observarse en el Apéndice 5, los nombres de los clados o grupos corresponde a los señalados en la figura 39 (árbol de consenso). 


\section{Flores estaminadas en el centro de la inflorescencia (1:1)}

La presencia de este carácter es usada como diagnóstico para el género Acicarpha. En el análisis combinado presenta un solo paso, de ausente a presente en el clado Acicarpha (Clado 1, excepto B. anthemoides) para el que representa una sinapomorfía y un caracter diagnóstico confiable.

\section{Estado de las brácteas del receptáculo (4:2)}

El estado "brácteas soldadas delimitando celdas" es usado tradicionalmente como caracter diagnóstico del género Gamocarpha. Sin embargo, todos los AMP del análisis combinado muestran que los taxones que presentan ese estado: Gamocarpha alpina, G. selliana, G. gilliesii y Boopis multicaulis integran una politomía en el Clado 5, en el árbol de consenso, junto a otras especies de Boopis y Nastanthus. Por su parte, este carácter es inaplicable para G. dentata y G. angustifolia por no poseer brácteas en el receptáculo, que quedan segregados del grupo e integran el clado 2 (véase Fig. 40).

El análisis morfológico mostró que: 1) el estado "libres en la base" es ancestral y predomina en casi todo el árbol, 2) Boopis raffaellii, Boopis graminea, Boopis australis y Boopis australis var. hickenii presentan brácteas libres y ocasionalmente soldadas por un lado del involucro, es decir, el estado "soldadas por uno de sus lados" se manifiesta de manera ambigua junto con el estado ancestral, apareciendo varias veces de forma independiente dentro del clado 5, y 3) B. multicaulis presenta brácteas soldadas delimitando celdas.

El genero Gamocarpha no es monofilético y los estados de este carácter no constituyen sinapomorfías para ningún grupo.

\section{Forma del receptáculo florífero (7:2)}

Este carácter se usa en taxonomía para distinguir al género Acicarpha, que posee el estado "columnar".

En el análisis combinado, el estado basal es "punctiforme" y está presente en casi toda la familia. El estado "globoso", que otorga más homoplasia al carácter, aparece independientemente 5 veces en el árbol: $C$. sympaganthera, B. pterocalyx, $C$. pulvinata, N. patagonicus y N. compactus (aunque también presentan este estado especies no incluidas en el análisis combinado, todas ellas no se agrupan en el análisis 
morfológico tampoco). El estado "columnar" aparece sólo una vez dentro del clado 1 y agrupa a A. obtusisepala + A. spathulata + A. tribuloides.

El estado "columnar" de este carácter representa una sinapomorfía para el género Acicarpha.

\section{Forma del receptáculo fructífero (5:3)}

Este caracter es usado como diagnóstico para distinguir dos géneros: Acicarpha, al que correponde el estado "columnar", y Nastanthus, al que corresponde el estado "globoso".

Los análisis combinado y morfológico muestran al estado "columnar" como una sinapomorfía del clado Acicarpha. Por su parte, el estado "globoso" aparece tres veces de forma independiente dentro del clado B: en B. pterocalyx (clado 3), en Nastanthus patagonicus (basal en el clado 5) y en N. scapigerus (politomía del clado 5).

El análisis morfológico de las especies de la familia mostró que: 1) Boopis itatiaiae presenta el receptáculo fructífero columnar y representa el taxón hermano del clado Acicarpha (véase Fig. 40, Clado 1), 2) el estado "globoso" aparece también en $B$. necronensis (asociado a B. pterocalyx y $N$. patagonicus en la filogenia morfológica) y en $N$. ventosus (asociado con $N$. scapigerus en la filogenia morfológica), y 3) a Boopis castilloni corresponde el nuevo estado del carácter, "discoideo", que no había sido descripto anteriormente en la familia.

El receptáculo fructífero "columnar" representa un carácter diagnóstico confiable para el grupo Acicarpha, aunque genera incertidumbre acerca del alcance taxonómico del género por su presencia compartida con B. itatiaiae y B. jürgensii.

El estado "globoso" se manifiesta independientemente en varios taxones de Nastanthus y Boopis y no constituye una sinapomorfía ni un carácter diagnóstico para ningún grupo.

El estado "discoideo" se manifiesta únicamente en Boopis castilloni (incluido sólo en el análisis morfológico) y representa una autapomorfía para dicho taxón.

De acuerdo al análisis combinado el estado ancestral es "punctiforme" (compartido por la mayoría de los taxones de Calyceraceae). Por su parte, el análisis morfológico propone a $B$. castilloni como taxón basal de la familia y al estado "discoideo" como ancestral. Para resolver esta diferencia, será necesario incluir caracteres moleculares de B. castilloni en futuros análisis filogenéticos. 
Boopis jürgensii se encontraría en una situación similar a la de $B$. itatiaiae dado que comparte el receptáculo fructífero columnar (aunque no fue incluido en ninguno de los análisis, véase apartado 2.5.1).

\section{Diferenciación del involucro (4:1)}

La ausencia de involucro es usado como carácter diagnóstico del género Moschopis. En el análisis combinado el estado "ausente", aparece sólo en tres taxones del género y de manera independiente: Moschopis leyboldi (clado A), M. monocephala (clado 4) y $M$. trilobata (clado 2). El estado ancestral del carácter en la familia Calyceraceae es "presente".

Este caracter demostró ser altamente homoplásico, y aunque el estado "ausente" aparece en los tres taxones mencionados más arriba, estas especies no están relacionadas filogenéticamente.

El análisis morfológico de las especies de la familia mostró que: 1) $M$. caleofuensis, $M$. subandina, $M$. rosulata y $M$. ameghinoi presentan un involucro diferenciable (aunque la taxonomía tradicional distinguió a Moschopis por no presentar un verdadero involucro principal), y 2) que el estado del carácter es ambiguo en Calycera pulvinata y en Nastanthus caespitosus porque el involucro es indiferenciable en la inflorescencia central pero se forma en los grupos cimosos externos e inflorescencias periféricas.

En resumen, el involucro es bien distinguible en varios taxones asignados a Moschopis e indiferenciable en taxones asignados a otros géneros. Este carácter no es diagnóstico de ningún grupo ni constituye una sinapomorfía para ningún clado.

\section{Cáliz fructífero espinoso (5:1)}

La presencia de sépalos espinosos en el cáliz fructífero es usada como caracter diagnóstico del género Calycera.

El estado "presente" aparece independientemente en los clados 1, 3, 4 y 5, del análisis combinado. Dentro del grupo Acicarpha del Clado 1, agrupa a A. spathulata, A. tribuloides, a los que se suma A. procumbens de acuerdo con el análisis de caracteres morfológicos. Dentro del grupo de las "Pubescentes", el carácter varía: hay especies con cáliz frutífero presente, otras con el estado ausente y otras polimórficas. En el clado 4, el caracter también se muestra ambiguo. Aquí, ambos estados del caracter alternan 
independientemente en las distintas terminales, manifestándose "ausente" en $M$. monocephala y C. crassifolia, "presente" en C. herbacea var. herbacea, C. pulvinata, C. calcitrapa, y permaneciendo ambiguos en Calycera herbacea var. sinuata.

El carácter es polimórfico para Boopis anthemoides (clado 1) y Gamocarpha alpina (clado 5), aunque en estos taxones el estado "presentes" aparece muy esporádicamente en algunos ejemplares.

El cáliz fructífero espinoso es una sinapomorfía para los clados 1 y 4, aunque como caracter diagnóstico es ambiguo y poco confiable, ya que en los dos grupos aparecen reversiones al estado "ausente".

\section{Polimorfismo de frutos $(5: 1)$}

En la taxonomía tradicional se usa el carácter "cipselas dimorfas" para identificar la presencia de cipselas espinosas e inermes sobre el mismo receptáculo, característica reservada para los géneros Calycera y Anomocarpus (= Calycera). Sin embargo, se trata en realidad de un carácter más complejo (véase apartado 3.2). Este carácter fue codificado como doble-estado: "ausencia de polimorfismo de frutos" y "presencia de polimorfismo de frutos".

En la base del clado A el carácter es ambiguo. En el clado 1, el carácter se manifiesta "ausente" en Boopis anthemoides y "presente" en el clado Acicarpha. En el grupo hermano del clado 1, el caracter se manifiesta "presente" en Boopis gracilis y “ausente" en el clado M. leyboldi + clado 2 "Glutinosas".

Dentro del clado B el estado ancestral es "presente" y así se manifiesta en $C$. sessiliflora y los grupos siguientes: clado 3 ("Pubescentes") y clado 4, aunque en este último el estado revierte a "ausente" en C. crassifolia. En el clado 5 el estado revierte a "ausente".

Probablemente, el estado ancestral para la familia sea "ausente", como muestran los grupos externos. Aunque el polimorfismo de frutos no se ajusta bien a la topología de los árboles, constituye una sinapomorfía para varios grupos:

- con estado "presente" en el clado Acicarpha, B. gracilis (sus dos variedades) y el clado B;

- con estado "ausente" en el clado M. leyboldi + clado 2 ("Glutinosas"), C. crassifolia (sus dos variedades) y el clado 5.

Sin embargo, no es un buen carácter para distinguir las especies de Calycera. 


\section{Pared del fruto $(5: 1)$}

La pared carenada del fruto se usa como diagnóstico del género Nastanthus. El estado ancestral del caracter es "liso" en los AMP del análisis combinado. El estado “carenado" aparece independientemente en A. obtusisepala, C. horrida, C. herbacea (sus dos variedades), C. calcitrapa y N. scapigerus. El análisis morfológico mostró que, además de los taxones mencionados, C. eryngioides, $N$. ventosus y $N$. caespitosus también presentan la pared del fruto costada.

Representa una sinapomorfía morfológica para el grupo formado por las variedades herbacea y sinuata de Calycera herbacea, taxón para el que no se registraba este carácter. Sin embargo, el análisis filogenético demuestra que tampoco constituye un carácter diagnóstico para el género Nastanthus.

Exceptuando el grupo formado por las dos variedades de C. herbacea, este carácter no representa sinapomorfía alguna para ningún otro clado.

Los caracteres de la taxonomía clásica usados en el análisis mostraron un grado variable de homoplasia.

Algunos caracteres aparecen en la historia evolutiva de la familia una sola vez y permanecen en todos los descendientes dentro de un clado. Por ejemplo, la existencia flores estaminadas en el centro de la inflorescencia (carácter 19) está presente en todas las especies del clado de Acicarpha y no aparece en ningún otro lugar del árbol. Lo mismo ocurre con los demás caracteres usados en taxonomía para distinguir a Acicarpha.

Entre los caracteres que mostraron mayor número de pasos, se observa que la diferenciación del involucro (carácter 30, utilizado para distinguir las especies de Moschopis) aparece independientemente en los clados 2, 4 y en A.

De los siete caracteres usados en taxonomía clásica, cuatro se distinguen como sinapomorfías: 19, 33, 35 y 36; aunque, como ya se ha señalado, el caracter 33 es ambiguo y poco confiable. De todos modos, y exceptuando a Acicarpha, ninguno de los géneros para los cuales se consideraban caracteres diagnóstico es monofilético. 
3.4.4. Descripción y discusión de otros caracteres morfológicos relevantes

Los números entre paréntesis son una medida del grado de homoplasia para cada caracter ( $\mathrm{N}^{\circ}$ de pasos en uno de los AMP : $\mathrm{N}^{\circ}$ mínimo de pasos para el carácter). La optimización de los caracteres discutidos en uno de los AMP puede observarse en el Apéndice 5, los nombres de los clados o grupos corresponde a los señalados en la figura 39 (árbol de consenso).

\section{Hojas glutinosas (2:1)}

El estado ancestral es "ausente". El estado "presente" pudo haber aparecido independientemente en los clados Gamocarpha dentata + Moschopis caleofuensis y $M$. rosulata + M. subandina + Boopis filifolia (por estar ausente en B. patagonica y polimórfico en $M$. trilobata, la optimización es ambigua en la base del Clado 2). Sin embargo el carácter podría estar "presente" en el ancestro del Clado 2 ("Glutinosas") con una reversión en Boopis patagonica, siendo entonces una sinapomorfía del Clado 2. La presencia de una secreción glutinosa un carácter muy importante en la filogenia de las Calyceraceae, y merece un estudio más detallado y profundo de su origen y composición, especialmente si consideramos que Hellwig (2007) observó la presencia de una sustancia resinosa en los tallos y las brácteas involucrales de Boopis gracilis, razón por la cual el Clado 2 pueda incluir también algunas especies externas más próximas. El carácter presente se ha detectado de manera ambigua en el grupo externo a las Caluceraceae.

\section{2. Ápice de las anteras $(5: 1)$}

Se revisó la optimización de este carácter en la filogenia de la familia debido a su valor taxonómico en tribus y géneros de Asteraceae (Roque et al., 2009). El estado ancestral del caracter para la familia Calyceraceae es "no apiculado". El estado "apiculado" aparece independientemente en B. filifolia (clado 2), C. sessiliflora (basal clado B), B. pusilla y C. sympaganthera + C. horrida (clado 3), y C. pulvinata (clado 4), mientras que se manifiesta ambiguo para M. rosulata (clado 2), B. australis y $B$. australis var. hickenii (clado 5).

El carácter no define grupos importantes y únicamente representa una sinapomorfía para el grupo C. horrida $+C$. sympaganthera. 


\section{Forma de la base de las anteras (5:1)}

Se revisó la optimización de este carácter en la filogenia de la familia debido a su valor taxonómico en tribus y géneros de Asteraceae (Roque et al., 2009). El estado ancestral del caracter para la familia Calyceraceae es "obtusa". El estado "sagitada" aparece independientemente cuatro veces: 1) en $M$. trilobata + M. rosulata $+M$. subandina + B. filifolia (subgrupo del clado 2 "Glutinosas"); 2 ) en B. pusilla $+C$. sympaganthera $+C$. horrida $+C$. leucanthema $+B$. pterocalyx (clado 3 "Pubescentes"); 3) B. australis var. hickenii + B. chubutensis (subgrupo del clado 5); 4) G. alpina + B. multicaulis + B. australis $+N$. compactus $+N$. scapigerus (subgrupo del clado 5).

El carácter representa una sinapomorfía para todo el clado 3 y para algunos subgrupos dentro de los clados 2 y 5 .

\section{Caudículas en las anteras $(7: 1)$}

Se revisó la optimización de este carácter en la filogenia de la familia debido a su valor taxonómico en tribus y géneros de Asteraceae (Roque et al., 2009). El estado ancestral del caracter para la familia Calyceraceae es "ausentes". El estado "presentes" aparece independientemente en $M$. leyboldi, B. filifolia, $C$. horrida $+C$. sympaganthera, C. herbacea var. sinuata y el clado 5 (con reversiones internas).

La optimización de este carácter no queda resuelta en el clado 5 debido a las diferentes resoluciones de la politomía del árbol consenso.

\section{Nectarios estaminales $(2: 1)$}

El estado ancestral del caracter para la familia Calyceraceae es "ausentes". La optimización es ambigua en la base del clado A. Sin embargo, es más parsimonioso suponer que haya aparecido una vez en el ancestro del Clado A con una reversión en $B$. anthemoides, siendo entonces una sinapomorfía del Clado A (Fig. 39). La naturaleza de los nectarios parece ser un carácter muy importante en la filogenia de las Calyceraceae, y merece un estudio más detallado y profundo de su estructura y desarrollo.

\section{Nectarios hipantiales $(2: 1)$}

El estado ancestral del caracter para la familia Calyceraceae es "ausentes". El estado "presentes" es una sinapomorfóa del clado B, solo compartida con $B$. anthemoides del caldo A. Tal como se explica en el carácter anterior, la naturaleza de 
los nectarios parece ser un carácter muy importante en la filogenia de las Calyceraceae, y merece un estudio más detallado y profundo de su estructura y desarrollo.

\section{Presencia de tubo estaminal (1:1) y 8 . Longitud del tubo estaminal $(7: 1)$}

El desarrollo de un tubo estaminal es raro en las Asteraceae (por ej. en algunas especies de Barnadesia, en Xanthium y Dicoria, Roque et al., 2009) pero está presente en las Calyceraceae. El análisis filogenético propone la presencia de un tubo estaminal como sinapomorfía de la esta familia, en coincidencia con lo sugerido por Lundberg \& Bremer (2003).

En cuanto a las diferentes longitudes del tubo estaminal en las Calyceraceae, estado ancestral es "desarrollado". El estado "reducido" aparece independientemente en M. leyboldi, Calycera sessiliflora, M. monocephala + C. pulvinata + C. calcitrapa $+C$. crassifolia, B. australis var. hickenii y B. multicaulis + B. australis+ Nastanthus compactus (parafilético).

El carácter no define grupos importantes y sólo representa una sinapomorfía para el grupo M. monocephala $+C$. pulvinata $+C$. calcitrapa $+C$. crassifolia (con sus dos variedades) dentro del clado 4.

\section{Nervadura central de los sépalos (6:1)}

El estado ancestral "presente" aparece en la base de los clados A y B. Dentro del clado A, el estado "presente" se mantiene en todo el clado 1, con la única excepción de A. obtusisepala (estado "ausente"), mientras que el estado se manifiesta "ausente" en el grupo hermano B. gracilis (con sus dos variedades) + M. leyboldi + clado 2 "Glutinosas".

Dentro del clado B, el estado "presente" se mantiene en $C$. sessiliflora, mientras que se manifiesta "ausente" en el ancestro del grupo hermano formado por clado 3 "Pubescentes" + clado 4 + clado 5. A su vez, dentro de este grupo, el caracter revierte al estado "presente" de manera independiente en una rama terminal ( $C$. sympagantera del clado 3 "Pubescentes") y en dos grupos: C. calcitrapa + C. crassifolia var. crassifolia + C. crassifolia var. spinulosa (clado 4), y B. australis var. hickenii + B. chubutensis (clado 5). 
A pesar de exceder el $n^{\circ}$ de pasos mínimos esperados, este caracter parece ajustarse bien a la filogenia y, en sus dos estados, representa una sinapomorfía para varios grupos:

- "presente" en C. clacitrapa + C. crassifolia (con sus dos variedades),

- "presente" en B. australis var. hickenii + B. chubutensis;

- "ausente" en B. gracilis (con sus dos variedades) + M. leyboldi + clado 2 "Glutinosas",

- "presente" en clado 3 "Pubescentes" + clado 4 + clado 5.

En el caso del clado 1, la presencia de nervadura central en los sépalos es una característica interesante que ayuda a distinguir este grupo del resto del clado A y de la mayoría de los grupos del clado B. Sin embargo, se trata de una simplesiomorfía, ya que el estado "presente" ya se encuentra en el ancestro común del clado A, en el del clado B y en el ancestro común a todo Calyceraceae. El mismo caso se repite en $C$. sessiliflora.

\section{Proporción del hipanto (10:2)}

Junto con el carácter 18, se revisó la optimización de la proporción del hipanto como una manera de analizar la importancia filogenética de la morfología del perianto dentro de las Calyceraceae, debido a su valor taxonómico en Asteraceae (Roque et al., 2009; si bien en las Asteraceae el hipanto se describe usualmente como tubo corolino). El estado ancestral permanece ambiguo para los clados A y B ya que los estados se intercalan en la filogenia y aparecen en más de un grupo de manera independiente.

Dentro del clado A, el estado del carácter permanece ambiguo en el clado 1 por manifestarse los estados "de igual longitud que la corola" (A. spathulata y A. obtusisepala) y "más largo que la corola, 1.5 veces o más" (B. anthemoides y $A$. tribuloides) de forma independiente en cada uno de los taxones terminales; mientras que en el grupo hermano, B. gracilis (con sus dos variedades) + M. leyboldi + clado 2 "Glutinosas", el estado se manifiesta "subnulo" con excepción de M. trilobata (con estado del caracter "de igual longitud que la corola").

Dentro del clado B, el estado "subnulo" constituye una autopomorfía para $C$. sessiliflora, mientras que en el ancestro del grupo hermano formado por clado 3 "Pubescentes" + clado 4 + clado 5 el carácter se manifiesta "de igual longitud que la 
corola". A su vez, dentro de este grupo, el carácter se menifiesta "más largo que la corola, 1.5 veces o más" de manera independiente en una rama terminal y dos grupos: C. herbacea var. herbacea, y C. calcitrapa $+C$. crassifolia var. crassifolia $+C$. crassifolia var. spinulosa, pertenecientes al clado 4, y el grupo terminal del clado 5.

En sus tres estados, este carácter representa una sinapomorfía para varios grupos:

- "subnulo": B. gracilis (con sus dos variedades) + M. leyboldi + clado 2 "Glutinosas";

- "de igual longitud que la corola": clado 3 "Pubescentes" + clado 4 + clado 5;

- "más largo que la corola, 1.5 veces o más": C. calcitrapa + C. crassifolia (con sus dos variedades).

\section{Proporción de la porción lobada de la corola (13:2)}

Junto con el carácter 17, se revisó la optimización de la proporción de la porción lobada de la corola como una manera de analizar la importancia filogenética de la morfología de la corola dentro de las Calyceraceae, debido a su valor taxonómico en Asteraceae (Roque et al., 2009). El estado ancestral "partida" es compartido por los clados A y B. Dentro del clado A, el estado "partida" se mantiene en los taxones basales del clado 1 y B. gracilis (con sus dos variedades), mientras que se manifiestan dos cambios de estado: a "secta" en A. spathulata + A. tribuloides (clado 1), y a "fida" en M. leyboldi + clado 2 "Glutinosas" (con reversión a "partida" en M. trilobata).

Dentro del clado B, el estado "partida" se mantiene en $C$. sessiliflora y en los taxones basales de los clados 3 ("Pubescentes"), 4 y 5.

Dentro del clado 3 se manifiestan dos cambios de estado: a "fida" en $C$. horrida, y a "secta" en $C$. leucanthema.

Dentro del clado 4 se manifiestan tres cambios de estado: a "fida" en $M$. monocephala, y a "secta" en $C$. herbacea var. herbacea y C. crassifolia (con sus dos variedades) de forma independiente. En C. herbacea var. sinuata el carácter es ambiguo para "partida" y "secta".

Dentro del grupo terminal del clado 5 (correspondiente a la politomía del análisis combinado) el estado experimenta cambios a los estados "fida" y "secta", y, en uno de los AMP, reversiones al estado "partida".

En general, este carácter no parece confiable para definir grupos ya que los estados "fida" y "secta" aparecen independientemente en varios grupos y taxones, y 
existen varias reversiones al estado "partida". No obstante, la proporción de la porción lobada de la corola puede considerarse sinapomorfía para A. spathulata + A. tribuloides ("secta") y M. leyboldi + clado 2 "Glutinosas" ("fida").

\section{Pubescencia en el pedúnculo (1:1)}

El estado ancestral de este carácter es "ausente" y el estado "presente" aparece una única vez en el ancestro del Clado 3 ("Pubescentes"): Boopis pusilla, B. pterocalyx, Calycera leucanthema, $C$. horrida y $C$. sympaganthera. La pubescencia no suele ser un carácter relevante para definir géneros o taxones de rango mayor, y no lo es para las Asteraceae. Llama la atención que en las Calyceraceae la presencia de pelos uniseriados, largos, conformando una pubescencia villosa a lanosa, sea una de las sinpomorfías de un clado con alto soporte $(\mathrm{Jm}=93 \%, \mathrm{Jc}=69 \%)$. Si bien este carácter se ha tomado para los pedúnculos, varias de las especies del Clado 3 tienen pubescencia en las hojas y los tallos, de modo que puede considerarse también una característica vegetativa.

\section{Brácteas del receptáculo $(5: 1)$}

Se revisó la optimización de este carácter en la filogenia de la familia debido a su valor taxonómico en tribus y géneros de Asteraceae (Roque et al., 2009). El estado ancestral "presente" se mantiene en casi todos los clados. El estado "ausente" aparece independientemente en $C$. sessiliflora, M. monocephala, $N$. compactus y en el grupo $M$. leyboldi + clado 2 "Glutinosas". En este último grupo, la existencia de algunos taxones con estado del carácter ambiguo ( $G$. dentata, M. subandina y B. filifolia) sumada a la reversión al estado "presentes" en M. caleofuensis, hacen de este carácter una sinapomorfía ambigua.

3.4.5. Discusión de las relaciones filogenéticas de los taxones no incluidos en el análisis combinado.

Algunos de los taxones no pudieron ser incluidos en el análisis filogenético de caracteres combinados por no contar con material para la extracción de ADN. A 
continuación, se discuten sus posibles relaciones filogenéticas de acuerdo a los resultados obtenidos a partir del análisis de caracteres morfológicos (figura 2).

Boopis castilloni aparece como taxón basal de la familia. Comparte con el resto de los taxones de Calyceraceae las sinapomorfías hipanto "presente" y tubo estaminal "presente", a las que se suma nectarios hipantiales "presentes". Esto último es un indicio de que la presencia de nectarios hipantiales sería el estado ancestral del carácter. B. castilloni queda separado del resto de Calyceraceae por poseer receptáculo fructífero "discoideo" (caracter no compartido con ningún otro taxón) y "ausencia" de polimorfismo de frutos.

Boopis bupleuroides y B. itatiaiae comparten varias similitudes morfológicas con el género Acicarpha: grupos cimosos internos al involucro "ausentes", grupos cimosos externos al involucro "ausentes" filamentos estaminales "ausentes", forma de la base de las anteras "obtusa", ápice de las anteras "ausente", caudículas en las anteras "ausentes", proporción del hipanto "1.5 veces el largo de la corola". Ambas especies ocupan una posición basal en el clado 1 y podrían representar una serie de estados intermedios entre $B$. anthemoides y el grupo Acicarpha. Sin embargo, existen evidencias que podrían distinguirlos dentro de un clado particular por fuera de $B$. anthemoides y de Acicarpha: el receptáculo florífero "punctiforme" y la presencia de "flores tetrámeras" (para $\mathbf{n}^{\mathbf{0}}$ de piezas por ciclo floral), probablemente constituyan sinapomorfías para estos dos taxones que debería ser probada en el marco de un análisis combinado. Además, $B$. bupleuroides posee receptáculo fructífero "punctiforme" y $B$. itatiaiae muestra una reversión al estado "presentes" para el caracter filamentos estaminales.

Por otro lado, B. jürgensii y B. itatiaiae comparten con el grupo Acicarpha el estado de caracter "columnar" para forma del receptáculo fructífero. B. jürgensii probablemente ocupe una posición similar, dadas sus similitudes con el clado 1. Sin embargo, no se cuenta con material de herbario ni con muestras de ADN para incluirlo en ningún tipo de análisis filogenético.

Acicarpha procumbens aparece incluido dentro del clado Acicarpha como taxón hermano del grupo A. tribuloides + A.spathulata. Comparte todas las sinapomorfías del grupo.

Moschopis ameghinoi aparece como grupo hermano del clado "Glutinosas" en el árbol de consenso de mayoría. Comparte con el grupo "Glutinosas": los nectarios 
estaminales "presentes", el tubo estaminal "desarrollado", los filamentos estaminales "ausentes" y la proporción del hipanto "subnula". Sin embargo, la cantidad de estados de carácter compartidos no es suficiente como para incluirla dentro de las "Glutinosas" y diferenciarla del resto de los taxones asociados. Es por eso que en el árbol de consenso estricto forma una politomía con M. leyboldi, B. gracilis, el clado Acicarpha, el clado "Glutinosas" y M. monocephala.

Por su parte, Gamocarpha angustifolia aparece incluida dentro del clado "Glutinosas", formando una politomía con $G$. dentata y el clado M. rosulata $+M$. caleofuensis + M. subandina + Boopis filifolia. Comparte todas las sinapomorfías del grupo.

Boopis necronensis aparece como taxón hermano de Nastanthus patagonicus formando un grupo terminal, asociado con B. pterocalyx y Calycera pulvinata. B. necronensis podría incluirse dentro del clado 5 por compartir el estado del carácter caudículas en las anteras "presentes" (sinapomorfía del clado 5 en el análsis combinado), y quedaría asociado a $N$. patagonicus formando un grupo terminal ya que ambos comparten la forma de los lóbulos corolinos "cuculados" (autapomorfía para $N$. patagonicus en el análisis combinado). Otros caracteres compartidos con $N$. patagonicus son: longitud del tubo estaminal "reducido" y forma del receptáculo fructífero "globoso".

Boopis raffaellii y $B$. graminea también aparecen incluidos dentro del clado 5 por compartir sus dos sinapomorfías: caudículas en las anteras "presentes" y polimorfismo de frutos "ausente", pero formando una politomía con el grupo terminal. También comparten con el clado 5 los nectarios hipantiales "presentes" y la forma de la base de las anteras "sagitada". B. raffaellii y B. graminea quedan incluidos dentro del peine del grupo terminal, con el que comparten la sinapomorfía proporción de la porción lobada de la corola "fida".

Por su parte, Nastanthus caespitosus y N. ventosus quedan incluidos dentro del subclado de la politomía terminal del clado 5 que los agrupa con $N$. scapigerus y $N$. compactus.

Calycera eryngioides aparece incluida dentro del clado "Pubescentes" (aunque este grupo también incluye a Calycera herbacea en el análisis de caracteres morfológicos con reversión al estado "ausente" para el caracter pubescencia del pedúnculo). Comparte todas las sinapomorfías del grupo. 


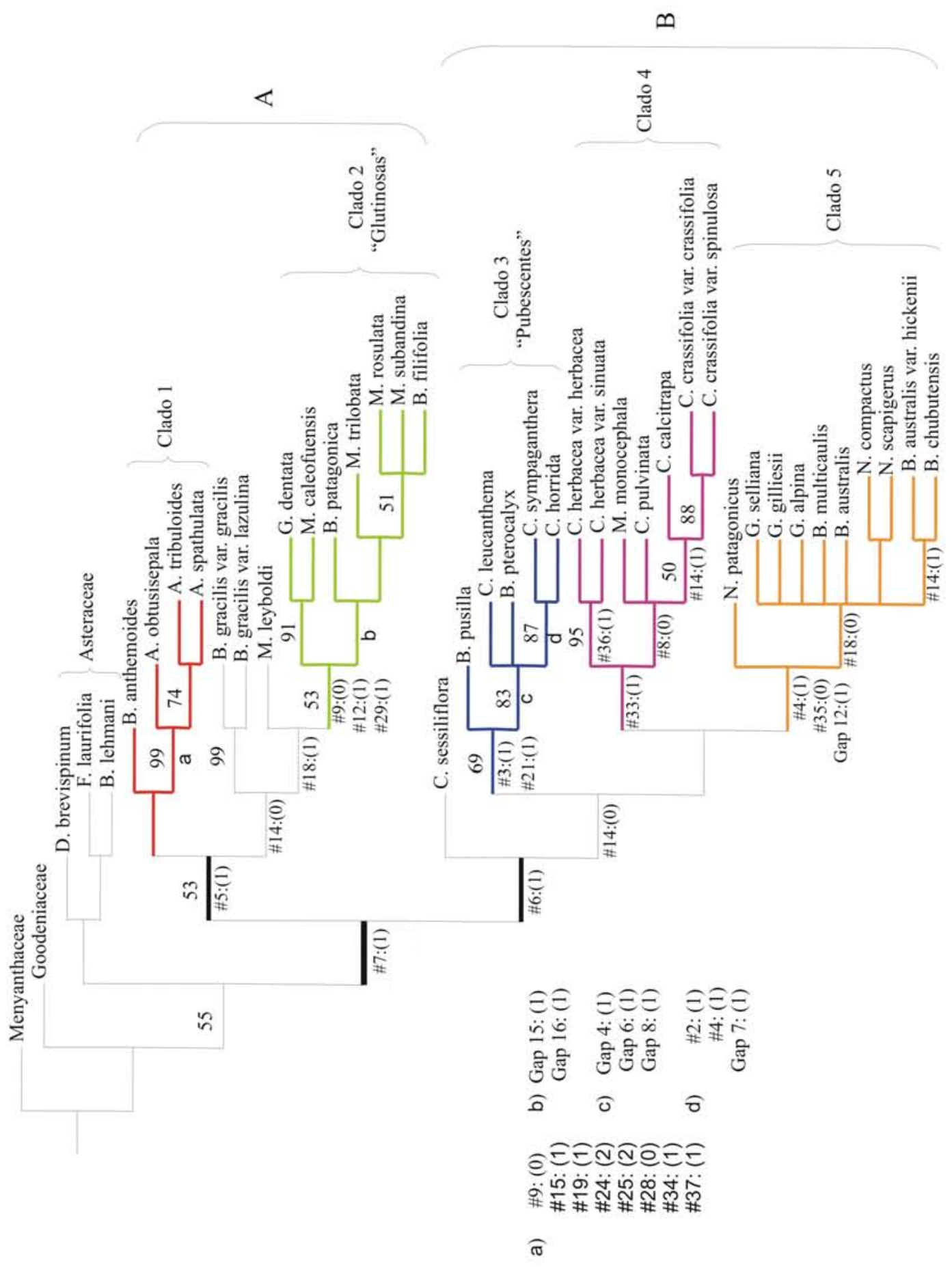

Figura 39. Árbol de consenso estricto de los AMP resultantes del análisis combinado. Los números sobre las ramas indican los soportes de Jackknife. Debajo de las ramas se indican, para cada clado, las sinapomorfias morfológicas y gaps comunes a todos los AMP. Las sinapomorfias para cada clado se indican con letras minúsculas de imprenta en caso de no haber espacio en el gráfico. 


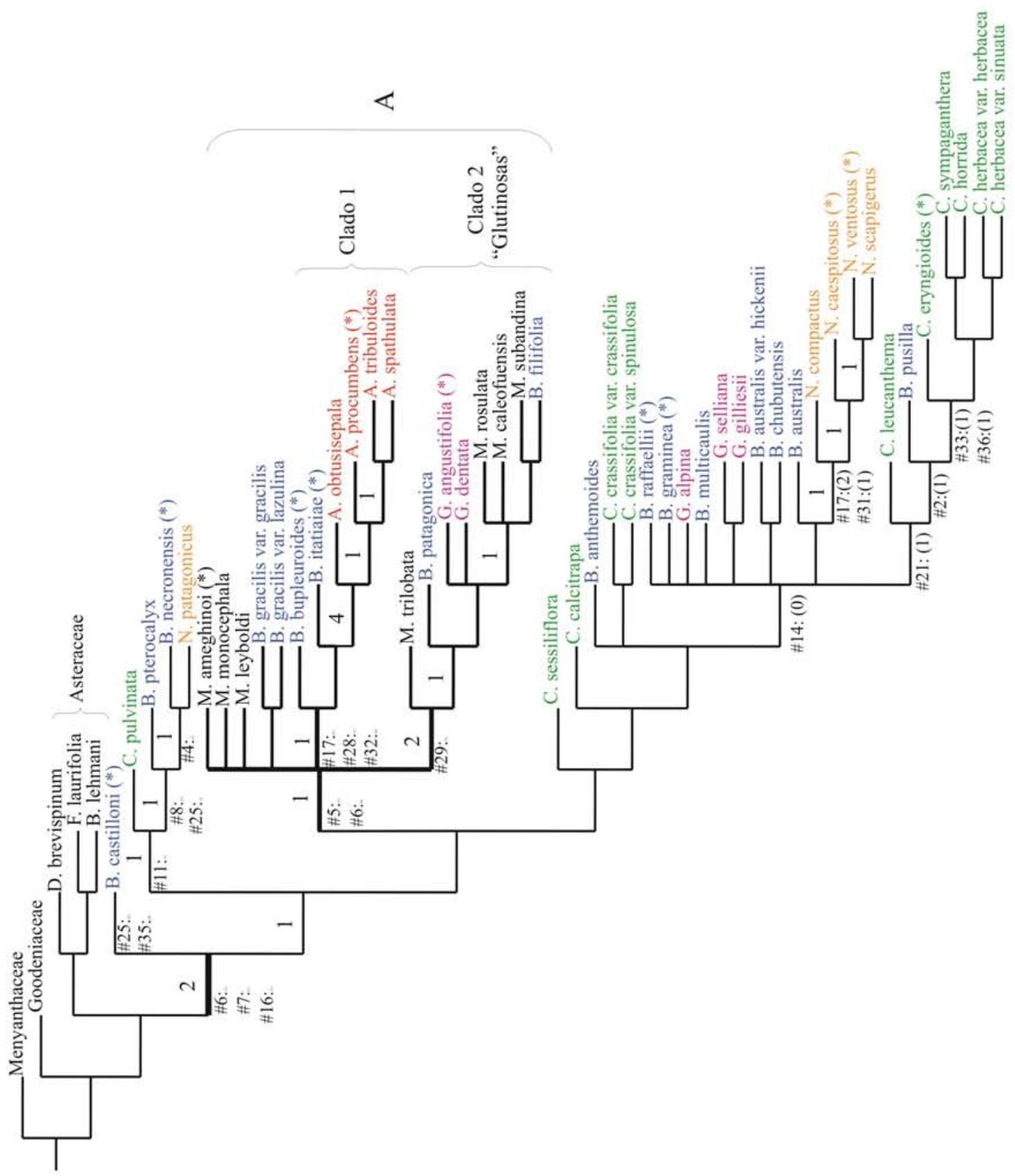

Figura 40. Árbol de consenso estricto de los AMP resultantes del análisis morfológico. Los números sobre las ramas indican los soportes de Bremer. Debajo de las ramas se indican las sinapomorfias morfológicas comunes a todos los AMP, para los clados que contienen los taxones no incluidos en el análisis molecular $(*)$. Los taxones pertenecientes al mismo género de acuerdo a la taxonomía clásica comparten el mismo color. 


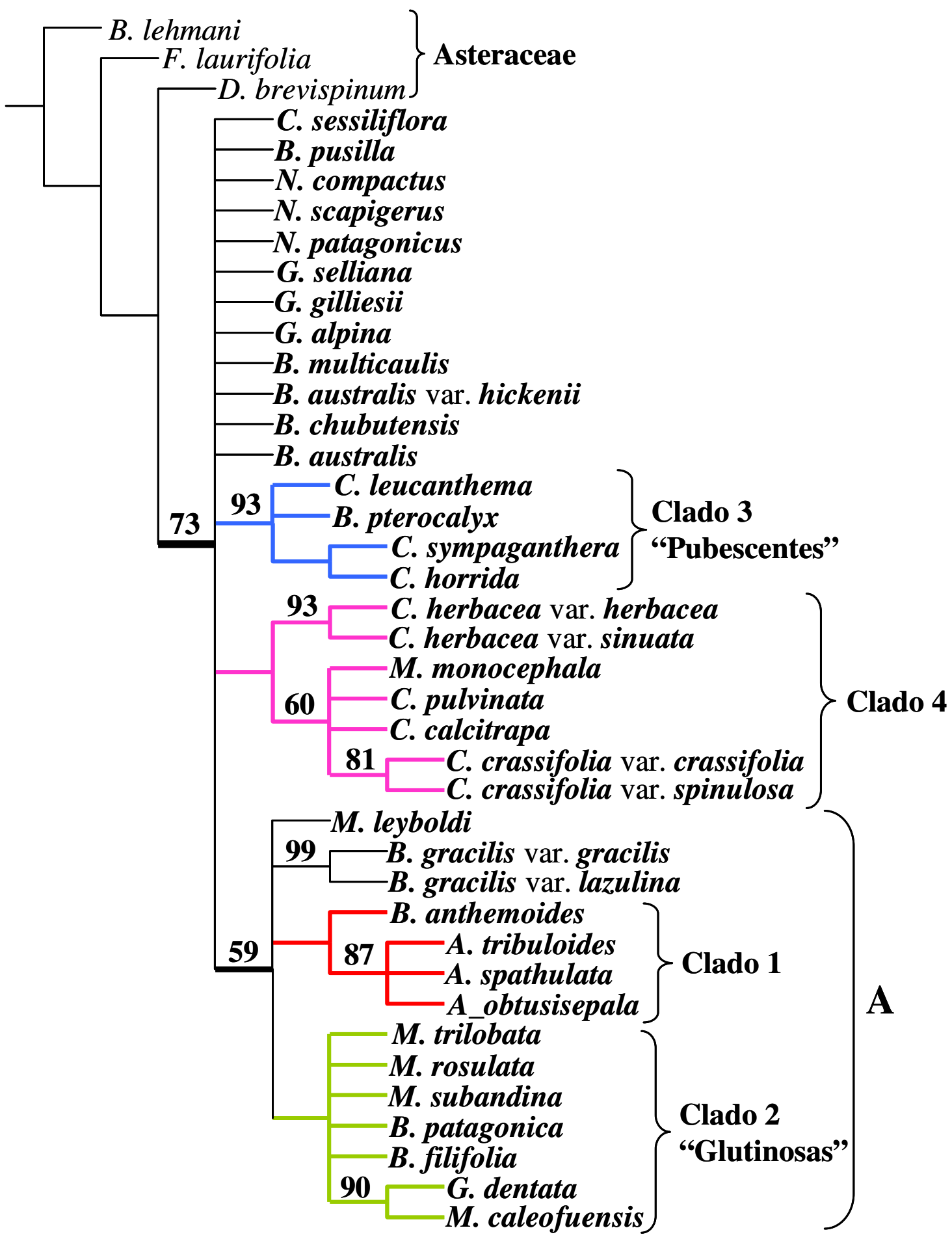

Figura 41. Árbol de consenso estricto de los AMP resultantes del análisis molecular. Los números sobre las ramas indican los soportes de Jackknife. Se muestran los clados coincidentes con el análisis combinado destacados en el mismo color. 
3.4.6. Apéndice 5. Optimización en uno de los AMP de los caracteres discutidos.
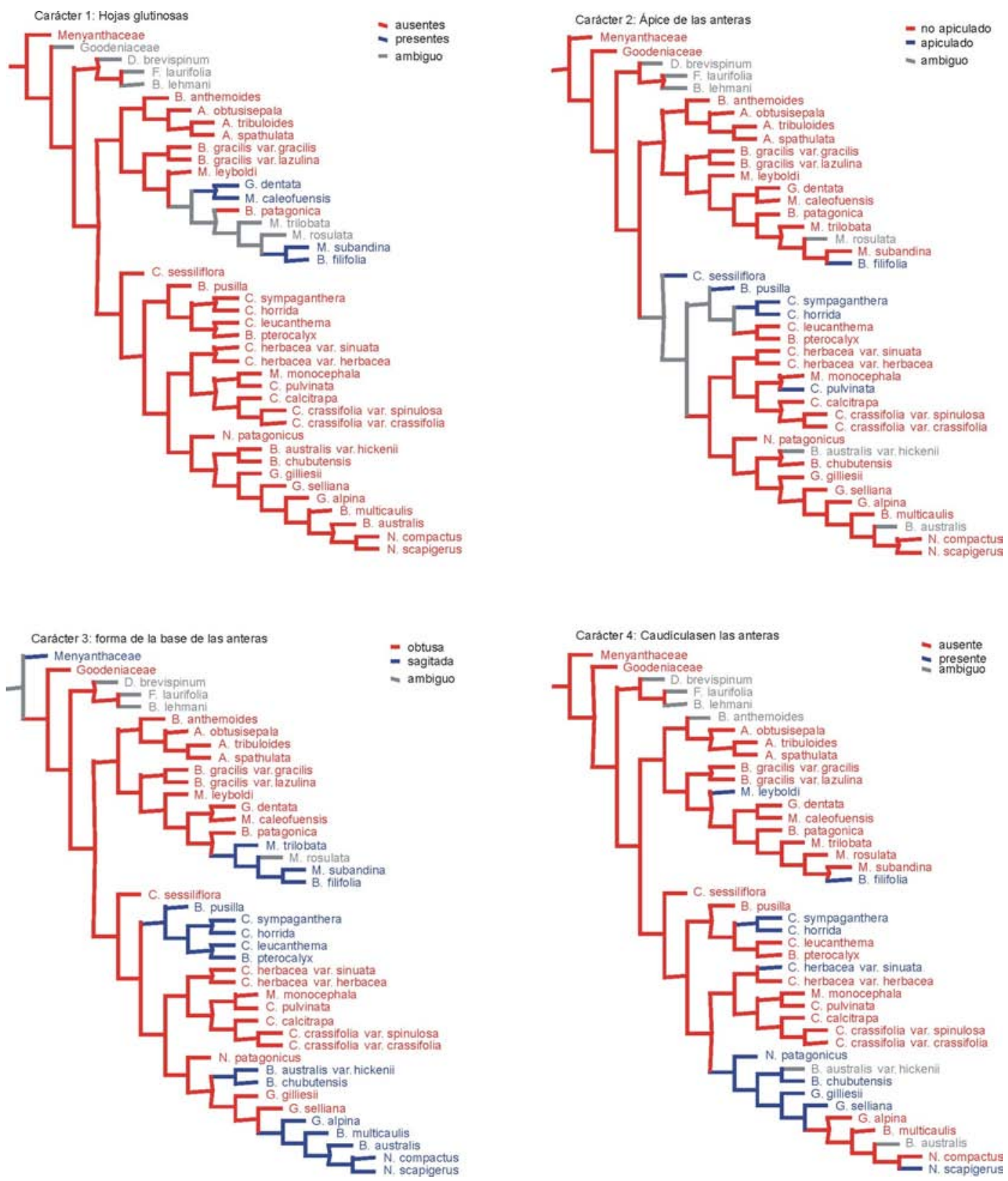

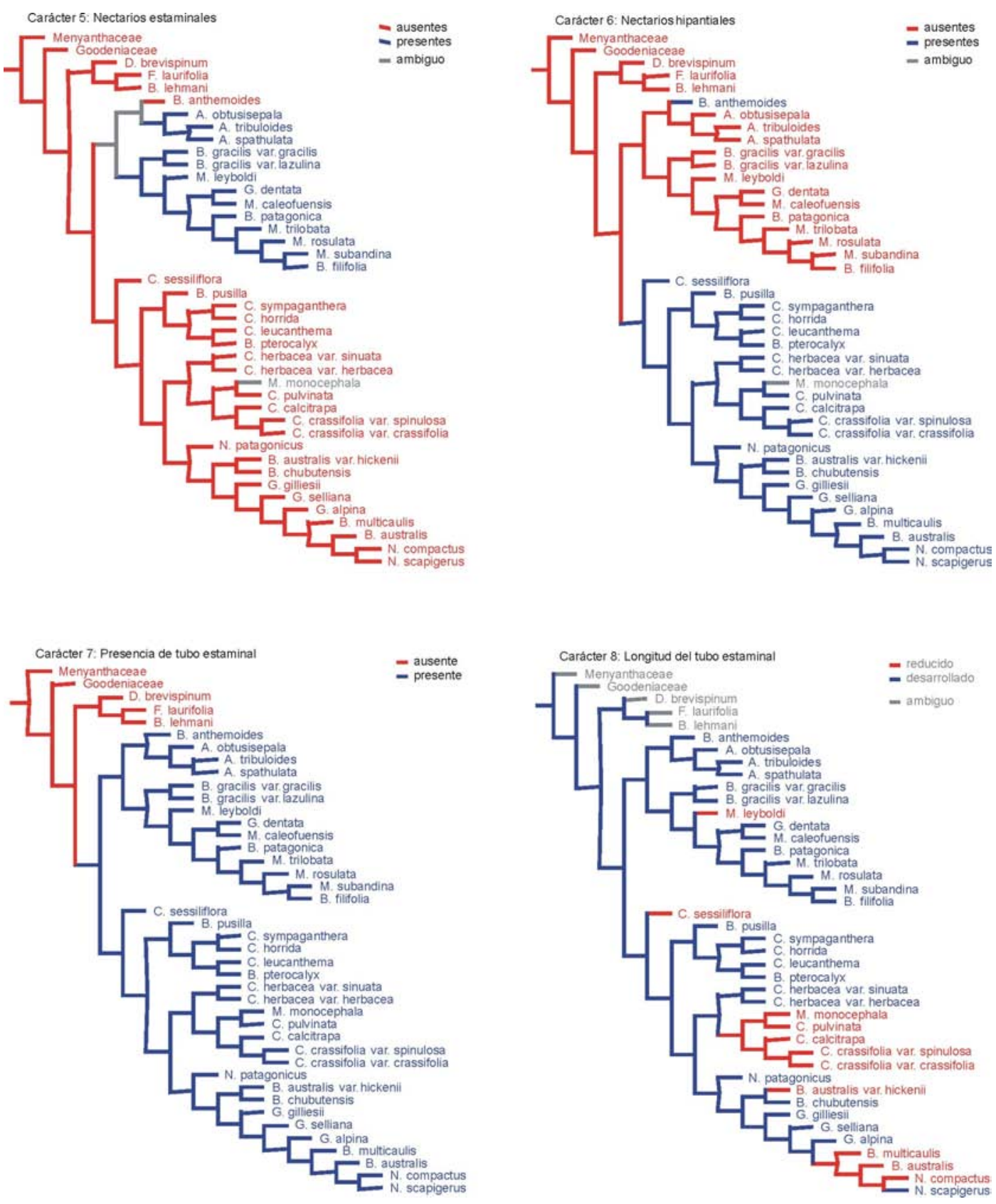

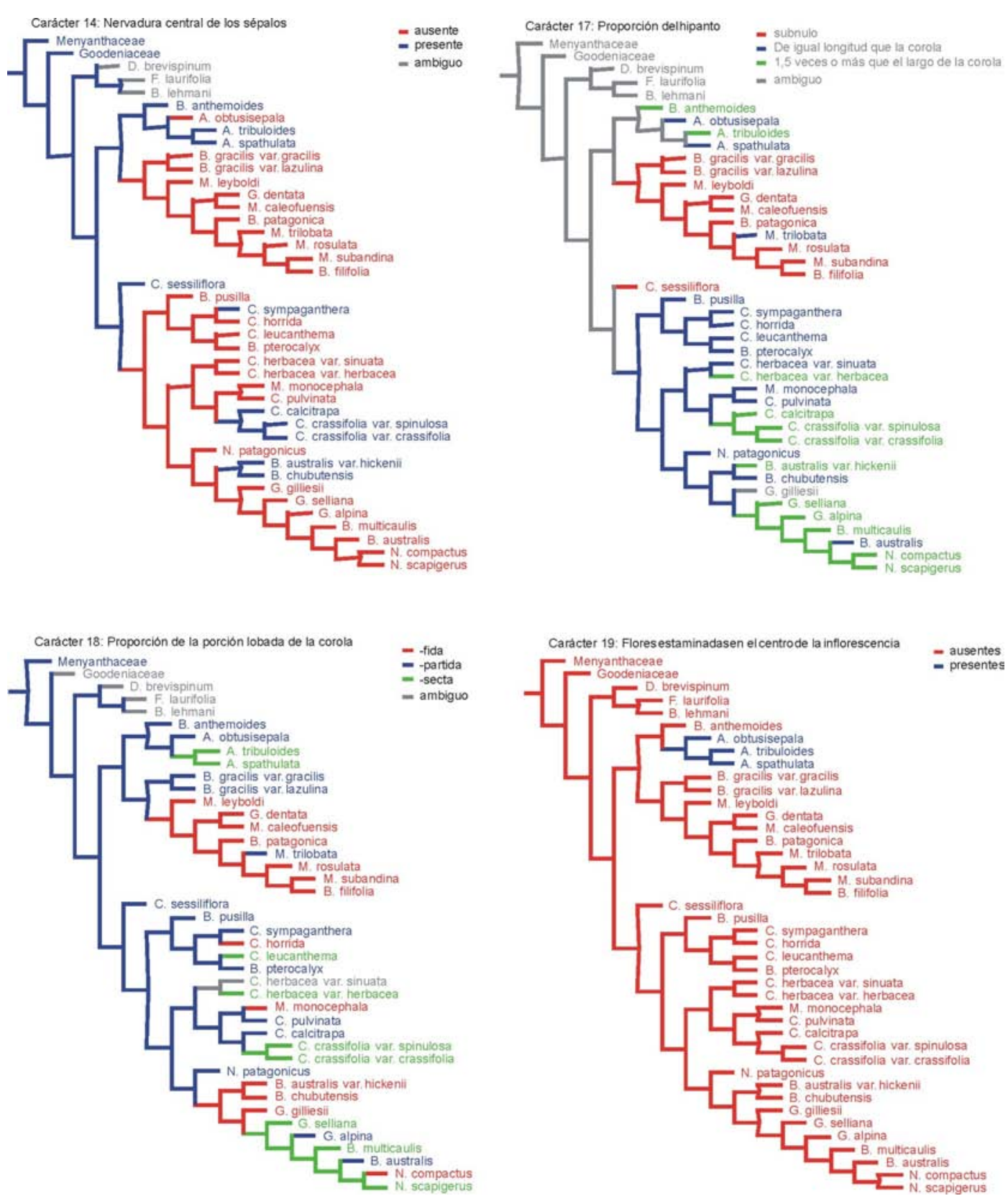

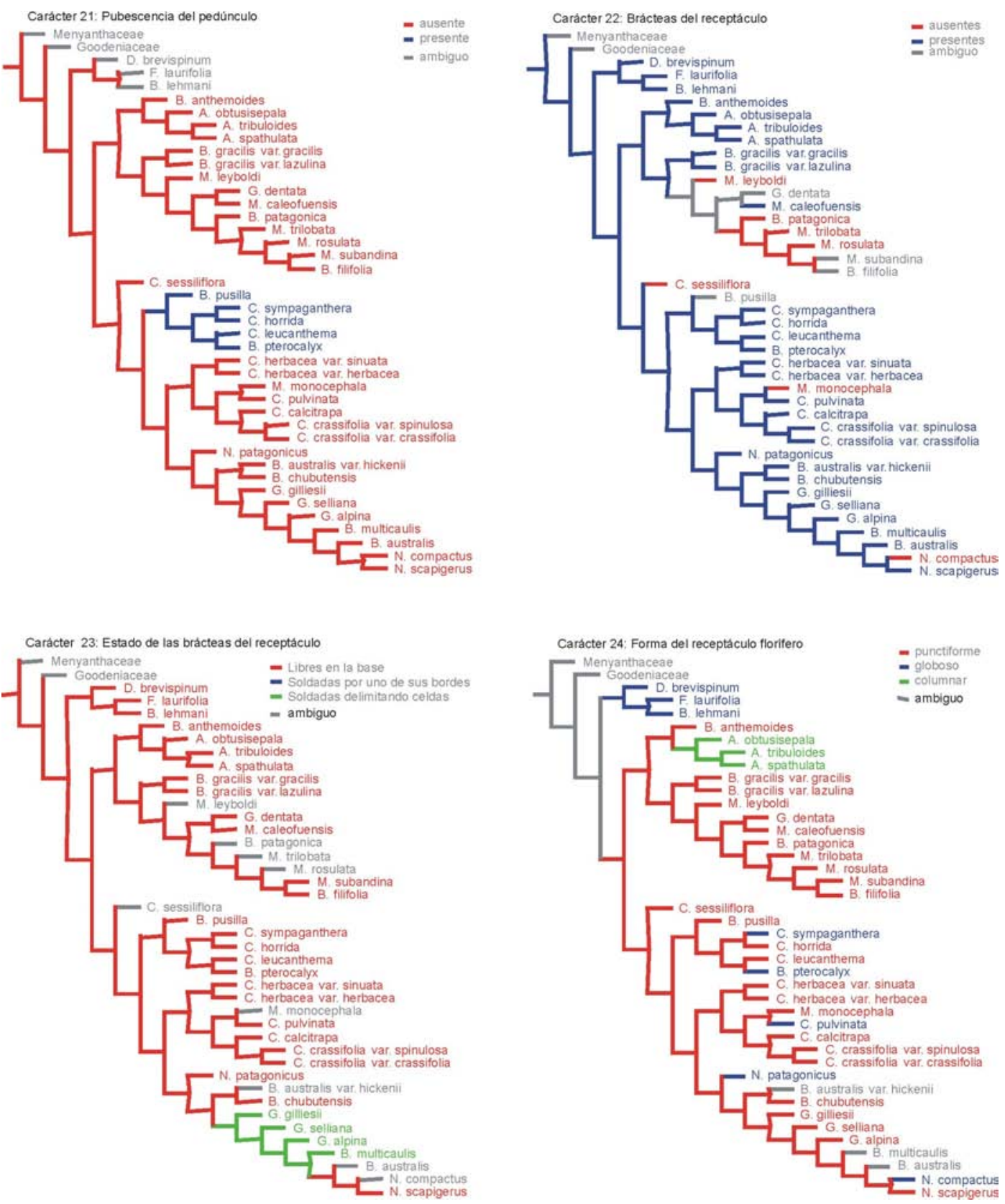

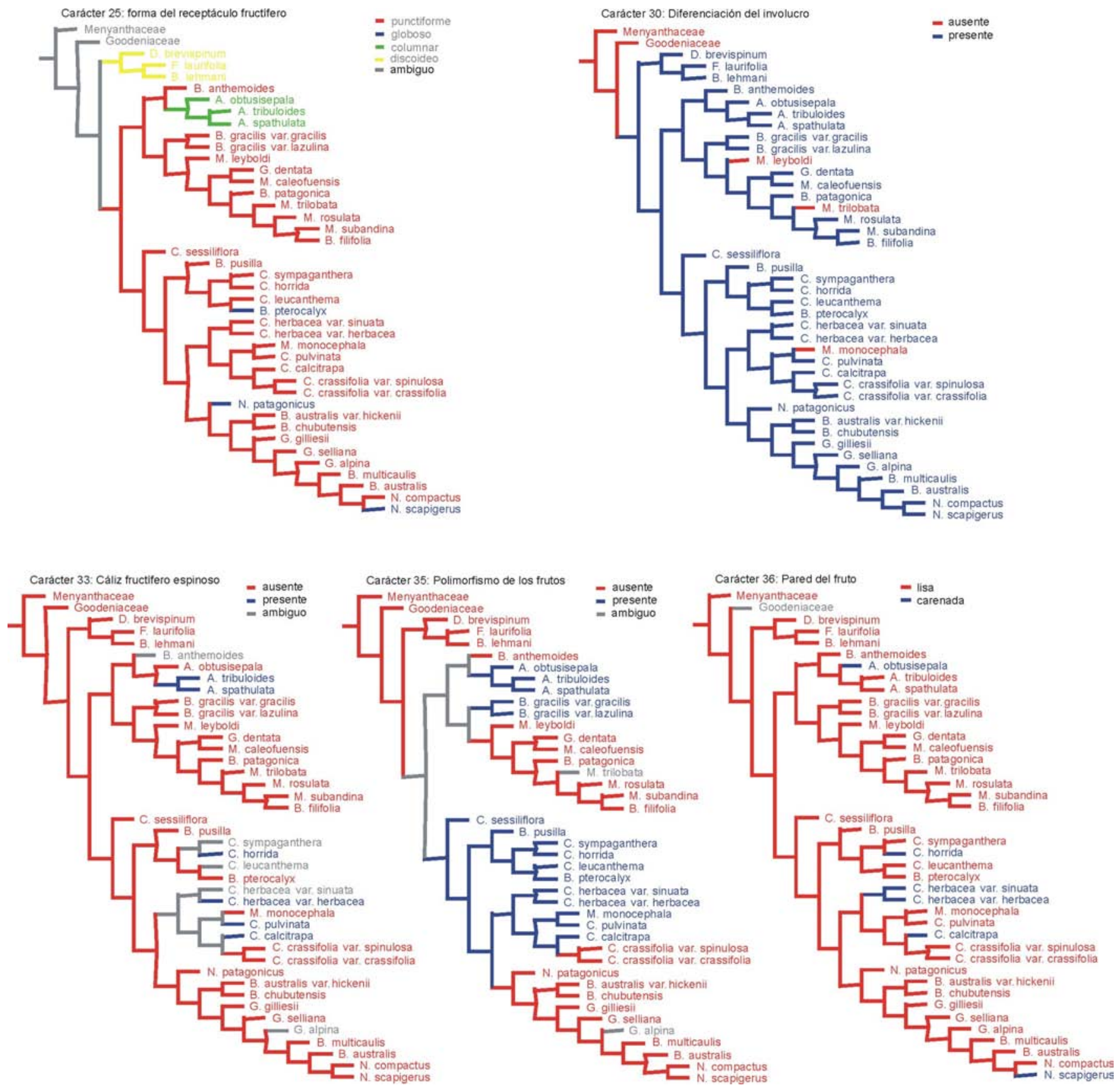


\subsection{Distribución y Hábitat}

3.5.1 Comentarios acerca de la distribución geográfica de las especies de Calyceraceae

La familia Calyceraceae es endémica del Cono Sur de Sudamérica. La mayor parte de las especies de esta familia se distribuye a lo largo y a lo ancho del Dominio Andino-Patagónico, ocupando numerosos ambientes: plena estepa; dunas costeras y playas marinas; ambientes ripícolas asociados a ríos, lagos y lagunas (a menudo en suelos salinos); ambientes pedemontanos y ambientes de alta montaña, sobre suelos arenosos y pedregosos de planicies y laderas de acarreo y asociadas a cursos de agua. Existe un pequeño grupo de especies endémicas que se distribuyen exclusivamente en zonas tropicales y templadas que incluyen: la costa atlántica del sur de Brasil y Uruguay, y algunas áreas restringidas de la Selva Paranaense y la Provincia Atlántica.

Entre las dos zonas mencionadas anteriormente, es decir, en el área fitogeográfica que corresponde al Dominio Chaqueño, habitan Boopis anthemoides y Acicarpha tribuloides, las dos especies más conocidas y difundidas de Calyceraceae, presentes en todos los pisos de vegetación comprendidos entre la llanura chacopampeana y la Prepuna ${ }^{1}$.

Existe, además, una especie microendémica, Nastanthus falklandicus, de la cual se conoce una única población en el extremo sudoeste de la isla Gran Malvina, área correspondiente a la Provincia Insular del Dominio Subantártico.

De las 47 especies reconocidas de Calyceraceae, sólo unas pocas alcanzan rangos extensos de distribución geográfica, entre ellos Boopis anthemoides y Acicarpha tribuloides (mencionados anteriormente), a las que pueden sumarse:

- Boopis australis: extendida por toda la meseta patagónica, desde la costa atlántico-magallánica hasta la cordillera del Viento y las primeras estribaciones andinas en la provincia de Neuquén;

- Boopis gracilis: su distribución comprende gran parte del centro y noroeste de la Patagonia, llega a ser muy abundante en la flora mendocina y alcanza las montañas de la región maulina (Chile), dispersándose a través de los valles intermontanos y portezuelos andinos;

\footnotetext{
${ }^{1}$ Aunque existen registros aislados de A. tribuloides para los bosques montanos de Podocarpus parlatorei (Pcia. de las Yungas) y los alrededores de Cuzco, Perú, a 3500 m s. m. (Pcia. Altoandina).
} 
- Calycera crassifolia (en sus dos variedades): se distribuye en dos áreas bien definidas: a lo largo de la costa atlántica argentino-uruguaya y en la zona pedemontana del Distrito de la Payunia, ambas conectadas por las cuencas de los ríos Colorado y Negro.

En general, las especies restantes poseen una distribución más restringida y se hallan circunscriptas a zonas mucho menos extensas, sujetas a requerimientos ambientales mucho más estrictos. Sin embargo, las poblaciones de Calyceraceae llegan a ser muy numerosas en sitios puntuales donde el ambiente es favorable, volviéndose muy comunes en la flora local. En este sentido, la apreciación del paisaje durante los viajes de colección realizados para esta tesis, sugiere que la mayoría de las especies de Calyceraceae son abundantes a escala local y escasas a escala regional.

Además, existen especies muy localizadas, sólo conocidas por la colección tipo o por no más de dos colecciones a lo largo de toda la historia del taxón. Este es el caso de Boopis jürgensii, Boopis pusilla, Moschopis ameghinoi, Boopis itatiaiae, Boopis bupleuroides y Acicarpha obtusisepala. Este fenómeno podría explicarse por: 1) la existencia de microendemismos con áreas de distribución sumamente restringidas, 2) la inaccesibilidad de las áreas de distribución y su consecuente escasez de muestreo, 3) la alteración humana del área de distribución, con la consecuente pérdida de hábitat y diversidad, y la ausencia de registros asociada, 4) la explosión demográfica súbita de las poblaciones durante cortos periodos favorables, seguida por largos periodos de dormancia durante la época desfavorable.

3.5.2 Comentarios acerca de la distribución geográfica de los clados discutidos en la hipótesis filogenética

Las especeis que integran los clados 1 a 5, descriptos en el análisis cladístico, muestran coherencia en su distribución geográfica:

El clado 1 (B. anthemoides+Acicarpha) se encuentra ampliamente distribuido en la porción cisandina del Cono Sur, ocupando la llanura chaco-pampeana en toda su extensión, desde la costa atlántica hasta la cumbre del sur de los Andes peruanos. Sin embargo, queda excluido de Chile (Fig. 42). 
El clado 2 ("Glutinosas") asociado con M. leyboldi y B. gracilis (con sus dos variedades), se distribuye a lo largo del extremo sur del Dominio Andino-Patagónico, alternando entre la Provincia Altoandina y la Provincia Patagónica, en la mayoría de los casos circunscripto al área andina y sus adyacencias (a veces en áreas muy puntuales, muchos endemismos), siendo escasos los taxones que amplían su distribución hacia el oriente patagónico (B. gracilis var. lazulina, B. filifolia, B. patagonica).

A su vez, la mayoría de las especies del subclado caracterizado por la presencia de los gaps 15 y 16 como sinapomorfías (M. trilobata, M. rosulata, B. patagonica y $B$. filifolia) se encuentran distribuidas en el extremo sur de la Patagonia (pcia. de Santa Cruz) con la única excepción de $M$. subandina, que habita en la región del lago Vintter Carrenleufú (pcia. de Chubut), llegando hasta el sur de la pcia. de Neuquén. Por su parte, las especies restantes ( $M$. caleofuensis y $G$. dentata) sólo habitan en el área septentrional del área de distribución de las "Glutinosas" (Fig. 42).

El clado 3 ("Pubescentes") es exclusivo de los Andes chileno-argentinos centrales y cordones montañosos extraandinos asociados. La mayoría de sus taxones son exclusivos para zonas puntuales y algunos han sido registrados pocas veces, con grandes periodos de tiempo entre las colecciones (Boopis pusilla, Calycera horrida). Están asociados a zonas fitogeográficas con gran riqueza de endemismos (Provincia Chilena Central; Payunia) (Fig. 43).

A pesar de que el clado 4 no tiene un alto soporte (Jackknife), aún así sus taxones muestran correspondencia en la distribución geográfica. Ocupan el área andina de Argentina que se extiende desde Neuquén hasta el noroeste y el área de pedemonte y cadenas montañosas extraandinas asociadas a su ladera oriental. Excepcionalmente, un taxón (Calycera crassifolia) llega a distribuirse por la costa atlántica, dispersándose por los valles de los ríos Colorado y Negro (Fig. 43).

Asimismo, el clado 5 tampoco está soportado, pero la distribución geográfica de sus taxones también muestra coherencia. Ocupa un área similar a la del clado 2, con el que llega a superponerse, pero su alcance geográfico sobrepasa el límite subandino, llegando a distribuirse por la Patagonia en toda su extensión (Fig. 43). 


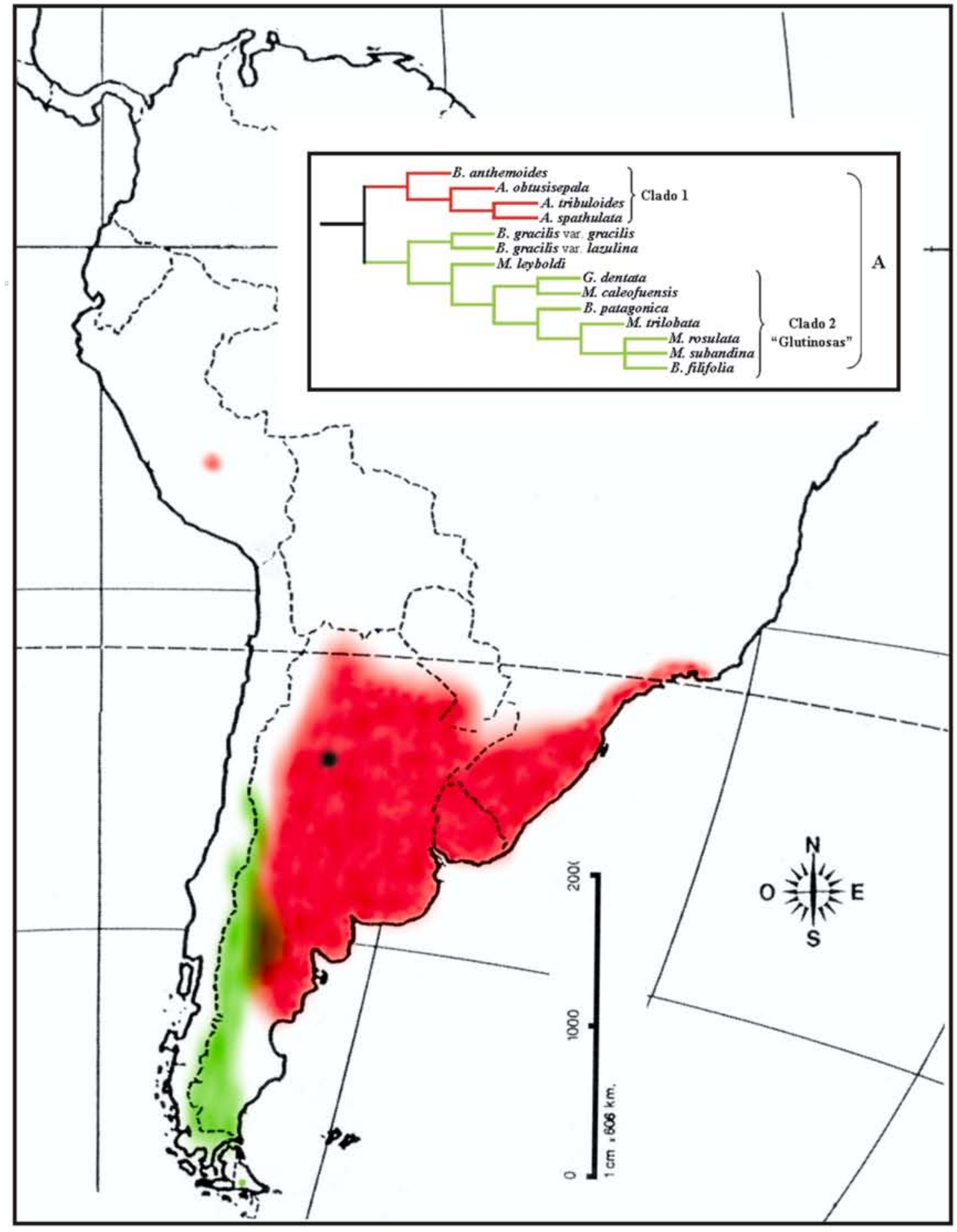

Figura 42. Mapa de distribución de los grupos que integran el clado A. En negro, distribución de Boopis castilloni (incertae sedis). 


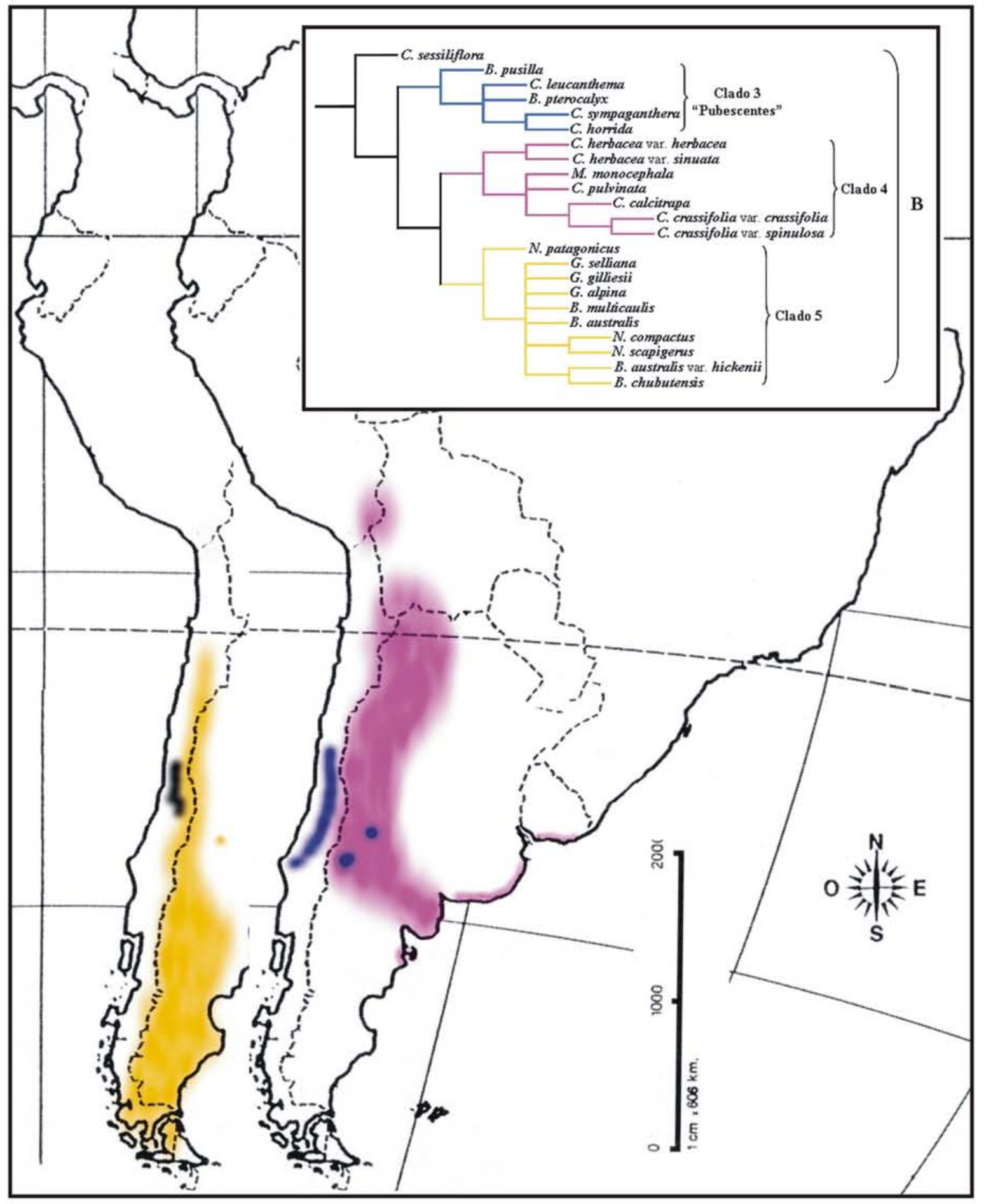

Figura 43. Mapa de distribución de los grupos que integran el clado B. En negro, distribución de Calycera sessiliflora. La porción occidental de Sudamérica se muestra repetida para una mejor visualización de las áreas superpuestas. 


\section{CONCLUSIONES}

Hipótesis 1) Los géneros de Calyceraceae son para- o polifiléticos: rechazada para Acicarpha, validada para el resto de los géneros.

- De los seis géneros tradicionalmente aceptados, sólo Acicarpha es monofilético; Boopis, Calycera, Gamocarpha, Moschopis y Nastanthus son grupos polifiléticos. Los resultados del análisis filogenético sugieren que son necesarios cambios profundos en la taxonomía de la familia, para delimitar nuevamente y enmendar los géneros que la integran.

Hipótesis 2) Los caracteres diagnósticos usados hasta ahora en taxonomía clásica para definir los géneros (estructura del fruto y la inflorescencia) son homoplásicos y no permiten definir grupos naturales: validada.

- Con excepción de la presencia de flores estaminadas en el centro de la inflorescencia y la forma columnar del receptáculo, que son sinapomorfías de Acicarpha, los restantes caracteres morfológicos usados tradicionalmente en la taxonomía de la familia son altamente homoplásicos y no permiten definir grupos naturales.

Hipótesis 3) Algunos caracteres florales no utilizados en la taxonomía tradicional de Calyceraceae y Asteraceae, como la posición de las glándulas nectarígenas, permiten definir a los principales linajes dentro de las Calyceraceae: validada.

- Se reconocen dos grandes grupos monofiléticos dentro de Calyceraceae, especies con glándulas nectaríferas estaminales (clado A) y especies con glándulas nectaríferas hipantiales (clado B).

- La nervadura de los sépalos posee cierta importancia filogenética ya que define grandes grupos de especies (Clado B excepto Calycera sessiliflora, y Clado $2+$ Boopis gracilis y Moschopis leyboldi).

Hipótesis 4) Algunos caracteres florales con valor taxonómico dentro de las Asteraceae, como la forma de la base y el ápice de las anteras, la brácteas del receptáculo y la morfología de la corola, también tienen valor taxonómico en las 
Calyceraceae: validada para la forma de la base de las anteras y las caudículas, rechazada para el ápice de las anteras, las brácteas del receptáculo, la proporción del hipanto y de la porción lobada de la corola (éstos dos últimos como indicadores de la estructura general de la corola).

\section{Conclusiones adicionales}

\section{Taxonómicas}

- Se reconocieron 47 nombres válidos de especies (4 de Acicarpha, 16 de Boopis, 9 de Calycera, 5 de Gamocarpha, 7 de Moschopis y 6 de Nastanthus) y 6 variedades (2 de Boopis gracilis, 2 de Calycera crassifolia y 2 de C. herbacea).

- Se describieron dos nuevas especies endémicas de Argentina: Boopis pterocalyx y Boopis necronensis.

- Se seleccionaron 21 lectotipos y un neotipo. Se lectotipificó un género (Anomocarpus). Se propuso una nueva combinación. Se establecieron 25 nuevos sinónimos, se enmendó una especie y se establecieron dos nombres dudosos.

- Se hallaron 35 nuevos ejemplares tipo nomenclaturales de Calyceraceae.

\section{Morfológicas}

- Se agrega como sinapomorfía de la familia Calyceraceae la estructura de la inflorescencia de tipo cefalodio y se confirma el tubo estaminal.

\section{Filogenéticas}

- Dentro del clado A se reconocen dos grupos monofiléticos: el clado 1, integrado por el género Acicarpha junto con su especie hermana Boopis anthemoides; y el grupo de plantas formado por las especies "Glutinosas" del clado 2 junto con su especie hermana Moschopis leyboldi y las dos variedades de Boopis gracilis (en su mayoría de distribución austral).

- Dentro del clado B se reconocen tres grupos monofiléticos: el grupo de especies "Pubescentes", que incluye especies de los géneros Boopis y Calycera (clado 3); el clado 4, que incluye a Moschopis monocephala y algunas especies del género Calycera; y el clado 5, que incluye varias especies de los géneros Nastanthus, Boopis y Gamocarpha. 
- Calycera sessiliflora se dispone como taxón basal del clado B, hermano del grupo monofilético formado por los clados 3,4 y 5.

\section{Biogeográficas}

- El clado 1 posee una distribución típicamente chaqueña, aunque se extiende hacia el Dominio Amazónico por el sur de Brasil (Provincias Paranaense y Atlántica) y el noroeste de Argentina (Provincia de las Yungas); y hacia el Dominio Andino-Patagónico por el sur de Argentina (Provincia Patagónica), donde se superpone con la distribución del grupo B. gracilis + M. leyboldi+clado 2.

- Existe un único registro aislado para el clado 1 en Perú, correspondiente a $A$. tribuloides.

- El grupo formado por B. gracilis+M. leyboldi+clado 2 posee una distribución típicamente andino-patagónica, aunque avanza sobre el Dominio Chaqueño por el sur de la Provincia del Monte, donde se superpone con el clado 1.

- Todos los subclados incluidos dentro del clado B poseen una distribución típicamente andino-patagónica, aunque el clado 4 se extiende hacia el Dominio Chaqueño por todo el oeste de Argentina, y especialmente por la Provincia del Monte (cuencas de los ríos Colorado y Negro), donde alcanza la costa atlántica. Por su parte, los clados 3 y 5 alcanzan a extenderse por la Provincia Subantártica (Dominio Subantártico, Región Antártica) a lo largo de sus zonas de contacto con la Provincia Chilena Central y con el Distrito Austral de la Provincia Altoandina, respectivamente. 


\section{PROYECCIONES}

Sobre la base de los resultados obtenidos en los análisis filogenéticos realizados y teniendo en cuenta la posición de las especies tipo de los géneros actuales dentro del cladograma combinado, se realizan comentarios acerca de una reasignación nomenclatural tentativa de los géneros de Calyceraceae.

El género Boopis podría quedar reducido a un taxón monotípico con su especie tipo (B. anthemoides), o bien extenderse a todo el grupo que actualmente se conoce como Acicarpha (clado 1, de mayor soporte), quedando este grupo reducido a un subgénero de Boopis. Sin embargo, para resolver este dilema nomenclatural, es preciso sumar a Boopis bupleuroides, B. itatiaiae y B. jürgensii al análisis combinado, especies estrechamente relacionadas con con ambos géneros, que representan valores intermedios de estados para los caracteres 24 y 25 (forma del receptáculo florífero y del receptáculo fructífero, punctiforme- columnar).

El género Moschopis (especie tipo: M. leyboldi) podría extenderse a todo el clado 2 ("Glutinosas") e incluir a Boopis gracilis; este clado no se encuentra bien soportado pero presenta una sinapomorfía morfológica (carácter 14: ausencia de nervadura central en los sépalos) y coherencia geográfica. Otra opción, dadas las diferencias morfológicas notables que distinguen a M. leyboldi de las restantes especies del caldo que integra (forma de vida geófita, ausencia de hojas glutinosas, ausencia de grupos cimosos externos, ausencia de involucro definido), es considerarlo un taxón monotípico, asignando un nuevo nombre genérico a las especies integrantes del clado 2 , con soporte, y ubicar a B. gracilis como un taxón incertae sedis.

Calycera sessiliflora es la especie basal del clado B y es la especie tipo de Anomocarpus, género que podría restablecerse como monotípico, caracterizado por: ápice de las anteras apiculado, tubo estaminal reducido, nervadura central de los sépalos presente, ausencia de brácteas del receptáculo, hipanto subnulo, grupos cimosos internos al involucro ausentes, y los cefalodios nutantes.

El clado 3, que agrupa a especies "Pubescentes", contiene a C. leucanthema. Entre los sinónimos de esta especie está Leucocera annua, especie tipo del género Leucocera, taxón que debería ser recategorizado a género y consecuentemente emendado. Este clado tiene soporte y presenta dos sinapomorfías morfológicas (caracteres 3 y 21). 
El clado 4 correspondería al género Calycera (contienen a la especie tipo: $C$. herbacea) con lo cual sólo restaría realizar la nueva combinación para Moschopis monocephala. Este clado no soportado presenta el carcter morfológico 33 como sinapomorfía.

Las especies integrantes del clado 5 quedarían posicionadas dentro del género Gamocarpha (contiene a la especie tipo G. poeppigii = G. alpina), que incluiría varias especies de los géneros Boopis y Nastanthus. Sin embargo, este clado no se recupera en el análisis molecular ni morfológico por separado y se hace evidente la necesidad de ampliar el muestreo de especies de Nastaanthus y Boopis para alcanzar una decisión acertada.

Para avanzar en la sistemática de la familia es necesario ampliar la evidencia morfológica y utilizar nuevos marcadores moleculares (incluyendo nucleares) que permitar obtener hipótesis más robustas sobre las relaciones de las especies. La anatomía del fruto, el análisis de caracteres polínicos [de los que se reconocen al menos dos grandes morfologías polínicas en la familia (Hansen, 1992)], así como la profundización en el estudio de los caracteres florales y de la inflorescencia, son referentes para la búsqueda de nuevos caracteres morfológicos que deberían tenerse en cuenta en estudios futuros. 


\section{BIBLIOGRAFÍA}

Avetisjan, E. M. 1980. Palynomorphology of the family Calyceraceae, in S. Zhilin (ed.), Systemtics and evolution of higher plants, pp. 57-64. Nauka: Leningrad.

Ball, J. 1886 [1884]. Contributions to the Flora of North Patagonia and the adjoining Territory. J. Linn. Soc., Bot. 21: 203-240.

Belem, C. I. F. 1977. Descriçao palinologica de especies dos generos Boopis e Acicarpha (Calyceraceae). Rev. Brasil Biol. 37(3): 611 - 614.

Bentham, G. \& J. D. Hooker. 1873. Genera plantarum ad exemplaria imprimis in herbariis kewensibus servata definita, Voluminis Secundi, Pars I, Sistens Dicotyledonum Gamopetalarum Ordines VI, Caprifoliaceas-Compositas. Londini: Lovell Reeve \& Co., Williams \& Norgate

Bremer, K. 1994. Branch support and tree stability. Cladistics 10: 295-304.

Cabrera, A. L. 1994. Regiones fitogeográficas argentinas (1ª reimpresión). Enciclopedia argentina de agricultura y jardinería 2(1):1-85. Buenos Aires: Editorial Acme S.A.C.I..

Cabrera, A. L. \& A. Willink. 1980. Biogeografía de América Latina, ed. 2. Secretaría General de la Organización de los Estados Americanos. Programa Regional de Desarrollo Científico y Tecnológico. Serie de Biología. Monografía no 13: 1-122.

Carlquist, S. \& M. DeVore. 1998. Wood anatomy of Calyceraceae with reference to ecology, habit, and systematic relationships. Aliso 17 (1): 63-76.

Carolin, R. C. 1967. The concept of the inflorescence in the order Campanulales. Proc. Linn. Soc. N. South Whales 92: 7-26.

Cavanilles, A. J. 1797. Icones et descriptiones plantarum quae aut sponte in Hispania crescunt aut in hortis hospitantur, vol 4. Madrid: Ex Regia Typographia.

Chiapella, J. 1999a. Calyceraceae, en M. N. Correa (ed.), Flora Patagónica. Colección Científica del INTA 8(6): 492-517.

Chiapella, J. 1999b. Calyceraceae, en F. O. Zuloaga \& O. Morrone (eds.), Catálogo de las Plantas Vasculares de la República Argentina II, Acanthaceae-Euphorbiaceae (Dicotyledoneae). Monogr. Syst. Bot. Missouri Bot. Gard. 74: 490-495.

De Candolle, A. P. 1836. Prodromus Systematis Naturalis Regni Vegetabilis, sive enumeratio contracta ordinum, generum, specierumque plantarum huc usque cognitarum, juxta methodi naturali normas digesta; Pars Quinta: Sistens 
Calycereas et Compositarum tribus priores. Parisiis: Sumptibus Sociorum Treuttel et Würtz, viâ dictâ de Lille, $n^{\circ} 17$. Venitque in eorumdem bibliopolio, Argentorati.

DeVore, M. 1994. Systematic studies of Calyceraceae. Ph. D. Thesis, Ohio State University, Columbus, $\mathrm{OH}$.

Domeyko, I. 1859. Publicaciones de algún interés hechas en Alemania i en Francia sobre la jeografia, jeolojia, historia natural e industria minera de América i especialmente de Chile. Anales Univ. Chile 16: 426-469.

Doyle, J. J. \& J. L. Doyle. 1987. A rapid DNA isolation procedure for small quantities of fresh leaf tissue. Phytochemical Bulletin 19: 11-15.

Edgar, R. C. 2004. MUSCLE: multiple sequence aligment with high accuracy and high throughput. Nucl. Acids Res. 32(5): 1792-1797.

Erbar, C. 1993. Studies on the floral development and pollen presentation in Acicarpha tribuloides with a discussion of the systematic position of the family Calyceraceae. Bot. Jahrb. Syst. 115(3): 325-350.

Erbar, C. \& P. Leins. 1995. Portioned pollen release and the syndromes of secondary pollen presentation in the Campanulales-Asterales-complex. Flora 190: 323-338.

Farris, J. S. 1983. The logical basis of phylogenetic analysis, en N. Platnick \& V. Funck (eds.), Proceedings of the Second Meeting of the Willi Hennig Society. Advances in Cladistics 2, pp. 1-47. New York: Columbia Univ. Press.

Farris, J. S. 1989. The retention index and the rescaled consistency index. Caldistics 5: 417-419.

Farris, J. S., V. A. Albert, M. Källersjö, D. Lipscomb \& A. G. Kluge. 1996. Parsimony jackknifing outperforms neighbor-joining. Cladistics 12: 99-124.

Galvão Magenta, M. A. \& J. R. Pirani. 2002. Calyceraceae, en M. G. L. Wanderley, G. J. Shepherd, A. M. Giulietti, T. Sant'Ana Melhem, V. Bittrich \& C. Kameyama (eds.), Flora Fanerogamica do Estado de São Paulo, vol. 2, pp. 67-69. São Paulo: FAPESP, Editora HUCITEC.

Goloboff, P., J. Farris \& K. Nixon. 2003. T.N.T.: Tree Analysis using new Technology. Cladistics 24: 774-786 (program and documentation, available from the authors and at http://www.zmuc.dk/public/phylogeny)

Gray, A. 1861. Five Hundredth Meeting. November 13, 1861. Statute Meeting; Notes upon a Portion of Dr. Seemann's Recent Collection of Dried Plants Gathered in the Feejee Islands; Characters of New or Obscure Species of Plants of Monopetalous 
Orders in the Collection of the United States South Pacific Exploring Expedition under Captain Charles Wilkes, U. S. N. with Occasional Remarks, \&c. Proc. Amer. Acad. Arts Sci. Vol. 5(May 1860 - May 1862): 313-352.

Grisebach, A. H. R. 1854. Systematische Bemerkungen über die Beiden Ersten Pflanzensammlungen Philippi's und Lechler's im Südlichen Chile und an der Maghellans-strasse. Göttingen

Gustafsson, M. H. G. 1995. Petal venation in the Asterales and related orders. Bot. J. Linn. Soc. 118: 1-18.

Gustafsson M. H. G. \& K. Bremer. 1995. Morphology and phylogenetic interrelationships of the Asteraceae, Calyceraceae, Campanulaceae, Goodeniaceae, and related families (Asterales). Amer. J. Bot. 82(2): 250-265.

Hall, T. A. 1999. BioEdit: a user-friendly biological sequence aligment editor and analysis program for Windows 95/98/NT. Nucl. Acids Symp. Ser. 41: 95-98.

Hansen, H.V. 1992. Studies in the Calyceraceae with a discussion of its relationship to Compositae. Nordic J. Bot. 12(1): 63-75.

Hansen, H. V. 1997. Studies in the Goodeniaceae and the Brunoniaceae with a discussion of their relationship to Asteraceae and Calyceraceae. Nordic J. Bot. 17 (5): 495-510.

Hardwicke, R. 1867. The miscellaneous botanical works of Robert Brown, Esq., D. C. L., F. R. S., foreign associate of the Academy of Sciences of the Institute of France, etc. Vol. 2, containing systematic memoires and contributions to systematic works. London: Ray Society by Robert Hardwicke, 192, Piccadilly.

Hauman-Merck, L. L. 1918. La végétation des hautes Cordillières de Mendoza (Suite et fin). Anales Soc. Ci. Argent. 86: 225-348.

Hellwig, F. H. 2007. Calyceraceae, en K. Kubitzki, J. W. Kadereit \& C. Jeffrey (eds.), The families and genera of vascular plants. Flowering plants - Eudicots. Asterales. Vol. 8, pp. 19-25. Berlin Heidelberg: Springer-Verlag.

Hicken, C. M. 1919. Calyceracearum Argentinarum Catalogus. Catálogo de las caliceráceas argentinas. Prim. Reun. Nac. Soc. Argent. Cien. Nat. [Tucumán, 1916]: 238-253.

Hieronymus, G. H. E. W. 1881. Sertum Sanjuaninum ó descripciones y determinaciones de plantas fanerógamas y criptógamas vasculares recolectadas por el Dr. D. Saile Echegaray en la Provincia de San Juan. Bol. Acad. Nac. Ci. (Córdoba) 4(1): 1-73. 
Hoffmann, A., M. K. Arroyo, F. Liberona, M. Muñoz \& J. Watson. 1998. Plantas altoandinas en la flora silvestre de Chile. Santiago de Chile: Ed. Fundación Claudio Gay.

Inoue, N. \& H. Tobe. 1999. Integumentary studies in Menyanthaceae (Campanulales sensu lato). Acta Phytotaxonomica et Geobotanica 50: 75-79.

Jussieu, A. L. 1803. Mémoire sur l'Acicarpha et le Boopis, deux genres nouveaux de plantes de la famille des Cinarocéphales. Ann. Mus. Natl. Hist. Nat. 2: 345-350.

King R. M. \& H. W. Dawson. 1975. Cassini on Compositae collected from the Dictionnaire des Sciences Naturelles. Vol. 1. New York: Oriole Editions.

Leins, P. \& C. Erbar. 1990. On the mechanisms of secondary pollen presentation in the Campanulales-Asterales-Complex. Bot. Acta 103: 87-92.

Leins, P. \& C. Erbar. 2010. Flower and Fruit. Stuttgart: Schweizerbar.

Lessing, C. F. 1831a. De plantis in expeditione speculatoria Romanzoffiana. Observatis disserere pergunt AD. de Chamisso et D. de Schlechtendal. Synanthereae Rich. (continuatio). Linnaea 6: 209-260.

Lessing, C. F. 1831b. De plantis in expeditione Romanzoffiana. Observatis disserere pergitur. Calycereae R. Br. Linnaea 6: 527-528.

Lundberg, J. 2009. Asteraceae and relationships within Asterlaes, en V.A. Funk, A. Susanna, T.F. Stuessy \& R.J. Bayer (eds.), Sistematics, evolution and biogeography of Compositae. Capítulo 10, pp. 157-169. Viena: International Association for Plant Taxonomy (IAPT).

Lundberg, J. \& K. Bremer. 2003. A phylogenetic study of the order Asterales using one morphological and three molecular data sets. Int. J. Plant. Sci. 164(4): 553-578.

McNeill, J.; F. R. Barrie, W. R. Buck, V. Demoulin, W. Greuter, D. L. Hawksworth, P. S. Herendeen, S. Kanpp, K. Marhhold, J. Prado, W. F. Prud'homme van Reine, G. F. Smith, J. H. Wiersema \& N. J. Turland. 2012. International Code of Nomenclature for algae, fungi and plants (Melbourne Code): adopted by the Eighteenth International Botanical Congress Melbourne, Australia, July 2011. Regnum Vegetabile 154. Königstein: Koeltz Scientific Books.

Meyen, F. J.F. 1834. Reise um die Erde ausgefürt auf them Königlich preussischen Seehandlungs-Schiffe Prinzess Louise, commandirt von Capitain W. Wendt, in den Jahren 1830, 1831 und 1832. Vol. 1. Berlin. 
Miers, J. 1860-1869. Contributions to botany, iconographic and descriptive, detailing the characters of plants that are either new or imperfectly described; to which are added remarks on their affinities. Vol. 2. London: Williams \& Norgate.

Moore, D.M. 1967. Further records for the vascular flora of the Falkland Islands. Bot. Not. 120: 17-25.

Müller, C. F. 1885. Calyceraceae, en C. F. P. S. Martius, Flora Brasiliensis. Vol. 6. London: Lovell Reeve.

Muñoz-Pizarro, C. 1960. Las especies de plantas descriptas por R. A. Philippi en el siglo XIX. Estudio crítico en la identificación de sus tipos nomenclaturales. Santiago de Chile: Ediciones de la Universidad de Chile.

Muradian, L. G. 1991. Carpologic character of Calyceraceae family (en ruso). Flora, rastitel'nost i rastit -el'nye resursy armenii, Sborn. Nauch. Trud. 13: 85-95.

Nixon, K. C. \& J. M. Carpenter. 1993. On outgroups. Cladistics 9: 413-426.

Pesacreta, T. C., V. I. Sullivan \& M. DeVore. 1994. The connective base and filament of Acicarpha tribuloides (Calyceraceae). Amer. J. Bot. 81(6): 753-759.

Philippi, R. A. 1856. Plantarum novarum Chilensium. Centuria tertia. Linnaea 28: 705752.

Philippi, R. A. 1860. Viage al Desierto de Atacama hecho de orden del gobierno de Chile en el verano 1853-54. Halle en Sajonia: Libreria de Eduardo Anton.

Philippi, R. A. 1861. Observaciones botánicas sobre algunas plantas recogidas en Chile por los señores don Ricardo Pearce i don Jerman Volckmann. Anales Univ. Chile 18: 43-69.

Philippi, R. A. 1862a. Viaje a los baños y al nuevo volcán de Chillán por don R. A. Philippi. Tercera Parte. Anales Univ. Chile 21(2): 377-389.

Philippi, R. A. 1862b. Sertum Mendocinum. Catálogo de las plantas recojidas cerca de Mendoza i en el camino entre ésta i Chile por el portezuelo del Portillo, por don Wenceslao Diaz en los años de 1860 i 1861. Anales Univ. Chile 21(2): 389-407.

Philippi, R. A. 1864-1865. Plantarum novarum Chilensium. Centuriae inclusis quibusdam Mendocinis et Patagonicis. Linnaea 33: 1-308.

Philippi, R. A. 1865. Excursión botánica en Valdivia desde los Cuncos en el departamento de la Unión, a través de la Cordillera de la Costa, hasta el mar, por Federico Philippi i descripción de las especies nuevas de plantas halladas en ella por Rodolfo Amando Philippi. Anales Univ. Chile 27(3): 289-351. 
Philippi, R. A. 1870. Sertum Mendocinum alternum, o sea, catálogo de las plantas recojidas cerca de Mendoza y en los caminos que conducen de Chile a esa ciudad. Anales Univ. Chile 36: 159-212.

Philippi, R. A. 1872. Descripción de las plantas nuevas incorporadas últimamente en el herbario chileno, por el doctor don R. A. Philippi. Anales Univ. Chile 41(1): 663746.

Philippi, R. A. 1891. Catalogus praevius plantarum in itinere ad Tarapaca a Friderico Philippi lectarum. Anales Mus. Nac., Santiago de Chile, Sección 2 (Botánica), Entrega 8: 1-96 + 2 lám.

Philippi, R. A. 1894. Plantas nuevas chilenas de las familias que corresponden al tomo III de la obra de Gay (Continuación). Anales Univ. Chile 85: 813-844.

Pilger, R. 1931. Eine neue Art von Boopis aus Südbrasilien. Notizbl. Bot. Gat. Berlin 11: $101-102$.

Pontiroli, A. 1963. Flora argentina. Calyceraceae. Revista Mus. La Plata (N. S.) 9 (Bot. 4): $175-241$.

Pontiroli, A. 1993. Calyceraceae, en A. L. Cabrera (ed.), Flora de la provincia de Jujuy. Colección Científica del INTA 12(9): 537-545.

Pozner, R., C. Zanotti \& L. A. Johnson. 2012. Evolutionary origin of the Asteraceae capitulum: insights from Calyceraceae. Amer. J. Bot. 99 (1): 1-13.

Reiche, C. 1900. Estudios críticos sobre la flora de Chile (continuación). Anales Univ. Chile 106: 965-1048.

Reiche, C. 1900 [1901]. Beiträge zur Systematik der Calyceraceen. Bot. Jahrb. Syst. 29: 107-119, Taf. 1.

Reiche, C. 1902. Flora de Chile. Tomo 3. Familias 47; 50-59. Santiago de Chile: Imprenta, litografía i encuadernación Barcelona.

Reitz, R. 1988. Caliceráceas, en R. Reitz (ed.), Flora ilustrada Catarinense. I Parte. Monografia. As plantas, pp. 1-18. Itajaí: Herbário "Barbosa Rodrigues".

Rémy, E. J. 1847 [1848]. Calicereas, en C. Gay (ed.), Historia física y política de Chile según documentos adquiridos en esta República durante doce años de residencia en ella. Botánica. Tomo 3. Paris: Imprenta de Fain y Thunot.

Richard, L. C. M. 1820. Mémoire sur une famille de plantes dites les Calycérées. Mém. Mus. Hist. Nat. 6: 28-82. 
Roque, N.; D. J. Keil \& A. Susanna. 2009. Illustrated glossary of Compositae, en V. A. Funk, A. Susanna, T. F. Stuessy \& R. J. Bayer (eds.), Systematics, Evolution and Biogeography of Compositae, pp.781-806. Austria: International Association for Plant Taxonomy.

Ruiz, H. \& J. Pavón. 1798. Flora peruviana, et chilensis, sive descriptiones, et icones plantarum peruvianarum, et chilensium, Secundum Systema Linnaeanum digestae, cum characteribus plurium generum evulgatorum reformatis, Tomus I. Typis Gabrielis de Sancha.

Simmons, M. P. \& H. Ochoterena. 2000. Gaps and characters in sequence-based phylogenetic analyses. Syst. Biol. 49(2): 369-381.

Skvarla, J. J., B. L. Turner, V. C. Patel \& T. A. Spencer. 1977. Pollen morphology in the Compositae and in morphologically related families, en V. H. Heywood, J. B. Harborne \& J. B. Turner (eds.), The Biology and Chemistry of the Compositae I, pp. 141-248. London: Academic Press.

Stafleu, F. A. \& R. S. Cowan. 1988. Taxonomic Literature. A selective guide to botanical publications and collections with dates, commentaries and types, 2nd edition. Volume 7. Bohn, Scheltema \& Holkema, Utrecht/ Antwerpen dr. W. Junk b.v., Publishers, The Hague/ Boston.

Stapf, O. 1929 [1932]. Acarpha laciniata. South America. Curtis's Bot. Mag. 155: Tab. 9272.

Thiers, B. [de actualización permanente, consulta 2013]. Index Herbariorum: a global directory of public herbaria and associated staff. New York Botanical's Virtual Herbarium, http://sweetgum.nybg.org/ih

Troll, W. 1964. Die Infloreszenzen: Typologie uns Stellung im Aufbau des Vegetationskörpers. Stuttgart: Gustav Fischer.

Turczaninow, N. 1848. II. Decades quarta et quinta generum adhuc non descriptorum. Flora, Neue Reihe 6 (44): 707-716.

Weddell, H. A. 1857 [1858]. Chloris Andina. Essai d'une flore de la région alpine des Cordillères de l'Amerique du Sud. Vol. 2. Paris: P. Bertrtand.

Zanotti, C. \& R. Pozner. 2007. Valor diagnóstico de la estructura del fruto de Boopis y Nastanthus (Calyceraceae). XXXI Jornadas Argentinas de Botánica, Corrientes. Bol. Soc. Argent. Bot. 42 (supl.): 142. 
Zanotti, C. A. \& R. E. Pozner. 2008. Calyceraceae, en F. O. Zuloaga, O. Morrone \& M. J. Belgrano (eds.), Catálogo de las plantas vasculares del Cono Sur (Argentina, sur de Brasil, Chile, Paraguay y Uruguay). Monogr. Syst. Bot. Missouri Bot. Gard. 107: $1844-1853$.

Zavala-Gallo, L., S. Denham \& R. Pozner. 2010. Revision of Nastanthus (Calyceraceae). Gayana Bot. 67 (2): 158-175.

Zavala-Gallo, L., S. Denham \& R. Pozner. 2011. Two new species of Boopis (Calyceraceae) from Argentina. Brittonia 63 (1): 113-117. 